\title{
21st Annual Congress ESGE September 11-14, 2012, Paris
}

107

\author{
A RARE CASE OF BILATERAL LIVE ECTOPIC PREGNANCY \\ Case Reports
}

A. Khali1*, R. Karmarkar, A. Sohail
${ }^{[1]}$ North Hampshire Hospital $\sim$ Basingstoke $\sim$ United Kingdom

Summary (4 lines): We present a case of bilateral live ectopic pregnancy after IVF treatment. Both fetuses showed cardiac activity on scan. The patient underwent bilateral salpingectomy to treat the condition.

Introduction: Ectopic pregnancy is known to be occurring more often after IVF and related assisted conception techniques. Rate of bilateral ectopic pregnancy is about 1 in 200,000. To the best of our knowledge this is the first reported case of bilateral live ectopic pregnancy.

A 34 year old women presented at five plus weeks after her IVF treatment. She had no risk factors for ectopic pregnancy and had unexplained subfertility. She attended for a dating and viability scan. She was completely asymptomatic. Trans-vaginal ultrasound scan revealed bilateral live ectopic pregnancy. Treatment options were discussed with the patient. She chose surgical option and underwent bilateral salpingectomy on the same day. She had an uneventful recovery.

Material and Methods: Written consent obtained from the patient. Results: Patient underwent laparoscopic bilateral salpingectomy.

Discussion: We used the surgical method of laparoscopic bilateral salpingectomy to treat bilateral live ectopic pregnancies. As the patient was having IVF treatment, it was felt that her chances of achieving a successful intrauterine pregnancy would be higher with the damaged tubes removed.

There was however, discussion about methotrexate, which was dropped as both fetuses showed cardiac activity and due to patient's wishes.

\section{4}

LAPAROSCOPIC UTERINE ANASTOMOSIS FOR TRAUMATIC SEPARATION OF CERVIX FROM THE UTERINE CORPUS CAUSED BY CLOSED PELVIC FRACTURE. A CASE REPORT AND LITERATURE REVIEW

Case Reports

S. Yao*

${ }^{[1]}$ The first affiliate hospital of Sun yat-sen university $\sim$ Guangzhou China
Summary (4 lines): Laparoscopic uterine anastomosis for traumatic separation of cervix from the uterine corpus caused by closed pelvic fracture was reported. The young girl resume a normal menstruation after surgery.

Introduction: The purpose of this case report is to describe the diagnosis and successful laparoscopic approximation of traumatic separation of the cervix from the uterine corpus.

Material and Methods: A 16-years girl with primary amenorrhea and cyclic abdominal pain was referred to our hospital. She had a car accident at the age of 2 years which resulted in closed pelvic fracture. A conservative management of pelvic fracture was given to her with a uneventful recovery. She has a cyclic abdominal pain in the past 3 years since the age of 13 . Ultrasonography and laparoscopy examination find a complete separation of the uterine corpus from the cervix and a endometrioma in the left ovary. The uterine corpus was approximated to the cervix with circumferentially placed sutures under laparoscopy. The endometrioma was also resected concomitantly.

Results: The cyclic menstruation resume 2 months after surgery. cyclic abdominal pain disappear after surgery.

Discussion: This case demonstrates the successful laparoscopic approximation of traumatic separation of the uterine corpus from the cervix which result primary amenorrhea. Normal menstruation can be resumed after surgery.

\section{4}

\section{A REVIEW OF MAJOR COMPLICATIONS ASSOCIATED WITH 832 OPERATIVE GYNAECOLOGICAL LAPAROSCOPIES Complications}

K. Niblock*, K. Johnston, D. Morgan, G. Mccracken

${ }^{[1]}$ Antrim Area Hospital $\sim$ Antrim, N.Ireland $\sim$ United Kingdom

Summary (4 lines): A large cohort of operative laparoscopic cases including a large number of advanced procedures demonstrating incidence, nature and management of major complications.

Introduction: Operative laparoscopic surgery is now proliferating in volume and complexity in the United Kingdom. With experienced, trained surgeons this approach offers well established benefits with very low major complication rates.

Material and Methods: Review of 832 operative laparoscopies from 2 centres over 4 yrs by 3 trained surgeons. Including 279 laparoscopic adnexal surgeries, 201 total laparoscopic hysterectomies, 135 laparoscopic excisions of endometriosis and, 63 pelvic floor repairs. Complications diagnosed intra and postoperatively were analysed. 
Results: Major complications namely visceral, ureteric, vascular injury and return to theatre occurred in $1.56 \%$ (13/832). Injuries were $4 / 832(0.5 \%)$ bowel, $1 / 832(0.1 \%)$ vascular, $1 / 832(0.1 \%)$ ureteric, 4/832 (0.4\%) bladder and 4/832 (0.5\%) return to theatre. Most 10/13 (77 \%) were recognised intra-operatively and all managed laparoscopically.

Discussion: Operative gynaecological laparoscopy is now well established; with appropriately trained surgeons it is associated with a very low major complication rate.

292

\section{EARLY CERVICAL CANCER: LAPAROSCOPIC APPROACH \& MAJOR COMPLICATIONS \\ Complications}

C. De Valle Corredor*, L. Fernandez Munoz, V. Garcia Pineda, M.D. Rodriguez Garnica, P. Valenzuela Ruiz, A. Couso Gonzalez, A. Zapico Goñi

${ }^{[1]}$ Hospital Universitario Principe de Asturias $~$ Alcalá de Henares $~$ Spain

Summary (4 lines): Laparoscopy (LPS) has shown to be a feasible alternative to laparotomy (LPM) in early stage cervical cancer (ESCC) with low complications rate

Introduction: Laparoscopic radical hysterectomy + pelvic lymphadenectomy $(\mathrm{RH}+\mathrm{PL})$ is the standard approach for ESCC. LPS is done on our patients to reduce morbidity.

Material and Methods: Retrospective \& descriptive study of 57 patients with ESCC (IA1, IA2, IB1, IIA1) and operated by LPS in our hospital between 2000-2011. Surgery outcomes and major complications are analyzed.

Results: The mean age were $49.2+-12,6(26-82)$ years. 51 $(89,5 \%)$ patients were scheduled for RH + PL: 44 (87,3 \%) had RH + PL and in 7 (12,7\%), pelvic nodes were positive in intraoperative frozen section whereby paraortic lymphadenectomy (PALN) was performed.

RH + PL had a mean hospital stay of 4,77 $\pm 2,101(2-11)$ days and its major surgical complications (MSC) were $2(4,5 \%)$ great vessel injuries (GVI) solved laparoscopically and 1 (2,2\%) GVI + ureteral lesion which required conversion into LPM.

No MSC were seen in PALN and its mean hospital stay was $2,14 \pm$ $0,378(2-3)$ days.

Major postoperative complications (MPC) happened in 3 cases $(6,8 \%)$ in RH + LP group [1 (33\%) intestinal injury which required colostomy on 5 th day postsurgery, 1(33,3\%) vesicovaginal fistula, $1(33,3 \%)$ ureteral estenosis after 2 months]. No MPC were seen in PALN group. Discussion: LPS is an excellent approach for treatment and staging with little morbidity and complications.

\section{2}

\section{HOW TO PREVENT TROCAR-RELATED COMPLICATIONS:} TROCAR USE AND FASCIAL CLOSURE

Complications

C. La Chapelle*, F.W. Jansen

${ }^{[1]}$ dept. of gynecology, Leiden University Medical Centre $\sim$ Leiden $~$ Netherlands

Summary (4 lines): As part of development of a multidisciplinary guideline on Minimally Invasive Surgery (MIS), we formulated evidence based recommendations for trocar use and fascial closure of trocarsites.
Introduction: Trocars are the most named in malpractice injury claims associated with MIS. Differences in trocar related complications (TRCs) may be attributable to different trocar types and fascial closure. Problem analysis for a guideline on MIS, revealed clinical heterogeneity in trocar use and fascial closure.

Material and Methods: A literature review of trocar use and fascial closure was performed. Conclusions were drawn, considerations discussed and multidisciplinary recommendations formulated.

Results: A meta-analysis of RCTs concluded that the use of radially expanding access (REA) vs cutting trocars leads to fewer trocar site bleedings (TSB). For secondary ports the use of REA vs cutting resulted in decreased postoperative pain. A retrospective study showed non-cutting vs cutting trocars lead to fewer TSBs and trocar site herniation (TSH). No studies showed a reduced risk for TSH after fascial closure. Two retrospective studies found increased prevalence of TSH in $12 \mathrm{~mm}$ vs $10 \mathrm{~mm}$ ports.

Evidence was balanced with other considerations

Discussion: To reduce the risk for TRCs, we recommend to -Use non-cutting trocars for $10-12 \mathrm{~mm}$ ports.

-Close the fascia of trocar ports $>10 \mathrm{~mm}$. Fascial closure of smaller ports could be considered.

\section{7}

\section{LAPAROSCOPIC EXCISION OF RV NODULES - SURGICAL OUTCOMES AND COMPLICATIONS. THE ADVANTAGES OF A COMBINED APPROACH.}

Complications

A. Kent*, T. Rockall
${ }^{[1]}$ MATTU $\sim$ Guildford $\sim$ United Kingdom

Summary (4 lines): A combined surgical approach was developed for the surgical management of severe DIE of the bladder, ureter and RV septum.

Introduction: We present the operative outcomes and complications of the combined surgical practice of a single Gynaecologist (Kent) and Colorectal Surgeon (Rockall), the two surgeons operating together. Surgery was carried out in two stages with down regulation. Outcomes were rates of laparoscopy; laparotomy; conservative surgery; clearance surgery; bowel resection; disc resection; rectal shaves; colostomy rates and complications. Material and Methods: Prospective study of 201 patients undergoing resection of bladder, ureteric, RV nodules, or combinations thereof.

The aim was to remove visible/palpable nodules. The energy of choice was Harmonic. The decision to carry out segmental or disc resection was made at the time of surgery.

Results: $(\mathrm{n}=201)$

Laparoscopy $96.0 \%(193)$

Laparotomy $4.0 \%(8)$

Conservative $69.5 \%(140)$

Clearance $30.5 \%(61)$

Resection $52.5 \%$ (105)

Disc $7.0 \%(14)$

Shave $40.5 \%(82)$

'ostomy $1.0 \%$ (2)

Bowel surgery post op cpx $3.5 \%$

Total major post op cpx $6.0 \%$

Discussion: A combined surgical two stage approach achieves a $96 \%$ laparoscopy rate to treat severe DIE regardless of whether bowel, ureteric or bladder resection is required. Requirement for defunctioning 'ostomy was under $1 \%$. There were no intra operative complications and no compromise in terms of major postoperative complication rates. 
23

\section{PREVENTION AND TREATMENT OF COMPLICATIONS OF OPERATIONS PROLIFT - MULTICENTER ANALYSIS OF OVER 1000 TRANSACTIONS \\ Complications}

A. Popov*, K. Abramyan, I. Krasnopolskaya, A. Fedorov, B. Slobodeniaouk, M. Ramazanov

${ }^{[1]}$ Moscow regional institute $O \mid G \sim$ Moscow $\sim$ Russian Federation

Summary (4 lines): operation with Prolift causes 20,2\% of general surgical and mesh-associated complications.

Introduction: One of the main arguments of opponents to use synthetic materials gives information about the large number of complications compared with traditional reconstructive operations.

Material and Methods: for the analysis of complications performed a multicenter retrospective study included 1131 patients with prolapse stage II-IV classification POP-Q, All patients were performed a colpopexy using PROLIFT sets at 2005-2011, including Prolift ant - 364 $(32,2 \%)$, post $-130(11,4 \%)$, total $-384(34 \%)$ ant \& post- 253 $(22,4 \%)$. All complications were divided into 2 groups:- general surgical and mesh-associated.

Results: Incidence of complications was $20.2 \%$. General surgical was $10.9 \%$, (urethral injury $0.2 \%$, bladder injury $1.4 \%$, injured ureter $0.08 \%$, rectal injury in $0.7 \%$, bleeding $1.9 \%$, hematoma $5 \%$, urinary retention $1.6 \%$. Mesh-associated complications were $9.3 \%$, which we attributed the formation of adhesions in the vagina $0.08 \%$, implant infection $0.7 \%$ of cases, implant shrinking $0.7 \%$ of cases, vaginal erosion $5.8 \%$ and erosion of the bladder, urethra-vaginal fistula $0.08 \%$ cases, pain in the perineum and buttocks $1.9 \%$.

Discussion: operation with Prolift causes 20,2 \% of general surgical and mesh-associated complications. Prolift operation cannot be recommended as an operation of choice for all forms of genital prolapse.

\section{5}

\section{REGRET FOLLOWING HYSTEROSCOPIC TUBAL} STERILIZATION: RETROSPECTIVE COHORT STUDY. Complications

L. Guillaume*, V. Marion, F. Hervé

${ }^{[1]}$ Hopital de Bicêtre - Assistance Publique des Hopitaux de Paris $\sim$ Le Kremlin Bicêtre $\sim$ France

Summary (4 lines): The regret rate after tubal hysteroscopic surgery using Essure procedure is a rare event occuring in less than $4 \%$.

Introduction: Women who undergo sterilization may later regret this decision. The regret rate after tubal hysteroscopic sterilization has not been evaluated yet. Our aim is to evaluate this regret after Essure procedure.

Material and Methods: Retrospective monocentric study from June 2002 to December 2010. All patients with hysteroscopic tubal sterilization with Essure microinserts were included. Patients with medical indication of sterilization and patients treated prior to IVF procedure in case of hydrosalpinx were excluded. All patients were sent a specific questionnaire to evaluate the regret.

Results: At all, 452 hysteroscopic tubal sterilizations were performed in our center. Three hundred seven patients $(68 \%)$ completed the questionnaire. Mean age at the procedure was 42.1 years. Mean follow-up was 43 months. Twelve patients over the 307 (3.91\%) expressed regret having the surgery. Seven patients $(2.28 \%)$ consulted in order to envisage a new pregnancy. Five patients $(1.63 \%)$ have thought to IVF procedure and $7(2.28 \%)$ have thought to adoption.
Finally, none of them have resorted to an IVF or to an adoption procedure.

Discussion: The regret rate after tubal hysteroscopic sterilization is a rare event (less than $4 \%$ ). Patient's selection and surgeons' experience may have influenced these results.

76

ARE DIGESTIVE SYMPTOMS IN WOMEN PRESENTING WITH PELVIC ENDOMETRIOSIS SPECIFIC TO LESION LOCALISATIONS? A PRELIMINARY PROSPECTIVE STUDY Endometriosis: Diagnosis

R. Horace*, N. Julie, S. Celine, S. Guillaume, G. Guillaume

${ }^{[1]}$ University Hospital $\sim$ Rouen $\sim$ France

Summary (4 lines): There is a high prevalence of digestive complaints in pelvic endometriosis sparing the rectum.

Introduction: We compared digestive symptoms in patients with different localisations of pelvic endometriosis and checked specific symptoms related to rectal stenosis.

Material and Methods: Prospective recording of data was done for women managed for stage 1 endometriosis, deep endometriosis, and deep endometriosis infiltrating the rectum. Patient characteristics and data from preoperative standardized questionnaires GIQLI, KESS and SF-36 were compared.

Results: 21, 42 and 53 women were included the three groups. The values of total KESS and total GIQLI score were comparable. Women presenting with rectal endometriosis were more likely to present cyclic defecation pain $(67.9 \%)$, cyclic constipation $(54.7 \%)$, and a significantly longer stool evacuation time, although these complaints were also frequent in other groups Among women with rectal endometriosis, $26.4 \%$ presented rectal stenosis and were more likely to report constipation, defecation pain, longer evacuation time and increased stool consistency.

Discussion: The high prevalence of digestive complaints in women presenting with pelvic endometriosis sparing the rectum suggests that digestive symptoms would be the consequence of cyclic inflammation and irritation of the digestive tract, rather than to actual infiltration of the rectum by the disease itself.

321

EPAQ-MPH: PATIENT EXPERIENCE OF AN ELECTRONIC QUESTIONNAIRE FOR THE ASSESSMENT OF MENSTRUAL, PAIN \& HORMONAL DISORDERS

Endometriosis: Diagnosis

M. Connor*, E. James, S. Radley

${ }^{[1]}$ Royal Hallamshire Hospital, Sheffield Teaching Hospitals $\sim$ Sheffield $\sim$ United Kingdom

Summary (4 lines): An electronic questionnaire for women with menstrual, pain $\&$ hormonal problems has undergone initial validation $\&$ psychometric testing.

Introduction: This study evaluates the psychometric properties of ePAQ-MPH (electronic Personal Assessment QuestionnaireMenstrual, Pain, Hormonal) a web-based instrument for the assessment and monitoring of outcomes in clinical practice.

Material and Methods: ePAQ-MPH was completed by 308 women. Factor analysis was undertaken to establish its domain structure and perform item reduction. Women also completed the Women's Health \& Menstrual Distress Questionnaires (WHQ \& MDQ) to evaluate criterion validity as well as a 10-item questionnaire (QQ-10) measuring 
womens' views of using ePAQ-MPH. Test-retest reliability was evaluated in 30 women.

Results: Factor analysis identified 18 domains (Cronbach's ? >0.7) and 17 redundant items. Significant correlations were found with salient WHQ and MDQ domain scores (Spearman's Rho $>0.5$ ). Test-retest analysis found acceptable intraclass correlations of $0.6-0.9(\mathrm{P}<0.05)$. QQ-10 found high value and low burden attributed to ePAQ use.

Discussion: ePAQ-MPH offers a user-friendly tool for clinical practice, providing reliable, valid comprehensive assessment of symptoms and quality of life for women with menstrual, pain and hormonal disorders. Further studies will evaluate patient \& clinician experience and its ability to measure outcomes in practice.

424

CONSERVATIVE SURGICAL TREATMENT FOR BOWEL ENDOMETRIOSIS

Endometriosis: Surgery

M. Veujoz*, E. Faller, C. Zacharopoulou, J. Albornoz, M. Puga, A. Wattiez

${ }^{[1]}$ Universitary Hospital of Strasbourg $\sim$ Strasbourg $\sim$ France

Summary (4 lines): This study compares discoid resection (DR) vs shaving/mucosal skinning for colorectal endometriosis.

Introduction: Rectum is affected in $2 / 3$ of cases of bowel endometriosis. Segmental resection is associated with significant complications. Literature provides few data comparing the different conservative techniques.

Material and Methods: A retrospective study between Jan. 2008 and Dec. 2010 included all cases of colorectal endometriosis conservative surgery. Ten patients underwent a DR (group 1, G1), and 30 patients had shaving or mucosal skinning (group 2, G2). Populations, diagnostic means, surgical data, and complications were compared.

Results: Populations were equivalent except for digestive symptoms (more frequent in G1 $\mathrm{p}=0,0006$ ). There was no case of conversion. Operative time was shorter in $\mathrm{G} 2(\mathrm{p}=0,0025)$. Length of urinary catheterisation, hospital stay and complications were equivalent. Complication rate was $22,5 \%$, with 2 grade $3 \mathrm{~B}$ complications (ClavienDindo classification). Depth of invasion was greater in $\mathrm{G1}(\mathrm{p}=0.006)$. Discussion: We report a low rate of $3 \mathrm{~B}$ complication $(5 \%)$. These complications were not related to the digestive procedure. We identified a tendency for more severe complications in $\mathrm{G} 1(\mathrm{p}=0,54)$.

Conservative treatment is feasible and safe. Shaving or mucosal skinning must be the first step, completed if needed by discoid resection. Long-term outcome for bowel dysfunction and fertility are data that are still not available (questionnaires on course).

484

\section{CABERGOLINE SIX MONTHS TREATMENT BEFORE ENDOMETRIOSIS SURGERY}

Endometriosis: Surgery

M. Iraci Sareri, G. Giunta*, I. Salemi, C. Clemente, R. Caltabiano, A. Cianci

${ }^{[1]}$ Policlinico Universitario G. Rodolico $\sim$ Catania $\sim$ Italy

Summary (4 lines): To evaluate 6 months Cabergoline (CB)treatment in reducing endometriotic peritoneal lesions before surgery

Introduction: Endometriotic lesions grow in areas with a rich vascularization, suggesting angiogenesis as a prerequisite for endometriosis development. $\mathrm{CB}$ inhibit the growth of endometriotic foci in nude mice, promoting VEGF receptor-2(VEGFR2) endocytosis. Besides
$\mathrm{CB}$ reduces ovarian permeability and ascites in ovarian hyperstimulation syndrome by VEGFR2 dephosphorilation. Basing on this we designed a 6 months $\mathrm{CB}$ treatment to reduce angiogenesis and vascularization of endometriotic lesions before surgery.

Material and Methods: 6 month before surgery 16 women with diagnosis of endometriosis where divided in two groups: GA(n8)received $0,25 \mathrm{mg}$ of $\mathrm{CB}\left(\right.$ Dostinex ${ }^{\circledR}$ Pfizer) three times a week, GB(n8) received a placebo. Major exclusion criteria where previous endometriosis surgery and use of hormonal treatment in the last 6 months. Every woman underwent SF-36 and VAS to evaluate QoL and pelvic pain. During surgery peritoneal endometriosis lesions where excised to asses proliferative activity, neoangiogenesis, VEGFR2 phosphorilation.

Results: Women from groupA had a significant improvement on QoL and pain relief, and a decrease in cellular proliferation index, neoangiogenesis and VEGFR2 phosphorolation than control.

Discussion: $\mathrm{CB}$ inhibits the growth of endometriotic peritoneal lesions through VEGFR2 inactivation and could be a potential agent to reduce peritoneal endometriosis.

\section{DEEP ENDOMETRIOSIS SURGERY AND OVARIAN RESERVE:} A CONFLICTUAL RELATION

Endometriosis: Surgery

F. Emilie*, A. Jaime, M. Pietro, P. Marco, W. Arnaud

${ }^{[1]}$ Universitary Hospital of Strasbourg iRCAD/EITS Strasbourg France

Summary (4 lines): Women with endometriosis are often young with a fertility desire: respect of the ovarian reserve must be a priority.

Introduction: The aim of this study was to evaluate the impact of deep pelvic endometriosis surgery performed by an expert on ovarian reserve, even in the absence of endometrioma.

Material and Methods: We conducted an observational study at the University Hospital of Strasbourg amongst 30 consecutive patients who had surgery for severe endometriosis with pain. Inclusion criteria were the following: patient with endometriosis stage III/IV AFSr score confirmed during surgery, maximum age 40 years old, presence of 2 ovaries. Exclusion criteria were as follows: adnexectomy or hysterectomy performed before or during surgery. Ovarian reserve was evaluated before and 3 months after surgery with measurement of Anti-Müllerian Hormone $(\mathrm{AMH})$ levels along with antral follicle count by ultrasound. Demographic data and previous surgery were noted. The surgical procedure was described to compare patients with or without major adhesiolysis, cystectomy, bowel or urinary procedure.

Results: in progress

Discussion: Deep endometriosis surgery is accurate for pain but surgeons must take into account the patient's fertility desire. In case of ovarian failure before surgery, the strategy must be adapted to preserve the patient's fertility. Additional prospective studies will be necessary to propose ovarian cryoconservation before surgery.

\section{3}

\section{ENDOMETRIOTIC ASCITES: TWO CASE REPORTS Endometriosis: Surgery}

F. Passos, G. Rodrigues, T. Diniz-da-costa*, S. Barata, I. Rato, C. Castro, C. Alho, F. Osório, C. Calhaz-jorge

${ }^{[1]}$ Hospital Santa Maria $\sim$ Lisboa $\sim$ Portugal

Summary (4 lines): Endometriotic ascites is a rare entity that requires differential diagnosis with peritoneal carcinomatosis. We present two 
case reports of severe ascitic endometriosis, one of them with thoracic involvement.

Introduction: We could find 63 reported cases of ascites secondary to endometriosis. This disease should be considered in a patient with ascites of unknown origin.

Material and Methods: Case 1: 32-years-old patient presenting with hemorrhagic ascites. She underwent diagnostic laparoscopy with drainage of the ascites and multiple biopsies. Afterwards she was submitted to another laparoscopy with drainage of $8500 \mathrm{ml}$ of ascitic fluid, adhesiolysis, excision of a rectovaginal endometriotic nodule, bilateral salpingectomy and excision of the pelvic parietal peritoneum. She remains under $\mathrm{GnRH}$-analogs with add-back therapy.

Results: Case 2: 26-years-old patient who presented with adnexal masses and ascites. A diagnostic laparoscopy was performed with drainage of hemorrhagic ascites and multiple biopsies. She was submitted later to laparoscopic adhesiolysis and excision of a rectovaginal endometriotic nodule and to a toracoscopy (aspiration of $1500 \mathrm{ml}$ of hematic fluid and pleurodesis). She remains under GnRH-analogs with add-back therapy.

Discussion: Endometriotic ascites is associated with a severe form of endometriosis disease, with high risk of recurrence. The best approach to this form of the disease is still unclear.

270

\section{EXTERNAL VALIDATION OF THE ENDOMETRIOSIS FERTILITY INDEX (EFI)}

Endometriosis: Surgery

C. Tomassetti*, B. Geysenbergh, C. Meuleman, D. Timmerman, T. D'Hooghe

${ }^{[1]}$ University Hospitals Leuven $\sim$ Leuven $\sim$ Belgium

Summary (4 lines): External validation of the Endometriosis Fertility Index $(\mathrm{EFI})$

Introduction: The EFI can be useful to counsel endometriosis patients about reproductive options after surgery, but requires external validation.

Material and Methods: Retrospective cohort study in a university hospital on 233 endometriosis patients attempting non-ART conception (ie. no IVF/ICSI) immediately after therapeutic surgery. The EFI was calculated from prospectively collected data. Postsurgical reproductive outcome was analyzed using Kaplan-Meier (KM) estimates; Cox regression analysis; performance with Brier score; areas under the receiver-operating curve (AUC), and global concordance index Ct.

Results: A significant association was observed between EFI and time to non-ART pregnancy $(\mathrm{p}=0.0004)$. The overall $\mathrm{KM}$ estimate of cumulative pregnancy rate at 12 months was $45.5 \%$ but was lower for women with EFI scores 0-3 (17\%) than for women with EFI scores 9$10(63 \%)$. Due to the linear relationship between EFI and pregnancy, an increase of EFI with 1 point results in an increase of pregnancy rate with $31 \%$. However, the predictive performance of the EFI was moderate (small decrease in Brier score; low estimates for $\mathrm{R}^{2}$ and $\mathrm{Ct}$ ). Discussion: The EFI, initially tested in the USA, was externally validated in an European population in this study, and can be used for postoperative reproductive counseling in women with endometriosis with respect to non-ART conception, regardless of the rAFS endometriosis stage.

48

FEASIBILITY OF CONSERVATIVE SURGERY IN ADVANCED DEEP ENDOMETRIOSIS INFILTRATING THE RECTUM: PRELIMINARY RESULTS OF ENDORE RANDOMIZED TRIAL Endometriosis: Surgery
R. Horace*, D. Emile, T. Jean Jacques

${ }^{[1]}$ Rouen University Hospital $\sim$ Rouen $\sim$ France

Summary (4 lines): We report preliminary data of ENDORE randomized trial comparing the colorectal resection to conservative surgery (either shaving or disc excision) in rectal endometriosis.

Introduction: We analyse the feasibility of surgical procedures and immediate postoperative outcomes.

Material and Methods: Data of women enrolled in ENDORE (NCT01291576) during 11 months.

Results: There were 13 women managed by conservative surgery ( 5 shaving and 8 full thickness) vs. 14 women managed by colorectal resection. Rectal involvement size was $32 \pm 7 \mathrm{~mm}$ (range22-50) vs. $32 \pm 10 \mathrm{~mm}$ (range $21-50$ ). Rectal stenosis was revealed in 11 ( $85 \%$ ) vs $12(86 \%)$. Multiple digestive nodules were found in $10(77 \%)$ vs $11(79 \%)$.

Operative time was $285 \pm 143 \mathrm{~min}$ vs. $261 \pm 66 \mathrm{~min}$. The main diameter of the rectal disc excised was $50 \mathrm{~mm}$ (range $40-60 \mathrm{~mm}$ ), while the length of the colorectal segment removed was $94 \mathrm{~mm}(30-180 \mathrm{~mm})$. As regard postoperative outcomes, in the conservative surgery arm were recorded 1 rectovaginal fistulae, 1 abdominal wall abscess and 2 de novo bladder atony requiring self catheterization. In the colorectal resection arm there were 1 de novo bladder atony requiring self catheterization, 1 pelvic abscess, 3 rectal haemorrhages, and 3 stenosis of the anastomosis.

Discussion: Preliminary data suggest that conservative surgery in advanced rectal endometriosis may reasonably be considered instead mandatory colorectal resection.

\section{7}

\section{IMPACT OF OVARIAN ENDOMETRIOMA REMOVAL ON IVF OUTCOME}

Endometriosis: Surgery

A. Vogler*, M. Ribic-pucelj, I. Virant-klun

${ }^{[1]}$ University Medical Centre $\sim$ Ljubljana $\sim$ Slovenia

Summary (4 lines): The impact of ovarian endometrioma removal on IVF outcome remains still controversial. Our study was designed to elucidate if bilateral ovarian endometrioma removal affects IVF outcome. Results show that prior ovarian surgery does not impair IVF outcome.

Introduction: Laparoscopic surgery in infertile patients with severe endometriosis leads to more than $60 \%$ pregnancy rate. For the rest of the patients IVF is the most appropriate treatment of choice.

Material and Methods: In 64 patients (group A) underwent IVF program the only cause of infertility was bilateral ovarian endometrioma which had been previously surgically removed. The control group (group B) represents 330 patients who underwent IVF procedure due to tubal infertility. Among others, following parameters were analyzed and compared between the both groups: pregnancy rate and take home baby rate (THBR) per cycle and per embryo transfer (ET).

Results: In none of the following parameters statistically significance was reached. In the group A pregnancy rate per cycle and per ET was $40.6 \%$ and $46.6 \%$ compared to the group B where was $43.3 \%$ and $48.2 \%$ respectively. THBR per cycle and per ET in the group A was $34.4 \%$ and $39.3 \%$, whereas in the control group was $36.1 \%$ and $40.1 \%$ respectively.

Discussion: Bilateral endometrioma removal does not affect IVF outcome. Appropriate surgical technique is of paramount importance to preserve functional ovarian tissue in patients with endometriomas leading to favourable IVF outcome if needed. 
340

\section{LAPAROSCOPIC NEUROLYSIS OF S1-3 NERVE ROOTS IN THE TREATMENT OF ENDOMETRIOSIS-RELATED SCIATICA.}

Endometriosis: Surgery

J. English*, J. Clark

${ }^{[1]}$ Brighton and Sussex University Hospital $\sim$ Brighton $\sim$ United Kingdom

Summary (4 lines): Laparoscopic dissection of nerve roots infiltrated by endometriosis is possible and should be undertaken when there is nerve involvement.

Introduction: Sciatic pain due to infiltration of the sacral nerve roots is a relatively rare symptom associated with severe endometriosis. Identification and dissection of the nerve roots can be difficult in the presence of endometriosis and especially so when there is disease wrapped about them thereby potentiating damage to the nerves with resultant sensori-motor loss.

Material and Methods: The presacral fascia was divided to reveal the nerve roots of S2-4 and these were dissected free from the surrounding disease. The pelvic splanchnics arising from S4 running to the bladder were dissected out and preserved as was the hypogastric nerve on the right. The resection of endometriosis included a repeat segmental rectal resection with J pouch formation. Due to DIE in the ureteric wall the diseased segment was excised and the ureter reimplanted into the bladder with an added psoas hitch.

Results: Infiltrated sacral nerve roots and their parasympathetic branches were successfully dissected out despite infiltration by disease. Discussion: Dissection of the sacral nerve roots and their splanchnic branches can be very difficult especially when surrounded by endometriosis. A sound knowledge of anatomy and adherence to anatomical dissection can make the dissection possible with resultant preservation of innervation.

483

LAPAROSCOPIC SURGICAL TREATMENT OF DIAPHRAGMATIC ENDOMETRIOSIS: A SEVEN-YEAR SINGLE-INSTITUTION RETROSPECTIVE REVIEW.

Endometriosis: Surgery

M. Ceccaroni, P. Giampaolino*, G. Roviglione, R. Clarizia, F. Bruni, G. Ruffo, T.S. Patrelli, G. De Placido, L. Minelli

${ }^{[1]}$ Gynecologic Oncology Division, International School of Surgical Anatomy, Sacred Heart Hospital, Negrar, Verona Italy

Summary (4 lines): About $0,6 \%$ of patients with deep endometriosis, present diaphragmatic endometriosis involvement.

Introduction: To analyze clinical aspects and surgical treatment of diaphragmatic endometriosis in a long-experience series of an Italian Referral Center for the Diagnosis and Treatment of endometriosis.

Material and Methods: A retrospective analysis of patients with diaphragmatic endometriosis treated by laparoscopic eradication from February 2004 to May 2012, was performed.

Results: 48 women with diaphragmatic endometriosis were reviewed, over a total of 2180 laparoscopies performed for suspected endometriosis $(2 \%) .70 \%$ of diaphragmatic nodules were multiple and $75 \%$ of them were defined as superficial; in 13 patients $(26 \%)$ nodules had diameter $=1 \mathrm{~cm} .7(13 \%)$ patients had hepatic, $1(2.1 \%)$ pericardial and pleural nodules. Surgical procedures included diathermocoagulation (36.9\%), argon coagulation (28.2\%), excision (23.9\%)and stripping in 1 case, $(2.1 \%)$ with conversion to abdominal route for massive left diaphragmatic, pleural and pericardial involvement. Intra-operative opening of the diaphragm occurred in 5 patients $(10.8 \%), 3$ of which were sutured laparoscopically and 2 had intra-thoracic drainage. 3 patients had intraoperative pneumothorax, spontaneously sort out. Discussion: Laparoscopic surgery, when performed by expert surgeons, can be safe and completely eradicative, with complete resolution of symptoms.

491

\section{LAPAROSCOPIC TREATMENT OF RARE FORMS OF ENDOMETRIOSIS.}

Endometriosis: Surgery

K. Puchkov*, J. Andreeva, N. Podzolkova, V. Korennaya

${ }^{[1]}$ Clinical and Experimental Surgery Center $\sim$ Moscow $\sim$ Russian Federation

Summary (4 lines): The rare forms of endometriosis are difficult to diagnose and to treat. We have successfully treated 125 patients with rare forms of the disease using the laparoscopic rout.

Introduction: Frequent sites of the endometriosis are the ovaries, uterine ligaments, serosa, fallopian tubes. The atypical sites of endometriotic lesions, such as invasive forms, bowel, urinary tract or a scar endometriosis-are rare, which makes them difficult to diagnose and to treat. Material and Methods: From 2008 till 2011 we have treated 556 patients with endometriosis, 125 of them had rare forms of the disease. Results: 39 of operated women had endometriosis of cul-de-sac and in $21 \%$ had undergone the vault resection. Recto-vaginal endometriosis was diagnosed in 76 patients, in $40 \%$ of the cases staged as 2 degree or more. Resection of the rectosigmoid part of the bowel was performed in $4 \%$. Ten women had undergone the surgery for the endometriosis of spleen, appendix or cecum.

Laparoscopic bladder resection $(n=4)$, ureter resection $(n=2)$ and ureterolysis $(\mathrm{n}=8)$ was performed for urinary tract endometriosis.

In one case we performed a resection of endometriosis of the uterus scar after the $\mathrm{C}$-section and in one patient the ablation of the endometriotic cyst of the uterus.

Discussion: The experienced, we have gained during these years and some surgical techniques that we have developed are useful for effective treatment of rare forms of endometriosis by the minimally invasive laparoscopic rout.

\section{9}

QUALITY OF LIFE AFTER LARGE BOWEL RESECTION FOR DEEP INFILTRATING ENDOMETRIOSIS (DIE)

Endometriosis: Surgery

S. Gocevska*, A. Bouggenout, J.C. Noel, A. Vincent

${ }^{[1]}$ Erasme Hospital, Université Libre de Bruxelles, ULB, $\sim$ Brussels $~$ Belgium

Summary (4 lines): Symptoms of deep bowel endometriosis are really harmful for the patient's quality of life. Colonic resections for DIE are difficult and time consuming operations. Laparoscopic assisted rectosigmoid resection for DIE is a safe and feasible intervention with good outcomes on the patient's QoL.

Introduction: To assess the impact of large bowel resection in a consecutive series of 117 cases severe pelvic endometriosis with symptomatic large bowel involvement on women's pain symptoms, analgesic intake, work absenteeism, work difficulties and some elements of sexual life before and after treatment.

Material and Methods: Approved quality of life questionnaires and visual analogue scale (1-10) on a prospective database of large bowel 
resection for highly symptomatic DIE, performed in Erasme Hospital between October 1997 and October 2012.

Results: Median age was $33 \pm 6$ years. Mean follow-up was $64 \pm$ 44 months. Significant statistical differences were found between preoperative and postoperative pain scores, percentages of women absent from work, percentages taking analgesics or nonsteroidal antiinflammatory drugs, and percentages having work difficulties due to pain. Also a significant difference was found in frequencies of sexual desire and coitus.

Discussion: Our findings support the beneficial role of the surgical treatment in relieving the symptoms of DIE and consequently in improving the QOL of these patients.

184

\section{REVERSE LAPAROSCOPIC TECHNIQUE FOR MANAGEMENT OF DEEP ENDOMETRIOSIS OF THE RECTOVAGINAL SEPTUM: PRELIMINARY EXPERIENCE IN CHILE}

Endometriosis: Surgery

D. Larraín, J. Prado, F. González*, F. Marengo, I. Rojas

${ }^{[1]}$ Clínica Santa María $\sim$ Santiago $\sim$ Chile

Summary (4 lines): Reverse laparoscopic technique is feasible and reproductible, however, it should be reserved to teams experienced in advanced laparoscopic surgery.

Introduction: Surgical resection of deep infiltrating endometriosis (DIE) is complex and it is associated with a high complication risk. Recently, the so-called reverse technique seems to be associated with lower a complication rate when compared to standard technique. The term "reverse" means that rectal shaving is performed after "en block" vaginal excision, and not before as in standard technique.

Material and Methods: Report of 5 cases from a prospective database.

Results: Mean age was 34,2 years (range 32-37). All patients had chronic pelvic pain and severe dyspareunia and 3 women had previous surgeries for endometriosis. Mean operative time was 313 minutes (range 180-450). All surgeries were completed by laparoscopy and no complications were noted. Histological analysis confirmed endometriosis in all cases. Mean follow-up was 4 months (range 2-8).

Discussion: Reverse laparoscopic technique is feasible and reproductible, however, it should be reserved to teams experienced in advanced laparoscopic surgery.

94

SELECTIVE USE OF ILEOSTOMY IN LAPAROSCOPIC LEFT BOWEL RESECTIONS FOR DEEP ENDOMETRIOSIS: LESSONS LEARNED FROM A RETROSPECTIVE REVIEW ON 41 PATIENTS

Endometriosis: Surgery

P. Messori*, E. Faller, J. Albornoz, J. Leroy, A. Wattiez

${ }^{[1]}$ IRCAD/EITS $\sim$ Strasbourg $\sim$ France

Summary (4 lines): Bowel segmental resection for endometriosis is performed on leakage low risk patients; when performed with all the principles of conservative benign surgery, the procedures can be done without a protective stoma

Introduction: The aim of this retrospective analysis is to verify the hypothesis that in the majority of patients segmental resections for endometriosis can be safely performed without a stoma.
Material and Methods: Between 2004 and 2011, 41 women with bowel endometriotic lesions underwent segmental resections.

Results: There were 6 sigmoid, 21 high, 10 low, 4 ultra-low rectal anterior resections. Four patients with an ultra-low anterior resection had a temporary ileostomy. There was 1 anastomotic leak, in a patient with an unprotected anastomosis which required a secondary stoma. Other post operative complications were 1 hemoperitoneum, 1 pelvic abscess, 1 pelvic collection.

Discussion: Given the fact that colon surgery is different in the context of endometriosis (no need for radical surgery on the mesorectum and colonic mesenteries, young and healthy patients), the indication for a protecting stoma, lacking intraoperative risk factors, should be questioned. In our opinion the resection level should not by itself justify the recourse to ileostomy, that should be reserved to particularly complex operations and suspected defective anastomosis.

\section{3}

\section{SEXUAL AND EMOTIONAL IMPACT OF DYSPAREUNIA IN WOMEN WITH ENDOMETRIOSIS: A MULTI-CENTRE STUDY}

Endometriosis: Surgery

N. Fritzer, A. Thomas, P. Oppelt, D. Haas, S. Renner, D. Hornung, M. Woelfler, M. Sillem, G. Fischerlehner, U. Ulrich, G. Hudelist*

${ }^{[1]}$ Alps-Adria-University Klagenfurt $\sim$ Klagenfurt $\sim$ Austria

Summary (4 lines): Dyspareunia as one of the most common symptoms in endometriosis has not only an impact on health, but also on relationship and quality of sex life.

Introduction: At the moment there is a lack of prospective studies evaluating sexual function and emotions from suffering women.

The current study aimed to evaluate the impact of dyspareunia on women's sex life and relationship.

Material and Methods: The present work was conducted as a questionnaire-based study among eight special centres for endometriosis in Austria and Germany. One-hundred and twenty endometriosispatients with dyspareunia were interviewed with the Female Sexual Function Index, the Female Sexual Distress Scale-Revised and a selfadministered questionnaire.

Results: Patients suffered averagely 4.76 years (SD: 4.9) from dyspareunia with a median pain intensity on the VAS of 5.52 (SD: 2.48 ) points during intercourse and 4.45 points (SD: 2.87 ) after it. $72.8 \%$ of patients experienced sexual distress during the past 30 days and in $25,2 \%$ of cases, results of the FSFI showed a sexual dysfunction. Sixty percent of patients were afraid of pain and $38 \%$ convulsed their body during intercourse. Furthermore, they had a reduced female-identity, feelings of guilt towards their partners and were afraid of getting divorced because of their complaints.

Discussion: Greater awareness among gynaecologists who are involved in the management of endometriosis would be beneficial.

\section{8}

\section{SHAVING OR MUCOSAL SKINNING FOR BOWEL ENDOMETRIOSIS: IS THERE A DIFFERENCE? \\ Endometriosis: Surgery}

F. Emilie*, A. Jaime, M. Pietro, P. Marco, W. Arnaud

${ }^{[1]}$ universitary Hospital of Strasbourg iRCAD/EITS Strasbourg France 
Summary (4 lines): The objectives is to evaluate the definition elaborated by experts in shaving and mucosal skinning for bowel endometriosis.

Introduction: A questionnaire was sent by e-mail to the main authors who have published on the subject of bowel endometriosis.

Material and Methods: A simple question was addressed to different authors: In your opinion, is there a difference between shaving and mucosal skinning? If yes, what is it?

Results: Eleven answers were obtained. Eight surgeons thought that there was a difference between shaving and mucosal skinning, and all answers were different. This shows that a clear definition has yet to be elaborated to clarify publications. Through a literature review, we propose to clearly define each term.

Discussion: The terms shaving and mucosal skinning are used in the literature but have no clear definition.

We propose to standardize these terms to clarify data found in the literature.

517

SURGICAL TREATMENT OF BOWEL ENDOMETRIOSIS: EVALUATION OF SYMPTOMS AND QUALITY OF LIFE BY A SELF-ASSESSMENT QUESTIONNAIRE FROM A CONTINUOUS SERIES OF 41 PATIENTS

Endometriosis: Surgery

P. Panel*, E. Bailly, F. Renouvel, A. Le Tohic, A. Fauconnier

${ }^{[1]}$ Centre Hospitalier de Versailles $\sim$ Versailles $\sim$ France

Summary (4 lines): Evaluate symptoms and quality of life before and after surgical treatment of endometriosis with bowel involvement.

Introduction: A 5 years continuous series, in a department of gynaecology and obstetrics of a French general hospital

Material and Methods: 41 patients operated for bowel endometriosis filled a self-assessment questionnaire. Pain was assessed using five visual analogical scales, symptoms using 26 questions with a four level Likert item, sexuality using the SEXACQ, and quality of life using the EHP-5 and the EQ-5D VAS.

Results: Follow-up was greater than 12 months for all patients. All VAS scores were significantly improved. Patients also reported improvement of SEXACQ score and quality of life scores EHP-5 and EQ-5D VAS. No difference was found between disk resection and segmental resection in terms of symptoms, sexuality and quality of life. Histological analysis suggests that when a segmental resection is done, the stage of the endometriosis bowel involvement is more advanced.

Discussion: Surgery of bowel endometriosis improves symptoms and quality of life.

When the stage of the bowel endometriosis is advanced, a segmental resection should be done. Moreover, self assessment questionnaire used at the CHV seems an appropriate tool to evaluate functional outcome.

277

TOTAL LAPAROSCOPIC COLORECTAL RESECTION WITH NATURAL ORIFICE SPECIMEN EXTRACTION (NOSE) : A TECHNIQUE PARTICULARLY ADAPTED TO BOWEL ENDOMETRIOSIS.

Endometriosis: Surgery

F. Emilie*, A. Jaime, M. Pietro, P. Marco, L. Joel, W. Arnaud

${ }^{[1]}$ universitary Hospital of Strasbourg $\sim$ iRCAD/EITS Strasbourg France
Summary (4 lines): Evaluate the possibility of a total intracorporeal anastomosis for bowel resection for deep endometriosis using the vagina like a NOSE.

Introduction: The aim of our study was to evaluate the feasibility of a total intracorporeal anastomosis for bowel resection.

Material and Methods: Retrospective analysis of 41 consecutive patients between 2004 and Forty-one patients who had a bowel resection for deep endometriosis.

Results: Thirty-two patients had a standard technique using a minilaparotomy and 9 patients had a NOSE technique. Two patients had a transanal approach, 5 patients had a transvaginal approach with In 5 cases, exteriorization of the bowel through the vagina. In 2 cases, the anastomosis was intracorporeal and transanal with a specimen extraction using the vagina There was no difference in operative time (about 220'), mean hospital stay (9,8 days). There was no conversion by laparotomy in the two groups. Complication rate was the same : 2 major complications in the NOSE group (22\%), and 7 complications in the abdominal group (22\%).

Discussion: NOSE technique to perform colorectal anastomosis seems to be as efficient as the abdominal technique. It theoretically can avoid cosmetic damage, orifice hernia, pain or infection. Bigger study are required to attest the benefit in a long term of the use of NOSE.

\section{5}

\section{VAGINAL AND TRANSRECTAL "NOTES" FOR OPERATIVE TREATMENT OF SEVERE RECTOVAGINAL ENDOMETRIOSIS}

Endometriosis: Surgery

S. Todorow*

${ }^{[1]}$ Klinik am Hofgarten $\sim$ Bayreuth $\sim$ Germany

Summary (4 lines): The vaginal approach via lens tube and successive insertion of a PVC ring/cuff system allows a contamination free diverting of the sigma in anterior resection of the rectum.

Introduction: The transanal, extraperitoneal resection of the rectum front wall by Longo is used as an intestinal wall resection in cases of rectum prolapse and ODS in the field of gynecology and surgery since a few years. The anastomosis dehiscence ratio of the stapler technique could be lowered to less than $1 \%$, though.

Material and Methods: A mini laparotomy is carried out in most cases of laparoscopic anterior rectum resection for inserting of the proximal stapler head. The associated diminished blood supply and trauma of the intestine results in anastomosis insufficiency rates fluctuating from 5 to $7 \%$. The overall morbididty rate exceeds $30 \%$. The rate for formation of fistula is serious.

Results: Technically we achieve an anterior wall disc resection but without opening of the gut lumen. This fact should reduce the incidence of complications significantly. The first cases showed no complications. This method is secure, easy and holds technical advantages compared to the so called "mucosal shaving" disc resection or the anterior rectum segment resection.

Discussion: We hope that this new intraperitoneal STARR technique will expand our operative capabilities in treating securely one of the most challenging conditions in gynecology and surgery: the rectovaginal endometriosis.

BARBED VS. STANDARD SUTURE: A RANDOMIZED TRIAL FOR LAPAROSCOPIC VAGINAL CUFF CLOSURE

Hysterectomy 
J.I. Einarsson*, S. Cohen, E. Sandberg, J. Gobern, D. Brown

${ }^{[1]}$ Brigham and Women's Hospital $\sim$ Boston $\sim$ United States

Summary (4 lines): This randomized trial compares vaginal cuff closure times, cuff healing and postoperative dyspareunia between barbed and traditional suture during total laparoscopic hysterectomy.

Introduction: 64 women who planned total laparoscopic hysterectomy for benign indications at two university hospitals are included.

Material and Methods: Subjects were randomized to vaginal cuff closure performed by either attending or trainee with either Quill or Vicryl suture. Time required for cuff closure was documented. A standardized sexual function questionnaire (FSFI) was administered preoperatively and at three months postoperatively and patients were examined at four weeks postoperatively.

Results: Baseline characteristics and indications for hysterectomy between barbed and Vicryl suture groups were similar. Mean cuff closure time was not significantly different $(9.6 \mathrm{~min}$. for vicryl vs. $10.4 \mathrm{~min}$. for barbed; $p=.49$ ); these findings were maintained with subgroup analyses of the attending and trainee surgeons. Two patients experienced bleeding from the vaginal cuff that required treatment (one from each group), three patients had dehiscence (two vicryl, one barbed) and two patients were treated for cuff cellulitis or abscess (one from each group).

Discussion: Barbed suture is a viable option for vaginal cuff closure during a total laparoscopic hysterectomy as it is not associated with higher complication or dyspareunia rates.

1

ELECTROSURGICAL BIPOLAR VESSEL SEALING VERSUS CONVENTIONAL CLAMPING AND SUTURING FOR VAGINAL HYSTERECTOMY: A RANDOMIZED CONTROLLED TRIAL Hysterectomy

M. Lakeman, M. Dijkgraaf, J. Roovers*

${ }^{[1]}$ AMC Amsterdam $\sim$ Amsterdam $\sim$ Netherlands

Summary (4 lines): Using vessel sealing during vaginal hysterectomy results in less post-operative pain, shorter operating time and similar 1) morbidity,2)pelvic floor function and 3) costs.

Introduction: To compare the effects of electrical bipolar vessel sealing (Ligasure Impact) and conventional clamping in women undergoing vaginal hysterectomy.

Material and Methods: A multi-center randomized controlled trial was performed including 100 patients scheduled for vaginal hysterectomy for benign conditions excluding pelvic organ prolapse. Interventions were compared for VAS pain scores, surgery time, blood loss, complications, quality of life related to pelvic floor function, and costs. Follow-up was 6 months.

Results: The evening after surgery, patients in the vessel sealing group reported significantly less pain (5.7 vs $4.5 \mathrm{p}=0.03$ ), but after that pain scores where similar. Operation duration was shorter for vessel sealing (60 vs 71 minutes $p=0.05$ ). Blood loss and hospital stay did not differ. We observed no major difference in costs between the two interventions. (2903 vs 3102 euro, $p=0.26$ ). Changes in micturition and defecation symptoms were similar.

Discussion: Although using ligasure vessel sealing results in a shorter operation duration, the costs of the Ligasure clamp are not completely compensated for. Patients undergoing hysterectomy appear to benefit from the use of a vessel sealing technique.

122

HOW TO AVOID LAPAROTOMY DURING HYSTERECTOMY Hysterectomy
O. Istre*, H. Springborg

${ }^{[1]}$ Aleris Hamlet Hospital, University of Southern Denmark Copenhagen $\sim$ Denmark

Summary (4 lines): Hysterectomy can always be managed with endoscopic technique low complication rate good follow up and control of data and Satisfied patients.

Introduction: Abdominal hysterectomy TAH is the main treatment for enlarged uterus in Denmark. Laparoscopic hysterectomy is only performed in $9 \%$ and the vaginal route is utilized in $30 \%$ in the smaller uterus. At our hospital we have offered TLH, LSH and VH as standard operation to all patients with no exception since November 2010. We have interviewed the patient about their expectation and their reaction to these new offers.

Material and Methods: Using a $5 \mathrm{~mm}, 30$ degree High Definition (HD) optic, we are able to perform port jumping, coming around the corners. Inserting the ports high up on the abdomen, also above the umbilicus we are able to treat the big fibroids, difficult endometriosis when hysterectomy is needed.

Results: Experience of our consecutive series of 150 hysterectomies with one abdominal conversion in $2,5 \mathrm{~kg}$ retroperitoneal fibroids and two operative complications. One bladder perforation sutured primarily one ureter lesion treated with $\mathrm{J}$ sten. All patients were discharged within 24 hours.

Discussion: Hysterectomy can be managed with endoscopic technique if you have modern equipment, dedicated nursing staff, smooth hospital environment, A "nurd" Doctor burning for it, low complication rate good follow up and control of data and Satisfied patients.

\section{6}

\section{IS LAPAROSCOPIC HYSTERECTOMY OVERTAKING VAGINAL HYSTERECTOMY \\ Hysterectomy}

M. Elessawy*, T. Schollmeyer, M. Lisolette, W. Jonat, I. Alkatout

${ }^{[1]}$ University hospitals Kiel, Germany $\sim$ Kiel $\sim$ Germany

Summary (4 lines): The aim of the study to investigate different operative route of hysterectomy.

Introduction: The art of surgery developed with the advancement of technology allowing a minimal invasive operation.

Material and Methods: 954 patient underwent hysterectomy from 2002-2010 at benign uterine disease in Kiel University hospital. Data were retrospectively collected and analysed.

Results: The hospital stay was longest by the $\mathrm{AH}$ representing 7,9 days, shortest by LASH 3,8, VH 6,7 and TLH 4,3. The operating time was significantly shortest by the $\mathrm{VH} 76,0$ minutes, longest by the TLH 130,2 and by LASH 106,5. The average uterus weight was by VH 127,7 , by LASH 244,9 and by TLH 205,1 . The average hemoglobin decrease was significantly low with LASH $0,7 \mathrm{~g} / \mathrm{dl}$, by the VH 1,2 and by the TLH 0,8 . The LASH showed the lowest complication rate $2,4 \%$ and $\mathrm{AH}$ showed highest complication rate $6,6 \%$, by $\mathrm{VH} 6,3 \%$, which is more than TLH 2,9\%. Highest intra-operative complication rate by AH 4,5 \%, fewer by TLH 1,5\%. Highest incidence of major complication was recorded by LAVH $6,4 \%$ and low incidence $1,2 \%$ by LASH. The lowest minor postoperative complication rate recorded by LASH 1,8 \% and highest by AH 8,4 \%.

Discussion: The choice of the hysterectomy route is dependent from many factors; the size, mobility of uterus, the BMI, preoperative history, anatomical variation, level of experience of the surgeon and the patient wish plays an important role. In our study $\mathrm{LH}$ have advantages over $\mathrm{VH}$ and $\mathrm{AH}$ by benign indication. 
159

\section{LAPAROSCOPIC HYSTERECTOMY (LH) AND ENHANCED RECOVERY PATHWAY (ERP) \\ Hysterectomy}

A. Kent*, N. Waters, W. Mitchell

${ }^{[1]}$ MATTU $\sim$ Guildford $\sim$ United Kingdom

Summary (4 lines): We present the outcome of our pathway combining LH and ER, achieving an LH rate of $>90 \%$, with $90 \%$ of LH ERP going home within $23 \mathrm{hrs}$. Complication rates were negligible.

Introduction: Uptake of LH remains low due to the perception that LH takes too long; requires high skill set; has limited application; has no benefit regarding hospital stay; is too costly; has high per-op complication rates. The purpose of this study was to assess the acceptability and safety of a $23 \mathrm{hr}$ stay pathway for patients undergoing LH. Material and Methods: A prospective study of 376 patients, 336 were judged suitable for LH ERP. A core surgical technique utilizing Harmonic ACE and the Valtchev Uterine Manipulator was used. The ERP key elements were pre-op preparation, intra-op management, post-op management, follow up, audit.

Results: 376 hysterectomies (336 LH ERP 40 TAH) $90 \%$ overall LH rate.

Mean Age 46. Mean BMI 27.5 (18-46).

Mean Op time 68 mins (10-150 mins).

Mean MBL $32 \mathrm{ml}$.

Mean uterine size $220 \mathrm{~g}(40-1300 \mathrm{~g})$.

$15 \%$ (38) simple LHi, $85 \%$ other lap procedures.

LH ERP conversion to TAH after initial lap $0.8 \%$.

Per-op cpx 1 bladder perf repaired.

Mean hosp stay 1.16 days (1-6) $90 \%$ home within $23 \mathrm{hrs}$.

RTT $2.3 \%$ approx 1 /year. Readmissions $7.8 \%$.

Discussion: Perceptions of LH (actual LH ERP)

- takes too long (mean 68 mins)

- high skill set required (not with LHi)

- limited application (flexible technique)

- no benefit regarding LOS (90\% 23 hr stay)

- expensive (cost effective)

- high per-op cpx rates $(<1 \%)$

110

LAPAROSCOPIC HYSTERECTOMY (LH) OR SUB-TOTAL HYSTERECTOMY (LSH) FOR BENIGN INDICATIONS: FEASIBILITY OF A DOUBLE BLIND, RANDOMISED CONTROLLED TRIAL (RCT) TO COMPARE POSTOPERATIVE RECOVERY.

Hysterectomy

A.H. Ahmed*, H. Wisa

${ }^{[1]}$ Medway Maritime Hospital $\sim$ Kent $\sim$ United Kingdom

Summary (4 lines): Feasibility of an RCT to compare 2 methods of Laparoscopic Hysterectomy was studied. 50 women were randomised \& treated over 17 months. We confirm feasibility \& present preliminary data. Introduction: Hysterectomy remains the last resort for a number of benign conditions. Case series suggest recovery after LSH occurs quickly. We conducted a pilot RCT to compare week by week recovery following LH and LSH.

Material and Methods: Premenopausal women scheduled for hysterectomy for benign indications were interviewed. Women were eligible if they were receiving routine cervical screening, had less than stage 2 utero-vaginal prolapse and the uterus was less than 16 weeks size.
Computer generated randomisation was performed at laparoscopy via www.sealed envelope. Primary outcome measures were recruitment \& obstacles to randomisation. Secondary outcome measures were quality of recovery, assessment of mood, urinary, bowel, vaginal \& sexual function.

Results: Recruitment was completed over 15 months. 65 women were interviewed. Surgery was randomised \& completed in 50 participants over 17 months.

Discussion: Recruitment was successful. Less than $14 \%$ of interviwees had trenchant views regarding cervical conservation. Our data confirm study feasibility \& show a trend towards quicker recovery with LSH. Further, we provide pilot data to inform power calculations.

\section{6}

\section{LAPAROSCOPIC HYSTERECTOMY IN ONE DAY SURGERY: PRELIMINARY RESULTS OF A PROSPECTIVE STUDY}

Hysterectomy

O. Donnez*, J. Mitchell, J. Donnez

${ }^{[1]}$ CHU Mont-Godinne $\sim$ Yvoir $\sim$ Belgium

Summary (4 lines): We present here preliminary results of our study about Laparoscopic Hysterectomy (LH) for benign disease in one day surgery,

Introduction: LH is now widely recognized. As the technique is now standardized, we decided to bring it to the one day surgery.

Material and Methods: After approval obtained from the Ethics Committee to conduct a prospective study of all women requiring LH for benign disease, 30 patients were actually eligible for the study. Standardized technique was performed in order to avoid any post operative pain. We analyzed pain score (VAS) from recovery room until one week after surgery, hospitalization length, re-hospitalization rate, satisfaction of the patients and complication rate.

Results: All surgeries were performed in less than one hour. All 30 patients were able to return back home within $5.08 \pm 1$ hours of hospitalization. After 4 hours, all patients had VAS $=4(1.85 \pm 1.05)$. No significant difference in VAS was observed in the evening (phone call) and after one day. $93.3 \%$ of the patients were satisfied and would recommend performing that surgery in one day. No complication occurred.

Discussion: Our results clearly show that performing LH in one day surgery is feasible with good clinical outcome. Standardized technique as well as coordination with anaesthetist and good compliance of the patient is mandatory. Specific indication must be identified in order to offer this possibility to our patients.

\section{8}

\section{POSTOPERATIVE PAIN AFTER VAGINAL AND LAPAROSCOPIC HYSTERECTOMY; SYSTEMATIC REVIEW}

Hysterectomy

\section{H. Haerizadeh*, J. Frappell \\ ${ }^{[1]}$ Department of minimal access gynaecology, Derriford Hospital Plymouth United Kingdom}

Summary (4 lines): In this review, literature has been searched for data comparing pain after vaginal and laparoscopic hysterectomy. Studies are assessed regarding methodology and validity of the results. The results can help gynaecologists in decision making and counselling of patients. Introduction: Significant evidence has shown the benefits of vaginal and laparoscopic approach compared to abdominal approach. 
However, it is less clear which of these two approaches are preferred in individual patients.

Material and Methods: The Cochrane Library, TRIP, Pub Med and trial databases were searched for relevant papers using medical subject heading (MESH) terms "hysterectomy" and "postoperative pain". This was combined with a keyword search including terms "laparoscopic" and "vaginal". Results: Initial search resulted in 465 articles which were narrowed down to 37.

One trial showed increased pain after laparoscopic hysterectomy. Two prospective studies and one trial failed to show any significant difference. However, one retrospective study, six randomised trials and one met analysis have shown laparoscopic hysterectomy is associated with less postoperative pain.

Discussion: Heterogeneity of techniques and patients, reasons for hysterectomy, and presence of prolapse has made most of the studies prone to bias. Moreover, pain is not a primary outcome in most of the studies. High quality studies comparing postoperative pain after vaginal and laparoscopic hysterectomy are needed.

22

\section{SURGICAL SAFETY ASPECTS OF TLH IN VERY OBESE PATIENTS}

Hysterectomy

M. Blikkendaal*, F. Burggraaf, A. Twijnstra, F.W. Jansen

${ }^{[1]}$ Leiden University Medical Center $\sim$ Leiden $\sim$ Netherlands

Summary (4 lines): Present study shows that total laparoscopic hysterectomy (TLH) in obese patients has a relatively high conversion rate, but the incidence of adverse events remains low.

Introduction: Nowadays, in obese patients, gynaecologists still seem to prefer an AH in stead of a TLH. We hypothesize that the well known advantages of TLH over AH also apply to patients with a BMI $>35 \mathrm{~kg} / \mathrm{m} 2$.

Material and Methods: We conducted a retrospective cohort study and included all patients with a BMI $>35 \mathrm{~kg} / \mathrm{m} 2$ who underwent an elective $\mathrm{AH}$ or TLH in a tertiary referral centre between January 2005 and December 2011. AH and TLH (including conversions) were compared.

Results: We included 22 AHs and 32 TLHs. 5 TLHs (15.6 \%) were converted to $\mathrm{AH}$ for strategic reasons. Mean BMI of the total cohort was $39.6 \pm 5.1 \mathrm{~kg} / \mathrm{m} 2$ (range $35-56$ ). In the AH group 7 adverse events $(31.8 \%)$ occurred; in the TLH group $5(15.6 \%)$, none within or after the converted procedures $(\mathrm{p}=.160)$. Operative time was similar (AH: $123 \pm 46 \mathrm{~min}$; TLH $132 \pm 32 \mathrm{~min}, \mathrm{p}=.403$ ). In the AH group blood loss was higher and length of hospital stay was longer (AH: $560 \pm 555 \mathrm{~mL}$; TLH: $200 \pm 182 \mathrm{~mL}, \mathrm{p}=.007$. AH: $5.1 \pm 1.3$ days; TLH: $3.6 \pm 1.5$ days, $\mathrm{p}=.001)$.

Discussion: Despite a relatively high conversion rate, adverse events tended to occur less frequent after TLH. Unlike literature, surgery for TLH did not take longer. Together with a lower blood loss and shorter length of hospital stay, also TLH in obese patients seems to have advantages if vaginal hysterectomy is not feasible.

\section{4}

THE TRANSVAGINAL OPERATIVE ENDOSCOPY (TVOE) AN AN AUTONOMOUS PROCEDURE OR IN VAGINAL HYSTERECTOMIES: A NEW TECHNIQUE AND EQUIPMENT Hysterectomy

\footnotetext{
S. Todorow*

${ }^{[1]}$ Klinik am Hofgarten $\sim$ Bayreuth $\sim$ Germany
}

Summary (4 lines): A new equipment allows the vizualization, preparation and removal of adnexa, ovarian cysts or appendix.

Introduction: In the age of "NOTES" the subumbilical route was mainly explored by surgeons and by gynecologists. The transvaginal route was used in the context of "hybrid approaches" for the extraction of laparoscopically removed organs. Hereby the combination of transvaginal endoscopy in cases of vaginal HEs expands the operative capabilities.

Material and Methods: A new equipment, based on a transrectal endoscopic surgery with a newly developed vaginal cuff, allows a secure visualization, preparation and removal of adnexa, ovarian cysts or appendix. The pelvic anatomy is explored after setting of a transvaginal pseudoperitoneum by transversal culdotomy or after vaginal circumcision in a typical vaginal $\mathrm{HE}$.

Results: The difference to the well known transvaginal hydrolaparocsopy is essential, because this procedure is a genuine innovation, allows a higher degree of complex 3D operative manipulation required during surgery and allowing the simultaneous use of three endoscopic instruments and a $10 \mathrm{~mm}$ optic.

Discussion: The paradigm of the typical gynecological vaginal surgery is widened by the consequent vaginal operative endoscopy without shifting to transumbilical techniques. The new technique amplifies the indication of vaginal surgery to a new dimension.

417

\section{TOTAL LAPAROSCOPIC HYSTERECTOMY WITHOUT} UTERINE MANIPULATOR.

Hysterectomy

A. Kavallaris*, D. Zygouris, N. Chalvatzas, E. Terzakis

${ }^{[1]}$ Department of Gynecologic Oncology, St. Loukas Hospital, Thessaloniki, $\sim$ Greece $\sim$ Thessaloniki $\sim$ Greece

Summary (4 lines): Total Laparoscopic hysterectomy without using manipulator seems to be a feasible, safe and practical surgical method.

Introduction: Hysterectomy remains the most common major gynecological surgery. This is the first study that describes a new technique of total laparoscopic hysterectomy, without using any kind of uterine manipulator. We present a case series of 119 cases of total laparoscopic Hysterectomy without uterine manipulator.

Material and Methods: In the period between October 2008 and March 2012, 119 patients underwent laparoscopic total hysterectomy without using any kind of uterine manipulator. We analyzed the surgical outcome and peri - operative morbidity for all the patients retrospectively. All the operations were performed by the same surgeon with one assistant.

Results: There were no major intraoperative or postoperative complications and the mean operative time was 48 minutes if an adnexectomy was performed and 40 minutes without adnexectomy. The average uterine weight was 280 grams.

Discussion: Total Laparoscopic hysterectomy without using manipulator seems to be a safe and practical surgical method, especially for patients with vaginal stenosis or large uterus. We believe that an advanced option for laparoscopic hysterectomy that reduces surgical time without major complications.

\section{2}

TRANSVERSUS ABDOMINAL PLANE BLOCK, AN EFFECTIVE ANALGESIC STRATEGY FOR PATIENTS UNDERGOING AMBULATORY TOTAL LAPAROSCOPIC HYSTERECTOMY: A RANDOMIZED CONTROLLED TRIAL. Hysterectomy 
G. Calle*, J.F. De Los Rios, C. Lopez, E. Sanchez, E. Serna, J. CastaÑeda, R. Vasquez, A. Arango

${ }^{[1]}$ CLINICA DEL PRADO MEDELLIN Colombia

Summary (4 lines): Radomized placebo controlled trial evaluating the effectiveness of Tranversus Abdominal Plane anesthetic block as an analgesic strategy in patients after total laparoscopic hysterectomy managed on ambulatory basis.

Introduction: The present study shows the results TAP Block applied to patients who underwent total laparoscopic hysterectomy in an outpatient setting.

Material and Methods: 211 women after total laparoscopic hysterectomy were randomized to receive either a bilateral infiltration with $40 \mathrm{~mL}$ of Bupivacaine $0,25 \%$ OR $40 \mathrm{~mL}$ of saline solution. Patients and investigators performing the blockage were blinded to group assignment. At discharge they were asked to rate their pain levels on visual analogue scale. Individuals were followed-up by phone calls at 24,48 and 72 hours after discharge by one of the investigators unaware of group allocation.

Results: Patients who underwent infiltration with anesthetic solution had a significant reduction in pain score at discharge time compared with the placebo group $(p=0,017)$. There were no statistically significant differences in opioid requirements after the procedure between the groups.

Discussion: TAP Block after total laparoscopic hysterectomy reduces the pain score at discharge compared with placebo and permits an optimal ambulatory management.

\section{1}

\section{VIKY UTERINE POSITIONER IN GYNAECOLOGICAL ROBOTIC SURGERY \\ Hysterectomy}

N. Akrivos, P. Barton-smith*

${ }^{[1]}$ Royal Surrey County Hospital Guildford United Kingdom

Summary (4 lines): The aim of this study is to collect data regarding use of the ViKY UP device for uterine or vaginal vault manipulation during robot-assisted gynaecological surgery.

Introduction: A pilot study of uterine manipulation with a new surgical robot for gynaeocological surgery.

Material and Methods: A prospective cohort study of the ViKY Uterine Positioner in combination with the V-Care manipulator.

Results: 36 cases were performed between July 2010 and February 2012. Mean was age 48, BMI 25.7, uterine weight $231 \mathrm{~g}$. Mean ViKY docking time once V-Care inserted, was $4.3 \mathrm{~min}$. No perioperative complications were observed. Adequate mobilization, visualization and range of movements was possible in 81,78 and $61 \%$ of cases respectively with most of the problems arising in cases with uterine weight $>350 \mathrm{~g}$. ViKY positioner was detached and an assistant was required in 3 cases, whilst V-Care came out of the uterus in 1 case. The learning curve led to various adjustments including optimizing patient position, increasing the device range of movement and adjusting device sensitivity.

Discussion: Pilot study results show ViKY UP to be very safe, effective and easy to learn.

508

ADNEXAL MASS PRIOR TO LAPAROSCOPIC EXPLORATION: MENOPAUSAL STATUS MAY GUIDE THE DIAGNOSTIC WORK-UP

Imaging
V. Julia*, R. Marc, D. Herbert, M. Anke, R. Ingo Bernard

${ }^{[1]}$ Jena University Hospital $\sim$ Jena $\sim$ Germany

Summary (4 lines): Assessment of an adnexal mass may be guided by the menopausal state.

Introduction: Assessment of adnexal masses focuses on accurate discrimination between benign and malignant lesions prior to laparoscopic exploration. We evaluated the discriminative power of commonly used diagnostic strategies.

Material and Methods: 1362 surgical explorations were included. Preoperative work-up comprised of an expert sonography and a CA125 measurement. We calculated sensitivity, specificity, PPV, NPV and Cohen's Kappa for each diagnostic test.

Results: In the premenopause, expert sonography reached the highest discriminative power with a kappa value of 0.53 , a PPV of 0.45 and a NPV of 0.99 . In the postmemopausal subgroup, the combinations of expert sonography with a CA 125 serum measurement or a RMI assessment showed the highest discriminative power: The combination of CA 125 and expert sonography reached a PPV of 0.89 and a NPV of 0.97, kappa yielded 0.84 . RMI combined with expert sonography as a triage strategy showed comparable results with a PPV of 0.89 , a NPV of 0.96 and a kappa value of 0.82 .

Discussion: Preoperative assessment of an adnexal mass may be guided by the patient's menopausal state: In premenopausal patients, expert sonography results in an acceptable preoperative differentiation. In postmenopausal patients, the use of triage strategies (CA125 serum measurement or RMI) can be recommended.

\section{6}

\section{APPLICATION OF HYPERSPECTRAL HYSTEROSCOPY IN THE INVESTIGATION OF THE ENDOMETRIAL POLYPS Imaging}

F. Gkrozou*, L. Lavasidis, A. Koutlas, T. Vrekousis, N. Georgiou, V. Kavvadias, I. Navrozoglou, T. Stefos, C. Balas, M. Paschopoulos

${ }^{[1]}$ University Hospital of Ioannina $\sim$ Ioannina $\sim$ Greece

Summary (4 lines): This work attempt to identify and categorize endometrial polyps to functional and non-functional, by using hyperspectral hysteroscopy according to tissue's optical properties.

Introduction: Endometrial polyps may be categorized as functional or non, based on histological analysis. The purpose of this study is the presentation of a novel methodology called hyperspectral hysteroscopy and its application to endometrial polyp characterization with respect to tissue's optical properties.

Material and Methods: Hyperspectral hysteroscopy images were acquired from 31 women with abnormal uterine bleeding who had ultrasonographic evidence of endometrial polyps. For each hyperspectral hysteroscopy image acquired a corresponding pseudocolour map consisting of various colours.

Results: Histological results identified 28 cases of polyps, of them 7 were functional and 21 non-functional. Hyperspectral hysteroscopy images were processed and two associated pseudocolour maps were extracted automatically. The first map consisting of green, red and yellow areas correspond to non functional polyps while the second consisting of purple and blue areas correspond to functional polyps. Hypespectral hysteroscopical analysis was able to recognize 28 cases of polyps, 8 functional and 20 nonfunctional.

Discussion: Hyperspectral hysteroscopy detected the presence of endometrial polyps and their type. This study presents early results of this novel methodology. 
281

\section{RECTOVAGINAL ENDOMETRIOSIS: MRI CORRELATION WITH APPEARANCE AND MANAGEMENT AT LAPAROSCOPY Imaging}

F. Shakir*, N. Waters, G. Marinakis, F. Hussein, A. Kent

${ }^{[1]}$ Royal Surrey County Hospital, Guildford $\sim$ Guildford, Surrey United Kingdom

Summary (4 lines): MRI evaluation of rectovaginal endometriosis can aid in surgical planning. We evaluate MRI correlation with appearance and management at laparoscopy.

Introduction: Assessment of rectovaginal endometriosis is helpful in planning surgical excision. We determine the capability of MRI in diagnosing rectovaginal endometriosis and establish its accuracy at detecting bowel involvement.

Material and Methods: 200 cases of severe endometriosis since 2004-present with rectovaginal involvement found at laparoscopy were evaluated to see if MRI was performed. This was a retrospective study and all cases were performed by the same gynaecologist and colorectal surgeon. The MRI images were interpreted by the same radiologist. 48 patients had MRI imaging and these cases were evaluated to see if MRI correctly identified disease and if so the degree of bowel involvement.

Results: MRI correctly identified disease in 33 cases (sensitivity $69 \%$ ) and of these the level of bowel involvement was correctly identified in $17(52 \%)$

Discussion: MRI maybe a useful adjunct to physical examination and can aid surgical planning in some cases. Delineation of bowel involvement can indicate whether an anterior resection/disc resection or shaving the disease off the bowel is required. However, sensitivity of both identifying disease and the level of bowel involvement is not high and as a consequence we carry out treatment of severe endometriosis as a two stage procedure.

54

CAN TRANSVAGINAL HYDROLAPAROSCOPY REPLACE THE DIAGNOSTIC LAPAROSCOPY WITH CHROMOPERTUBATION IN THE DIAGNOSIS OF TUBAL PATHOLOGY?

Infertility and Reproductive Medicine

R. Coenders-tros*, M.A. Van Kessel, P.E. Adriaensen, B. Mol, C. Kok ${ }^{[1]} V U$ Medisch Centrum $\sim$ Amsterdam $\sim$ Netherlands

Summary (4 lines): Transvaginal hydrolaparoscopy (THL) is used for tubal testing for over 10 years in MMC. With a low complicationrate and a positive predictive value of $80 \%$, THL is a safe and reliable method.

Introduction: THL is used as a method of first choice in tubaltesting in fertility-workup. We compared the findings of an abnormal THL with the findings at laparoscopy.

Material and Methods: In MMC Veldhoven, the Netherlands, THL is used for tubal testing. In this retrospective study, we evaluated all women who received a THL in the last 10 years. The findings of THL were categorized as tubal pathology, endometriosis or adhesions. We compared the findings of laparoscopy in patients with abnormal THL.

Results: We included 677 women who underwent a THL. Access to the pouch of Douglas and visualization was achieved in 643 patients $(95 \%)$. Complications were seen in 5 patients $(0,7 \%$. Of the 643 women who underwent a THL, $197(31 \%)$ were abnormal $(n=107$ tubal pathology, $n=36$ endometriosis, $n=30$ adhaesions and in 18 both.
An incomplete image was seen in 6 cases. A total of 51 (26\%) of the group of abnormal THL subsequently underwent a laparoscopy. The primary diagnosis was confirmed by laparoscopy in 41 cases. That gives the THL a positive predictive value of $80 \%$.

Discussion: THL is a safe and reliable method in the diagnostic workup for subfertility. When THL is abnormal, diagnostic laparoscopy rarely gives new insights.

\section{0}

\section{FERTILITY OUTCOMES AFTER RECTAL SURGERY FOR} SEVERE ENDOMETRIOSIS

Infertility and Reproductive Medicine

N. Waters*, F. Shakir, P. Haines, T. Rockall, A. Kent

${ }^{[1]}$ Royal Surrey Hospital $\sim$ Guildford $\sim$ United Kingdom

Summary (4 lines): Fertility is markedly reduced in endometriosis due to multiple factors. In severe endometriosis it is compounded by adhesions, disruption of anatomy and distortion of the ovarian capsule by endometriomas.

Introduction: We have identified 134 cases of fertility sparing surgery for severe rectovaginal endometriosis. 53 patients presented with primary infertility as an indication for the procedure.

Material and Methods: $53 \%$ of patients had rectal endometriosis shaved off, $40 \%$ of patients had a segmental resection and $7 \%$ had a disc resection.

Results: 27 (pregnancy rate $53 \%$ ) patients had a pregnancy. 22 patients had spontaneous pregnancy with 6 miscarriages, 1 ectopic pregnancy, 13 caesarean sections and 2 vaginal deliveries. There were 5 IVF pregnancies, which resulted in 2 miscarriages and 6 live births ( 1 set of twins and 1 set of triplets). Live term pregnancy birth rate was $33 \%$ and all of pregnancies occurred within first 12 months after surgery.

Discussion: The pregnancy rate and live term pregnancy birth rate is high considering these patients had very severe endometriosis. Most benefit seems to be in the first 12 months following surgery, possibly due to restoration of anatomy, particularly of the fallopian tubes, ovaries and reconstructing a normal Pouch of Douglas. However, these results do not reflect the fact that many women have not been able to conceive and have elected to proceed to pelvic clearance due to severe symptoms.

\section{6}

HYSTEROSCOPIC FINDINGS IN INFERTILE PATIENTS SUBMITTED TO IVF: SHOULD HYSTEROSCOPY BE CONSIDERED A FIRST-STEP TOOL?

Infertility and Reproductive Medicine

A. Ubeda*, M. Simon, E. Fernandez, P. Barri, A. Vazquez

${ }^{[1]}$ Institut Universitari Dexeus $\sim$ Barcelona $\sim$ Spain

Summary (4 lines): Diagnostic hysteroscopy shoul be considered a routine tool in managemnet of infertile pacients prior to any assisted reproductive technique, aminly IVF.

Introduction: We analize our own results in primary infertile couples submitted to IVF in vitro fertilization) procedures, both before and after first IVF cycle.

Material and Methods: In group A, 632 patients submitted to an IVF cycle along two years were previously performed an office hysteroscopy. In group B, hysteroscopy was carried out in 88 patients with a previous failed IVF cycle.

Results: In group A, pathologic findings were diagnosed in 219 hysteroscopies. (34.7\%), being suspicion of endometritis (13.9\%), 
endometrial polyps $(9.3 \%)$ and uterine abnormalities $(6.8 \%)$ the most prevalent pathologies. After either medical or surgical treatment, pregnancy rate of $40.6 \%$ was achieved in previous pathologic hysteroscopies, compared to $31.0 \%$ rate in women with a normal hysteroscopy. In group B, pathologic findings were recorded in 34 cases $(38.6 \%)$, with endometritis suspicion as the most frequent diagnosis $(19.3 \%)$. After suitable treatment, patients with abnormal hysteroscopy reached a $41.2 \%$ pregnancy rate, in front of $29.6 \%$ in women qualified as having normal hysteroscopy.

Discussion: We plead for hysteroscopy to be considered a first-step procedure in the management of patients sent to IVF procedures as treatment of pathologic hysteroscopies allow better pregnancy rates.

\section{4}

\section{OVARIAN FUNCTION AFTER ANTEROGRADE OR RETROGRADE SALPINGECTOMY}

Infertility and Reproductive Medicine

R. Mocciaro*, R. Venturella, M. Morelli, F. Zullo

${ }^{[1]}$ Obstetrics and Gynecology Department, "Magna Graecia" University $\sim$ Catanzaro $\sim$ Italy

\section{Summary (4 lines):}

Introduction: Laparoscopic salpingectomy is considered the procedure of choice to treat tubal ectopic pregnancy (EP). Salpingectomy can be performed by both anterograde or retrograde procedure, but no randomized studies have compared these two techniques yet. The aim of this study was to compare the fertility outcomes after laparoscopic anterograde and retrograde salpingectomy in women with EP.

Material and Methods: This is a retrospective analysis of 47 women submitted to IVF and treated for EP, 24 patients underwent to laparoscopic anterograde (LAS) and 23 patients retrograde (LRS) salpingectomy.

Results: FSH levels were lower and AMH levels were higher in LAS than in RAS group, but this difference were not statistically significant. There were no difference in the E2 levels between groups. Furthermore, median basal antral follicle number, median ovarian diameter and median peak systolic velocity were not significantly different between the groups. No significant difference in term of operative time between groups was found. ? Hb was higher in LAS than LRS, but this difference was not statistically significant. None surgical complications were recorded in the two groups.

Discussion: Anterograde and retrograde salpingectomy techniques are both safe in terms of preservation of ovarian function and surgical complications. The anterograde salpingectomy, however, should be probably preferred because it seems to allow resection as close as possible to tubal surface.

\section{3}

\section{PATHOGENETICALLY SUBSTANTIATED METHOD OF SURGICAL TREATMENT OF POLYCYSTIC OVARY SYNDROME (PCOS)}

Infertility and Reproductive Medicine

D. Simrok-starcheva*, V. Simrok

${ }^{[1]}$ State Institution "Lugansk State Medical University" Lugansk Ukraine

Summary (4 lines): This research paper presents the results of ovarian reserve estimation for 72 women with the Polycystic Ovary Syndrome (PCOS) who have undergone thermokauterisation and drilling by laser (Ho-Yag). Gentlest method of surgical treatment is drilling by HoYag laser.
Introduction: The operation is a successful approach to the treatment of patients with PCOS, which allows to reduce the severity of hirsutism, to repair menstrual rhythm and fertility in most patients.

Material and Methods: We inspected 72 women of reproductive age with PCOS who underwent endoscopic treatment. The main group included 39 patients who were operated by laser drilling with Holmium Laser (Ho-Yag), the comparison group (TC) included 33women, who underwent by the method of thermal cauterization of ovaries (TCO). we ivesigated level of follicle-stimulating hormone, luteotropin hormone, prolactin hormone, estradiol, progesterone, dehydroepiandrosterone sulfate, the level of anti-Müllerian hormone and the level of inhibin-A.

Results: in the early follicular phase the levels of inhibin A in the main group remained low, and then began to rise at the end of follicular phase and peaked in the middle of luteal phase.

Discussion: The advantage of laser drilling is the most efficient and attenuated method of surgical intervention on ovaries

151

RETROSPECTIVE ANALYSIS OF TRANS-ABDOMINAL
CERVICAL CERCLAGE: COMPARISON OF OPEN VERSUS
LAPAROSCOPIC APPROACH Infertility and Reproductive Medicine

S. Cohen*, E. Sandberg, C. Hill-lydecker, G.M. Jonsdottir, F. Vree, J.I. Einarsson

${ }^{[1]}$ Brigham and Women's Hospital $\sim$ Boston $\sim$ United States

Summary (4 lines): This retrospective study aims to compare the outcomes of transabdominal cerclage placement done via laparoscopy to the ones done via laparotomy.

Introduction: 77 patients who underwent a transabdominal cerclage placement done via laparoscopy $(n=48)$ or laparotomy $(n=29)$ between 2007 and 2012 at Brigham and Women's Hospital are included. Material and Methods: Charts were reviewed for baseline and perioperative characteristics, and a follow-up mixed-mode survey regarding pregnancy outcomes was administered.

Results: Initial survey response rate was $31.17 \%$; twenty patients had at least one documented pregnancy with their cerclage in place (7 laparotomy patients, $(24.13 \%) ; 13$ laparoscopic patients, $(27.08 \%)$ ). The pregnancy events were as follows: for the cases done via laparotomy (C-section, $n=9$, miscarriage, $n=2)$ and for the ones done via laparoscopy (C-section, $n=9$, miscarriage, $n=3$ and $n=1$ still pregnant at the time of the study). As of preliminary analysis, no significant differences were found between the two groups regarding baseline characteristics (age, BMI, race, parity) or perioperative outcomes (operative time, estimated blood loss, intraoperative or postoperative complications).

Discussion: Transabdominal cerclage placement done via laparoscopy a safe alternative to approach via laparotomy with similar pregnancy outcomes.

\section{1}

\section{UNCATEGORISED VAGINAL AND UTERINE CONGENITAL ANOMALIES}

Infertility and Reproductive Medicine

B. Molnár G.*
${ }^{[1]}$ Dept. of $O B / G Y N$ University of Szeged $\sim$ Szeged $\sim$ Hungary

Summary (4 lines): Two cases of uncategorised vaginal and uterine congenital anomalies are discussed. 
Introduction: The Müllerian duct and the lower genital tract malformations are often discovered only when the patient's infertility or sterility has to be investigated. The grouping of malformations makes it easier to establish a comparable diagnosis. Such a grouping has been created by the AFS; widely used but too detailed and therefore impractical. The new categorisation of the uterine malformations by the ESGE Working Group is much easier to use. However, there are cases that do not fit either the categorisation system of AFS or of ESGE. Among these cases two will be discussed by analysing some of the difficulties in diagnosis and the therapeutic options.

Material and Methods: During the last twenty years the author performed approximately 3000 laparoscopies and 1000 hysteroscopies. Most of the patients suffered from infertility. In this patient population there were two cases with vagina fully divided by a septum and uteruses similar to uterus duplex, but no abnormality by laparoscopy.

Results: If one of the uterine cavities is bigger than the other, embryos should be transferred to the larger cavity. Even patients with serious congenital abnormality can be able to carry pregnancy to term.

Discussion: These two cases also point out the importance of endoscopic examination of infertile patients prior to IVF-ET.

\section{4}

\section{UTERINE MYOME (FIBROID) AS A FACTOR FOR FALLOPIAN TUBES AND UNEXPLANED INFERTILITY.}

Infertility and Reproductive Medicine

G. Brodsky*

${ }^{[1]}$ Practice Obestetrics and Gynaecology $\sim$ Munich $\sim$ Germany

Summary (4 lines): Our objective was to investigate the effekt of uterine myoma (fibroid) on the morphological structure of the uterine tube.

Introduction: Uterine fibroids are the most common tumors found in women. However, the effekt of their presence on fertility is still subject to controversy.

Material and Methods: A retrospective, comparative study has been carried out on 100 uterine tubes of 50 women at reproductive age in uterus myoma group. The data was compared with data from 60 cases from control group without genital tract pathology.

Results: In the preliminary analysis of the 50 cases (100 Failopian tubes) with uterine myoma, we found morphological abnormalities Fallopian tubes, which were classified, as mild, moderate and severe. We found evidence of severe morphoiogical abnormalities in $50 \mathrm{Fal}-$ lopian tubes $(50 \%)$. In 48 Fallopian tubes $(48 \%)$ we observed moderate abnormalities, and in 2 Fallopian tubes (2\%) - mild grade of abnormalities.

Discussion: We conclude, that evidence based changes in morphological structutres of the uterine tube in uterus myoma group could play a significant role as a morphological substrat for clinical data associated with significantly lower outcome of assisted reproductive technology. Our findings have implication on the use of diagnostic and therapeutic strategies in women with uterus myoma who seek fertility assesment and treatment.

\section{0}

\section{ADVANTAGES OF NEW MINIATURIZED INSTRUMENTS Innovation in Surgery}

G. Gubbini, G. Gonzales*

${ }^{[1]}$ Division of Obstetrics and Gynaecology - Hospital Madre Fortunata Toniolo $\sim$ Bologna $\sim$ Italy
Summary (4 lines): The"Mini Hystero-Resectoscope Gubbini" has introduced a new way to perform operative procedures. This tool reduce operative time and discomfort in the patient and no cervical dilatation is performed.

Introduction: The Mini Hystero-Resectoscope offers the advantage that can be used for ambulatory procedures (outpatient settings) as well under general anaesthesia (inpatient settings).

Material and Methods: The "Gubbini System" is a continuous-flow hysteroscope or a mini-resectoscope (Tontarra Medizintechnik GmbH Germany). The outer sheath is a $16 \mathrm{Fr} / 5.4 \mathrm{~mm}$ diameter and $194 \mathrm{~mm}$ length. Introducing into the outer sheath a working sheath with $5 \mathrm{Fr}$. instrument channel and a $2.9 \mathrm{~mm}$ diameter/ $30^{\circ}$ telescope, semi-rigid instruments (biopsy forceps, scissors, mono or bipolar electrodes) can be used as office hysteroscope. Mini-resectoscope is assembled introducing into the outer sheath an inner sheath together with a Bi-power working element with a $2.9 \mathrm{~mm}$ diameter/ $0^{\circ}$ telescope and mono or bipolar electrodes or cold loops.

Results: In inpatient settings the dilatation of cervix uteri is avoid, reducing any possible complication. In outpatient settings surgical gesture is easier and the removal of debris by the loop is quicker if compared to hysteroscopic surgery; therefore surgical times are reduced as well as patient discomfort.

Discussion: This tool combines the advantages of the resectoscopic surgical technique with the versatility of a mini-instrument. Specific indications are endocervical diseases (polyps and polyposis) and isthmocele because of their unfavourable anatomic position. Endometrial polyps, submucous myomas or uterine septa/subsepta, synechiae or metropathia haemorragica can be as well treated. The limit depends on the size of the pathology and the skill of the surgeon. In narrow uteri, where the surgical area is reduced, in virgo patients (vaginal approach and no cervical dilatation), in external os stenosis.

87

\section{DIRECT LAPAROSCOPE TROCAR INSERTION WITHOUT PRIORPNEUMOPERITONEUM}

Innovation in Surgery

R. Markovic*, L. Vuckovic, V. Stamenic

${ }^{[1]}$ General Hospital Uzice $\sim$ Uzice $\sim$ Serbia

Summary (4 lines): The results of direct trocar insertion without prior establishment of pneumoperitoneum are documented in 1000 consecutive laparoscopies.

Introduction: Direct trocar insertion without previous pneumoperitoneum was reported to be a safe alternative to Veress needle insertion. We carried out this study to compare the ease of use, safety, and efficacy of direct trocar insertion with elevation of anterior abdominal wall with the non-dominant hand and both assistants hands and blind insertion of the Veress needle in laparoscopic surgery.

Material and Methods: From January2010 to April 2012 we retrospectively studied 1000 consecutive patients.

Results: Creation of pneumoperitoneum with DTI was feasible in $100 \%$ of patients. There were no cases of technical failure. No complication.

Discussion: We conclude that, DTI at laparoscopy is a safe alternative to the VN technique. Direct trocar insertion with elevation of anterior abdominal wall with the non-dominant hand and both assistants hands is an easy, safe, fast and effective technique.

\section{5}

LAPAROSCOPIC UTERINE SLING, USING MERSILENE TAPE, FOR FERTILITY SPARING TREATMENT OF UTERINE PROLAPSE: FIRST REPORTED CASES OF PREGNANCY OUTCOME AND FOLLOW UP

Innovation in Surgery 
P. Wipplinger*, A. Cutner, D.L. Byrne

${ }^{[1]}$ Royal Cornwall Hospital $\sim$ Truro $\sim$ United Kingdom

Summary (4 lines): Laparoscopic uterine sling corrects prolapse and preserves fertility. We present three successful pregnancies with postnatal follow up.

Introduction: Utero-vaginal prolapse requiring treatment is common. Laparoscopic sacrohysteropexy with mesh encircling the cervix, will correct prolapse but may compromise the uterine blood supply in pregnancy. In 2007 laparoscopic uterine sling was registered as a new procedure with NICE and undertaken on women at the Royal Cornwall Hospital with severe uterine prolapse who wished to conceive.

Material and Methods: The procedure involves exposure of the sacral promontory bilaterally. A Mersiline tape is passed through the back of the cervix twice, looped around each uterosacral ligament and anchored with titanium screws to the promontory.

Results: Three patients have spontaneously conceived. One is in her 3rd trimester with a well-supported uterus. Two patients delivered by elective caesarean section. Intra-operative findings were a peritonealised tape, no adhesions and a well-supported uterus. One patient's 6 month follow-up confirmed the uterus in the normal position.

Discussion: Laparoscopic sacrohysteropexy is safe and effective. Busby $\&$ Broome presented a successful pregnancy in 2010 on a woman who had a laparoscopic sacrohysteropexy. These are the first 3 cases in the world with pregnancies following a Mersilene tape uterine sling procedure.

26

TRANSVAGINAL NATURAL-ORIFICE TRANSLUMINAL ENDOSCOPIC SURGERY (NOTES) IN ADNEXAL PROCEDURES Innovation in Surgery

K. Wu*, C. Lee, H. Su, C. Yen

${ }^{[1]}$ Chang Gung Memorial Hospital at Linkou Tao-Yuan Taiwan, Province of China

Summary (4 lines): Natural orifice transluminal endoscopic surgery (NOTES) is a newly developed surgery but there are few reports in gynecology. Thus, we presented our experience of NOTES in adnexal surgery to evaluate its feasibility and safety.

Introduction: NOTES uses the natural orifices of the body surface as surgical channels to avoid scars on abdominal wall. With experiences in laparoendoscopic single-site surgery (LESS), we used this technique to achieve a vaginal access and made the concept of transvaginal NOTES a reality.

Material and Methods: NOTES was started with posterior colpotomy then a port in the vagina was established, as used in LESS. Conventional laparoscopic instruments were used. Tubal sterilization, salpingectomy, or enucleation was done as in LESS. The procedure was ended with closure of the colpotomy wound.

Results: From August 2010 to June 2011, 10 patients were enrolled, including tubal sterilization in 3 , salpingectomy because of ectopic pregnancy in 3, and ovarian tumor enucleation in 4. NOTES was completed in 9 and failed in one with a parietal peritoneal tumor. Operative time was in 18 to 30 minutes in tubal sterilization, 62 to 116 in salpingectomies, and 64 to 162 in enucleation with blood loss $<50 \mathrm{~mL}$.

Discussion: In conclusion, our method offers a new vision of vaginal surgery, and avoids wounds on the abdominal wall. As transvaginal NOTES is still in its infancy, it should be used only in highly selected patients.
57

\section{AQUADISSECTION OF MYOMA WITHOUT VASOPRESSIN} USE

Myomectomy

R. Vukasin*

${ }^{[1]}$ Health Center Valjevo $\sim$ Valjevo $\sim$ Serbia

Summary (4 lines): Laparoscopic dissection of uterine myoma performed with aquadissection without vasopressin was reviewed. Use of saline irrigation after instrumental dissection appears to be sufficient by avoiding adverse effects of vasopressin.

Introduction: Vasopressin can be used during myomectomy for a blood loss reduce. Severe hemodynamic complications can arise. Tissue coagulation and section followed by aquadisection helps once instrumentally started excision to be faster.

Material and Methods: Patients (N 43) in a reproductive age with a different size and localisation of myoma uteri underwent laparoscopic myomectomy. Aquadissection with bipolar energy coagulation\&section during operation without vasopressin administration was performed.

Results: It was easier to achieve complete dissection by performing aquadissection only after right identification of space between myometrial layer and myoma. Time of operation was $20 \%$ shorter. Even a very big "iceberg" myomas were succesfully dissected without harm to a endometrial tissue. Bleeding was succesfully controled.

Discussion: There is a need to respect anatomic features during any kind of operation procedure. It is unlike that complet dissection of any tissue will be obtained only with high presure solutions instillation. Aquadissection after initial identification of tissue structures throughout use of energy supplied laparoscopic instruments can override few time gaps during intervention.

264

\section{CLINICAL APPLICATION OF HIGH INTENSITY FOCUSED ULTRASOUND (HIFU) THERAPY ON UTERINE MYOMAS Myomectomy}

J. Rodriguez*, J. Isern, A. Pessarrodona, E. Vallejo, J. Cassadó, L. Quintas

${ }^{[1]}$ University Hospital Mutua Terrassa $\sim$ Barcelona $\sim$ Spain

Summary (4 lines): We evaluate our results in a 263 patients series with uterine myoma treated with ultrasound-guided focused ultrasound surgery (USgFUS).

Introduction: USgFUS is a completely non-invasive, out-patient procedure for conservative treatment of uterine myomas.

Material and Methods: Three hundred and eight treatments in 263 patients were done. After treatment, we used contrast-enhanced MR images to measure the mean nonperfused volume ratio. The volume change ratio was calculated at 3, 6, 12 and 24 months. Symptom severity score (SSS) was examined before and after treatment at 3, 6, 12 and 24 months. Complications and need of surgical procedure after HIFU treatment were also recorded.

Results: Treatment was achieved in 251 patients $(95.5 \%)$ and it wasn't achieve in 12 patients $(4.5 \%)$. In $82 \%$ of patients $(206 / 251)$ treatment was effective and $18 \%(45 / 251)$ required a surgical procedure. The mean myoma volume was $212 \mathrm{~cm} 3$. The average nonperfused volume was $55 \%$ immediately after treatment. Treated myomas decreased in volume by $48 \%, 55 \%$ and $62 \%$ at 6,12 and 24 months, respectively. There was a $38 \%$ reduction in median SSS at 6 months and $58 \%$ at 12 
and 24 months posttreatment. Of 40 patients who had complications only one had a major complication (third-grade skin burn).

Discussion: According to our experience HIFU therapy seems to be an effective and safe treatment for ablation of uterine myomas.

\section{6}

\section{LAPAROSCOPIC ASSISTED TRANSVAGINAL MYOMECTOMY AFTER HYSTERECTOMY \\ Myomectomy}

\author{
L. Meglic*, B. Cvjeticanin, S. Drusany \\ ${ }^{[1]}$ Klinicni center Ljubljana, Ginekološka klinika $\sim$ Ljubljana $\sim$ Slovenia
}

Summary (4 lines): Laparoscopic assisted transvaginal myomectomy still has benefits of minimally invasive surgery. In the case report we are describing colpotomy and myomectomy after hysterectomy.

Introduction: Parasitic myomas can be detached from the uterus and can be found after morcellations or they can grow without any connections to the uterus or previous surgeries.

Material and Methods: A 47- year old multiparous woman came again to our office because her gynecologist found a mass $6 \mathrm{~cm}$ in diameter on ultrasound in her lower abdomen on the right side, looking like myoma. She had abdominal hysterectomy without adnexa fourteen years ago because of myomathose uterus but she also had lymphoma excised on the back couple of years ago. Because of her history it was uncertain if that is a benign process.

Results: We inspected the peritoneal cavity laparoscopicaly, located the myoma near lig. rotundum on the right side. We extracted the myoma from retoperitoneum. Because of the previous diseases we didn't morcelate it. We opened a vagina between bladder and rectum and extracted the tumor. The histology after showed that it was myoma.

Discussion: Laparoscopic assisted transvaginal has been first described in 1997. It offers advantages to removing myomas trough laparotomy after laparoscopy. In this case the colpotomy was a bit more complicated because years ago hysterectomy was performed and the path between bladder and rectum was not clearly outlined.

511

LAPAROSCOPIC MYOMECTOMY IN PERI- AND POSTMENOPAUSAL WOMEN IS SAFE, EFFICACIOUS AND ASSOCIATED WITH LONG-TERM PATIENT SATISFACTION

Myomectomy

R. Marc, R. Mathias*, M. Anke, D. Herbert, R. Ingo Bernard

${ }^{[1]}$ Jena University Hospital Jena $\sim$ Germany

Summary (4 lines): Complication rates for laparoscopic myomectomy appear to be comparably in pre- and postmenopausal patients.

Introduction: Women in the peri- and post-menopause may ask for uterus-preserving therapy options when presenting with benign uterine disorders such as symptomatic fibroids. The aim of this study was to analyze safety and outcome of laparoscopic myomectomy (LM) beyond the reproductive period.

Material and Methods: We evaluated the outcome for a total of 451 patients: 85 peri- or post-menopausal women as group $\mathrm{A}$ and, for reference, 366 premenopausal women in group B, who received LM.

Results: Number of fibroids removed and size of the leading fibroid were similar in both groups. Time of surgery was different in group A, at $102.8 \mathrm{~min}$, compared to group B, $128.6 \mathrm{~min}(\mathrm{p}<0.01)$. Rates of intra- $(2.21 \%$ overall) and post-operative complications $(6.22 \%$ overall) were comparable for both groups. Patient's satisfaction was high in both subgroups with a significantly lower number of relapses in group A $(3.5 \%)$ compared to group B $(11.2 \%)$.

Discussion: Data from our study indicate that LM in the peri- and postmenopause is a safe and efficient treatment option if a patient opts to preserve her uterus.

512

\section{LONG-TERM RISK OF RECURRENCE OF FIBROIDS AFTER LAPROSCOPIC MYOMECTOMY \\ Myomectomy}

R. Marc*, D. Herbert, M. Anke, R. Ingo Bernard

${ }^{[1]}$ Jena University Hospital $\sim$ Jena $\sim$ Germany

Summary (4 lines): Patients with multiple myomas should be counseled thoroughly about the risk of recurrence prior to LM.

Introduction: The aim of this study was to estimate the risk of recurrence after laparoscopic myomectomy (LM) and to identify factors, which contribute to the rate of fibroid relapse.

Material and Methods: Retrospective cohort study at a tertiary referral center. A total of 331 patients treated by LM from 1996 through 2003. 224 patients consented to participate in our follow-up survey in 2009. Clinical symptomatic recurrence of a leiomyoma was defined as a relapse. Recur-rence rates were compared by using the Cox regression model for factors such as patients' age at time of surgery, number of fibroids, seize of leading fibroid removed, anatomical local-ization of fibroids, duration of surgery and BMI

Results: In 224 patients, we observed 75 recurrences. The cumulative risk of recurrence was $4.9 \%$ at 24 months, $21.4 \%$ at 60 months and $31.7 \%$ at 96 months. Patients' age and anatomical degree of severity in leiomyoma manifestation at time of surgery were identified as the main risk factors for a symptomatic recurrence after LM.

Discussion: Patients with multiple myomas should be counseled thoroughly about the risk of recurrence prior to LM. The low observed recurrence rate in peri -and postmenopausal patients of our study, might serve as an argument to offer LM as an uterus-preserving surgical alternative beyond the reproductive period.

\section{4}

\section{UTERINE LEIOMYOMAS: SONOGRAPHY IN PREDICTION OF COMPLICATIONS DURING OR AFTER MYOMECTOMY Myomectomy}

\author{
S. Peetermans, J. Verguts* \\ ${ }^{[1]}$ UZ Leuven $\sim$ Leuven $\sim$ Belgium
}

Summary (4 lines): Transvaginal sonography can be used in the prediction of complications during and after myomectomy.

Introduction: To evaluate the value of transvaginal sonography in the prediction of complications during or after myomectomy.

Material and Methods: Retrospective study of myomectomies in the Leuven University Hospital from 2009 till 2011. Hysteroscopic, laparoscopic and laparotomic myomectomies were analyzed (group 1, 2, 3). 5 myomal characteristics were collected: number of myomas, volume, vascularization, location and calcifications. We registered surgical time, bloodloss, embolisms, hospitalstay, pre- and postoperative $\mathrm{Hb}$-value, visceral damage (i.e. perforation), fever, hemorrhage, blood transfusion and second-look surgery.

Results: 295 hysteroscopic, 60 laparoscopic and 41 laparotomic myomectomies were analyzed. Complication rates were $11.86 \%$, $16.67 \%$ and $36.59 \%$ respectively. In group 1 , association with 
the volume and outcome were found. With higher vascularization, the blood loss during surgery increased. In group 2, with increasing size the difference between pre- and postoperative $\mathrm{Hb}$-value and the hospital stay increased. In group 3, number of removed myomas was predictable for length of hospital stay and risk of complications.

Discussion: With hysteroscopic or laparoscopic resection the volume of the myoma is a significant predictor of complications. With laparotomic resection, the number of myomas to be removed, can predict the outcome.

\section{8}

\section{CONFIRMATION TEST FOR ESSURE@ TUBAL STERILISATION IN FRANCE.}

Office \& Diagnostic Hysteroscopy

P. Panel*, S. Heckel, J.B. Engrand, R. Hsiung, A. Agostini, V. Villefranque, R. Kutnaorsky, P. Lopes, H. Martigny, F. Marchand, C. Chis, J. Coudray, C. Dhainault, H. Fernandez

${ }^{[1]} \mathrm{CH}$ de Versailles $\sim$ versailles $\sim$ France

Summary (4 lines): This is part of a French multicentre cohort study on Essure ${ }^{\circledR}$ in 11 facilities from September 2008 through Mars 2010. Introduction: Aim: to analyse modalities and results of control at three months of hysteroscopic tubal sterilisation by Essure ${ }^{\circledR}$. Standard control is based on X ray for normal procedure or hysterosalpingography (HSG) in case of difficulties during Essure ${ }^{\circledR}$ placement, or when X ray didn't reach acceptable criteria.

Material and Methods: 2535 patients attempted placements of Essure $^{\circledR}$, successfully in 2366 case. $2149(90.8 \%)$ patients had control at three month. X ray was performed in 1812 cases $(84.3 \%)$ and HSG in 332 cases $(15.4 \%)$. Nevertheless, ultrasound was performed alone in 144 cases $(6.7 \%)$ and associated with $\mathrm{X}$ ray for 959 women (44.6\%).

Results: For bilateral standard cases, HSG should have been performed in $19.2 \%$ of cases. When $\mathrm{X}$ ray didn't meet criteria, ultrasound confirm good placement in $71.2 \%$ of the cases.

For bilateral difficult cases, half patients had Xray + ultrasound. When $\mathrm{X}$ ray was correct, ultrasound showed a wrong placement of implants for 3 patients ; when $\mathrm{X}$ ray didn't meet criteria, ultrasound confirm good placement in $2 / 3$ of the cases.

Discussion: Physicians concluded to a good result in 2099 cases (97.7\%).

If ultrasound was systematically associated with X ray, HSG should be required in only $8.7 \%$ of bilateral cases $(5.8 \%$ of normal placements and $27.4 \%$ of difficult placements).

\section{8}

\section{DIAGNOSTIC STRATEGY FOR RECURRENT POST- MENOPAUSAL BLEEDING}

Office \& Diagnostic Hysteroscopy

P. Smith*, J. Clark

${ }^{[1]}$ Birmingham Women's Hospital $\sim$ Birmingham $\sim$ United Kingdom

Summary (4 lines): Hysteroscopy based diagnostic pathway for those patients that present with recurrent post-menopausal bleeding (PMB). Introduction: For most, first line investigation for PMB will involve ultrasound of the endometrial thickness which will then dictate whether further investigation, normally in the form of an endometrial biopsy. The management of patients that re-present with PMB is more ambiguous. All investigations for PMB carry a false negative rate so it is important to re-investigate. However, evidence on how they should be investigated and at what time interval is lacking.

Material and Methods: Data was prospectively collected for 1938 consecutive patients in PMB clinic. Of these, 106 had previously been investigated.

Results: The chance of having cancer or hyperplasia with atypia is significantly less if a patient re-presents after having normal investigations ( 0 cases from 106 patients presenting with recurrent PMB). The patients are significantly more likely to have endometrial polyps $28.3 \%$ ( $p$ value 0.02 ). Discussion: Although our results suggest the risk of endometrial cancer in PMB is extremely low in patients who have previously had normal investigations, it is important to appreciate that all investigations carry a false negative rate and this is reflected in other research in PMB. Although polyps are less serious than cancer or hyperplasia, we believe a treatment pathway should be used that would aim to detect and ultimately treat all these pathologies.

\section{0}

ESSURE® IMPLANTS FOR TUBAL STERILISATION IN FRANCE : SUCCES II

Office \& Diagnostic Hysteroscopy

P. Panel*, S. Heckel, J.B. Engrand, R. Hsiung, A. Agostini, V. Villefranque, R. Kutnaorsky, P. Lopes, H. Martigny, F. Marchand, C. Chis, J. Coudray, C. Dhainault, H. Fernandez

${ }^{[1]} \mathrm{CH}$ de Versailles $\sim$ Le Chesnay $\sim$ France

Summary (4 lines): This prospective observational cohort study covered 11 facilities from September 2008 to Mars 2010 and collected data on placement, confirmation test, long term outcome and satisfaction. Introduction: The aim of this study was to evaluate terms of use, failure and pain predictive factors, confirmation test modalities and 5 years follow-up of sterilization with Essure ${ }^{\circledR}$.

Material and Methods: This study included 2573 patients and 2535 attempted placements of Essure ${ }^{\circledR}$ micro-insert. In 38 cases $(1,5 \%)$, procedure didn't occur because of lack of visibility.

Results: The procedure was done with no anaesthesia in $81 \%$ of cases. Before procedure, analgesics were given to $86 \%$ of patients (NSAID: $42,4 \%$; combination with NSAID: $44,7 \%$; non NSAID: $12,8 \%$ ). Uterus was retroverted in $18 \%$ of cases. Both ostia were visualised in $91,2 \%$ of cases. In 2366 cases (93,3\%), the first placement attempt was successful. VAS pain measurement at the time of hysteroscope introduction in the cervix was $<=3$ for $72 \%$ of women. VAS pain measurement at the time of implant placement was $<=3$ for $58 \%$ of women. Predictive factors for pain were past tubal surgery, PID, endometriosis, no use of analgesic or NSAID alone. Predictive factors for failure were lack of ostia visualisation, retroverted uterus, hospital, insufficient premedication and pain. $97 \%$ of women were satisfied.

Discussion: Whereas placement rate and satisfaction was high, Analgesia for selected patients may improve satisfaction.

\section{5}

ESSURE $®$ PERMANENT BIRTH CONTROL EFFECTIVENESS: A SEVEN YEARS SURVEY

Office \& Diagnostic Hysteroscopy

S. Jost, C. Huchon, G. Legendre, A. Le Tohic, H. Fernandez, P. Panel* ${ }^{[1]}$ Department of Obstetrics and Gynecology, Centre Hospitalier de Versailles $\sim 78150$ Le Chesnay $\sim$ France

Summary (4 lines): As Essure sterilization appears to be most effective, pregnancy still may occur. 
Introduction: The aim of this study is to report French pregnancies after Essure hysteroscopic sterilization.

Material and Methods: Retrospective national survey between January 2003 to September 2010. Data were collected in two ways:

- a mail-in questionnaire addressed to surgeons performing Essure procedures in France

- pregnancies reported directly to the manufacturer.

Results: Results:

The surgeons contacted in our study were responsible for $63.4 \%$ of all Essure procedures in France. 58 cases of unintended pregnancies after Essure sterilization were reported during the study period. The main causes of unintended pregnancies were patients non-compliance to the procedure (22 pregnancies, $38 \%$ of cases) and misinterpretation at the 3rd-month confirmation test (19 cases, $33 \%)$. Other causes were physician's deviation from protocol (10 cases) and undetected pre-procedure pregnancy (3 cases). Three pregnancies happened before the 3-months confirmation test. Regarding the number of kits sold during the period, the estimated pregnancy rate was between 1.07 (CI95\%; 0.8-1.5) and 1.09/1000 CI95\%; 0.9-1.4).

Discussion: Conclusion: Improving patient education, modalities and physician knowledge concerning the third month confirmation test, may reduce the pregnancy risk after hysteroscopic sterilization.

327

\section{LEARNING CURVE OF HYSTEROSCOPIC PLACEMENT OF TUBAL STERILIZATION MICRO-INSERTS BY 15 GYNAECOLOGISTS IN THE NETHERLANDS \\ Office \& Diagnostic Hysteroscopy}

J. Janse, T. Pattij*, R. Eijkemans, F. Broekmans, S. Veersema, H. Schreuder

${ }^{[1]}$ University Medical Centre Utrecht $\sim$ Utrecht $\sim$ Netherlands

Summary (4 lines): Prospective multicenter cohort study, assessing the learning curve of the Essure ${ }^{\mathbb{R}}$ sterilization method of 15 gynaecologists in the Netherlands. A learning curve was seen in a significant decrease of procedure time with increasing experience.

Introduction: The aim of this study is to evaluate the learning curve of hysteroscopic placement of tubal sterilization micro-inserts.

Material and Methods: Fifteen gynaecologists from ten community hospitals in the Netherlands recorded all Essure ${ }^{\circledR}$ sterilizations from their very first placement. A total of 631 women were included and data concerning patient, placement and follow-up were available. Statistical analysis was executed by a Generalised Estimating Equation and adjustment for possible confounding factors was performed.

Results: Bilateral successful placement was achieved in $479(77.8 \%)$ patients at first attempt and in $44(7.1 \%)$ at second attempt. Mean procedural time was 8.49 min $(\mathrm{SD} \pm 3.9)$. A learning curve was seen in a significant decrease of procedure time. The effect extends to $16-20$ cases and is followed by a plateau phase of the subsequent 60 cases. No clear learning curve was found for successful bilateral placement, pain scores or complication rate.

Discussion: A learning curve for hysteroscopic tubal sterilization was seen for procedure time, but successful placement, pain scores and complication rate were not influenced by increasing experience.

\section{2}

OFFICE DIAGNOSTIC-OPERATIVE HYSTEROSCOPY: ANALYSIS OF OVER 5800 CASES

Office \& Diagnostic Hysteroscopy
J. Santos*, A. Sousa, C. Ferreira, N. Nogueira Martins, A. Pipa, F. Nogueira Martins

${ }^{[1]}$ Hospital São Teotónio Viseu Portugal

Summary (4 lines): The authors present the results of eleven years of experience in outpatient hysteroscopy.

Introduction: Hysteroscopy is a well-known endoscopic method of evaluation of the uterine cavity. Diagnostic and operative hysteroscopy (in selected procedures) can be accomplished in an outpatient setting, avoiding patient hospitalization and use of general anesthesia. The ability to convert diagnostic hysteroscopy in an operative procedure is a valuable asset in an office setting.

Material and Methods: From May 1996 to April 2012, 5817 women were submitted to outpatient hysteroscopy at our Department. Procedures were performed through vaginoscopic approach, using saline distension medium, without anesthesia.

Results: Among the 5817 women who underwent hysteroscopy, 4300 presented an intracavitary anomaly. Biopsy was performed in 2248 patients and 901 were treated for benign intrauterine pathology in the same office hysteroscopic procedure. Indications for hysteroscopy included suspected intracavitary lesion (3429), postmenopausal metrorrhagia (961) and abnormal uterine bleeding in non-menopausal women (647). With this minimally invasive procedure 122 cases of endometrial adenocarcinoma were diagnosed and histologically confirmed.

Discussion: An adequate technique performed by an experienced team enables not only diagnosis of intrauterine pathology but also several operative procedures in an office setting with excellent cost-benefit, patient acceptance and high efficacy.

\section{7}

\section{THE ROLE OF HYSTEROSCOPY IN ULTRASONOGRAPHIC ENDOMETRIAL THICKENING \\ Office \& Diagnostic Hysteroscopy}

C. Ferreira, J. Santos*, V. Silva, N. Nogueira Martins, A. Pipa, F. Nogueira Martins

${ }^{[1]}$ São Teotónio Hospital Viseu Viseu Portugal

Summary (4 lines): Retrospective study of hysteroscopic findings in 1375 women with thickened endometrium.

Introduction: Endometrial thickening on ultrasound is a marker for intra-uterine pathology. If present, a diagnostic hysteroscopy should be offered. This study aimed to evaluate the hysteroscopic findings in thickened endometrium patients.

Material and Methods: Retrospective study of 1375 women with thickened endometrium on ultrasound, who underwent diagnostic hysteroscopy. Endometrial sampling was performed whenever indicated.

Results: The mean age was 59.9 years. At hysteroscopy, the endometrium had a normal appearance in 259 cases $(18.8 \%)$. The main hysteroscopic findings were polyps ( $\mathrm{n}=719 ; 52.3 \%$ ), endometrial hypertrophy $(\mathrm{n}=178 ; 12.9 \%)$, submucosal fibroids $(\mathrm{n}=72 ; 5.2 \%)$, malignancy $(n=63 ; 4.6 \%)$ and uterine malformations $(n=32 ; 2.3 \%)$. Of the 403 conclusive endometrial samplings, normal features were present in 141 cases $(34.9 \%)$. Polyps were diagnosed in 130 cases (32.3\%), endometrial hyperplasia in $78(19.4 \%)$, endometrial carcinoma in $52(12.9 \%)$ and carcinoma of the endocervix in $2(0.5 \%)$. Of the 54 cases of carcinoma diagnosed bysampling, 41 were suspected on hysteroscopy (sensitivity: $75.9 \%$ )

Discussion: Polyps were the most common hysteroscopic and histological findings. Hysteroscopy had a high sensitivity for endometrial carcinoma, which was diagnosed histologically in $12.9 \%$ of cases. Hysteroscopy is a well-tolerated and effective method for the assessment of women with endometrial thickening. 
466

THE USE OF OFFICE HYSTEROSOCPY TO TREAT PATIENTS WITH SYMPTOMS OF PROLONGED POSTMENSTRUAL UTERINE BLEEDING CAUSED BY ISTHMIC MUCUS POUCH (ISTHMOCELE)

Office \& Diagnostic Hysteroscopy

J.W. Meyer*, F. Meyer, M. Barros, J. Martinez De Oliveira, J. Moutinho

${ }^{[1]}$ Centro Hospitalar Cova da Beira $\sim$ Covilha $\sim$ Portugal

Summary (4 lines): Hysteroscopy technique to treat 5 patients with symptomatic isthmic mucus pouch.

Introduction: 5 women aged between 42 and 48, complaining of postmenstrual uterine bleeding, dysmenorrhoea and prolonged menstrual cycles were submited to transvaginal ultrassound evaluation that revealed cystic image in the endocervical/endometrial line compatible with a mucus pouch. Moreover, they had a very narrow (punctate) cervical ostium. They had at least one previous cesarean delivery and none of them had any previous vaginal delivery.

Material and Methods: Hysteroscopy with vaginoscopic approach was performed in outpatient setting. With a Twizzle Versapoint Electrode, the punctate cervical ostium was expanded with a cross shape cut and a new and larger cervical ostium was constructed. When reached the mucus pouch, a light brown thick fluid spilled out through the canal, and the pouch was "washed" with a a continuous flow system.

Results: Transvaginal ultrasound was performed 4 weeks and 6 months later and revealed complete resolution of the mucus pouch and menstrual symptoms.

Discussion: Vaginoscopic hysteroscopy might represent a valuable technique to treat symptomatic isthmic mucus pouch and the etiology of these pouches may be linked not only to the fragility of the uterus wall (caused by cesarean scar)but also to the increasing pressure caused by inadequate menstrual flow in punctate cervical ostia

384

100 CASES OF MINILAPAROSCOPIC TREATMENT FOR EARLY STAGE ENDOMETRIAL CANCER: COMPARISON WITH CONVENTIONAL LAPAROSCOPY

Oncology

G. Bogani*, S. Uccella, A. Cromi, J. Casarin, G. Marchitelli, G. Fabio

${ }^{[1]}$ university of Insubria $\sim$ varese $\sim$ Italy

Summary (4 lines): Miniaturized $3 \mathrm{~mm}$ laparoscopic instruments have been developed to reduce incision-related morbidity of conventional laparoscopy. We report our encouraging experience of 100 cases of minilaparoscopic staging for early stage endometrial cancer (EC).

Introduction: We investigated the feasibility of minilaparoscopy in the surgical treatment of EC and to compare its outcomes with those of conventional laparoscopy.

Material and Methods: 100 patients who underwent minilaparoscopic staging for EC were compared with a control group of 195 women who had staging with conventional laparoscopy.

Results: Demographic characteristics were similar between groups. Operative time, blood loss and hospital stay were lower in the minilaparoscopy than in the conventional group $(\mathrm{p}<0.01)$. No conversion to conventional laparoscopy or open surgery occurred in the experimental group, while 6 conversions to open surgery were recorded among patients in the control group $(\mathrm{p}=0.09)$. No difference in complication rate was recorded.

Discussion: Our data suggest that minilaparoscopy is a safe and adequate surgical option for EC patients, with the potential to further decrease invasiveness of the conventional surgery. The beneficial effects of $3 \mathrm{~mm}$ instruments shown in this series are likely determined by the expertise of the surgeon since minilaparoscopic was introduced at our department after having acquired adequate background in conventional laparosopy.

93

BORDERLINE TUMORS OF THE OVARY: KEY HOLE SURGERY OFFERS THE KEY FOR THEIR IN TIME DIAGNOSIS

Oncology

G. Pados, S. Masouridou*, D. Tsolakidis, A. Makedos, T. Zarampoukas, B. Tarlatzis

${ }^{[1]} 1$ st Department of Obstetrics \& Gynaecology, School of Medicine, Aristotle University of Thessaloniki, "Papageorgiou General Hospital" Thessaloniki Greece

Summary (4 lines): Early detection of borderline tumors and staging is essential as these tumors usually involve women of childbearing age thus offering the opportunity of fertility-sparing surgery. Laparoscopy provides accurate diagnosis, staging and simultaneous treatment.

Introduction: Our aim was to examine the value of diagnostic/operative laparoscopy in identifying unexpected borderline tumors in women who underwent laparoscopy for benign ovarian tumors and to review the characteristics of the women.

Material and Methods: From 10/1992 to 12/2010, 1,332 laparoscopies were done and 1,838 ovarian cysts removed. In case of suspicious findings in the U/S, tumor markers, color Doppler and MRI followed. During laparoscopy, any solid component or papilla was sent for rapid frozen section.

Results: Borderline tumors were diagnosed in 8 out of 1,332 women $(0.6 \%)$. Bilateral tumors were found in 2 women. In 2 cases, diagnosis was done during diagnostic laparoscopy, while in 6 women during operative laparoscopy. The mean age of the 8 patients was $28.75 \pm$ 9.27 years and mean diameter of the ovarian cysts was $5.1 \pm 1.7 \mathrm{~cm}$.

Discussion: Risk of failure to diagnose malignancy could be minimized with careful patient selection preoperatively. Adequate training in laparoscopic oncology contributes in accurate diagnosis of unexpected ovarian borderline tumors encountered in diagnostic/operative laparoscopy, as well as synchronous safe and complete oncologic surgical management.

\section{7}

\section{CONVERSIONS TO LAPAROTOMY IN A GYNECOLOGY ONCOLOGY SERIES \\ Oncology}

T. Bernal Arahal*, R. Ruiz Sautua, M. Avlia Calle, C. Goiri Little, L. Goyeneche Lasaga, L. Martínez Gallardo

${ }^{[1]}$ Hospital Universitario Donostia $\sim$ San Sebastian $\sim$ Spain

Summary (4 lines): Laparoscopic access is a viable option in gynecologic oncological surgery. We report a low rate of laparotomic conversion: $1,64 \%$.

Introduction: We review the indications for conversion to laparotomy in our oncologic surgery series.

Material and Methods: Between November 2007 and December 2011 we performed 366 laparoscopies for gynecological cancer. This 
included 158 bilateral pelvic lymphadenectomies, 29 transperitoneal and 104 retroperitoneal aortocava lymphadenectomies.

Results: Six patients requiered conversion to laparotomy for the following indications:

1: transperitoneal aortocava lymphadenectomy impossible due to obesity.

2: hemorrage during biopsy in advanced ovarian cancer.

3: previously irradiated myomatous uterus. Weight: $923 \mathrm{~g}$.

4: uterus with leiomiosarcoma, impossible to lateralize.

5: vena cava lesion.

6: severe pulmonary hypertension.

Discussion: Our conversion rate is $1,64 \%$. In 2 cases the cause was uterine size, which complicates the procedure, especially as morcellation is contraindicated.

One patient with a BMI of 36 required laprotomy to complete a transperitoneal aortocava lymphadenectomy, which is made more difficult by obesity. There were 2 surgical complications that required laparotomy: one vena cava lesion and a hemorrage after biopsy in an advanced ovarian cancer. The sixth case can be attributed to inadequate preanesthetic evaluation. Our results support the feasibility of laparoscopy in gynecologic oncological surgery.

\section{2}

\section{ENDOMETRIAL CANCER REVIEW IN OUR COMMUNITY HOSPITAL: FROM 1996 TO 2011. \\ Oncology}

M.D. Rodríguez Garnica*, V. García Pineda, C. De Valle Corredor, A. Zapico Goñi, V. Marcos González

${ }^{[1]}$ Príncipe de Asturias University Hospital $\sim$ Alcalá de Henares, Madrid. Spain

Summary (4 lines): We reviewed 301 patients with endometrial carcinoma treated in our hospital.

Introduction: We reviewed our case-serie of endometrial cancer.

Material and Methods: We executed a descriptive and retrospective study in 301 patients with endometrial carcinoma in the Príncipe de Asturias University Hospital, Alcalá de Henares, Madrid, between 1996 and 2011.

Results: $82 \%$ (247) had an early stage, $18 \%$ presented advanced stages (54). $4 \%$ had endometrioid adenocarcinoma (263).

The surgery in $77.8 \%$ consisted of hysterectomy, bilateral salpingooophorectomy and lymphadenectomy (234). The mean number of lymph nodes obtained was $8.10 \pm 0.361(0-28)$. The surgical approach used in $72.1 \%$ of cases (217) was by laparoscopy and by laparotomy in $23.6 \%$ (71), with a conversion to laparotomy rate of $7 \%$ (21).

Our surgical protocol is: by laparoscopic approach we carry out a hysterectomy and bilateral salpingo-oophorectomy. Uterus is sent to intraoperative study. If we have a stage Ia G1G2 a pelvic lymphadenectomy is not performed. In case of a stage $\mathrm{Ib}$ G1G2 a pelvic lymphadenectomy is performed with intraoperative study of lymph nodes. If they are positive a para-aortic lymphadenectomy is performed.

In the follow up, $14.3 \%$ of patients died (43), $7.3 \%$ had relapses (22) and $78.4 \%$ are disease free $(236)$.

Discussion: The laparoscopic surgical treatment should be considered today as the first option.

360

EVOLUTION OF THE TECHNIQUE IN NERVESPARING LAPAROSCOPIC RADICAL HYSTERECTOMY: FEASIBILITY AND MORBIDITY THROUGH THE LEARNING CURVE

Oncology
J. Gilabert-estelles*, J.J. Rodenas, A. Viegas, S. Costa, F. Coloma, J. Gilabert-aguilar

${ }^{[1]}$ Hospital General Universitario $\sim$ Valencia $\sim$ Spain

Summary (4 lines): We describe the modifications in our laparoscopic technique through our learning curve, the feasibility and the clinical outcomes in the treatment of the early stage cervical cancer.

Introduction: Alteration of bladder, rectal and sexual function in patients after radical hysterectomy for cervical cancer is usually observed if no preservation of pelvic autonomic nerves is performed.

Material and Methods: We evaluated a retrospective cohort of patients affected of cervical cancer. Since 2001 and 20048 patients (Group A) were treated by type II combined laparoscopic-assisted radical vaginal hysterectomy (LARVH). 22 patients (Group B) were treated by total laparoscopic radical hysterectomy (TLRH). Since January 2006, 45 patients (Group C), were treated by TLRH with pelvic splachnic nerve preservation.

Results: Recovery of the rectal voiding function was faster in group $\mathrm{C}$ in comparison with groups A and B. Group A presented a higher recurrence rate but no other difference was observed in other oncologic parameters between groups. Patient satisfaction using a validated scale for cervical cancer showed better results in the nervesparing group. Discussion: Preservation of pelvic splachnic nerves at the cardinal ligament offers a faster recovery of the rectal function and a better satisfaction in the patients affected of cervical cancer.

\section{9}

LAPAROSCOPIC NERVE - SPARING RADICAL HYSTERECTOMY IN PATIENTS WITH CERVICAL CANCER FIGO STAGE IB2 - IIA.

Oncology

A. Kavallaris*, D. Zygouris, N. Chalvatzas, E. Terzakis

${ }^{[1]}$ Department of Gynecologic Oncology, St. Loukas Hospital, Thessaloniki, Greece

Summary (4 lines): Laparoscopic nerve sparing radical hysterectomy is feasible for cervical cancer FIGO stage Ib2 - II.

Introduction: Despite the widespread application of Papanicolaou cytology examination in preventing cancer cervical cancer, a large number of cases are diagnosed at an advanced stage.

Material and Methods: We present five cases of patients with cervical cancer FIGO stage Ib2 - IIa. Four patients were stage Ib2 (volume $5 \mathrm{~cm}, 4.5 \mathrm{~cm}, 4.5 \mathrm{~cm}$ and $8 \mathrm{~cm}$ respectively) and one patient was stage IIa (vaginal infiltration and tumor size $4 \mathrm{~cm}$ ). All the patients underwent total laparoscopic nerve sparing radical hysterectomy and pelvic and para - aortic lymphadenectomy.

Results: The mean operative time was 270 minutes and estimated blood loss was in all cases $<200 \mathrm{ml}$. The number of lymph nodes removed was 41 (1 infiltrated), 48 (0 infiltrated), 36(0 infiltrated) and 36 ( 3 infiltrated) and 41 (no infiltration) respectively, while the surgical margins were free of disease. The catheter was removed the third post operative day, with residual urine volume less than $50 \mathrm{ml}$.

Discussion: The surgical treatment seems to be feasible for cervical cancer FIGO stage Ib2 - II. Laparoscopic nerve sparing radical hysterectomy is a safe approach, minimizing the complications rate and morbidity. It provides oncological safety and the removal of tumors larger than $4 \mathrm{~cm}$, with disease free surgical margins in a reasonable operative time.

272

LAPAROSCOPIC SENTINEL LYMPH NODE (SLN) DETECTION IN CERVICAL CARCINOMA

Oncology 
M. Perovic*, A.J. Papadopoulos, M. Gautam, M. Kodampur, S. Attard-montalto, J. Donaldson, O. Devaja

${ }^{[1]}$ Department of Gynaecological Oncology, West Kent Cancer Centre Maidstone $\sim$ Maidstone $\sim$ United Kingdom

Summary (4 lines): Laparoscopic SLN detection is safe and accurate in assessing the lymph node status in cervical cancer.

Introduction: The study aim was to assess the detection rate for laparoscopic sentinel lymph node (SLN) in cervical carcinoma.

Material and Methods: This was a prospective 5 year study recruiting 50 consecutive women undergoing surgery for cervical carcinoma at the West Kent Gynaecological Oncology Centre, Maidstone, UK. SLN detection was performed using the combined method (Tc-99 $\mathrm{m}$ and Methylene blue dye). All patients underwent SLN removal with bilateral pelvic lymphadenectomy (BPLND) along with surgical treatment of the primary tumour.

Results: The SLN was detected using the Gama camera in $48 / 50$ (96\%), the Gamma probe in 48/50 (96\%) and blue dye alone in 46/ 50 patients $(92 \%)$. The combined method detected the SLN in all women $50 / 50(100 \%)$. The SLN were detected bilaterally in $38 / 50$ women (76\%). The median SLN count was 3 nodes (range1-7) and the median non-sentinel lymph node count was 19 nodes (range $8-35$ ). All 50 women with sentinel node detection also underwent BPLND, and in none of these cases was a benign sentinel node associated with a malignant non-sentinel node (100\% negative predictive value).

Discussion: Laparoscopic SLN detection is safe and accurate in assessing the lymph node status of women with cervical carcinoma undergoing surgical staging. The combined method utilising Tc-99 $\mathrm{m}$ and Methylene blue dye injection is the most reliable method.

\section{9}

\section{LAPAROSCOPIC VS. OPEN RADICAL HYSTERECTOMY AFTER NEOADJUVANT CHEMOTHERAPY FOR CERVICAL CANCER}

Oncology

S. Uccella*, A. Cromi, G. Bogani, J. Casarin, G. Marchitelli, F. Ghezzi

${ }^{[1]}$ univerisity of Insubria $\sim$ Varese $\sim$ Italy

Summary (4 lines): Laparoscopic approach seems a valuable alternative to open surgery for patients with locally advanced cervical cancer who receive neoadjuvant chemotherapy (NACT)

Introduction: Radical hysterectomy in patients treated with NACT can be technically challenging. We investigated whether surgical outcomes of laparoscopic radical hysterectomy (LRH) and open radical hysterectomy (RAH) are equivalent in the setting of NACT

Material and Methods: Five gynecologic oncology service prospective databases were searched for stage IB2-IIB cervical cancer patients undergoing surgery after NACT. LRH and RAH patients were compared with respect to perioperative outcomes and mid-term survival. Adjustment for potential selection bias in surgical approach was made with propensity score (PS) matching

Results: LRH cases $(n=68)$ were associated with lower stage, lower grade tumors compared with ARH group $(n=273)$. When patients were grouped by stage at presentation (IB2-IIA and IIB), complication rates and perioperative outcomes were equivalent between groups. LRH offered less blood loss, lower transfusion rate, and shorter hospitalizations. These differences remained significant after PS matching. In the PS matched cohort survival did not differ by surgical group

Discussion: Laparoscopy following NACT allows the same complication rates and survival than open radical hysterectomy, with significant benefits in terms of blood loss, transfusion rate and hospital stay
175

\section{LAPAROSCOPY IN EVALUATION OF OPTIMAL RESECTABILITY IN ADVANCED OVARIAN CANCER Oncology}

B. Kobal*, B. Cvjeticanin, M. Barbic, L. Meglic, M. Jakimovska

${ }^{[1]}$ University medical centre $\sim$ Ljubljana $\sim$ Slovenia

Summary (4 lines): Evaluation of laparoscopy as a predictor for optimal resectability in patients with advanced ovarian cancer.

Introduction: Optimal cytoreduction remains the most important prognostic factor for survival in patients with advanced ovarian cancer (AOC). Material and Methods: 39 patients with clinically AOC had laparoscopic evaluation of disease spread in pelvis (adnexa, peritoneum, serosis and mesenterium of sigmoid colon) and upper abdomen (peritoneum, diaphragm, omentum, paracolic regions, serosa and mesenterium of intestines). The same regions were examined at laparotomy. Degree of agreement using kappa value was calculated. and accuracy of laparoscopic prediction for not optimal cytoreduction was measured by the area under the ROC curve.

Results: Kappa values $>0.80$ were present for adnexal pathology, upper abdomen peritoneum, diaphragm and omentum. The kappa values for pelvic peritoneal infiltration, serosa of sigmoid colon, appendix, paracolic regions and serosa of of intestines were $0.67,0,55$, 0,53, 053 and 0, 68 respectivelyt. Laparoscopic evaluation of sigmoid colon mesenterium and mesenterium of small intestines expressed low kappa values ie; 0,33 and 0,25 when compared to laparotomy finding. The area under the ROC curve for laparoscopic prediction of not optimal cytoreduction was 86,6 evaluating method as good (B).

Discussion: According to our results laparoscopy can be included in pretreatment evaluation of optimal resectability.

\section{1}

\section{LESSONS FROM OUR FIRST 100 RETROPERITONEAL AORTOCAVA LYMPHADENECTOMIES}

Oncology

C. Goiri Little*, R. Ruiz Sautua, M. Avlia Calle, T. Bernal Arahal, L. Goyeneche Lasaga

${ }^{[1]}$ Hospital Universitario Donostia $\sim$ San Sebastian $\sim$ Spain

Summary (4 lines): We present the results of our first 100 retroperitoneal aortocava lymphadenectomies (RACL) and technical tips that we have found useful and use systematically.

Introduction: Aortocava lymphadenectomy is a common procedure in gynecologic oncologic surgery. It can be performed laparoscopically with either a transperitoneal or retroperitoneal access. The latter is less frequently used, as it requires specific technical knowledge, though it provides certain advantages.

Material and Methods: We include 100 consecutive RACL performed between May 2008 and December 2011. There were no conversions to laparotomy. Data were collected prospectively.

Results: Average lymph node (LN) yield was 12,8 . Average hospital stay was 4 days (other procedures were also performed). Obesity did not influence results. With experience, LN node yield increased. Vascular complications remained similar.

Discussion: Most gynecologists are unfamiliar with retroperitoneal access, though it presents multiple advantages for aortic LN dissection: it does not require Trendelenburg and the surgical field is well defined and not impaired by intestines.

However it obliges us to learn a different access technique and new anatomical references. 
Good results can be achieved with relatively few cases.

In this video we present some tips that aid in adequate trocar insertion and completing adequate dissection up to the renal vein

\section{6}

\section{NERVE-SPARING MINILAPAROSCOPIC VS. CONVENTIONAL LAPAROSCOPIC RADICAL HYSTERECTOMY PLUS SYSTEMATIC PELVIC LYMPHADENECTOMY IN CERVICAL CANCER PATIENTS \\ Oncology}

S. Uccella*, G. Bogani, A. Cromi, G. Formenti, N. Piazza, F. Ghezzi

${ }^{[1]}$ Univerisity of Insubria $\sim$ Varese $\sim$ Italy

Summary (4 lines): Nerve-sparing minilaparoscopic radical hysterectomy (NS-mLRH) + pelvic lymphadenectomy (PL) represents an option to reduce surgical related morbidity in cervical cancer patients. Introduction: To present our experience with NS-mLRH + PL for the surgical treatment of cervical cancer and to compare outcomes with conventional laparoscopy.

Material and Methods: Data of 94 women who underwent minimally invasive radical hysterectomy for cervical cancer were prospectively collected. 17 consecutive NS-mLRHs + PL (entirely performed using $3 \mathrm{~mm}$ ancillary ports) were compared with the previous 77 patients who had standard laparoscopy.

Results: NS-mLRH was successfully accomplished in all cases with no conversion to standard laparoscopy or open surgery. 2 $(2.6 \%)$ conversions to open surgery occurred in the standard laparoscopy group. Surgical characteristics and complication rates were similar between the two groups. No differences in the amount of parametrial and vaginal tissue removed were observed. The number of lymph nodes retrieved through minilaparoscopy was higher than conventional laparoscopy (30[26-38] vs. 22[849]; $\mathrm{P}=0.002$ ). However, no difference was observed when the analysis was restricted to the last 10 conventional procedures (30[26-38] vs. 29 [24-49]; $\mathrm{P}=0.8$ ).

Discussion: Our data show that, in experienced hands, NS-mLRH with $\mathrm{PL}$ is a feasible and safe procedure.

170

\section{PREOPERATIVE STAGING OF ENDOMETRIAL CANCER Oncology}

V. García Pineda*, M.D. Rodríguez Garnica, C. De Valle Corredor, Á. Zapico Goñi, L. Fernández Múñoz

${ }^{[1]}$ Príncipe de Asturias Hospital $\sim$ Spain $\sim$ Spain

Summary (4 lines): The aim of this research is study the infradiagnosis rate of preoperative staging of endometrial carcinoma

Introduction: Preoperative evaluation of myometrial invasion by magnetic resonance imaging (MRI) and histologic grade analysis allows patient selection for pelvic or para-aortic lymph node sampling while obviating the need for radical surgery in patients with low risk disease.

Material and Methods: In this retrospective research we study 86 women operated of endometrial cancer with IaG1-2 preoperative stage, from 1996 to 2011. We performed hysterectomy, double adnexectomy and pelvic limphadenectomy in $83,7 \%$ of patients $(n=72)$, and hysterectomy and double adnexectomy in the remaining $16,3 \%$. We calculate the underdiagnosis rate of preoperative staging by MRI and endometrial biopsy.

Results: Regarding the definitive study of myometrial invasion, the underdiagnosis rate of MRI (>Ia stage) is $27,9 \%(n=24)$. The intraoperative evaluation of myometrial invasion reduces this rate to $23,2 \%(n=20)$. With respect to definitive histological grade, the underdiagnosis rate, just considering G3, is $7 \%(n=6)$. The combination of both factors at preoperative staging, suppose an underdiagnosis rate of $27,9 \%(n=24)$.

Discussion: According to our results, it would have been necessary reoperate to $27,9 \%$ of patients for lymph node evaluation, despite combine diagnostic methods such as MRI, endometrial biopsy and intraoperative evaluation at preoperative staging.

\section{1}

\section{RADICAL VAGINAL TRACHELECTOMY COMBINED BY LAPAROSCOPIC ABDOMINAL CERCLAGE \\ Oncology}

L. Meglic*, T. Tomazevic, M. Cavic, B. Kobal, B. Cvjeticanin, M. Barbic

${ }^{[1]}$ University clinical center Ljubljana, Dep. Ob/Gyn $\sim$ Ljubljana Slovenia

Summary (4 lines): Radical vaginal trachelectomy (RVT) is a fertilitypreserving procedure for treating cancers of the cervix. Complications following RVT are second trimester miscarriage and premature delivery. Our solution is laparoscopic abdominal cerclage (LAC). All our patients, who wanted to conceive, delivered healthy child at 38 th week of gestation. Introduction: RVT with laparoscopic pelvic lymphadenectomy is a fertility-preserving procedure for treating small invasive cancers of the cervix. To avoid second trimester miscarriage and premature delivery some centers perform closure of the cervix after conception. We perform LAC before conception.

Material and Methods: Only patients with cervical cancer stages IA1, IA2 and partially IB1 were recruited for RVT. It was performed in younger patients who wanted to preserve fertility. In the cases of second trimester miscarriages vaginal cerclage (Shirodkar suture) were replaced by LAC with tape.

Results: We performed RVT in 14 patients. 2 of them wanted to concieve.

In first patient pregnancy ended in 17th week in second in 18th week of gestation with miscarriage.

LAC was performed in both patients, both delivered healthy child in 38 th week of gestation by cesarean section.

We found no recurrences of cervical cancer.

Discussion: In our cases LAC proved to be efficient procedure to overcome second trimester miscarriage. More cases are needed for combination of procedures (RVT and LAC) to be proven as a treatment of choice.

380

\section{REPRODUCTIVE AND ONCOLOGIC OUTCOMES OF MINIMALLY INVASIVE FERTILITY-SPARING TREATMENT OF EARLY OVARIAN CANCER}

Oncology

G. Bogani*, S. Uccella, A. Cromi, G. Formenti, N. Piazza, F. Ghezzi ${ }^{[1]}$ Univerisity of Insubria $\sim$ Varese $\sim$ Italy

Summary (4 lines): Young women affected by early stage ovarian cancer (EOC) who undergo minimally-invasive fertility-sparing surgery have reproductive potential preservation without compromising survival. Introduction: We investigated safety and reproductive outcomes of fertility-sparing comprehensive staging for apparent EOC patients undergoing laparoscopy. 
Material and Methods: Between 2001 and 2011 data about consecutive women undergoing fertility sparing laparoscopic surgery for EOC were prospectively collected.

Results: 40 cases (9 malignant and 31 borderline ovarian cancers) were included. No conversion to open surgery was necessary. Intra-operative rupture of the mass occurred in $6(15 \%)$ cases. 2 $(22.2 \%)$ patients with invasive cancer and 4(12.9\%) with borderline tumors were upstaged. $6(15 \%)$ patients had adjuvant chemotherapy. All women reported normal menstrual cycle after surgery. $12(30 \%)$ pregnancies occurred in $11(27.5 \%)$ women. 3 $(25 \%)$ pregnancies were obtained with assisted reproduction. The median time between surgery and pregnancy was 6 months (range,2-18). 1(11.1\%) and 2(6.4\%) recurrences of disease were observed in women affected by malignant and borderline EOC, respectively $(\mathrm{p}=1.0)$. No patient died of disease.

Discussion: Our results suggest that conservative laparoscopic surgery for young EOC patients preserves fertility potential. Moreover, minimally invasive fertility-sparing surgery does not seem to affect survival outcomes.

\section{9}

\section{SURGICAL MANAGEMENT OF OVARIAN CYSTS IN PREMENOPAUSAL WOMEN \\ Oncology}

C. Aiken, N. Deakin*, M. Mehasseb, P. Baldwin

${ }^{[1]}$ Addenbrooke's Hospital $\sim$ Cambridge $\sim$ United Kingdom

Summary (4 lines): We evaluated current practice in managing ovarian cysts in premenopausal patients in light of recently published standards (RCOG/BSGE Guideline No.62). The high incidence of laparoscopic management is encouraging, and does not appear to confer a high risk of unexpected malignancy.

Introduction: The majority of premenopausal ovarian cysts are benign, creating difficulty in accurately detecting the small but important minority who may have malignant disease.

Material and Methods: We reviewed 250 premenopausal women who underwent primary surgery for ovarian cysts in a tertiary centre over 2 years. The ultrasound score (USS) and risk of malignancy index (RMI) were calculated retrospectively.

Results: $4.8 \%(12 / 250)$ patients had a malignant diagnosis. $77 \%$ of patients were managed laparoscopically with only 3 patients who had unexpected malignancy. Of the 13 laparotomies performed in patients with a high risk of malignancy, 6 ultimately had a benign diagnosis. RMI was not clearly documented in clinical correspondence but retrospective calculation was possible for $54 \%$ - mainly through lack of US data ( 79 cases) and lack of CA125 (36 cases). Inappropriate measurement of CA125 was performed in 27 of 50 women with simple cysts $<5 \mathrm{~cm}$.

Discussion: Adoption of new BSGE guidelines will reduce overtreatment of ovarian cysts where conservative management is appropriate. Adequate ultrasound records, appropriate biochemical tests and calculation of RMI will facilitate management decisions

472

FERTILITY AND OBSTETRICAL OUTCOME AFTER BIPOLAR HYSTEROSCOPIC SEPTOPLASTY: A SERIES OF 70 PROCEDURES

Operative Hysteroscopy

J. Sroussi*, M. Perrin, L. Pratt, A. De Quillacq, M. Piketty, J. Benifla

${ }^{[1]}$ Hopital Trousseau $\sim$ Paris $\sim$ France
Summary (4 lines): Septate uterus negatively affects reproductive outcome. In a retrospective series of 70 bipolar hysteroscopic septoplasty in 52 women, we reported an improve of fertility and obstetrical outcome after surgery.

Introduction: In the general population, the uterine septum prevalence is probably underestimated, but was respectively reported to $3 \%$ and $1 \%$ in women population with recurrent abortion and infertility. The aim of our study was to evaluate fertility and obstetrical outcome before and after bipolar hysteroscopic septoplasty.

Material and Methods: In this retrospective study, we included 70 procedures in 52 women with septate uterus from 2003 to 2011. All surgeries were performed with Versapoint ${ }^{\circledR}$ electrodes.

Results: In all pregnancies, live birth rate was increased after surgery $(25.3 \%$ versus $73.7 \%, p<0.0001)$ and miscarriage rate was decreased $(55.4 \%$ versus $15.8 \%, \mathrm{p}<0.0001)$. Obstetrical prognosis is improved after surgery with a decrease of cesarean section $(57.1 \%$ versus $28.6 \%, \mathrm{p}=0.038)$ and fetal malpresentations $(57.1 \%$ versus $10.7 \%$, $\mathrm{p}<0.0001)$. A complete uterine septum section in the first surgery was performed in $71.2 \%(37 / 52)$. A second-look hysteroscopy was realized in 13 patients. Only one uterine perforation was diagnosed in our series $(1.4 \%)$.

Discussion: Bipolar hysteroscopic septoplasty improves overall pregnancy prognosis, with low complication rate.

335

HYSTEROSCOPIC REMOVAL OF INTRAUTERINE POLYPS AND SUBMUCOSAL FIBROIDS WITH THE USE OF MYOSURE MORCELLATOR VERSUS TRUCLEAR. THE GREEK EXPERIENCE

Operative Hysteroscopy

D. Mathiopoulos*

${ }^{[1]}$ Leto - Rea - Iaso Maternity Hospitals $\sim$ Athens $\sim$ Greece

Summary (4 lines): A new technique, the Hysteroscopic Morcellator using mechanical cutting to reduce the tumour into small chips out of the uterine cavity by aspiration

Introduction: The aim of this presentation is to report our experience of the use MyoSure (Hollogic )Morcellator for removal intrauterine myomas and submucous fibroids type $0 \& 1$ versus Truclear (Smith\& Nephew) Morcellator.

Material and Methods: This study involved 50 patients with abnormal uterine bleeding (menomitroragia, metroragia) and infertility who were diagnosed with intrauterine myomas or submucous fibroids type 0 \& 1. in 25 women underwent operative hysteroscopy with MyoSure (Hollogic )Morcellator $6 \mathrm{~mm}$ and in 25 patients underwent hysteroscopy with Truclear (Smith\& Nephew) Morcellator $9 \mathrm{~mm}$ in diameter Results: Evaluation and comparison criteria were operative time, fluid loss visibility, serious complications, learning curve, feasibility and cost effectiveness. In all these, the MyoSure proved superior to Truclear

Discussion: The demonstrated evaluation of MyoSure has to be validated in a prospective randomised trial

\section{5}

HYSTEROSCOPIC MORCELLATOR: A NEW APPROACH TO REMOVAL OF PLACENTAL REMNANTS.

Operative Hysteroscopy

T. Hamerlynck*, M. Blikkendaal, B. Schoot, M.H. Emanuel, F.W. Jansen

${ }^{[1]}$ University Hospital $\sim$ Gent $\sim$ Belgium 
Summary (4 lines): The hysteroscopic morcellator appears to be an effective and safe alternative in removing placental remnants.

Introduction: The aim of this study is to evaluate the use of the hysteroscopic morcellator (HM) as an alternative treatment modality in the management of placental remnants.

Material and Methods: We retrospectively reviewed all medical charts of women diagnosed with placental remnants after miscarriage, termination of pregnancy, or delivery who were treated with the HM in three hospitals in the Netherlands between January 2005 and May 2010.

Results: We analyzed 128 procedures in 124 different women. In $69 \%$ of the cases, treatment with the HM was a secondary last resort intervention. A markedly high percentage of patients (13\%) had either a history of Asherman's syndrome, or was diagnosed with, and treated for, intrauterine adhesions at the time of the hysteroscopic procedure. In $91 \%$ of the cases the placental remnants were removed successfully. Eighty-six percent of procedures were uneventful. Second look hysteroscopy showed occurrence of intrauterine adhesions in 19 out of 77 patients, of whom 8 had a history of Asherman's syndrome.

Discussion: The HM is an effective and safe alternative in the management of placental remnants, even in patients with a history of Asherman's syndrome. Prospective research comparing hysteroscopic techniques is needed to further investigate the role of different treatment modalities.

260

HYSTEROSCOPIC TREATMENT (ISTHMOPLASTY) OF CESAREAN DELIVERY-INDUCED ISTHMOCELE: TECHNIQUE EVOLUTION

Operative Hysteroscopy

G. Gubbini, E. Marra, M. Filippeschi, P. Florio*

[1] "Madre Fortunata Toniolo" Hospital Bologna Italy

Summary (4 lines): The hysteroscopic treatment of isthmocele is resectoscopic.

Introduction: The aim was to evaluate the outcomes associated with hysteroscopic treatment of isthmocele, by using a new continuous flow mini-resectoscope $16 \mathrm{Fr}$ by vaginoscopic approach, with $0^{\circ}$ angle of view rod lens optic, monopolar and pure cutting electrical current, and sorbitol-mannitol solution as a medium of distension.

Material and Methods: This is a prospective observational controlled study in tertiary referral centers for gynecologic care. 160 symptomatic pts (28-42 yrs)with isthmocele were enrolled (Apr 2009-May 2012). They underwent isthmoplasty under general anaesthesia, and office hysteroscopic evaluation after 3 months. Main outcome measure was the improvement of postmenstrual AUB(PAUB), heavy menstrual bleeding (HMB), suprapubic pain, and secondary infertility.

Results: No intraoperative complications were recorded. Office hysteroscopy confirmed the correction of the anatomical defect, and the absence of postsurgical adhesions. Correction of isthmocele was reached in the first attempt in 153/160 pts. Resolution of PAUB, $\mathrm{HMB}$, and pelvic pain was obtained in all cases. The $85.7 \%$ of pts (42/49)with secondary infertility becames pregnant spontaneously within 12-24 months, and delivered at term by CS without any complications.

Discussion: The use of the mini-resectoscope was safe and useful in perform isthmoplasty, ensuring resolution of symptoms and restoring fertility.

\section{1}

\section{IMPACT OF FIBROIDS ON THE RESULTS OF TRANSCERVICAL RESECTION OF THE ENDOMETRIUM.}

Operative Hysteroscopy
C. Christoffersen*, S. Stampe, H. Jensen

${ }^{[1]}$ Herlev Hospital $\sim$ Copenhagen $\sim$ Denmark

Summary (4 lines): Dataextraction regarding the outcome of transcervical resections of the endometrium. Comparison between uteri with and without fibroids.

Introduction: Data extraction from the Danish Quality Database for Operative Hysteroscopy and Transcervical Surgery, (Hyskobase). The paper investigates longterm hysterectomy rates following Transcervical Resection of the Endometrium (TCRE) in the presence and absence of intracavitary fibroids.

Material and Methods: Standardized data based on a total of 1894 women between the age of 23 and 59 years old from 2001 to 2004. The womenwere tagged as having either fibroids or no fibroids and both groups were subject to statistical analysis. Detailed information on fibroid size and intramural involvement (Type 0,1 and 2) was collected.

Results: 456 women with fibroids met the inclusion requirements and of those $17,98 \%$ were later hysterectomised. 1438 women without fibroids were included in the control group, $19,75 \%$ of which were hysterectomised. Following TCRE, Type 2 fibroids were found to have a significantly higher risk of hysterectomy than type 0 fibroids $(\mathrm{P}=$ 0,036 ), and fibroids with a diameter of $>3,6 \mathrm{~cm}$ were found to have a significantly higher risk of hysterectomy than of fibroids of smaller sizes including controls, $(\mathrm{P}=0,012)$. Save for the above mentioned fibroid type and sizes, the presence of intracavitary fibroids did not worsen the outcome of TCRE as to the risk of subsequent hysterectomy.

Discussion: -

249

RECURRENCE AND REPRODUCTIVE RESULTS AFTER HYSTEROSCOPIC MYOMECTOMY

Operative Hysteroscopy

S. Campos*, M.J. Carvalho, G. Carvalho, L. Almeida E Sousa, F. Falcão, I. Torgal

${ }^{[1]}$ Centro Hospitalar e Universitário de Coimbra $\sim$ Coimbra $\sim$ Portugal

Summary (4 lines): Retrospective study of the recurrence and reproductive results after hysteroscopic myomectomy

Introduction: Submucous fibroids constitute $5.5-16.6 \%$ of all uterine fibroids and are associated with abnormal uterine bleeding (AUB) and infertility. Hysteroscopic myomectomy (HM) is a safe and effective procedure in most cases. The objective of our study was to evaluate recurrence and reproductive results after HM.

Material and Methods: Retrospective study of 119 patients submitted to HM from 2006 to 2010 in our Unit.

Results: Mean age of the patients: $43 \pm 10[23-80]$ yrs. Main indications: AUB $76.5 \%$, infertility $14.3 \%$, assymptomatic abnormal US $8.4 \%$, pelvic pain $0.8 \%$. No. of excised myomas: 1 in $80 \%,>=2$ in $20 \%$. Mean size of the myomas: $24 \pm 10 \mathrm{~mm}$. Mean procedure time: $37 \pm$ $16 \mathrm{~min}$. A second intervention was needed in $3.4 \%$. Complication rate: $9.2 \%$. US control possible in $59.7 \%$ (mean $42 \pm 9$ months after surgery) with $35.2 \%$ of recurrences. Comparing recurrent vs nonrecurrent groups, 1 myoma was excised in $56 \%$ vs $67 \%,>=2$ in $60 \%$ vs $19 \%(p<0,001)$. Of the 17 infertile women, $42 \%$ got pregnant, mean $13 \pm 9$ months after surgery. C-section rate: $71.4 \%$. Comparing pregnancy vs non-pregnancy groups, 1 myoma excised in $57 \%$ vs $90 \%,>=2$ in $43 \%$ vs $10 \%(\mathrm{p}=\mathrm{ns})$.

Discussion: Recurrence was more frequent in patients with several excised myomas. HM is safe and effective, improving the obstetric outcome in cases of infertily associated with submucous fibroids. 
188

\section{REPRODUCTIVE OUTCOME FOLLOWING RESECTION OF UTERINE SEPTUM: A RETROSPECTIVE STUDY \\ Operative Hysteroscopy}

S. Gundabattula, L. Marakani, S. Dasari, N. Yanamandra*, P. Nirmalan

${ }^{[1]}$ Fernandez Hospital $\sim$ Hyderabad $\sim$ India

Summary (4 lines): Reproductive outcomes in women with infertility and prior pregnancy losses were compared before and after uterine septal resection. This study demonstrates reduction in miscarriage rates and significant improvement in reproductive performance following septal resection.

Introduction: Mullerian duct anomalies can have significant implications on fertility and obstetrical issues. While for many women with uterine septum good reproductive outcome can be achieved, some may need surgical correction to enhance their reproductive outcome.

Material and Methods: This is a retrospective study between 2003 and 2010. Septoplasty was performed using Resectoscope or Versapoint. All operations were performed under general anaesthesia.

Results: The study included 124 women. Major indications for septal resection were infertility and prior pregnancy losses. 74 women $(59.7 \%)$ had an incomplete septum and 50 (40.3\%) had complete septum. Post-resection cervical cerclage was done in $33 \%$ women. Over three fourths of pregnancies that had cerclage carried pregnancy to term. Miscarriage and live birth rates were $93 \%$ \& $4 \%$ pre-resection and $32 \& 61 \%$ postresection respectively.

Discussion: Inadequate septal vascularization, abnormal placentation and incoordinated contractility of septum are possible mechanisms that hinder embryo implantation and pregnancy outcome. We found a post resection pregnancy rate of $75 \%$ and take home baby rate of $61 \%$ in our study population.

37

\section{SUCCESSFUL INTRODUCTION OF PROPOFOL SEDATION FOR EXTENSIVE HYSTEROSCOPIC SURGERY IN AN OUTPATIENT SETTING}

Operative Hysteroscopy

H. Kok*, R. Kraima, P. Van Kesteren, E. Bakkum

${ }^{[1]}$ Onze Lieve Vrouwe Gasthuis Amsterdam $~$ Netherlands

Summary (4 lines): Moderate sedation with Propofol for extensive hysteroscopic surgery in an outpatient setting.

Introduction: Recently, we introduced moderate sedation with Propofol administered by a certified non-anaesthesiologist sedation practitioner in an outpatient setting for extensive hysteroscopic surgery in those patients previously requiring general or spinal anaesthesia as a safe and patient friendly alternative.

Material and Methods: The implementation process was analysed by a prospective risk analysis.

In a pilot study, 26 patients were operated under Propofol sedation.

Results: Transcervical myomectomies of type 0/I myomas up to $3 \mathrm{~cm}$ and (multiple) polypectomies were successfully performed. All procedures in this pilot were completed and patients' feedback showed very high satisfaction. No prolonged stay in dayor clinical care was required. No adverse advents were recorded. Discussion: Propofol sedation in extensive hysteroscopic surgery is a novel treatment mode which fits with the current trend to shift clinical care towards outpatient care. To date, there is no literature on systematic use of Propofol sedation for hysteroscopic surgery. We hypothesize that this treatment mode leads to increased patient satisfaction and less costs without concessions on quality of care and patient safety. A randomized trial is planned to assess medical- and cost-effectiveness along with patient satisfaction and patient preference.

\section{2}

TAILOR MADE PROFICIENCY CURVES IN LAPAROSCOPIC HYSTERECTOMY: ENHANCING PATIENT SAFETY USING CUMULATIVE SUM ANALYSIS

Operative Risk Management

J. Frank Willem*, B. Mathijs, V.Z. Erik, D.K. Cor, T. Andries

${ }^{[1]}$ Leiden University Medical Center $\sim$ Leiden $\sim$ Netherlands

Summary (4 lines): With proposed validated and risk adjusted CUSUM graphs surgeons are able to continuously monitor their surgical performance in laparoscopic hysterectomy, consequently identifying suboptimal factors, which allows improvement of their surgical outcomes and further enhancement of patient safety.

Introduction: We aimed to develop a risk adjusted real-time quality control system in laparoscopic hysterectomy with respect to blood loss, operative time and adverse events in order to signal derailing surgical performance in a timely fashion.

Material and Methods: Uterus weight, body mass index, number of surgeons, prior abdominal surgery and type of laparoscopic hysterectomy were identified as significant covariates predicting successful surgical outcome. Cut-off values were set at blood loss $<200 \mathrm{~mL}$, operative time $<120$ minutes and no adverse event. CUSUM analysis was selected as a possible tool for performance analysis.

Results: Risk adjusted CUSUM graphs were constructed. In order to detect unacceptable failure rates in surgical performance within 20 procedures, as a result competent surgeons will be flagged once in every 70-75 procedures without justified bad performance.

Discussion: With risk adjusted CUSUM graphs surgeons are able to continuously monitor their surgical performance in laparoscopic hysterectomy.

427

\section{ORTHOTOPIC TRANSPLANTATION OF CRYOPRESERVED OVARIAN TISSUE VIA ROBOT-ASSISTED LAPAROSCOPY} Robotics

\author{
C. Tomassetti*, H. Afschrift, C. Spiessens, T. D'Hooghe \\ ${ }^{[1]}$ University Hospitals Leuven $\sim$ Leuven $\sim$ Belgium
}

Summary (4 lines): Orthotopic transplantation of cryopreserved ovarian tissue via robot-assisted laparoscopy, in a patient with premature ovarian failure (POF) after multiple chemotherapy, resulted in recovery of oestradiol production and follicular development.

Introduction: Patients who need chemotherapy can be counselled for ovarian cryopreservation to preserve fertility. In case of POF, autotransplantation can be done.

Material and Methods: A patient with POF after chemotherapy and stem cell transplantation for nephroblastoma in remission, underwent autotransplantation of ovarian cortex on the decorticated contralateral remnant ovary. Preop analysis of the cryopreserved specimen showed no malignancy and a large number of primordial follicles. Peroperative analysis of the cortex of the contralateral ovary only showed fibrosis and foreign-body reaction but no follicles. Transplantation was performed with robot- 
assisted laparoscopy as the specimens were very small $(1 \times 1 \times$ $10 \mathrm{~mm}$ ).

Results: 5 months after the transplantation, recovery of ovarian function was demonstrated disappearance of menopausal symptoms, elevated serum oestradiol levels, follicular development and withdrawal bleeding. However, up to now progesterone levels remain low, and no pregnancy has occurred.

Discussion: Laparoscopic ovarian orthotopic cryotransplantation was feasible with robot-assistance, especially when transplanted fragments are very small, and resulted in recovery of ovarian function.

142

\section{ROBOTIC-ASSISTED SINGLE-INCISION SACROCOLPOPEXY: EARLY EXPERIENCE}

Robotics

V. Etienne, G. Pierre, S.L. Delphine, K. Aminata*, V. Richard

${ }^{[1]}$ hopital des Diaconesses Croix St Simon $\sim$ Paris $\sim$ France

Summary (4 lines): Sacrocolpopexy can be performed safely with single-site port using the daVinci-Si robotic system.

Introduction: Laparoendoscopic single-site surgery (LESS) decrease postoperative pain with better cosmesis. Instrument conflict minimization often requires the crossing of articulating instruments, which can be intuitively facilitated using the daVinci-Si robotic system.

Material and Methods: Three robotic single-incision double meshes sacrocolpopexy were performed using the daVinci-S robotic system using string suture. The procedure was performed using Da Vinci Single-Site Port through a single $35 \mathrm{~mm}$ incision around the umbilicus with three robotic arms, a $8.5 \mathrm{~mm}$ camera and two $5 \mathrm{~mm}$ semi-rigid instruments introduced in specialized curved cannulae. A 5 or $10 \mathrm{~mm}$ straight cannula for non robotic help is also used in the same single site port.

Results: Three consecutive sacrocolpopexy were performed successfully (one with a non conservative subtotal hysterectomy, and one with bilateral ovario salpingectomy) in average operating times of $230 \mathrm{~min}$ range to $210-240 \mathrm{~min}$ without intraoperative or postoperative complications. All patients were discharged on day 2 .

Discussion: Robotic-assisted single-incision sacrocolpopexy can be successfully and safely performed using the daVinci-S robotic system. By crossing the robotic instruments, the robot help to perform these procedures safely in a more confortable way compared to conventional single site laparoscopy.

311

ROBOTIC SINGLE-SITE HYSTERECTOMY: FEASIBILITY, LEARNING CURVE AND SURGICAL OUTCOME

Single Access Surgery

N. Pluchino*, L. Freschi, G. Simi, V. Cela

${ }^{[1]}$ Ob-Gyn - Pisa University Hospital Pisa Italy

Summary (4 lines): Single-Site hysterectomy combines the advantages of single incision surgery with the surgical technology of robotic assistance.

Introduction: The robotic single-site surgery is the hybridization of robotic technology with laparoendoscopic single-site surgery

Material and Methods: Perioperative informations of 12 robotic single-site hysterectomy (R-SSH) were collected to evaluate the surgical feasibility and the possible influence of the BMI and the uterine weight on operative times.
Results: The mean operative time was $85 \pm 33$ minutes (range 355 to 149 minutes), the mean docking time was $9 \pm 3$ minutes and the mean console time was $76 \pm 33$ minutes. The mean blood loss was $80 \pm 18 \mathrm{~mL}$ and the median weight of resected uteri was $220 \pm 45 \mathrm{~g}$. No serious post-operative complications occurred. The CUSUM learning curve was observed to consist of two different phases: phase 1 (the initial 6 cases) and phase 2 (the last 6 cases) with significant reduction in operative and console time observed between the two phases. As far as BMI no correlation was found with operative times, console times and docking times and no correlation was found between uterine weight and operative time

Discussion: This series, identifying two different phases of the learning curve and suggesting that the initial learning phase for the procedure can be achieved after 6 cases, confirms the feasibility of robotic single-site hysterectomy.

469

ADNEXAL MASSES TREATED USING A COMBINATION OF THE SILSTM PORT AND NON-CURVED STRAIGHT LAPAROSCOPIC INSTRUMENTS

Single Access Surgery

P. Dursun*, C. Gulumser, T. Tezcaner, H. Zeyneloglu, A. Ayhan

${ }^{[1]}$ Baskent University $\sim$ Ankara $\sim$ Turkey

Summary (4 lines): Concomitant surgical procedures together such as cholecystectomy and adnexal masses removal are able to perform with same single umbilical port.

Introduction: The study included 14 women with symptomatic and persistent adnexal masses.

Material and Methods: A prospective case series. Intervention: Removal of adnexal masses via single-incision laparoscopic surgery using a combination of the SILSTM port and straight non-roticulating laparoscopic instruments.

Results: Mean age was 38 years. Mean duration of surgery was 71 min (range: 45-130 min). Mean adnexal mass' diameter was $6 \mathrm{~cm}$. Totally, 3 unilateral salpingo-oophorectomy, 1 bilateral salpingooophorectomy, 1 myomectomy + USO, 5 cystectomy, and 2 salpingectomy were performed. Of those 2 subjects concomitantly had cholecystectomy and appendectomy via same umbilical incision. All pathology reports were benign. None of the operation needs to convert to laparotomy. All the patients were discharged on postoperative day one. After the surgery, all subjects were satisfied with their incision and cosmetic results.

Discussion: This procedure can reduce the cost of treatment and comparable with laparoscopic approach regarding duration of operation and patient satisfactions that may eventually lead to more widespread use of the SILSTM port approach. Furthermore, concomitant surgical procedures are able to perform with same single umbilical port.

487

\section{FROM SILS OOPHORECTOMY TO MICRO ORGAN OVARIAN TRANSPLANTATION}

Single Access Surgery

A. Revel*
${ }^{[1]}$ Hadassah Hebrew University hospital $\sim$ Jerusalem $\sim$ Israel

Summary (4 lines): Despite efforts to reduce the impact of cancer treatment on fertility, women with cancer remain at risk for impaired reproductive potential. We present the possibility and methods to remove ovarian cortex for cryopreservation. 
Introduction: Ovarian cryopreservation should no longer be presented as an experimental procedure seeing that more than 20 children were born worldwide following ovarian transplantation. Patients should be counseled as to the risks and benefits of laparoscopic surgery for fertility preservation.

Material and Methods: Ovarian cryopreservation has been performed for over 15 years at our institution. During the last 2 years we have modified our method to SILS single port oophorectomy. The transplantation method has been adapted to the micro organ technique.

Results: For patients over 10 y old we used covidien SILS port. For younger patients we used an h laparoscope and one $5 \mathrm{~mm}$ port. All patients started chemotherapy with 48 hours of surgery. Mean patient age was 17 (2-34). 14 ampoules were preserved per patient. Growing oocytes were vitrified separately as we previously published. The mean number of vitrified oocytes was $6(0-43)$. In one patient postoperative infection of the SILS scar occurred.

Discussion: Patient satisfaction from the SILS approach was high. Following transplantation of micro organ ovarian cortex we have restored fertility in 2 women who have conceived and delivered healthy babies following bone marrow transplantation.

\section{8}

IMPLEMENTATION OF LESS IN GYNAECOLOGICAL SURGICAL PRACTICE: IS IT EVIDENCE BASED?

Single Access Surgery

C. La Chapelle*, M. Van Den Tweel, P. Van Kesteren, B. Risseeuw, A. Thurkow, F.W. Jansen

${ }^{[1]}$ Leiden University Medical Centre $\sim$ Leiden $\sim$ Netherlands

Summary (4 lines): LESS surgery is gradually implemented in dutch gynecological practice. We present a case-series and review the evidence. Introduction: Since 2008 Laparoendoscopic Single-Site (LESS) surgery has been implemented for a variety of gynecologic procedures. We compiled an inventory in the Netherlands and reviewed the evidence.

Material and Methods: 1)Chart study was performed in 3 dutch centres.

2)Literature of LESS in gynecological surgery is systematically reviewed.

Results: 68 gynecological LESS procedures were performed in 3 training hospitals. Including: total hysterectomy $(\mathrm{TH})(\mathrm{n}=41)$, adnexal surgery $(n=23)$ and others $(n=4)$. Mean surgery duration for TH was $133(\mathrm{SD} \pm 38)$, for adnexal surgery $88(\mathrm{SD} \pm 32)$ min. Median estimated blood loss in TH was 100 and in adnexal surgery $<50 \mathrm{ml}$. 5 complications were registered.

The review focusses on hysterectomy and adnexal surgery and included 11 studies comparing LESS to conventional laparoscopy. Evidence suggests advantages of LESS in terms of postoperative pain for LAVH and adnexal surgery and in cosmetics for TH and adnexal surgery. No difference was found for complications, blood loss, hospital stay and conversions.

Discussion: LESS is gradually implemented, though a solid evidence base is lacking. Adequate studies comparing LESS to conventional laparoscopy are sparse. Evaluation in larger trials with long follow up is required, focussing on relevant outcomes. To highlight this, our new COSTLESS-study will be presented.

471

LAPAROENDOSCOPIC SINGLE-SITE SURGERY WITH BENIGN ADNEXAL DISEASE AND OPTIMIZING THE ENTRY METHOD IN PREGNANT PATIENTS

Single Access Surgery
P. Dursun*, C. Gulumser, H. Zeyneloglu, A. Haberal, A. Ayhan

${ }^{[1]}$ Baskent University Ankara Turkey

Summary (4 lines): To report the feasibility of laparoendoscopic single port surgical approach for the management of huge adnexal cyts and adnexal torsion during pregnancy. To discuss the optimal method of entry into the peritoneal cavity for pregnant patients.

Introduction: First patient was diagnosed with ovarian cyst in $18 \mathrm{~cm}$ diameter on left ovary on 12th weeks of pregnancy. The other patient had IVF twin pregnancy and presented with left adnexal torsion on 25th weeks of pregnancy.

Material and Methods: Single-port laparoscopic surgery was performed on both patients.

Results: No fetal and maternal complication was diagnosed during both pregnancies. And both patients delivered their babies uneventfully.

Discussion: There is still no clear consensus on optimal method of entry into the peritoneal cavity. Irrespective of major complications, in pregnant patients, rupture of uterus, fetal complications can also occur in particular if the Veress Needle is used for the entry. Therefore we believe that using an open-entry technique compared to Veress Needle reduces the risk of such pregnancy related and fetal complications with pregnant patients. In conclusion, single port laparoscopy with openentry technique might be better surgical options for the pregnant patients with persistant adnexal mass and pregnant women with adnexal torsion. However, further studies with larger sample size needed to reach clear conclusion.

473

\section{SUPRACERVICAL HYSTERECTOMY BY SINGLE PORT ACCESS WITH TRANSCERVICAL MORCELLATION}

Single Access Surgery

J.P. Estrade*, M. Marcelli, P. Crochet, B. Gurriet, A. Lazard, A. Agostini

${ }^{[1]}$ La Conception Hospital $\sim$ marseille $\sim$ France

Summary (4 lines): Supracervical hysterectomy by single port access with transcervical morcellation is a feasible and safe procedure. Limitation of this procedure is the duration of morcellation.

Introduction: To evaluate faesability of supracervical hysterectomy (SPH) by single port access (SPA) with LESS system with transcervical morcellation.

Material and Methods: From September 2010 to March 2012, 40 patients who required hysterectomy for fibroma were included in this study. All procedures were performed by 5 surgeons in two departments in Marseille, France (Bouchard Clinics and La Conception Hospital).

Results: Nine patients had a previous cesarean section $(22,5 \%)$ and 19 a laparotomy $(47,5 \%)$. Mean of BMI was $24,2+/-4,3$. Mean duration of procedure was $128+/-55 \mathrm{~min}$. and uterus weight was $310+/-214 \mathrm{~g}$. Eleven patients had an bilateral annexectomy $(27,5 \%)$. Two patients required a second laparoscopy for pelvic abccess and remaining fibroma (5\%). Two patient required coagulation of endocervix for bleeding at day 6 and 10 after surgery.

Discussion: SPH by SPA with transcervical morcellation is feasible with a low risk of complications and benefits of SPA. Limitation of the procedure is the time required for morcellation.

A NATIONAL SURVEY OF THE MANAGEMENT OF TUBAL PREGNANCY: THE UNITED KINGDOM EXPERIENCE Teaching and Training 
M. Taheri*, R. Bharathan, A. Subramaniam, T. Kelly

${ }^{[1]}$ Queen Charlotte's and Chelsea Hospital $\sim$ London $\sim$ United Kingdom

Summary (4 lines): Our national survey demonstrates increased use of medical and laparoscopic management of ectopic pregnancy in the UK.

Introduction: $1 \%$ of UK pregnancies are ectopic. Laparoscopy is the recommended surgical approach in stable patients. In the UK in $2000,35 \%$ of cases were managed by laparoscopy, $63 \%$ by laparotomy and $1 \%$ with medication. A recent review in the USA revealed increasing rates of medical management and decreasing rates of laparotomy; a trend driven by both cost effectiveness and patient choice.

Material and Methods: 132 early pregnancy units were surveyed regarding the nature and management of the 3 most recent cases of ectopic pregnancy; in addition feasibility of training residents was also requested.

Results: The response rate was $26 \%$; participants reported on 100 cases with a median of 5 cases per month per department. $59 \%$ of cases were managed laparosocpically, $31 \%$ medically, $6 \%$ by laparotomy and $5 \%$ conservatively. All tertiary centres reported that they can manage all ectopics laparosocpically with 3/9 doing so in unstable patients. 22/34 centres have the facilities for laparoscopic training and early pregnancy USS.

Discussion: We report an increasing rate of laparoscopic, medical and conservative management with a fall in open surgery. Influencing factors included early pregnancy diagnostic services, patient choice, co-morbidities, clinical urgency, suitability for laparoscopic surgery and the availabilty of a competent surgeon.

\section{8}

EFFECTS OF A HOME-BASED SIMULATION PROGRAMME Teaching and Training

C. Harrity*, R. O'Sullivan, W. Prendiville

${ }^{[1]}$ RCSI $\sim$ Dublin $\sim$ Ireland

Summary (4 lines): Use of a video simulator at home significantly improves laparoscopic suturing skills, with a correlation between performance and practise.

Introduction: Laparoscopic skills training by simulation is effective. As working hours decrease, training at home has to potential to help improve psychomotor skills outside the operating theatre or skills laboratory. Laparoscopic suturing is difficult to master, involving depth perception, visuospatial awareness, co-ordination, fine motor skill, and non-dominant hand use. Suturing can be divided into several steps requiring different techniques, and is often considered a marker of proficiency in laparoscopy.

Material and Methods: 30 students with no prior experience enrolled on a single day laparoscopic skills course, ending with the ability to suture. A video simulator was provided for use at home over a 6-week period, with access to online videos. Suturing was timed on day 1 and week 6 , and correlated with the amount of practice.

Results: Mean suture time at the end of D1 was $612.5 \mathrm{~s} \mathrm{(sd} \mathrm{294.2),} \mathrm{and}$ was significantly reduced to $433.9 \mathrm{~s}$ ( $\mathrm{sd} 195.5)$ by week $6(\mathrm{p}<0.0001$, paired t-test). The mean number of home simulator hours per week was 2.33 (sd 1.27), with a significant correlation between improvement and practice (Pearson $0.861, \mathrm{p}<0.0001$ )

Discussion: This study demonstrates that simulation shortens the learning curve and improves psychomotor skills in laparoscopic suturing. The hypothesised correlation between practice and improvement has been shown.
332

\section{IMPACT OF INSTRUCTOR FEEDBACK ON PERFORMANCE IN A VIRTUAL REALITY SIMULATOR AT SIX MONTHS FOLLOW-UP}

Teaching and Training

F. Bjerrum*, M. Maagaard, C. Rifbjerg Larsen, J. Led Soerensen, C. Ringsted, B. Ottesen, O. Jeanett

${ }^{[1]}$ Rigshospitalet, University Hospital of Copenhagen $\sim$ Copenhagen Denmark

Summary (4 lines): A randomised trial examining long term retention after instructor feedback when training on a laparoscopic virtual reality trainer.

Introduction: A randomised trial to investigate if instructor feedback has any impact on long-term retention after training a complex operation on a virtual reality simulator.

Material and Methods: Medical students $(n=91)$ trained a laparoscopic salpingectomy to a proficiency level on a virtual reality simulator. The intervention group received standardized instructor feedback and the control group did not. After six months participants trained the same operation to the same proficiency level.

Results: Initially the intervention group used an average of 162 minutes (min.) and 29 repetitions (rep.) to reach the proficiency level. The control group used $342 \mathrm{~min}$. and 65 rep. $(\mathrm{p}<0,0005)$. At follow-up $(n=65)$ the intervention group used $83 \mathrm{~min}$. and 20 rep. to reach the same level and the control group used $73 \mathrm{~min}$. and 21 rep. No significant difference was found regarding time and repetitions at follow-up. Both groups used significantly fewer repetitions and less time $(p<0,01)$ to reach the same level after six months.

Discussion: Feedback has shown to increase efficiency when training on a virtual reality simulator. After six-months both groups show the same level of retention, underlining the need for proficiency based training.

\section{8}

\section{INSTRUCTOR FEEDBACK IMPROVES PERFORMANCE IN A VIRTUAL REALITY SIMULATOR: A RANDOMIZED TRIAL}

Teaching and Training

J. Oestergaard, F. Bjerrum*, M. Maagaard, P. Winkel, C. Rifbjerg Larsen, C. Ringsted, C. Gluud, T. Grantcharov, B. Ottesen, J. Led Soerensen ${ }^{[1]}$ Rigshospitalet, University Hospital of Copenhagen $\sim$ Copenhagen $\sim$ Denmark

Summary (4 lines): A randomised trial investigating the impact of instructor feedback when training a laparoscopic salpingectomy on a virtual reality simulator.

Introduction: To investigate the impact of instructor feedback versus a self-directed approach when training a complex task on a laparoscopic virtual reality simulator, including focus on potential gender differences and self-perception.

Material and Methods: A randomized trial. The intervention group received standardized instructor feedback (10 minutes) a maximum of three times. The control group received no instructor feedback. Both groups had to reach a predefined proficiency level for at laparoscopic salpingectomy on a virtual reality simulator. Participants: senior medical students $(\mathrm{n}=99)$ without prior laparoscopic experience, but familiar with the operational procedure.

Results: Time (minutes) and repetitions were significantly reduced in the intervention group (162 vs. 342 min., $p=0.0005)$ and ( 29 vs. 65 rep., $\mathrm{p}=0.0005)$ respectively. Men used less time $(\mathrm{p}=0.037)$. 
Participants in the intervention group had higher self-perception regarding surgical skills after the trial $(p=0.011)$.

Discussion: Instructor feedback is highly effective regarding time, repetitions and self-perception when training complex operational tasks on a virtual reality simulator. Two feedback sessions were sufficient to reach proficiency level. This is crucial knowledge when building a surgical curriculum.

\section{4}

INTRACORPOREAL KNOT TYING IN A BOX TRAINER AS PREDICTOR OF SURGICAL OUTCOME IN ADVANCED LAPAROSCOPIC PROCEDURES: HOW PROFICIENT IS IN VITRO EVALUATION IN EXPERTS?

Teaching and Training

T. Andries*, H. Ellen, B. Elzelyne, E. Van Zwet, D.K. Cor, J. Frank Willem

${ }^{[1]}$ Leiden University Medical Center $\sim$ Leiden $\sim$ Netherlands

Summary (4 lines): Proficiency in advanced laparoscopic surgery cannot be appropriately monitored by economy of movement analysis in box trainers.

Introduction: Economy of movements of intracorporeal knot tying in laparoscopic box trainers can differentiate between novices, intermediates and experts. In search of predictive models for quality of care we researched the applicability of motion-analysis parameters of knot tying in box trainers in experts as predictors for surgical outcome.

Material and Methods: Time, path length and motion in depth of a standardized intracorporeal knot tying task were compared to average risk-adjusted primary outcomes (blood loss and operative time) of a one year consecutive analysis of laparoscopic hysterectomies. Surgical outcomes were corrected for patient factors.

Results: Experience with laparoscopic hysterectomy correlated with efficient knot tying in the box trainer. After correction for patient mix in 50 expert surgeons, motion-analysis of intracorporeal knot tying could not significantly discriminate surgical outcome skills in advanced laparoscopic surgery.

Discussion: Proficiency in advanced laparoscopic surgery cannot be appropriately monitored by economy of movement analysis in box trainers. Instead, we suggest monitoring of real-time risk adjusted surgical outcomes of experts.

\section{1}

\section{TRAINING LAPAROSCOPIC MIOMECTOMY IN ANIMAL} MODEL

Teaching and Training

A.C. Lou*, A. Borque, P. Gonzalez, Á. García De Jalón, J.M. Sánchez, J. Gómez-arrué, S. Repollés, M. Díaz, C. Pastor

${ }^{[1]}$ Department of Urology. Hospital "Ernest Lluch". Aragonian Group of Formation in Experimental Surgery (Laparoscopic Section). Calatatud $\sim$ Spain

Summary (4 lines): We present an animal model for training in laparoscopic myomectomy (LM).

Introduction: Laparoscopic myomectomy is becoming more popular in gynecologic laparoscopy. The need of hemostatic suture of uterine parenchyma is probably its main difficulty. We present partial nephrectomy (PN) in animal model as a practice of hemostatic parenchymal suture for training in LM.

Material and Methods: One sow, variety LW. Laparoscopic dissection of renal pedicle, selective arterial clamping, partial excision of renal parenchyma, internal hemostatic intracorporeal suture of renal parenchyma, external intracorporeal suture, unclamp renal artery and test hemostasis. Descriptive video will be showed.

Results: Laparoscopic parenchymal excision during PN can reproduce the resection of intramural myoma in LM. The two layers suture in PN with hemostatic goal reproduces the same objective in LM. We could not reproduce the selective enucleation of nodular mass as it happens in LM, as a limitation of our animal training model.

Discussion: The main difficulties of LM can be trained in animal model of PN: intramyometrial resection and intracorporeal hemostatic sutures. Interrupted or running intracorporeal sutures can be trained. Selective nodular enucleation was not reproduced.

\section{2}

\section{TRAINING UROLOGICAL INJURIES IN LAPAROSCOPIC GYNECOLOGICAL SURGERY}

Teaching and Training

A. Lou-mercadé*, A. Borque-fernando, P. González-ramos, A. García De Jalón-martínez, J. Sánchez-zalabardo, J. Gómez-arrué-azpiazu, S. Repollés-lasheras, M. Díaz-vega, C. Pastor-oliver

${ }^{[1]}$ Department of Obstetrics and Gynecology. Hospital Clínico Universitario "Lozano Blesa". Aragonian Group of Formation in Experimental Surgery (Laparoscopic Section). $\sim$ Zaragoza. $\sim$ Spain

Summary (4 lines): We present laparoscopic models in training solution of urologic injuries (UI) during laparoscopic gynecological surgery (LGS). Introduction: Laparoscopic hysterectomies for benign disease present bladder (2.01\%) or ureteric injuries (1.21\%) (Nieboer TE, 2009). Increased experience decreases these rates but the more surgical complexity increases them. That low frequency difficults to gain experience in solving UI. We show experimental models of UI during LGS to gain that experience. Material and Methods: One female sow, variety LW / Model 1, BLADDER INJURY: Hole the bladder, catheter indwelling, bladder closure, closure test / Model 2, URETERAL INJURY: Ureteral dissection, coagulation and cut, ureteral management, catheter placing, laparoscopic suture / Model 3, PSOAS HITCH BLADDER AND URETERAL REIMPLANTATION: Retroperitoneal dissection of psoas muscle, transversal bladder section, bladder hitch, uterus horn used for ureteral reimplantation, ureteral catheter placing, longitudinal bladder closure. Three descriptive videos will be showed.

Results: Our three training models of UI reproduce laparoscopic human surgery. The low frequency but high morbidity of UI makes our models a way to gain experience in their repairing. Porcine uterus is used as ureter. Discussion: UI in LGS can be trained in experimental surgery avoiding conversion to laparotomy. The models reproduce the human surgery. A single animal is used in a cost-effectiveness way. These models are easily reproducible.

432

VIRTUAL REALITY TRAINING FOR LAPAROSCOPIC TUBAL SURGERY IS VALID AND EFFECTIVE

Teaching and Training

R. Bharathan*, S. Vali, T. Setchell, T. Miskry, A. Darzi, R. Aggarwal ${ }^{[1]}$ Imperial Coolege $\sim$ London $\sim$ United Kingdom

Summary (4 lines): Virtual reality (VR) training module in laparoscopic tubal surgery demonstrates construct validity. This is an effective tool for novice gynaecologists.

Introduction: VR training enables skill transference to the OR. Validation of VR laparoscopic training for the management of tubal pregnancy is required in order to enhance training. 
Material and Methods: 9 experienced, 9 intermediate and 16 novice gynaecologists were recruited. All subjects performed 2 sessions of salpingectomy and salpingotomy on a VR simulator to assess construct validity (CV). 9 novices performed 10 such sessions to enable assessment of the learning curve (LC). The face validity (FV) of the 2 procedures, as reported by the experienced and intermediate level surgeons was compared.

Results: Salpingectomy demonstrated CV in terms of time taken $(p=0.003)$ and movements $(\mathrm{p}=0.045)$. Salpingotomy demonstrated CV in terms of time taken $(p=0.002)$, movements $(p=0.005)$ and path length $(p=0.05)$. LC analysis for Salpingectomy displayed a plateau in time taken at session (S) 9 , and movements at S5. Salpingotomy displayed a plateau in time taken at S8, movements at S8 and path length at S6. Cognitive load as assessed by NASA TLX displayed a correlation with these LC's. The FV scores were not significantly different between the two procedures $(p=0.619)$.

Discussion: The LAP Mentor VR simulator is a valid and effective tool for training novice surgeons in the laparoscopic removal of ectopic pregnancy.

143

PHASE II AND PHASE III QUALITY-OF-LIFE AND SAFETY OUTCOMES AFTER RADIOFREQUENCY VOLUMETRIC THERMAL ABLATION OF SYMPTOMATIC FIBROIDS IN 206 PATIENTS

Technical Tricks and New Instrumentations

K. Isaacson*

${ }^{[1]}$ Harvard Medical School Boston United States

Summary (4 lines): Laparoscopic radiofrequency volumetric thermal ablation (RFVTA) of symptomatic fibroids is safe, provides significant improvement in quality of life (QOL), and rapid recovery.

Introduction: Patient QOL outcomes, reported device/procedure-related adverse events (AEs), and recovery rates for laparoscopic RFVTA.

Material and Methods: Retrospective analysis of 206 consecutive cases of RFVTA of symptomatic fibroids performed on an outpatient basis under 2 phase II studies ( 2 sites, $n=69$ ) and 1 phase III study (11 sites, $n=137$ ). Results: From baseline to 12 mo: Phase II symptoms reduced from a mean score of 53.9 to 8.8 and health-related quality-of-life (HRQL) scores improved from 48.5 to $92.0(n=58 ; p<.001)$ with one $(1 / 69 ; 1.4 \%)$ device/procedure-related $\mathrm{AE}$ (abdominal bleed) and return to normal activities in $4 \mathrm{~d}(1-11 \mathrm{~d} ; \mathrm{n}=65)$ and to work in $4 \mathrm{~d}(2-10 \mathrm{~d}$; $\mathrm{n}=39$ ). Phase III symptoms reduced from a mean score of 61.4 to 26.4 and HRQL scores improved from and 37.1 to $80.0(n=129$; $\mathrm{p}<.001)$ with four $(4 / 137 ; 2.9 \%)$ device-related AEs (pelvic abscess, abdominal pain, superficial serosal burn, postprocedural hemorrhage) and return to normal activities in $9 \mathrm{~d}(2-60 \mathrm{~d} ; \mathrm{n}=$ $133)$ and return to work in $5 \mathrm{~d}(0-29 \mathrm{~d} ; \mathrm{n}=88)$.

Discussion: RFVTA of symptomatic myomas provides significant improvement in subject QOL without requiring serosal and myometrial suturing which are often deemed technical surgical obstacles.

397

INSIDE-OUT VERSUS OUTSIDE-IN TRANSOBTURATOR TRAJECTORIES FOR THE TREATMENT OF FEMALE STRESS INCONTINENCE: RADIOGRAPHIC CONSIDERATIONS

Urogynaecology

P. Hinoul*, A. Kirkemo, M. Walters, F. Brian, E. Henk

${ }^{[1]}$ Ethicon Inc. $\sim$ Sommerville $\sim$ United States

Summary (4 lines): 3-D CT scan imaging of midurethral-sling (MUS) trajectories demonstrate a more acute angulation of the hammock formed by the inside-out tape than in the outside-in approach.
Introduction: 3-D configuration of MUS is difficult to demonstrate in traditional anatomical dissections. The aim was to assess differences in angulations in MUS positioning.

Material and Methods: Two independent surgeons performed their usual MUS placement on 1 side each of 10 consecutive fresh cadavers. The sides were alternated in sequential cadavers. Tantalum wires threaded through the polypropylene-tapes rendered them radio-opaque. Following placement CT scans were obtained and then analyzed.

Results: The mean angle formed by the in-out-MUS measured 122 degrees (95\%CI: 107-136); versus 144 degrees $(95 \%$ CI: $131-151)$ for the out-in-MUS $(p=0.02)$. The variability of the angle was greater for the in-out (90-148) than the out-in placement (62-122). There was no correlation between either approach and BMI or angle of the pubic arch. The images revealed that the tapes lie as a band posterior/dorsal to the urethra rather than inferior.

Discussion: The clinical implications (efficacy/safety) of the more horizontal positioning after the out-in approach remain to be determined in a clinical setting. The dorsal position of the MUS suggests a passive (non obstructive) role in achieving continence.

\section{9}

LAPAROSCOPIC SACROCOLPOPEXY (LSCP) VS TRANSVAGINAL MESH (TVM) REPAIR: COMPARISON OF ANATOMICAL AND FUNCTIONAL RESULTS IN THE MEDIUM TERM. BI-CENTER RETROSPECTIVE STUDY ABOUT 122 CASES

Urogynaecology

B. Georges*, H. Cyrille, P. Alain, F. Arnaud

${ }^{[1]}$ Poissy University Hospital $\sim$ POISSY $\sim$ France

Summary (4 lines): The aim was to compare the medium term anatomical and functional results of Laparoscopic Sarcocolpopexy (LSCP) and Transvaginal mesh (TVM)for POP repair.

Introduction: LSCP is considered to be the reference technique for POP repair. TVM techniques offer satisfactory anatomical results but seem to be associated with higher morbidity.

Material and Methods: Bi-centric retrospective study comparing two groups of 61 patients after matching on age criterion $(55+/-5$ years $)$, operated for POP, respectively by abdominal (LSCP) and vaginal routes (TVM).

Results: The mean age was comparable in both groups $(p=0.1)$. Population characteristics were similar. The mean operative time of LSCP was significantly higher $(\mathrm{p}=0.001)$. The rate of intraoperative complications was higher in the TVM group $(p=0.36)$. The anatomical results were equivalent in both groups at 8.5 months of surgery. At 18 months, satisfaction rate was higher in the LSCP group. De novo dyspareunia was higher in the TVM group $(\mathrm{p}=0.015)$. Postoperative constipation was higher in the LSCP group $(p=0.02)$. The rate of reoperation for complications (was higher $(p=0.27 \mathrm{NS})$ in TVM group. The rate of surgery for recurrent POP was strictly identical in both groups $(\mathrm{p}=1)$.

Discussion: The LSCP does provide a better QOL and a higher satisfaction rate with less morbidity compared with TVM.

\section{4}

LAPAROSCOPIC SACROCOLPOPEXY IN PELVIC ORGAN PROLAPSE TREATMENT : A RETROSPECTIVE STUDY OF 178 PATIENTS

Urogynaecology

A. Richter-marot*, C.Y. Akladios, A. Wattiez

${ }^{[1]}$ Centre Hospitalo-Universitaire Strasbourg $\sim$ Strasbourg $\sim$ France 
Summary (4 lines): Our retrospective study of 178 laparoscopic sacrocolpopexies shows the effectiveness of the technique which may deserve to be considered as the gold standard in POP-treatment.

Introduction: Pelvic organ prolapse (POP) is a common disease of women, undermining their quality of life. The goal of this study was to evaluate the effetiveness of laparoscopic sacrocolpopexy, a twenty-yearold technique, in the surgical management of genital organ prolapse.

Material and Methods: We retropectively studied 178 laparascopic sacrocolpopexies, performed from January 2004 to December 2010 in the Department of Gynecology at the University of Strasbourg.

Results: The mean operative duration was 188 minutes (70-360 min) with a conversion rate of $0,6 \%$. Early re-operation occurred in $1,7 \%$. With a mean follow-up of 35,1 months (7-84 months), we found $2,7 \%$ of reoperation for prolapse recurrence. Erosion of mesh was reported in 3,3\%. Overall, $97,4 \%$ of patients were globally satisfied with the operative result. Discussion: Our study confirms that sacrocolpopexy is a highly effective technique of POP-treatment, with good anatomic and functional results, associated with a very low rate of complication and of recurrence. It is the only minimally invasive surgical approach allowing a global and reconstructive repair of the prolapse with low morbidity.

364

\section{OHVIRA: CASE REPORT}

Case Reports

E. Villegas MuÑoz*, M.E. Valdivia De Dios, P. Barri Soldevilla, A. Ubeda Hernandez

${ }^{[1]}$ Carlos Haya Regional Hospital Malaga Spain

Summary (4 lines): A case of OVHIRA Syndrome diagnosed and operated in our hospital.

Introduction: OVHIRA (Obstructed HemiVagina and Ipsilateral Renal Anomaly). A consequence of an anomaly in the development of the paramesonephric and mesonephric ducts and its associated with the uterovaginal duplication, obstructed hemivagina and ipsilateral agenesis. Material and Methods: A 13 year woman with hypogastric pain getting worse in the last menstruations. Examination: Right vagina wall is inflating and deforming the vaginal route. Small orifice in the right vagina wall that drains chocolate-brown coloured material. U1trasound and NMR: Complete fusion defect of the Müllerian ducts. Didelphys uterus. Vaginal partial agenesis, with important hematometra. Cranial displacement of the left horn. Right renal agenesis. EUA and diagnostic laparoscopy: Double uterus, moved cranially. Normal ovaries. Parietal peritoneum soaked with chocolate-brown coloured menstrual reflux. Great swelling of the vagina that protrudes in abdominal cavity. Drained hematocolpo through the vagina and access to the right blind vagina. Marsupialisation of the left vagina.

Results: Patient currently asymptomatic.

Discussion: The OVHIRA syndrome is a very rate malformation. Should always be kept in mind in the differential diagnosis, the progressive severe dysmenorrhoea in young women with renal agenesis that present urinary retention or pelvic mass. The early diagnose and treatment of vaginal septum is crucial to avoid any complications at long term and also to preserve fertility.

243

12 WEEK TUBAL ECTOPIC PREGNANCY AFTER A SURGICAL TERMINATION OF PREGNANCY (STOP). WHAT CAN BE LEARNT

Case Reports

A.Z. Davison, H. Jan*, J. Ross, N. Narvekar

${ }^{[1]}$ Kings College Hospital $\sim$ London $\sim$ United Kingdom
Summary (4 lines): A case who presented following a surgical termination of pregnancy with a ruptured live 12 week tubal ectopic pregnancy managed laparoscopically.

Introduction: We highlight the importance of transvaginal ultrasound, recognising absence of products of conception and laparoscopic management.

Material and Methods:

Results: The patient was referred following a STOP where no products of conception were identified. Transvaginal ultrasound showed an empty uterus with no uterine anomalies. A 12 week live ectopic pregnancy was seen separate to the uterus with significant haemoperitoneum. The patient was haemodynamically stable. Laparoscopy confirmed a tubal ectopic with a 3 litre haemoperitoneum. A laparoscopic right salpingectomy was performed.

Discussion: This highlights the importance of transvaginal ultrasound in early pregnancy. Current RCOG training for ST2 includes abdominal scan between $8-12 / 40$, however revision to transvaginal should be considered. The uterus should be visualised from cervix to fundus including interstitials to exclude uterine anomalies and interstitial ectopics. Large advanced ectopic pregnancies are rare but occur in the isthmic or iterstitial portion of the tube, the ovary or abdominally, therefore ectopic pregnancy cannot be excluded by gestational age alone. Laparoscopic management is appropriate in ruptured and advanced ectopic pregnancies and should be considered where the patient is haemodynamically stable.

\section{9}

\section{A CASE OF A MESONEPHRIC CYST CAUSING PELVIC PAIN Case Reports}

F. Shakir*, B. Jones, N. Waters, T. Rockall, A. Kent

${ }^{[1]}$ Royal County Surrey Hospital, Guildford $\sim$ Guildford, Surrey United Kingdom

Summary (4 lines): A rare case of a mesonephric cyst causing pelvic pain in a woman who had previously undergone hysterectomy and bilateral salpingo-oopherectomy for severe endometriosis.

Introduction: The mesonephric duct, also known as the Wolffian duct is a paired organ found in humans and other mammals during embryogenesis. In males the mesonephric duct develops into the epididymis, vas deferens and the seminal vesicles. However, in the female the absence of testosterone causes the mesonephric duct to regress, although inclusions and particularly cysts, may persist or develop as a remnant.

Material and Methods: A 40 year old woman presented with recurrent right iliac fossa pain having previously undergone hysterectomy and bilateral salpingo-oophorectomy. She was investigated with CT and MRI scans, both of which did not show any abnormality. However because of ongoing symptoms, a diagnostic laparoscopy was performed. Results: At laparoscopy a round ligament cyst was seen on the right pelvic side wall. Excision of the cyst was performed with monopolar diathermy. Histology of the specimen revealed a mesonephric cyst. The patient was seen four weeks post-operatively with resolution of her symptoms.

Discussion: Pelvic pain has multiple aetiologies presenting a challenge to healthcare professionals in its diagnosis and ongoing management. It was therefore important in this case to act upon the patient's ongoing symptoms, despite normal imaging, as rare causes such as this can be present.

\section{4}

\section{A CASE OF BOWEL OBSTRUCTION DUE TO ENDOMETRIOSIS} Case Reports

H. Lee* ${ }^{[1]}$ THE CATHOLIC UNIVERSITY OF KOREA $\sim$ SEOUL $\sim$ Korea,
Republic of 
Summary (4 lines): Although intestinal endometriosis is common, it is rarely manifested as an acute bowel obstructions We have experienced a case of intestinal obstruction due to endometriosis. It may involve intestinal wall and protrude into its lumen and patients with severe intestinal involvement usually require surgical resection.

Introduction: Among women with intestinal endometriosis, bowel endometriosis is an uncommon cause of intestinal obstruction.

Material and Methods: Clinical manifestations are not specific, making the preoperative diagnosis difficult to establish. An emergency laparoscophy was performed.

Results: The pathologic examination of the resected sample revealed endometriosis of sigmoid colon. Endometriosis can involve the intestinal tract extensively, causing a variety of clinical symptoms, and can result in a spectrum of mucosal alterations.

Discussion: This report highlights the importance of histopathological findings of resected specimens in the diagnosis of sigmoid colon obstruction due to intestinal endometriosis.

\section{8}

\section{A CASE REPORT OF THE MANAGEMENT OF OVARIAN TORSION IN PREGNANCY}

Case Reports

D. Crosby*, Y. O'Brien, A. Mullally, G. Von Bunau

${ }^{[1]}$ Coombe Women and Infants University Hospital, Cork Street, Dublin $8 \sim$ Dublin Ireland

Summary (4 lines): We present an interesting case of ovarian torsion in pregnancy due to a dermoid cyst and its successful laparoscopic management.

Introduction: We present the case of LF, a 31 year old primigravida who presented to the emergency room at 23 weeks gestation with an 8 hour history of a dull right iliac fossa pain with associated vomiting. Material and Methods: Mid-stream urine was positive for ketones. Her white cell count was raised to $15.0 \times 10 * 9 / \mathrm{L}$. Her bloods including full blood count, urea and electrolytes and C-reactive protein were normal. Ultrasound of pelvis showed a solid/cystic lesion with an irregular outline on the right ovary measuring $4.21 \times 4.15 \times 6.32 \mathrm{~cm}$. It was mixed echogenic and hypovascular in appearance suggestive of a dermoid cyst.

Results: She was brought to theatre and a laparoscopic right salpingooopherectomy was performed under general anaesthesia. Findings at laparoscopy showed an enlarged uterus with a torted tubo-ovarian necrotic mass to the right of the uterus.

Discussion: Post-operatively, she was continued on intravenous coamoxiclav for 3 days and intravenous atosiban for 24 hours. Fetal well being was assessed following the procedure. She had an uneventful post-operative course. She was discharged on day 3 post-operatively with antenatal clinic follow up three days later.

The histology revealed an ovarian dermoid cyst with no suspicious features.

\section{9}

\section{ADENOMATOID TUMOR WITH COMPLAINS OPERATED LAPAROSCOPICALLY: A CASE REPORT \\ Case Reports}

J. Alves*, A. Setubal, F. Faustino, D. Vilarinho, J. Faria

${ }^{[1]}$ Hospital da Luz $\sim$ Lisbon $\sim$ Portugal

Summary (4 lines): A 21-year old woman with dysmenorrhea, dyspareunia and dyschezia with MRI suggestive of endometriosis and a subserosal leiomyoma. Resection of uterosacral ligament and tumor removal was performed with rare adenomatoid tumor found. 6 month later patient has no complains.
Introduction: Adenomatoid tumors are distinctive neoplasms of mesothelial origin that occur both in men and women and in different parts of the body. Usually are incidental findings in Hysterectomy for different causes. No specific symptoms have been attributed to them and they usually are benign. Material and Methods: We present a 21-year old woman with dysmenorrhea (7/10), dyspareunia (5/10) and dyschezia (3/10). At observation there was pain touching pouch of Douglas and utero fundus, tenderness, pain and thickness of right utero sacral ligament. MRI revealed adenomiosis and a subserosal leiomyoma.

Results: Laparoscopic surgery was performed with resection of right uterosacral ligament and the right subserosal tumor described as leiomyoma. Histology described an adenomatoid tumor and inflammatory reaction of uterosacral ligament. After surgery, 6 months follow up the patient is symptom free.

Discussion: Adenomatoid tumors usually do not justify patient complains like dysmenorrhea and others. We present a rare case report of a young women with complains likely of suspicious endometriosis that turn out to be an adenomatoid tumor, that improved with resection.

\section{6}

\section{ADIANA HYSTEROSCOPIC TUBAL OCCLUSION DEVICE FOR THE TREATMENT OF HYDROSALPINX PRIOR TO IN VITRO FERTILIZATION : A CASE REPORT \\ Case Reports}

L. Guillaume*, G. Vanessa, L. Jean-marc, F. Hervé

${ }^{[1]}$ Hôpital de Bicêtre Assistance Publique des Hôpitaux de Paris France

Summary (4 lines): Tubal occlusion of hydrosalpinx prior to in vitro fertilization by Adiana matrix allows pregnancy and live birth.

Introduction: Recently a hysteroscopic approach to proximal occlusion of a hydrosalpinx using Essure microinsert coupled with IVF cycle has been proposed in order to increase pregnancy rate as an alternative to laparoscopic salpingectomy or laparoscopic tubal occlusion. If only few small series have described tubal occlusion with Essure device in this context, results are promising with 25 live birth reported (from 61 patients). To our knowledge, this case report is the first case of Adiana tubal sterilization prior to IVF in a context of hydrosalpinx and also the first case of pregnancy and live birth with this silicone matrix.

Material and Methods: We report the case of a 32-year-old nulligravid patient with pelvic adhesive disease and unilateral hydrosalpinx.

An unilateral proximal occlusion of the right fallopian tube with Adiana microinsert was performed.

The Main Outcome Measure was the success of the placement of the Adiana device and pregnancy.

Results: The patient underwent a successful occlusion of the hydrosalpinx by Adiana matrix with a pregnancy after IVF cycle.

Discussion: Adiana tubal occlusion device can be used prior to IVF and seems to be an alternative to Essure ${ }^{\circledR}$ procedure. The theoretical advantage of Adiana is the ability to maintain a uterine cavity free of all foreign matter.

\section{6}

BILATERAL BORDERLINE PAPILLARY SEROUS TUMOR OF THE FALLOPIAN TUBE: A CASE REPORT

Case Reports

M.I. Conca Rodero*, E. Cazorla Amorós, R. Betoret Gustems, C. Muñoz Ruiz

${ }^{[1]}$ Hospital del Vinalopo $\sim$ Elche $\sim$ Spain

Summary (4 lines): We present a case of bilateral PSBT of the fallopian tubes with unusual presentation 
Introduction: Papillary serous borderline tumor of the fallopian tube (PSBT) is a very rare gynecological entity. So there is limited experience about its behaviour and treatment.

Material and Methods: Case report of a 18-year-old patient with bilateral PSBT

Results: CASE REPORT

A 18-year-old patient presented with lower abdominal pain, abnormal bleeding and abdominal distension for the last two months. Ultrasound and CT scans revealed ascites and bilateral adnexal masses measuring, $80 \times 51 \mathrm{~mm}$ the left one and $92 \times 56 \mathrm{~mm}$ the right one. Serum CA 125 level was $108 \mathrm{U} / \mathrm{ml}$. A diagnostic paracentesis was done with report of inflammatory changes (not malignant cells). A laparoscopy was performed with findings of pelvic inflammatory disease with bilateral hydrosalpinx. Bilateral salpingectomy was done. Definitive pathologic report was bilateral PSBT of the fallopian tubes

Discussion: DISCUSSION

Review of the literature suggests salpingectomy as the optimal treatment for patients with borderline tumors of the fallopian tubes. This is a very unfrequent gynaecological disease that can present with atypical symptoms.

73

\section{BILATERAL OVARIAN METASTASIS IN A PATIENT WITH BREAST CANCER \\ Case Reports}

G.G. Germano*, I. Posada, L. Belza, M. Gimer

${ }^{[1]}$ Hospital Central de las Fuerzas Armadas Montevideo Uruguay

Summary (4 lines): We report a case presenting with an ovarian bilateral metastasis in a patient with lobular breast cancer.

Introduction: A 58 years old patient with previous breast lobular carcinoma Stage I, high risk in 1998, treated with mastectomy and linphadenectomy is presented. In 2007 presented bone metastasis, treated with chemotherapy.

Material and Methods: In December 2011 a control ultrasound showed oversized ovaries, irregular superficies, and inhomogeneous structure, with normal vascularization and mild ascitis. Tumor markers (CEA; CA19-9; CA125; and CA15-3) were normal. A laparoscopic approach was performed, finding oversized, brain-shaped, solid, ovaries. A bilateral adnexectomy was performed.

Results: The intraoperative pathologist consult, showed bilateral metastasis of breast cancer. No other localizations of tumor were found in the abdominal-pelvic laparoscopic exploration.

Discussion: Ovarian metastasis in a breast cancer frequency is very low. The type that more often present metastasis is infiltrating lobular carcinoma. The differentiation between primary or metastatic ovarian cancer only can be made by biopsy, thus the pathological study of the specimen in the operating room is mandatory. The accepted treatment for this pathology is the bilateral adnexectomy.

92

CAESAREAN SCAR PREGNANCY TREATED SUCCESSFULLY SURGICALLY FOLLOWING FAILED MEDICAL THERAPY - A CASE REPORT

Case Reports

X. Ong*, M. Mathur

${ }^{[1]}$ KK Women's and Children's Hospital $\sim$ Singapore $\sim$ Singapore

Summary (4 lines): We report a case of Caesarean scar ectopic pregnancy successfully treated by laparoscopic guided evacuation of uterus following failed methotrexate therapy in our centre.
Introduction: Implantation of an ectopic pregnancy within a previous Caesarean section scar is a rare condition. However, its incidence is increasing worldwide due to the increased number of Caesarean sections performed. We report a case of Caesarean scar ectopic pregnancy treated surgically following failed medical therapy.

Material and Methods: -

Results: A 29 year old Chinese woman with one previous Caesarean section was diagnosed with a Caesarean scar pregnancy. Initial treatment with intramuscular methotrexate failed. A repeat scan showed an increase in the size of the scar ectopic and increasing bhCG levels. Diagnostic laparoscopy and evacuation of the uterus was performed. She remained clinically well upon discharge and on follow up, with falling beta-hCG levels. Discussion: There is limited scientific data on the pathophysiology, predisposing factors, recurrence rate and optimal treatment for this rare but potentially catastrophic complication of a previous Caesarean birth. Current data indicate that expectant treatment is rarely successful and delayed diagnosis and treatment may lead to serious morbidity and mortality. We aim to follow up this case study by conducting a retrospective review of the cases treated both medically and surgically in our centre.

\section{8}

\section{CASE REPORT - MANAGEMENT OF AN ABDOMINAL PREGNANCY USING THE PLASMAJET DEVICE}

Case Reports

Y. Diab*, F. Shakir

${ }^{[1]}$ Royal Surrey County Hospital, Guildford Guildford, Surrey United Kingdom

Summary (4 lines): Abdominal pregnancy is rare, reported as $1 \%$ of all ectopic pregnancies. We present a case of an abdominal pregnancy which was successfully treated with the plasmajet device.

Introduction: A 33 year old woman who had IVF ICSI with 2 embryos transferred presented with pelvic pain and vaginal bleeding one month later. An ultrasound performed demonstrated a mass in the right adnexae assumed to be an ectopic pregnancy.

Material and Methods: A laparoscopy was performed which did not demonstrate any ectopic pregnancy but an ill defined mass in the right side wall, assumed to be a nodule of endometriosis, which was left uninterrupted. She represented as her symptoms did not resolve. Repeat ultrasound suspected an abdominal pregnancy so another laparoscopy was arranged.

Results: At repeat laparoscopy there was a right pelvic side wall haematoma with adherent tissue. The area of tissue was vascular when avulsion was performed and bleeding difficult to control. The area was fully cauterised with the plasmajet device which achieved complete vaporisation of residual tissue and haemostasis. The histology from the tissue revealed chorionic villi.

Discussion: Abdominal pregnancy is a rare event and can lead to severe morbidity and mortality if missed. We advocate a detailed pelvic and abdominal survey during laparoscopy if the pregnancy is clearly not intrauterine. The use of plasmajet was useful leading to vaporisation of residual chorionic villi and haemostasis.

\section{6}

CASE REPORT AND LITERATURE REVIEW: MANAGEMENT OF A LARGE CELL NEUROENDOCRINE CARCINOMA OF THE UTERINE CERVIX

Case Reports

${ }^{[1]}$ Newcastle University $\sim$ Newcastle Upon Tyne $\sim$ United Kingdom 
Summary (4 lines): We present the case of a large cell neuroendocrine carcinoma (LCNEC)of the uterine cervix in view of the existing literature including tumour histology, prognosis, and management options.

Introduction: Neuroendocrine carcinomas of the cervix are a rare and aggressive subtype of cervical carcinomas first described by AlboresSaavedra in 1972. LCNECs have a reported incidence of $<0.2 \%$ of cervical cancers. LCNECs typically metastasise and recur early despite intensive multimodal treatment; survival beyond 2 years is rarely reported. Material and Methods: A $23 \mathrm{yr}$ underwent caesarean section when she was found to have a pelvic mass. Histology showed large cells with vesicular nuclei, a mitotic index in excess of $40 / 10 \mathrm{HPF}$, and geographical necrotic areas. Immunohistochemistry showed positivity for synaptophysin, chromogranin and neuron-specific enolase. The proliferation marker Ki67 was almost $100 \%$.

Results: The FIGO stage IIB tumour was treated with a dose dense chemotherapy regimen of Cisplatin and Etoposide, 50 Gy radiotherapy and 40 Gy brachytherapy. She remains in remission 18 months after diagnosis.

Discussion: Since the clear classification of neuroendocrine carcinomas in 1997 there have been $\sim 80$ cases of LCNECs reported in literature. However, optimal management is yet to be delineated. Our case supports growing evidence of the efficacy of cisplatin and etoposide and should provide a valuable guide to future practice.

\section{4}

\section{CASE REPORT: A RARE CASE OF INTRAMYOMETRIAL ENDOMETRIOSIS \\ Case Reports}

A. Recas*, M. Cosan, P. Cavallé Busquets, J. Salinas, L. Martínez

${ }^{[1]}$ Hospital Universitario Sant Joan de Reus $\sim$ Reus $\sim$ Spain

Summary (4 lines): Patient 18 years old who presents dysmenorrhea and left colic renal pain coincident with menstruation, which has been evolving for 2 years.

Introduction: Physical examination of the woman is completely normal. On pelvic examination does not show remarkable changes.TPAL 0000. Begins study of pelvic pain

Material and Methods: US: image suspected of double uterus. CT: hypodense image in the left uterine side wall very close to left uterine artery, suggestive of endometrioma of $23 \times 27 \mathrm{~mm}$. MRI: Altered uterine endometrial cavity double presence that communicates in the $1 / 3$ posterior lower uterine highly suggestive of incomplete double uterus. Compression could condition the left ureter. Hysteroscopy: Single externe orifice and single cervical canal. Normal uterine cavity. Results: It is considered diagnostic uterine wall endometrioma. Laparoscopy is performed where intramyometrial endometrioma observed. Capsule excision is performed in its entirety without going into uterine cavity. Peritoneal implant surface active endometriosis is removed

Discussion: The combination of imaging and diagnostic hysteroscopy reduces the diagnostic possibilities in cases of certain uterine malformations, especially when there is discrepancy between tests. Laparoscopy allows visual clinical diagnosis and extent of injury, being possible the optimal treatment of the same, decreasing the morbidity associated with laparotomy, being for this reason an optimal surgical approach in young patients.

\section{1}

\section{CERVICAL CANCER: CASE SERIE UPDATE SINCE 2000-2011} Case Reports

C. De Valle Corredor*, L. Fernandez Muñoz, M.D. Rodriguez Garnica, V. Garcia Pineda, P. Valenzuela Ruiz, A. Couso Gonzalez, A. Zapico Goñi ${ }^{[1]}$ Hospital Universitario Príncipe de Asturias $\sim$ Madrid $\sim$ Spain
Summary (4 lines): Laparoscopy (LPS) has low morbidity and complications rate for staging and treating cervical cancer (CC).

Introduction: Staging leads clinical approach and prognosis in 2nd most prevalent cancer in women.

Material and Methods: Retrospective \& descriptive study of 108 patients with CC between 2000-2011. Early stage group (ESG) (IA1, IA2, IB1, IIA1) has 57(52,8 \%) patients and advanced stage group (ASG) has 51 (47,2\%). Surgical staging and treatment, major surgical complications (MSC) and outcomes are analyzed.

Results: Surgery is done in $55(96,4 \%)$ patients in ESG.

LPS is performed in $44(80 \%)$ radical hysterectomy + pelvic lymphadenectomy $(\mathrm{RH}+\mathrm{PL})$ and 7 (12,7 \%)pelvic \& paraaortic lymphadenectomy (PALN). Conservative simple histerectomy ( $\mathrm{SH}$ ) was done in $4(7,3 \%)$.

MSC appeared in $3(5,5 \%)$ RH + PL [2 (66 \%) great vessel injury (GVI) which didn't require conversion into laparotomy (CIL) and 1 $(33,3 \%) \mathrm{GVI}+$ ureteral lesion which needed CIL].

Catch-up SH is done in $10(19,6 \%)$ ASG patients after neoadjuvant treatment. Staging retroperitoneal laparoscopy (SRL) was done in 14 $(27,5 \%)$, paliative surgery is done in $1(2 \%)$. An intestinal lesion happened in $1(4 \%)$ patient which was solved laparoscopically. In ESG follow-up accounted 11 (19\%) relapses, 6(10,3\%) deaths vs ASG which had $7(14 \%)$ relapses and $12(24 \%)$ deaths.

Discussion: LPS is a good way to approach CC for treatment and for staging.

MSC rate were low in both groups.

No were differences in the relapse rate between ESG \& ASG and the death rate was increased in ASG.

273

\section{COMPARTMENT SYNDROME IN DEEP ENDOMETRIOSIS SURGERY}

Case Reports

E. Fernández Miranda*, P. Barri Soldevila, C. Cárdenas Nylander, M.T. Cusidó, A. Úbeda

${ }^{[1]}$ IU Dexeus $\sim$ Barcelona $\sim$ Spain

Summary (4 lines): Compartment syndrome is defined as an increased hydrostatic pressure $(>30 \mathrm{mmHg})$ in an osteofascial closed space, which leads to a decrease of perfusion muscles and nerves of compartment.

Introduction: A case of a 33 year-old woman who underwent surgery with a diagnosis of deep pelvic endometriosis. In the immediate postoperative a tumescent erythematous area is observed, with severe pain and paresthesias in the posterolateral compartment of the lower external limb. Pressure in the compartment is $31 \mathrm{~mm} \mathrm{Hg}$, so that the diagnosis is a compartment syndrome.

Material and Methods: Fasciotomy was performed with a unique lateral incision that does not close. After 4 days a revaluation proceeds, noting an area of decreased contractility of medial gastrocnemius muscle, so that the extirpation of the affected area and fasciotomy closure are performed.

Results: A correct gynaecological position in long duration surgeries is of vital importance. Anomalous positions, together with hypotension, hypovolemia and peripheral vasoconstriction as well as venous disorders or other risk factors such as obesity, can be a cause of this complication.

Discussion: Therefore we must take those possible preventive measures, such as the correct position of the limb, the use of brackets padded, avoiding the Trendelenburg end, avoiding hypotension, hypovolemia and vasoconstrictor drugs, and we must perform legs moving every 2 hours when a surgery of more than 4 hours is expected. 
339

\section{DETECTION OF A CERVICAL DEFECT AT PRE-IVF HYSTEROSCOPY}

Case Reports

C. Van Turnhout*, P. Puttemans, S. Gordts, R. Campo, M. Valkenburg, S. Gordts

${ }^{[1]}$ Leuven Institute of Fertility and Embryology $\sim$ Leuven $\sim$ Belgium

Summary (4 lines): A 29-year old patient in our IVF program presented with a defect of the cervix at hysteroscopy, leaving only a layer of visceral peritoneum between the endocervical canal and the pouch of Douglas. We discuss the difficulties at embryo transfer and the obstetrical implications. Introduction: Routine pre-IVF hysteroscopy can reveal abnormalities that are important for further treatment.

Material and Methods: We report a case of an unusual hysteroscopy several years after laser-laparoscopy for AFS grade IV endometriosis. Results: A 29-year old patient consulted for primary infertility of 2 years. She already had an operative laparoscopy for severe endometriosis. After adhesiolysis in the obliterated Douglas, bilateral ovarian endometriomas were removed with the $\mathrm{CO} 2$ laser, as well as a nodule of deep rectovaginal endometriosis.

Office mini hysteroscopy revealed a defect in the posterior wall of the endocervical canal just below the os internum. Only a thin layer of peritoneum was separating the endocervical canal from the pouch of Douglas. As a consequence the embryo transfer had to be performed under ultrasound guidance with a firm catheter to ensure correct positioning of the tip. Moreover, it was decided not to perform any reconstructive surgery. Meanwhile patient conceived, her pregnancy was uneventful and she gave birth to a healthy child at 38 weeks by C-section.

Discussion: Systematic pre-IVF hysteroscopy surely helps to anticipate difficult embryo transfers.

\section{9}

\section{DIAGNOSIS AND MANAGEMENT OF LIFE THREATENING UTERINE ARTERIOVENOUS MALFORMATION Case Reports}

R. Viswanatha*, J. Clarke, R. Hamid, S. Sankaranarayanan

${ }^{[1]}$ Croydon University Hospital $\sim$ London $\sim$ United Kingdom

Summary (4 lines): We present a case of acquired uterine arteriovenous malformation (AVM) causing life threatening hypermenorrhagia. Appropriate and timely investigation and treatment saved her life and fertility. Introduction: An Young nulliparous lady presented with a history of heavy bleeding following a surgical termination of pregnancy. She was managed in various hospitals with hormonal and non hormonal medications and Mirena coil with no improvement in her symptoms and eventually causing her to develop depression.

Material and Methods: She was admitted 4 times with very low hemoglobin requiring blood transfusions. She had stopped working and was even prepared to have a hysterectomy to return to normal life. A Doppler Ultrasound of the pelvis and hysteroscopy showed a pulsating lesion in the posterior uterine wall just below the endometrium. MRI pelvis confirmed uterine AVM and she was successfully treated with uterine artery embolization (UAE).

Results: Four weeks following the UAE bleeding ceased and all her medication were stopped.

Discussion: Das et al, 2008, described AVM as a life threatening condition. Uterine AVM can be congenital where it may be isolated or associated with AVM in other organs. Acquired AVM is usually secondary to uterine trauma. O Brien et al, 2006 showed that the Doppler ultrasound is the key investigation of choice. Systematic review on acquired AVM by Peitsidis et al, 2011, showed that UAE was the most commonly used method of treatment.
202

\section{DIFFERENTIAL DIAGNOSIS OF RIGHT-SIDED PELVIC MASSES Case Reports}

L. Pineda*, J.L. Alcazar

${ }^{[1]}$ Clinica Universidad de Navarra $\sim$ Pamplona $\sim$ Spain

Summary (4 lines): We report on a case of mucinous cystadenoma of the appendix mimicking an adnexal mass.

Introduction: A 79-year-old woman that attended our office due to new onset of right adnexal mass with tumour markers within normal range. She referred no symptoms but mild inespecific epigastric pain. Material and Methods: Review of clinical, sonographic and histopathologic data.

Results: US revealed cystic right adnexal mass of $79 \times 39 \mathrm{~mm}$, compatible with benign process and free liquid in Douglas pouch with no other remarkable findings. Laparoscopic surgery was performed showing a $5 \mathrm{~cm}$ mucin-filled apendicular mass and macroscopically normal uterus and adnexa. No other sings of pathology were found. Laparoscopic exeresis of appendix and adnexa was performed. Definitive histopathological diagnosis was mucinous cystadenoma of the appendix. After 5 months of follow up, the patient has a full recovery.

Discussion: Appendiceal mucoceles are a rare entity found in only 0 ' $3 \%$ of appendiceal specimens and from those, $63 \%$ are mucinous cystadeomas. About half of the patients are asymptomatic and the other half present inespecific abdominal symptoms. Eventhough US is the primary diagnostic tool, there is no specific method to determine the diagnosis with certainty preoperatively. Surgical excision of mucocele of appendix can be treated safely with laparoscopic approach, obteining advantages such as good exposure and evaluation of the entire abdominal cavity as well as rapid recovery and better cosmetic outcome.

\section{0}

\section{ENDOMETRIAL OSSEOUS METAPLASIA: 2 OWN CASES} Case Reports

Y. Goncharova*, I. Sudoma, O. Solovyov, T. Zadorozhna

${ }^{[1]}$ Clinic of reproductive medicine NADIYA $\sim$ Kyiv $\sim$ Ukraine

Summary (4 lines): We present 2 own cases of endometrial osseous metaplasia.

Introduction: Endometrial osseous metaplasia (EOM) is a rare clinical entity with the presence of mature or immature bone in endometrium. Most of the cases clinically present with secondary infertility following an abortion. Hysteroscopy thought to be a gold standard for its diagnosis and treatment. We present 2 own cases of EOM.

Material and Methods: Both patients were referred to the clinic for infertility treatment. The first patient, 29 y.o., had secondary infertility and hysteroresectoscopical myomectomy in the past. The second patient, 40 y.o., had primary infertility and endometriosis.

Results: EOM in the first case was suspected on ultrasound and confirmed during hysteroscopy and hystologically. Fully formed bone fragment resembling coral was found in uterine cavity. In the second case EOM was suspected on ultrasound and confirmed by histological exam, while hysteroscopical findings were not convincing: endomerium was thin, with slightly unusual opalescence of the surface, without any visible bone-like fragments. Hystologically EOM was diffuse. After 1 IVF failure this patient had spontaneous conception but missed pregnancy with chromosomal anormaly.

Discussion: Hysteroscopy has been shown to be effective in the diagnosis and treatment of EOM associated with infertility but sometimes ultrasound may show more prominent changes and only histological exam plays crucial role in correct diagnosis. 
331

HYSTEROSCOPIC REMOVAL OF MATURE CYSTIC TERATOMA IN ENDOMETRIAL CAVITY WITH THE USE OF MYOSURE MORCELLATOR : CASE REPORT

Case Reports

D. Mathiopoulos*, T. Panoskaltsis, Patmanidis, J. Vlaxos, N. Vlahos, T. Tsiaousi

${ }^{[1]}$ LETO MATERNITY HOSPITAL ATHENES $\sim$ Greece

Summary (4 lines): Teratomas are the commonest germ cell tumours and are most frequently found in the testes and ovary. Extragonadal teratomas are rare and mainly occur in midline structures.

Introduction: Mature cystic teratomas (commonly called 'dermoid cysts') are cystic tumors composed of well-differentiated tissue derived from at least two of the three germ cell layers (ectoderm, mesoderm and endoderm). Teratomas are the commonest germ cell tumours and are most frequently found in the testes and ovary. Extragonadal teratomas are rare and mainly occur in midline structures. Uterine teratomas are extremely rare with only 17 cases reported. Teratomas of the uterus can occur in the uterine cavity, cervical canal, and uterine surface. The majority of the tumors described in the literature were mature teratomas, only two immature teratomas $\mathrm{h}$ been reported.

Material and Methods: We present the 8th case of endometrial teratoma ever reported in the literature. It occurred in a 37 year old nulliparous woman who presented with abnormal uterine bleeding. The Transvaginal ultrasound showed an endometrial mixed texture mass measuring $3 \times 2 \mathrm{~cm}$. The mass was excised with the MyoSure Morcellator.

Results: Histology revealed a mature teratoma

Discussion: The MyoSure Morcellator proved to be an effective tool for remove entirety even this kind of tumor. Except for its already its reported use by us with polyps and fibroids.

499

\section{HYSTEROSCOPIC TREATMENT OF INTRAUTERINE ADHESIONS}

Case Reports

M. Djakonovic Maravic, M. Dokic, Z. Vilendecic, M. Mitrovic*, S. Vidakovic

${ }^{[1]}$ Clinic for Gynaecology and Obstetrics, Clinical Centre of Serbia Belgrade $\sim$ Serbia

Summary (4 lines): We used hysteroscopy as a diagnostic and therapeutic tool in infertile patient with intrauterine adhesions.

Introduction: Evaluation of uterine cavity is a standard part of infertility work-up. If intrauterine pathology is suspected, differential diagnosis usually include endometrial polyps, submucous myomas and intrauterine adhesions.

Material and Methods: We present a case of an infertile patient in whom hysteroscopy was performed as a diagnostic and therapeutic procedure.

Results: A 32-year-old patient with secondary infertility was admitted to our institution due to an intracavitary lesion diagnosed by hysterosalpingography. In contrast, ultrasound exam failed to demonstrate intrauterine pathology. Hysteroscopy was performed and a single voluminous intrauterine adhesion between anterior and posterior uterine wall was diagnosed. We used bipolar electrode as a treatment option.

Discussion: Intrauterine adhesions may interfere with normal reproduction. If surgical treatment is indicated, results are improved if the surgery is performed under direct visualization using a hysteroscope.
268

\section{HYSTEROSCOPIC TUBAL STERILIZATION: OUR EXPERIENCE WITH THE ESSURE® SYSTEM}

Case Reports

A. Regalo*, L. Caseiro, F. Fernandes

${ }^{[1]}$ Hospital Espirito Santo $\sim$ Évora $\sim$ Portugal

Summary (4 lines): The aim of this study is to update the evidence of efficacy and safety of the Essure ${ }^{\circledR}$ system.

Introduction: Female sterilization has undergone changes in the last decade. Besides laparoscopic tubal occlusion, the Essure ${ }^{\circledR}$ system is nowadays a viable option.

Material and Methods: A retrospective case-series report of 191 women undergoing Essure ${ }^{\circledR}$ coil insertion between July of 2008 and December of 2011, in an ambulatory setting in a Portuguese secondary hospital. The procedures used a $5 \mathrm{~mm}$ single channel operative hysteroscope and efficacy was based on the confirmation imagiologic test. Follow up concerning presumptive pregnancies were also taken into account. Results: The women mean age was 32 years, mean parity was 1,9 children. The majority of procedures occurred on the 11th day of the menstrual cycle. Essure ${ }^{\mathbb{R}}$ was successfully placed on the first attempt in 187 of $191(98 \%)$ and the mean duration was 5.4 minutes. 1 patient required a second attempt to complete placement and 3 patients required laparoscopic tubal sterilization after an unsuccessful Essure ${ }^{\circledR}$. Between July of 2008 and December of 2011, 191 women underwent the Essure ${ }^{\mathbb{R}}$ procedure, 13 were excluded for absence of the confirmation test $(\mathrm{n}=178)$. Our efficacy, confirmed at 3 month follow up, is $97 \%$ (173/178), with no reported pregnancies.

Discussion: The Essure ${ }^{\circledR}$ procedure in an ambulatory setting is safe, well tolerated and the least invasive method for female sterilization. Our success rate is $97 \%$.

38
INTRA-MYOMETRIAL PREGNANCY: A RARE LOCALIZATION OF ECTOPIC PREGNANCY
Case Reports

Y. Ait Benkaddour*, K. El Abdi, A. Aboulfalah, H. Asmouki, A. Soummani

${ }^{[1]}$ Department of obstetrics and gynecology, University hospital of Marrakesh, Cadi Ayyad University Marrakesh $\sim$ Morocco

Summary (4 lines): We report a rare case of intra-myometrial pregnancy following IVF. The diagnosis was done intra-operatively when patientwas admitted for a genital hemorrhage. The case illustrate the diagnostic challenge of this rare condition.

Introduction: Intra-myometrial pregnancy is a rare form of ectopic pregnancy. It makes a diagnostic and therapeutic challenge. If misdiagnosed the intra-myometrial pregnancy can cause a uterine rupture and become life-threatening condition.

Material and Methods: We report a case of intra-myométrial pregnancy in twin pregnancy following IVF with spontaneous abortion of the first twin At 9 weeks of gestation. The 10 weeks scan showed a normal fetus which was described to be highly localized in the uterus but the diagnosis of intra-myometrial pregnancy was not suspected.

Results: The patient was admitted at 14 weeks of gestation with pelvic pain, hemorrhage and shock. She was operated and the diagnosis of ruptured intramyometrial pregnancy was done and managed conservatively.

Discussion: This case illustrates the diagnostic difficulties of intramyometrial pregnancy. We discuss pathophysiology, diagnosis and treatment of this exceptional form of ectopic pregnancy. 
325

\section{LAPAROSCOPIC GENITAL REASSIGNMENT SURGERY FOR FEMALE-TO-MALE TRANSSEXUALISM}

Case Reports

\author{
S. Ribeiro*, A. Valentim Lourenco, A. Henriques, A.L. Ribeirinho \\ ${ }^{[1]}$ Centro Hospitalar Lisboa Norte $\sim$ Lisboa $\sim$ Portugal
}

Summary (4 lines): Female-to-male transsexualism has a demanding treatment that traditionally includes combined vaginal and abdominal genital reassignment surgery. In this case-series we report on an alternative to this procedure, in which the vaginectomy is also performed laparoscopically. Introduction: We report on an alternative procedure to the traditional female-to-male transsexualism genital reassignment surgery in which the vaginectomy is also performed laparoscopically.

Material and Methods: We reviewed the charts of all (15 cases) patients submitted to genital reassignment surgery in our department between November 2007 and March 2011. In all cases the vaginectomy was also performed laparoscopically in continuum with the hysterectomy and bilateral salpingo-oophorectomy.

Results: The average operating time was 222 minutes. No serious complications were observed. Patients were released no later than 3 days after the procedure, except in 2 cases with prolonged postoperative urinary retention who were released on the seventh postoperative day with no need for bladder catheterization. One patient had a perineal hematoma 1 month after surgery, which resolved spontaneously.

Discussion: Total laparoscopic genital reassignment surgery appears to be an appropriate alternative to the traditional combined abdominal/vaginal approach. This procedure provides better exposure of the anatomical structures and has the advantage of being executed in a continuous fashion.

\section{0}

\section{LAPAROSCOPIC INTRASAC METHOTREXATE INJECTION FOR TREATING A CORNUAL ECTOPIC}

Case Reports

A. Mchugh*, N. Purandare, N. Maher, P. Mcparland

${ }^{[1]}$ The National Maternity Hospital, Holles Street, $\sim$ Dublin $2 \sim$ Ireland

Summary (4 lines): Cornual ectopic pregnancies are rare accounting for $<0.01 \%$ of all pregnancies.

Introduction: We present a case of a cornual ectopic pregnancy managed using laparoscopic intrasac injection of methotrexate in a $35 \mathrm{yr}$ old. Material and Methods: A suspected cornual ectopic pregnancy was noted on ultrasound scan at 9 weeks gestation. The gestational sac measured $15 \times 21 \times 17 \mathrm{~mm}$ with a fetal pole of $4 \mathrm{~mm}$ identifiesd within the sac. The patient was asymptomatic. Serum BhCG was 27,471 IU/L. At laparoscopy, the right cornua appeared enlarged, consistant with an unruptured right cornual ectopic pregnancy. Intraoperatively the gestational sac was injected with methotrexate. The rent from the needle was sealed with bipolar diathermy. There were no immediate post operative complications. Methotrexate injections were repeated on post-op day3 following confirmation of normal liver function. Serum BhCG levels were repeated regularly until $<2 \mathrm{miu} / \mathrm{ml}$ at 4 months post operative.

Results: Day (D)- Serum BhCG miU/ml Pre-op-27471, D3-14016, D5-13628, D7-7996, D14-2622, D21-864,4 months $<2$.

Discussion:Cornual ectopic pregnancies can pose a therapeutic challenge. Traditionally, the gold standard of treatment was radical surgery often including hysterectomy. Currently, more conservative laparoscopic treatment can be accomplished with great success. Our case illustrates the effective management of a cornual ectopic pregnancy using laparoscopic intrsac methotrexate injection.
464

\section{LAPAROSCOPIC MANAGEMENT OF A HUGE STRUMA OVARII Case Reports}

A. Hosseini, A. Moeini*

${ }^{[1]}$ Department of Obstetrics and Gynecology, ShohadaTajrish Hospital, Shahid Beheshti University of Medical Science $\sim$ Tehran $\sim$ Iran, Islamic Republic of

Summary (4 lines): We report a 43-year-old female with a giant size of SO presenting with palpable lower abdominal mass. Removal of the mass was achieved via a laparoscopic approach.

Introduction: Struma Ovarii (SO) is a variant of dermoid tumors of the ovary in which thyroid tissue components is the major constituent.

The largest size of this tumor reported by Ezon was $30 \mathrm{~cm}$ in greatest dimension.

The nature of disease is benign, so laparoscopic technique could be performed.

Material and Methods: A 43-year-old virgin female complained from abdominal vague pain. Computed tomography (CT) of the abdomen demonstrated a large complex cystic lesion measuring $28 \times 22 \mathrm{~cm}$ originating from the right ovary.

The results of CA-125, AFP, B-HCG, CEA and TFT were normal.

Results: Laparoscopic resection of the adnexal mass was performed due to the benign appearance of the mass on $\mathrm{CT}$ and the negative laboratory studies. Drainage of the cyst was done under direct visualization with caution to prevent spillage of the cyst contents.

After complete decompression of the mass, a right salpingooophorectomy was performed using a laparoscopic vascular stapler.

Immunohistochemistry of the cyst was compatible with SO.

Discussion: SO tumors vary in size but usually measure less than $10 \mathrm{~cm}$ in diameter. In the two reports by Ezon and Savelli the largest dimension were 30 and $20 \mathrm{~cm}$ respectively.

Our patient also had a tumoral mass measuring $28 \times 22 \mathrm{~cm}$ which was treated by a laparoscopic total right salpingo-oopherectomy.

\section{3}

\section{LAPAROSCOPIC MANAGEMENT OF PRIMARY SIGNET RING CELL CARCINOMA OF THE OVARY}

Case Reports

M. Nicholson*, P. Athanasias, N. Pisal, C. Podesta

${ }^{[1]}$ Whittington Hospital $\sim$ London $\sim$ United Kingdom

Summary (4 lines): Primary signet ring cell carcinoma of the ovary is an extremely rare gynaecological pathology. We report a rare case of a signet ring cell ovarian carcinoma with typical features of a primary presenting as a unilateral ovarian torsion

Introduction: Signet ring cell carcinoma accounts for 1-2 \% of ovarian tumours. Historically this characteristic morphology is recognised as a feature of metastatic carcinoma, with the primary often located in the GI tract.

Material and Methods: A 46 year old woman presented with acute onset right iliac fossa pain and vomiting. She had no other associated symptoms and no risk factors for ovarian cancer. Transvaginal scan showed a complex right adenexal structure and free fluid in the pelvis. Ca125 was 127

Results: With US strongly suggestive of ovarian torsion, emergency laparoscopic right salpino-oophorectomy took place. Histology confirmed signet ring cell ovarian adenocarcinoma confined to the capsule with no evidence of local spread. Pelvic MRI, CT pancreas and chest, colonoscopy, gastroscopy and CT pneumocolon took place but no other tumour or distal deposits were identified. Laparoscopic appendectomy and an omental biopsy followed. 
Discussion: This case report supports previously identified clinical features typical of a primary signet ring cell ovarian carcinoma. It stresses the necessity of thorough investigation to exclude primary carcinoma elsewhere and illustrates the clinical challenges this poses.

343

\section{MANAGEMENT OF OVARIAN BORDERLINE TUMOR DISCOVERED INCIDENTALLY DURING EXPLORATORY LAPAROSCOPY FOR INFERTILITY \\ Case Reports}

D. Grigoras*, L. Pirtea

${ }^{[1]}$ UMF "Victor Babes" Timisoara Romania

Summary (4 lines): It is presented a case of a 30 years old nuligesta, investigated for infertility. On ultrasound bilateral ovarian cysts with papillary projections (smooth margins, reduced blood flow) were found. Exploratory laparoscopy was performed and extracapsular vegetations were observed at the surface of both ovaries. Touch imprint exam revealed borderline areas on the surface of right ovary. Right adnexectomy and left cystectomy, peritoneal washing, omentum and peritoneal biopsy were performed. The morfopathological exam revealed borderline areas on the left cyst. The disease was staged IC acording to FIGO. Restaging by laparoscopy was performed after 3 months. Complete staging was performed no upstaging was decided. Introduction: Borderline ovarian tumors can be discovered incidentally during laparoscopy. In nuliparous women fertility sparring is required. Restaging can be recommended in cases with conservative treatment

Material and Methods: Data from the ultrasound, touch imprint and morfopathological exam as well as intra-operative description from both surgeries are provided.

Results: The tumors were discovered incidentally during surgery Touch imprint exam found one lesion, but missed the other one Laparoscopic restaging was performed, no upstaging.

Discussion: Borderline ovarian tumors can be found incidentally during laparoscopy for presumed benign disease.

Value of restaging in cases where fertility was preserved.

\section{MANAGEMENT OF PATIENTS WITH ETOPIC PREGNANCY OR} OVARIAN BLEEDING WITH MASSIVE HEMOPERITONEUM BY LAPAROSCOPIC SURGERY

Case Reports

M. Kim*, J. Ko, C. Lee

${ }^{[1]}$ 1. Sanggye Paik Hospital $\sim$ Seoul $\sim$ Korea, Republic of

Summary (4 lines): Even in women with massive hemoperitoneum, laparoscopic surgery can be safely conducted.

Introduction: Traditionally, laparoscopic surgery was contraindicated in patients with massive hemoperitoneum. We performed retrospective study to evaluate the feasibility and safety of surgical laparoscopy with ectopic pregnancy or ovarian bleeding with massive hemoperitoneum.

Material and Methods: From January 2008 through April 2012, One hundred and fifty-seven women with ectopic pregnancy and one hundred and forty-two hemorrhagic corpus luteum were treated by laparoscopic surgery. Twenty-four patients who demonstrated more than $500 \mathrm{cc}$ of intraabdominal bleeding were classified as having massive hemoperitoneum (ectopic pregnancy: 14, hemorrhagic corpus luteum; $10)$ and retrospectively analyzed.

Results: Site of pregnancy in 4 patients with massive hemoperitoneum was isthmic: 3 ; and ampullary: 11 . The mean amount of estimated intraabdominal bleeding, which was calculated by the difference between the volumes of aspirated and irrigated fluids, was $1016.2 \pm 484.2 \mathrm{cc}$. In all cases of massive hemoperitoneum, there was no need for laparotomic conversion. There were no treatment related complications.

Discussion: Even in women with ectopic pregnancy or ovarian bleeding with massive hemoperitoneum, laparoscopic surgery can be safely conducted by experienced laparoscopists if hemodynamic stability is achieved by perioperative management.

425

\section{OVARIAN CANCER ARISING IN ENDOMETRIOID CYSTS: A CASE REPORT \\ Case Reports}

A. De Almeida Rodrigues*, J. Sequeira Alves, C. Castro, A. Rodrigues, C. Alho, F. Osório, C. Calhaz-jorge

${ }^{[1]}$ Hospital de Santa Maria - CHLN, EPE $\sim$ Lisboa $\sim$ Portugal

Summary (4 lines): We present a case of ovarian adenocarcinoma arising in an endometrioid cyst.

Introduction: Endometriosis is a common gynaecological disease with an estimated prevalence of $5-15 \%$ in women of reproductive age. Although benign, it represents a serious clinical problem because of its potential for adverse sequelae, including infertility, pain and risk of development of ovarian cancer, existing evidence that suggest an association between endometriosis and ovarian cancer, with an estimated prevalence of $0.3-0.8 \%$.

Material and Methods: (Not applicable).

Results: A 48 year-old woman with a right ovarian endometrioma known for 9 months presented with dysmenorrhoea, dyspareunia and chronic pelvic pain. She was submitted to laparoscopic hysterectomy and right adnexectomy. The pathologic analysis of the ovarian cyst revealed mucinous ovarian adenocarcinoma. Surgical staging was performed including laparoscopic left adnexectomy; no tumor tissue was identified. A multidisciplinary team decided that there was no indication for adjuvant therapy. Currently, the patient is well, distressed only from vasomotor symptoms.

Discussion: The relationship between endometriomas and ovarian tumors is highly discussed, and if it exists, it seems to have meaning for the clear cell carcinoma. We present a case of coexistence of a mucinous ovarian tumor in a patient with endometrioma.

\section{4}

\section{PELVIC PAIN AFTER TRANSCERVICAL TUBAL OCCLUSION: A CASE REPORT}

Case Reports

M. Simón Coll*, A. Úbeda, M. Cusido

${ }^{[1]}$ Dexeus $\sim$ Barcelona $\sim$ Spain

Summary (4 lines): Essure procedure failure.

Introduction: Transcervical tubal occlusion (TTO) with the Essure ${ }^{\circledR}$ procedure causes tubal obstruction by a fibrosis of the intratubal canal through dacron fibers. Placement must be a standardized process and control is currently authorized in the FDA and EEC with a simple pelvic X-ray of pelvis within three months.

Material and Methods: A pregnancy and pelvic pain after TTO

Results: 47 year-old patient with a spontaneous miscarriage, vaginal delivery in 1990 and TTO 2008. After an unexpected pregnancy in 2010, she underwent an uneventful laparoscopic bilateral tubal binding (BTB). In October 2010, she complained of a two years chronic pain. Ultrasound showed the absence of the intramural portion of the device. She was suggested to undergo a diagnostic laparoscopy. During surgery both devices were found to be subserous at the level of both uterine 
cornual regions. They were both easily removed and bilateral salpingectomy was performed. The patient is currently totally asymptomatic.

Discussion: Chronic pelvic involves up to $15 \%$ of women, being $20 \%$ of gynecologic origin (endometriosis and pelvic adhesions). TTO is a good office method of definitive contraception, well tolerated, safe and effective. Any kind of difficulty or adverse event during insertion, or pelvic pain after the procedure should be sent to a hysterosalpingography to discard unadverted expulsion, and to ensure correct placement and tubal obstruction.

\section{5}

\section{PREGNANCY OF UNKNOWN LOCALISATION - ENDOSCOPIC APPROACH}

Case Reports

Z. Vilendecic*, D. Sljivancanin, M. Dokic, M. Djakonovic Maravic, S. Vidakovic

${ }^{[1]}$ Clinic for Gynaecology and Obstetrics, Clinical Centre of Serbia Belgrade Serbia

Summary (4 lines): Laparoscopy was utilized as the final diagnostic and therapeutic tool in a misdiagnosed case of ruptured corpus luteum cyst in early intrauterine pregnancy.

Introduction: There is an overlap of values in early intrauterine pregnancy and in ectopic pregnancy which raises a concern that procedures done in unclear cases can harm normal pregnancies.

Material and Methods: We present a case of ruptured corpus luteum cyst in early intrauterine pregnancy which was misdiagnosed as an ectopic pregnancy.

Results: A 35-year-old woman was admitted due to lower abdominal pain. Ultrasound showed a heterogeneous formation in the right adnexal region, hCG was $2550 \mathrm{IU} / 1$. Repeated scan showed increased amount of free abdominal fluid and additional formation in the right adnexal region. The main findings on laparoscopy were ruptured cyst of right ovary and hematoperitoneum. We performed laparoscopic cystectomy and suture of right ovary. Postoperatively, hCG value was $4668 \mathrm{IU} / 1$ and ultrasound demonstrated gestational sac within uterine cavity.

Discussion: Serial hCG testing and repeated ultrasound exams would eventually classify pregnancies of unknown localisations. Unfortunately, discriminatory levels are without a clear cut-off values, ultrasound can be non conclusive or misleading and uterine curettage could terminate normal pregnancy. We utilized diagnostic and therapeutic potential of laparoscopy. Those features are underlined particularly in cases with uncertain preoperative diagnosis.

\section{5}

\section{PRINCIPLES OF SAFE OVARIAN SURGERY}

Case Reports

V. Zaporozhan, I. Gladchuk, N. Rozhkovska, V. Marychereda, A. Volyanska, O. Aleksandrov*, Y. Petrovskyi

${ }^{[1]}$ Odessa National Medical University $\sim$ Odessa Ukraine

Summary (4 lines): Surgeons performing laparoscopic intervention for pelvic organs should know basics of functional ovarian surgery, mechanisms of prevention of adhesive processes of women reproductive organs.

Introduction: Surgeons performing urgent endoscopic ovarian surgery or simultaneous intervention should be expert of physiology and pathology of female reproductive system at different age periods. They should know basic principles of safe ovarian surgery to avoid iatrogenic complications (infertility and disability).

Material and Methods: A total of 186 females (13-45 years) underwent laparoscopic surgery for presumed benign ovarian cyst.
Results: The mean age-28,2 years. At laparoscopic surgery 30,3\% had endometriomas, 16,7 \% - dermoid cysts, 13,3\%-paraovarian cysts; 97 (29,7\%)-simple ovarian cysts, $4 \%$-mucinous cystadenomas and $5 \%$ serous cystadenomas, 4 cysts were malignant- $1,4 \%$. Cystectomy was performed in $73 \%$, fenestration- $27 \%$. The operative diagnosis was highly correlated with final pathological reports $(\mathrm{P}<0,001)$. There were no operative and postoperative complications in our series.

Discussion: With a careful preoperative screening, laparoscopic surgery of ovarian cyst is efficient and safe treatment for adolescents and young women. Endoscopist should know principles of using different types of surgical energies (mono-, bipolar electrosurgery, ultrasonic, laser) on the reproductive system of women, indications and contraindications for organ-sparing and radical intervention on women pelvic organs.

74

\section{PYOMETRA IN A 15 YEARS OLD GIRL WITH DYSFUSED UTERUS}

Case Reports

A. Achard Algorta, G. Germano*

${ }^{[1]}$ Centro Hospitalario Pereira Rossell $\sim$ Montevideo $\sim$ Uruguay

Summary (4 lines): We report a case with double uterus, complicated with pyometra in the obstructed uterus.

Introduction: The importance of this fact is that the patient can present acute complications, and a poor prognosis in her reproductive life.

Material and Methods: A 15 years old girl, presents with fiber, leucocitosis and vaginal purulent discard, being interpreted as PID. In the ultrasound evaluation a double uterus was seen, and the absence of the right kidney. She is sent for Laparo/Histero evaluation. In the laparoscopic approach, we found a double uterus, each one with its normal adnexe. Some light adhesions in Douglas, and a few endometriosis superficial lesions in the right side. A peri Hepatitis (Stajano syndrome) like seen in Clamydia infections was found. In the edocervical canal, there was a bifurcation, leading: in the left side to a hemicavity with a horn and left ostium; and in the right side, an obstructed channel, wich was opened with Bettocchi System. A great amount of purulent, fetid material was drained. An intravenous antibiotic double plan was indicated with excellent evolution.

Results: the endoscopic approach was able to assess the diagnostic and classification of congenital uterine malformation. Also, we could treat the two complications in the same time: the endometriosis lesions, the pyometra. Discussion: By opening the cervical canal, the menstrual fluid will come out without resistance, minimizing the risk of infection complications, and the progress of endometriosis.

\section{0}

\section{ROBERT'S UTERUS WITH MENSTRUAL RETENTION IN THE BLIND CAVITY: A CASE REPORT \\ Case Reports}

L. Herter, C. Paim, D. Gassen, M. Milagre, S. Pessini*, R. Deyl

${ }^{[1]}$ Universidade Federal de Ciencias da Saude de Porto Alegre $\sim$ Porto Alegre $\sim$ Brazil

Summary (4 lines): Case report of a 13 years old patient with Robert's uterus.

Introduction: Robert's uterus is a malformation characterized by two cavities, one of which blind.

Material and Methods: Female 13 years old with dysmenorrhea. Physical examination revealed pubertal development appropriate and pain. A pelvic US identified uterus with a cystic formation in proximity 
to the endometrial cavity. CT scan showed uterus with two hypodense formations. MR imaging identified uterus with elongated cavity.

Results: The patient was given the continuous contraceptive pills, to reduce the pain and to investigate a possible hematometra. After some weeks, she started to have spotting and the pain returned. The hysteroscopy identified lack of the right tubal ostium, the uterine cavity slightly skewed to the left, normal left tubal ostium and a bulging in the right lateral uterine wall. The laporoscopy identified a uterus of normal external appearance, with a increase in the right hemiuterus. The right tube was absent, the ovaries were normal. The right hemiuterus was punched with withdrawal of dark blood. We indicated the removal of accessory horn. The patient progressed well, with regular cycles and no dysmenorrhea. Discussion: Patients with Robert Uterus have asymmetric septate uterus, with one blind cavity. This malformation cause increased risk of endometriosis and pregnancy complications. It's important to think about obstructing uterine malformation in all cases of dysmenorrhea

\section{7}

\section{SENTYNEL NODES BIOPSY AND ENDOMETRIAL CANCER} Case Reports

A. Zapico*, A. Couso, P. Valenzuela, P. Fuentes, V. Garcia Pineda

${ }^{[1]}$ Principe de Asturias Hospital $\sim$ Alcalá de Henares $\sim$ Spain

Summary (4 lines): Sentynel nodes biospsy for endometrial cancer shows controversial data. Doble tracer technique seems to be the most reliable procedure

Introduction: Sentynel nodes biopsy has been widely used in breast cancer. However, controversial data are showed in endometrial cancer. Material and Methods: From september 2010 to march 2012, 22 patient with endmetrial cancer were managed by Sentinel node biopsy. Patent blue was used in all cases. In 8 cases $(36,3 \%)$ Tc technique was also used.

Results: Detection rate was $64,5 \%, 9$ cases, when patent blue was the only tracer. When a doble tracer (Tc and patent blue ) was used detection rate went up to $100 \%$. However, in 2 cases detection was made based on a single tracer, Tc or blue. Single blue tracer sentynel nodes identified average was 2,07+0,34 (1-4) while doble tracer got $2,75+0,35$ (1-4). Bilateral nodes were seen in $42,8 \%$ y $37,5 \%$. Micrometastasis was seen in one IAG2 case. The remaining cases were negative. No false negative was seen.

Discussion: Doble tracer allows better nodes identification. Due to the low rate of metastasic nodes in endometrial cancer higher case serie are needed for validation.

\section{9}

\section{THE USE OF LAPAROSCOPY IN AN APPROACH TO TUBO-OVARIAN ABSCESSES}

Case Reports

\section{O. Shevchenko*}

${ }^{[1]}$ Odessa National Medical University $\sim$ Odessa $\sim$ Ukraine

Summary (4 lines): In this study laparoscopic approach to tubo-ovarian abscesses was evaluated. Laparoscopic surgery has significant advantage in terms of preserving hormonal and reproductive function.

Introduction: TOA is a severe complication of PID which also can result in pyosalpinx and peritonitis. Findings indicate that TOA develops in up to $30 \%$ of women hospitalized for PID. The approach to TOA is still highly debatable.

Material and Methods: There were 96 women with TOA observed. The mean age was $30 \pm 7,2$ years. All patients were operated using laparoscopic access. The mean time of an operation was $49 \pm 13,5$ min.
Results: All patients were treated during 48 to 72 hours before operation using broad-spectrum antibiotics, infusion therapy ( $>2,5$ liters per day), anti-inflammatory drugs, vitamin supplement, etc. There was performed lysis of pelvic adhesions, drainage and irrigation of pyosalpinges and TOA with irrigation of the pelvic cavity with 3-4 liters of physiologic saline in all cases. Removing of a unilateral infectious complex and resection of ovary was done for 31 patients. We were focused on saving ovarian tissue as much as possible for infertile and nulliparous women. There was not revealed any complications after using this therapy.

Discussion: Laparoscopic surgery which diminishes postoperative complications should be the first choice in the managing of TOA. However it is crucial to provide adequate preoperative treatment and postoperative rehabilitation.

\section{0}

\section{TUBAL MOLAR PREGNANCY: A CASE REPORT.} Case Reports

\author{
F. Marengo, D. Larraín, F. Gonzalez*, I. Rojas \\ ${ }^{[1]}$ Clínica Santa María Santiago $\sim$ Chile
}

Summary (4 lines): Case report of succesfull laparoscopic treatment of molar tubal pregnancy and follow-up

Introduction: Tubal molar pregnancy is very a rare occurence if we consider that the incidence of hydatidiform moles is 1 per 1,000 pregnancies and that of ectopic pregnancy is 20 per 1,000 pregnancies.

Material and Methods: Case report of tubal molar pregnancy

Results: We report a case of a 32-years old pregnant woman with 7 weeks of amenorrhea who presented vaginal bleeding and lower abdominal pain. She had a previous history of vaginal delivery and laparoscopic kystectomy for ovarian endometrioma. Pelvic ultrasound revealed an irregular echogenic mass in the left adnexa and hemoperitoneum. Beta hCG was $50000 \mathrm{mU} / \mathrm{L}$. We performed a laparoscopic salpingectomy because of an ruptured ampullary ectopic pregnancy. The histopathological examination identified a molar pregnancy. The patient was followed weekly with Beta -hCG titers until three successive negative levels and then monthly for six months.

Discussion: Although rare, molar pregnancy can occur at any site. It's a good practice take always histological examination of all specimens for a better treatment and surveillance.

\section{9}

\section{TWO CASES OF PREGNANCY FOLLOWING HYSTEROSCOPIC STERILIZATION \\ Case Reports}

C. Ferreira, N. Nogueira Martins*, A. Pipa, F. Nogueira Martins ${ }^{[1]}$ São Teotónio Hospital Viseu Viseu $\sim$ Portugal

Summary (4 lines): Two cases of failure with Essure ${ }^{\circledR}$ hysteroscopic sterilization

Introduction: Hysteroscopic sterilization with Essure ${ }^{\circledR}$ is a reliable alternative to laparoscopic non-reversible contraception. At present, the risks of the device to any woman who becomes pregnant or to the fetus are unknown.

Material and Methods: Report of two cases of pregnancy after hysteroscopic sterilization with Essure ${ }^{\circledR}$.

Results: Case 1:36 year-old woman, who got pregnant 11 months after Essure ${ }^{\mathbb{R}}$ placement; pregnancy continued without complications until the vaginal delivery of a healthy newborn. Case 2:40 year-old hypertensive woman, who got pregnant 6 months after Essure ${ }^{\mathbb{R}}$ placement; pregnancy was complicated by gestational diabetes and worsening of hypertension, 
but ended with a vaginal delivery of a healthy newborn at $37 \mathrm{w}$ gestation. Both cases followed the existing protocol, which revealed a normal transvaginal scan and pelvic radiography at the 3 months' appointment.

Discussion: Pregnancies after Essure ${ }^{\circledR}$ hysteroscopic sterilization are uncommon. With properly positioning of the Essure ${ }^{\mathbb{R}}$ device and strictly complying with the follow-up protocol the risk of pregnancy after hysteroscopic sterilization is very low. Taking into account the uprise in popularity, it is increasingly important to understand the effects of the device on pregnancy when the procedure fails. Documentation of uneventful pregnancies may create the chance to perform assisted reproductive techniques after hysteroscopic sterilization in the future.

\section{9}

\section{ULTRASOUND DIAGNOSE AND SUCCESSFUL LAPAROSCOPIC TREATMENT OF 7 WEEK CORNUAL HETEROTROPHIC PREGNANCY- A CASE REPORT. Case Reports}

W. Pieta*, A. Wilczynska, T. Makowski, R. Gardocki, P. Swietlicki ${ }^{[1]}$ Miedzyleski Specialistic Hospital Warsaw Poland

Summary (4 lines): The cornual pregnancy is a rare kind of heterotrophic pregnancy. It should be recognized as soon as possible to avoid complication as hemorrhage even deaths. Usually it is treated with surgery or of puncturing and aspirating pregnancy under transvaginal ultrasound guidance. Treatment by drugs is usually not enough.

Introduction: The cornual pregnancy is a diagnostic and therapeutic challenge with a height risk of complications such as rupturing and bleeding. It is mostly recognized between 9 and 12 week.

We present a left cornual pregnancy and explain how we dealed with this case laparoscopically.

Material and Methods: We report a case of 7 weeks pregnancy. We removed pregnancy during laparoscopy with small damage of uterus and with minimal blood loss.

We diagnosed it ultrasonografically in a 25 -year-old multigravida. Bhcg was $1800 \mathrm{mIU} / \mathrm{ml}$. Patient had a pelvic pain. She delivered once 2 years ago and had a history of Chlamydia trachomatis infections. We visualized in ultrasound a $14 \mathrm{~mm}$ gestation sac in left corner of uteri.

Results: Patient was qualified for laparoscopy treatment. We extracted pregnancy with small part of left corner of uteri. Blood loss was $5 \mathrm{ml}$. Patient went from hospital after 2 days of observation with no complication.

Discussion: Laparoscopic surgery for cornual pregnancy is a safe procedure. However it may be in the future reason of uterine rupture during the next pregnancy and labor.

\section{6}

\section{UNUSUAL PRESENTATION OF PSEUDOMYXOMA PERITONEI}

Case Reports

C. Frendo-balzan*, A. Abdelmagied

${ }^{[1]}$ Royal Gwent Hospital $\sim$ Newport $\sim$ United Kingdom

Summary (4 lines): $50 \mathrm{yr}$ old presents with irregular vaginal bleeding. Hysteroscopy and biopsy shows mucinous metaplasia. Laparoscopy confirms suspicion of pseudomyxoma peritonei (PMP) of appendix. Introduction: $50 \mathrm{yr}$ old with mirena presents with troublesome irregular mucus-type bleeding and post-coital bleeding. On examination there was fullness in the right adnexa. Apart from a high BMI and epilepsy the patient was otherwise well and asymptomatic.

Material and Methods: Pipelle biopsy shows intestinal type metaplasia of endometrium which was followed up by a hysteroscopy and curettage reported atypical mucinous metaplasia with goblet cells. A follow up pipelle confirms the same therefore a TLH/BSO was planned. Laparoscopy revealed mucin filling the abdomen and pelvis with evidence of widespread disease involving omentum, liver and diaphragm, with an enlarged appendix. Ovaries and tubes looked normal, uterus was slightly enlarged.

Results: Tumour markers were slightly raised at CA125:71, CEA 12. Biopsies confirm the diagnosis of PMP from appendeceal tumour.

Discussion: PMP is a rare condition, and presentation usually involves signs and symptoms of increasing abdominal girth and ovarian mass rather than mucous bleeding per vaginam. Intestinal type metaplasia of endometrium is extremely rare and usually associated with adenocarcinoma of cervix, endometrium or ovary and not of the appendix and is easily overlooked.

\section{3}

\section{URETERAL STUMP METASTASIS OF RENAL CELL CARCINOMA TO GENITOURINARY TRACT}

Case Reports

L. Ajori, A. Moeini*

${ }^{[1]}$ Department of Obstetrics and Gynecology, ShohadaTajrish Hospital, Shahid Beheshti University of Medical Science $\sim$ Tehran $\sim$ Iran, Islamic Republic of

Summary (4 lines): We report a 32-year-old female with a huge metastatic involvement of the ureteral stump and utero-cervical structures about 2 years after a left radical nephrectomy. She underwent enbloc resection of the mass in conjunction with total ureterectomy, bladder cuff excision and hysterectomy.

Introduction: Renal cell carcinoma (RCC) has the characteristics of unpredictable presentation and propensity for early metastasis.

Material and Methods: A 32-year-old female presented with a onemonth pelvic pain. She had history of left radical nephrectomy.

Physical examination showed a large round shaped, hard, immobile mass detected in the left adnexal region on pelvic.

Magnetic resonance (MR) study revealed the mass was in close vicinity of bladder base, uterus cervix and lower segment of body, separate from intact left ovary.

The results of tumor markers were in normal range.

Results: The mass was identified in the posterior aspect of the bladder with adhesion to the antero-lateral portion of uterine body and cervix. En-bloc resection of the mass in conjunction with total ureterectomy and bladder cuff excision were adopted. Due to the mass adhesion to uterus, also a hysterectomy was performed for her.

Pathologic serial sections revealed Fuhrman grade 3/4 clear cell type of RCC.

Discussion: Although RCC metastasis to genitourinary tract is a rare phenomenon, this does not hinder the need for adequate and regular surveillance including pelvic organs.

\section{7}

\section{UTERUS DIDELPHYS WITH A BLIND HEMIVAGINA COMPLICATED BY PYOCOLPOS}

Case Reports

H.J. Lee*, H. Lee

${ }^{[1]}$ Sungae Hospital $\sim$ Seoul $\sim$ Korea, Republic of

Summary (4 lines): we report a case of uterus didelphys with a blind rudimentary hemivagina complicated by pyocolpos due to Pediococcus infection. Complete resection of the vaginal septum and laparoscophy was performed. 
Introduction: A woman was referred due to severe pelvic pain. Vaginal examination showed a severely protruding left vaginal wall with severe tenderness.

Material and Methods: This report presents a rare case of uterus didelphys with a blind rudimentary hemivagina complicated by pyocolpos due to Pediococcus infection. The findings in this case suggest that uterus didelphys complicated by abscess such as pyocolpos should be considered as a possible cause of acute abdomen.

Results: After creating an opening in the left vaginal wall and inserting a Foley catheter through the opening into the vaginal wall for drainage with antibiotic therapy, the patient's condition rapidly improved. A culture of the pus drained from the vaginal wall showed Pediococcus species. Resection of the vaginal septum and laparoscophy was performed.

Discussion: Although uterus didelphys with a blind hemivagina and Pediococcus infection are rare, they may induce serious conditions before precise diagnosis can be made. Therefore, should be aware of uterus didelphys with blind hemivagina and consider uterus didelphys complicated by severe infection as a possible cause of acute abdomen.

\section{3}

\section{UTERUS DIDELPHYS WITH A BLIND HEMIVAGINA COMPLICATED BY PYOCOLPOS \\ Case Reports}

H. Lee*

${ }^{[1]}$ THE CATHOLIC UNIVERSITY OF KOREA SEOUL Korea, Republic of

Summary (4 lines): we report a case of uterus didelphys with a blind rudimentary hemivagina complicated by pyocolpos due to Pediococcus infection. Complete resection of the vaginal septum and laparoscophy was performed.

Introduction: A woman was referred due to severe pelvic pain. Vaginal examination showed a severely protruding left vaginal wall with severe tenderness.

Material and Methods: This report presents a rare case of uterus didelphys with a blind rudimentary hemivagina complicated by pyocolpos due to Pediococcus infection. The findings in this case suggest that uterus didelphys complicated by abscess such as pyocolpos should be considered as a possible cause of acute abdomen.

Results: After creating an opening in the left vaginal wall and inserting a Foley catheter through the opening into the vaginal wall for drainage with antibiotic therapy, the patient's condition rapidly improved. A culture of the pus drained from the vaginal wall showed Pediococcus species. Resection of the vaginal septum and laparoscophy was performed.

Discussion: Although uterus didelphys with a blind hemivagina and Pediococcus infection are rare, they may induce serious conditions before precise diagnosis can be made. Therefore, should be aware of uterus didelphys with blind hemivagina and consider uterus didelphys complicated by severe infection as a possible cause of acute abdomen.

\section{4}

\section{UTERUS DIDELPHYS WITH A BLIND HEMIVAGINA COMPLICATED BY PYOCOLPOS Case Reports}

H. Lee*

${ }^{[1]}$ The Catholic University of Korea $\sim$ Seoul $\sim$ Korea, Republic of

Summary (4 lines): we report a case of uterus didelphys with a blind rudimentary hemivagina complicated by pyocolpos due to Pediococcus infection. Complete resection of the vaginal septum and laparoscophy was performed.
Introduction: A woman was referred due to severe pelvic pain. Vaginal examination showed a severely protruding left vaginal wall with severe tenderness.

Material and Methods: This report presents a rare case of uterus didelphys with a blind rudimentary hemivagina complicated by pyocolpos due to Pediococcus infection. The findings in this case suggest that uterus didelphys complicated by abscess such as pyocolpos should be considered as a possible cause of acute abdomen.

Results: After creating an opening in the left vaginal wall and inserting a Foley catheter through the opening into the vaginal wall for drainage with antibiotic therapy, the patient's condition rapidly improved. A culture of the pus drained from the vaginal wall showed Pediococcus species. Resection of the vaginal septum and laparoscophy was performed.

Discussion: Although uterus didelphys with a blind hemivagina and Pediococcus infection are rare, they may induce serious conditions before precise diagnosis can be made. Therefore, should be aware of uterus didelphys with blind hemivagina and consider uterus didelphys complicated by severe infection as a possible cause of acute abdomen.

\section{7}

\section{UTROSCT: 2 CASES REPORT Case Reports}

S. Calzolari*, E. Castellacci, F. Zolfanelli, V. Dubini

${ }^{[1]}$ Palagi Hospital- Florence- Italy $\sim$ Florence $\sim$ Italy

Summary (4 lines): We report 2 cases of rare tumor (uterine tumor resembling ovarian sex cord tumor) histologically diagnosticated after hysteroscopic polipectomy.

Introduction: Uterine tumors resembling ovarian sex cord tumors (UTROSCTs) are rare neoplasms of uncertain malignancy, affecting either pre or menopausal women.

Material and Methods: 2 women 50 and 55 years old came to our attention for hypermenorrea. They were subjected to ultrasound examination showing endometrial pathology and underwent to office polipectomy. Histology showed monomorphic proliferation of cellular elements arranged in a delicate taste and bays and surrounded by eosinophilic stroma, suggestive for sex cord derivation, like UTROSCT.

According to both patients, clinical and diagnostic examinations were performed at 3 and 6 months.

All the examinations were negative except for the office hysteroscopy at 6 month that showed a new polip at the site of the former polip.

The histological examination confirmed the previus diagnosis of UTROSCT.

Abdominal hysterectomy with bilateral salpingo-oophorectomy were performed.

Results: Histological examination of the surgical specimen shows secretive endometrium with diffuse adenomiosis area without further neoplastic proliferation.

Discussion: In our cases the diagnosis of UTROSCT was made by hystological examination and the managment in line with previous cases described in the literature.

265

\section{AIREMBOLISM DURING HYSTEROSCOPIC MYOMECTOMY Complications}

J. Verguts*, S. Peetermans, A. Teunkens, S. Devroe ${ }^{[1]}$ UZ Leuven $\sim$ Leuven $\sim$ Belgium

Summary (4 lines): Airembolisme during hysteroscopic myomectomy is not reported so far; we present 7 cases with suspicion of airembolism and possible mechanisms. 
Introduction: Airembolism is a possibly fatal complication during surgery; it is so far never described during hysteoscopic surgery.

Material and Methods: We retrospectively reviewed all our cases ( $n=$ 295) of hysteroscopic myomectomy from 2009 till 2011 in the university hospital Leuven and selected patients with suspicion of airembolism during surgery.

Results: Seven patients encounterd an airembolism during hysteroscopic myomectomy. The mean volume in these cases was $22.78 \mathrm{~cm}^{3}$ compared to $17.40 \mathrm{~cm}^{3}$ in the hysteroscopic resections without occurrence of a gas embolism. In 6/7 cases the myoma wasn't calcificated. Only in 3/7 cases an anterior located myoma was removed.

Discussion: The mechanism by which airembolisms may occur during surgery and possible methods to prevent this possible fatal complication will be discussed.

\section{1}

BOWEL OBSTRUCTION WITH A 5MM PORT SITE HERNIA Complications

H. Jan*, G. Araklitis, A. Al-khatib, A. Davison, N. Narvekar

${ }^{[1]}$ King's College Hospital $\sim$ London $\sim$ United Kingdom

Summary (4 lines): A case of bowel obstruction through a $5 \mathrm{~mm}$ laparoscopic port site

Introduction: 41 year old underwent laparoscopic myomectomy for $15 \mathrm{~cm}$ pedunculated fibroid. 4 ports, $2 \times 10 \mathrm{~mm}$ and $2 \times 5 \mathrm{~mm}$ ENDOPATH $^{\circledR}$ XCEL $^{\circledR}$ Bladeless Trocar were used for access and 1:20 vasopressin was used for haemostasis. The fibroid was then morcelated with PlasmaSORD ${ }^{\mathrm{TM}}$ Bipolar Morcellator. The rectus sheath for the $10 \mathrm{~mm}$ umbilical and right lateral ports were closed with J-vicryl and skin with monocryl. The surgical time was 194 minutes. There was recurrent reinsertion of trocar due to large BMI. No drains were used and the patient was discharged 2 days later.

Material and Methods:

Results: She was re-admitted 10 days later with intractable vomiting and abdominal pain. Plain x-ray revealed air under the diaphragm and CT abdomen showed a loop of small bowel within the abdominal wall at the $5 \mathrm{~mm}$ left lateral port site. Emergency laparotomy confirmed incarcerated strangulated small bowel within the port site and a straightforward resection anastomosis was performed. The patient was discharged 9 days later.

Discussion: The RCOG guidelines advise closure of $7 \mathrm{~mm}$ or larger lateral ports and $10 \mathrm{~mm}$ or larger midline ports. This case demonstrated that smaller ports are not necessarily risk free. In gynae-oncology herniation through $5 \mathrm{~mm}$ port sites has been shown particularly when drains have been left in. This benign gynaecology case shows there is a risk and one should be vigilant in port closure and be aware of this potential complication.

\section{6}

\section{CLINICAL ANALYSIS OF LAPAROSCOPIC SURGERY OF TUBOOVARIAN ABSCESS IN ACUTE PELVIC INFLAMMATORY DISEASE \\ Complications}

\author{
H.J. Lee* \\ ${ }^{[1]}$ THE CATHOLIC UNIVERSITY OF KOREA $\sim$ SEOUL Korea, \\ Republic of
}

Summary (4 lines): To evaluate the clinical characteristics between patients with tuboovarian abscess and those presenting with nontuboovarian abscess in acute pelvic inflammatory disease according to the operation findings.
Introduction: Our results suggest that some variables noted between the tuboovarian abscess group and acute pelvic inflammatory disease group. Material and Methods: The patients were divided into 2 groups based on the operation findings at laparoscopic surgery;

Results: There were no significant differences between the two groups considering the percentage of age, parity, mean abortion rate and rate of the history of previous pelvic inflammatory disease. But there were differences in mean white blood cell count, erythrocyte sedimentation rate and C-reactive protein level. A higher number of patients having intrauterine devices were observed in the tuboovarian abscess group than in the non-tuboovarian abscess group. Also, statistical significance was noted in hospital stay after operation but there was no difference in number of sick days prior to operation.

Discussion: These results make easier to calculate diagnostic accuracy of patients prone to developing tuboovarian abscess and prevent subsequent complications from the delay of treatment if sensitivity, specificity, negative and positive predictive values were assessed.

288

\section{CLINICAL ANALYSIS OF LAPAROSCOPIC SURGERY OF TUBOOVARIAN ABSCESS IN ACUTE PELVIC INFLAMMATORY DISEASE \\ Complications}

H. Lee*

${ }^{[1]}$ THE CATHOLIC UNIVERSITY OF KOREA $\sim$ SEOUL $\sim$ Korea, Republic of

Summary (4 lines): o evaluate the clinical characteristics between patients with tuboovarian abscess and those presenting with nontuboovarian abscess in acute pelvic inflammatory disease according to the operation findings.

Introduction: The patients were divided into 2 groups based on the operation findings at laparoscopic surgery; 56 patients diagnosed with evidence of tuboovarian abscess and 74 patients diagnosed with acute pelvic inflammatory disease without tuboovarian abscess.

Material and Methods: Those were compared with respect to patient characteristics, clinical and laboratory findings depending on the significant differences.

Results: There were no significant differences between the two groups considering the percentage of age, parity, mean abortion rate and rate of the history of previous pelvic inflammatory disease. But there were differences in mean white blood cell count, erythrocyte sedimentation rate and $\mathrm{C}$-reactive protein level. A higher number of patients having intrauterine devices were observed in the tuboovarian abscess group than in the non-tuboovarian abscess group. Also, statistical significance was noted in hospital stay after operation but there was no difference in number of sick days prior to operation.

Discussion: Our results suggest that some variables noted between the tuboovarian abscess group and acute pelvic inflammatory disease group.

\section{7}

\section{CLINICAL ANALYSIS OF LAPAROSCOPIC SURGERY OF TUBOOVARIAN ABSCESS IN ACUTE PELVIC INFLAMMATORY DISEASE}

Complications

H. Lee*

${ }^{[1]}$ THE CATHOLIC UNIVERSITY OF KOREA $\sim$ SEOUL $\sim$ Korea, Republic of

Summary (4 lines): To evaluate the clinical characteristics between patients with tuboovarian abscess and those presenting with non- 
tuboovarian abscess in acute pelvic inflammatory disease according to the operation findings.

Introduction: Our results suggest that some variables noted between the tuboovarian abscess group and acute pelvic inflammatory disease group. Material and Methods: The patients were divided into 2 groups based on the operation findings at laparoscopic surgery.

Results: There were no significant differences between the two groups considering the percentage of age, parity, mean abortion rate and rate of the history of previous pelvic inflammatory disease. But there were differences in mean white blood cell count, erythrocyte sedimentation rate and C-reactive protein level. A higher number of patients having intrauterine devices were observed in the tuboovarian abscess group than in the non-tuboovarian abscess group. Also, statistical significance was noted in hospital stay after operation but there was no difference in number of sick days prior to operation.

Discussion: These results make easier to calculate diagnostic accuracy of patients prone to developing tuboovarian abscess and prevent subsequent complications from the delay of treatment if sensitivity, specificity, negative and positive predictive values were assessed.

\section{1}

\section{COMPLICATIONS DUE TO MYOMA SCREW AS UTERINE MANIPULATOR IN TOTAL LAPAROSCOPIC HYSTERECTOMY}

Complications

A. Magdum*, H.R. Padiyath, A. Puthiyidom, N. Trehan, A. Gupta, S. S

${ }^{[1]}$ Sunrise Hospital, Kakkanad $\sim$ Ernakulam $\sim$ India

Summary (4 lines): Myoma screw is an effective and cost-effective instrument for uterine manipulation for total laparoscopic hysterectomy. Introduction: Uterine manipulation plays vital role during total laparoscopic hysterectomy (TLH). Myoma screw is good uterine manipulator. We present complications with it.

Material and Methods: In this retrospective study from January 2008 till 29 February 2012, complications related with use of myoma screw during TLH were analysed.

Procedure:

$5 \mathrm{~mm}$ myoma screw was inserted through trocar in right iliac fossa for uterine manipulation. Bowel injuries were managed conservatively or with suturing depending on extent of injury.

Results: $40 \%$ of study population $(\mathrm{n}=2880)$ had uterus larger than 20 weeks size. In $231(8 \%)$ cases there was minor difficulty to operate on the right side of uterus. Additional trocar was inserted in 12(0.4\%) cases for difficulty in uterine manipulation due to excessively enlarged uterus. In $9(0.3 \%)$ cases two myoma screws were used. In 16 cases there was bowel injury, $10(0.34 \%)$ serosal and6( $0.2 \%)$ complete thickness of wall. Bleeding from omental vessels occured in one case. Bipolar coagulation was required in $44(1.5 \%)$ cases for bleeding from myoma screw site. Uterine manipulation was difficult in $2 \%$ of atrophic uteri due to size. Discussion: Conclusion:

Myoma screw is one of the most effective and cheapest uterine manipulators which can be used during TLH safely.

\section{9}

\section{COMPLICATIONS OF GYNECOLOGIC LAPAROSCOPY: 1990-2011 REVIEW \\ Complications}

V. García Pineda*, M.D. Rodríguez Garnica, C. De Valle Corredor, Á. Zapico Goñi, V. Marcos González

${ }^{[1]}$ Príncipe de Asturias Hospital $\sim$ Spain $\sim$ Spain
Summary (4 lines): The aim of this research is study the laparoscopic complications in the last 21 years of our experience.

Introduction: It is estimated that the overall complication rate ranges from $0.2-10.3 \%$.

Material and Methods: We perform a retrospective study in 2066 patients operated by laparoscopic approach from 1990 to 2011, distributed into oncological cases $(n=295)$ and non oncological cases $(n=$ 295). We calculate the rate of intraoperative complications (minor and major), considering the events on learning curve in the last 21 years, the rate of conversion to laparotomy and the prevalence of postoperative complications at the follow up of 1601 patients $(80,4 \%)$ in both groups.

Results: The overall intraoperative complications rate is $9,0 \%(\mathrm{n}=$ 186) being in oncological cases of $17,3 \%(n=51)$ and in non oncological cases of 7,6 \% $(n=135)$. The major complications rate is $5,1 \%$ in oncological group $(n=15)$ and in non oncological one is $0,6 \%$ $(n=12)$. The rate of intraoperative complications by group of patients and year is influenced by four learning curve events with a peak of prevalence in each one. Postoperative complications rate of oncological patients is $18,7 \%(\mathrm{n}=30)$ and in non oncological group $7 \%$ $(\mathrm{n}=101)$.

Discussion: Incorporation of new complex procedures such as new laparoscopic surgery indications, mainly in oncological field, increases complications rate due to the complexity of the surgery procedures and the limited experience of the surgeon.

\section{1}

\section{GYNECOLOGIC LAPAROSCOPY: IS IT REALLY A SAFE PROCEDURE? \\ Complications}

\section{A. Gonçalves*, C. Marques, M. Simões, F. Ribeiro, C. Marques \\ ${ }^{[1]}$ Maternidade Dr. Alfredo da Costa $\sim$ Lisboa $\sim$ Portugal}

Summary (4 lines): Retrospective study that determined and described complications in gynecologic laparoscopic procedures in a tertiary center.

Introduction: Laparoscopic surgery is the first choice for most gynecologic procedures. Concerns over patients' safety have emerged and the study of the operative complications is essential.

Material and Methods: Retrospective study of laparoscopic procedures over the adnexa in which complications were described, during a three-year interval.

Demographic, clinical and surgery-related variables were collected. Complications were classified as intraoperative (approach and technique-related) or postoperative (pain, gastrointestinal complications, infection and hemodynamic instability). Conversions to laparotomy and the need for re-hospitalization were recorded.

Results: Of 374 laparoscopies, 18 complications occurred (4,8 \%), at a mean age of 47 years[25-85].

The most frequent procedure was adnexectomy $(n=10)$.

Fourteen complications were intraoperative, 2 postoperative and in two cases both a intra and postoperative complication was recorded. Five intraoperative complications were during the approach and 10 technique-related. Five surgeries were converted $(1,3 \%)$. Postoperative complications were pain $(n=1)$ and intestinal tract-related $(n=2)$; two needed rehospitalization. One patient died.

Discussion: The most frequent complications must be accessed. Surgeons' technical skills are essential for overcoming any difficulty and guarantee patients' safety throughout the procedure. 
32

\section{MANAGEMENT OF LATE RECTAL EROSION AFTER TRANSVAGINAL RECTOCELE MESH REPAIR IN A 37 YEAR OLD WOMAN \\ Complications}

B.E.M. Ali*, V. Béatrice, J. Stéphanie, B. Georges

${ }^{[1]}$ Poissy Univeristy Medical center $\sim$ POISSY $\sim$ France

Summary (4 lines): These last few years, non-absorbable prostheses have come to play a prominent role in vaginal prolapse surgery. Late complications have begun to emerge.

Introduction: We present a case of late erosion in the lower rectum after transvaginal rectocele repair in a 37 year old woman.

Material and Methods: A few cases have been reported in literature about this type of late complication with a Polypropylene macroporous mesh.

This case can probably be explained by late rectal erosion or unrecognized intraoperative perforation of the rectal wall.

Results: Prosthesis was totaly removed by progressive transanal traction. It was surrounded by a granulomatous inflammation without collected abscess. There was a well organized fistula in the right rectovaginal space, without communication with the vaginal wall or perineal skin.

Discussion: Non-absorbable prostheses are certainly an option for the treatment of vaginal prolapsed, especially for rectocele repair in young women. However, complications with these implants should be recognized and patients should be advised of the risks involved.

\section{5}

\section{OUR EXPERIENCE WITH OPEN LAPAROSCOPIC ENTRY \\ Complications}

T. Bernal Arahal*, C. Goiri Little, R. Ruiz Sautua, M. Avlia Calle, A. Lekuona Artola, L. Martínez Gallardo

${ }^{[1]}$ Hospital Universitario Donostia $\sim$ San Sebastian $\sim$ Spain

Summary (4 lines): Hasson entry provides safe access to the abdominal cavity. It may especially benefit women who are obese or have undergone previous surgeries or chemoradiotherapy. Vascular or intestinal complications are a rare occurrence.

Introduction: Insertion of the first trocar is a critical step for safe laparoscopic surgery.

Entry related injuries are rare (3 per 1000 reported), but potentially life threatening.

Several techniques have been developed, none of which is exempt of complications. We describe our experience with Hasson entry.

Material and Methods: Between October 2007 and December 2011 we have performed 366 laparoscopies using Hasson entry. Data were collected prospectively.

Results: Mean patient age was 56,63 years (range 16-88). Mean BMI was 27,01 (range 17-53). Mean hospital stay was 4,25 days.

18 patients $(4,92 \%)$ had previously recived chemo-radiation; $8(2,19 \%)$ had had neoadjuvant radiotherapy and $74(20,22 \%)$ had had previous abdominal surgery.

Our only entry-related complication was a jejunal perforation, which was immediately diagnosed and repaired laparoscopically.

Entry to the abdominal cavity was achieved in all cases.

Discussion: We systematically use Hasson entry. We find it simple to perform and it provides more security in previously operated patients.
The trocar can be easily removed and put back in place to extract material from the abdominal cavity.

373

\section{QUILL BARBED SUTURE-RELATED COMPLICATION. 2 CASE REPORTS}

Complications

P.N. Barri-soldevila*, S. Rombaut, A. Vazquez, A. Ubeda, S. Baulies

${ }^{[1]}$ Instituto Universitario Dexeus $\sim$ Barcelona $\sim$ Spain

Summary (4 lines): We report 2 cases of major complication in a laparoscopic myomectomy using a barbed suture for the suturing procedure.

Introduction: Case 1: We present the case of a 30 years old women affected by a symptomatic uterine myoma. The surgical procedure performed was laparoscopic myomectomy. Three weeks after myomectomy, the patient was admitted with a diagnosis of pseudoobstruction. Diagnostic laparasocopy revealed a small bowel strangled by the barbed suture. Once dissected, normal colour was restored. The patient had re-established peristaltism and was discharged asymptomatic. Material and Methods: Case 2: 39 years old woman underwent laparotomic multiple abdominal myomectomy. She was reoperated two weeks later for bowel obstruction that required adhesiolisis between the uterus and a bowel loop, and nasogastric aspiration for two days. Closure of the uterus was made by using the barbed suture Quill SRS in both cases.

Results: Laparoscopic myomectomy with barbed suture is a fast and efficient procedure. It is important to emphasize the need to proceed with this suture technique following the care recommendations and paying special attention to not leaving free suture in the abdominal cavity.

Discussion: However, in our experience, the frequency of bowel occlusion after myomectomy has reached an unacceptable rate and we recommend the discontinuation of the use of barbed suture.

\section{0}

\section{RISK OF UNPLANNED OOPHORECTOMY AT LAPAROSCOPIC OVARIAN CYSTECTOMY FOR CLINICALLY BENIGN CYSTS Complications}

R. Mesquita Pinto*, G. Michos, G. Papageorgiou, G. Halmos, M.D. Dacco', M. Moustafa, A. Magos

${ }^{[1]}$ Minimally Invasive Therapy Unit and Endoscopy Training Centre University Department of Obstetrics and Gynaecology, Royal Free Hospital $\sim$ London $\sim$ United Kingdom

Summary (4 lines): Our aim was to find out the risk of oophorectomy as a possible complication of laparoscopic ovarian cystectomy for benign ovarian cysts.

Introduction: Laparoscopic surgery is the preferred approach in women with ovarian cysts and a low risk of malignancy, and the aim in young women should be to preserve the ovary. We are not aware of any data on the success of conservative surgery in preserving the affected ovary.

Material and Methods: We have reviewed the medical notes of the 123 women who underwent surgery for clinically benign ovarian cyst between November 2004 and May 2012. The median age of patients was 34 years (range 13-50) and 20 patients had previous abdominal surgeries. The median cyst diameter was $5.8 \mathrm{~cm}$ (range 1-17).

Results: The operative procedures performed were ovarian cystectomies $(n=119)$, salpingo-oophorectomy $(n=2)$ and fenestration $(n=2)$. 
There was one conversion to laparotomy and four perioperative complications (3.2\%): three superficial bowel injuries and one omental injury. The histopathological results were: endometrioma $(37.4 \%)$, benign cystic teratoma (27.6\%), serous cyst $(7.3 \%)$, corpus luteal cysts $(7.3 \%)$, cystadenofibroma $(4.1 \%)$, mucinous cystadenoma $(4.1 \%)$, serous cystadenomas $(3.3 \%)$, other benign cysts $(10.6 \%)$ and 2 cases of borderline serous cystadenofibroma.

Discussion: Planned laparoscopic ovarian cystectomy is usually successful with only $1.6 \%$ of patients in our series undergoing oophorectomy.

\section{2}

\section{UTERINE ARTERY PSEUDOANEURYSM FOLLOWING LAPAROSCOPIC HYSTERECTOMY. AN UNUSUAL CAUSE OF DELAYED HEAVY VAGINAL BLEEDING. \\ Complications}

D. Miligkos*, K. Louden, A. Page, R. Behrens

${ }^{[1]}$ Royal Hampshire County Hospital $\sim$ Winchester $\sim$ United Kingdom

Summary (4 lines): Uterine artery pseudoaneurysm is a rare complication of laparoscopic hysterectomy that can present with delayed heavy vaginal bleeding. Correct diagnosis is important as uterine artery embolisation rather than surgery is the treatment of choice.

Introduction: Uterine artery pseudoaneurysms are vascular malformations that can rarely develop following pelvic surgery. They usually present with unexpected bleeding after spontaneous rupture.

Material and Methods: This is the case of a 36 year old woman who underwent total laparoscopic hysterectomy and left salpingoophorectomy for pelvic pain. The procedure was uncomplicated but she was readmitted 22 days post-operativelly with sudden onset heavy vaginal bleeding. There was active bleeding from the vaginal vault and she was transferred to theatre for resuturing of the vault.

Results: Complete haemostasis was achieved vaginally but the following day she had further vaginal bleeding. She underwent an emergency pelvic angiogram which confirmed a pseudoaneurysm of the left uterine artery. This was successfully embolised with complete occlusion of the pseudoaneurysm.

Discussion: Uterine artery pseudoaneurysm should be considered whenever delayed heavy vaginal bleeding occur post laparoscopic hysterectomy. Embolisation is the treatment of choice rather than surgery. Surgery should be avoided as it can increase morbidity by applying stitches in a recently operated pelvis.

\section{3}

\section{A CASE OF BOWEL OBSTRUCTION DUE TO ENDOMETRIOSIS} Endometriosis: Diagnosis

H. Lee*

${ }^{[1]}$ THE CATHOLIC UNIVERSITY OF KOREA SEOUL Korea, Republic of

Summary (4 lines): Although intestinal endometriosis is common, it is rarely manifested as an acute bowel obstructions We have experienced a case of intestinal obstruction due to endometriosis. It may involve intestinal wall and protrude into its lumen and patients with severe intestinal involvement usually require surgical resection.

Introduction: Among women with intestinal endometriosis, bowel endometriosis is an uncommon cause of intestinal obstruction.

Material and Methods: Clinical manifestations are not specific, making the preoperative diagnosis difficult to establish. An emergency laparoscophy was performed.
Results: The pathologic examination of the resected sample revealed endometriosis of sigmoid colon. Endometriosis can involve the intestinal tract extensively, causing a variety of clinical symptoms, and can result in a spectrum of mucosal alterations.

Discussion: This report highlights the importance of histopathological findings of resected specimens in the diagnosis of sigmoid colon obstruction due to intestinal endometriosis

90

\section{DETERMINATION OF VGEF IN THE PERITONEAL FLUID - CLINICAL SIGNIFICANCE IN THE PATIENTS SUFFERING ENDOMETRIOSIS}

Endometriosis: Diagnosis

V. Evdokimova*

${ }^{[1]}$ Odessa National Medical University Odessa Ukraine

Summary (4 lines): The study was dedicated to the assessment of the clinical significance of angiogenesis factors in the cases of endometriosis.

Introduction: Endometriosis is one of the most common gynecological disorders. It affects $12-50 \%$ of females in the reproductive age. The pathogenesis of endometriosis is still unknown but there is strong evidence that angiogenesis is a key pathobiological process in its development.

This study was aimed to assess the expression of VEGF in the perioneal fluid of the patients with endometriosis.

Material and Methods: There were analysed the samples of peritoneal fluid obtained from 86 females with verified external genital endometriosis during routine diagnostic laparoscopic surgery. The concentration of VEGF was determined using ELISA method. Statistical processing was conducted using Statistica 8.0 (StatSoft Inc., USA) software.

Results: The concentration of VEGF in the peritoneal fluid was variable $(23.1 \pm 1.3 \mathrm{ng} / \mathrm{ml})$ and was correlated with the severity of endometriosis lesions $(\mathrm{r}=0,61 \mathrm{p}<0,05)$. There was determined the dependence of VEGF concentration on the phase of menstrual circle - the highest levels $(30.5 \pm 0.7 \mathrm{ng} / \mathrm{ml})$ were characteristic for the proliferative phase.

Discussion: There is considered that the determination of VEGF expression in the peritoneal fluid of the patients with endometriosis could be valuable biomarker of prognosis and susceptibility for this disease.

\section{DIAGNOSTIC DELAY OF ENDOMETRIOSIS IN AUSTRIA AND GERMANY - CAUSES AND POSSIBLE CONSEQUENCES Endometriosis: Diagnosis}

G. Hudelist*, N. Fritzer, A. Thomas, C. Niehues, P. Oppelt, D. Haas, A. Tammaa, H. Salzer

${ }^{[1]}$ Wilhelminen Hospital Vienna $\sim$ Vienna $\sim$ Austria

Summary (4 lines): Previous studies have demonstrated that the length of the time interval from onset of symptoms to diagnosis is surprisingly long. Introduction: The aim of the present study was to investigate the reasons and length of diagnostic delay of endometriosis in Austria and Germany

Material and Methods: The present work was designed as a crosssectional study conducted in Austria and Germany. One-hundred and seventy-one patients completed a 26 item questionnaire in their own language following surgical diagnosis of endometriosis. 
Results: Mean age at the time of diagnosis was 32 (SD 6.0) years. The median interval from the first onset of symptoms to diagnosis was 10.42 (SD: 7.91) years. Seventy-four percent of patients received at least one false diagnosis. Patients with misdiagnosis, women whose mothers considered menstruation as a negative event and normalization of dysmenorrhea by patients had a significant negative impact on diagnostic delay. An association between the patient's impression not to be taken seriously by the gynecologist seeked and prolonged diagnostic delay was also observed.

No association was found between superficial and deep infiltrating endometriosis and oral contraceptive use and prolongation of diagnosis.

Discussion: Measures such as training programs to enhance diagnostic skills and public awareness initiatives could possibly reduce diagnostic delay in Central European countries.

\section{1}

\section{IS OVARIAN ENDOMETRIOMA A RISK FACTOR FOR OVARIAN CANCER?}

Endometriosis: Diagnosis

A. Siozos*, E. Manzo, T. Majmudar, H. Abdel-rahman

${ }^{[1]}$ Hinchingbrooke Health Care Trust $\sim$ Huntingdon $\sim$ United Kingdom

Summary (4 lines): To evaluate a possible association between ovarian cancer and ovarian endometrioma.

Introduction: A retrospective review of 54 cases of ovarian cancer and 34 cases of histologically confirmed endomentrioma.

Material and Methods: Women aged over 18 diagnosed with ovarian cancer or endometrioma over a 5 year period. Previous history of endometriosis in ovarian cancer patients or concomitant diagnosis of ovarian cancer and endometrioma were considered significant findings.

Results: Of the 54 cases of ovarian cancer only 3 had a previous history of endometriosis. In 2 cases endometriosis was diagnosed incidentally at surgery. The patients age was 35,37 and 42 respectively. Endometriosis was the indication for hysterectomy in one case. Of the 34 cases of endometrioma, none was associated with malignancy.

Discussion: Patients with ovarian cancer were older and postmenopausal. Information relating to past pelvic pathology was scanty and a diagnostic laparoscopy for pelvic pain had seldom been performed. Patients with current endometrioma were younger and in some cases had undergone several surgical procedures for pelvic pathology. Our observational study was unable to demonstrate a link between ovarian cancer and ovarian endoemtrioma. The biggest limitation of our study is the demographic diversity of the groups being studied. This reflects how medical practice and patients expectations have changed over the last 20 years.

482

\section{OUR EXPERIENCE IN LAPAROSCOPIC DIAGNOSIS AND TREATMENT OF PELVIC ENDOMETRIOSIS}

Endometriosis: Diagnosis

A. Gvenetadze*, T. Sabakhtarashvili, L. Dzotsenidze
${ }^{[I]}$ Tbilisi State University, ZS Reproductive clinic $\sim$ Tbilisi $\sim$ Georgia

Summary (4 lines): The retrospective study is performed of the data from january to June 2012 on 130 patients. The study shows that laparoscopy is the best approach to diagnose and treat pelvic endometriosis.

Introduction: the purpose of this retrospective study is to demonstrate the efficacy of laparoscopy in the diagnosis and treatment of pelvic endometriosis.

Material and Methods: in our new clinic (RCZS), that is functioning since January 2012 we performed 130 laparoscopy during 6 months.119 of them were performed in combination with hysteroscopy, 10 of them was a preparation for IVF. Indications for operation were: suspected pelvic endometriosis 58/130 $(43.52 \%)$; ovarian endometriomas 27/130(20.77\%) infertility $115 / 130(73.07 \%)$ including tubal factor infertility $20 / 130$ $(15.38 \%)$;

Results: In 45/58(77.58 \%)woman laparoscopy confirmed the presence of pelvic endometriosis.11/58 18.96\%)-I stage, 21/58(36.20\%)II stage, $9 / 58$ (15.51\%)-III stage, $3 / 58$ (5.17 \%)-IV stage); in 13/58 $(22.42 \%)$ excluded it; in 25/27(81.48 \%) we confirmed endometriomas ( 2 of them was the kissing cysts); In $2 / 27(7.40 \%$ ) we found small dermoid cist of ovary. In addition in 7/72(9.72\%)women where were no suspect to endometriosis we found it during laparoscopy. In 49/130 $(37.69 \%)$ woman were found various grades of pelvic peritoneal adhesions;

Discussion: Our materials confirm once again that laparoscopy is a gold standard for the diagnosis and treatment of pelvic endometriosis.

\section{9}

\section{SERUM HUMAN EPIDIDYMIS PROTEIN 4(HE4) \\ LEVELS ARE LESS FREQUENTLY ELEVATED THAN CA125 IN WOMEN WITH DIFFERENT TYPES OF ENDOMETRIOSIS}

Endometriosis: Diagnosis

M. Rius*, M.Á. Martínez-zamora, P. Fusté, R. Molina, J. Balasch, F. Carmona

${ }^{[1]}$ Hospital Clinic $\sim$ Barcelona $\sim$ Spain

Summary (4 lines): HE4 is elevated less frequently than CA125 in endometriosis patients. Ovarian endometrioma (OE)alone-patients present with higher HE4 levels than other types of endometriosispatients.

Introduction: Elevations of CA125 are a problem in premenopausal endometriosis women. HE4 is a new biomarker for ovarian cancer. We evaluated serum concentrations of HE4 and CA125 in women with various types of endometriosis.

Material and Methods: CA125 and HE4 serum concentrations were determined in 37 patients with OE alone, 30 with DIE alone and 47 patients with OE and DIE before laparoscopic surgery.

Benign endometriosis was confirmed in all cases during surgery and they were classified as having ovarian endometrioma (OE)alone, deep infiltrating endometriosis (DIE)alone or both.

Results: 43 patients had serum CA125 levels higher than $40 \mathrm{U} /$ $\mathrm{mL}$ (17OE alone, 7DIE alone and 19OE + DIE) and 13 patients had serum HE4 levels higher than $150 \mathrm{pmol} / \mathrm{L}(7 \mathrm{OE}$ alone, 3DIE alone and $3 \mathrm{OE}+\mathrm{DIE})(\mathrm{p}<0.0001)$. The comparison of serum concentrations of HE4 between the 3 groups showed higher levels of HE4 in OE alone patients compared to DIE alone patients $(\mathrm{p}=0.04)$. The comparison of serum concentrations of CA125 between the 3 groups showed no statistical difference $(\mathrm{p}=0.2)$.

Discussion: HE4 is elevated less frequently than CA125 in endometriosis patients. Patients with $\mathrm{OE}$ alone have more frequently increased HE4 levels than patients with DIE alone. 
194

\section{ACTIVATION OF FIBRINOLYSIS DURING BENIGN ADNEXAL LAPAROSCOPIC SURGERY \\ Endometriosis: Surgery}

M. Martínez-zamora*, D. Tàssies, J.C. Reverter, J. Balasch, F. Carmona

${ }^{[1]}$ Hospital Clínic of Barcelona $\sim$ Barcelona $\sim$ Spain

Summary (4 lines): Benign adnexal laparoscopy seems to activate plasma fibrinolysis. Fibrinolysis studies can stratify the thromboembolic risk in patients undergoing laparoscopy.

Introduction: Laparoscopic surgery appears to be less traumatic than open procedures but the risk of thrombosis after laparoscopic surgery is not well known. We analyzed changes of systemic fibrinolysis in patients who underwent elective benign adnexal laparoscopic surgery (uni or bilateral cystectomy/adnexectomy).

Material and Methods: Blood samples were drawn on admission (S1), during surgery (S2) and on the first postoperative day (S3), in 45 patients. Samples were evaluated for clot lysis time (CLT) and plasmin-alpha2-antiplasmin complexes (PAP) as global functional tests of fibrinolysis.

Results: No patient presented with thromboembolic events before or after surgery. CLT (minutes) was shorter in S2 although differences were only statistically significant between S2 and S3 $(p=0.02)$ $(\mathrm{S} 1: 64.2 \pm 13.0 ; \mathrm{S} 2: 59.6 \pm 15.4 ; \mathrm{S} 3: 68.9 \pm 15.2)$. PAP was statistically higher in $\mathrm{S} 2$ compared to $\mathrm{S} 1(\mathrm{p}=0.001)$ and $\mathrm{S} 3(\mathrm{p}<0.0001)(\mathrm{S} 1: 375 \pm$ 190; S2:912 $\pm 654 ; \mathrm{S} 3: 296 \pm 153)$.

The pre and postoperative CLT was longer in older, multiparous, obese patients and in users of hormonal treatments.

Discussion: Benign adnexal laparoscopy transitionally activate plasma fibrinolysis. Older patients, obese and/or multiparous women, and users of hormonal treatments, should be considered at high thromboembolic risk.

\section{4}

BOWEL FUNCTIONAL SYMPTOMS AFTER LAPAROSCOPIC EXCISION OF DEEP INFILTRATION ENDOMETRIOSIS WITH SEGMENTAL BOWEL RESECTION

Endometriosis: Surgery

J. Albornoz*, E. Faller, P. Messori, M. Puga, A. Wattiez

${ }^{[1]}$ IRCAD Strasbourg France

Summary (4 lines): Bowel resection for endometriosis improves gynaecological and digestive symptomatology, without important increase in bowel functional symptoms.

Introduction: Report rate of bowel functional symptoms after bowel resection for endometriosis.

Material and Methods: Retrospective study in thirty-tree patients complaining from dysmenorrhea, dyspareunia, chronic pelvic pain (CPP), dyschezia and rectorragy who underwent laparoscopic endometriosis excision with segmental bowel resection and primary anastomosis.

Results: Mean age of patients was 33-years-old. According to distance from anal verge, bowel anastomosis was performed at high rectum $(>10 \mathrm{~cm})$ in $54.5 \%$, low rectum $(=10 \mathrm{~cm})$ in $39.3 \%$ and at the sigmoid in $6 \%$ of the cases. After 25.7 month mean follow up, main postoperative bowel functional symptoms were uncomfortable intestinal gas emission (36.4\%), frequent depositions $(27.3 \%)$, meteorism $(24.2 \%)$, diarrhea $(21.2 \%)$ and constipation (18.2\%). We observed a $72.5 \%$ decrease in pain score (VAS) for dysmenorrhea, $57.9 \%$ for dyspareunia, $50 \%$ for dysuria, $65.2 \%$ for CPP and $61.4 \%$ for dyschezia.
Discussion: Laparoscopic excision of pelvic endometriosis with segmental bowel resection improves gynaecological and digestive symptomatology, without important increase in bowel dysfunction symptoms.

502

\section{DEEP INFILTRATING ENDOMETRIOSIS. HOW DO YOU TREAT YOURS? \\ Endometriosis: Surgery}

T. Smith Walker*, D.L. Byrne

${ }^{[1]}$ Royal Cornwall Hospital $\sim$ Truro $\sim$ United Kingdom

Summary (4 lines): In order to see how different hospitals manage deep infiltrating endometriosis an online survey was conducted. The results highlight the variation in hospital set up, treatment and follow up with regards to deep infiltrating endometriosis.

Introduction: The management of deep infiltrating endometriosis (D.I.E) requires a multidisciplinary approach to manage this complex condition optimally. There is, however, no standardised surgical approach. Our survey was designed to see how different units are set up and how their surgical approaches vary.

Material and Methods: An online survey was sent out to all British Society of Gynaecological Endoscopy (BSGE) members via 'survey monkey'. The survey used questions to identify the multidisciplinary team used managing D.I.E, their surgical approach and whether GnRH analogues were used as part of there treatment regime.

Results: Initial analysis of the results show that there is a varied approach to treating these challenging patients. So far 42 units across the UK and overseas have responded, final results will be presented in Paris

Discussion: This is a preliminary investigation in the identification of the 'gold standard' treatment of deep infiltrating endometriosis. The next step is to look at the longterm results these units are having. This can only be done if we record our outcomes using a central database such as the one used by the BSGE. It is crucial, when analysing such data, that we know the approach used to achieve their outcomes.

\section{0}

\section{DOES LAPAROSCOPIC TREATMENT OF ENDOMETRIOSIS IMPROVE QUALITY OF LIFE?}

Endometriosis: Surgery

V. Minas*, G. Brierley, T. Dada

${ }^{[1]}$ Stoke Mandeville Hospital $\sim$ Aylesbury $\sim$ United Kingdom

Summary (4 lines): We conducted a survey to investigate the effect of laparoscopic treatment of endometriosis to patients' quality of life. Patients reported significant improvement in quality of life scores.

Introduction: Complete surgical eradication is considered the mainstay of treatment for endometriosis. Still, the duration of therapeutic benefit varies and the effect on the women's quality of life has not been studied extensively. The aim of the study was to investigate patients' own assessment of whether their laparoscopic treatment made a difference to their quality of life, as well as to assess local recurrence rates.

Material and Methods: The notes of 50 women who had laparoscopic treatment for endometriosis at Stoke Mandeville Hospital between 2008 and 2010, were reviewed. Patients were sent the EHP-5 questionnaire and were asked to score their quality of life in relation to endometriosis symptoms, prior to the surgery and presently. 
Results: The response rate was $80 \%$. The group of 50 patients was divided in subgroups of patients with stage I/II or stage III/IV disease, as well as in the subgroups of 12-24, 24-36 and 36-48 months of follow up. All subgroups reported improvement in quality of life scores. The overall recurrence rate was $18 \%$.

Discussion: In our study, we conclude that patients post laparoscopic treatment of endometriosis, experience significant quality of life improvement regardless of stage. This appears to persist for up to 3648 months following surgery.

402

\section{ENHANCED RECOVERY AFTER RECTAL RESECTION FOR SEVERE ENDOMETRIOSIS}

Endometriosis: Surgery

N. Waters*, F. Shakir, A. Day, R. Smith, T. Rockall, A. Kent

${ }^{[1]}$ Royal Surrey Hopital $\sim$ Guilford $\sim$ United Kingdom

Summary (4 lines): Traditionally open rectal resection was associated with significant morbidity and prolonged hospital stay (10 days). In our institution $99 \%$ of rectal resection for severe endometriosis is performed laparoscopically (national average $25 \%$ ). The introduction of enhanced recovery further improved complication rate, hospital stay and early return to normal function

Introduction: The main aspects of enhanced recovery program is carbohydrate and protein loading, tailored anaesthesia and postoperative analgesia, maintaining high oxygen concentration and normothermia, avoiding peri-operative fluid overload and early postoperative mobilisation

Material and Methods: 12 cases of severe endometriosis patient's undergoing laparoscopic rectal resection were enrolled into an enhanced recovery program. They received either a spinal or epidural analgesia and their outcomes were collected. Their outcomes were compared with patients undergoing laparoscopic rectal resection with a standard care pathway

Results: The average hospital stay was 4.5 days. There were no cases of prolonged ileus and bladder retention in the enhanced recovery group. The average operation time was 154 minutes and the average time to bowel sounds was 1.4 days and there were no colorectal complication

Discussion: Enhanced recovery together with a laparoscopic approach improves patients mobilisation, reduces ileus and improves their return to normal activities

\section{4}

\section{LAPAROSCOPIC MANAGEMENT OF ENDOMETRIOSIS}

Endometriosis: Surgery

P. Stanciu*, M. Ionescu, S. Pantea, D. Grigoras, M. Craina

${ }^{[1]}$ University Clinic of Obstetrics and Gynecology Bega $\sim$ Timisoara Romania

Summary (4 lines): The purpose of this paper is to analyze 487 cases of endometriosis, whom underwent laparoscopic surgery in University Clinic of Obstetrics - Gynecology Bega, Timisoara, Romania.

Introduction: Endometriosis is a condition characterized by the presence of functional endometrial tissue outside the uterine cavity. It frequently affects women of reproductive age.

Material and Methods: During February 2007 to February 2012 were performed a number of 3270 laparoscopies, 487 (14,89\%) of them for endometriosis. The types of surgery that were performed: $71 \%$ of cases diatermocoagulation of various sites of endometriosis, $34 \%$ of cases ovarian cystectomy, $9 \%$ of cases partial resection of the ovary, $28 \%$ of cases adesiolysis and $4 \%$ of cases adnexectomy.

Results: Outbreak of endometriosis in the study group has the following repartition: Douglas cul-de-sac and utero-sacral ligaments - 305 cases, visceral peritoneum of bladder and uterus - 81 cases; ovaries 217 cases. Staging cases of endometriosis by ASRM classification: stage I - 198 cases (40,7 \%), stage II - 138 cases (28.3\%), stage III - 113 cases $(23.2 \%)$ and stage IV - 38 cases $(7,8 \%)$.

Discussion: Laparoscopic surgery is currently the most appropriate method of treatment for endometriosis. Laparoscopy set the fastest diagnosis of endometriosis, applies sanction ablation therapy for endometriosis cysts or cauterization for endometriosis sites and along with hormonal treatment corrects fertility and prevents endometriosis recurrence.

\section{1}

\section{LAPAROSCOPIC NERVE - SPARING RADICAL TREATMENT OF DEEP INFILTRATING ENDOMETRIOSIS.}

Endometriosis: Surgery

A. Kavallaris*, D. Zygouris, N. Chalvatzas, E. Terzakis

${ }^{[1]}$ Department of Gynecologic Oncology, St. Loukas Hospital,

$\sim$ Thessaloniki, $\sim$ Greece $\sim$ Athens $\sim$ Greece

Summary (4 lines): Identification of the inferior hypogastric nerve and plexus is feasible and performed in acceptable operative time in treatment of deep infiltrating endometriosis.

Introduction: The radical surgery of the deep infiltrating endometriosis of the rectovaginal septum and the uterosacral ligaments can cause urinary retention and the need of self-catheterization.

Material and Methods: We present a case series of 40 patients, which underwent laparoscopic nerve sparing surgery of deep infiltrating endometriosis. In 30 patients a bilateral identification of the inferior hypogastric nerve and plexus was performed nad in 10 unilateral.

Results: The mean operative time was $42 \mathrm{~min}$. All patients underwent unilateral or bilateral resection of the uterosacral ligaments. Dysmenorrhoea, pelvic pain, dyspareunia and bladder bleeding in two patients disappeared postoperatively in all patients. Postoperatively, the overall time to resume voiding function was 2 days and the residual urine volume was in all patients $<50 \mathrm{ml}$ in two ultrasound measurements.

Discussion: Identification of the inferior hypogastric nerve and plexus was in our study feasible and performed in acceptable operative time. The comparison with the older non nerve sparing surgical technique that many patients needed bladder self-catheterization for a long time or more worse for a life time, confirmed the importance of the nerve sparing surgical procedure.

\section{0}

\section{LAPAROSCOPIC RESECTION OF DEEPLY INFILTRATING ENDOMETRIOSIS OF THE BLADDER: THE HUNGARIAN EXPERIENCE \\ Endometriosis: Surgery}

B. Attila*, N. Péter, R. János

${ }^{[1]}$ Semmelweis University 1st dept OB/Gyn $\sim$ Budapest $\sim$ Hungary

Summary (4 lines): The first-line treatment of the deeply infiltrating endometriosis of baldder is laparoscopic partial bladder resection in some cases bladder nodules can be resected extramucosally as well. Introduction: Involvement of the urinary tract by deeply infiltrating endometriosis (DIE) is estimated in 1-2 \% of all cases, most frequently affecting the bladder. The treatment's goal is the alleviation of the patient's symptoms and prevention of renal insufficiency. 
Material and Methods: Between 10/07/2009 and 20/06/2012 at the 1st Dept. of OB/GYN, Semmelweis University, Budapest a series of 13 laparoscopic partial bladder resection and 20 extramucosal resection was performed for bladder DIE.

Cystoscopy was frequently ineficient because lesions are often leaving the mucosa free, in some of these cases extramucosal bladder resection could be performed. Transvaginal sonography was routinely performed to evaluate the pelvis and diagnosing bladder endometriosis.

Ureterolysis was performed in 19, colorectal resection in 6 cases. On postoperative day 7 the Foley catheter was removed in all but 3 cases when it was left until the 10th day.

Results: Postoperative outcomes showed a significant improvement in painful symptoms and an absence of urinary complaints in all cases.

Discussion: There was no difference regarding the intra- and postoperative complications in patients who underwent mucosal skinning when compared to the partial cystectomy.

\section{9}

\section{LAPAROSCOPIC SURGERY IN URINARY TRACT ENDOMETRIOSIS \\ Endometriosis: Surgery}

C. Castro*, J. Alves, A. Rodrigues, T. Leitão, C. Alho, F. Osório, C. Calhaz-jorge

${ }^{[1]}$ Hoispital de Santa Maria $\sim$ Lisboa $\sim$ Portugal

Summary (4 lines): Review of 10 cases of laparoscopic surgery for bladder/ureteral endometriosis.

Introduction: Endometriosis of the urinary tract affects $1 \%$ of women with pelvic endometriosis.

Material and Methods: We reviewed 10 cases of patients undergoing laparoscopic surgery for bladder/ureteral endometriosis in our Department. Variables recorded were: age, previous abdominopelvic surgery, preoperative symptoms (pain visual scale - a score 0 to 10), other structures involvement, type of surgery, complications and pain intensity during follow-up.

Results: Six patients had bladder endometriosis and 4 ureteral endometriosis. Mean age was 34 years. Six patients had previous pelvic surgery ( 4 for endometriosis). Dysmenorrhea was present in all patients (improved 6 points), dyspareunia in 6 (improved 4 points), dysuria in 5 (improved 4 points) and dyschezia in 6 (improved 3 points). All patients had recto-vaginal lesions, 4 endometrioma, 7 extensive adhesions and 6 intestinal wall involvement. In all cases of bladder endometriosis, transmural partial cystectomy was performed with double laparoscopic suture. Average diameter of lesions was $2.5 \mathrm{~cm}$. Segmental ureteral resection was performed in 1 case and ureterolysis with excision of superficial lesions in 3 . One patient had a postoperative complication - pyelonephritis.

Discussion: Our results show that laparoscopic surgical treatment of urinary tract endometriosis improves patients quality of life.

\section{8}

\section{LAPAROSCOPIC TREATMENT OF ENDOMETRIOMA}

Endometriosis: Surgery

L. Vuckovic*

${ }^{[1]}$ Medical Center $\sim$ Uzice $\sim$ Serbia

Summary (4 lines): Postoperative hormonal therapy show better results in the combined way of treatment of endometriosis and infertility.

Introduction: Endometriosis is one of the most frequent causes of infertility. Therapy can be hormonal and operative.
Material and Methods: In the period between 2003-2011, there were 4049 interventions. $78 \%$ were operated with laparotomy and $22 \%$ laparoscopically. $1.8 \%$ were operated due to endometrioma. We applied retrospective and prospective study.

Results: Endometrioma extirpation was performed in $94 \%$ of cases, ovariectomy in $4 \%$ and hysterectomy with bilateral adnexectomy in $2 \%$ of cases.

All patients received postoperative hormonal therapy. $25 \%$ received preoperative hormonal therapy with $10 \mathrm{mg}$ progestron pills on a daily basis continuously for 3 months. Postoperatively, $31 \%$ received continuously progesteron pills of $10 \mathrm{mg}$ for 3-6 months, and the remaining $69 \%$ received subdermally GnRH analogue for 6 months.

Out of totally 72 patients, pregnancy occurred in $55 \%, 7 \%$ was lost during the process of following. $7 \%$ patients were sent to assissted reproduction. $27 \%$ succeeded in getting pregnant after pre and post operative therapy and $73 \%$ succeeded in getting pregnant after postoperative hormonal therapy.

Discussion: The number of performed laparoscopic procedures increased due to better diagnostics and expanded selection criteria

\section{2}

\section{MINIMALLY INVASIVE SURGICAL TREATMENT OF ENDOMETRIOSIS: EXPERIENCE OF THE LAST 3 YEARS Endometriosis: Surgery}

A. De Almeida Rodrigues*, J. Alves, C. Castro, C. Alho, F. Osório, C. Calhaz-jorge

${ }^{[1]}$ Hospital de Santa Maria - CHLN, EPE $\sim$ Lisboa $\sim$ Portugal

Summary (4 lines): We present the experience of our Department on minimally invasive surgical treatment of endometriosis since 2009. Introduction: Laparoscopic ablation of endometriosis lesions and restoration of normal pelvic anatomy result in improvement of quality of life. It is a complex procedure with a significant risk of complications, requiring a high degree of differentiation and experience.

Material and Methods: Retrospective clinical study of a total of 64 cases including ovarian endometriosis $(n=26)$, recto-vaginal septum infiltration $(n=9)$, an association of the previous two $(n=18)$, bladder and ureter $(\mathrm{n}=10)$ and abdominal endometriosis $(\mathrm{n}=1)$. All cases were operated by the same surgeon.

Results: There was an overall improvement of pain in $79.6 \%$ of patients. Complications included 2 cases of bowel injury (diagnosed and treated during surgery, one with conversion to laparotomy), 2 vaginal vault dehiscences, 3 infectious complications ( 1 major, 2 minor), and 1 seroma of abdominal wall. Surgery was conservative (preserving at least one ovary) in $89 \%$ of cases. 9 patients were reoperated, one for staging of an ovarian cancer diagnosed in the specimen of an endometrioma, the others for disease recurrence after a mean of 12 months.

Discussion: These results regarding pain relief, associated with a low rate of complications, allows us to say that this is a successful approach in the treatment of endometriosis.

\section{6}

\section{MRI SCANNING FOR THE DIAGNOSIS OF DEEPLY INVASIVE ENDOMETRIOSIS (DIE) \\ Endometriosis: Surgery}

\author{
J. Joy, D. Hunter* \\ ${ }^{[1]}$ Belfast Health Trust $\sim$ Belfast $\sim$ United Kingdom
}

Summary (4 lines): This study assesses the accuracy of MRI scanning in diagnosing DIE. 
Introduction: Magnetic Resonance Imaging (MRI) is a recognised noninvasive technique for assessing DIE.

Material and Methods: 30 patients with endometriosis undergoing MRI scanning and surgery were identified and had MRI findings and operative data examined. The accuracy of the MRI to detect DIE was determined by descriptive statistics.

Results: DIE involving the uterosacral ligaments, rectovaginal septum, rectum, bladder were documented in $36.7 \%, 33.3 \%, 30 \%$ and 0 cases. Adenomyosis was diagnosed by MRI in $7(23.3 \%)$ cases and confirmed in $2(6.7 \%)$ cases by hystrectomy. The sensitivity, specificity, positive likelihood ratio ( $+\mathrm{LR})$ and negative likelihood ratio ( $-\mathrm{LR})$ respectively for MRI was $9 \%, 100 \%, 8$ and 0.9 for uterosacral ligament endometriosis; $87.5 \%, 72.7 \%, 3.2,0.17$ for endometriosis of rectovaginal septum; $100 \%, 76 \%, 4.2,0$ for rectal endometriotic involvement and $84 \%, 64 \%, 2.3,0.25$ for ovarian endometriosis.

Discussion: MRI had high specificity and positive likelihood ratio for detecting uterosacral ligament endometriosis, but very low sensitivity. The sensitivity and specificity for detecting rectovaginal septal, rectal and ovarian endometriosis was high and comparable to other studies

\section{5}

\section{PRIMARY REFERRAL TO SPECIALIST ENDOMETRIOSIS CENTRES REDUCES MORBIDITY IN PATIENTS WITH SEVERE ENDOMETRIOSIS}

Endometriosis: Surgery

\author{
L. Forster*, T. Smith-walker, P. Wipplinger, D. Byrne \\ ${ }^{[1]}$ Royal Cornwall Hospital $\sim$ Cornwall $\sim$ United Kingdom
}

Summary (4 lines): Three indicative cases of severe endometriosis with poor outcomes following treatment in a generalist setting, which required secondary referral to a specialist endometriosis centre. Primary referral to a specialist centre would have reduced patient morbidity.

Introduction: Deep infiltrating endometriosis is a complex disease causing significant morbidity. Patients often experience a delay in diagnosis and suboptimal treatment.

Material and Methods: We reviewed three cases referred to the endometriosis service following initial suboptimal treatment.

Case 1: Laparoscopy \& diathermy and subsequent hysterectomy. Required extensive surgery to remove residual endometriosis.

Case 2: Two laparoscopies and laser treatment, hysterectomy, subsequent bowel obstruction secondary to endometriosis requiring right hemicolectomy. Case 3: Endometrial ablation, subtotal hysterectomy. Requires further surgery for ongoing pain and adhesions secondary to residual endometriosis.

Results: The specifics of each case will be discussed in the presentation. In each case the patients received suboptimal initial treatment and required further surgery.

Discussion: Additional surgery would not have been required if these patients had undergone initial treatment in a specialist centre. This illustrates the importance of education regarding the value of the specialist service. A low threshold for referral is fundamental in facilitating optimum patient care.

516

\section{PROSPECTIVE EVALUATION OF URINARY DYSFUNCTION AFTER SURGICAL RESECTION OF DEEP ENDOMETRIOSIS Endometriosis: Surgery}

J. Niro*, C. Vilette, A. Le Tohic, P. Panel

${ }^{[1]}$ centre hopsitalier de versailles $\sim$ le chesnay $\sim$ France

Summary (4 lines): Evolution of urinary symptoms among patients who underwent surgery for a deep endometriosis. USL lesions are mainly found on patients with urinary symptoms. Surgical resection improves these symptoms.

Introduction: Evolution of urinary symptoms among patients who underwent surgery for a deep endometriosis and locate the injuries.

Material and Methods: Cohorte of 51 patients underwent surgery for deep endometriosis. Locations of the surgical injuries were reported into three categories: anterior (bladder), posterior (UteroSacral Ligaments, rectovaginal and colorectal) and mixt. Each patient filled a selfassessment questionnaire before and after surgery.

Results: 2 patients had isolated anterior injuries, 4 had mixt injuries and 45 had posterior injuries (15 isolated USL, 9 rectovaginal, and 21 colorectal). Among the posterior group of patients, the USL injuries was most often found among the patients who had urinary symptoms and this for every symptom taken separately. After surgery, painful needs $(\mathrm{p}=0,001)$, pain while urinating $(\mathrm{p}=0,0001)$, urgency $(\mathrm{p}=0,031)$ and urinary frequency $(p=0,0004)$ significantly decreased whereas burning on urination $(p=$ 0.079 ) didn't. Analysis of all symptoms intensity show a significant decrease $(p=0,0003)$. The average study time is 44 weeks.

Discussion: USL lesions are mainly found on patients with urinary symptoms. Curative surgical resection on deep endometriosis improves urinary symptoms.

\section{3}

SHOULD ADENOMYOSIS BE MORCELLATED?

Endometriosis: Surgery

H. Krentel*

${ }^{[1]}$ Hospital Eisenhüttenstadt $\sim$ Eisenhüttenstadt $\sim$ Germany

Summary (4 lines): Based on a pubmed analyse the use of electric morcellation on adenomyotic tissue in laparoscopic subtotal hysterectomy is discussed.

Introduction: Laparoscopic subtotal hysterectomy with morcellation of the utero has become a standard procedure in gynecologic surgery. The morcellation of the uterus means to shred a histologically unknown variety of tissues in the abdomen. Publications have shown the correlation between morcellation and postoperative peritoneal endometriosis and adenomyosis. It still remains unclear if the incidence of postsurgical complications depends on the histology of the morcellated tissue.

Material and Methods: Pubmed analyse of recent publications using the keywords subtotal hysterectomy, adenomyosis and morcellation.

Results: Case reports show a great variety of postsurgical complications by tissue implants after morcellation. Respective studies are still missing. Discussion: As the pathogenesis of peritoneal endometriosis remains still unclear, it seems questionable that the morcellation of adenomyosis is a common practice. Further studies have to show that this procedure is not correlated with a higher incidence of postsurgical endometriosis and adenomyosis. The questions if a presurgical histology of the posterior uterine wall should be taken by resectoscopy, if adenomyosis could be a contraindication for subtotal hysterectomy, and how safe a histologic report of more than $500 \mathrm{gr}$. of morcellated tissue can be, will be discussed.

\section{0}

\section{SURGICAL MANAGEMENT OF RECTO-SIGMOIDAL ENDOMETRIOSIS \\ Endometriosis: Surgery}

\author{
A. Mccoubrey, D. Hunter*, K. Khosraviani \\ ${ }^{[1]}$ Belfast Health Trust $\sim$ Belfast $\sim$ United Kingdom
}

Summary (4 lines): The management of severe endometriosis should be undertaken in centres offering treatment by experienced surgeons 
Introduction: $5-10 \%$ of women with endometriosis have stage IV disease with involvement of the colon (rectum/rectosigmoid junction in approximately $75 \%$ ). Medical treatment often fails: surgery is the preferred management option. The study reviews the experience of segmental bowel resection for severe endometriosis in a regional unit. Material and Methods: Data was collected over a 5 year period (20062011) for 18 women who underwent surgery for endometriosis under the joint care of a colorectal surgeon (KK) \& a gynaecologist (DH).

Results: Age range 26 - 46 years (mean 33 years). 11 patients $(61 \%)$ underwent laparoscopic resection, 2 were converted to open procedures (18\%). 7 underwent laparotomy. 14 (78\%) had a segmental bowel resection performed.10 patients underwent concurrent hysterectomy \& salpingo-oophorectomy. Mean length of stay was 8 days. In those undergoing colonic resection, pathology confirmed endometriosis in all cases. 2 patients experienced post-operative complications (11\%).3 patients had stoma formation, 1 of which has since been reversed. The majority of patients reported significant improvement in symptoms postoperatively. 3 patients (of 6 with fertility issues) became pregnant.

Discussion: Segmental bowel resection for severe endometriosis is safe and feasible with significant reduction in symptoms post-operatively.

\section{9}

\section{SURGICAL TREATMENT OF RECTOVAGINAL ENDOMETRIOSIS}

Endometriosis: Surgery

\author{
J. Alves*, C. Castro, A. Rodrigues, F. Osorio, C. Calhaz Jorge \\ ${ }^{[1]}$ Hospital de Santa Maria $\sim$ Lisbon $\sim$ Portugal
}

Summary (4 lines): We present a retrospective analysis of 36 patients with rectovaginal endometriosis treated with laparoscopic surgery. There was a significant improvement in dysmenorrhea, dyspareunia, dyschezia and dysuria.

Introduction: Rectovaginal endometriosis can cause intense dysmenorrhea, dyspareunia and/or dyschezia. Surgical treatment is preferably by laparoscopy allowing skinning of the bowel wall in most superficial lesions and segmental resection if deep infiltration is present. A series of 36 patients is presented.

Material and Methods: Retrospective evaluation of medical files of patients operated between April 2009 and December 2011. Parameters registered: pain complains before and after surgery, complications related and necessity to re-operate. Wilcoxon sign rank test was used. Results: Average age was 33 years; half of patients had previous surgeries for endometriosis. All had dysmenorrhea and dyspareunia, half presented dyschezia and $38 \%$ dysuria; all improved after surgery $(\mathrm{p}<0.001) .63 \%$ had also endometriomas and $33 \%$ vesical involvement. Rectal or bowel skinning was performed in $89 \%$. In 2 patients intra-operatively detected intestinal perforations occurred. Five patients were re-operated within one year: two anexectomies and three bowel resections were performed.

Discussion: There was a significant improvement in pain complains, leading to a better quality of life. This plus the small number of complications and reduced number of re-operations are encouraging results.

\section{9}

\section{THE EFFICACY OF PREOPERATIVE TREATMENT FOR PATIENTS WITH ENDOMETRIOSIS: WHAT COULD BE DONE BEFORE LAPAROSCOPY}

Endometriosis: Surgery

\author{
T. Lunko, O. Aleksandrov* \\ ${ }^{[1]}$ Odessa National Medical University $\sim$ Odessa $\sim$ Ukraine
}

Summary (4 lines): This research shows high effectiveness of preoperative treatment with semisynthetic, steroidal progestogen - Dienogest for women with endometriosis and infertility.

Introduction: Among women with pelvic pain, the prevalence of endometriosis ranges up to $21 \%$ and it is diagnosed in $17 \%$ of women with primary infertility.

Material and Methods: There were 97 women (mean age 29,7 $\pm 2,3$ ) who had endometriosis associated with chronic pelvic pain and infertility (primary and secondary) more than 1 year. All women were prescribed Dienogest ( $2 \mathrm{mg}$ per day) during 3 month before laparoscopy was performed. There was confirmed endometriosis: mild- $42 \%$, moderate$37 \%$, severe- $21 \%$. The mean time of operation was $(50 \pm 15 \mathrm{~min})$ with excision, coagulation and ovarian resections to be performed.

Results: We did not reveal any complications after these operations. There were $42(43,3 \%)$ patients who get pregnant in 3-4 month after operation. Chronic pelvic pain significantly reduced among 34 women with 63(64,9\%)who did not complain of it.

Discussion: The results have proved high effectiveness of preoperative treatment by steroidal progestogen which has anti-adhesive, antiinflammatory and anti-proliferative effects. This reduces operational time, improves quality of life and reproductive outcome.

URINARY DYSFUNCTION AFTER LAPAROSCOPIC SURGERY FOR CHRONIC PELVIC PAIN RELATED TO PELVIC ENDOMETRIOSIS; POSTOPERATIVE EVALUATION AND MANAGEMENT

Endometriosis: Surgery

K. Ki*, J. Lee

${ }^{[1]}$ Kyung Hee University Hospital $\sim$ Seoul $\sim$ Korea, Republic of

Summary (4 lines): laparoscopic surgery for chronic pelvic pain and resection of deep infiltrating pelvic endometriosis are source of urinary dysfunction whatever the surgical procedure.

Introduction: To evaluate urogenital dysfunction before and after laparoscopic surgery for chronic pelvic pain.

Material and Methods: From April 2009 through May 2011, laparoscopy was performed to evaluate any pathologic condition in 163 women with chronic pelvic pain, median age was 41 years (range 23-66) received.

Results: Laparoscopic and histopathologic findings of the pelvis, any associated factors to the pain, as well as subjective pain score and urinary symptoms after laparoscopic management. Most patients have significantly improved pain symptoms after laparoscopic adhesiolysis and excision of endometriosis. However Urinary dysfunction, mainly dysuria, was observed in $19 \%$ of cases postoperatively. Surgical stimulation of pelvic autonomic nerve during adhesiolysis is the main cause of urinary dysfunction. Pelvic autonomic nerves are especially at risk in cases of deep infiltrating endometriosis during pelvic sidewall dissection.

Discussion: Structured education with regard to pelvic neuroanatomy is might be the key to improving functional outcome for these patients.

\section{7}

\section{A RETROSPECTIVE STUDY OF 175 WOMEN UNDERGOING UTERINE ARTERY EMBOLISATION \\ Hysterectomy}

M. Hardway*, S. Rees, N. Amso

${ }^{[1]}$ University hospital of Wales $\sim$ cardiff $\sim$ United Kingdom

Summary (4 lines): Long-term follow-up suggests major surgery can be avoided in the majority of patients after UAE. Fibroid position and presenting symptoms in patient choice ensure good outcomes post UAE. 
Introduction: UAE is associated with minor complications but still the need for additional surgical intervention. This study examines further surgical intervention cases post UAE.

Material and Methods: Retrospective analysis of research data prospectively collected for the British Society of Interventional Radiologist Fibroid registry in 1 participating institution from 175 women with symptomatic uterine fibroids during 2001-10.

Results: Total Re-intervention rate was $15.43 \%$, hysterectomy performed in 19 cases a mean time of 3.5 yrs post UAE with poor correlation to patient satisfaction noted.

11 could be reviewed showing minimal blood loss with benign fibroids. 67 of 104 women with urinary symptoms 1 year post-UAE had complete resolution of symptoms and compared with fibroid position revealed nearly $40 \%$ with dominant fibroid posteriorly

Discussion: Long term follow-up of 25 women $>10$ yrs only 3 had hysterectomy meaning $88 \%$ of women avoided major surgery. UAE mean age of 42.3 yrs and average menopause in early 50 s mean risk of further surgery for symptomatic fibroids is low.

Average blood loss for TAH for fibroids is $436 \mathrm{ml}$ but post-UAE due to decreased vascularity of fibroids was minimal, UAE can at least benefit patients in reduced morbidity in terms of haemorrhage.

\section{3}

\section{CHANGING TRENDS IN THE SURGICAL MANAGEMENT OF DUB}

Hysterectomy

E. Pavlidis*, D.H. Hunter

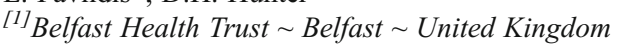

Summary (4 lines): The paper examines the changing trends in the daycase and inpatient management of patients with DUB.

Introduction: The introduction of Mirena and the 2nd generation endometrial ablation devices has had a profound impact on the treatment of women with DUB. A comparison of treatment numbers in 2000 and 2010 is presented.

Material and Methods: Theatre ledgers for the calendar years 2000 and 2010 were examined. All surgical procedures undertaken for DUB were recorded.

Results: In 2000, 112 inpatient and daycase procedures (27 Mirena $(24.1 \%), 16$ ablations $(14.3 \%), 69$ hysterectomies $(61.6 \%))$ were undertaken for DUB. In 2010, 232 inpatient and daycase procedures were performed (69 Mirena (29.7\%), 115 ablations (49.6\%), 48 hysterectomies $(20.7 \%))$.

Discussion: The surgical management of DUB has changed significantly in ten years with the introduction of minimally invasive techniques for treatment of DUB. A move to outpatient treatments will skew this figure further.

\section{6}

\section{COMPARATIVE STUDY OF DIFFERENT APPROACHES IN HYSTERECTOMY}

Hysterectomy

E. Fernández Miranda*, C. Barbed, M.T. Cusidó, A. Úbeda, P. Barri Soldevila

${ }^{[1]}$ IU Dexeus $\sim$ Barcelona $\sim$ Spain

Summary (4 lines): Compare the different surgical approaches to hysterectomy (abdominal, vaginal, laparoscopic total and subtotal). The variables analyzed were operating time, hospital stay, complications and indications. The mean age of patients was 50 years.
Introduction: Hysterectomy is the partial or total removal of the uterus by any means. The most frequently used routes are the abdominal, vaginal, laparoscopic or combined.

Material and Methods: This retrospective study on 591 hysterectomies performed for benign disease between January 2008 and December 2011 which compares the different surgical approaches.

Results: The most common indication for hysterectomy was uterine fibroids (43.3\%), followed by pelvic floor disorders (30.1\%).

Mean hospital stay was shorter in laparoscopic surgery with an average of 2.73 days $8.9 \%$ of the patients had any complications, the percentage increased by abdominal $(14.8 \%)$ and lowest for vaginal (6 \%). Major complications such as vesicovaginal fistula, unilateral or bilateral ureteral injury, bladder injury or intestinal perforation were more frequent in total laparoscopic hysterectomy ( $9.5 \%$ of all complications).

Discussion: The road that had a lower rate of complications was vaginal and laparoscopic subtotal hysterectomy. The average hospital stay and complications were lower in the laparoscopic approach.

118

\section{DOES ROUTE OF HYSTERECTOMY AFFECT OUTCOME IN ADENOMYOSIS PATIENTS? \\ Hysterectomy}

R. Solernou*, F. Barranco, M.A. Martínez-zamora, J. Balasch, F. Carmona

${ }^{[1]}$ Hospital Clínic of Barcelona $\sim$ Barcelona $\sim$ Spain

Summary (4 lines): The vaginal and laparoscopic hysterectomies had shorter hospital stay and lower blood loss than the laparotomic route in adenomyosis patients, although laparoscopic surgery was significantly longer.

Introduction: Hysterectomy (HST) in adenomyosis (AD) patients may be more difficult and increase the risk of complications. We compared the surgical outcomes of AD patients having HST according to the route (vaginal (VG), laparoscopic (LPS) or laparotomic (LPT)) of the procedure.

Material and Methods: We analyzed the surgical outcome in 108 patients with AD who underwent VG HST(Group 1; $\mathrm{n}=26)$, total LPS HST (Group 2; $\mathrm{n}=60$ ) or LPT HST(Group 3; $\mathrm{n}=22$ ).

Results: The mean surgical time was significantly longer in Group 2 (Mean?SD in minutes: Group 1:60?19; Group 2:177?185; Group 3:120? $39 ; \mathrm{p}=0.003)$. There were not statistical differences in surgical complications among groups (Group $1 \mathrm{n}=1$; Group $2 \mathrm{n}=3$; Group $3 \mathrm{n}=2$; $\mathrm{p}=0.4$ ). Similarly, there were not statistical differences among groups regarding postsurgical complications (Group $1 \mathrm{n}=2$; Group $2 \mathrm{n}=7$; Group $3 \mathrm{n}=5$; $p=0.7)$. Three patients in Group 3 received blood transfusion $(p=0.02)$. The mean hospital stay (days) was significantly longer in Group 3 (Group 1:3.2?1.5; Group 2:3.3?2.4; Group 3: 5.8?4.3; $\mathrm{p}=0.001$ ).

Discussion: The VG and LPS routes provided shorter hospital stay and lower blood loss than the LPT route, although LPS surgery was significantly longer. The risk of complications was not different among the three routes analyzed.

313

\section{EVOLUTION OF THE SURGICAL ROUTE AND THE LEARNING CURVE OF LAPAROSCOPIC HISTERECTOMY IN HOSPITAL DE LA SANTA CREU I SANT PAU Hysterectomy}

M. Arqué Gonzalez*, L. Alcoverro Bedós, M. Campillo Ajenjo, C. Baitg Rosó, R. Rovira Negre

${ }^{[1]}$ Hospital de la Santa Creu i Sant Pau $\sim$ Barcelona $\sim$ Spain 
Summary (4 lines): Laparoscopic histerectomy is a safe, effective and reproducible surgical technique after the appropriate training, and with a current technique, has a low rate of complications.

Introduction: Laparoscopic histerectomy is an efficient alternative to laparotomy in the treatment of benign uterine disorders; however, it is necessary to use an standarised technique and reach the "plateau" of the learning curve to guarantee optimal results with minimum complications.

Material and Methods: Descriptive retrospective study, that included 157 patients who underwent laparoscopic histerectomy between 2005-2011.

Results: Evolution of the technique was analysed in two periods: 2005-2008; 2009-2011.

There is a decrease of laparotomic histerectomies, from $62,7 \%$ in 2007 to $26,6 \%$ in 2011 ; an increase of laparoscopic histerectomies, from $8,27 \%$ in 2007 to $40,8 \%$ in 2011 . Vaginal histerectomy remains steady along the studied period (32\%).

The inflexion point of both tendencies coincides with the implantation of a programme to acquire skills in laparoscopic surgery in 2008 .

Discussion: Comparing periods, there is an increase of laparoscopic histerectomy and a decrease of laparotomic histerectomy, due to the implantation of a programme to acquire skills in laparoscopic surgery.

Results show that the more experience, the more capacity to deal with more complex surgeries with a lower rate of complications.

\section{0}

\section{INCIDENCE AND CHARACTERISTICS OF PATIENT WITH VAGINAL STUMP DISRUPTION AFTER TOTAL LAPAROSCOPIC HYSTERECTOMY}

Hysterectomy

S. Park*, Y. Lee

${ }^{[1]}$ Hallym University $\sim$ Seoul $\sim$ Korea, Republic of

Summary (4 lines): Vaginal stump disruption should be considered when abdominal pain and vaginal spotting appear after total laparoscopic hysterectomy, mostly due to early coitus.

Introduction: We design this study to estimate incidence and characteristics of patients with vaginal stump disruption after total laparoscopic hysterectomy.

Material and Methods: We reviewed medical records retrospectively from Jan. 2007 to Jan. 2010 of all patients with vaginal stump disruption after total laparoscopic hysterectomy.

Results: Twelve of 740 patients were identified with vaginal stump disruption after total laparoscopic hysterectomy(incidence $1.6 \%$ ). Early coitus was the triggering event of vaginal stump disruption in 9 patients(75 \%) and spontaneous rupture occurred in 3 patients (25\%). Abdominal pain and vaginal spotting were the most common symptom in the patients with vaginal stump disruption(75\%). Bowel evisceration was associated in four patients(33.3\%). Median time to presentation was 86.5 days. Nine cases were repaired by abdominal approach $(75 \%)$. One case was repaired by laparoscopic suture $(8.3 \%)$ and two cases were repaired by vaginal approach $(16.7 \%)$.

Discussion: Vaginal stump disruption should be considered in patient with abdominal pain and vaginal spotting after total laparoscopic hysterectomy, most commonly caused by early coitus. Contributing factors remains unknown but thermal effect and vaginal closure technique probably play major role.
77

\section{LAPAROSCOPIC HYSTERECTOMY - OUR EXPERIENCE Hysterectomy}

P. Reis, R. Sousa, R. Maciel, C. Lourenço, C. Marques, R. Cubal, H. Ferreira*

${ }^{[1]}$ Centro Hospitalar do Porto $\sim$ Porto $\sim$ Portugal

Summary (4 lines): The goal of our study was to analyze laparoscopic hysterectomy indications, the clinicopathological factors of each patient such as age, parity and history of previous surgery, operative time, estimated blood loss, surgical complications and conversion to laparotomy and length of hospital stay.

Introduction: Hysterectomy is one of the most common surgical procedures.

There has been a significant increase in the numbers of laparoscopic hysterectomies over the last years.

Material and Methods: A retrospective study was performed to analyze 102 cases of total laparoscopic hysterectomy (TLH) that took place in our hospital between November 2009 and December 2011.

Results: The most common indication was leiomyomas (43\%) followed by abnormal uterine bleeding (38\%), pelvic organ prolaps $(12 \%)$, endometrial hyperplasia $(5 \%)$ and cervix pathology $(2 \%)$. The mean age of the patients was 50 years. $11 \%$ were nulliparous. $41 \%$ had undergone previous abdominal surgery.

Mean operative time was $104 \mathrm{~min}$. The mean blood loss was $150 \mathrm{ml}$ (range 30-300).

There was 1 case of ureteric injury.

Conversion to laparotomy was required in 3 cases.

The average duration of hospital stay was 2 days.

Discussion: Laparoscopic hysterectomy is a feasible and safe procedure with a very low complication rate for experienced laparoscopic surgeons.

477

\section{LAPAROSCOPIC HYSTERECTOMY - SIX-YEARS EXPERIENCE \\ Hysterectomy}

S. Tomov*, G. Gorchev, L. Tanchev, G. Radinski

${ }^{[1]}$ Gynecologic Oncology Clinic, Medical University $\sim$ Pleven $\sim$ Bulgaria

Summary (4 lines): Laparoscopic hysterectomy ( $\mathrm{LH})$ is an inniovative operative method which is still object of extensive research.

Introduction: The autors' aim is to analyse the perioperative factors of LH and to evaluate its clinical importance for treatment of gynaecological diseases.

Material and Methods: For a six-year period (2004-2010) at the Gynecologic Oncology Clinic, Medical University, Pleven, and "St. Marina” Hospital of Obstetrics and Gynecology, Pleven, Bulgaria, laparoscopic hysterectomy was accomplished on 635 patients as follows: on $168(26 \%)$ - laparoscopically-assisted vaginal hysterectomy (LAVH), on 435 (69\%) - laparoscopic hysterectomy with uterine artery ligation (LH (a)), and on 32 (5\%) - total laparoscopic hysterectomy (TLH).

Results: The most common indication for LH was the myoma of the uterus ( $n=462,73 \%)$, followed by cervical carcinoma in situ $(n=75$, $12 \%)$ and benign ovarian tumors $(n=26,4 \%)$. The patients with precancerous diseases and those with malignant tumors FIGO I stage formed $23 \%$ of all cases. TLH had significantly the lowest mean operative time $(65,78 \pm 25 \mathrm{~min})$ and the highest postoperative hemoglobin $(117,66 \pm 14 \mathrm{~g} / \mathrm{l})$; and $\mathrm{LH}(\mathrm{a})$ had significantly the shortest hospital stay $(3 \pm 1,01$ days $)(p<0,05)$. There was no significant relationship between the type of LH and the total rate of complications. 
Discussion: The laparoscopic hysterectomy takes an important place in the operative treatment of benign, precancerous and malignant diseases in current gynecological practice.

\section{7}

\section{LAPAROSCOPIC HYSTERECTOMY FOR LARGE UTERUS Hysterectomy}

H. Jan*, G. Araklitis, N. Narvekar

${ }^{[1]}$ King's College Hospital $\sim$ London $\sim$ United Kingdom

Summary (4 lines): Laparoscopic hysterectomies for large uterus is safe and has a learning curve

Introduction: Laparoscopic hysterectomy in women with large fibroid uterus can be technically challenging

Material and Methods: A review of consecutive cases of laparoscopic hysterectomy for women with uterus $>16$ weeks size performed by 1 surgeon from July 2008-March 2012

Results: 17 patients between 2008-2012 with mean (sd) age of 47.2 (8.2)years, median (range) uterine size of 16 (16-22) weeks, median (range) fibroid number $2(1-7)$, mean (sd) diameter of largest fibroid $62.8(21.7) \mathrm{mm} .88 \%$ were for heavy menstrual bleeding. 2 (12\%)cases were converted to open for technical difficulty. The mean surgical time was 183 (46.7) minutes. Median hospital stay was 2 (1-5) days. Mean uterine weight was 371.4 (185.9) m. 11/15 (73\%) uteruses were morcellated vaginally and there were no major complications. The results from 2011-2012 were analyzed separately to assess the impact of learning curve and modifications to surgical technique. For the 8 patients in this group, the median uterine size was 18 (18-20) weeks and mean uterine weight 460 (217.5) g. The mean operating time was 154.6 (31.9) minutes and median hospital stay 1 day.

Discussion: Laparoscopic hysterectomy for large fibroid uterus is feasible and safe. The operative technique has a clear learning curve. It needs to be individualised with appropriate use of high ports, good uterine traction, safe treatment of the uterine vessels and a clear plan for uterine morcellation

280

\section{LAPAROSCOPIC HYSTERECTOMY IN A DISTRICT GENERAL HOSPITAL IN THE UK. \\ Hysterectomy}

D. Miligkos*, R. Behrens, K. Louden

${ }^{[1]}$ Royal Hampshire County Hospital $\sim$ Winchester $\sim$ United Kingdom

Summary (4 lines): Laparoscopy is the standard approach for benign non-prolapse hysterectomy in our department. The rate of complications is low and with increasing experience we provide training in advanced laparoscopic surgery.

Introduction: Laparoscopic hysterectomy (LH) was introduced in our department in 2009. The aim of this study was to compare the outcomes of the first 50 cases of LH with the outcomes of cases performed within the last 12 months.

Material and Methods: This is a prospective audit of women who have undergone LH at RHCH. Data were collected from medical notes and post-operative patient questionnaires.

Results: Ninety-four LH have been performed in our department. All first 50 cases were performed by consultants whereas in the second group, 24/44 hysterectomies were performed by trainees. There was no conversion to laparotomy, bowel, bladder or ureteric injury. One patient in the second group returned to theatre 22 days post-operatively.
The number of day cases was significantly higher in the 2nd group. In both groups the average interval to return to work was 6 weeks.

Discussion: There has been a significant increase in the number of $\mathrm{LH}$ in our department and this is now the standard route for benign hysterectomy. The rate of complications was low. As the technique has become embedded there has been an increased number of day cases and cases performed by trainees. We expect an improvement on the recovery time with implementation of the enhanced recovery programme

\section{7}

\section{LAPAROSCOPICAL RADICAL HYSTERECTOMY - IT IS COMPARABLE WITH LAPAROTOMICAL APPROACH? Hysterectomy}

\author{
G. Mecejus*, A. Milaknyte \\ ${ }^{[1]}$ Vilnius City Clinical Hospital, Vilnius University Vilnius $\sim$ Lithuania
}

Summary (4 lines): Laparoscopic radical hysterectomy is enough radical and no worse than laparotomic.

Introduction: Laparotomy is still gold standard in oncology of abdominal cavity. Aim of this study - to compare radicality and early complication rate of total laparoscopic versus laparotomic hysterectomy performed for endometrial cancer.

Material and Methods: Laparotomy is still gold standard in oncology of abdominal cavity. Aim of this study - to compare radicality and early complication rate of total laparoscopic versus laparotomic hysterectomy performed for endometrial cancer.

Results: 7 cases of laparoscopic and 10 cases of laparotomic total hysterectomies were analyzed. Mean blood loss was less in laparoscopic group than in laparotomic $(71,4 \pm 188,9$ and $71,4 \pm 188,9$ respectively, $p>0,05$ ). Mean number of removed lymph nodes is large in laparoscopic group (15,3 $\pm 9,1$ and $11,1 \pm 5,3$ respectively). More lymph nodes were removed in left than in right side $(8,7 \pm 5,5$ and $6,6 \pm 3,9$ respectively).

Discussion: Laparoscopic surgery in cases of endometrial cancer is enough safe and radical. Some authors demonstrated some doubts about number of lymph nodes removed laparoscopicaly, but our results show more advantages.

60

\section{MINILAPAROTOMY : A MINIMALLY INVASIVE ALTERNATIVE APPROACH FOR MANAGEMENT OF UTERINE AND ADENXAL LESIONS IN PATIENTS WITH HIGH BODY MASS INDEX, PROSPECTIVE RANDOMIZED STUDY}

Hysterectomy

M. Shams*
${ }^{[1]}$ mansoura university hospital $\sim$ mansoura $\sim$ Egypt

Summary (4 lines): Minilaparotomy was a feasible surgical approach in different gynecological diseases in patients with high body mass index.

Introduction: attempts to explore less invasive Tran abdominal incision, this study was to assess the feasibility and the clinical outcome of minilaprotomy in a large series of patients compare the results obtained using the laprotomy, laparoscopy and minilaparotomy approaches for for mangment of uterine and adenxal lesions in patients with high body mass index,

Material and Methods: this is prospective randomized study 81 patients randomized undergoing surgery by modified minilaprotomy 
for adenxal or uterine disease in patients with high body mass index. Surgical treatment included total abdominal hysterectomy. Mymectomy uni or bilaeral salpigoophrectomy and adenxal surgery, 46 cases with laprotomy, 38 laproscopy.

Results: ; the mean population age are 41,4 years(+14,3SD.), respectively the mean operative time was $70,2+24,64 \mathrm{~s} 1,22 \mathrm{sd}$, there is no significantly blood loss, with significantly lower duration o recovery in the group of simple adenxal surgery with respect to the others $(p=$ $0,0001)$ no severe early post operative morbidity was observed Discussion: Minilaparotomy was a feasible surgical approach in different gynecological diseases in patients with high body mass index

\section{3}

\section{NOCICEPTIVE AND STRESS HORMONAL STATE DURING ABDOMINAL, LAPAROSCOPIC AND VAGINAL HYSTERECTOMY AS PREDICTORS OF POSTOPERATIVE PAIN PERCEPTION \\ Hysterectomy}

T. Andries*, D. Albert, T.K. Moniek, J. Frank Willem

${ }^{[1]}$ Leiden University Medical Center $\sim$ Leiden $\sim$ Netherlands

Summary (4 lines): Intraoperative pain indicators and pain perception during the first postoperative hours are comparable between vaginal, abdominal and laparoscopic hysterectomy. The minimally invasive approach shows a faster decline in pain scores.

Introduction: Objective is to compare pain perception during and after surgery between abdominal hysterectomy (AH), laparoscopic hysterectomy (LH), and vaginal hysterectomy ( $\mathrm{VH})$.

Material and Methods: Intraoperative nociceptive state (by means of pulse transit time; PTT), heart rate (HR) and stress hormone levels (SH-level; adrenalin and noradrenalin) were correlated with postoperative pain scores and SH-levels and demand for postoperative analgesics such as morphine.

Results: Intraoperative PTT levels as well as perioperative and postoperative $\mathrm{SH}-$ levels did not differ significantly between $\mathrm{AH}, \mathrm{LH}$ and VH. One day postoperatively, LH patients reported significantly lower pain scores, compared to AH patients. High intraoperative SH-levels predicted a significant higher demand for morphine postoperatively, accompanied with higher pain scores. Low mean PTT and high HR levels did not predict a higher demand for postoperative analgesics.

Discussion: If $\mathrm{VH}$, the gold standard in hysterectomy, is not applicable, $\mathrm{LH}$ proves to be advantageous over $\mathrm{AH}$ with respect to a faster decline in pain scores.

\section{8}

OUTCOME OF DIFFERENT HYSTERECTOMY TECHNIQUES Hysterectomy

M. Shahin*, H. Alhadithi, O. Chappatte, E. Kovoor

${ }^{[1]}$ Maidstone Tunbridge Wells Hospital $\sim$ kent $\sim$ United Kingdom

Summary (4 lines): A retrospective review for the procedure details, outcome and complication rate for hysterectomies done by vaginal, laparoscopic and abdominal route.

Introduction: Hysterectomy can be done by different routes with variable operating time, recovery and complications.

Material and Methods: 114 cases were reviewed and detailed information about the demography, operating details, intra-operative and post-operative complications rates were analyzed. Operations were done by 10 different consultants in one unit during one calendar year.
Results: LH was done generally for higher BMI. Longer operating time was compensated for by quicker recovery. Laparoscopic Hysterectomy had the least intraoperative and postoperative complication rate.

Discussion: Laparoscopy is currently a safe and available route for performing hysterectomy for a growing indications

\section{0}

\section{PATIENT SATISFACTION AND REDUCTION OF PELVIC PAIN AFTER LAPAROSCOPIC SUPRACERVICAL HYSTERECTOMY.}

Hysterectomy

E. Berner*, M. Lieng, E. Qvigstad

${ }^{[1]}$ Oslo University Hospital $\sim$ Oslo $\sim$ Norway

Summary (4 lines): A prospective observational study of women scheduled for laparoscopic supracervical hysterectomy (LSH) in a Norwegian university teaching hospital.

Introduction: The objective was to explore outcome of LSH in terms of pelvic pain and patient satisfaction.

Material and Methods: 124 premenopausal women who were scheduled for LSH and who preoperatively reported to suffer from pelvic pain, were included in the study. The study participants underwent LSH, with 12 months follow-up. The main outcome measures were reduction of pelvic pain measured by a visual analogue scale (VAS) 0 10 and patient satisfaction (VAS 0-10) 12 months after the procedure. Results: Nine women were lost to follow-up. Consequently, 115 women $(92.7 \%)$ were followed up 12 months after LSH. The mean intensity of pelvic pain was significantly reduced from 5.6 (SD 2.4) preoperatively to 0.7 (SD 1.6) 12 months after the procedure on the VAS scale. A mean reduction of pelvic pain of 4.9 (SD 2.8), $\mathrm{p}<0.001$. The mean patient satisfaction score was 9.3 (SD 1.5) on a VAS $0-10$, where 10 indicated completely satisfied. There were no differences in outcome of pelvic pain or patient satisfaction 12 months after LSH when subgroups of women with different intensities of preoperative pelvic pain, or with different histopatological results were compared. Discussion: The patient satisfaction after LSH is very high. LSH reduce pelvic pain to a minimum.

495

RATES \& PREDICTORS OF PROGRESSION TO SURGERY FOR HMB: RESULTS FROM THE ECLIPSE TRIAL

Hysterectomy

L. Gennard*

${ }^{[1]}$ Birmingham University $\sim U K \sim$ United Kingdom

Summary (4 lines): Uncertainty surrounds the rate and predictive factors of progression to surgery after initial medical treatment for heavy menstrual bleeding (HMB). We show rates are low, independent of initial treatment \& associated with no requirement for contraception.

Introduction: The ECLIPSE Trial randomised 571 women with HMB to usual medical treatment (mainly mefenamic/tranexamic acid) or the levonorgestrel releasing intrauterine system (LNG-IUS). Women were followed for a median of $2.0 \mathrm{yrs} \&$ progression to hysterectomy or endometrial ablation recorded.

Material and Methods: Rates between groups were compared using a chi-squared test. Multivariable survival analysis examined age, symptom duration, incl of hormonal treatment, dysmenorrhoea, BMI \& contraceptive requirement as predictors. 
Results: 19/286 women in the medical treatment group (7 \%) \& 21/ 285 in the LNG-IUS group ( $7 \%$ ) had hysterectomies $(\mathrm{p}=0.7)$, whilst the corresponding numbers for EAs were $21(7 \%) \& 17(6 \%)(p=0.5)$. Median time to surgery was $1.5 \mathrm{yrs}$. The only conclusive predictor of surgery was no pre-trial need for contraception (HR 3.2, 95\% CI: 1.4 to $7.5 ; \mathrm{p}=0.02$ ).

Discussion: Rates did not differ between groups \& were unexpectedly low. This may reflect trial eligibility as those with fibroids and other pathologies were excluded. Further investigation of reasons for medical treatment failure is warranted. Given the long natural history of HMB, we intend to assess the same study outcomes at $5 \& 10$ yrs.

190

\section{RISK OF LATE COMPLICATIONS OF VAGINAL VAULT AFTER TOTAL LAPAROSCOPIC HYSTERECTOMY: A COMPARISON BETWEEN THE VAGINAL ROUTE AND LAPAROSCOPIC SUTURE. \\ Hysterectomy}

L. Ferrer Ventura*, M. Martínez-zamora, F. Barranco, R. Solernou, J. Balasch, F. Carmona

${ }^{[1]}$ Hospital Clinic i Provincial de Barcelona $\sim$ Barcelona $\sim$ Spain

Summary (4 lines): We compare the risk of late complications after vaginal cuff closure(VCC) in patients undergoing a total laparoscopic hysterectomy(TLH). We conclude vaginal or laparoscopic sutures are safe.

Introduction: TLH may increase the risk of late $(>15$ days after surgery) vaginal vault complications. The objective of this study was to compare the risk of late complications after vaginal route or laparoscopic suture for VCC in patients undergoing a TLH.

Material and Methods: We analyzed the surgical outcome and risk of late heavy bleeding of VCC in 190 consecutive patients who underwent TLH due to benign illnesses: the VCC was performed laparoscopically in 131 patients(Group L) and vaginally in 54(Group V). Results: Mean surgical time(Mean \pm SD minutes:L:141 \pm 48 ; V:152 \pm $70 ; \mathrm{p}=0.2$ )and weight of the uterus was similar in both groups $($ Mean \pm SD gms:L:253 $\pm 176 ; \mathrm{V}: 279 \pm 212 ; p=0.4)$. There were no conversions to laparotomy and no statistical differences in surgical complications $(p=0.5)$. There were no dehiscences of the vaginal vault. 8 patients of $\mathrm{L}$ and 3 patients of $\mathrm{V}$ required new hospital stay due to late heavy bleeding of vaginal vault $(\mathrm{p}=1)$. No patient required transfusion due to this bleeding. There were not statistical differences in the mean hospital stay(days:L:2.9 $\pm 1.2 ; \mathrm{V}: 3.1 \pm 2.3 ; \mathrm{p}=0.4$ ).

Discussion: We conclude that vaginal or laparoscopic sutures are safe and feasible for vaginal cuff closure in patients undergoing TLH.

\section{7}

\section{SATISFACTION RATE AND OUTCOMES OF SEXUAL FUNCTION AFTER INTRAFASCIAL LAPAROSCOPIC HYSTERECTOMY}

Hysterectomy

N. Waters*, F. Shakir, W. Mitchell, A. Kent

${ }^{[1]}$ Royal Surrey Hospital $\sim$ Guildford $\sim$ United Kingdom

Summary (4 lines): Women who underwent laparoscopic hysterectomy report very high satisfaction rate and improvement of their sexual function. Introduction: A questionnaire was sent to women following laparoscopic intrafascial (conserving vault support) hysterectomy, which assessed recovery, satisfaction and sexual function.
Material and Methods: 180 patient's (out of 250) completed their questionnaires ( $72 \%$ response rate) and these were analysed. The average age of women in our study was 46 years.

Results: $99 \%$ of women rated preoperative care and information given as excellent, very good or good. $93 \%$ were very satisfied or satisfied with their care and $2 \%$ were dissatisfied. $93 \%$ of women felt it was worthwhile and would recommend laparoscopic hysterectomy to a friend $1.6 \%$ did not feel the operation was worthwhile and $4 \%$ were uncertain. 99 $(55 \%)$ women reported sexual problems before surgery and in $74 \%$ their function improved. $8 \%$ reported worsening of sexual function although a majority of these had oophorectomy and associated their problems with loss of libido and one woman reported prolapse as a cause $26 \%$ of women reported no sexual problems before surgery but still had improvement in sexual function after. If they were sexually active the average time to resume intercourse was 7 weeks.

Discussion: Laparoscopic hysterectomy achieved high patient satisfaction rate with improved sexual function.

\section{0}

\section{TOTAL LAPAROSCOPIC HISTERECTOMY - OUR EXPERIENCE}

Hysterectomy

C. Castro*, I. Rato, S. Barata, C. Alho, F. Osório, C. Calhaz-jorge

${ }^{[1]}$ Hospital de Santa Maria $\sim$ Lisboa $\sim$ Portugal

Summary (4 lines): Review the outcomes of patients submitted to total laparoscopic hysterectomy(TLH).

Introduction: Laparoscopy has increased as a surgical approach to hysterectomy worldwide

Material and Methods: Retrospective study of TLH $(\mathrm{N}=152)$ performed in our Department between April 2009 and December 2011. Patients age, previous abdominal surgery, surgery indication, operative time, morcellation, complications, conversion, uterus weight and length of hospital stay were recorded.

Results: Patients mean age was 47.8 years. The most frequent hysterectomy indication was uterine myoma(s) and menorrhagia/ metrorrhagia $(n=94)$, followed by ovarian mass and uterine pathol$\operatorname{ogy}(n=24)$, adnexal mass $(n=9)$, uterine malignancy $(n=5)$, urogenital prolapse $(n=5)$, menorrhagia/metrorrhagia $(n=5)$, recurrent endometrial polyposis $(n=5)$, surgical castration $(n=2)$, complex endometrial hyperplasia $(n=2)$ and ovarian malignancy $(n=1) .51 .3 \%$ uni/bilateral salpingo-oophorectomy were performed. Mean operative time was 83 minutes. Vaginal morcellation was performed in $20.4 \%$ and vaginal/laparoscopic in $2 \%$. In 1 case conversion to open surgery was decided. There was 1 case of intra-operatory need for blood transfusion and 1 of vaginal vault dehiscence. Mean uterus weight was $232.7 \mathrm{~g}$; mean length of hospital stay was 2.3 days.

Discussion: Regarding operative time, complications and length of hospital stay our results are positive when compared to previously published studies. In our opinion, TLH is an alternative to total abdominal hysterectomy.

150

\section{TOTAL LAPAROSCOPIC HYSTERECTOMY IN ADENOMYOSIS PATIENTS: RISK OF COMPLICATIONS AND CONVERSION \\ Hysterectomy}

F.J. Barranco*, R. Solernou, M.Á. Martínez Zamora, J. Balasch, F. Carmona

${ }^{[1]}$ Hospital Clínic de Barcelona $\sim$ Barcelona $\sim$ Spain 
Summary (4 lines): The risk of conversion and complications in total laparoscopic hysterectomy is not higher in adenomyosis patients.

Introduction: Laparoscopic surgery produce less scars and faster recovery but in adenomyosis patients may be more difficult and increase the risk of complications and conversion. We analyzed these risks in total laparoscopic hysterectomy(TLH)in adenomyosis patients.

Material and Methods: We analyzed the risk of conversion and complications after TLH in 60 patients with adenomyosis (Group1)and 44 control patients with other benign illnesses (Group2).

Results: The mean surgical time was similar between both groups (Mean?SD [minutes]:Group1:178?185; Group2 131?52; p=0.08). There were only two conversions to laparotomy in Group1 due to severe adhesions $(p=0.5)$. There were not statistical differences in surgical complications between both groups( 6 patients in Group1 and 3 in the Group2, including two intestinal lesions in Group1 and two bladder lesions in Group2; $\mathrm{p}=0.5$ ). Similarly, there were not statistical differences between both groups regarding postsurgical complications (Group1 $n=8$ and Group2 $n=4 ; p=0.4$ ). Two patients received blood transfusion( 1 of each group). There were not statistical differences in the mean hospital stay(days)(Group1:3.3?2.4; Group2:2.8?0.8; $\mathrm{p}=0.06$ ).

Discussion: TLH is a feasible and safe technique in patients with adenomyosis. The risk of conversion and complications do not seem to be higher in adenomyosis patients.

\section{0}

\section{TOTAL LAPAROSCOPIC HYSTERECTOMY VERSUS TOTAL} ABDOMINAL HYSTERECTOMY

Hysterectomy

S. Nurullaeva*

${ }^{[1]}$ Queen Elizabeth the queen mother hospital $\sim$ margate $\sim$ United Kingdom

Summary (4 lines): This study shows that total laporoscopic hysterectomy might take longer time but takes less hospital stay, lesser complication and ultimately affects the cost of the procedure in favor of laporoscopic TLH compared to abdominal hysterectomies.

Introduction: To identify differences in the operative time and post operative hospital stay and in women undergoing hysterectomy for benign conditions performed either by total abdominal hysterectomy or by total laparoscopic hysterectomy.

Material and Methods: Retrospective observational study of 100 women undergoing total abdominal hysterectomy for benign gynaecological conditions and 100 undergoing total laparoscopic hysterectomy. The duration of the procedure and postoperative number of days in hospital before discharge and of both groups were compared.

Results: Although total laparoscopic hysterectomy took longer 80 - $89 \mathrm{~min}$ versus 56-62 $\mathrm{min}$, the women undergoing this procedure had a shorter time 1-2 days in recovery and discharge from hospital. The cost of the laparoscopic-assisted procedure was greater during the operation with longer operating time and cost of disposable interments.

However, the total cost of treatment was less in this group because of shortened post-operative stay.

Discussion: Discussion The study showed that total laparoscopic hysterectomy is a cost-effective procedure for women requiring a hysterectomy for benign gynaecological conditions compared to total abdominal hysterectomy.
319

TOTAL LAPAROSCOPIC HYSTEREKTOMY VS. VAGINALE SUPRACERVICAL LAPAROSCOPIC HYSTERECTOMY: COMPARISON OF POSTOPERATIVE QUALITY OF LIFE UND SEXUALITY

Hysterectomy

J. Radosa*, I. Kastl, M. Radosa, R. Mavrova, A. Rody, S. Baum, E. Solomayer

${ }^{[1]}$ University Hospital Homburg Homburg Germany

Summary (4 lines): There seems to be little diffences between total laparoscopic hysterectomy and suprazervical hysterectomy in regard to postoperative improvement of quality of life and sexuality

Introduction: In comparison to total laparoscopic hysterectomy (TLH) LASH is easier to perform and quicker. Furthermore the rate of postoperative descensus uteri, mictionary disorders seem to be less frequent and postoperative sexuality seems to be better. However there is no sufficient data about the postoperative quality of life and sexuality after total and supracervical hysterectomy.

Material and Methods: 170 patients undergoing TLH $(n=90)$ or LASH $(n=80)$ in our department between 2010 and 2011 where asked 6 month after surgery using a standarized questionnaire. Content of the questionnaire were pre - and postoperative quality of life (EQ-5D) and sexual satisfaction (Female Sexual Function Index (FSFI-d))

Results: Both groups showed a significant increase in life quality after and FSFI-d Index laparoscopic hysterectomy. There where no differences between TLH and LASH regarding these aspects.

Discussion: Patients suffering from symptomatic uterus myomatosus or endometriosis clearly profit from laparoscopic hysterectomy in regard to life quality and sexuality. The surgical technique which is used seems to play a minor role on the outcome of these two parameters

185

\section{WHEN SECOND GENERATION ENDOMETRIAL ABLATION FAILS!}

Hysterectomy

K. Niblock*, M. Abdelrahman, K. Johnston

${ }^{[1]}$ Antrim Area Hospital $\sim$ Antrim, N.Ireland $\sim$ United Kingdom

Summary (4 lines): A study to identify contributing factors in patients requiring hysterectomy after 2 nd generation endometrial ablation, aiming to improve outcome, reduce failure rates through better patient selection.

Introduction: Global endometrial ablation has dramatically reduced hysterectomy rate, however up to a quarter still have definitive surgery. Can gynaecologists better identify the cohort where hysterectomy should be first line?

Material and Methods: Retrospective review of 190 patients who underwent ablation (July 2007 to Aug 2011). Data including; number of hysterectomies, interval from ablation, Body Mass Index (BMI), prior caesarean section $(\mathrm{C} / \mathrm{S})$, uterine weight, operative findings and uterine histopathology.

Results: Post ablation (32/190) $17 \%$ of women required hysterectomy. Mean time to hysterectomy was 14 months range (5-50). Average BMI was 29 range (18-44). $25 \%(8 / 32)$ had previous C/S. Pathology was identified in $84 \%(27 / 32)$ of hysterectomies. Mean uterine weight was $141 \mathrm{~g}$ range $(95-450 \mathrm{~g})$. Adenomyosis was confirmed in (16/27) $59 \%$ of uteri, (4/27) $15 \%$ had fibroids and (7/27) $26 \%$ had both fibroids and adenomyosis. 
Discussion: Hysterectomy rate post endometrial ablation was $17 \%$. Pathology was confirmed in $84 \%$ of patients. Potentially pre-operative imaging such as MRI / ultrasound may better help identify patients particularly adenomysosis were ablation is more likely to fail and hysterectomy a better option.

362

\section{D AND3D ULTRASONOGRAPHICAL TOOLS VERSUS HYSTEROLAPAROSCOPY IN THE DIFFERENTIAL DIAGNOSIS OF SEPTATE, BICORNUATE AND ARCUATE UTERI \\ Imaging}

\author{
L. Artur*, L. Inga, P. Kazimierz, B. Tomasz, K. Anna \\ ${ }^{[1]}$ Chair of Gynaecology and Obstetrics, Departament of Gynaecology \\ and Oncology, Jagiellonian University $\sim$ Krakow $\sim$ Poland
}

Summary (4 lines): $100 \%$ diagnostic accuracy of 3D-SIS and only slightly lower of 3D-TVS and 2D-SIS questions the need of endoscopy in the differential diagnosis of the most common congenital uterine anomalies.

Introduction: Aim of study were to evaluate the diagnostic accuracy of 3D-SIS,3D-TVS,2D-SIS and 2D-TVS(initial/expert) compared to hysterolaparoscopy(HL) in the differential diagnosis of septate, bicornuate and arcuate uteri.

Material and Methods: Prospective study involved 117 women with RA or infertility and 2D-TVS initial diagnosis of septate, bicornuate or arcuate uterus. Subsequently ROC analysis was used to estimate test sensitivity, specificity, PPV and NPV, all of which were calculated separately for patients with arcuate, bicornuate and septate anomalies by comparing the US-tools results with the HL-generated diagnosis. The AUC was used to evaluate the statistical significance.

Results: 3D-SIS showed a perfect ACC(100.0\%) in general detection of uterine abnormalities compared to initial 2D-TVS(77.8\%), expert 2D-TVS(90.6\%), 2D-SIS(94.0\%) and 3D-TVS(97.4\%). In overall diagnosis of uterine anomalies all of the diagnostic methods had statistically significantly better diagnostic value from the initial $2 \mathrm{D}$ TVS( $p<0.001)$, whereas 3D-SIS, as the only method was better form the expert $2 \mathrm{D}-\mathrm{TVS}(\mathrm{p}<0.001)$

Discussion: The use of endoscopic methods can be limited to their therapeutic use during metroplasty of the uterine septum.

\section{6}

\section{A REVIEW OF PATIENTS DIAGNOSED WITH TUBO-OVARIAN ABSCESS (TOA) ON ULTRASOUND SCAN Imaging}

G. Araklitis, H. Jan*, M. Naidu, T. Bracewell-milnes, N. Narvekar

${ }^{[1]}$ king's College Hospital $\sim$ London $\sim$ United Kingdom

Summary (4 lines): Retrospective observational study reviewing patients diagnosed with tubo-ovarian abscess (TOA) on ultrasound scan

Introduction: Tubo-ovarian abscess (TOA) develops in $34 \%$ of patients requiring admission for PID (Rizk, 1995)

Material and Methods: A retrospective review of ultrasound diagnosis of TOA at a tertiary referral center.

Results: 50 patients with TOA were identified from a review of medical records from 2000 to 2011. Mean age at presentation was $34.8(\mathrm{SD}=8.7)$ years. $89 \%$ presented with abdominal pain, $49 \%$ with fever and $25 \%$ with vaginal discharge. On ultrasound scan, $31 \%$ had right TOA, $46 \%$ had left TOA and $23 \%$ had bilateral TOA. All patients had antimicrobial therapy with $79 \%$ prescribed intravenous antibiotics. 10 out of 50 patients $(20 \%)$ had surgical intervention including 3 laparotomy, 2 laparoscopy and 5 ultrasound guided vaginal drainage. Median length of stay was 6 (range1-19) days. 7 patients were readmitted. 2 had previous surgical intervention. All readmissions were managed conservatively with anti-microbial therapy and discharged home after a median of 4 (range 1-14) days of hospital stay. Discussion: A diagnosis of TOA should be considered in all women of reproductive age presenting with abdominal pain and fever. Medical management with IV antibiotics is successful in majority of patients and organ-sparing surgical intervention should be considered for all patients with intractable symptoms.

\section{CLINICAL AND ANATOMIC FEATURES OF FEMALE GENITAL TUBERCULOSIS, A MOROCCAN EXPERIENCE Imaging}

Y. Ait Benkaddour*, B. Fakhir, A. Aboulfalah, K. Fichtali, L. Boukhanni, K. Harrou, A. Bassir, H. Asmouki, A. Soummani ${ }^{[1]}$ Department of obstetrics and gynecology, University hospital of Marrakesh, Cadi Ayyad University Marrakesh Morocco

Summary (4 lines): Tuberculosis continues to be a global health problem in the developing countries. Female genital tuberculosis is rare Introduction: We report a study presenting clinical and anatomic features of female genital tuberculosis

Material and Methods: Retrospective review of 28 cases of genital tuberculosis treated in gynecology obstetrics department of the university hospital of Marrakesh.

Results: Mean age of our study group was 33 years. Pelvic pain was the major symptom followed by infertility. Preoperative evaluation was performed mainly by pelvic ultrasound and hysterosalpingogram. The most frequent finding were hydrosalpinx, tubal obstruction, pelvic mass and ascites. Nineteen patients underwent laparoscopy and 7 had laparotomy. Tow patients underwent hysteroscopy with endometrial biopsy, it shows endometrial hypertrophy. Tubal tuberculosis localization is the most frequent $(53.57 \%)$, it is associated with peritoneal infection in $32,14 \%$ of cases, endometrial localization was seen in $7,4 \%$ of cases and ovarian tuberculosis was found in $4,5 \%$ of cases. After medical treatment only one patient had a pregnancy and it was a tubal ectopic pregnancy. One patient had synechia surgical cure followed by tow IVF failures.

Discussion: Female genital tuberculosis presents a big variety of clinical and anatomic presentations. It is a diagnosis to keep in mind in front of infertility problems or adnexal mass or isolated ascitis especially in developing countries.

192

\section{REPRODUCIBILITY OF 3D CORONAL VIEW OF THE UTERUS TO EVALUATE POSITIONNING OF ESSURE® 3 MONTHS AFTER PROCEDURE}

Imaging

L. Irene, C. Perrine*, F. Florent, L. Jean-marc, L. Guillaume, P. Pierre, C. Guillemette, V. Vincent, F. Hervé

${ }^{[1]}$ Bicetre hospital $\sim$ Le Kremlin Bicetre $\sim$ France

Summary (4 lines): Evaluation of sonographic coronal 3D view for position of intratubal insert.

Introduction: Essure ${ }^{\circledR}$ procedure is becoming the procedure of choice for tubal occlusion. Initial recommended method to confirm position of 
the microinsert was hysterosalpingography but disponibility and acceptability of this procedure led to developement of alternative method to assess good positionning (pelvic X-ray, 2D or 3D sonography).

Sonography with $3 \mathrm{D}$ acquisition is one of these alternatives and the aim of this study is to evaluate if the coronal view can allow diagnosis of position of the insert.

Material and Methods: Fifty coronal view performed by a referent were used, each with 2 sides leading to evaluation of positioning of a hundred microinsert. Three hysteroscopic surgeons and two sonographists read them. Results of these reading were compared to result of the referent sonographist using k-value as for reproducibility.

Results: K-value was disparate from 0.26 to 0.82 . It appears that the only explaining factor for these differences was anterior knowledge of the classification used (Legendre et al 2011); persons used to the classification (independently from his practice (sonographist or surgeon)) had a higher reproducibility.

Discussion: Sonographic 3D coronal view of the uterus is a good way to determine the good position of intratubal microinsert as soon as readers are used to the classification.

\section{7}

\section{STUDY TO ASSESS CORRELATION BETWEEN DIAGNOSTIC IMAGING AND HISTOLOGY OF ADENOMYOSIS AT HYSTERECTOMY Imaging}

R. Banerjee*, E. Ofuasia

${ }^{[1]}$ Croydon University Hospital $\sim$ Surrey $\sim$ United Kingdom

Summary (4 lines): The aim of the study was to co-relate imaging with the actual diagnosis established at histology during hysterectomy Introduction: Women with adenomyosis may present with nonspecific symptoms Establishing an accurate diagnosis preoperatively is very important so that the women can be appropriately counselled.

Material and Methods: 100 case notes of women who had hysterectomy between January 2010 and December 2010 who had proven adenomyosis were reviewed. The diagnosis was compared to the results of imaging.

Results: $70 \%$ of the women were found to be multiparous. The women were mostly in the premenopausal age group between 40 50 years and had TAH with BSO as the definitive treatment. In 12 women with large fibroids pre-operative diagnosis was missed. In case of MR imaging 5 out of 79 women who had MRI, pre-operative diagnosis was missed; all of them had large fibroids. $65 \%$ of women had associated fibroids. Adenomyosis was an incidental finding at vaginal hysterectomy for prolapse in 10 women. In most women conservative methods of treatment led to partial relief of symptoms only.

Discussion: In our study the sensitivity of TV USS was found to be $86 \%$; and specificity of $95 \%$ and MRI being $93.6 \%$ and $96 \%$ respectively. The diagnostic accuracy of both the imaging modalities was comparable

309

ULTRASONOGRAPHIC EVALUATION OF MESH IMPLANTS AFTER VAGINAL WALL PROLAPSE REPAIR Imaging

G. Mecejus*, A. Marcinkute

${ }^{[1]}$ Vilnius City Clinical Hospital Vilnius $\sim$ Lithuania
Summary (4 lines): Failure of vaginal wall prolapse repair is under observation using ultrasound examination for women after surgery.

Introduction: We tried to investigate whether postoperative ultrasonography is useful for identification of polypropylene mesh, assessment of mesh retraction and defining factors determining recurrence and complications after prolapse repair using transvaginal mesh.

Material and Methods: Transvaginal two-dimensional ultrasound examination was performed to identify the polypropylene mesh and measure four distances in the midsagittal plane: length and thickness of the mesh, distance from the mesh to the vaginal mucosa (A), distance from the anterior mesh to the bladder neck (B).

Results: The mean follow-up time was 42 months. There were two cases $(8,6 \%)$ of cystocele recurrence and two cases $(8,6 \%)$ of mesh erosion. Patients with cystocele recurrence had increased distance B (9.7 vs $6.9 \mathrm{~mm})$. Mesh retraction was observed, equally in patients with and without recurrence. Patients with mesh erosion had decreased distance A (2.5 vs $3.9 \mathrm{~mm})$.

Discussion: According to several researchers, distance B and mesh retraction are associated with prolapse recurrence. We observe that recurrence is associated with the distance B though it is not associated with mesh retraction. Postoperative ultrasonography appears to be useful for polypropylene mesh evaluation. Erosion is associated with the depth of mesh implantation.

\section{4}

\section{ULTRASOUND DIAGNOSIS OF ADENOMYOSIS, LEIOMYOMA OR COMBINED WITH HISTOPATHOLOGICAL CORRELATION}

Imaging

\section{Hanafi* \\ ${ }^{[1]}$ Saint Joseph's Hospital of Atlanta, Emory Healthcare Atlanta, Georgia United States}

Summary (4 lines): Objective: To evaluate the accuracy, sensitivity and specificity of the diagnosis of adenomyosis, leiomyoma and combined adenomyosis with leiomyoma by the use of transvaginal ultrasound (TVS) in correlation to the histopathological findings.

Introduction: To evaluate the use of ultrasound method as a tool to diagnose adenomyosis, leiomyoma and combined

Material and Methods: Retrospective study of 163 patients with a preoperative transvaginal ultrasound diagnosis of adenomyosis, leiomyoma and combined All patients underwent surgery for the treatment of adenomyosis, leiomyoma, or both. A histopathological examination was performed by the hospital pathologists.

Results: The sensitivity, specificity of TVS in the diagnosis of adenomyosis was $84.55 \% 43.40 \% 71.17 \%$ respectively. TVS diagnosis of adenomyosis is sensitive but not specific. Histopathological confirmation of the TVS diagnosis of leiomyoma $(n=134)$ was positive in 133 patients $(99.25 \%)$ and negative in 1 patient $(0.75 \%)$. The sensitivity, specificity, and accuracy of TVS in the diagnosis of combined adenomyosis with leiomyoma was $96.38 \%$, $96.00 \%$ and $96.32 \%$ respectively. TVS was sensitive, specific, and accurate in the diagnosis of leiomyoma and combined adenomyosis with leiomyoma.

Discussion: Ultrasound is sensitive in the diagnosis of adenomyosis only but not specific. It is sensitive and specific in the diagnosis of leiomyoma and combined adenomyosis with leiomyoma. 
153

\section{AN AUDIT OF THE IUI SERVICE AT A LARGE DISTRICT GENERAL HOSPITAL}

Infertility and Reproductive Medicine

\author{
K. Hall* \\ ${ }^{[1]}$ Newcastle University $\sim$ Newcastle $\sim$ United Kingdom
}

Summary (4 lines): To undertake an audit of the intra-uterine insemination (IUI) service at a large district general Hospital against the national average.

Introduction: Infertility currently affects 1 in 6 couples in the UK and is defined as an inability to conceive after two years of regular unprotected intercourse. Intra-uterine insemination (IUI) is a method of fertility treatment for unexplained infertility.

Material and Methods: Retrospective analysis of 342 cycles of IUI undertaken at the Assisted Conception Unit (ACU) at a large district general hospital. Data was kept confidential and variables observed included information on the patient's age, cause of infertility, cycle number at the time of treatment, whether any medication was used and the outcome of the treatment.

All patients had patent fallopian tubes assessed either by a hysterosalpingogram or a laparoscopy and dye test. The audit did not include patients aged over 40 and couples who had used donor sperm.

Results: The overall pregnancy rate was $19.8 \%$ per cycle of IUI $(n=342)$ consistent with the national average of which $78 \%$ of pregnancies went on to deliver healthy babies. Approximately $90 \%$ of all pregnancies occurred in the first 3 cycles of treatment.

Discussion: Three cycle of IUI is sufficient to increase the chance of pregnancy for infertile couples and across all age groups the IUI service at Bishop Auckland is meeting the national average.

59

\section{CONNECTIVE TISSUE DYSPLASIA MANIFESTATIONS IN PATIENTS WITH PELVIC PERITONEAL ADHESIONS.} Infertility and Reproductive Medicine

E. Dubinskaya*, A. Gasparov, O. Barabanova

${ }^{[1]}$ Peoples' Friendship University of Russia $\sim$ Moscow $\sim$ Russian Federation

Summary (4 lines): The results of the study suggest that pelvic adhesions formation relates with connective tissue dysplasia.

Introduction: The aim of the study was to evaluate clinical markers of connective tissue dysplasia in patients with pelvic peritoneal adhesions.

Material and Methods: 200 patients with laparoscopically verified pelvic adhesions were included in the study. 35 patients without adhesions were recruited as the control group.

Results: Among patients with I-II stage of adhesions compared to patients with III-IV stages clinical manifestations of DCT included scoliosis (28,4 and 62,4\%), platypodia (50,7 and $88 \%$ ), disorders of growth and density of teeth $(32,8$ and $58,6 \%)$, thin and easily damaged skin (34,3 and 55,6\%), multiple pigmented spots (13,4 and 26,3\%), kelloid scars (23,9 and 48,1\%), myopia (38,8 and 64,6\%), hematoma formation after minimal damage $(37,3$ and $77,4 \%)$, varicose veins $(12$ and $36,8 \%$ ).

Discussion: Our data suggest that patients with severe and moderate stages of pelvic adhesions have more connective tissue disorders external manifestations compared to patients with minimal and mild stages and the control group. One patient with stage III-IV adhesions has 6 and more phenotype stigmas of connective tissue disorders (unclassified phenotype). The results proved the role of genetic factors in pelvic adhesion formation and connective tissue disorders as endogenous risk factor of pelvic adhesion formation.

468

\section{EFFECT OF OVARIAN CYST ON OVARIAN RESERVE QUANTIFIED USING ANTRAL FOLLICLE COUNTS MEASURED BY SONOAVC \\ Infertility and Reproductive Medicine}

S. Deb*, B. Campbell, N. Raine-fenning

${ }^{[1]}$ Nottingham University Hospitals $\sim$ Nottingham $\sim$ United Kingdom

Summary (4 lines): Benign ovarian cysts, overall do not affect the ovarian reserve as measured using small antral follicles. Endometrioma however appears to reduce the ovarian reserve of diseased ovary.

Introduction: Aim was to quantify the effect of ovarian cyst on ovarian reserve measured using small antral follicles.

Material and Methods: Women diagnosed with an ovarian cyst of benign nature on ultrasound scan were prospectively recruited.3D transvaginal ultrasound scan was performed and antral follicles of various sizes in each ovary measured using sonography-based automated volume calculation (sonoAVC). Paired samples $t$ test was used to analyse variance between two ovaries.

Results: 38 women were included in final analysis. 15 had endometrioma, 18 had dermoid cyst and 5 had simple functional cyst. A nonsignificant variation in small $(2-6 \mathrm{~mm})$, large $(>6-10 \mathrm{~mm})$ and total $\mathrm{AFC}$ was noted between the ovary with cyst and contra-lateral normal ovary $(\mathrm{P}>0.05)$. On analysis of cyst types however, a significantly lower number of small antral follicles was noted with endometrioma $(\mathrm{P}=0.042)$.

Discussion: The small antral follicles show minimal inter-ovarian variation and excellent correlation with $\mathrm{AMH}$ and were therefore used to measure and compare ovarian reserve between normal and diseased ovary. There was no significant difference in the small and total AFC between ovaries. Endometrioma however appears to reduce the ovarian reserve of diseased ovary.

470

\section{ENDOMETRIOMAS AND REDUCED OVARIAN RESERVE IN WOMEN WITH ENDOMETRIOSIS}

Infertility and Reproductive Medicine

V. Kovacevic*, S. Spremovic-radjenovic, L. Andjelic, G. Lazovic-radonjic ${ }^{[1]}$ General Hospital of Subotica $\sim$ Subotica $\sim$ Serbia

Summary (4 lines): Our results suggest that decrease in ovarian reserve should be taken into account in patients indicated for cystectomy, especially for bilateral endometriomas.

Introduction: To correlate the ovarian reserve changes six months after laparoscopic endometrioma stripping surgery with size and number of endometriomas (bilat or unil), using serum antimullerian hormone (AMH), Inhibin B concentration, and AFS score

Material and Methods: prospective study, one center, one surgeon method is used. 30 reproductive- aged women underwent 
laparoscopic stripping surgery for endometrioma. 6-months postoperatively second look laparoscopy was performed. Concentrations of $\mathrm{AMH}$, inhibin B and CA 125 were measured on the day 3 of the cycle, pre- and 6 months post-operatively. Results were analyzed by standard software SPSS.

Results: Laparoscopic findings: endometrioma(s) unil 20 (66, $66 \%$ ), bilat10 (33, $33 \%)$. ASRM stageIII: 7 women(23, $33 \%)$, IV: 23 women(76, $66 \%)$. Second look laparoscopy: in 2 women endometrioma $(3-4 \mathrm{~cm})$ were found in contra lateral unoperated ovary. In 1 woman endometrioma $(3 \mathrm{~cm})$ were found in the same ovary. Ovarian reserve evaluation: concentration of $\mathrm{AMH}<1 \mathrm{ng} / \mathrm{ml}$ had 8 (26, $7 \%)$ patients pre-operatively, and $16(53,3 \%)$ post-operatively (Mc Nemar test, $p=0,021)$. The Mean AMH was in unilateral endometriomas $2.8 \pm 1,8 \mathrm{ng} / \mathrm{ml}$ prior surgeries and $1.4 \pm 1.1$ post-surgery, and in bilateral endometriomas $1.7 \pm 1.1$ prior and $0.8 \pm 0.5 \mathrm{ng} / \mathrm{ml}$ post surgery.

Discussion: No

\section{8}

\section{EXOGENOUS RISK FACTORS OF PELVIC ADHESIONS FORMATION.}

Infertility and Reproductive Medicine

E. Dubinskaya*, A. Gasparov, N. Lapteva

${ }^{[1]}$ Peoples' Friendship University of Russia $\sim$ Moscow $\sim$ Russian Federation

Summary (4 lines): In this study exogenous risk factors of pelvic adhesions were analyzed.

Introduction: The objective of this study was to analyze the risk factors affecting adhesions formation and to see how they relate to their stage.

Material and Methods: 1000 hospital discharges in gynecological department from the most recent data (2008-2011) were analyzed. 752 women were discharged with a diagnosis that included the specific diagnosis of pelvic adhesions. Among them 372 $(49,5 \%)$ patients had previous surgery and 355 (47,2\%) patients had pelvic inflammatory diseases. We evaluated 79 factors connecting with previous surgery (and 57 factors connecting with inflammation.

Results: We determined significant risk factors of pelvic adhesions formation connecting with surgery: types of surgery (myomectomy $(f=0,478, p<0,01)$, adhesiolysis $(f=0,455, p<0,01)$, laparotomy $(\mathrm{f}=0,439, \mathrm{p}<0,01)$, repeated surgeries $(\mathrm{f}=0,355, \mathrm{p}<$ $0,01)$, peritoneal drainage $(\mathrm{f}=0,324, \mathrm{p}<0,01)$, emergency of previous surgery $(\mathrm{f}=0,219, \mathrm{p}<0,01)$ and inflammation: sexually transmitted infections ( $\mathrm{f}=0,854, \mathrm{p}<0,0001)$, IUD using ( $\mathrm{f}=0,337$, $\mathrm{p}<0,01), 2$ and more abortions $(\mathrm{f}=0,432, \mathrm{p}<0,01)$, pregnancy complications connecting with pelvic inflammatory diseases $(\mathrm{f}=$ $0,641, \mathrm{p}<0,01)$.

Discussion: These results could be useful for preoperative evaluation of adhesions stage as an addition to physical and ultrasound examination. Surgery that includes aggressive risk factors needs antiadhesions barrier usages.

\section{3}

\section{HOSPITAL-BASED PREVALENCE OF POLYCYSTIC OVARIAN SYNDROME AMONG OMANI WOMEN Infertility and Reproductive Medicine}

\footnotetext{
M. Al Khaduri*, Y. Al Farsi

${ }^{[1]}$ SULTAN QABOOS UNIVERSITY $\sim$ MUSCAT $\sim$ Oman
}

Summary (4 lines): A study of prevalence of polycystic ovary syndrome in Omani women showed that it was low compared to other countries.

Introduction: The aim is to estimate the hospital prevalence of PCOS among Omani women in reproductive age group (12-45) years who attended the Obstetrics and Gynecology facility at a tertiary hospital in Oman.

Material and Methods: A retrospective cross-sectional study that included all cases of PCOS from 2006 to 2010. The data was collected from patients' electronic health records. Diagnoses of PCOS were made as per Rotterdam criteria. Prevalence estimates were standardized to the mid-year population of Omani women in year 2009.

Results: A total of 255 cases were enumerated among 90,608 women, indicating an overall prevalence of 2.8 cases per 1000 women. The highest prevalence estimate (7.1 per 1000) was obtained among women aged 25-34 years. Regionally, the highest prevalence (4.4 per 1000) was found in Muscat. The majority of PCOS cases were from Al-Batinah $(63$ cases) followed by AlDakhelia (38 cases) regions.

Discussion: This study shows low prevalence estimates of diagnosed PCOS cases in Oman relative to other countries. This finding highlights the national need for more diagnostic facilities and for the establishment of record-based surveillance of ovarian dysfunction in primary and tertiary hospitals. It also highlights the need for future research to carry out population-based assessment of PCOS prevalence.

\section{INFLUENCE OF SURGICAL TREATMENT OF ENDOMETRIOSIS ON SUBSEQUENT PREMATURE OVARIAN FAILURE}

Infertility and Reproductive Medicine

A. Gudovic, S. Spremovic- Radjenovic*, G. Lazovic, R. Sparic

${ }^{[1]}$ Clinic for Gynecology and Obstetrics, Clinical Center of Serbia Belgrade Serbia

Summary (4 lines): Influence of POF is very serious, on the reproductive possibilities, on the quality of life, and on the general health. Very careful analysis of follicular reserve, indications for operation and surgical technique would be of benefit in jatrogenc POF prevention, esspecially in patients with endometriomas.

Introduction: Compare premature ovarian failure (POF) frequency according to the background pathology.

Material and Methods: Open, prospective, single center study. Patient: amenorrhoea before age of 40 years, with follicle stimulating hormone above $30 \mathrm{U} / 1$ on at least two occasions in the presence of low estrogens. Exclusion criteria: abnormal karyotyping results.

Results: Study included 66 women, 50 with primary and 16 with iatrogenic POF, 9 of which undergone laparoscopic surgery for endometriosis, 2 for cystadenoma, one for dermoid ovarian tumour, one had ovarian drillling, and 3 had chemotherapy for other than ovarian causes.

Discussion: Of 13 surgically treated patients ending with POF, 9 were endometriosis cases. Surgical excisions of remnant ovary are larger in endometriosis than in other diseases. Laparoscopic excision of endometriosis improves fertility outcome in grade I-II endometriosis. Presence of serious endometriosis in ovary affects ovarian reserve, as extended surgical procedure could endanger ovarian tissue. In recurrent endometriosis surgery should be 
avoided if possible. Extensive ovarian drilling could accelerate ovarian aging.

19

\section{LAPAROSCOPIC MANAGEMENT OF ACUTE PELVIC PAIN (APP)}

Infertility and Reproductive Medicine

\author{
V. Radojicic* \\ ${ }^{[1]}$ Health Center Valjevo $\sim$ Valjevo $\sim$ Serbia
}

Summary (4 lines): Usefulness of early laparoscopic approach in women with APP was reviewed. It was shown that urgent endoscopic procedure is appropriate treatment for acute lower abdominal pain of unknown etiology.

Introduction: Variety of conditions are responsible for occurens of acute pelvic pain (APP). Laparoscopy provides diagnosis in a first $24 \mathrm{~h}$ after admission and includes best results by giving more in a fertility outcome.

Material and Methods: In a two years 76 patients of reproductive age with APP underwent urgent laparoscopic intervention. After ultrasound examination, CBC including serum beta HCG and CRP dosage patients were operated.

Results: Ectopic pregnancy, PID, adnexal torquation and a rupture of ovarian cyst were most frequent cause of APP. Acute appendicitis, ovulation or endometriosis were a reason for APP too. Treatment was performed with preservation of fertility where possible. In a $64,4 \%$ patients, clinical diagnosis corresponded to a operative one. Definitive diagnosis was made in a $98,6 \%$. No complication was seen. No reintervention was done. In a one case reason for APP remained unknown.

Discussion: Aim of laproscopic approach in APP is to set quickly a right diagnosis. There is a good reason to use laparoscopy as first line procedure. Safe and accurate as it is, endoscopy excludes expectative approach, gives more for fertility preservation and reduce a risk of a serious complications.

103

\section{LAPAROSCOPIC-ASSISTED HYSTEROSCOPIC METROPLASTY FOR THE TREATMENT OF COMPLETE UTERINE SEPTUM WITH TWO CERVICES AND VAGINAL SEPTUM}

Infertility and Reproductive Medicine

L. Mencaglia, L. Mereu, H. Khalifa*, G. Carri, I. Arena, T. Zampetoglou

${ }^{[1]}$ Centro Oncologico Fiorentino $\sim$ Firenze $\sim$ Italy

Summary (4 lines): Laparoscopic hysteroscopic alternated transillumination metroplasty in case of mullerian malformations

Introduction: The prevalence of müllerian malformations is $1 / 200$. Correct diagnosis and treatment of the malformation is often difficult. Even if septate uterus is not proven to be a cause for infertility, performing hysteroscopic metroplasty can diminish the chance of miscarriage and improves pregnancy rates.

Material and Methods: A 31 year-old infertile woman affected by Mullerian malformation with 2 cervices and longitudinal vaginal septum was scheduled for diagnostic laparoscopy and hysteroscopic metroplasty. Diagnostic laparoscopy showed a normal shape uterine fundus and diagnostic hysteroscopy detected the presence of 2 uterine cavities, divided by a complete septum. A $22 \mathrm{Fr}$ bipolar resectoscope was inserted in the larger cavity and a diagnostic hysteroscopy in the 2nd cavity. Septum resection has been performed using bipolar resectoscope and guided by both laparoscopic and hysteroscopic alternated transillumination.

Results: Complete septum resection was achieved in $53 \mathrm{~min}$, no intra or postoperative complications.

Discussion: Laparoscopic hysteroscopic alternated transillumination metroplasty in case of major mullerian malformations minimize complications and improving outcome. Transillumination is fundamental to individuate the spatial position of the 2 cavities and to realize a successful surgery.

451

\section{LAPAROSCOPY IN INFERTILE PATIENTS AFTER FIRST AND SECOND MISCARRIAGE}

Infertility and Reproductive Medicine

M. Panevska-gareva*

${ }^{[1]}$ University Hospital "Maichin dom' Sofia Bulgaria

Summary (4 lines): Many medical and obstetric factors associated with an increased risk of miscarriage.

Introduction: Is it necessary and when do we have to perform laparoscopy in infertile couple with miscarriage.

Material and Methods: We analyzed retrospectively the data of 290 patients after operative laparoscopy from January 2010 to February 2012. Sixty three patients of them had miscarriages until 12 weeks of gestation. The patients were divided in 2 groups, First group of 48 women with one miscarriage and 15 women with two miscarriages. The period between miscarriage to laparoscory in first group was from 1 to 16 years and this period in second group was from 1 to 14 years

Results: In first group abnormal findings were as follow; $37.5 \%$ had endometriosis, $29.1 \%$ had myoma, $25 \%$ had hydrosalpinx., $16.6 \%$ had PCOS, $12.5 \%$ had adhesions, $10.4 \%$ had PID, $8.3 \%$ had cyst of ovary (one dermoid ). Only 3 patients were without abnormal findings. ln second group the abnormal findings were as follow: $33.3 \%$ had endometriosis, $13.3 \%$ had myoma, PCOS, adhesions and $6.6 \%$ had cyst of ovary. After diagnosis and treatment pregnancy rate was $38.09 \%$.

Discussion: Our retrospective report for the last two years demonstrate a high persentige of pathology after first miscarriage. The data confirm that laparoscopy have to perform not late than a year after miscarriage.

\section{4}

\section{LAPAROSCOPYC TREATMENT OF INCOMPETENT UTERINE SCAR AFTER CESAREAN SECTION. \\ Infertility and Reproductive Medicine}

A. Popov, A. Fedorov*, L. Logutova, T. Manannikova, N. Puchkova, M. Chechneva, R. Barto

${ }^{[1]}$ Moscow regional institute $O \mid G \sim$ Moscow $\sim$ Russian Federation

Summary (4 lines): The most often reason of uterine scar oncompetence after cesarean section is puerperal endometritis.

22 times we used laparotomyc access, 11 cases of laparoscopyc access.

Introduction: The WHO estimates the rate of Caesarean sections at between $10 \%$ and $15 \%$ of all births in economic developed countries. Among 2177 deliveries in 2010 after cesarean section was 203(9,3\%) patients. Scar incompetence was detected in 79 (38,9\%. Puerperial endometritis - as a main reason of scar incompetence. 
Material and Methods: in 2006-2011 89 patients with scar incompetence after cesarean section was treated in our institute. 45 patients were undergoing reconstructive surgery at first 40 days after childbirth. 11 times hysterectomy was performed. 33 women were treated before next pregnancy after 18-24 months. In all cases we did ultrasound investigation with measurement of blood flow and scar condition.

Results: at pregnancy planning stage (18-24 months) we performed lower segment reconstruction. 22 times we used laparotomyc access, 11 cases of laparoscopyc access.

After surgical treatment 13 patients were successfully delivered by cesarean section.

Discussion: The most often reason of uterine scar oncompetence after cesarean section is puerperal endometritis. Ultrasound investigation of scar condition with hysteroscopy allowed to identify patients who can be treated with uterus preservation in puerperal and delayed period by laparotomyc and laparoscopyc aproaches.

453

\section{OFFICE HYSTEROSCOPY WITH MULTIPLE ENDOMETRIAL BIOPSIES AND ENDOMETRIAL BRUSHING IMPROVES EMBRIO IMPLANTATION IN SPONTANEOUS CONCEPTION OR BY ASSISTED REPRODUCTIVE TECHNIQUES IN INFERTILE PATIENTS OR FAILED BEFORE}

Infertility and Reproductive Medicine

E. Castellacci*, S. Calzolari, F. Cammilli, C. Becattini, E. Valagusta

${ }^{[1]}$ Palagi Hospital- Florence- Italy $\sim$ Florence $\sim$ Italy

Summary (4 lines): We report a new technical procedure in office hysteroscopy to improve the implantation rate in infertile women or failed ART techniques before.

Introduction: Local injury of the endometrium induces an inflammatory resonse that may facilitate the preparation of the endometrium for implantation

Material and Methods: a prospective study started in January 2012. 40 infertile women treated with office hysteroscopy and endometrial biopsies and brushing in proliferative or secretive phase of mestrual cycle.

Results: $25 \%$ of women treated with office hysteroscopy and endometrial biopsies and brushing underwent to ART techniques or spontaneus cycle, went on to develop pregnancy.

Discussion: office hysteroscopy and endometrial biopsies and brushing may facilitate the preparation of the endometrium for implantation.

479

PERCENTAGE EXPRESSION OF NATURAL KILLER CELL SUBTYPES IN PATIENTS WITH FAILED AND SUCCESSFUL IMPLANTATION FOLLOWING INTRACYTOPLASMIC SPERM INJECTION (ICSI)

Infertility and Reproductive Medicine

M. Shahin*, M. Omran, A. Hashaad, F. Salama

${ }^{[1]}$ Ain Shams University $\sim$ Cairo $\sim$ Egypt

Summary (4 lines): Quantification of the percentage of expression of peripheral blood natural killer cell subtypes.

Introduction: Several studies have demonstrated that natural killer cells play an important role in the success of pregnancy.

Material and Methods: A prospective observational study conducted in Ain Shams University Maternity hospital. 46 patients were included and subdivided into 2 Groups: 26 Patients with primary infertility with at least one previous failed ICSI and Control group including 20 healthy fertile women.

Results: Women with previous implantation failure who failed to achieve pregnancy after repeat IVF treatment have significantly higher levels of activated CD69+ NK cells, and have significantly higher levels of activated CD69+/CD56dim natural killer cells.

There was a significant higher percentage of CD69+ and CD69+/ CD56dim natural killer cells among infertile population with previous unexplained implantation failure Also, there was a significantly higher percentage of the total natural killer expressing CD56+ve surface marker in women with previous implantation failure who had a successful ICSI cycle.

Discussion: Quantification of the percentage of expression of peripheral blood natural killer cell subtypes has a promising role to offer to be integrated with various measures to predict the outcome of ICSI cycle especially those with previous implantation failures.

314

REPRODUCTIVE OUTCOMES FOLLOWING SURGICAL MANAGEMENT OF ENDOMETRIOSIS - AN AUDIT

Infertility and Reproductive Medicine

J. Mckenna*, D. Hunter

${ }^{[1]}$ Royal Jubilee Maternity Hospital $\sim$ Belfast $\sim$ United Kingdom

Summary (4 lines): Current NICE guidelines support surgical treatment of all grades of endometriosis as this improves the chance of pregnancy. This audit looked at the reproductive outcomes following surgery to treat disease of all stages. Results were in keeping with current literature values.

Introduction: This audit looked at the reproductive outcomes of women following surgery to excise their disease. The audit aimed to support the development of a local database to facilitate clinical information gathering.

Material and Methods: A manual search of theatre ledgers was performed from Jan 2011 to Dec 2011. Fifty prospective cases were selected and charts reviewed. Information was recorded on a proforma and subsequently transcribed onto a spreadsheet table.

Results: In approximately half of cases infertility was the indication for surgery. The stage of disease ranged from minimal to severe in both groups.

At follow-up the majority of patients were on a waiting list for IVF in the six months to one year follow-up period from their surgery. A minority of patients had already undergone fertility treatment with equal measure of successful and failed outcomes.

Discussion: Evidence exists to support the surgical ablation and excision of disease. Reproductive outcomes are improved both with surgery alone and surgery and assisted reproductive techniques in combination. The small numbers reviewed support current evidence.

415

\section{SAFETY OF ESSURE STERILIZATION IN FIRST CASE SERIES}

Infertility and Reproductive Medicine

B. Verdnik Golob*, H. Šavc

${ }^{[1]}$ General Hospital Slovenj Gradec Slovenj Gradec Slovenia 
Summary (4 lines): Analysis of the first case series of 94 women with Essure sterilization done in General Hospital Slovenj Gradec in Slovenia was performed. The results confirm that Essure sterilization is safe, very well accepted and tolerated method of permanent sterilization.

Introduction: Since November 2010 to March $201243 \%$ of all tubal sterilizations done in General Hospital Slovenj Gradec were Essure sterilizations. The objective of the study was to asses the safety, placement efficacy, women's tolerance and satisfaction.

Material and Methods: 112 women scheduled for Essure sterilization have been treated in line with own clinical pathway, including assesment of pain and satisfaction. The vaginoscopy approach under premedication with oral analgetics was performed.

Results: Microinsert placement was attempted in 94 women. Bilateral placement success was achieved in 83 of 94 women $(88.3 \%)$ and unilateral in 4 women $(4.25 \%)$. In 7 women $(7,45 \%)$ the placement was not achieved. Transvaginal ultrasound at 3 months confirmed both implants in good cornual position in 40 women (48.2\%), in 47 patients $\mathrm{X}$-RAY and/or HSG were done. One case of perforation happened and has been discussed in detail.

Discussion: Essure sterilization is safe and very well accepted and tolerated method of permanent sterilization. Education, practical skills and strict following the Essure confirmation test protocol are the most important factors to prevent and diagnose the perforation in time.

363

\section{THE DIAGNOSTIC AND THERAPEUTIC USE OF HYSTEROSCOPY IN UTERINE INFERTILITY OUTSIDE} IVF. A CLINICAL STUDY

Infertility and Reproductive Medicine

R. Socolov*, S. Butureanu, O. Neumann, D. Socolov

${ }^{[1]}$ Department Obstetrics Gynecology, University of Medicine and Pharmacy Gr T Popa Iasi Romania

Summary (4 lines): The uterine infertility is increasing among the causes of female infertility- in our statistics $38 \%$. Etiologies are: synechiae $44 \%$, malformations $19 \%$, fibroids $29 \%$ and polyps $8 \%$. At one-year follow up, pregnancy rate was $22 \%$, with $42 \%$ from those with therapeutic gestures.

Introduction: The uterine infertility is becoming more and more prevalent in the causes of female infertility. We analyze hysteroscopy use in the cases of these diseases.

Material and Methods: There were 141 female infertility patients admitted in our service of Iasi- Romania, during 2 years. We separated uterine and non-uterine infertility cases.

Results: We found 54 cases of uterine-only infertility, with $59 \%$ secondary infertility, compared to $31 \%$ in non-uterine infertility group; $35 \%$ had previous termination of pregnancy. The uterine pathology described included: intrauterine adhesions (44\%), uterine malformation (19\%), uterine fibroids $(29 \%)$ and polyps $(8 \%)$. Therapeutic gestures were performed in $52 \%$ of cases. Pregnancy rate at 1 year follow-up was with 12 pregnancies in the uterine infertility group (5 after synechiae, 4 after malformations, 3 after fibroid/ polyps) representing $22 \%$, and $42 \%$ from those with therapeutic gestures.

Discussion: As uterine infertility becomes more prevalent in female infertility, hysteroscopy is a first line investigation, not only in IVF cases, but also as basic evaluation.
467

\section{THE INTER-CYCLE VARIATION IN THE NUMBER \\ OF ANTRAL FOLLICLES STRATIFIED BY SIZE \\ USING THREE-DIMENSIONAL ULTRASOUND \\ AND IN THE ENDOCRINE MARKERS OF OVARIAN RESERVE IN WOMEN WITH NORMAL OVULATORY MENSTRUAL CYCLES}

Infertility and Reproductive Medicine

S. Deb*, B. Campbell, J. Clewes, C. Pincott-allen, N. Raine-fenning

${ }^{[1]}$ Nottingham University Hospitals $\sim$ Nottingham $\sim$ United Kingdom

Summary (4 lines): Small AFC, AMH and total AFC show inter-cycle stability and an excellent correlation over a period of 12 months.

Introduction: The objective was to quantify the inter cycle variation in antral follicle count(AFC) and anti-m?llerian hormone(AMH)over 12 months in normo-ovulatory healthy volunteers to examine their predictive ability over time.

Material and Methods: 3D transvaginal ultrasound scans were performed and bloods taken in the early follicular phase of menstrual cycle(days 2-5) at $0,1,3,6$, and 12 months. Repeated measures analysis was used to assess inter-cycle variation.

Results: The variation in mean total $\mathrm{AFC}(2-10 \mathrm{~mm})$ over 12 months was not significant $(\mathrm{P}=0.147)$. Small $\mathrm{AFC}(2-6 \mathrm{~mm})$ and large $\mathrm{AFC}$ $(>6-10 \mathrm{~mm})$ both showed a non-significant variation over 12 months $(\mathrm{P}=0.326$ and $\mathrm{P}=0.484$ respectively). Total $\mathrm{AFC}$ and small $\mathrm{AFC}$ showed excellent correlation across 5 cycles in 12 months $(0.876, \mathrm{P}<$ 0.001 and $0.916, \mathrm{P}<0.001$ respectively). Antral follicles measuring $>6$ $10 \mathrm{~mm}$ showed a significant but poorer correlation than small AFC $(0.379, \mathrm{P}=0.027)$.

Discussion: Ovarian reserve measured using small AFC and $\mathrm{AMH}$ show least inter-cycle variation and an excellent within subject correlation. A comparable inter-cycle stability of total AFC is perhaps due to small AFC forming the major proportion of the count.

\section{5}

\section{THE RECURRENCE RATE OF HYDROSALPINGES AFTER NEOSALPINGOSTOMY.}

Infertility and Reproductive Medicine

J.J. Dekker*, I. Melgers

${ }^{[1]}$ Free University Medical Center $\sim$ Amsterdam $\sim$ Netherlands

Summary (4 lines): A treatment for hydrosalpinx is a neosalpingostomy. For good counselling of patients knowledge of the recurrence rate of a hydrosalpinx is important. We found a recurrence rate of $13 \%$

Introduction: In case of ultrasonographically visualised hydrosalpinx, salpingectomy performed prior to IVF improves pregnancy rates significantly. An alternative treatment is neosalpingostomy. Spontaneous pregnancies can still occur. A drawback is the risk of recurrence. No sufficient data are available in literature. We evaluated the recurrence rate of hydrosalpinx after neostomy in our clinic.

Material and Methods: In a retrospective cohort study all patients who underwent uni- or bilateral neosalpingostomy between 2004 and 2011 were included. Neostomy was performed laparoscopically by means of CO2-laser. Recurrence of hydrosalpinges was based on midcycle ultrasound. 
Results: Of 32 patients identified, 30 patients and 37 tubes could be evaluated. Management was expectant/IUI in 20 patients and primarily IVF in 10 . in the first group pregnancy occurred in $8(40 \%)$ patients. Of these, $4(20 \%)$ were ongoing pregnancies, $2(10 \%)$ clinical miscarriages, $1(5 \%)$ ectopic pregnancy and 1 biochemical pregnancy. In all patients recurrence was suspected for 5 tubes in 4 subjects. After re-operation, 4 hydrosalpinges were confirmed, resulting in a recurrence rate of $13 \%$.

Discussion: Our findings of a recurrence rate of $13 \%$ can be used in the counselling of patients for either salpingectomy or neosalpingostomy.

\section{8}

\section{TRANSVAGINAL HYDROLAPAROSCOPY - ASSESSMENT OF ABILITY OF SONOSALPINGOGRAPY IN EVALUATION OF FERTILITY OF TUBO-OVALIAL COMPLEX}

Infertility and Reproductive Medicine

I. Mgaloblishvili*, M. Mgaloblishvili, K. Tabutsadze

${ }^{[1]}$ Center for Reproductive Medicine and Infertility $\sim$ Tbilisi $\sim$ Georgia

Summary (4 lines): One-stop evaluation of tubo-ovarial complex by sonosalpingography(SSG) and transvaginal hydrolaparoscopy(THL) allowed defining advantages and disadvantages of SSG in an assessment of tubo-ovarial system of subfertile women.

Introduction: THL-minimally invasive endoscopic method allows direct visualization of tubo-ovarial complex, practically in a physiologic manner. At the same time, SSG represents non-invasive, routine, method of evaluation tubal patency and tubo-ovarial complex, abilities and disadvantages of which aren't still revealed.

Material and Methods: During 2011, we performed fertiloscopic assessment of 276 subfertile women aged 17-40 years using fertiloscope (Karl Storz, Tuttlingen, Germany).

Results: We assessed 276 women with patent or restored after recanalization uterine tubes(537) by SSG and THL. In 81,2\%(436/ 537), SSG-ic assessment of a tubo-ovarial complex coincided with real condition of reproductive system detected during THL. In 7,8\%(42), SSG of distal segments was difficult to perform and in $10,6 \%(57)-$ showed false results.

Discussion: Main false results in SSG-ic examination of uterine tubes $89.5 \%(51 / 57)$ is due to subtle string-like adhesions in infundibular segments that was not detectable sonographically. When adequately filling cul-de-sac with Saline and with corresponding skills, mostly it is possible to evaluate adequately distal tubal segments and its orientation to the ovary.

\section{8}

\section{USE OF ESSURE@ MICRO-INSERTS FOR TREATMENT OF HYDROSALPINX PRIOR TO IN VITRO FERTILIZATION: A MULTICENTER ITALIAN EXPERIENCE}

Infertility and Reproductive Medicine

M. Franchini*, P. Litta, M. Luerti, G.L. Marchino, P. Florio, A. Di Spiezio Sardo, S. Calzolari

${ }^{[1]}$ Freestanding Palagi Hospital $\sim$ Florence $\sim$ Italy

Summary (4 lines): Placement of the Essure micro-inserts in patients with hydrosalpinges provides a minimally invasive and successful option for proximal tubal occlusion prior to IVF
Introduction: To evaluate the proximal tubal occlusion rate with Essure devices in women with hydrosalpinx prior to undergoing in vitro fertilization (IVF), and to observe pregnancy and delivery rates post-IVF. Prospective multicenter clinical study.

Material and Methods: In a prospective multicenter clinical study, 25 women with uni- or bilateral hydrosalpinges prior to IVF underwen an off-label hysteroscopic placement of Essure devices in an office setting Results: 23 patients had successful placement of the Essure devices without any complications. Two unsuccessful placement occurred. Proximal tubal occlusion was confirmed by hysterosalpingography or hysterosalpingocontrast sonography in all 23 patients. 4 live births and no miscarriages were observed in 8 women after three IVF cycles. In 4 patients the first two cycles were unsuccessful. 11 women have not yet begun IVF.

Discussion: Placement of the Essure micro-inserts in patients with hydrosalpinges provides a minimally invasive and successful option for proximal tubal occlusion prior to IVF

\section{0}

\section{USE OF LAPAROSCOPY IN INFERTILITY: 7-YEAR EXPERIENCE OF A GYNECOLOGY DEPARTMENT Infertility and Reproductive Medicine}

J. Santos, R. Pinto*, C. Andrade, N. Nogueira Martins, A. Pipa ${ }^{[1]}$ Hospital São Teotónio $\sim$ Viseu $\sim$ Portugal

Summary (4 lines): Laparoscopy is a useful tool in diagnostic and therapeutic approach of selected infertile patients. We present our experience in evaluation and management of these patients.

Introduction: Transabdominal laparoscopy is useful in the approach of infertile patients, particularly if endometriosis or pelvic adhesions/tubal disease are suspected, as it provides a panoramic view of the pelvic cavity and good access if surgical treatment is needed in the same procedure.

Material and Methods: From January 2005 to December 2011, 177 women from Infertility outpatient clinic were submitted to diagnostic laparoscopy.

Results: Diagnostic laparoscopy revealed pelvic pathology in 123 patients. Endometriosis and pelvic adhesions were detected and treated in 27 and 24 patients, respectively. Other procedures were also performed: 7 salpingostomies, 47 ovarian drillings and 17 ovarian cystectomies. Bilateral tubal patency was verified in 136 patients and 10 bilateral obstructions were detected. Pregnancy was the outcome for 34 women and other 35 were referred to assisted reproductive techniques. Two minor complications occurred and had successful outcome after conservative management: one uterine perforation and one moderate bleeding in the trocar insertion point.

Discussion: Although hysteroscopy and hysterosalpingography can assist in the evaluation of infertile patients, laparoscopy remains the preferred approach if pelvic pathology requiring surgical treatment is suspected.

236

\section{A REVIEW OF A SINGLE CENTRE'S EXPERIENCE OF LAPAROSCOPIC ADNEXAL SURGERY}

Innovation in Surgery

R.W.K. Kung*, K. Johnston

${ }^{[1]}$ Antrim Area Hospital $\sim$ Antrim $\sim$ United Kingdom

Summary (4 lines): Review of a single centre's laparoscopic adnexal surgical experience. 
Introduction: Laparoscopic approach to adnexae in benign gynaecology should be the "gold standard". Unexpected borderline/malignant pathology is low. It is feasible in women with previous laparotomies and increased BMI.

Material and Methods: Retrospective chart review of laparoscopic adnexal operations from $03 / 10 / 2007$ to $20 / 02 / 2012$. To identify number of laparoscopic procedures versus equivalent open operations, surgery performed, pathology, indications, demographics, bed stay and complications.

Results: 77 laparoscopic adnexal surgeries were identified vs. 206 adnexal surgeries by laparotomy over same period. Mean age was 46 yrs (range 17-79), average BMI 26 (range 18-43). Operations performed; Bilateral Salpingoophrectomy 51/77 (66.2\%), ovarian cystectomy 25/77 (32.5\%) and 1/77 patient (1.3\%) underwent unilateral salpingoophrectomy. Pain was commonest indication 51/77 (66.2\%). Prophylactic surgery 16/77 (20.8\%). Previous laparotomies existed in 17/77 $(22.1 \%)$ of patients. Commonest pathologies were serous, mucinous cystadenoma and endometriomas. Unexpected borderline 2/77 (2.6\%) and frank malignancy diagnosed in 2/77 (2.6\%). Discharge before 24 hours achieved in 54/77 (70.1\%). No major complications occurred. Discussion: Only about 1/3 adnexal surgery in our unit was performed laparoscopically, there is a now a drive to improve upon this.

\section{5}

\section{DIAGRAM TO MAP THE LOCATIONS OF ENDOMETRIOSIS Innovation in Surgery}

R. Lasmar, B. Lasmar*, C. Pillar

${ }^{[1]}$ Fluminense Federal University Niterói $\sim$ Brazil

Summary (4 lines): The objetive is to develop and test a visual map that corresponds practically and objectively to the anatomical areas affected by endometriosis.

Introduction: One of the major challenges of making a diagnosis in women with suspected endometriosis is to assess the extent of the disease and its functional consequences for the pelvic or extra-pelvic organs. Moreover, it is difficult to create a common language among specialists that allows standardized diagnosis and reatment.

Material and Methods: The present study is a descriptive study of a case series. A total of 150 questionnaires concerning 10 clinical cases of endometriosis presented as visual diagrams were distributed during 3 scientific gynecology meetings. At each meeting, the questionnaires were handed out to 50 different gynecologists.

Results: The analysis of the responses showed that the endometriosis mapping diagram is easily understood and can describe the location of the affected sites in an objective and clear way. It may prove to be a useful tool for gynecologists because it reproduces the clinical data and images, is helpful in management and follow up of endometriosis patients, and can act as a map for surgical purposes.

Discussion: A graphic mapping system of areas affected by endometriosis can be used as the basis for therapeutic decision making and follow up of patients. The present study proposed a mapping diagram for everyday clinical practice, both at the initial approach before surgery and at follow up.

15

\section{EXPERIENCE WITH THE VIZABLATE® SYSTEM} AT AN ACADEMIC MEDICAL CENTER IN KÖLN Innovation in Surgery

R. Bends*, T. Römer, U. Müller-lung, D. Toub

${ }^{[1]}$ Evangelisches Krankenhaus Köln-Weyertal $\sim$ Köln $\sim$ Germany
Summary (4 lines): We report our safety and efficacy experience with the VizAblate ${ }^{\circledR}$ System.

Introduction: The VizAblate ${ }^{\circledR}$ System is a new, CE-marked transcervical device for radiofrequency ablation (RFA) of fibroids. VizAblate images fibroids via a built-in intrauterine ultrasound probe and ablates them with a needle electrode array.

Material and Methods: Nonrandomized, single-center case series. Postablation MRI was performed on most patients. Women who desired fertility were excluded except when potential risks were outweighed by those of standard therapy.

Results: Patient recruitment is ongoing, with 11 patients having been treated as of 19/4/12. Two women desired future fertility. In 4 $(36 \%)$ of the patients, concomitant procedures were performed for fibroid management. A total of 11 myomata were treated with VizAblate. Median fibroid size was $3.1 \mathrm{~cm} \pm 2.2 \mathrm{~cm}$ (range $1 \mathrm{~cm}-$ $8 \mathrm{~cm}$ ). There have been no complications and no postembolization syndrome. Contrast-enhanced MRI imaging was obtained in 7 patients. MRI performed soon after treatment revealed ablation of all or most of the fibroid volume. MRI imaging performed several weeks after ablation demonstrated reduction in fibroid volumes. Follow-up to date of treated patients indicated good symptomatic relief.

Discussion: Early commercial use of the VizAblate System demonstrates that this new, CE-Marked device produces targeted, safe ablations within fibroids, resulting in symptomatic relief.

\section{6}

\section{FORCE-TORQUE MEASUREMENTS DURING LAPAROSCOPIC PRIMARY PORT INSERTION FOR ROBOTIC PERITONEAL ENTRY TERNAMIAN A, MD FRCSC, BAKIRTZIAN A, MHSC, BENHABIB B, PHD, TREMBLEY L, PHD.}

Innovation in Surgery

A. Ternamian*, A. Bakirtzian, L. Tremblay, B. Benhabib

${ }^{[1]}$ Department of Mechanical and Industrial Engineering University of Toronto $\sim$ Toronto $\sim$ Canada

Summary (4 lines): Summary: Force-Torque magnitude required to cannulate a layered foam is dictated by the friction between the foam and the external surface of the Threaded Visual Cannula; EndoTIP. Additionally, the magnitude of friction is determined by the material properties of individual layers.

Introduction: Introduction: Despite advances in endoscopy, primary peritoneal entry remains one of the most critical aspects of laparoscopy.

Material and Methods: Objective: To establish a reproducible platform for entry Force-Torque measurement, when applying the TVCEndoTIP cannula.

Materials \& Methods: Force-Torque profile is generated by measuring torque required to cannulate the TVC through a layered foam, mimicking anterior abdominal tissues. Penetration was synchronously captured by digital imaging.

Results: Results: A reproducible method of measuring entry ForceTorque, during TVC application is established.

Discussion: Discussion: Irrespective of peritoneal entry method (conventional, single port or robotic), primary port placement remains mostly blind, requires a central sharp-pointed trocar, recruits inordinate linear uncontrolled Penetration Force, is susceptible to push-through trocar overshoot, and remains inadaptable of incremental, remote or automated access. Robotically assisted surgery offers important innovations to include remote and automated peritoneal entry. 
299

\section{HOW TO SET UP AN AMBULATORY GYNAECOLOGY UNIT IN A DISTRICT GENERAL HOSPITAL}

Innovation in Surgery

\author{
E. Manzo*, T. Majmudar, H. Abdel-rahman \\ ${ }^{[1]}$ Hinchingbrooke Health Care Trust $\sim$ Huntingdon $\sim$ United Kingdom
}

Summary (4 lines): To improve patient experience and make costefficiency savings to the health service we set up an ambulatory gynaecology unit.

Introduction: A clinician led scoping exercise involving stakeholders concluded that hysteroscopic sterilisation, endometrial ablation, termination of pregnancy and cystoscopy were suitable for the ambulatory setting.

Material and Methods: Clinical and information governance for each step of the patient pathway were explored involving each staffing group. A business case was not required as the activity would be added to the plan for 2012-13. Allocation of facilities was negotiated with unit managers and team leaders. Time-tables of medical, nursing and support staff were revisited to minimize costs. A care pathway booklet, a drug chart, and a sedation protocol have been implemented upon approval by the drugs and therapeutic committee. Doctors and nurses training in Advanced Life Support was updated

Results: The unit will be launched at the beginning of August

Discussion: Procedures carried out in the ambulatory setting allow for improved patients experience, reduced morbidity and quicker return to daily activities. Cost efficiency savings, by reducing the use of operating theatre, general anaesthetics and the newly introduced best practice tariff, further favor the use of the ambulatory setting. Further expansion of the service will include termination of pregnancy using manual vacuum aspiration.

\section{7}

\section{INNOVATIONS IN CONSERVATIVE SURGICAL MANAGEMENT OF HMB \\ Innovation in Surgery}

T. Adedipe*

${ }^{[1]}$ MYH NHS Trust $\sim$ Wakefield $\sim$ United Kingdom

Summary (4 lines): Conservative options allow for reduced morbidity to the patient, resulting in improved quality of life as a result of decreased disruption of work, home-life demands particularly for the women who simply want to avoid major surgery. Potential drawbacks include lack of long-term data. Fertility can not always be guaranteed whilst recurrence of the fibroids may occur. The most appropriate, available and safe intervention following due counselling should be offered.

Introduction: $40 \%$ of women present to their GPs in the UK on account of menorrhagia: accounting for $12 \%$ of all gynaecology clinic referrals. Hysterectomies, previously the bane of surgical treatment, has halved in incidence over a 10-year period in the U.K., with a concomitant rise in endometrial ablations and the Mirena IUS use. As more user- and patient-friendly, uptake is expected to be on the rise. Material and Methods: Literature search using Pubmed, Medline and Cochrane databases. Material from ESGE 2011.

Results: Different interventions at varying levels of clinical trials are described.

Discussion: These new surgical options avoiding the need for major surgeries include: Doppler guided uterine artery occlusion device, Laparoscopic uterine artery occlusion, Cryomyolysis, MRI guidedfocussed ultrasound, Viz-Ablate. The following endoscopic devices targetingfibroids and polyps include Integrated Bigattishaver, Truclear morcellator, Myosure hysteroscopic tissue removal, Transcervical vaporisation of myomas, $\mathrm{BIOH}$ (Bettochi).

\section{5}

\section{MESH-LESS LAPAROSCOPIC HYSTEROPROMONTOFIXATION FOR GRADE IV PELVIC ORGANE PROLAPSE \\ Innovation in Surgery}

A.R. Mothes*, M.P. Radosa, I.B. Runnebaum

${ }^{[1]}$ Jena University Hospital $\sim$ Jena $\sim$ Germany

Summary (4 lines): We present a new technique of uterine fixation to the promontory by laparoscopic sutures without mesh in selected massive genital prolapse.

Introduction: The multi-morbid patient suffering grade IV uterine prolapse is increasingly becoming a challenge for surgeons in an aging population.

Material and Methods: In two patients presenting massive uterovaginal prolapse application of two permanent laparoscopic prolene sutures to the uterine isthmocervical junction and the anterior longitudinal ligament at the promontorial site was performed. Anatomic results were evaluated by POP-Q values, functional outcome/subjective cure by interview.

Results: We report on two multi-morbid, senior patients age 80/76 years presenting extremely large POP (tvl 18/21; C +18/+21). Co-morbidities included renal and liver failure, pyelonephritis, ascites. In one case laparoscopic procedure was combined with vaginal repair. Hysterofixation as such including the preparation of the ligament lasted 20 minutes. Mean $\mathrm{Hb}$-decline was 0,25 . Procedures were successfully finished. No complication occurred. One patient could be followed up after 22 months. The anatomic, functional and subjective result was excellent.

Discussion: Laparoscopic hysteropromontofixation without mesh appears to be feasible in cases of very large genital prolapse and may be advantageous for multi-morbid patients because of minimal trauma and short operating time. Prospective evaluation of a larger patient cohort is on-going.

317

\section{NEXT GENERATION ADHESION PREVENTION PRUDUCT-LABS ADHESION BARRIER SYSTEM Innovation in Surgery}

\section{K. Skinner*, L. Jenkins \\ ${ }^{[1]}$ Actamax Surgical Materials LLC $\sim$ Wilmington $\sim$ United States}

Summary (4 lines): The LABS ${ }^{\mathrm{TM}}$ Adhesion Barrier System is a sprayable tissue adherent hydrogel adhesion barrier. LABS was evaluated in a rat sidewall defect model and was successful in reducing number of adhesions.

Introduction: Surgical adhesions continue to be a problem and no therapy significantly prevent adhesions especially following laparoscopic procedures. LABS is comprised of dextran-aldehyde, methoxypolyethylene glycol amine and an eight -arm star PEG amine polymer. When sprayed simultaneously on to tissue, the components crosslink to form a hydrogel.

Material and Methods: We tested the effectiveness of LABS in a rat sidewall defect model. In 60 adult female $\operatorname{rats}(\mathrm{n}=10$ per group), a $1 \mathrm{~cm} \times 1 \mathrm{~cm}$ peritoneal made along the abdominal wall the defect was enclosed with \#3-O silk suture to induce ischemia. The animals were 
randomized to LABS groups (two doses- $.25 \mathrm{~g} / \mathrm{cm}^{2}$ or. $38 \mathrm{~g} / \mathrm{cm}^{2}$ ) or non-treatment control group. At week 1 , and 2 we scored for percentage of animals adhesion free.

Results: At week 1, both doses of LABS were $90 \%$ adhesion free as compared to controls which were $20 \%(\mathrm{p}<.05)$ At week 2 , the high dose $(100 \%)$ and low dose $(80 \%)$ had significantly greater percentage of adhesion free animals as compared to the control $(20 \%, \mathrm{p}<.05$.

Discussion: These results indicate that LABS Adhesion Barrier may be useful adjuvant for adhesion prevention in open and laparoscopic surgical procedures. The effectiveness and safety of LABS will be tested in human clinical studies.

\section{7}

\section{REVIEW OF PATIENT DEMOGRAPHICS, ENDOMETRIAL HISTOPATHOLOGY AND FAILURE RATE OF 190 ENDOMETRIAL ABLATIONS}

Innovation in Surgery

\section{A. Abdelrahman*, K. Niblock, K. Johnston \\ ${ }^{[1]}$ Antrim Hospital $\sim$ Northern Ireland $\sim$ United Kingdom}

Summary (4 lines): Retrospective study of 190 endometrial ablation patients demographics endometrial ablation including failure rate over a 5 year period.

Introduction: To identify demographics, failure rate and histopathological results of large cohort of endometrial ablation patients.

Material and Methods: Retrospective chart review who underwent endometrial ablation (Thermablate Balloon Ablation \& Novasure) from July 2007-August 2011. Analysed: age, parity, caesarean $(\mathrm{C} / \mathrm{S})$, Body Mass Index (BMI), previous use of levonorgestrel-releasing intrauterine system (Mirena), subsequent hysterectomy.

Results: Average age was 42 yrs (range 21-55); average parity was 2 (range 0-7). Average BMI was 31 (range 17-54). WHO classification of "obese" applied to 89/190 (47 \%). In total 40/190 $(21 \%)$ had a previous C/S and 18/190 (9\%) had $=2 \mathrm{C} / \mathrm{S}$. Previous Mirena was attempted in 66/190 (35\%). Endometrial hyperplasia occurred in only 4/190 (2\%) and complex atypia in 1 patient. Hysterectomy was carried out in 32/190 (17 \%), mean post ablation interval 14 months

Discussion: Almost 1/2 of our patients were "obese", approximately 1/ 5 had a previous caesarean and $1 / 3$ had previously tried Mirena. Complex atypical hyperplasia was rare $(0.5 \%)$. Subsequent hysterectomy rate was $17 \%$.

101

\section{SAFETY OF LAPAROSCOPY IN THE MANAGEMENT OF HUGE ADNEXAL CYSTS \\ Innovation in Surgery}

A. Alobaid*, L. Aldakhil, S. Alobaid
${ }^{[1]}$ King Fahad Medical city $\sim$ Riyadh $\sim$ Saudi Arabia

Summary (4 lines): The aim of our study is to evaluate the safety, effectiveness and feasibility of operative laparoscopy in the management of huge adnexal cysts reaching above the umbilicus.

Introduction: Laparoscopic management of huge adnexal cysts has been described in previous case reports Despite of this, most patients with huge adnexal cysts are managed by laparotomy The aim of our study is to evaluate the safety, effectiveness and feasibility of operative laparoscopy in the management of huge adnexal cysts reaching above the umbilicus.

Material and Methods: A retrospective chart review was done for all patients who had laparoscopy for huge adnexal cysts.

Results: 12 patients were included in the study the mean age of the patients was 32.4 (range 16-69) the mean cysts diameter was $24.16 \mathrm{~cm}$ (range 20-42), the mean amount of fluid drained intraoperatively from the cyst was $4158 \mathrm{ml}(1000-12000)$, the mean operative time was 107.6 minutes (76-190), all the surgeries were done using three ports only except for one patient as she LAVH, the Pathology reports were benign cysts, the blood loss was minimal, five patients had cystectomies, one had $1 \mathrm{AVH}$, one salpingectomy, and five had salpingooopherectomies. there was no operative or post-operative complications

Discussion: With proper patients selection and availability of experts in gynecologic endoscopy, it is possible to remove huge cyst by laparoscopy.

\section{6}

THE MAYER-ROKITANSKY-KUSTER-HAUSER AND 45,X/46,XX MOSAICISM IN A WOMEN WHO UNDERWENT LAPAROSCOPIC PERITONEAL VAGINOPLASTY

Innovation in Surgery

J. Namkung*, J. Kim, M. Kim

${ }^{[1]}$ The Catholic University $\sim$ Seoul $\sim$ Korea, Republic of

Summary (4 lines): Laparoscopic peritoneal vaginoplasty based on modified Davydov's technique is simple, less invasive operation in MRKH syndrome.

Introduction: The MRKH syndrome is characterized by congenital aplasia of the uterus and the upper part $(2 / 3)$ of the vagina in women showing a normal 46, XX karyotype. In this report, we describe a 35 -year-old woman with primary amenorrhea caused by MRKHS. The interest in this case is that MRKHS is associated with $\mathrm{XO} / \mathrm{XX}$ mosaicism. External examination reveals completed puberty with normal secondary female sexual characteristics and normal external genitalia. The vagina was short, rudimentary, and blind-ended.

Material and Methods: Laparoscopy revealed two compact muscular buds, extending from the centre laterally. The ovaries were in their usual position and were macroscopically normal. Our patient underwent laparoscopic peritoneal vaginoplasty based on modified Davydov's technique.

Results: At 6 months after surgery she was sexually active, and functional success was obtained. The quality and structure of the neovaginal epithelium were evaluated by visual inspection, without biopsy, during gynecologic examinations.

Discussion: Laparoscopic peritoneal vaginoplasty based on modified Davydov's technique is simple, less invasive operation in MRKH syndrome.

VERSAPOINT ENDOMETRIAL ABLATION: PRESENTATION OF A NOVEL TECHNIQUE AND ITS OUTCOME AS TREATMENT FOR MENORRHAGIA

Innovation in Surgery

T. Manias*, J. Wynn

${ }^{[1]}$ University Hospital of South Manchester $\sim$ Manchester $\sim$ United Kingdom 
Summary (4 lines): The present study introduces the 1 st generation endometrial ablation technique we have developed and evaluates its effectiveness in our case series.

Introduction: In our unit we have developed this technique as an adjunct to the global endometrial ablation procedures (Novasure/ MEA).

Material and Methods: This is a retrospective study of 10 patients (age 30-51) who underwent Versapoint endometrial ablation as treatment for menorrhagia. They all had US pelvis and one injection of Zoladex $(3.6 \mathrm{mg}) 1$ month prior to the procedure. Initially, we perform a routine saline hysteroscopy dilating to Hegar 8 under general anaesthetic. We subsequently use the spring tip of the Gynecare Versapoint electrode (Vaporization P: $130 \mathrm{~W}$, Desiccation P: $60 \mathrm{~W}$ ) as a means to ablate the entire endometrial surface under direct hysteroscopic guidance (refer to HD images of the presentation for operative technique). All the patients were discharged the same day and were followed-up at 3 months.

Results: On follow-up 7 out of 10 patients reported subjective improvement of their menstrual symptoms and did not require any further treatment. 3 in 10 were listed for hysterectomy the histology of which confirmed adenomyosis.

Discussion: Our initial results show that there is further scope for the development of this technique, which could be useful for units exclusively using the Versapoint system for operative hysteroscopy.

437

\section{WHY HAEMODYNAMICALLY UNSTABLE PATIENT WITH ECTOPIC PREGNANCY SHOULD NOT AVAIL THE BENEFIT OF LAPAROSCOPIC SURGERY? \\ Innovation in Surgery}

R.(.Saha*

${ }^{[1]}$ Heart of England NHS Foundation Trust $\sim$ BSGE, UK $\sim$ United Kingdom

Summary (4 lines): Laparoscopic approach to surgical management of ectopic pregnancy should be considered for patients with unstable haemodynamic condition. RCOG guidance should be amended.

Introduction: RCOG (Royal College of Obstetricians and Gynaecologists, Green Top Review 2010) recommends laparoscopic approach for surgical management of tubal ectopic pregnancy in haemodynamically stable patient, Grade $1 \mathrm{~A}$ evidence. However there was Grade $\mathrm{C}$ evidence to suggest laparotomy should be the choice in haemodynamically unstable patients. The author shares his experience in dealing haemodynamically unstable patients

Material and Methods: Two patients aged 35 and 37 years attended the Emergency Dept, resuscitation unit via ambulance. Both patients required active resuscitation.

Results: Patients were counselled for laparoscopic surgery with possibility of laparotomy. Massive haemoperitoneum was noted with ruptured tubal ectopic. Contralateral tube were normal. Ligasure was used in one patient and the bipolar electrosurgery on the other to perform total salpingectomy. The blood clots were removed by suction irrigation following haemostasis. The estimated blood losses were $>2500 \mathrm{ml}$. Intraoperative blood loss was minimal. Video will be presented.

Discussion: Laparoscopic surgery should be considered also in haemodynamically unstable patients with ectopic pregnancy. The procedure is safe, takes less time, less blood loss than open surgery (laparotomy).
500

\section{BLOOD LOSS REDUCTION DURING LAPAROSCOPIC MYOMECTOMY BY INTERNAL ILIAC ARTERIES TEMPORARY OCCLUSION}

Myomectomy

K. Puchkov*, J. Andreeva, N. Podzolkova, V. Korennaya, A. Dobychina ${ }^{[1]}$ Clinucal and Experimental Surgery Center $\sim$ Mscow $\sim$ Russian Federation

Summary (4 lines): Internal iliac arteries temporary occlusion (IIATO) during laparoscopic myomectomy is effective and feasable method of blood-loss reduction.

Introduction: Laparoscopic myomectomy can be a difficult procedure and result in excessive blood loss. Therefore, a reliable method of bleeding control is highly needed.

Material and Methods: We applied a technique of IIATO with smooth vascular clamps in 2008. The peritoneum above the iliac arteries is opened and vascular clamps "De Bakey" are applied on both sides. Then the standard procedure of myomectomy is performed. The clamps are removed after the procedure completion.

Results: 302 patients have been treated by the described method. Almost $70 \%$ of them had myomas of $50-80 \mathrm{~cm} 2$ volume and/or "inconveniently" located. The operative time was $65 \pm 15 \mathrm{~min}$. Time, spent on clamp placement was $15 \pm 10 \mathrm{~min}$. Blood loss was $40 \pm 10 \mathrm{ml}$. The early therapeutic outcomes showed shorter recovery period, less amount of analgetics needed and no thrombotic complications. No blood transfusion was needed. Scar formation was controlled by the ultrasonic examination in 7, 30, 60 and 180 days after surgery and didn ${ }^{\prime} t$ differ from patients treated conventionally. Trough out this period we have had two complications (iliac vein and internal illiac artery trauma) wich we managed laparoscopicaly.

Discussion: IIATO is acceptable, feasible and doesn't prolong the operation time sagnificantly.

\section{5}

\section{BLOOD-LOSS PREVENTING PROCEDURES IN CONSERVATIVE MYOMECTOMY THROUGH LAPAROTOMY \\ Myomectomy}

A. Shaparnev, S. Vardanyan, S. Vlasenko, B. Tsivyan*

${ }^{[1]}$ State City Hospital \# 40, Sestroretsk $~$ Saint- Petersburg $\sim$ Russian Federation

Summary (4 lines): The effect of different types of temporary block of uterine blood supply on blood-loss in conservative myomectomy. Introduction: The decrease of blood loss in conservative myomectomy is still actual and can be achieved by temporary block of uterine blood supply.

Material and Methods: A retrospective study of 25 cases of conservative myomectomies performed for large fibroids through laparotomic approach. The age of patients was from 24 to 37 years, the size of fibroids was from 4 to $15 \mathrm{sm}$, the number of myomas was from 1 to 8 . In 10 cases (group1)no blood - supply block was used, in 10 cases(group2)temporary arterial block was used: by tourniquettes on ascending branches of a. uterinae and arteries of infundibulo-pelvic ligaments ( 8 cases), artery of round ligament and infundibulo-pelvic ligament( 1 case)and clipping of a. iliaca interna on both sides ( 1 case). In 5 cases(group3)temporary uterine artery embolisation was performed the day before surgery.

Results: The mean operating time in group 1 was $134+-14$ min, in group 2-181+-18 min, in group 3-128+-12 min, the mean blood loss was $215+-15 \mathrm{ml}, 141+-18 \mathrm{ml}$ and $128+-14 \mathrm{ml}$ respectively. 
Discussion: Temporary blood-supply block demonstrates blood-loss decrease for $30 \%$, the duration of operations is longer in cases of blood vessels ligation. UAE gives the comparable effect of blood loss decrease, but it needs additional anestesy and work of surgeons and is accompanied by severe pain syndrome and fever.

310

\section{CAN WE COMPARE LAPAROSCOPIC AND LAPAROTOMIC} MYOMECTOMIES

Myomectomy

G. Mecejus*, I. Kuropatkinaite, E. Baužyte

${ }^{[1]}$ vilnius City Clinical Hospital, Vilnius University $\sim$ Vilnius $\sim$ Lithuania

Summary (4 lines): Laparoscopic approach for myomectomy is safe and enough radical with less perioperative complications and shorter hospitalization time

Introduction: Some gynecologists have doubts is laparoscopic myomectomy acceptable for the duration and perioperative safety comparing with laparotomy. Aim of study - to compare methods of laparoscopic (LSM) and laparotomic (LM) myomectomies and perioperative period.

Material and Methods: Retrospective analysis of case histories of women, who underwent myomectomy in 2011 by laparoscopical approach (I gr.) and in 2005-2007 by abdominal approach (II gr.). Data for research: size of enucleated myomas, duration of operation and postoperative hospitalization, perioperative complications.

Results: 59 cases in each group. Removed myomas size was large in II gr $8,6 \pm 3,7$ vs $6,8 \pm 1,9 \mathrm{~cm}, \mathrm{p}<0,05$. Operation time was significantly greater in I gr. $(88,9 \pm 43,3$ min. - I gr., $67,3 \pm 20,9$ min. - II gr., $p<$ $0,05)$, but postoperative period was longer in II gr. $(25,5 \pm 8,3$ and $118,2 \pm 68,8 \mathrm{~h}$ respectively, $\mathrm{p}<0,05)$. Complications were more frequent in II gr. (I gr. $-7(11,7 \%)$, II gr. - 10(16,9\%)). Myoma's weight is related only with complication rate in II gr. $(r=0,665, p<0,05)$.

Discussion: Myomectomy is acceptable and safe in both methods, but laparoscopic myomectomy has more advantages because of few perioperative complications and shorter hospitalization time.

\section{0}

\section{COMBINED USE OF VASOPRESSIN AND UTERINE ARTERY LIGATION FOR HEMOSTASIS IN LAPAROSCOPIC MYOMECTOMY WITH LARGE UTERINE SIZE IN LIMITED RESOURCE SETTING. \\ Myomectomy}

A. Magdum*, H.R. Padiyath, A. Puthiyidom, S. S, N. Trehan, A. Gupta

${ }^{[1]}$ Sunrise Hospital, Kakkanad $\sim$ Ernakulam $\sim$ India

Summary (4 lines): combination of vasopressin and uterine artery ligation is an effective method of hemostasis during laparoscopic myomectomy for large uteri.

Introduction: Laparoscopic myomectomy (LM) is standard of care for symptomatic uterine leiomyoma who want to conserve the uterus. We present combined intra-operative use of vasopressin and uterine artery ligation (UAL) for hemostasis during LM for large fibroids in limited resource center.

Material and Methods: This is a retrospective analysis of 86 laparoscopic myomectomies (uterine size 24 to 30 week's size) at limited resource center. Study group had vasopressin injection followed by myomectomy then UAL at its origin. In control group women underwent LM with vasopressin \&/or UAL which was done before myomectomy. Size of uterus, parity, need for intra-operative and postoperative blood transfusion, length of hospital stay and complications were analyzed and compared.

Results: The need for blood transfusion (nil v/s $12.9 \%$ ) and length of hospital stay (nil v/s $12.9 \%$ ) was significantly less in study group $(\mathrm{n}=$ 32) compared with control group $(n=54)$.

Discussion: During laparoscopic myomectomy for large uteri where size of uterus makes uterine artery ligation difficult, combined method of use of vasopressin and UAL with changed sequence of procedures is effective method for hemostasis in limited resource center.

53

\section{LAPAROSCOPIC MYOMECTOMY - OUR EXPERIENCE Myomectomy}

R. Sousa, P. Reis, L. Coutinho, G. Inocêncio, R. Cubal, H. Ferreira* ${ }^{[1]}$ Centro Hospitalar do Porto $\sim$ Porto $\sim$ Portugal

Summary (4 lines): Analyze myomectomy indications, surgical complications, number of fibroids removed, their localization and average diameter of all laparoscopic myomectomies carried out at our centre. Introduction: Uterine fibroids are the most common benign gynaecological tumor. Surgical treatment can be recommended when myomas become symptomatic, presenting with abnormal uterine bleeding, pain, causing infertility and abortions or when asymptomatic but growing rapidly. Laparoscopic myomectomy is a valid surgical option in many cases, offering many benefits such as lower postoperative pain and shorter hospitalization time when compared to laparotomy.

Material and Methods: Retrospective study of 36 patients submitted to laparoscopic myomectomy at our centre in 2011.

Results: Mean age:35 years (range 22-46); Main indication: abnormal uterine bleeding, lower abdominal pain and infertility; Number of fibroids removed: in 30 cases only one fibroid was removed (maximum of four); Mean fibroid size:5 $\mathrm{cm}$ (maximum of $9 \mathrm{~cm}$ ); Localization: in the majority of cases $(81 \%)$ the fibroids presented an intramural/subserous component; Mean time of surgery:64 min; Mean hospitilization time: 2 days; Complications: no intra or postoperative major complications were registered and there was no need for laparotomic conversion.

Discussion: Laparoscopic myomectomy is a safe procedure, presenting a shorter hospitalization time, a low complication risk, with good overall results and should therefore be a valid surgical procedure recommended in selected cases.

\section{1}

\section{LAPAROSCOPIC MYOMECTOMY IN PERI-} AND POST-MENOPAUSAL WOMEN IS SAFE AND FEASIBLE Myomectomy

P. Đordevic*

${ }^{[1]}$ Department for Gynecology and Obstetrics, Clinical Hospital Center Rijeka, Medical Faculty University of Rijeka, Croatia Rijeka Croatia

Summary (4 lines): In order to assess efficacy and safety of laparoscopic myomectomy in women after the reproductive period, we compared peri- and post-menopausal women with women of reproductive age who underwent laparoscopic myomectomy. We found similar surgical outcome in both groups.

Introduction: An increasing number of peri- and post-menopausal women with symptomatic myoma seek an alternative surgical procedure to hysterectomy wishing to retain their uteri. The aim of the study 
was to analyze safety, feasibility and outcome of laparoscopic myomectomy beyond the reproductive period.

Material and Methods: All patients who underwent laparoscopic myomectomy in our department during a 15 year period were analyzed. Patients were divided in two groups; group A included women of reproductive age $(n=396)$ and group B peri- and post-menopausal women $(\mathrm{n}=90)$.

Number and size of fibroids, surgical outcome and hospital stay were evaluated in both groups.

Results: No significant difference was noted in number of fibroids and size of the leading fibroid removed between groups. Number of laparotomy conversions, surgery time, blood loss during surgery, opening of the uterine cavity and post-operative complications, hospital stay and the amount of analgesics received within the first 48 hour period were similar in both groups.

Discussion: Laparoscopic myomectomy in peri- and post-menopausal women is a feasible and safe procedure and therefore could be considered as a treatment option.

418

\section{LAPAROSCOPIC MYOMECTOMY OF LARGE UTERINE MYOMAS.}

Myomectomy

\author{
A. Kavallaris*, D. Zygouris, N. Chalvatzas, E. Terzakis \\ ${ }^{[1]}$ Department of Gynecologic Oncology, St. Loukas Hospital, \\ Thessaloniki, $\sim$ Greece $\sim$ Thessaloniki $\sim$ Greece
}

Summary (4 lines): Laparoscopic myomectomy of large myomas is a feasible and safe method.

Introduction: Laparoscopic myomectomy is a minimal invasive method, for management of myomas in women of reproductive age. As a result the procedure is used more frequently for small- and medium sized myomas and there are only a few series of myomectomies for large myomas. We report a case series of xxx patients with uterine myomas bigger than $8 \mathrm{~cm}$, who underwent total laparoscopic myomectomy.

Material and Methods: $\mathrm{n}$ the period between March 2011 and March 2012, 18 patients underwent total laparoscopic myoctem with at least one myoma of $8 \mathrm{~cm}$ diameter. We analyzed the surgical outcome and perioperative morbidity for all the patients retrospectively. All the operations were performed using a PK (Gyrus) morcelator, without tissue or blood spillage in the abdomen. The uterine defect was closed with an absorbable suture in 2 layers with intra corporeal knots.

Results: There were no major intraoperative or postoperative complications and the mean estimated blood loss was $250 \mathrm{ml}$. The mean myoma's weight was 410 gr (180-620 gr) and there was not needed any blood transfusion intra - or post - operatively. Six patients needed one unit of PRBC blood transfusion.

Discussion: We believe that this is the best minimally invasive choice for fertility preservation even in cases of large myomas considering the other methods that have been proposed, such as mini - laparotomy or gasless laparoscopic myomectomy.

137

LAPAROSCOPIC MYOMECTOMY: A SAFE ALTERNATIVE? Myomectomy

I. Siebert*, R. Lourens, K. Van Der Merwe, T. Kruger

${ }^{[1]}$ Stellenbosch University $\sim$ Tygerberg $\sim$ South Africa

Summary (4 lines): Background: The safety of laparoscopic myomectomy has been questioned especially in the case of intramural fibroids.
Introduction: Aim: To assess the safety of a laparoscopic myomectomy for intramural fibroids and study the effect on fertility.

Material and Methods: Methods and Settings: A retrospective study of a single private reproductive biology centre specialising in laparoscopic myomectomy.

Results: Results: 58 Patients were studies in which there were no major complications. The conversion rate from laparoscopy to laparotomy was $3 / 58(5 \%)$ due to multiple fibroids. There was one case of uterine perforation during hysteroscopy. The overall pregnancy rate was $25 / 44(57 \%)$ with a spontaneous pregnancy rate of $14 / 25(56 \%)$.

Discussion: Conclusions: Laparoscopic myomectomy can be regarded as a safe alternative to abdominal myomectomy in the hands of the experienced surgeon. We are also of the opinion that if an intramural fibroid is suspected of being a causative factor for infertility and fits within the set criteria for removal. The removal of an intramural fibroid will most likely improve fertility.

113

\section{OUR EXPERIENCE OF PERFORMING LAPAROSCOPIC CONSERVATIVE MYOMECTOMY}

Myomectomy

A. Shaparnev, B. Tsivyan*, S. Vardanyan, S. Vlasenko

${ }^{[1]}$ Hospital $40 \sim$ Sestroretsk, Sankt-Petersburg $\sim$ Russian Federation

Summary (4 lines): laparoscopic myomectomy is a safe procedure. Introduction: To present our results of laparoscopic management of patients with fibroids.

Material and Methods: A retrospective study of 148 cases of patients with fibroids managed in our department by laparoscopic myomectomy from 2009 till 2012. Indications for surgical management were following: presence of symptoms, rapid growth, size of myoma more than $5 \mathrm{sm}$ in patients before pregnancy and subfertility.

Results: The average age of patients was 36,1 years ( $23-45$ years). The mean operating time and blood loss were $126 \mathrm{~min}(30-240 \mathrm{~min})$ and $174 \mathrm{ml}(35-350 \mathrm{ml})$ respectively. The mean number of removed fibroids was $3(1-8)$, size $6.5 \mathrm{~cm}(1-13 \mathrm{~cm})$.

10 cases of large fibroids were combined with preoperative temporary uterine artery embolisation (by hemostatic sponge) the day before surgery. 6 patients underwent temporary laparoscopic clipping of a.iliaca interna by placing the "bulldog" clamps on both sides with good hemostatic effect.

Myomectomy was performed using ultrasonic energy Sonosurg or bipolar forceps. Laparoscopic repair of uterine defect was carried out by monofilament sutures.

In 2 cases with large fibroids laparoconversion became necessary. No patients required blood transfusion.

Discussion: laparoscopic myomectomy is a safe procedure in experienced hands even for removal of large fibroids. Transient blocking of uterus blood supply is feasible and can be performed safely.

\section{5}

\section{OUTPATIENT HYSTEROSCOPIC LASER TREATMENT OF MYOMAS: RESULTS OF THIS NOVEL TECHNIQUE Myomectomy}

E. Velasco Sanchez*, J.E. Arjona Berral, L. Nieto Pascual, B. Povedano Cañizares, J.A. Monserrat Jordan

${ }^{[1]}$ Hospital Universitario Reina Sofia $\sim$ Cordoba $\sim$ Spain 
Summary (4 lines): To describe a novel technique for submucous myoma with laser that allows the treatment in the outpatient basis of this pathology. To describe the technique of this novel approach.

Introduction: 5-10 \% are submocous myoma, and they are the most symptomatics, requiring most of them surgical treatment.

Material and Methods: For this approach we use a Wolf hysteroscope of optic fiber with a worksheet of 9 French, and a Myotwister laser fiber de Biolitec ${ }^{\circledR}$ that allows the Selective Live Vaporization. Patients are pretreated with $10 \mathrm{mg}$ diazepam and $600 \mathrm{mg}$ ibuprofen 1 hour before procedure, and paracervical anaesthesie during procedure. They undergo with oral contraceptives. Indications for the procedure are: symptomatic myoma less than $4 \mathrm{~cm}$, G0-G1-G2 of Wamsteker.

Results: Number of procedures 22. Age of patients is 42,45. Last of procedure $51 \mathrm{~min}$. Size of myoma 30,38 mm: G0 47,8 \% (11), G! $13,0 \%$ (3)and G2 39,1 \% (9). Complete resection in 76,2 \% of cases: persit myoma 2 months later in $33,3 \%$ of cases, and after 4 months in $13,8 \%$ of women. Pain during procedure: no pain $(72,7 \%)$, mild pain $(13,6 \%)$, moderate pain $(9,1 \%)$, and sever $(4,5 \%)$. Vasovagal syndrom: $0 \%$

Discussion: The vaporesection of myomas in our center with this novel technique is feasible and the advantages are: no need of hospitalization, no need of general anaethesie, a quickly recovery, and a better management of the operation room.

\section{8}

\section{PREGNANCY OUTCOMES AFTER HIGH INTENSITY FOCUSED ULTRASOUND (US GFUS) "HIFU SYSTEM" TREATMENT OF UTERINE FIBROIDS.}

Myomectomy

L. Quintas Marques*, A. Pessarrodona Isern, E. Vallejo Barnosell, J. Isern Quitllet, J. Rodriguez Gonzalez, J. Cassadó Garriga

${ }^{[1]}$ University Hospital Mutua de Terrassa Terrassa, Barcelona $\sim$ Spain

Summary (4 lines): We report all pregnancies after USgFUS treatment of uterine fibroids

Introduction: USgFus is a not invasive new treatment for uterine fibroids based on High intensity focused ultrasound that doesn't interfere in myometrium nor uterine vascular supply.

Material and Methods: All patients that got pregnant from a 250 myomas series treated with HIFU from February 2008 to December 2011

Results: In this period thirteen patients got pregnant, nine of them gave birth normally. We got ten healthy newborns (one twin pregnancy). 8/ 10 deliveries were vaginal and two were Caesarean sections for bad foetal position.

The average weight of newborns was of $3.197 \mathrm{gr}$.

There were two miscarriages at first trimester, one therapeutic abortion and one legal abortion.

During full-term pregnancies there were no complications attributable to ultrasound treatment.

The average time from treatment to gestation was of 11,3 month. The average volume of treated fibroids was of $175 \mathrm{cc}$. The average treated area was of $64,16 \%$.

Discussion: Preliminary pregnancy experience after US g FUS is encouraging, a high rate of full-term pregnancies were observed. In our short experience HIFU technique is safe and effective in patients with gestational desire to treat uterine fibroids. The treatment seems doesn't interfere in myometrium and endometrium; no placentation disorders (placenta previa o acretismus) were observed, no uterine rupture was seen in our series.
162

\section{SECOND LOOK HYSTEROSCOPY FINDINGS IN PATIENTS AFTER MYOMECTOMY WITH INTRAUTERINE PENETRATION AND MYOMECTOMY FOLLOWING UTERINE ARTERY EMBOLIZATION \\ Myomectomy}

K. Kubinova*, M. Mara, D. Kuzel

${ }^{[1]}$ Department of Obstetrics and Gynecology, 1st Medical Faculty of Charles University $\sim$ Prague $\sim$ Czech Republic

Summary (4 lines): Evaluation of intrauterine findings in patients after myomectomy with peroperative uterine cavity penetration and patients after myomectomy and preceding UAE.

Introduction: A study was conducted to evaluate the second-look (SL) hysteroscopic findings for the presence of intrauterine abnormalities in patients after myomectomy with peroperative uterine cavity penetration (IUP) and to compare them with second-look hysteroscopic findings of patients after myomectomy and preceding uterine artery embolization (UAE)

Material and Methods: Patients after myomectomy with IUP and patients after elective myomectomy for the persistence of clinically significant myoma following UAE were included. Patients underwent SL hysteroscopy in 3-6 months following myomectomy

Results: Total of 84 SL hysteroscopic procedures $(75$ after myomectomy-group A, 9 after myomectomy following UAE-group B) were performed. Negative intrauterine finding for the presence of synechiae was significantly higher in group A (62 cases; $83 \%$ ) than group B ( 4 cases; $45 \%$ ), $p=0,019$. Severe abnormalities were present in 2 patients $(22 \%)$ after UAE compared to 2 patients (3\%) after myomectomy $(\mathrm{p}=0,055)$

Discussion: IUP does not represent a risk of intrauterine abnormalities. Preceding UAE is associated with high rate of intrauterine pathologies. UEA should not be therefore indicated in patients with future reproductive plans

\section{5}

\section{THE BENEFITS OF V-LOC SUTURE DURING LAPAROSCOPIC MYOMECTOMY \\ Myomectomy}

A. Iefimenko*, V. Bondarchuk, V. Zhegulovich, O. Shevchenko, Y. Zhegulovich, I. Nohovska

${ }^{[1]}$ National O.O. Bohomolets medical university $\sim$ Kyiv $\sim$ Ukraine

Summary (4 lines): The results of this study showed a significant advantage of unidirectional sutures during laparoscopic myomectomy that is evident in blood loss and operation time reduction and provides a good scar formation.

Introduction: The aim of our study was to evaluate the benefits of unidirectional suture material during laparoscopic myomectomy.

Material and Methods: During 6 months we have operated 47 patients with myomas, average age is 31.4 . Of those, for 22 operations we were using ordinary suture material, and for 25 operations-V-loc suture. Size of fibroids varied from 3 to $7 \mathrm{~cm}$, intramural, noninvolving the cavity. The technique of laparoscopic myomectomy was typical. The average operation time using conventional suture was $74.2 \mathrm{~min}$, and for v-lock suturing-53.9 min. Total blood loss was $150 \pm 30 \mathrm{ml}$, and $90 \pm 20 \mathrm{ml}$ respectively.

Results: All patients were discharged on the third day, duration of stay in hospital did not differ. There were no complications in early and late postoperative period. Ultrasound monitoring of the uterine scar after 3 - 
6 months did not show any fundamental differences in the consistency of the scar using different suture materials. Pregnancies did not occur (not recommended at least for 6 months).

Discussion: The use of unidirectional sutures during laparoscopic myomectomy reduces the operation time for average 20 minutes, and the amount of blood loss due to more rapid suturing, while not affecting the properties of the uterine scar, which is very important.

179

\section{A DOUBLE RANDOMIZED CONTROLLED TRIAL OF ORAL MISOPROSTOL BEFORE DIAGNOSTIC HYSTEROSCOPY}

Office \& Diagnostic Hysteroscopy

C. Sofoudis*, P. Bakas, M. Creatsa, M. Kalampokas, V. Grigoriou, N. Vlahos

${ }^{[1]}$ 2ND DEPARTMENT OF OBSTETRICS AND GYNECOLOGY UNIVERSITY OF ATHENS ARETAIEION HOSPITAL ATHENS Greece

Summary (4 lines): Oral use of $400 \mathrm{mg}$ of oral misoprostol is more effective than $200 \mathrm{mg}$ preoperatively and the control group.

Introduction: The objective of our study is to evaluate the efficiacy and safety of oral misoprostol for cervical priming.

Material and Methods: 54 patients divided in three groups were examined. The first group $(\mathrm{n}=18)$ had oral $200 \mathrm{mg}$ misoprostol twelve hours before the procedure received. The second group $(n=18)$ had oral $400 \mathrm{mg}$ misoprostol twelve hours before the procedure received. The third group $(n=18)$ was the contoll group which did not receive any cervical priming agent. The initial Hegar dilatation, the used Hegar dilatator, cases of fever, the time of cervical dilatation, any cases of diarrhoia, cases of lacerations and the pain score (1-10) after the procedure was noted in each case.

Results: The study revealed a significant difference between the two groups and the control group. The mean Hegar dilatation for the two groups was 5,5 and for the control group 3. There was not any cases of fever or diarrhoia. The mean time for cervical dialatation was 3 minutes for all groups. There were lacerations in all groups notified. The mean pain score was for the two study groups 3 in addition to the control group which was 5 .

Discussion: HYSTEROSCOPY IS ONE OF THE MOST COMMON METHODS FOR DIAGNOSING INTRUTERINE PATHOLOGY.

\section{5}

\section{A SYSTEMATIC REVIEW AND META-ANALYSIS OF RANDOMIZED STUDIES COMPARING MISOPROSTOL VERSUS PLACEBO FOR CERVICAL RIPENING PRIOR TO HYSTEROSCOPY}

Office \& Diagnostic Hysteroscopy

F. Gkrozou*, G. Koliopoulos, T. Vrekousis, A. Koutlas, L. Lavasidis, I. Navrozoglou, T. Stefos, M. Paschopoulos

${ }^{[1]}$ University Hospital of Ioannina $\sim$ Ioannina $\sim$ Greece

Summary (4 lines): This study presents the results of a systematic review and meta-analysis of randomized studies comparing misoprostol versus placebo for cervical ripening prior to hysteroscopy.

Introduction: Hysteroscopy is an effective diagnostic method for intrauterine cavity, with some limitations, such as the occasional need for cervical dilatation. Misoprostol is an agent that is routinely used for cervical dilatation, but has not gained wide acceptance for use before hysteroscopy.

Material and Methods: This review includes randomized controlled trials, which compare the use of misoprostol versus placebo by different routes and doses. Electronic search of MEDLINE database and Cochrane Central Register of Controlled Trials was performed for articles published from January 1970 to April 2011. The outcome measures studied were related either to the facilitation the hysteroscopic procedure or to the medication side effects.

Results: The administration of vaginal misoprostol reduced the need of cervical dilatation in population of pre- and postmenopausal women in a statistically significant degree. Most other outcomes relating to the facilitation of the procedure did not reach statistical significance. The side effects in the misoprostol group were significantly more frequent than in placebo group.

Discussion: There is not sufficient evidence to recommend the routine use of misoprostol before every hysteroscopy.

322

\section{ARE UTERINE POLYPS ASSOCIATED WITH ABNORMAL} UTERINE BLEEDING?

Office \& Diagnostic Hysteroscopy

H. Walshe*, N. Cooper, J. Clark

${ }^{[1]}$ Birmingham Women's Hospital $\sim$ Birmingham $\sim$ United Kingdom

Summary (4 lines):

Introduction: It is unclear whether uterine polyps cause abnormal uterine bleeding or are just coincidental findings. This study aimed to determine the incidence of endometrial polyps in women of all ages who had presented with abnormal uterine bleeding (AUB) against those who had no symptoms of AUB.

Material and Methods: This study was undertaken over a four year period, running from April 2008 to April 2012 in the one-stop hysteroscopy clinic at Birmingham Women's Hospital. AUB included intermenstrual bleeding, postmenopausal bleeding, postcoital bleeding and menorrhagia. Those women not presenting with AUB were being investigated for incidental findings on USS, pelvic pain, vaginal discharge or as follow-up from previous treatment. Data was collected prospectively by documenting when hysteroscopy diagnosed a women with a polyp and it was then recording whether she had symptoms of AUB or not.

Results: 855 uterine polyps were diagnosed. 780 (91\%) were associated with AUB and $75(9 \%)$ were not associated with AUB. The figures show a clear difference in the incidence of uterine polyps found between the two groups of women.

Discussion: Uterine polyps were more prevalent in women with abnormal uterine bleeding than those without symptoms. This suggests a causal relationship and supports the current practice of removing uterine polyps when detected.

\section{2}

\section{DIAGNOSIS OF POLYP BY OFFICE ENDOMETRIAL BIOPSY: IS THERE AN ULTRASOUND- HYSTEROSCOPY RELATIONSHIP?}

Office \& Diagnostic Hysteroscopy

C. Del Valle Rubido, J.A. Solano Calvo*, V. Marcos González, I. Heras Sedano, J.J. Delgado Espeja, A. Zapico Goñi, R. Cajal Lostao

${ }^{[1]}$ Hospital Universitario Príncipe de Asturias $\sim$ Alcalá de Henares $\sim$ Spain

Summary (4 lines): It is necessary to perform hysteroscopy when we diagnose polyps by office endometrial biopsy even if ultrasound is negative, because of the low sensitivity of this technique.

Introduction: Our objective is to study the relationship between pathology, hysteroscopy (HSC) and ultrasound (US) of the endometrial 
polyps diagnosed by endometrial biopsy in Hospital Príncipe de Asturias in the last 2.5 years.

Material and Methods: We conducted an analytic retrospective study verifying the presence or absence of endometrial polyp by HSC in those endometrial biopsies reported as polyp, and comparing them to the US findings in these patients. The purpose is to establish the necessity or not of performing HSC at the pathologic finding of polyps that do not have an US relationship.

Results: We found 58 endometrial biopsies diagnosing polyps, 23 $(39.7 \%)$ of which were confirmed as polyps during HSC. A contingence test proved that $27.6 \%$ of US did not detect polyps that were afterwards found in HSC (US false negative ratio: $36 \%$ - Sensitivity: $30.4 \%$ ). However, all the polyps that were not detected by US were smaller than $10 \mathrm{~mm}$.

Discussion: Polyp diagnosis by office endometrial biopsy requires hysteroscopic confirmation, even though there is no US relationship, as in these cases US has low sensitivity, at the expense of small-sized polyps.

164

\section{DIAGNOSTIC OF ATYPICAL ENDOMETRIAL POLYPS VIA} OFFICE HYSTEROSCOPY

Office \& Diagnostic Hysteroscopy

I. Ilic*, Y. Cunarro Lopez, A. Marti Colombas, F. Vicandi Plaza, R. Moreno Molina, L. Ortiz Quintana

${ }^{[1]}$ Gregorio Maranon University Hospital Madrid $\sim$ Spain

Summary (4 lines): Evaluation of indications for office hysteroscopy in endometrial polyp diagnosis and biopsy results in our unit.

Introduction: We aim to analyze the office hysteroscopy diagnosis of endometrial polyps in our center and try to evaluate the justification of indicating the procedure according to current practice guidelines.

Material and Methods: We analyzed 653 patients from 15/12/2010 $01 / 04 / 2012$ with office hysteroscopy performed in our center.

Results: Median age 50.1 \pm 13.1 years, ranging 25-84 years. Menopausal were $44.0 \%$. We have had 275 patients with biopsy specimen of endometrial polyp. Only a fraction, $22.9 \%$ reported symptoms related to their pathology (menopause uterine bleeding, spotting, excessive menstrual bleeding, etc). We encountered $40.7 \%$ atrophic, $16.0 \%$ hyperplastic, $19.3 \%$ non-categorized benign, $3.6 \%$ cervical, $0.7 \%$ placental and $1.1 \%$ atypical polyps (3 cases). Atypical polyps were categorized during hysteroscopy as suspicious of atypia in $2 / 3$ cases. Patients were 31,52 and 53 years old. All three presented symptoms related to polyps, excessive menstrual bleeding in young woman, and menopause bleeding the rest. Menopause patients were obese and 1 received hormonal replacement therapy.

Discussion: Atypical polyps are scarce pathology in our series. Excessive number of hysteroscopies needed to diagnose this pathology might be reduced by meticulous anamnesis and sound clinical judgment.

456

\section{EMBRIOSCOPY: NEW CLINICAL AND DIAGNOSTIC PROSPECTIVES OF THE OFFICE HYSTEROSCOPY IN THE EMBRIO DESEASES}

Office \& Diagnostic Hysteroscopy

E. Castellacci*, S. Calzolari, E. Periti, L. Rosignoli, V. Dubini

${ }^{[1]}$ Palagi Hospital- Florence- Italy $\sim$ Florence $\sim$ Italy

Summary (4 lines): The embrioscopy represents a new technic for the study of the ebrio desease diagnosticated by prenatal diagnosis center.
Introduction: the embrioscopy represent a valid diagnostic support to confirm and complete the study of suspected morfological abnormalities diagnosticated by ultrasound imaging.

Material and Methods: a case report of a woman at 10 weeks pregnancy, with an ultrasound suspect of chromosomal abnormalities.

Results: the transcervical embrioscopy confirm the presence of embrional signs of chromosomal abnormalities suspected with ultrasound imaging and confirmed by chromosomal exam (chorionic villus sampling).

Discussion: The office hysteroscopy can help to define and study the pathologies diagnosticated by ecographic images or invasive procedures (cvs) of the baby in first thrimester of life.

\section{9}

\section{ENDOMETRIAL POLYP ON ULTRASOUND- IT'S DIAGNOSIS AND IMPORTANCE IN PRE-MENOPAUSAL WOMEN? A 5-YEAR RETROSPECTIVE STUDY}

Office \& Diagnostic Hysteroscopy

N. Barros*, C. Pardal, E. Fernandes, L. Braga, A. Rocha, J. Pena
${ }^{[1]}$ Hospital of Braga $\sim$ Braga $\sim$ Portugal

Summary (4 lines): Endometrial polyps are becoming a clinical challenge in terms of orientation.

Introduction: Our aim was to compare the ecographic diagnosis of endometrial polyp(EP) with the histologic result in both symptomatic and asymptomatic premenopausal women and asses it's clinical importance.

Material and Methods: Retrospective study of all premenopausal women submitted to hysteroscopy from January2006 until December2010 because of ecographic diagnosis of EP and comparison with the histological definitive diagnosis.

Results: The study population was composed by 105 cases. We formed 2 groups -symptomatic (26 cases-24,8\%) and asymptomatic (79 cases-75,2 \%) women. From the total of 105 cases diagnosed ecographically with EP, histology confirmed EP in 83 cases $(79,1 \%)$. In the other 22 cases $(20,9 \%)$ the histology didn' $\mathrm{t}$ confirm EP and revealed:12(11,4\%) normal endometrium, 6 $(5,7 \%)$ submucous myoma, 2(1,9\%) atrophic cystitis, 2(1,9\%) endometrial hyperplasia without atypia. We didn't find any statistically significant difference between the symptomatic and asymptomatic groups in terms of histologic definitive diagnosis $(\mathrm{p}>0,05)$.

Discussion: EP is a frequent hysteroscopy indication in both pre and postmenopausal women. The ultrasound misdiagnosed 22 cases $(20,9 \%)$ of benign cases as being EP. The results show that the decision of doing hysteroscopy in woman with ecographic diagnosis of EP, especially in asymptomatic pre menopausal woman is questionable.

355

ESSURE HYSTEROSCOPIC STERILISATION: EXPERIENCE FROM BOLTON, UK

Office \& Diagnostic Hysteroscopy

S. Das*, J. Tomlinson
${ }^{[1]}$ Bolton NHS Foundation Trust $\sim$ Bolton, Greater Manchester $~$
United Kingdom

Summary (4 lines): Essure procedure can be easily performed in the outpatient setting and is set to become the first line in female sterilisation. 
Introduction: Essure has been widely used in the USA and Europe and introduced more recently in the UK. Our unit is one of the first few centres in UK to offer this procedure. We share the experience and challenges that new operators might encounter.

Material and Methods: Data from the first 110 Essure procedures performed in Bolton Hospital, UK.

Results: The procedure is performed in the out-patient setting. Pain score was 5 or less in $50 \%$ of cases. $5 \%$ stayed in hospital for 60 minutes or longer for pain relief. $8.8 \%$ procedures were abandoned due to pain or failure of device to pass into ostium. $69 \%$ procedures completed in less than 10 minutes. To date, 94 women have completed follow-up imaging to confirm successful sterilisation and 10 still awaiting imaging. No pregnancies have yet been reported. The rate of successful cannulation of both ostia at the first attempt was $95 \%$.

Discussion: From our experience, in the majority of women the procedure is straightforward and results are comparative with published data. Existing outpatient hysteroscopy setting lends itself to accommodate the procedure. It is popular with women. A common database agreed by all operators in the unit is important to produce consistent data.

198

\section{FAILURE TO PERFORM OFFICE DIAGNOSTIC HYSTEROSCOPY: FAILURE RATE AND CONTRIBUTING FACTORS - OUR EXPERIENCE.}

Office \& Diagnostic Hysteroscopy

I. Ilic*, J.A. De Leon Luis, M. Urdaci Elizondo, P. Menendez De Luarca Menendez, Y. Cunarro Lopez, R. Martinez Huertas, L. Ortiz Quintana

${ }^{[1]}$ Gregorio Maranon University Hospital Madrid Spain

Summary (4 lines): We aim to analyze the factors that make impossible office diagnostic hysteroscopy and define failure rates.

Introduction: Defining factors that contribute to the failure of the office diagnostic hysteroscopy might help improve our efficacy.

Material and Methods: We study 643 patients who underwent office diagnostic hysteroscopy from 15/12/2010 - 01/04/2012 in our unit. We use logistic regression to define risk factors for technical failure.

Results: Median age 50.2 \pm 13.1 years, ranging 25-84 years. Nuliparous, (no vaginal birth) $36.1 \%$, menopause $44.2 \%$. Most $(88.0 \%$ ) procedures were done by the 3 most experienced operators $(>100$ procedures each). We were unable to complete $5.3 \%$ of total procedures (cervical stenosis $59.9 \%$; pain $23.5 \%$; bleeding $11.8 \%$ ). Patient age did not influence success rate (Sig 0.783), neither did years of menopause (Sig. 0.528). Most likely not to be completed were procedures in nuliparous (RR 3.598, IC $95 \%$ 1.641-7.892), menopause patients (RR 2.285, IC $95 \% 1.052-4.962$ ), and those performed by less experienced hysteroscopist (RR 4.436, IC $95 \% 2.024-9.722$ ).

Discussion: Nuliparous menopause patients in the hands of inexperienced hysteroscopist are most likely not to have office diagnostic hysteroscopy successfully completed. The failure rate remains relatively low.

\section{2}

\section{HISTEROSCOPIC FINDINGS IN POSTMENOPAUSAL WOMEN}

Office \& Diagnostic Hysteroscopy

V. Ferreira, P. Almeida, C. Moreira, H. Nascimento, M. Oliveira*

${ }^{[1]}$ Centro Hospitalar e Universitário de Coimbra $\sim$ Coimbra $\sim$ Portugal
Summary (4 lines): Compare results of ambulatory hysteroscopy in postmenopausal women with and without uterine bleeding. 2 study groups were formed: G1 asymptomatic women $(n=70)$ and G2symptomatic women $(n=69)$.

Introduction: Hysteroscopy has become the gold standard test for evaluating the uterine cavity, providing direct visualization of endometrium and eye-guided biopsy sampling.

Material and Methods: Retrospective descriptive study carried out on 139 postmenopausal women who underwent diagnostic hysteroscopy, during 2011, in Centro Hospitalar Baixo Vouga Aveiro.

Results: Mean age in G1 was 64.07 (SD 9,99) and 62.01 (SD $10,57)$ in $\mathrm{G} 2(\mathrm{p}>0.05)$. Transvaginal ultrasound revealed (G1 vs G2): focal thickening ( $n=50$ vs $n=27)$, diffuse thickening ( $n=19$ vs $n=50)$ and intracavitary liquid $(n=2, G 1)$. No ultrasound changes were revealed in $5.8 \%$ of patients in G2 vs $1.43 \%$ in G1. Endometrial polyp was the more frequent hysteroscopic finding as well as the most frequent histological result in both groups. Endometrial carcinoma occurred in $7.24 \%$ of patients in G2 and there were no cases in G1. Mean age of women with endometrial carcinoma was 62.1 [53,83] (SD 9,7) and all cases showed abnormal ultrasound. Hysteroscopic findings associated with this histological result were: endometrial polyps $(n=3)$, submucous myoma $(n=1)$ and endometrial hypertrophy $(n=1)$.

Discussion: The incidence of endometrial carcinoma in endometrial polyps was $17,9 \%, 8,7 \%$ in submucous myoma and $15,9 \%$ in endometrial hypertrophy.

\section{HYSTEROSALPINGO-FOAM-SONOGRAPHY AS A CONFIRMATION TEST AFTER HYSTEROSCOPIC TUBAL OCCLUSION WITH ESSURE®}

Office \& Diagnostic Hysteroscopy

\author{
L. Massimo*, F. Cellani \\ ${ }^{[1]}$ Department of Gynecology, Istituto Clinico Città Studi Milan \\ Italy
}

Summary (4 lines): The study evaluates the reliability of hysterofoam-sonography with $\operatorname{ExEm}^{\circledR}$ Foam Kit as a confirmation test of proper placement of Essure micro-inserts and of tubal occlusion.

Introduction: Both the standard flat pelvic X-ray and Transvaginal Ultrasound (TVU) are recommended to confirm proper placement of Essure micro-inserts and Hysterosalpingogram (HSG) to confirm tubal occlusion. Hysterosalpingo-contrast-sonography (HyCoSy) is a potential alternative to X-ray and TVU, but the optimal contrast solution has not yet been identified.

Material and Methods: Confirmation test of both placement of Essure micro-inserts and tubal occlusion has been conducted in 50 cases by gel instillation (ExEm-gel) diluted with $10 \mathrm{~mL}$ purified water to create a foam that is sufficiently stable to show echogenicity for at least $5 \mathrm{~min}$ and sufficiently fluid to pass through patent tubes.

Results: Hysterosalpingo-foam-sonography (HyFoSy) appeared as reliable as $\mathrm{HSG}$ in the confirmation of proper placement and tubal occlusion after Essure insertion, with less pain and costs.

Discussion: HyFoSy appears to be a possible confirmation test both of proper placement and of tubal occlusion after hysteroscopic tubal occlusion with Essure micro-inserts. Further investigations are required to propose this test as the gold standard in the confirmation test after Essure micro-inserts insertion. 
174

\section{HYSTEROSCOPIC VISION OF TUBAL METHAPLASIA \\ Office \& Diagnostic Hysteroscopy}

I. Heras Sedano, C. Del Valle Rubido, V. Marcos González, J.A. Solano Calvo*, R. Cajal Lostao, J.J. Delgado Espeja, A. Zapico Goñi ${ }^{[1]}$ Hospital Universitario Príncipe de Asturias $\sim$ Alcalá de Henares $\sim$ Spain

Summary (4 lines): 32 hysteroscopies (HSC) were indicated after diagnosis of tubal methaplasia by office endometrial biopsy (EB), in $25 \%$ of which hyperplasic endometrium was found.

Introduction: We have analised the findings in HSC after an office EB with tubal methaplasia.

Material and Methods: We have conducted a descriptive retrospective study of 32 women that underwent diagnostic HSC after the diagnosis of tubal methaplasia by EB, in Hospital Universitario Príncipe de Asturias between the years 2009 and 2011.

Results: The findings during the $32 \mathrm{HSC}$ carried out after diagnosing tubal methaplasia in EB were: $25 \%$ hyperplasic endometrium, $21.9 \%$ functional endometrium, $18.75 \%$ endometrial polyp, $15.6 \%$ nonvaluable, $6.25 \%$ uterine malformation, 9.38 atrophic endometrium and $3.13 \%$ submucous fibroid. $25 \%$ of the EB performed during the HSC showed hyperplasia. There were no cases of endometrial cancer. Discussion: Of the 32 patients that were studied, $25 \%$ had hyperplasic endometrium in HSC. We have tried to relate tubal methaplasia with a higher risk of endometrial hyperplasia or occult cancer, but there is no evidence in literature to support this hypothesis because of the low number of studies and cases. The results of our study are similar to the series found in literature.

\section{9}

\section{HYSTEROSCOPIC DIAGNOSIS OF ENDOMETRIAL POLYPS} Office \& Diagnostic Hysteroscopy

P. Almeida, V. Ferreira, H. Nascimento, C. Moreira, M. Oliveira*

${ }^{[1]}$ Centro Hospitalar e Universitario de Coimbra $\sim$ Coimbra Portugal

Summary (4 lines): Endometrial polyps are the most common uterine intracavitary pathology.

Introduction: The frequency of diagnosis of endometrial polyps has increased in recent years due to easier access to uterine cavity by ultrasound and hysteroscopy. However, hysteroscopic image does not exclude malignant or premalignant disease in many cases. Although the majority is benign, incidence of malignant tumors confined to endometrial polyps is up to $4.8 \%$. Precancerous lesions have been documented in $3 \%$ of hyperplasic polyps and evolutionary potential to malignant tumors is still uncertain.

Material and Methods: Retrospective descriptive study of 140 cases of hysteroscopic diagnosis of endometrial polyp.

Results: Endometrial polyps accounted for $36 \%$ of diagnostic hysteroscopy. Average age was 54 years; $51 \%$ were postmenopausal and $55 \%$ asymptomatic. Abnormal uterine bleeding occurred independently of hormonal status. Histopathological results $(n=96)$ showed no changes in 11 cases (11.5\%), 7 fibroids (7.3\%), 4 isolated endometrial hyperplasia $(4.1 \%), 46$ benign polyps $(47.9 \%), 25$ polyps with hyperplasia without atypia $(26 \%)$ and $3.1 \%$ of endometrial carcinoma in the polyp.

Discussion: Hysteroscopy easily identifies and allows guided biopsy of uterine cavity focal lesions. Nevertheless, it may fail in visual differential diagnosis between benign and premalignant/malignant lesions. Histologic diagnosis is recommended. Therapeutic approach is also controversial, but removal of polyps has very low associated morbidity.

454

HYSTEROSCOPIC ENLARGMENT OF CERVICAL CANAL BY TWIZZLE GYNECARE® TO IMPROVE THE FEASIBILITY OF THE POLIPECTOMY IN OFFICE HYSTEROSCOPY. Office \& Diagnostic Hysteroscopy

S. Calzolari*, E. Castellacci, V. Dubini, F. Zolfanelli

${ }^{[1]}$ Palagi Hospital- Florence- Italy $\sim$ Florence $\sim$ Italy

Summary (4 lines): We report a new technical procedure in office hysteroscopy to improve the removal of endometrial polyps $<20 \mathrm{~mm}$ en bloc or sectioned in few parts throw a minimal enlargement of cervical canal by Twizzle instrument

Introduction: We know very well that a limit of the office hysteroscopy can be the measure of the endocavital neoformation, expecially in terms of time procedure and discomfort of the patient.

Material and Methods: 20 cases of enlargement of cervical canal by twizzle Gynecare ${ }^{\circledR}$ to remove en bloc endocavital neoformation.

Results: In all cases the polipectomy was possibile, reducing operative time and patient discomfort.

Discussion: The hysteroscopic enlargment of cervical canal by twizzle Gynecare ${ }^{\mathbb{R}}$ improves the feasibility of the office operativity

133

HYSTEROSCOPIC ESSURE® STERILIZATION: FIVE YEARS' EXPERIENCE IN A SINGLE CENTRE

Office \& Diagnostic Hysteroscopy

C. Andrade*, R.M. Pinto, N. Nogueira-martins, J. Damasceno, A. Pipa, F. Nogueira-martins

${ }^{[1]}$ São Teotónio Hospital Viseu Portugal

Summary (4 lines): Hysteroscopic sterilization using Essure ${ }^{\circledR}$ has been used since 2006 at the authors' Department with a global success rate of $98.1 \%$.

Introduction: The placement of tubal micro-implants as non-reversible contraception has become the initial method of choice, namely due to wider range of applicability. It is a highly effective and well-tolerated procedure, with a fast recovery, avoiding incisions or general anesthesia. Material and Methods: Retrospective study including all the patients who underwent Essure ${ }^{\mathbb{B}}$ sterilization in office hysteroscopy setting at our Department, in a period between January, 2006 and December, 2011.

Results: The mean age was 38 years old (24-54). From the 562 women undergoing hysteroscopic sterilization, 544 (96.8 \%) had their surgical procedure successfully completed. A total of 534 (98.2\%) women complied with the 3-month follow-up. The rate of successful bilateral obstruction was $98.1 \%$ (524 out of 534). The most frequent perioperative and postoperative complications were: pain $(10.4 \%)$, technical complications $(3.3 \%)$, vagal reaction $(2.0 \%)$ and ten cases of expulsion $(1.8 \%)$, two of which into the abdominal cavity. A total of 3 women $(0.55 \%)$ became pregnant.

Discussion: With a global success rate of $98.1 \%$, hysteroscopic sterilization proved to be a safe and effective method. This success seems to be dependent on a strict protocol designed for this group of patients, leading to a highly successful application rate and a low rate of complications. 
138

\section{HYSTEROSCOPIC EVALUATION OF UTERINE CAVITY AF- TER OPEN MYOMECTOMY}

Office \& Diagnostic Hysteroscopy

R. Mesquita Pinto*, G. Halmos, M.D. Dacco', G. Michos, G. Papageorgiou, A. Magos

${ }^{[1]}$ Centro Hospitalar de Tondela-Viseu, Hospital S.Teotónio, Viseu VISEU Portugal

Summary (4 lines): Intrauterine adhesions are a possible complication after open myomectomy. Our aim was to evaluate the uterine cavity after open myomectomy with hysteroscopy.

Introduction: Myomectomy is the treatment of choice for symptomatic fibroids and open myomectomy remains the principle treatment for multiple, large fibroids when preservation of fertility is desired. In such cases, the functional integrity of the uterus is an important surgical outcome but there is very little information about it.

Material and Methods: We reviewed the clinical records of 15 women who underwent hysteroscopic control 3 months after open myomectomy. All the patients were premenopausal; $40 \%$ were nulliparous. An average of 19 fibroids were removed at myomectomy (range: 261), weighing $378 \mathrm{~g}$ (range:68-986). Most of the excised fibroids were intramural. The uterine cavity was entered in 11 cases, and in $73 \%$ of these we injected Hyalobarrier ${ }^{\mathbb{R}}$. All surgeries were carried out using triple tourniquets to control intra-operative bleeding.

Results: The uterine cavity appeared normal in 13 cases. There were 2 cases of intrauterine adhesions and 2 of fibroids. Based on our findings, 2 women were booked for hysteroscopic myomectomy and 1 for hysteroscopic adhesiolysis.

Discussion: Previous but limited data have shown that less than $2 \%$ of women develop intrauterine adhesions after open myomectomy. Although our sample size is currently small, it suggests that its incidence may be considerably greater.

2

\section{HYSTEROSCOPIC FINDING IN INFERTILE WOMEN UN-} DERGOING CONCOMITANT LAPAROSCOPY

Office \& Diagnostic Hysteroscopy

Y. Ait Benkaddour*, A. Aboulfalah, B. Fakhir, K. Fichtali, K. Harrou, L. Boukhanni, A. Bassir, H. Asmouki, A. Soummani

${ }^{[1]}$ Department of obstetrics and gynecology, University hospital of Marrakesh, Cadi Ayyad University Marrakesh $\sim$ Morocco

Summary (4 lines): Hysteroscopy is considered the gold standard for diagnosis of intrauterine lesions. We report the hysteroscopic finding in the subpopulation of infertile patients who have an indication for diagnosis and therapeutic laparoscopy.

Introduction: Intra-uterine lesions are common in infertile women. However, evidence supporting systematic hysteroscopy in all infertile patients is lacking.

Material and Methods: Objective: To assess hysteroscopic findings in patients undergoing laparoscopy for infertility.

Design: Retrospective, observational study.

Interventions: Diagnostic and operative hysteroscopy and laparoscopy.

Results: Eighty one infertile women underwent hysteroscopy and concomitant laparoscopy. The mean age of patients was 32.8 years. Hysteroscopy was abnormal in $80.2 \%$. The most frequent lesions in patients with secondary infertility were uterine adhesions $(61.5 \%)$ while endometrial polyps were more frequents in primary infertility
(73.3 \%). Pre-operative ultrasound was normal in $59 \%$ of patients. All cases of uterine adhesions (11 patients) have been missed by ultrasound examination and $40 \%$ of lesions diagnosed at hysteroscopy were missed at hysterosalpingography.

Discussion: Intra-uterine lesions seem to be frequents in infertile patients undergoing laparoscopy. Systematic hysteroscopy can be considered as useful in this population. Studies with more number of patients are needed to confirm this finding.

\section{1}

\section{HYSTEROSCOPIC STERILIZATION WITH ESSURE® - 6 YEARS OF OUR EXPERIENCE}

Office \& Diagnostic Hysteroscopy

N. Oliveira*, A.C. Santos, M. Moreira, C. Moreira, M. Oliveira ${ }^{[1]}$ Centro Hospitalar do Baixo Vouga - Aveiro $\sim$ Aveiro $\sim$ Portugal

Summary (4 lines): Successful tubal occlusion was achieved in $98.3 \%$ women who underwent hysteroscopic sterilization in a single centre. Introduction: Essure ${ }^{\circledR}$ is a permanent sterilization procedure that has become widely used. Hysteroscopic sterilization, performed by the bilateral placement of the Essure ${ }^{\circledR}$ micro-inserts into fallopian tube lumens, is a safe and effective method of permanent contraception.

Material and Methods: Retrospective analysis of medical records of patients who underwent hysteroscopic Essure ${ }^{\circledR}$ sterilization between February 2006 and March 2012.

Results: This device was applied in 363 patients. The mean age was $38.0+/-4.6$ (26-48). Among women who underwent pelvic radiography after 3 months of follow up, $85.1 \%$ were considered to have satisfactory micro-insert placement and 2 presented an abdominal ectopic placement of the device. Forty-five women underwent pelvic ultrasound which identified 6 unsatisfactory placements. Hysterosalpingography was performed in 54(14.9\%) cases of unsatisfactory placement and confirmed bilateral tubal occlusion in 47. Successful bilateral occlusion was achieved in $98.3 \%$ of women; 344 cases during the first attempt and 7 cases on the second attempt. Fifty-six per cent of the procedures were performed in an outpatient setting. There was only 1 case of pregnancy $(0,28 \%)$.

Discussion: In our series, Essure ${ }^{\circledR}$ hysteroscopic tubal sterilization with proved to be an effective and safe procedure with a high rate of tubal occlusion.

\section{9}

\section{HYSTEROSCOPIC TUBAL STERILIZATION:EFFICIENCY OF ESSURE® INTRATUBAL DEVICE}

Office \& Diagnostic Hysteroscopy

I. Heras Sedano, C. Del Valle Rubido, V. Marcos González, J.A. Solano Calvo*, R. Cajal, J.J. Delgado Espeja, A. Zapico Goñi

${ }^{[1]}$ Hospital Universitario Príncipe de Asturias $\sim$ Madrid $\sim$ Spain

Summary (4 lines): The rate of failure of Essure despite correct placement and ultrasound justifies the use of hysterosalpingography to confirm impermeability

Introduction: Objective: to evaluate the efficiency of hysteroscopic sterilization with Essure after 2.5 years of experience in Hospital Príncipe de Asturias

Material and Methods: We conducted a retrospective descriptive analysis of the patients with tubal permeability after the insertion of 312 Essure. Correct placement was verified with 3D ultrasound and tubal occlusion confirmed by hysterosalpingography in 52 patients 
3 months after the insertion. Those showing permeability where tested again 6 months post-insertion

Results: We found 6 patients with tubal permeability $(11.54 \%)$. The insertion was successful in 3 patients, with both ostium visible and permeable. In 2 patients one ostium was sealed by adhesions; in another the cavity was occupied by an IUD.

The mean number of visible spirals of the devices was 6.12 (range of correct insertion: 2-12).

Hysterosalpingography was performed 6 months post-insertion. $50 \%$ showed impermeable tubes, which lowered the failure rate to $5.77 \%$. Discussion: Essure is a definitive contraceptive method that provides important advantages to conventional tubal ligation. The rate of failure, similar to other series, makes confirmation of its efficiency by hysterosalpingography advisable.

Ultrasound provides information about the placement of the devices but is not enough to ensure efficiency.

\section{4}

HYSTEROSCOPICAL FINDINGS IN ASYMPTOMATIC INFERTILE WOMEN PRIOR TO IN VITRO FERTILIZATION

Office \& Diagnostic Hysteroscopy

O. Gregoriou, P. Bakas, C. Grigoriadis*, V. Gregoriou, L. Aravantinos, N. Vlahos, G. Creatsas

${ }^{[1]}$ University of Athens, 2nd Department of Obstetrics and Gynecology, Aretaieion Hospital $\sim$ Athens $\sim$ Greece

Summary (4 lines): The aim of this study was to examine if hysteroscopy is necessary or not, prior to in vitro fertilization in asymptomatic women without pathological ultrasound or hysterosalpingography findings.

Introduction: Uterine factors represent only 2-3\% of infertility reasons, but intra-uterine lesions are more common among infertile women. These lesions can interfere with spontaneous fertility and can compromise pregnancy rates in assisted reproduction.

Material and Methods: This was an eight-year retrospective study. All records of asymptomatic women, without pathological ultrasound findings who underwent hysteroscopy because of infertility prior to in vitro fertilization between January 2003 and December 2010 were analyzed.

Results: Totally, 173 asymptomatic infertile women were included in the study. No intra-uterine abnormality was detected in $60.1 \%$ of the cases. In the rest cases hysteroscopy revealed: endometrial polyps $(14.5 \%)$, submucosal leiomyomas $(11 \%)$, uterine septa $(9.2 \%)$, and synechia $(5.2 \%)$. Surgical treatment of these lesions by operative hysteroscopy followed. No intraoperative complications occurred and the hospitalization time was less than 8 hours in all cases.

Discussion: Office hysteroscopy revealed intra-uterine pathology in $39.9 \%$ of asymptomatic infertile patients with normal hysterosalpingography. Therefore, we recommend routine hysteroscopy prior to in vitro fertilization.

285

IMPORTANCE OF MYCROHISTEROSCOPY IN HIGH RISK PATIENTS. DETECTING EARLY ENDOMETRIAL CANCER AND PREMALIGNANT LESIONS

Office \& Diagnostic Hysteroscopy

J.E. Dotto*, P. Da Graca Mila, M.A.J. Bigozzi, B. Lema, J.E.J. Dotto, J. Hamou

${ }^{[1]}$ Instituto Argentino de Diagnóstico y Tratamiento $\sim$ Ciudad de Buenos Aires Argentina
Summary (4 lines): Our objective with this work was to determine the importance of the use of hysteroscopy in high-risk patients. Design: retrospective multicentric double blind study.

Introduction: Microhysteroscopy is a safe and non-traumatic technique that provides a satisfactory assessment of the uterine cavity, and it is an effective tool for the detection of premalignant lesions and early stages of endometrial cancer.

Material and Methods: 4120 patients were studied with microhysteroscopy with a Hamou no. 1,4-mm, rigid hysteroscope. 420 patients ere studied by using a continuous Bettocchi office hysteroscope. In all cases we obtained biopsy samples that were sent to hystopathological examination Patients were divided into 3 groups: 1668 high-risk group, asymptomatic: 540 (group A) and symptomatic: 1128 (group B). Group C: consisted of 2872, low-risk symptomatic patients.

Results: High risk patients (symptomatic and asypmtomatic): $\mathrm{n}=1668$. Findings: atypical hyperplasia 12, carcinoma 18 and Metastases 1. High risk patients (asymptomatic) $n=540$. Findings: atypical hyperplasia 4, carcinoma 6 and metastases 1. Early detection of lesions/screening of asymptomatic patients was $11 / 540(2.03 \%)$. In this group we found a lobular breast carcinoma metastases.

Discussion: Our proposal is to use microhysteroscopy as a screening method in high risk patients.

INFLUENCES ON PATIENT LED CHOICE OF TREATMENT FOR HEAVY MENSTRUAL BLEEDING (HMB) IN A ONESTOP MENSTRUAL DISORDERS CLINIC IN SCOTLAND

Office \& Diagnostic Hysteroscopy

C.A. Higgins*, C.R. Taggart, M.W. Rodger

${ }^{[1]}$ University of Glasgow $\sim$ Glasgow $\sim$ United Kingdom

Summary (4 lines): Women with HMB have a number of options for symptom management. We found previous treatment experience had the greatest influence upon patient led choice of treatment.

Introduction: Guidelines for the management of women with HMB are well established in the UK. However, little is known about the influences on a patients' choice of treatment.

Material and Methods: We conducted a retrospective case note review of a one-stop menstrual disorder clinic. Data included age, symptoms, previous management and O\&G history. All had normal pelvic anatomy and endometrial histology where appropriate. Data was analysed using ANOVA or Pearson Chi-squared tests, $\mathrm{p}<0.05$ considered significant. Results: 88 women, mean age 42.5(SD 7.1)years were analysed in groups according to treatment chosen in clinic. Treatment choice was not significantly affected by age, symptom duration, cycle irregularity, dysmenorrhoea, parity, mode of delivery, contraception or abnormal smears $(p>0.07$ for all). Previous management was associated treatment choice $(\mathrm{p}=0.02)$; LNG-IUS use had the greatest influence $(\mathrm{p}<$ $0.01) ; 48.7 \%(\mathrm{n}=36)$ opting for LNG-IUS with no previous use, and $87.5 \%(n=7)$ of those opting for surgery with previous LNG-IUS use. Discussion: Patient selection of treatment for HMB in a dedicated onestop menstrual disorder clinic is influenced by previous treatment experience, particularly treatment with LNG-IUS.

\section{6}

INITIAL EXPERIENCE WITH ESSURE STERILISATION IN A TEACHING HOSPITAL

Office \& Diagnostic Hysteroscopy

C. Daly*, D. Hunter

${ }^{[1]}$ Belfast Health and Social Care Trust $\sim$ Belfast $\sim$ United Kingdom 
Summary (4 lines): ESSURE introduced January 2011.97 procedures have been performed. Follow up undertaken 67. Data with regards to procedure details and follow up is included.

Introduction: All sterilsiations were performed laparoscopically in this unit. Many patients remained in hospital overnight. An alternative to this invasive surgery was sought.

Material and Methods: Prospective data collection on 97 patients undergoing ESSURE sterilisation from January 2011 to date. Demographics, operative details and post procedure follow-up were recorded and analysed.

Results: Bilateral placement was achieved in $93 \%$ of cases. A further $3 \%$ with initial unilateral placement subsequently achieved bilateral placement. The procedure was well tolerated: average overall pain score of 3.5 and device placement time of 240 seconds. Average number of trailing coils: 4.10 . Satisfactory device placement was noted on plain X-ray film in $64 \%$ of cases. 16 cases had HSG to confirm tubal occlusion and satisfactory device placement $(100 \%$ confirmation). Difficulties with follow up were more frequent in patients having concomitant endometrial ablation(HSG could not be performed). No serious adverse events or complications (1 case of post ESSURE/ Novasure haematometra)were encountered in this series and no pregnancies have been reported in our case series.

Discussion: ESSURE is a safe, effective and well tolerated procedure for women seeking permanent contraception in this population.

\section{9}

INTRODUCTION OF AMBULATORY GYNAECOLOGY CLINIC IN A DISTRICT GENERAL HOSPITAL. OUTCOMES AND PATIENT EXPERIENCE.

Office \& Diagnostic Hysteroscopy

D. Morgan*, J. Magee

${ }^{[1]}$ Antrim Hospital $\sim$ Antrim $\sim$ United Kingdom

Summary (4 lines): Our experience of introducing office hysteroscopy has been beneficial for our hospital and our patients. Our study shows high levels of success and patient satisfaction.

Introduction: Providing procedures in an outpatient setting has clinical and economic benefits. We introduced this service and undertook a study to ascertain the outcomes, discharge at first visit rate and patient satisfaction

Material and Methods: 47 consecutive patients attending the service were included. $64 \%$ underwent hysteroscopy using no touch vaginoscopic method. Hysteroscopic instruments were available. Patients undergoing hysteroscopy were asked to complete an anonymous questionnaire

Results: Post menopausal bleeding (53\%) and abnormal bleeding (26\%) were the commonest referrals. $90 \%$ of hysteroscopies were completed satisfactorily. $6.7 \%$ were abandoned due to pain. $3.3 \%$ were unsatisfactory due to menstruation and menses. The relative risk of failure in post menopausal women $(1.5295 \% \mathrm{CI} 0.15-15.10)$ was not significant. $57 \%$ of women were treated and discharged at first visit. $64 \%$ reported pain. The mean pain score on a scale of 1-10 was 3.5. When asked if they would recommend the procedure $100 \%$ said yes Discussion: The clinic has enabled most women to be managed in one visit. The comments from women have been positive and there were no complications. The results support the development of the service to include other procedures e.g. sterilization.

166

IS 3D ULTRASOUND ENOUGH TO CONTROL PLACEMENT OF ESSURE® DEVICE AND CONFIRM PERMANENT TUBAL STERILIZATION?

Office \& Diagnostic Hysteroscopy
J.A. Solano Calvo*, H. García Briz, C. Del Valle Rubido, J. González Hinojosa, P. Juez, A. Zapico Goñi, J.J. Delgado Espeja

${ }^{[1]}$ Hospital Universitario Príncipe de Asturias $\sim$ Alcalá de Henares $\sim$ Spain

Summary (4 lines): It is necessary to perform hysterosalpingography to all patients undergoing hysteroscopic tubal sterilization with Essure ${ }^{\circledR}$, as 3D ultrasound is not able to predict tubal occlusion.

Introduction: Our objective is to find the relation between the number of spirals left in the uterine cavity and the length of the intracavitary devices measured by 3D ultrasound and the presence or absence of contrast in hysterosalpingography (HSG).

Material and Methods: Observational prospective study of 53 women sterilized by insertion of Essure intratubal device by hysteroscopy. 3 months post-insertion they underwent 3D ultrasound to confirm the correct placement of the devices. The intracavitary component of the devices was measured and they were classified following Legendre's classification. HSG was performed to confirm permanent tubal occlusion.

Results: $60.71 \%$ of cases were classified as perfect, $25 \%$ distal, $7.24 \%$ proximal and $7.14 \%$ very distal, following Legendre's criteria. We were not able to relate the number of spirals to the intracavitary length, due to the low number of cases (Rho Spearman), as well as the device placement and HSG. The 2 cases of bilateral tubal permeability had a perfect placement.

Discussion: Despite having to increase the number of cases of our study, because of the absence of relation between tubal definitive occlusion and the position of Essure devices in 3D ultrasound, it is necessary to perform HSG to all patients.

\section{9}

\section{MEDGYN ENDOSAMPLER VS ENDOCURETTE IN AN OUT- PATIENT HYSTEROSCOPY CLINIC}

Office \& Diagnostic Hysteroscopy

\author{
A. Khalid, C. Burke* \\ ${ }^{[1]}$ Cork University Maternity Hospital $\sim$ Cork $\sim$ Ireland
}

Summary (4 lines): MedGyn Endosampler and Endocurette endometrial biopsy devices were compared in an outpatient hysteroscopy clinic setting.

Introduction: Literature comparing outcomes such as pain and sample adequacy between MedGyn Endosampler and Endocurette is currently lacking.

Material and Methods: A prospective, randomised trial of MedGyn Endosampler and Endocurette in pre-and postmenopausal women attending our Outpatient Hysteroscopy Clinic. Pain scores, number of device insertions and sample adequacy were recorded among other outcomes.

Results: 106 women participated: 55 pre- and 51 postmenopausal. Mean pain scores were 5.83 and 4.58 in pre- and postmenopausal groups using the MedGyn Endosampler and 4.69 and 4.88 for pre-and postmenopausal patients in the Endocurette group. More premenopausal women in the MedGyn Endosampler group reported pain scores $>5$ ( $p$ 0.02). Repeated $(>2)$ insertions were eight times higher in the Endocurette vs MedGyn Endosampler groups in premenopausal women ( $p$ 0.0001). There was no difference in the rate of inadequate samples between premenopausal groups. Postmenopausal women had more inadequate samples compared to premenopausal women ( $\mathrm{p} 0.0025)$.

Discussion: Significantly fewer device insertions were required to obtain a satisfactory sample with MedGyn Endosampler resulting in a shorter procedure and a potentially lower risk of post-procedural infection, but with higher pain scores. 
452

\section{OUR EXPERIENCE IN OUTPATIENT OPERATIVE HYSTEROSCOPY}

Office \& Diagnostic Hysteroscopy

C. Vasile*, G. Sacco

${ }^{[1]}$ Hospital Mirano $\sim$ Mirano Venice $\sim$ Italy

Summary (4 lines): Nowadays new small caliber instruments and techniques are achieved in order to increase patients compliance during outpatient hysteroscopy.

Introduction: The development of miniistruments and tecniques determined an increased patients compliance with subsequent performance of a larger number of outpatient procedures.

Material and Methods: Between Sept 2008 and Sept 2011142 minioperative hysteroscopic procedures performed without anaesthesiologic support, in an outpatient setting, using a $2.9 \mathrm{~mm}$ optic $30^{\circ}$, a 5 Fr operative channel with mechanical or bipolar non disposable electrods (Storz). The uterine distension was achieved with saline solution. Pain level was determined using an analogic pain score.

Results: 142 patients with a median age of 46 yrs (range 23$74)$ underwent operative HYS. The procedures were: 80 polypectomies( 65 endometrial and 15 endocervical),30 permanent sterilizations Essure, 8 adhesyolisis and 24 endometrial focal resections. The mean operative time was 14.3 minutes(range 5-19 minutes). No complication was revealed and the mean pain score was 3 (range0-6).

Discussion: An attentive patients' selection in association with the use of adequate miniinstruments and a good level of HYS"no touch" vaginoscopic training are mandatory in order to perform quick, painless, safe procedures and reduce the inpatient hysteroscopies.

255

OUTPATIENT HYSTEROSCOPY IN THE MANAGEMENT OF PERSISTENT PREGNANCY OF UNKNOWN LOCATION (PPUL) Office \& Diagnostic Hysteroscopy

K. Afors*, J. Inder Reiden, P. Bulmer

${ }^{[1]}$ St George's Hospital NHS Trust $\sim$ London $\sim$ United Kingdom

Summary (4 lines): PPUL can be a failed intrauterine pregnancy or an ectopic, both of which can be managed expectantly. If the PPUL is an ectopic, expectant management could lead to tubal rupture. We have used outpatient hysteroscopy to locate the pregnancy and offer personalized management for patients.

Introduction: PUL is a descriptive term used when a woman with a positive pregnancy test does not have a pregnancy visualized on TVS. Most of these will be a failed IUP; a small number will be ectopic pregnancies. Whilst expectant management is the norm some women will receive methotrextae therapy with the rationale that an ectopic cannot be excluded. We propose a novel use of outpatient hysteroscopy to identify those PPUL's which are a failed intrauterine pregnancy. Those with an empty uterus will be assumed to have an ectopic pregnancy.

Material and Methods: Study design: prospective cohort pilot study. Subject: women with a PPUL with a suboptimally rising BHCG (rate of change $<35 \%$ over 48 hours).

Methods: Patients are recruited and undergo immediate outpatient hysteroscopy and targeted biopsy.

Results: In the first patient recruited to this study we identified intrauterine trophoblastic tissue which was confirmed on histology. The patient was successfully managed expectantly.
Discussion: We have demonstrated that outpatient hysteroscopy is a diagnostic test able to identify intrauterine trophoblastic tissue if present, enabling us to personalize treatment.

318

OUTPATIENT HYSTEROSCOPY IS THE MOST COSTEFFECTIVE INVESTIGATION FOR HEAVY MENSTRUAL BLEEDING

Office \& Diagnostic Hysteroscopy

N. Cooper*, P. Barton, M. Breijer, K. Khan, B. Mol, J. Clark

${ }^{[1]}$ University of Birmingham $\sim$ Birmingham $\sim$ United Kingdom

Summary (4 lines): An economic analysis of investigations for heavy menstrual bleeding (HMB) found outpatient hysteroscopy (OPH)was the most cost-effective test.

Introduction:

Material and Methods: A model-based economic evaluation compared 13 strategies for investigation of HMB. Data were derived from systematic reviews, individual patient data, focused searches and the consensus of an expert clinical panel. The model reflected current service provision in a one-stop clinic where all indicated tests would be available during a single visit. The tests evaluated were $\mathrm{OPH}$, transvaginal scan (TVS), saline infusion scan (SIS) and endometrial biopsy (EBx). The tests were evaluated alone and in combination. The outcome measure was cost effectiveness based upon incremental cost per patient satisfied.

Results: Two cost-effective testing strategies were identified; OPH alone and used in combination with EBx. The strategy OPH $+\mathrm{EBx}$ was more effective than OPH alone but cost $£ 21000$ more per additional satisfied patient. OPH alone cost only $£ 360$ per additional satisfied patient when compared to insertion of a levonorgestrel intrauterine system (LNG-IUS) without investigation. Testing with $\mathrm{OPH}$ was also the most cost-effective approach for women wishing to preserve fertility and for women whose symptoms were refractory to treatment with a LNG-IUS.

Discussion: OPH should be the first line diagnostic test for the investigation of women presenting to secondary care with HMB.

447

PELVIC PAIN AND ESSURE®

Office \& Diagnostic Hysteroscopy

B. Povedano Cañizares*, B. Rodriguez Jimenez, J. Arjona Berral, E. Velasco Sanchez

${ }^{[1]}$ Hospital Universitario Reina Sofia $\sim$ Cordoba $\sim$ Spain

Summary (4 lines): Nowadays there is a volumen of literatura related to the feasibility of the Essure device, but, no previous study with the appearance of pelvic pain in this women as a mothos complication.

Introduction: The main objective is to analyse the development of chronic pelvic pain in women with Essure.

Material and Methods: Review of medical hystory of 7 women with pelvic chronic pain after Essure placement registered in our database. Women underwent procedure between 2005 and 2011.

Results: In 7 patients $(0.16 \%)$ of 4274 women who underwent Essure hysteriscipic sterilization during this period, started with symptoms of pelvic chronic pain that required the microinserts withdrawal. $57.2 \%$ classified pain during procedure as medium-high. Media of exterior coils in uterine cavity were 5 in both tubes. $85.7 \%$ of patients didn't required analgesia after procedure and only in one case it is registered a vasovagal syncope. In three $(42.85 \%)$ of the 7 cases, surgeon 
described the procedure as difficult it is so that an hysterosapingogram was performed as control method. In $100 \%$ of cases, both devices were retired improving all women of symptoms.

Discussion: Hysteroscopic tubal sterilization it's a safe technique but in few cases could be related with the appearance of pelvic pain. In our review, $100 \%$ of patients improve the symptoms after the withdrawal of the devices. In $85 \%$ of cases, this withdrawal was performed by laparoscopy.

\section{2}

\section{PERMANENT CONTRACEPTION WITH ESSURE DEVICE IN WOMEN WITH SUSPECTED NIQUEL ALLERGY}

Office \& Diagnostic Hysteroscopy

E. Velasco Sanchez*, L. Nieto Pascual, J.E. Arjona Berral, B. Povedano Cañizares

${ }^{[1]}$ Hospital Universitario Reina Sofia $\sim$ Cordoba $\sim$ Spain

Summary (4 lines): To confirm that Essure device can be performed in women with niquel allergy after a prospective study of 45 patients suspicius for this allergy.

Introduction: To confirm that Essure insertion in patients with suspected niquel allergy it's safe.

Material and Methods: Prospective study of 45 patients suspicius for niquel allergy between 2003 to 2012. 5234 procedures were performed in our unit during this period. Patients with less than six months of follow up are excluded(5). A pach test was performed to all of them to confirm the allergy. Personal survey was performed to all of them. Five patients didn't accept the pach test.

Results: 24 (80 \%)patients have a positive result for niquel allergy. Media of time of surveillance after insertion is 3,77 years. In 29 $(96,7 \%)$ of patients no symptoms were found. Only 1 of them refers perimenstrual pain treated with normal analgesia. Since insertion, no patient have had allergies symptons. No device has been removed. $100 \%$ of patients would performed again the procedure. The incidence of niquel allergy in our serie is less tan $1 \%$, and in $80 \%$ of suspected allergy its confirmed.

Discussion: Suspicied other confirmed niquel allergy should not be a contraindication for women desiring permanent contraception with Essure microinserts. We recomend in this cases leave between 5 and 7 coils remaining in uterine cavity and if symptoms appear, a hysteroscopic outpatient removal of devices could be performed.

\section{3}

\section{PREGNANCY AFTER TUBAL STERILIZATION WITH ESSURE ${ }^{\circ}$ DEVICE}

Office \& Diagnostic Hysteroscopy

V. Marcos González, C. Del Valle Rubido, I. Heras Sedano, J.A. Solano Calvo, J.J. Delgado Espeja*, R. Cajal Lostao, A. Zapico Goñi

${ }^{[1]}$ Hospital Príncipe de Asturias $\sim$ Alcalá de Henares $\sim$ Spain

Summary (4 lines): A woman who was sterilised with Essure device became pregnant despite having tubal occlusion confirmed by hysterosalpingography

Introduction: Tubal sterilization with Essure device is highly effective. However, there are cases of failure after its execution

Material and Methods: We have performed a systematic review of our patient's medical record

Results: A 33-year-old woman had bilateral tubal occlusion with Essure by hysteroscopy at Hospital Príncipe de Asturias, following the normal procedure (alternative contraception before and after the insertion).
The insertion had no incidents, leaving 3 visible spirals at the right ostium and 4 at the left one. She was on the 26th day of cycle.

Three months afterwards the patient was pregnant with her last menstrual period in the same cycle of the procedure. She had a voluntary interruption of pregnancy outside our hospital.

During her follow-up we conducted 2D and 3D ultrasound which showed normal placement of the devices (by Legendre's classification). She underwent hysterosalpingography 4 months post-insertion showing non-permeable tubes, and was discharged.

Seven months afterwards the patient became pregnant again Discussion: Hysterosalpingography is the gold-standard technique to evaluate tubal permeability. Non-fulfillment of alternative contraception may explain the first pregnancy. However, the reason of her second pregnancy is still unknown. Our patient became pregnant with normal imaging techniques and hysterosalpingography

315

\section{PROSPECTIVE EVALUATION OF ENDOMETRIAL ABLATION WITH THERMABLATE®}

Office \& Diagnostic Hysteroscopy

B. Koullali*, A. Vollebregt, M.H. Emanuel, E. Hijmans

${ }^{[1]}$ Spaarne Ziekenhuis Hoofddorp Hoofddorp Netherlands

Summary (4 lines): Prospective study measuring satisfaction and effect of the Thermablate Endometrial Ablation System (TEAS) in treatment of menorrhagia. The majority of women were satisfied, only $22 \%$ required additional surgical treatment.

Introduction: TEAS is one of the newest endometrial ablation devices to treat menorrhagia in an office setting. The aim of this study was to evaluate the acceptability and efficacy of TEAS.

Material and Methods: In this prospective cohort study women with menorrhagia were treated with TEAS after former conservative therapy failed. Excluded were women with submucosal fibroids, polyps, desire to preserve fertility and suspicion of endometrial malignancy. Main outcome was satisfaction and effect of TEAS, measured by questionnaires at 3,6 or 12 months and recording additional therapies during follow up.

Results: The majority ( $92 \%$ ) of the 249 women included was treated at the outpatient clinic. A positive effect on menstruation was reported by $82 \%$. Complete amenorrhea was achieved in $18 \%$. $194(78 \%)$ women were satisfied with the procedure and $210(84 \%)$ would recommend it to a friend. After a mean follow up of 13 months (range $2-46), 55$ women $(22 \%)$ required additional surgical treatment. These women were significantly younger compared to women who did not require additional surgical treatment ( 43 vs. 45 years, $p=0.02$ ).

Discussion: TEAS appears to be a successful outpatient procedure. Further studies should focus on long term efficacy and predictors of success.

\section{7}

\section{RESECTOSCOPIC TREATMENT OF ISTHMOCELE}

Office \& Diagnostic Hysteroscopy

A. Haxhihyseni*, D. Vercuni

${ }^{[1]}$ Regional Durres Hospital $\sim$ Durres $\sim$ Albania

Summary (4 lines): Isthmocele is an anatomic defect like e reservoirlike pouch on the anterior wall of the uterine isthmus, the treatment of isthmoceleis with resectoscopic surgery. We study 711 cases, found 23 ishtmocele treated with resectoscopy.

Introduction: Isthmocele is an anatomic defect. All women examined had postmenstrual discharge of dark red or brown material from 2 to 
12 days. Our study concern the frequency of isthmocele, the treatment resectoscopic surgery and the results to postmenstrual abnormal uterine bleeding as main symptoms.

Material and Methods: Our 711 patients was selected occasionally throw the patients that hade one to three cesarean delivery. We made diagnostic hysteroscopy and found 34 isthmocele, 11 of isthmocele were asimptomatice. 23 women with simptomatic isthmocele underwent resectoscopic treatment.

Results: We from 711 women studied found 34 cases of isthmocele. $23 / 34$ of them had simptomps - postmenstrual abnormal uterin bleeding.11/34 was asimptomatic. The cases with simptomes was treated resectoscopically. Symptoms disapeard in 21 of them. Histologic diagnosis

Discussion: gynecologist doctor ought to think for possibility of isthmocele. Can be diagnosed with hysteroscopic examination. The best way to treat the anatomic defect of isthmocele is resectoscopic surgery that eliminate the simptoms in $91 \%$ of cases

167

\section{RISK FACTORS FOR ENDOMETRIAL POLYP IN POSTMENOPAUSAL WOMEN.}

Office \& Diagnostic Hysteroscopy

M.E. Santos*, S.T. Matsumura, J. Moura, E. Chagas, F. Adami, C.P. Barbosa, Â. Nimwegen

${ }^{[1]}$ Faculdade de Medicina do ABC $\sim$ Santo André $~$ Brazil

Summary (4 lines): The authors investigated the risk factors of endometrial polyp among postmenopausal women from ambulatory hysteroscopy clinic of Faculdade de Medicina do ABC, Brazil. The risk factors for polyp were advanced age, obesity and hypertension.

Introduction: The risk factors of endometrial hyperplasia and carcinoma in postmenopausal women are well-known. However, it is not well defined whether these risks can also be applied to all endometrial polyps, independently of these lesions being associated to malignization.

Material and Methods: Retrospective study. Clinical and demographics data were collected from medical records of 1102 patients submitted to hysteroscopy between August 2007 and September 2009. Indications of hysteroscopy were: endometrial thickness $>4 \mathrm{~mm}$ and/ or postmenopausal bleeding. Hysteroscopic diagnoses were: normal ( $\mathrm{n}=434)$, polyp (636), other results (32). Statistical analyses were made between normal- and polyp-group by Poisson regression.

Results: The risk factors identified were advanced age (OR 1,10), obesity (OR 1,23) and hypertension (OR 1,11).

Discussion: Endometrial diseases may be regarded as a consequence of imbalance between proliferation and apoptosis in the endometrial cells due to disturbances of the hormone-metabolic pathways and the decline in immunity with increasing age. However, benign and malign diseases may not have the same etiology, as well as, the same risk factors

\section{4}

RISK OF MALIGNANCE OF ENDOMETRIAL POLYPS

Office \& Diagnostic Hysteroscopy

B. Povedano Cañizares*, I. Partera Tejero, J. Arjona Berral, E. Velasco Sanchez, J. Monserrat Jordan

${ }^{[1]}$ Hospital Universitario Reina Sofia $\sim$ Cordoba $\sim$ Spain

Summary (4 lines): To evaluate the premalignant and malignant condition of endometrial polyps and the possible risk factors associated with carcinoma.
Introduction: Endometrial polyps are a frequent finding during hysteroscopy, most of them asymptomatic. It's a frequent finding also in women during control.

Material and Methods: Retrospective cohort study of 2404 polypectomies performed in our Hysteroscopy Outpatient Unit between june 2005 till december 2011. Patients are divided in premenopausal and postmenopausal condition, with or without bleeding disorders. Data were analyzed with spss 15.0 for Windows.

Results: $55,14 \%$ of polyps (1324) were found in postmenopausal patients. In $56,70 \%$ of this patient bleeding was the reason for hysteroscopy and in 36,20\% was an ultrasound finding without symptoms. Percentage are similar for premenopausal women: $56,90 \%$ and $33,80 \%$. Hystopathology of polyps were: $1,3 \%(17)$ premalignant polyps and $0,9 \%$ (13) malignant in postmenopausal patients; and $0,3 \%$ ( 3 ) and $0,1 \%$ (1) in premenopausal women. $0,5 \%$ of the asymptomatic patients had a malignant or premalignant disease, all of them postmenopausal, versus $1,22 \%$ of symptomatic patients. $(\mathrm{p}<$ $0,05)$.

Discussion: There is no risk of potential malignancy in asymptomatic patients with polyps and premenopausal condition. Statystical differences are shown between the risk of malygnancy, the size, hormonal status and symptoms. Nevertheless, risk of potential malygnancy is very low $(0,6 \%)$.

442

\section{TAMOXIFEN USE AND ENDOMETRIAL LESIONS: HYSTEROSCOPIC, ANATOMOPATHOLOGICAL AND IMMUNOISTOCHEMICAL FINDINGS IN POSTMENOPAUSAL WOMEN WITH BREAST CANCER}

Office \& Diagnostic Hysteroscopy

R. Dibi, G. Silveira, C. Zettler, S. Pessini*, A. Ayub, S. Almeida

${ }^{[1]}$ Complexo Hospitalar Santa Casa $\sim$ Porto Alegre $\sim$ Brazil

Summary (4 lines): Endometrial findings in patients using tamoxifen. Introduction: Tamoxifen is an agonist on the endometrium and increase expression of Ki-67 and steroid receptors. The aim is to evaluate the hysteroscopic, histological and immunohistochemical profiles of postmenopausal females taking tamoxifen.

Material and Methods: Forty postmenopausal patients using tamoxifen submitted to hysteroscopy and histology between were evaluated. Clinical and pathological data were analyzed and ki-67, ER and PR were determined.

Results: The most common histological finding was atrophic endometrium $(22.5 \%)$ and hysteroscopic lesion was endometrial polyp. IHC showed that $85 \%$ of the cases were PR-positive, $75 \%$ ER-positive and $50 \% \mathrm{Ki}-67$ positive. Endometrial polyp and polyp associated with atrophic endometrium were ER-positive $(p=0.019)$. A negative ER was more often found in the atrophic endometrium $(p=0.01)$. The longer the time of menopause, the lesser the ki-67 expression in the endometrium. Ki-67 showed greater expression in the endometrium of younger postmenopausal women $(\mathrm{p}<0.05)$.

Discussion: We found a high expression of steroid receptors in the endometrium. The most common hysteroscopic finding was the endometrial polyp. All cases of endometrial polyp were ER-positive. Although most histological findings were benign, only $22.5 \%$ were atrophic.

462

THE EFFECT OF CONTRACEPTIVE METHOD ON ESSURE® MICRO-INSERT PLACEMENT

Office \& Diagnostic Hysteroscopy 
P. Pérez, P. Pérez*

${ }^{[1]}$ University Hospital Complex of Vigo Vigo (Pontevedra) Spain

Summary (4 lines): MAIN TARGET: Evaluate the influence of the contraceptive method in the ESSURE ${ }^{\circledR}$ insertion difficulty.

Introduction: the display of the ostia tubal has been proposed as the factor that limits the placement of the essure.

Material and Methods: Method: Ambulatory tubal oclusion histeroscopy with ESSURE ${ }^{\circledR}$ Micro-insert.

Main variables: Contraceptive method. Channeling difficult.

Visualization

Results: 334 patients

Channeling was easy in $74 \%$ women with hormonal contraceptive methods, difficult in $21,3 \%$ and failed $4,7 \%$. Intrauterine device patients was easy in 56,4\%, difficult in $35,9 \%$ and failed in $6,7 \%$. So, channeling was easy in first attempt in 316 women(94,6\%), and in the second attempt were 331(99,1\%) Ostium Visualization in barrier method patients was good in $67,7 \%$, an poor in $33,3 \%$. In the contraceptive hormonal groups methods was good in $73,6 \%$ and poor in $26,4 \%$. Referring to IUD women carriers was good in $28,2 \%$ and poor in $71,8 \%$, being statistic considerable significative. Referring to pain, with the failed technique, there were no pain in $61,1 \%$, and some pain in $65,3 \%$. If the channeling was easy. Pain was not reported by $71.8 \%$, and some soft disturbs in 28,2 .

All women said absolute satisfaction in $98,8 \%$, and no satisfaction in $1,2 \%$. Discussion: Worst ostium visualization has not significant influence in the insert difficulty.

Higher channeling difficulty it takes more pain.

Micro-insert introduction difficulty was absolutely independent on thecontraceptive method.

THE FIRST EXTRAHOSPITALARY HYSTEROSCOPY UNIT IN BARCELONA: RESULTS OF 500 AMBULATORY INSERTIONS OF ESSURE

Office \& Diagnostic Hysteroscopy

S. Haimovich*, G. Mancebo, G. Mancebo, J.M. Sole, S. Agramunt, C. Serra, R. Carreras

${ }^{[1]}$ Del Mar University Hospital $\sim$ Barcelona $\sim$ Spain

Summary (4 lines): In 2009 we placed the hysteroscopy unit outside the hospital, in a primary health center(PHC), where we started to perform female sterilization with Essure.

Introduction: The Essure, a device used for female sterilization, approved by the FDA in 2002. Initially the technique was performed in surgery room and anesthesia was used. We placed the hysteroscopy unit outside the hospital, in a PHC.

Material and Methods: Between January 2009 and January 2012 we performed 502 procedures. The microinserts were placed through the 5 F-working channel of a 5 -mm, continuous- flow, $30^{\circ}$ _ hysteroscope with use of a saline distension medium. We didn't use aspeculum in any case and no anesthesia. Ten minutes after the procedure was finished, the patient was given a questionnaire in which we evaluated, pre procedure information, medication taken, visual analogue scale (VAS)for evaluating pain, satisfaction

Results: A total of 502 patients were enrolled in the study. The average (SD) age of participants was 37.1 (6.2) years, with the majority being immigrants. Successful placement occurred in 487 (97\%). The overall average pain reported for the procedure was 2.2 (SD 2.05; $95 \%$ CI 2.3 , 2.9).

Discussion: After the first 500 insertions of Essure microinserts with an office hysteroscopy procedure without anesthesia in a PHC, our results support high tolerability and satisfaction.
163

WHAT ARE THE CHANCES OF FINDING MALIGNANT ENDOMETRIAL DISEASE IN BLEEDING MENOPAUSE WOMEN?

Office \& Diagnostic Hysteroscopy

I. Ilic*, Y. Cunarro Lopez, P. Menendez De Luarca Menendez, M. Urdaci Elizondo, M.M. Munoz Chapuli, R. Mendizabal Castellanos, L. Ortiz Quintana

${ }^{[1]}$ Gregorio Maranon University Hospital Madrid Spain

Summary (4 lines): We aim to describe the utility of uterine bleeding in menopause women to predict or overrule malignant endometrial pathology.

Introduction: Criteria for indicating office diagnostic hysteroscopy in menopause women is much debated issue, and we aim to evaluate the role of uterine bleeding as a sole marker for malignant endometrial pathology. Material and Methods: We study 278 menopause patients who underwent office diagnostic hysteroscopy from 15/12/2010 - 01/04/2012 in our unit.

Results: Median age $60.0 \pm 9.9$ years, ranging $42-84$ years. Uterine bleeding was present in 101 patients, $36.3 \%$. No biopsy or insufficient specimen was the case in 56 patients $(20.1 \%)$, most of which $(69.6 \%)$ had hysteroscopy described as atrophic endometrium. We encountered 7 cases of adenocarcinoma $(2.5 \%), 4$ complex hyperplasia $(1.4 \%)(3$ with atypia), 2 atypical polyps $(0.7 \%)$.

Sensitivity of uterine bleeding in menopause women as a sole marker for malignant endometrium condition is of $76.9 \%$ (IC $49.7-91.8 \%$ ), specificity $65.7 \%$ (IC 59.8-71.1\%), PPV 9.9\% (IC 5.5-17.3\%), NPV $98.3 \%$ (IC 95.1-99.4\%). Diagnostic odds ratio is 6.4 (IC 1.7-23.7). Prevalence of $4.7 \%$.

Discussion: Prevalence of malignant endometrial pathology in bleeding menopause women in our series of patients is low, and it was highly unlikely to diagnose this pathology in women without uterine bleeding. Most of the patients without biopsy specimen were catalogued as atrophic endometrium during hysteroscopy.

\section{8}

\section{A NEW HELPFUL TOOL IN LARAROSCOPIC APROACH OF OVARIAN MASSES Oncology}

M. Brito*, J. Ribeiro, N. Nogueira Martins, F. Nogueira Martins ${ }^{[1]}$ Hospital São Teotonio-Viseu $\sim$ Viseu $\sim$ Portugal

Summary (4 lines): Risk of Ovarian Malignancy Algorithm (ROMA)is a dual biomarker combination of HE4 and CA125. Is it helpful in our clinical practice?

Introduction: Discrimination between benign and malignant ovarian neoplasms is essential to prepare adequate surgical approach. The authors study the impact of ROMA in the preoperative evaluation of ovarian masses.

Material and Methods: During the past year, patients diagnosed with ovarian masses scheduled for surgery were prospectively included $(\mathrm{n}=$ 109). Blood samples were collected and tested for CA125 and HE4 markers. ROMA was then calculated using cut-off values of $7.4 \%$ and $25.4 \%$ for premenopausal and postmenopausal respectively. A cut-off of $75 \mathrm{pM}$ was used for HE4 and $35 \mathrm{U} / \mathrm{ml}$ for CA125. The sensibility and specificity to distinguish between malignant versus benign ovarian mass were calculated for each parameter.

Results: Our sample revealed malignant $(n=17)$, borderline $(n=2)$, benign $(n=89)$ ovarian neoplasms and one fibroid. The sensibility achieved for CA125, HE4 and ROMA was $0.89,0.94$, and 0.94 
respectively. ROMA specificity was the highest $(0.90)$ and its negative predictive value was $98.7 \%$.

Discussion: ROMA has revealed to slightly increase the sensibility and specificity of CA125, especially in premenopausal women presenting with endometriosis. The high negative predictive value presented by ROMA is now a helpful tool to prepare our surgical team, increasing the possibility of a laparoscopic approach.

\section{0}

\section{ACCEPTABLE INDICATIONS OF LAPAROSCOPY IN THE OVARIAN CANCER \\ Oncology}

J. Molero Vílchez*, E. Martínez Lamela, S. Sancho Garcia, J.L. Prieto Alonso, Y. Expósito Lucena, E. Gallego Pastor, F.J. Salazar Arquero

${ }^{[1]}$ Hospital Universitario Infanta Leonor $\sim$ Madrid $\sim$ Spain

Summary (4 lines): A serie of 69 cases of ovarian cancer treated from April of 2008 to February of 2012 is rewied and a descriptive study about the value of laparoscopy is made.

Introduction: The laparoscopic valuation of operability in advanced stages of ovary cancer avoids suboptimal surgeries.

Material and Methods: Average age of presentation was 56.75 years. Serosopapilar cistoadenocarcinoma (21 cases, $30.4 \%$ ) was the most frecuent histologyc type. Ovarian borderline tumors constituted $21.7 \%$, being seroropapilar the most frequently extirpated. Stages of presentation IA and IC (16 cases respectively) and stage IV (13 cases) were the most frequent in our series. 57 patients were surgically treated. Previous laparoscopic was chosen for evaluation of operability for primary surgical staging procedure by opened surgery (46 cases), interval surgery ( 5 cases) and rescue debulking surgery (6 cases).

Results: Laparoscopic staging of initial stages tumors or borderline was made (6 cases). Complete or optimal surgery was obtained in 48 cases $(84.21 \%)$ and only suboptimal surgery in $15,7 \%$ of the cases. With respect to the ganglionary extirpation, pelvic and lumboaortic lymphadenectomy was made in 24 cases and in 3 cases only paraortic one. Average of the extirpated pelvic nodes was 10.61 and 11.4 for paraortic nodes.

Discussion: The laparoscopic approach is safe and with little morbidity in treatment of the cancer of invasive ovary in initial stages, tumors borderline and in fertility sparing.

\section{4}

\section{CONDITIONING OF THE ABDOMINAL CAVITY REDUCES TUMOR IMPLANTATION IN A LAPAROSCOPIC MOUSE MODEL}

Oncology

M.M. Binda*, R. Corona, F. Amant, P. Koninckx

${ }^{[1]}$ KULeuven $\sim$ Leuven $\sim$ Belgium

Summary (4 lines): Tumour implantation increases following $60 \mathrm{~min}$ pneumoperitoneum using standard dry $\mathrm{CO} 2$. This was prevented by full conditioning.

Introduction: Conditioning of pneumoperitoneum (PP)reduced postoperative adhesions. We aimed to demonstrate the damaging effect of dry $\mathrm{CO} 2 \mathrm{PP}$ and the beneficial effect of the conditioning upon tumor implantation in a laparoscopic model.

Material and Methods: Experiment 1: dose response curve with the tumor cell line CT-26 in control groups (without surgery) and in mice with 60 min dry CO2 PP. Experiment 2: cells were injected in control group without surgery (I) or after a $60 \mathrm{~min}$ PP (II to VI). To the dry
$\mathrm{CO} 2$ group(II), humidification(III), $10 \% \mathrm{~N} 2 \mathrm{O}(\mathrm{IV}), 4 \% \mathrm{O} 2(\mathrm{~V})$ and cooling(VI: conditioning) were sequentially added.

Results: In experiment 1, tumors increased after dry CO2 PP in both abdominal cavity $(\mathrm{p}=0.018)$ and on the wall $(\mathrm{p}<0.0001)$. In experiment $2,60 \mathrm{~min}$ of dry CO2PP confirmed the tumor increased in the abdominal cavity(IvsII: $p=0,026)$ which was dropped by full conditioning (IIvsVI: $\mathrm{p}=0.030$ ). For the abdominal wall, tumors increased by 60 min dry CO2 PP (IvsII: $p=0,003$ ) and this was reduced using humidified CO2 (IIvsIII; 0.032) or conditioning (IIvsVI: p=0,026).

Discussion: Similar to the observations made for adhesion formation, tumour implantation increases following a $60 \mathrm{~min}$ of dry CO2PP, and this was prevented by full conditioning. A less traumatic PP during laparoscopic oncological surgery is possibly beneficial.

171

\section{ENDOMETRIAL CARCINOMA APPROACH: LAPAROTOMY OR LAPAROSCOPY \\ Oncology}

V. García Pineda*, C. De Valle Corredor, M.D. Rodríguez Garnica, Á. Zapico Goñi, L. Nebreda Calvo

${ }^{[1]}$ Príncipe de Asturias Hospital $\sim$ Spain $\sim$ Spain

\section{Summary (4 lines):}

Study the advantages of laparoscopic approach of endometrial cancer. Introduction: The aim of our study is analyze the advantages of laparoscopic versus laparotomy approach.

Material and Methods: We performed a retrospective study in 298 patients operated of endometrial carcinoma by laparoscopic $(n=217)$ and laparotomy $(n=71)$ approach from 1996 to 2011 . We calculated the body mass index (BMI), the survival and the rate of intraoperative and postoperative complications in both groups. Data analysis was performed with SPSS program 15.0 version.

Results: In laparotomy group, the BMI main was $32,3 \pm 6,7 \mathrm{~kg} / \mathrm{m} 2(22$ 49), the intraoperative complications rate was $11,3 \%(\mathrm{n}=8)$ and postoperative rate was $38 \%(\mathrm{n}=27)$. The most prevalent complication was surgical wound infection, 33,3\% $(n=9)$. The mean days of hospitalization (days) was 7,96 $\pm 5,4$ days (3-33)

In laparoscopy group, the BMI mean was $30,78 \pm 6,2 \mathrm{~kg} / \mathrm{m} 2(18-52)$, the intraoperative and postoperative rates were $17,5 \%(n=38)$ and $38,2 \%(\mathrm{n}=83)$ respectively. The most frequent complication was anemia $(44,5 \%, n=37)$. The mean days of hospitalization (days) was $5,31 \pm 7,2$ days (2-65). With respect to survival rate, in laparoscopy group it was $90,3 \%$ and in laparotomy group it was $74,6 \%(p=0,076)$. Discussion: Using laparoscopy as main approach is the current trend because it shows advantages and similar effectiveness than laparotomy

\section{6}

\section{ENDOMETRIAL CARCINOMA IN PATIENTS WITH ELEVATED BMI IN OUR COMMUNITY: REVIEW FROM 1996-2011. Oncology}

M.D. Rodríguez Garnica*, C. De Valle Corredor, V. García Pineda, V. Marcos González, A. Zapico Goñi

${ }^{[1]}$ Príncipe de Asturias University Hospital. Alcalá de Henares, Madrid. Spain

Summary (4 lines): We reviewed 78 patients with elevated BMI treated for endometrial cancer.

Introduction: We reviewed our case-series of endometrial cancer in patients with elevated BMI. 
Material and Methods: We executed a descriptive and retrospective study in 301 patients treated in the Príncipe de Asturias University Hospital, Alcalá de Henares, Madrid (1996-2011).

Results: $25.9 \%$ of patients had a body mass index equal to or more than $35\left(\mathrm{~kg} / \mathrm{m}^{2}\right)(78)$.

Median age was $63.7 \pm 0.9$ age (48-83). $85.9 \%$ had endometrioid adenocarcinoma (67) and $14.1 \%$ non endometrioid carcinoma (11). $91 \%$ of cases had an early stage (Ia-Ib). Surgical approach was by laparoscopy in $59 \%$ (46), in $32 \%$ by laparotomy (25) and vaginally in $9 \%$ (7). The mean number of lymph nodes obtained was $6.21 \pm 0.7(0-28)$. The number of intraoperative complications and the rate of conversion to laparotomy was similar to the patients with normal BMI, $16.7 \%$ (13) versus $15.3 \%(46), 7.7 \%$ (6) versus $6.2 \%$ (12). $55.1 \%$ (43 cases) had a postoperative complication, anemia was the most frequent $(10.3 \%, 8)$, the average stay was higher, $8.05 \pm 1.3$ (2-65) versus $5.82 \pm 0.41$ (2-65). Disease free survival was $97.4 \%$ but the overall survival was lower, $78.2 \%$ versus $90.2 \%$ of patients with normal BMI $(\mathrm{p}=0.014)$ although we did not find significant difference that justified it.

Discussion: Laparoscopic approach is possible in most cases with a conversion to laparotomy rate similar to patients with normal BMI.

\section{8}

\section{FIRST GREEK REGISTRY BY GYNAECOLOGICAL ONCOLOGISTS ON LAPAROSCOPY IN ENDOMETRIAL CANCER \\ Oncology}

T. Panoskaltsis*, Z. Voulgaris, G. Pistofidis, D. Mathiopoulos, D. Xasiakos, K. Pavlakis

${ }^{[1]}$ Gynaecological Oncology Unit, 2nd University OB/GYN Department, Athens, $\sim$ Athens $\sim$ Greece

Summary (4 lines): FIRST GREEK REGISTRY BY GYNAECOLOG ICAL ONCOLOGISTS ON LAPAROSCOPY IN ENDOMETRIAL CANCER

Introduction: Establishing the role of laparoscopy in endometrial cancer. Material and Methods: We describe characteristics - outcome of first 18 cases. Preoperative histology had to be grade 1 or 2 endometrioid adenocarcinoma and, on imaging, no evidence of extrauterine disease. High BMI and age were not exclusion criteria. All patients underwent complete staging, except for one young patient (ovaries conserved) and another, who underwent radical hysterectomy (cervical involvement on MRI - clear cell adenocarcinoma on final histology).

Results: There were no anaesthetic complications and no conversions to laparotomy. Median age was 58.2 years, median BMI 28.5, mean hospital stay 2.1 days, median number of lymphnodes 19 . One ureterovaginal fistula was treated conservatively with pigtail insertion. A bladder perforation was sutured laparoscopically. There was accordance of clinical and surgical staging in all cases (Ia), except for two Ib and one II. After a mean follow-up of 14 months (4-33), all patients are well.

Discussion: Training in gynaecological oncology and experience both in open surgery and laparoscopy are prerequisites before selecting to treat laparoscopically oncological patients and, in such case, the outcome is excellent.

239

\section{INFLUENCE OF OBESITY ON BILATERAL PELVIC LYMPHADENECTOMY \\ Oncology}

R. Ruiz Sautua*, M. Avlia Calle, C. Goiri Little, A. Lekuona Artola, L. Martínez Gallardo, T. Bernal Arahal

${ }^{[1]}$ Hospital Universitario Donostia $\sim$ San Sebastian $\sim$ Spain
Summary (4 lines): Obesity does not influence the outcome of bilateral pelvic lymphadenectomy.

Introduction: Obesity complicates many surgical procedures. We analyze its influence on lymph node yield and mean hospital stay in our series of 152 bilateral pelvic lymphadenectomies.

Material and Methods: We performed 152 pelvic lymphadenectomies between October 2007 and December 2011, in combination with other oncologic procedures. We divide patients in 2 groups according to BMI, using 26 as a cutoff point and compare results. Data were collected prospectively.

$\begin{array}{ll}\text { Results: } & \text { A: } \mathrm{BMI}<=26 \mathrm{~B}: \mathrm{BMI}>26 \\ \text { Patients } & 7973 \\ \text { BMI range } & 17-2627-47 \\ \text { Mean stay } & 4,394 \\ \text { Mean BMI } & 23,1931,71 \\ \text { Total LN } & 1054959 \\ \text { Mean LN } & 13,3413,32 \\ \text { Mean age } & 56,7364,83\end{array}$

Discussion: Obesity compromises access to the pelvis. Laparoscopic access is also complicated by obesity. The Trendelenburg position may not be well tolerated. However, our results support the feasibility of laparoscopic pelvic lymphadenectomy in obese patients.

We find no differences in lymph node yield or hospital stay when comparing obese patients with those with a $\mathrm{BMI}<26$. We could not compare surgical times due to the additional procedures performed in many of the patients.

\section{0}

\section{INFLUENCE OF OBESITY ON RETROPERITONEAL AOR- TOCAVA LYMPHADENECTOMY \\ Oncology}

C. Goiri Little*, T. Bernal Arahal, R. Ruiz Sautua, M. Avlia Calle, L. Goyeneche Lasaga, L. Martínez Gallardo

${ }^{[1]}$ Hospital Universitario Donostia $\sim$ San Sebastian $\sim$ Spain

Summary (4 lines): Obesity does not compromise the results of retroperitoneal aortocava lymphadenectomy for gynecological cancer. This should be the first choice for aortovaca lymphadenectomy in obese patients.

Introduction: Obese patients pose a challenge to transperitoneal aortocava lymphadenectomy, be it open or laparoscopic.

We analyze the influence of obesity on the results of retroperitoneal aortocava lymphadenectomy (RACL) in a series of 102 consecutive patients.

Material and Methods: Between May 2008 and December 2011 we performed 102 RACL for gynecological cancer. We divide patients in 2 groups according to BMI, with a cutoff point, of 26 and compare results. Results: $\mathrm{A}: \mathrm{BMI}<=26 \mathrm{~B}: \mathrm{BMI}>=27$

Number of patients 4953

Mean age 56,21 67,02

Mean LN number 1313

Mean hospital stay 4,33 4,29

BMI 23,21 32,31

BMI range 19-26 27-41

Discussion: We observe that the number of periaortic lymph nodes is equal in both groups, which suggests that obesity may not influence results in terms of adequate dissection and number of $\mathrm{LN}$ obtained.

Mean hospital stay was also similar, though we must note that in many cases other procedures were also performed. Patients in Group A were on average 10 years younger than those in the BMI $>27$ group. This reflects the correlation between increasing age and obesity, as well as the greater prevalence of cervical cancer in this group, for which obesity is not an important risk factor. 
71

LAPAROSCOPIC BOARDING FOR RADICAL SURGICAL TREATMENT OF THE HIGH RISK ENDOMETRIAL CANCER Oncology

J. Molero Vílchez*, E. Martínez Lamela, Y. Exposito Lucena, M.T. Moro Martin, J.L. Prieto Alonso, T. Rivera Garcia, M.A. Lara Alvarez

${ }^{[1]}$ Hospital Universitario Infanta Leonor $\sim$ Madrid $\sim$ Spain

Summary (4 lines): A descriptive study and a critical valuation of the laparoscopic surgery boarding in the treatment of high risk endometrial cancer (EC).

Introduction: The chosen surgical technique (54.5\%) was extraperitoneal lumboaortic lymphadenectomy, transperitoneal pelvic lymphadenectomy, laparoscopic hysterectomy and bilateral salpingooforectomy. Peritoneal biopsies. omentectomy and appendicectomy were performed when macroscopic infiltration suspicion.

Material and Methods: A serie of 94 cases of EC was revised, where 27 cases were high risk EC. Average age was 68.2 years old. The most frequent histological type was the endometrioid (type I) of high risk (IbG3, II G3, III-IV stage) (13 cases). Carcinosarcoma was the most frequent of 14 cases of high degree (type II) histological types. Staging surgery was able to be performed in $86.36 \%$ of the cases, the laparoscopic route was the chosen technique. Other surgery routes was performed by medical comorbility

Results: In 15 cases, lymphadenectomy was performed with an average extirpated from pelvic node of 17.06 (7-32) and from paraaortic nodes of 10.18 (4-16). The average of extirpated pelvic nodes was greater by laparoscopic route (21.1 vs 10.2$)$ and extirpated paraortics nodes was greater by laparotomic route (14.2 vs 10.2$)$.

Discussion: The surgery of maximal debulking improves the global survival. The radicality was similar by laparoscopic than laparotomic route, but decrease the postsurgical morbility.

\section{4}

LAPAROSCOPIC DEBULKING OF BULKY LYMPH NODES IN PATIENTS WITH CERVICAL CARCINOMA: FEASIBILITY OF THE TECHNIQUE AND SURGICAL OUTCOMES.

Oncology

\author{
Z. Liang*, H. Xu, Y. Chen \\ ${ }^{[1]}$ Southwest Hospital $\sim$ Chongqing $\sim$ China
}

Summary (4 lines): Laparoscopic surgery is safe for cervical carcinoma patients with bulky lymph nodes.

Introduction: To investigate the feasibility of technique and surgical outcomes of laparoscopic debulking of bulky lymph nodes in patients with cervical carcinoma.

Material and Methods: From 2001 to 2008, a total of 655 patients with cervical carcinoma underwent laparoscopic lymphadenectomy. Of all the patients, 159 patients were confirmed with lymph node metastasis. 37 underwent laparoscopic resection of bulky pelvic lymph nodes (BLN group), 122 underwent lymphadenectomy for micro-metastasis lymph nodes (MLN group) at the time of radical hysterectomy.

Results: All patients successfully underwent laparoscopic surgery. Operating time, estimated blood loss, the number of lymph nodes yielded, and the resection rate of lymph nodes was similar in the two groups. Intra-operative and post-operative complications were no different in the two groups. In the BLN group, pathologic lymph node size was significantly larger than MLN group patients. After surgery, all patients in the BLN and MLN groups received post-operative adjuvant therapy.
Discussion: The results of this study suggest that, for the cervical carcinoma patients with bulky lymph nodes, the laparoscopic debulking of macroscopically positive lymph nodes may afford an accuracy evaluation of nodal status with the advantage of an early start of adjuvant treatment.

302

\section{LAPAROSCOPIC HYSTERECTOMY IN ENDOMETRIAL CANCER \\ Oncology}

A. Swaminathan, P. Harris*, N. Murthy, S. Satchitanantham, S. George, R. Macdonald, J. Kirwan

${ }^{[1]}$ Liverpool Women's Hospital $\sim$ Liverpool $\sim$ United Kingdom

Summary (4 lines): LAVH is a safe procedure and has less postoperative stay and less operating time as compared to TAH with less post operative complications.

Introduction: A comparison of operative outcomes of laparoscopic assisted vaginal hysterectomy (LAVH) compared to total abdominal hysterectomy (TAH) and vaginal hysterectomy $(\mathrm{VH})$ for patients of endometrial cancer numbering 144 treated in a tertiary cancer centre in the UK for one year.

Standard staging included total hysterectomy with bilateral salphingooopherectomy with peritoneal washings and selective sampling of abnormal lymphnodes

Material and Methods: Analysis of data from database

Results: Mean age of patients for TAH was 67, for VH was 70.5 and LAVH was 66.1. The mean BMI for TAH was 32.6 , for VH was 44.7 and LAVH was 32.4. The mean operating time was 95.6 minutes for TAH, 58.8 for VH and 86.2 for LAVH. The mean postoperative stay for TAH was 5.1 days, 2.3 days for $\mathrm{VH}$ and 2.4 days for LAVH. The average estimated blood loss varied between $250 \mathrm{mls}$ for $\mathrm{VH}, 359 \mathrm{mls}$ for TAH and $368 \mathrm{mls}$ for LAVH. There were 2 visceral injuries in LAVH group. There were 2 conversions to laparotomy one for bowel injury and the other for intraoperative bleeding. There were no readmissions in this group. In the TAH group 8 patients had wound infections, 2 developed abscesses, 1 vault haematoma and 1 bowel obstruction and there were 5 readmissions.

Discussion: LAVH is a safe alternative to TAH and has less postoperative stay and less operating time

\section{6}

\section{LAPAROSCOPIC MANAGEMENT OF EARLY OVARIAN CANCER: SURGICAL AND SURVIVAL OUTCOME Oncology}

A. Couso Gonzalez, A. Zapico Goñi*, P.L. Valenzuela Ruiz, P. Fuentes Castro

${ }^{[1]}$ Principe de Asturias Hospital $\sim$ Madrid $\sim$ Spain

Summary (4 lines): This study reports the application of laparoscopic approach for the surgical staging of early ovarian cancer.

Introduction: The objective of this study is analyze the roll of laparoscopy in the ovarian cancer citorreduction surgery.

Material and Methods: A retrospective review of laparoscopic staging procedures for early-stage ovarian cancer in eleven patients was conducted.

Results: A total of eleven patients underwent laparoscopic staging for presumed stage I ovarian cancer.

Mean operation time was 166 minutes (130-210). There has been no intraoperative and postoperative complications. 
The mean estimated blood loss was $190 \mathrm{ml}(30-500)$. Only one patient required a postoperative blood transfusion.

Mean number of pelvic nodes was 12 (5-17), and mean number of paraaortic nodes was $5(3-8)$.

Mean duration of follow-up was 17 months (2-59), only one patient had peritoneal recurrence.

Six cases were invasive epithelial tumors $(55,5 \%)$, and the others were 5 cases of borderline tumors $(45,5 \%$.

8 cases were stage IA $(72,7 \%, 1$ case was IIIA $(9,1 \%) 1$ case was IIIB $(9,1 \%)$ and 1 case was IIIC $(9,1 \%)$.

There has been no trocar site metastasis.

Discussion: Laparoscopic staging of early-stage ovarian cancers appears to be feasible and comprehensive without compromising survival when performed by gynaecologic oncologists experienced with advanced laparoscopy.

\section{5}

\section{LAPAROSCOPIC RADICAL HYSTERECTOMY WITH PELVIC LYMPHADENECTOMY \\ Oncology}

P. Stanciu*, M. Ionescu, D. Grigoras, M. Craina, S. Pantea

${ }^{[1]}$ Obstetrics and Gynecology Department, University Of Medicine and

Pharmacy "Victor Babes" Timisoara Romania

Summary (4 lines): The aim of this paper is to present the surgical technique and to evaluate the oncological outcome of laparoscopic radical hysterectomy with pelvic lymphadenectomy in cervical cancer. Introduction: Minimally invasive surgery set the fastest trend in all the fields of medicine especially in oncological genital pathology.

Material and Methods: From January 2008 to January 2012 we performed 24 radical hysterectomies with pelvic lymphadenectomy in patients diagnosed with cervical cancer. The mean age was 46.5 years. According to the International Federation of Gynaecology and Obstetrics (FIGO) staging of cervical cancer, 6 patients had stage IA, 10 patients stage IB and 8 patients stage IIA.

Results: The operation was performed by laparoscopy in all the patients, without conversions. The mean operation time was $178 \mathrm{~min}$. The medium blood loss was $230 \mathrm{ml}$. The mean number of extracted lymph nodes was 24.2. The surgical margins were free of disease in all the cases. The medium hospital stay was 3.6 days. Postoperative complications occurred in 4 patients $(16.7 \%)$ : one deep venous thrombosis, two ureteral fistula and one prolonged lymph leakage.

Discussion: Laparoscopic lymphadenectomy dissection has the advantage of greater accuracy than open surgery. For early cervical carcinoma laparoscopic approach may offer the advantages of mini invasive surgery and achieve optimal control of the radical principles.

66

\section{LAPAROSCOPIC STRATEGIES IN TREATMENT OF ENDOMETRIOID EPITHELIAL ENDOMETRIUM CARCINOMA OF LOW AND INTERMEDIATE RISK Oncology}

E. Martínez Lamela*, J. Molero Vílchez, R. Lorente Ramos, I. Casado Fariñas, A. Martin Marino, M.A. Lara Alvarez, J.J. Hernández Aguado ${ }^{[1]}$ Hospital Universitario Infanta Leonor $\sim$ Madrid $\sim$ Spain

Summary (4 lines): Describe of the paper of the intraoperating biopsy by frozen section (FS) for valuation of miometrial infiltration in low risk endometrium carcinomas (LR-EC), and of the pelvic lymphadenectomy in intermediate risk EC (IR-EC).
Introduction: Tumors Committee decided :In group of LR-EC (endometrioid EC, type I, Ia G1-2 stage), hysterectomy and double salpinogooforectomy (TAH-BSO) with FS for the valuation of the miometrial invasion and the degree of histological differentation; in group of IREC (endometrioid CE, type I, stages IAG3, IV G1-G2, II G1-G2), TAH-BSO and pelvic lymphadenectomy, with FS of the pelvic nodes. Material and Methods: We reviewed our series of 94 cases of EC. The chosen technique was the LPS (74 \%).

Results: The discordance in the presurgical staging respect to the final stage is of $37 \%$, but with the FS descended to $17.24 \%$. If FS informed a miometrial invasion more of $50 \%$ (9 cases), pelvic lymphadenectomy was performed with FS from extirpated node being negative in all the cases. In group of IR-EC, LPS was made in 29 cases $(65,5 \%)$. No case had node affectation in FS and in definitive study.

Discussion: The FS of the hysterectomy in LR-EC and of the pelvic lymphadenectomy in IR- EC allows a laparoscopic boarding with smaller morbility without changing the prognosis of the disease in the long term.

\section{8}

\section{LAPAROSCOPIC TREATMENT OF EARLY STAGE CERVICAL CANCER. A 40-CASE SERIES Oncology}

C. Goiri Little*, R. Ruiz Sautua, M. Avlia Calle, L. Martínez Gallardo, I. Jaunarena Marín, T. Bernal Arahal

${ }^{[1]}$ Hospital Universitario Donostia $\sim$ San Sebastian Spain

Summary (4 lines): We present the results of our first 40 laparoscopies for early cervical cancer.

Introduction: Laparoscopy provides adequate access in early stage cervical cancer $(<4 \mathrm{~cm})$

Material and Methods: From Jan 2009 to Dec 2011 we performed 40 laparoscopies for early cervical cancer. The first step is pelvic lymphadenectomy and intraoperative lymph node analysis. If negative, we proceed to radical hysterectomy. If positive, we perform aorto-cava lymphadenectomy and refer the patient for chemo-radiotherapy.

Results: Average age was 51 (31-81). Average BMI was 24,85 (17-39). Pelvic LNs were positive in $8(20 \%)$ cases. They underwent aortocava lymphadenectomy. There was one intraoperative false negative.

We performed 2 total hysterectomies for microinvasive tumors. We performed 2 radical trachelectomies: one fertility sparing surgery on a 31 -year old woman and one on a woman with a previous subtotal hysterectomy. The remaining 27 patients had radical hysterectomies.

Average hospital stay was 4 days. All urinary catheters were removed within 24 hours; there were no urinary retentions. There were no conversions to laparotomy or reinterventions. Average surgical time was $280 \mathrm{~min}$

Discussion: Early cervical cancer can be adequately treated via laparoscopy.

With intraoperative LN analysis we select patients who will not benefit from radical hysterectomy. This allows us to refer them for chemoradiotherapy with reduced risk of adhesion-related complications.

250

\section{PARA-AORTIC LYMPHADENECTOMY BY EXTRAPERITONEAL APPROACH: OUR EXPERIENCE Oncology}

M. Guzmán Muñoz, Á. Zapico Goñi*, A. Couso González, S. Heron Iglesias, E. Martínez Gómez

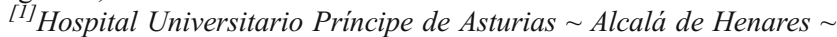
Spain 
Summary (4 lines): The para-aortic lymphadenectomy can be performed by extraperitoneal approach.

Introduction: The objective is to describe our experience and analyzing the results of this technique, demonstrating the advantages of the extraperitoneal approach.

Material and Methods: We have performed an analytical, descriptive and retrospective study comprised by 22 women with cervical cancer, endometrial or ovarian cancer treated at the Department of Gynecology and Obstetrics at our hospital. All of them underwent para-aortic lymphadenectomy for extraperitoneal approach during the execution of surgical staging. The data were obtained after review of medical records and statistically analyzed using SPSS 15.0.

Results: The number of patients with cervical cancer, endometrial and ovarian cancer was 11, 6 and 5 respectively. The mean age of patients was 51 years (30-80 years) with a BMI of 27 (19-37). The mean duration of surgery was 100 minutes. The average number of nodes removed was 11 . In no case was required conversion to open surgery. The only complication observed was a case of peritoneal tear which was fixed in the same surgical procedure. We didn't observed postoperative complications.

Discussion: The para-aortic lymphadenectomy by extraperitoneal approach is a way of analyzing the lymph nodes in the para-aortic area that offers many advantages over other routes because it has been associated with fewer intraoperative and postoperative complications.

\section{1}

\section{PARA-AORTIC LYMPHADENECTOMY BY EXTRAPERITONEAL OR RETROPERITONEAL WAY: WHICH IS BETTER? \\ Oncology}

M. Guzmán Muñoz, Á. Zapico Goñi*, A. Couso González, S. Heron Iglesias, E. Matínez Gómez

${ }^{[1]}$ Hospital Universitario Príncipe de Asturias $\sim$ Alcalá de Henares $~$ Spain

Summary (4 lines): The para-aortic lymphadenectomy can be performed by extraperitoneal or retroperitoneal approach for evaluating para-aortic lymph nodes.

Introduction: The aim is to analyze the indications, results and complications of para-aortic lymphadenectomy by extraperitoneal and transperitoneal way.

Material and Methods: We have done an analytical, descriptive and retrospective study comprised by 27 women with cervical cancer, endometrial or ovarian cancer treated at the Department of Gynecology and Obstetrics at our hospital. All of them underwent para-aortic lymphadenectomy. The data were obtained after review of medical records.

Results: The number of patients operated by extraperitoneal and retroperitoneal was 22 and 5 respectively. The mean age was 54 years with a BMI of 26 . The mean length of retroperitoneal surgery was 100 minutes and extraperitoneal was 60 minutes. The number of nodes removed was 11 in the extraperitoneal way and 10 in the transperitoneal. The blood loss was higher in the transperitoneal approach objectifying the biggest difference between preoperative and postoperative hemoglobin (1.9 vs. 1.45). We observed any intraoperative or postoperative complications.

Discussion: The para-aortic lymphadenectomy for extraperitoneal approach is more appropriate in patients with higher BMI. Also it has been observed lower risk of postoperative adhesions and intestinal lesions although the results of our study are similar in both groups.
52

\section{PELVIC AND PARA-AORTIC LYMPHADENECTOMY IN HIGH RISK ENDOMETRIAL CANCER (HREC)}

Oncology

M.F. Perelló*, M.J. Martínez, S. Martínez, A. Torné, P. Fusté, J. Pahisa

${ }^{[1]}$ Hospital Clínic Barcelona $\sim$ Barcelona $\sim$ Spain

Summary (4 lines): The role of lymphadenectomy in overall survival of EC and if its extension should include para-aortic lymphadenectomy until Left Renal Vein (LRV) or Inferior Mesenteric Artery (IMA) is controversial.

Introduction: The risk of lymph node metastasis in EC is based on myometrial invasion, hystology and tumor grade (HR criteria: GIII, M $>50 \%$, carcinosarcoma, clear cell, papillary serous cancer). The aim of the study is to report a high rate of para-aortic metastasis in patients with HREC, and to support our protocol of performing a lymphadenectomy until LRV.

Material and Methods: 120 women with HREC underwent surgery in Hospital Clínic (March 1999-March 2012). Pelvic and para-aortic laparoscopic lymphadenectomy until LRV was performed. We did a retrospective review of demographic, lymph node metastasis, anatomopathology and recurrence data. The mean follow-up was 30 months (1-127).

Results: The mean age was 66(36-84). The mean BMI was 27,7(15,3$47,6) .23,3 \%$ had positive lymph nodes $(57,9 \%$ of pelvic and $42,1 \%$ of para-aortic). $11,4 \%$ had isolated lymphatic metastasis in para-aortic region. AP: endometrioid (63,5\%), clear cells $(5,9 \%)$, papillary serous cancer $(23,6 \%)$ and carcinosarcoma $(6,8 \%)$. We observed $11,7 \%$ of recurrence and $16,7 \%$ of extranodal metastasis.

Discussion: The high rate of para-aortic nodal metastasis either isolated or combined with pelvic nodes suggests the enormous importance of performing a lymphadenectomy until LRV in patients affected of a HREC.

\section{0}

\section{POSTMENOPAUSAL BLEEDING AMONG OMANI WOMEN: RISK FACTORS PROFILE}

Oncology

\author{
M. Al Khaduri*, Y. Al Farsi \\ ${ }^{[1]}$ SULTAN QABOOS UNIVERSITY MUSCAT Oman
}

Summary (4 lines): A study of causes and risk factors for postmenopausal bleeding (PMB) which found that the commonest cause was polyp and that there was a significant association with obesity.

Introduction: The aim is to find the causes of PMB, associated risk factors and the proportion of endometrial cancer.

Material and Methods: A retrospective case-control study conducted at Sultan Qaboos University Hospital, Oman. Target population consisted of Omani women diagnosed with PMB. The control group included postmenopausal women who did not report history of PMB. The targeted case-to-control ratio was 1:2. Risk factors of interest were: age of menopause, BMI, diabetes, hypertension, parity, estrogen replacement therapy and family history of ovarian cancer. Chi-square test and odds ratio were used to find the association between PMB and potential risk factors with P-value less than or equal to 0.05 as a significant level of association.

Results: Polyp (63\%) was a common cause for PMB, followed by atrophy $(45 \%)$. The proportion of endometrial cancer was $18 \%$. Obesity (OR 3.9, $95 \%$ CI 1.6, 9.7) was significantly associated with PMB $(\mathrm{P}=0.010)$. Other factors were not significantly associated with PMB. 
Discussion: This study shows a significantly high proportion of PMB in Omani women is due to polyps. The proportion of endometrial cancer among PMB Omani women is higher than other countries and there is a significant association between obesity and PMB among Omani women as a risk factor.

519

\section{PROPHYLACTIC SALPINGECTOMY DOES NOT AFFECT SHORT AND LONG TERM SURGICAL OUTCOMES WHEN ASSOCIATED TO SURGERY FOR BENIGN INDICATIONS. Oncology}

R. Venturella*, R. Mocciaro, M. Morelli, F. Zullo

${ }^{[1]}$ University Magna Graecia $\sim$ Catanzaro $\sim$ Italy

Summary (4 lines): Adding salpingectomy to TLH, VH or LS do not affect any surgical outcomes.

Introduction: Serous ovarian cancers seems to originate from the distal Fallopian tube. Our objective was to demonstrate that adding a prophylactic salpingectomy to hysterectomy for benign pathology doesn't modify short and long term surgical outcomes.

Material and Methods: This is a retrospective analysis of 21 patients submitted to total laparoscopic hysterectomy (TLH), 12 to vaginal hysterectomy (VH) and 9 to laparoscopic sterilization (LS) in which prophylactic salpingectomy was added. Data for these patients were compared with those of 21 women treated by standard TLH (sTLH), 12 by standard $\mathrm{VH}(\mathrm{sVH})$ and 9 by laparoscopic sterilization with coagulation (cLS), matched for uterine volume and surgical difficulty.

Results: No significant differences were observed between the groups in terms of mean operative time, mean difference between pre- and postoperative hgb, mean hospital stay and mean return to normal activities. No complications were encountered and there was no conversion to laparotomy in all groups.

Discussion: Adding salpingectomy to TLH, VH or LS do not affect surgical outcomes. There is no known benefit of retaining the postreproductive Fallopian tube, whereas preserving ovaries reduces the significant negative effect surgical menopause, even probably reducing the risk for serous pelvic cancer.

\section{9}

\section{SUCCESSFUL PROTOCOL ON CONSERVATIVE MANAGEMENT OF ENDOMETRIAL CANCER Oncology}

T. Panoskaltsis*, Z. Voulgaris, D. Mathiopoulos, D. Xasiakos, K. Pavlakis

${ }^{[1]}$ Gynaecological Oncology Unit, 2nd University Department OB/ GYN, Athens Greece

Summary (4 lines): SUCCESSFUL PROTOCOL ON CONSERVATIVE MANAGEMENT OF ENDOMETRIAL CANCER

Introduction: Strict protocol to preserve fertility in young nulliparous patients with Endometrial Cancer or Complex Atypical Hyperplasia (EIN). Material and Methods: Protocol was applied to first 11 patients (5 had EIN) and included hysteroscopic resection of lesion, insertion of MIRENA, laparoscopy to exclude double primary ovarian neoplasm, Megace $160 \mathrm{mg} /$ day, aspirin $100 \mathrm{mg} /$ day, hysteroscopy every 3 months. Treatment lasted 6-9 months, according to severity of lesion and response to treatment. After treatment, patients were strongly advised to become pregnant soon (natural methods-IVF).

Results: There were no cases with myometrial invasion, complete regression observed in all. After mean follow-up of 33 months, 3 pregnancies out of 6 who tried (2 IVF pregnancies, 1 singleton, 1 twin and 1 spontaneous miscarriage). Two patients underwent laparoscopic hysterectomy (recurrence of cancer). All adhered appropriately to protocol and tolerated well treatment.

Discussion: Correct identification of patients and strict adherence to protocol produce satisfactory results for lesion regression and fertility outcome. Patients should know that success is not guaranteed and, after treatment, to immediately become pregnant. Hysterectomy is eventually unavoidable (high chance of recurrence).

\section{4}

\section{SURVIVAL OF ENDOMETRIAL CARCINOMA IN OUR COMMUNITY: REVIEW FROM 1996 TO 2011. \\ Oncology}

M.D. Rodríguez Garnica*, V. García Pineda, C. De Valle Corredor, A. Zapico Goñi, S. Heron Iglesias

${ }^{[1]}$ Príncipe de Asturias University Hospital, Alcalá de Henares, Madrid. Alcalá de Henares, Madrid. Spain

Summary (4 lines): We analyzed the overall survival in 301 patients with endometrial carcinoma

Introduction: We analyzed the overall survival in endometrial carcinoma in our community according to the stage, histological type, surgical approach and the BMI.

Material and Methods: We executed a descriptive and retrospective study in 301 patients treated in the Príncipe de Asturias University Hospital, Alcalá de Henares, Madrid (1996-2011).

Results: The overall survival rate was $85.7 \% \%$ with a median of $139.8 \pm 3.932$ (132.1-147.5) months. The disease free survival rate was $92.7 \%$.

We divided patients according to:

The histological type: $87.4 \%$ with endometrioid adenocarcinoma (263) had an overall survival rate of $87.1 \% .12 .6 \%$ with non endometrioid carcinoma (38), had an overall survival rate of $76.3 \%(p=$ 0.002 ).

Clinical stages: $82 \%$ with early stage (247) had an overall survival rate of $88.7 \% .18 \%$ Patients with advanced stages (54) had an overall survival rate of $72.2 \%$.

Surgical approach: $72.1 \%$ by laparotomy with an overall survival rate of $74.6 \%$. $23.6 \%$ by laparoscopy with an overall survival rate $90.3 \%$ $(\mathrm{p}=0.007)$.

BMI: $25.9 \%$ with a BMI higher than $35 \mathrm{~kg} / \mathrm{m}^{2}$ (78) with an overall survival rate of $78.2 \%$. The overall survival rate with death due to endometrial cancer was $87.2 \%(p=0.029)$

Discussion: Patients in which surgical approach was performed by laparotomy and those with higher BMI had lower survival.

372

\section{SYMTOMATIC LYMPHOCYST NEEDING SURGERY Oncology}

T. Bernal*, C. Goiri, L. Martinez, M. Arrue, A. Lekuona, M. Gorostidi ${ }^{[1]}$ Hospital Universitario Donostia $\sim$ San Sebastian $\sim$ Spain

Summary (4 lines): We describe the resolution of one big symptomatic lymphocyte after robotic lymphadenectomy with laparoscopic peritoneal marsupialization with figures and video.

Introduction: Lymphoceles are the most frequent complication after lymphadenectomy. Most are asymptomatic and do not require treatment. We present a case of a symptomatic lymphocele requiring surgery.

Material and Methods: CASE 
A 66-year old female BMI 37 underwent TLH-BSO, robotic retroperitoneal paraaortic lymphadenectomy and transperitoneal pelvic lymphadenectomy for endometrial carcinoma. There were no intraoperative complications. 29 negative nodes were obtained.

5 months after surgery she developed urinary incontinence and was admitted to Intensive Care with fever and renal failure. A CT scan showed hydronefrosis and a $12 \mathrm{~cm}$ lymphocele in the right pelvis. She initially recovered after antibiotic treatment and $\mathrm{CT}$ guided drainage. The lymphocele reappeared, and was treated with laparoscopic drainage and peritoneal marsupialization of the lymphocyst.

Results: DISCUSSION

The first option for lymphocele management is conservative. If drained, a pig-tail catheter should be placed for continuous drainage. Surgical treatment, either open or laparoscopic, consists of a broad incision on the lymphocele and excision of as much of the pseudocapsule as possible.

Discussion: CONCLUSIONS

Lymphocysts after lymphadenectomy are very frequent. Only symtomatic lymphocyst need to be treated.

\section{1}

\section{THE NEW THEORY OF CARCINOGENESIS_ _ THE THEORY OF GENE MULTIPLE HITS Oncology}

H. Xu*

${ }^{[1]}$ Department of internal and emergency medicine $\sim$ Clinical Institute, Workers hospital of Nanyang textile corporation, Nanyang city Changjiang RD No.200, Henan province, China. $\sim$ China

Summary (4 lines): The author has found a new mechanism of carcinogenesis, the theory of gene multiple hits.

Introduction: To enhance the achievement of cancer research by creating the new theory for the mechanism of cancer development.

Material and Methods: Referenced the scientific achievements of cancer research up to now, summarizing and creating the new theory of carcinogenesis.

Results: The gene multiple hits, the new theory of carcinogenesis, has been created. We have known that cancer development is caused by long time effecting of carcinogens. The carcinogens include environmental or chemical factors; biological factors; physical factor; factors of psychological, social, behavioral, emotional and spiritual issues and hereditary factors, et al. All these complicated, lots kinds of carcinogens, through many different ways, finally damage many different genes on the chromosomes. Which can be said those carcinogens damaging are gene multiple hits. The oncogenes and tumor suppressive genes or anti-oncogenes are hitting by lots of the carcinogens many times. Which lead to develop cancer.

Discussion: The new theory can explain well the research findings for mechanism of cancer development. The gene multiple hits of new theory provides the best directive references for the further cancer research, prevention, early diagnosis, early cure and cancer treatment.

\section{6}

\section{UNEXPECTED MALIGNANCY IN LAPAROSCOPIC ADNEXAL SURGERY \\ Oncology}

R.W.K. Kung*, G. Dorman, D. Morgan, K. Johnston
${ }^{[1]}$ Antrim Area Hospital $\sim$ Antrim $\sim$ United Kingdom

Summary (4 lines): Review of unexpected malignancy cases, Risk of Malignancy Index (RMI), Transvaginal Ultrasound (TVS),
CA125 and other malignant predictors in a laparoscopic adnexal surgical series.

Introduction: Unexpected malignancy rate at laparoscopic adnexal surgery is low. Evidence for Fallopian tube malignancy as a possible precursor to ovarian carcinoma is mounting. This calls into question the role of RMI.

Material and Methods: Retrospective chart review over 5 yrs. Pathology, TVS, CA125, personal/family history of breast/ovarian cancer and BRCA $1 / 2$ status analysed.

Results: 77 patients were identified. Age range 17-79 yrs. RMI performed in 51/77 (66 \%) none exceeded 200. BRCA 1/2 carriers accounted for 4/77 (5\%), 15/77 (19\%) had breast/ovarian cancer in personal/ family history. CA125 was performed in 51/77 (66\%), elevated in 15/51 (29\%). Prophylactic BSO was the surgical indication in 16/77 (20.8\%). One borderline ovarian tumour 1/77 (1.3\%) and 2/ $77(2.6 \%)$ fallopian tube carcinomas were identified. RMI was indicated in 1 of these 3 cases (borderline tumour) and was 154. In the 2 malignant cases, patients were aged $58 \& 59$ yrs, (TVS) was normal, one had a family of history of breast carcinoma and one a BRCA 2 carrier.

Discussion: Laparoscopic approach is still reasonable as unexpected malignancy rate is low, $2.6 \%$ in our series. Research and development into a preoperative screening tool for fallopian tube carcinoma now seems prudent.

441

\section{VAGINAL RADICAL TRACHELECTOMY - RECURRENCE AND PREGNANCY RATES \\ Oncology}

S. Pessini*, G. Silveira, M.C. Anselmi

${ }^{[1]}$ Universidade Federal de Ciencias da Saude de Porto Alegre $\sim$ Porto Alegre $\sim$ Brazil

Summary (4 lines): Radical trachelectomy follows the current concepts of conservative and minimal invasive surgery.

Introduction: Daniel Dargent proposed and began the radical trachelectomy with laparoscopic pelvic lymphadenectomy and parametrectomy. The surgery is indicated for patients who desire to preserve the fertility, tumor less $2 \mathrm{~cm}$, FIGO IA2 to IB1, epidermoid or adenocarcinoma histology without lymphovascular invasion. The aims are to describe our cases and to analyze the oncologic and obstetrics outcomes.

Material and Methods: From october 2000 to march 2012 we elected 13 patients. The surgery was aborted in one patient by positive sentinel node and in another by involvement of the up cervical channel. Age, parity, tumor hystology, FIGO stage, lymph nodes, oncologic and obstetrics results were the factors analyzed.

Results: Patients age ranged from 19 to 35 years. Eight patients didn't have children, and three had one. The histology was epidermoid in nine, and adenocarcinoma in two patients. FIGO stage was IB1 in ten patients and IA2 in one. The mean of lymph nodes was 21,9 (4 to 29). Oncologic results: One recurrence.

Obstetrics results: 7 pregnancies in 4 patients, with 3 live births (baby at home) and 4 with concept loss. Five patients attempting pregnancy (with anticonception).

Discussion: The radical trachelectomy with laparoscopic lymphadenectomy and parametrectomy, in early invasive carcinoma of the cervix has a raisonable pregnancy rate, according the international data.

241

VASCULAR COMPLICATIONS IN LAPAROSCOPIC
ONCOLOGIC SURGERY
Oncology


R. Ruiz Sautua*, M. Avlia Calle, C. Goiri Little, L. Martínez Gallardo, I. Jaunarena Marín, T. Bernal Arahal

${ }^{[1]}$ Hospital Universitario Donostia $\sim$ San Sebastian $\sim$ Spain

Summary (4 lines): We describe the vascular complications encountered in our series of laparoscopic oncologic procedures and how they were resolved.

Introduction: Vascular lesions in laparoscopy can endanger the life of the patient and require a prompt response.

Material and Methods: We describe the vascular complications in our series of 287 laparoscopic oncological procedures. Data were collected prospectively.

We performed 191 hysterectomies (31 of them radical), 156 bilateral pelvic lymphadenectomies, 100 retroperitoneal and 29 transperitoneal aorto-cava lymphadenectomies.

Results: We recorded a total of 16 vascular complications (5,58 \%)

- 6 vena cava: 2 electrocoagulation, 1 vascular clip and Floseal and 3 Floseal.

- 4 external iliac vein: 3 repaired with clips and 1 sutured

- 2 ovarian artery: bipolar electrocoagulation

- Left lateral aorta: Floseal

- Inferior mesenteric artery: coagulation and section

- Right hypogastric vein: clip and Floseal

- Right primitive iliac artery: bipolar coagulation.

All cases were resolved laparoscopically. There were no entry-related lesions. All vascular incidents occurred during lymphadenectomy.

Five patients required blood transfusions.

Discussion: Vascular lesions occur most frequently during lymphadenectomy. They are potentially grave and require rapid action to prevent bleeding from impairing vision, identify the lesion and repair it.

Several different hemostatic products are available. We have used Floseal with good results.

\section{4}

\section{VIDEO-ASSISTED THORACOSCOPIC SURGERY (VATS) FOR RECURRENT OVARIAN CANCER WITH A METASTATIC MEDIASTINAL MASS \\ Oncology}

K. Ki*, J. Lee

${ }^{[1]}$ Kyung Hee University $\sim$ Seoul $\sim$ Korea, Republic of

Summary (4 lines): VATS should be considered a viable option for the treatment of recurrent ovarian cancer with mediastinal metastasis.

Introduction: Although primary cytoreductive surgery for epithelial ovarian cancer is well accepted as the cornerstone of initial management, the benefits of cytoreductive surgery for patients with recurrent ovarian cancer remains unclear.

Material and Methods: A 29-year-old woman was admitted to our hospital due to suspicion of recurrent ovarian cancer. Four years previously, she was first diagnosed with an ovarian papillary serous adenocarcinoma stage IIIc. She underwent primary debulking surgery followed by six cycles of adjuvant chemotherapy, which consisted of paclitaxel $(175 \mathrm{mg} / \mathrm{m} 2)$ and carboplatin $(\mathrm{AUC}=5)$. A physical examination revealed a palpable inguinal mass and multiple enlarged pelvic lymph nodes. In addition, a mediastinal mass with a well-defined margin was visualized by chest CT. Video-assisted thoracoscopic surgery (VATS) was utilized for the cytoreduction of the recurrent ovarian cancer with solitary mediastinal metastasis.

Results: At the 20-month follow-up the patient was without evidence of disease.

Discussion: VATS may be attempted as a component of secondary cytoreductive surgery for the optimal debulking of macroscopic disease, and should be considered a viable option for the treatment of recurrent ovarian cancer with mediastinal metastasis.

\section{0}

\author{
A DOUBLE RANDOMIZED CONTROLLED TRIAL OF \\ VAGINAL MISOPROSTOL BEFORE OPERATIVE \\ HYSTEROSCOPY \\ Operative Hysteroscopy
}

\section{Sofoudis*, P. Bakas, M. Creatsa, M. Kalampokas, V. Grigoriou, N. Vlahos \\ ${ }^{[1]} 2 N D$ DEPARTMENT OF OBSTETRICS AND GYNECOLOGY UNI- VERSITY OF ATHENS ARETAIEION HOSPITAL ATHENS Greece}

Summary (4 lines): Vaginal application of $400 \mathrm{mg}$ misoprostol 12 hours preoperatively before operative hysteroscopy is more effective than $200 \mathrm{mg}$.

Introduction: Objective of this study was to assess the efficacy of preoperative use of vaginal misoprostol as cervical priming agent before operative hysteroscopy.

Material and Methods: 54 patients divided in three groups were examined. The first group $(\mathrm{n}=18)$ had vaginal $200 \mathrm{mg}$ misoprostol twelve hours before the procedure received. The second group $(n=18)$ had vaginal $400 \mathrm{mg}$ misoprostol twelve hours before the procedure received. The third group $(n=18)$ was the contoll group which did not receive any cervical priming agent. The initial Hegar dilatation, the used Hegar dilatator, cases of fever, the time of cervical dilatation, any cases of diarrhoia, cases of lacerations and the pain score (1-10) after the procedure was noted in each case.

Results: The study revealed a significant difference between the two groups and the control group. The mean Hegar dilatation for the first group was 6,5 the second group 5,5 and for the control group 4. There was not any cases of fever or diarrhoia. The mean time for cervical dialatation was 3 minutes for all groups. There were lacerations in all groups notified. The mean pain score was for the two study groups 2 in addition to the control group which was 4 .

Discussion: WE ASSESS THE THE EFFECTIVENESS OF VAGINAL MISOPROSTOL

47

\section{ASSESSMENT OF ULTRASOUND GUIDANCE IN HYSTERO- SCOPIC TREATMENT OF UTERUS SEPTA \\ Operative Hysteroscopy}

V. Solène*, L. Guillaume, L. Jean-marc, F. Hervé

${ }^{[1]}$ Hôpital de Bicêtre - Assistance Publique des Hopitaux de Paris $\sim$ Le Kremlin Bicêtre France

Summary (4 lines): The ultrasound guidance for hysteroscopic section of uterine septum limits complications and increases one-step procedure. Introduction: To assess the ultrasound guidance in operative hysteroscopic treatment of uterus septum to limit complications (such as uterine perforation) or need for a second intervention.

Material and Methods: Retrospective unicentric study from June 2009 to May 2012. All patients with operative hysteroscopy procedure of uterus septum were included. The ultrasound guidance was performed according to surgeron's choice. Complications and postoperative outcomes were evaluated.

Results: At all, 83 hysteroscopic procedures were performed. 33 of them had an ultrasound guidance and 50 with no ultrasound guidance. A second intervention was needed for nine patients in the group without guidance versus one in the group with guidance $(\mathrm{p}<0,05)$. 
Two uterine perforations occurred in our series, both of them were in the group without ultrasound guidance.

Discussion: The ultrasound guidance for hysteroscopic section of uterine septum limits complications and increases one-step procedure.

97

\section{DEMOGRAPHIC CHARACTERISTHICS AND TYPE OF ANESTHESIA IN HYSTEROSCOPIC POLYPECTOMY \\ Operative Hysteroscopy}

M. M*, R. Nonell, M. Cardona, M. Pons, F. Carmona

${ }^{[1]}$ Hospital Clínic $\sim$ Barcelona $\sim$ Spain

Summary (4 lines): In patients with demographic characteristics associated with an increased risk of glycine absorption, local anesthesia with sedation was the preferred anesthetic option.

Introduction: In this study we aim to analyze demographic characteristhics, type of anesthesia and quantify glycine absorption in women who underwent hysteroscopic polypectomy.

Material and Methods: This retrospective study includes 109 women who underwent hysteroscopic polipectomy between 2010-2011. The absorption of glycine was compared among general anesthesia (GA; $\mathrm{n}=56)$ and local anesthesia with sedation (LA; $n=53)$. Demographic, clinical and surgical characteristics were as well analyzed.

Results: The mean absorption was $256.6 \mathrm{ml}$ (10th-90th centile: 98.4 $499.4 \mathrm{ml}$ ) for the LA and $222.5 \mathrm{ml}$ (10th-90th centile: $116.7-325 \mathrm{ml}$ ) for the GA $(\mathrm{p}=0.005)$. Clinically significant absorption of glycine $(>500 \mathrm{ml})$ was increased in the LA group $(9.4 \%$ vs $0 \%$ in $\mathrm{GA} ; \mathrm{p}=0.02)$. Patients who underwent LA were older (55.9 vs 46.8 years; $p=0.04$ ), postmenopausal status was more frequent $(73.2 \%$ vs $30 \%$; $=0.001)$ weighted more ( 75.3 vs $62.5 \mathrm{Kg}$; $\mathrm{p}=0.036)$, had higher BMI (29.1 vs $\left.23.9 \mathrm{~kg} / \mathrm{m}^{2} ; \mathrm{p}=0.02\right)$ and longer operative time (19.3 vs $13.3 \mathrm{~min} . ; \mathrm{p}=$ $0.002)$.

Discussion: In patients with demographic characteristics associated with an increased risk of glycine absorption, LA was the preferred anesthetic option.

68

\section{DO REPEATED ENDOMETRIAL DESTRUCTION PROCEDURES DECREASE ABNORMAL UTERINE BLEEDING, AND DOES IT REDUCE THE NUMBER OF HYSTERECTOMY IN THIS INDICATION? \\ Operative Hysteroscopy}

L. Yael*, L. Guillaume, F. Hervé

${ }^{[1]}$ Hôpital de Bicêtre - Assistance Publique des Hôpitaux de Paris $\sim$ Le Kremlin Bicêtre France

Summary (4 lines): Hysteroscopic endometrial resection or destruction in the indication of abnormal uterine bleeding represent an alternative to hysterectomy, as it carries a lower morbidity rate. In case of failure of such procedure though, hysterectomy will most often be proposed as a second line of treatment. The place of the repetition of an endometrial destruction procedure has

Introduction: The aim of our study is to evaluate the efficiency and the satisfaction after two consecutive techniques of endometrial destruction in case of abnormal uterine bleeding.

Material and Methods: Nineteen patients with abnormal uterine bleeding underwent a second attempt of endometrial destruction, in our department, between 2004 and 2011.

Results: Sixteen of the nineteen patients included answered a questionnaire. The mean delay since the second procedure was 22 months.
Among them, 8 (i.e. $50 \%$ ) later underwent a hysterectomy, with 5 of them (31.25\% of all 16 patients) being directly attributed to treatment failure.

Patients said to be satisfied with the management of their condition in $68.75 \%$ of cases, and $93.75 \%$ of them would recommend it to a friend. Discussion: We thus suggest proposing a second conservative endometrial procedure in case of recurring abnormal uterine bleeding, especially in patients with surgical contra-indications, or patient willing to keep their uterus.

\section{9}

\section{ENDOMETRIAL DESTRUCTION TECHINQUES \\ Operative Hysteroscopy}

B. Sedlan*, J. Knez, M. Pakiž, B. Žegura

${ }^{[1]}$ University Medical Centre, Clinic for Gynaecology and Obstetrics Maribor $\sim$ Slovenia

Summary (4 lines): The comparison of efficiency and subjective success rate between two procedures used in heavy menstrual bleeding (HMB) treatment was done.

Introduction: Transcervical resection of endometrium (TCRE) and Thermal ballon endometrium ablation (TBA) were compared as treatment of HMB. Both approaches resulted in a comparable patient satisfaction.

Material and Methods: The questionnaires were sent to all the women treated for HMB with TCRE or TBA without any other endometrial pathology. The patients were asked about the procedure satisfaction, menstrual bleeding before and after the procedure, dysmenorrhea, need for oral hormonal contraceptives (OC), levonorgestrel IUS insertion or operation after the procedure.

Results: 29 women after TCRE and 50 women after TBA procedure completed the questionnaires. Both groups did not differ in mean age (42.72 vs 48.0 years, $p>0.05$ ). Satisfaction rate and time without symptoms after both procedures were comparable $(86.2 \%$ vs $86.0 \%$ and 46.4 vs 36.3 months, respectively; $p>0.05$ ). We determined that dismenorrhoea and HMB were lower after both procedures $(75,9 \%$ vs $92,0 \%$ and $92,1 \%$ vs $92,0 \%, p>0,05)$. Approximately $10 \%$ of patients required some form of of hormonal therapy afterwards (OC or levonorgestrel IUS). Repeated surgerical procedure after TCRE was needed in $10 \%$ and after TBA in $8 \%$ of cases.

Discussion: Our results show that patients are equally satisfied with both techniques. More than $90 \%$ of patients showed improvement in menstrual bleeding after both procedures.

443

ENDOMETRIAL POLYPS IN POSTMENOPAUSAL WOMEN: MALIGNANCY AFTER HYSTEROSCOPY RESSECTION

Operative Hysteroscopy

R. Dibi, C. Vanin, K. Rocha, S. Almeida, A. Ayub, S. Pessini*

${ }^{[1]}$ Complexo Hospitalar Santa Casa $\sim$ Porto Alegre $\sim$ Brazil

Summary (4 lines): Malignancy may occur in endometrial polyps without hysteroscopic suspicion vision

Introduction: Endometrial polyp (EP) is a benign lesion, with low potential for malignancy. Highest incidence occurs between 51 and 70 years. The aim is to determine the frequency of malignancy in polypoid lesions without suspicion in postmenopausal women.

Material and Methods: A cross-sectional study with review of hysteroscopic sheets and pathology reports. 238 post-menopausal patients 
submitted to hysteroscopic resection of endometrial polyps, from January 1999 to October 2006 in our Department.

Results: Malignant and pre malignant histopathology occurred in 3 patients $(1,2 \%)$ : adenocarcinoma in $2 / 238(0,8 \%)$ and atypia in $1 / 238$ $(0,4 \%)$.

Discussion: Because of the absence of a reliable non-invasive method to make the difference between benign from malignant cases, the complete resection of EP is the only safe way to obtain a definitive diagnosis despite of the low incidence of malignance in these lesions.

271

EVALUATION OF DEMOGRAPHICS AND OUTCOME OF INITIAL 50 CASES OF "ESSURE® HYSTEROSCOPIC STERILISATION

Operative Hysteroscopy

K. Connor*, K. Niblock, D. Morgan, K. Johnston

${ }^{[1]}$ Antrim Area Hospital $\sim$ Antrim $\sim$ United Kingdom

Summary (4 lines): ESSURE ${ }^{\circledR}$ is a fast, safe, effective method of permanent contraception with high levels of patient satisfaction and rapid return to normal activity.

Introduction: To evaluate introduction of ESSURE ${ }^{\circledR}$ Hysteroscopic Sterilization. The first 50 cases reviewed demographics, procedure, confirmatory test, hospital stay, patient satisfaction and complications. Material and Methods: Retrospective case series with follow up patient questionnaire

Results: Patient demographics included mean age 37, parity 2.8 , BMI 28.6. $90 \%$ were completed successfully, $10 \%$ converted to laparoscopic sterilization. Mean follow up 19 months. Procedure performed under general anaesthetic in $98 \%$. Bilateral placement of implants in $90 \%$, optimal bilateral placement in $90 \% .89 \%$ women attended for Hysterosalpingogram, 37.5\%described pain at HSG as severe. Bilateral tubal occlusion confirmed in all cases. $93 \%$ of patients reported procedure satisfaction, with a mean pain recall score of 3.2 and return to normal activities 5.2 days. $96 \%$ of women would recommend ESSURE $^{\circledR}$. One patient went on to have IVF, conceived then miscarried, no other pregnancies occurred. One patient had a perforation of device. 1 patient was treated for endometritis

Discussion: Hystersalpingogram as a confirmatory test appears to be poorly tolerated in over a third of patients and therefore an alternative imaging technique should be sought

232

\section{HYSTEROSCOPIC "ESSURE" VERSUS LAPAROSCOPIC STERILISATION: A RETROSPECTIVE COHORT COMPARATIVE STUDY \\ Operative Hysteroscopy}

K. Niblock*, K. Connor, D. Morgan, K. Johnston

${ }^{[1]}$ Antrim Area Hospital $\sim$ Antrim, N.Ireland $\sim$ United Kingdom

Summary (4 lines): Essure ${ }^{\circledR}$ is a fast, safe, successful and potentially cost effective method of permanent contraception and should be the primary surgical procedure in female sterilisation.

Introduction: The average woman spends three decades actively avoiding pregnancy. Essure ${ }^{\mathbb{R}}$ avoids laparoscopy and associated complications in women with additional risk factors $\&$ is 3.5 times more effective.

Material and Methods: Retrospective cohort comparative study of 70 patients. Charts were reviewed systematically for pre operative counselling, operative time, intraoperative complications, details of application of Essure ${ }^{\circledR}$ or Filshie ${ }^{\circledR}$ clips \& hospital stay. Patients were followed up \& a questionnaire completed.

Results: There is a statistical difference in favour of Essure ${ }^{\circledR}$ for pain, operative time, return to work \& hospital stay with no difference in complications or pregnancy rate. Hysterosalpingogram demonstrated $100 \%$ tubal occlusion but was poorly tolerated.

Discussion: Essure ${ }^{\mathbb{R}}$ is a safe and effective alternative to laparoscopic sterilisation with significantly less procedure related pain, shorter operative time, less hospital stay $\&$ faster return to normal activity makes Essure $^{\circledR}$ is economically superior for women.

69

\section{HYSTEROSCOPIC MYOMECTOMY FOR PATIENTS WITH COMBINATION OF LARGE SUBMUCOUS FIBROIDS AND INFERTILITY \\ Operative Hysteroscopy}

O. Aleksandrov*, L. Popova

${ }^{[1]}$ Odessa National medical university $\sim$ Odessa $\sim$ Ukraine

Summary (4 lines): This research shows effectiveness of hysteroresectoscopy with absence of complications and good reproductive outcome for young patients with large submucous fibroids and infertility Introduction: Myomas are common benign tumor found in women, as they affect $20 \%$ of women in the reproductive age group. When we use hysteroscopic myomectomy for removing of large fibroids $>3 \mathrm{~cm}$ it schould be controlled by laparoscopy

Material and Methods: There were 53 women 27-38 years olds with large submucous fibroid $>3 \mathrm{~cm}$ observed. All patients had primary and secondary infertility caused by different factors. The mean term of infertility $(2,6 \pm 1,4$ years). Hysteroscopic myomectomy was performed in all cases. In 8 cases the tumor size $>5 \mathrm{~cm}$ this procedure was controlled by laparoscopy to prevent uterine perforation. The mean time of operation was $25 \pm 4,9 \mathrm{~min}$

Results: We did not reveal any complications after these operations. Two-step operations were performed in 11 cases where we could not remove whole fibroid tissue properly. Such women were operated repeatedly. 28 women have become pregnant during 2 years and they had deliveries through the natural passages with 6 women whom caesarean section was performed

Discussion: The results have proved high effectiveness of hysteroresectoscopy which has to be more largely used for such patients. Diagnostic laparoscopy enables to avoid many possible complications and perform laparoscopic myomectomy if it is required

62

HYSTEROSCOPIC TREATMENT OF ATYPICAL POLYPOID ADENOMYOMA DIAGNOSED IN A YOUNG WOMAN

Operative Hysteroscopy

E. Martínez Lamela*, J. Molero Vilchez, F.J. Salazar Arquero, Y. Mena Buendía, M. Gimeno Aranguez, J.J. Hernández Aguado

${ }^{[1]}$ Hospital Universitario Infanta Leonor $\sim$ Madrid $\sim$ Spain

Summary (4 lines): Atypical polypoid adenomyoma (APA) is a rare lesion and radical treatment is necessary.

Introduction: For those women wishing to remain fertile, we can offer a conservative treatment once they have been informed regarding the associated risk of relapse and degeneration. To describe hysteroscopic resection of an APA in a young patient.

Material and Methods: A 24 years-old female with finding during routine examination a $1 \mathrm{~cm}$ of endocervical tumor, with diagnosis of 
APA. Tumor Committee proposed hysteroscopic resection of the lesion: adenomyosis in the front site of the isthmus wich affects the right side and some part of the back side of $2 \mathrm{~cm}$ covered by hypervascularized secretory endometrium.

Results: Resection with double tract resectoscope with $5 \%$ glycine solution and monopolar energy is performed. Resection in layers according to usual technique to removal of the APA, removal of endometrium adjacent to the APA, removal of the myometrium underlying the APA and multiple random endometrial biopsies. The lesion was completely resected and resulted complex gland proliferation with atypia compatible with APA and Microinvasion of the myometrium The patient followed progestative treatment and underwent regular ultrasound examination and hysteroscopic monitoring every 3 months. The results were no evidence of disease.

Discussion: Conservative hysteroscopic surgery may be a good treatment option for APA in patients wishing to remain fertility.

\section{8}

\section{MINI-SCISSOR VERSUS BIPOLAR TWIZZLE IN OFFICE HYSTEROSCOPIC METROPLASTY: A PROSPECTIVE RAN- DOMIZED STUDY}

Operative Hysteroscopy

S. El-tawab*, M. Kamel, O. Al Ashkar, M. Ismail

${ }^{[1]}$ Shatby maternity university hospital $\sim$ alexandria $\sim$ Egypt

Summary (4 lines): office hysteroscopic metroplasty, using mechanical or electrosurgical resection are equally feasible and safe. But, electrosurgical resection causes significant more pain.

Introduction: To compare between mechanical (mini-scissor) and bipolar electrosurgical resection (twizzle) in terms of feasibility and pain scoring, in office hysteroscopic metroplasty of short narrow based uterine septum.

Material and Methods: 40 patients, with recurrent abortion or infertility, diagnosed with short narrow based uterine septum $<2 \mathrm{~cm}$ were included.

A mini-hysteroscope (versascope ${ }^{\circledR}$ )with vaginoscopic approach was used in all patients. Either a 7Fr mini-scissor (group A: mechanical resection)or 5Fr bipolar twizzle(group B: electrosurgical resection) was used at random.

Results: No significant difference was found between both groups in term of operative time and fluid deficit $(12.68 \pm 2.08$ versus $12.08 \pm 2.62)$ and $(519 \pm 118.59$ versus $480 \pm 106.01)$ respectively. Pain scores were significantly higher in group B (bipolar twizzle) than in group A (mini-scissor), $(4.01 \pm 0.68$ versus $6.98 \pm 1.28)$ with $\mathrm{p}$ significant $<0.05$.

Discussion: Office hysteroscopic metroplasty is a reliable option in treating short narrow based uterine septum. Mechanical or bipolar resection are equally effective and safe; but bipolar electrosurgical resection causes significant more pain which may necessitate cautious selection in patients with known low pain threshold.

\section{0}

OUR EXPERIENCE IN HYSTEROSCOPIC DIAGNOSIS AND TREATMENT OF UTERINE INTRACAVITARY PATHOLOGIES Operative Hysteroscopy

A. Gvenetadze*, T. Sabakhtarashvili, L. Dzotsenidze

${ }^{[1]}$ Tbilisi State University, ZSRC $\sim$ Tbilisi $\sim$ Georgia

Summary (4 lines): The retrospective study shows: hysteroscopy is the best approach to diagnose and treat uterinal intracavitary pathology.
There was no significant difference between intraoperative and hystomorfological diagnosis.

Introduction: Objective: To study the efficacy of hysteroscopy for diagnosis and treatment of uterine intracavitary pathologies.

Material and Methods: in our new clinic, that is functioning since January 2012 we performed 132 hysteroscopy during 6 months. All of them were intrapatient with general anaesthesia. $85 \%$ of hysteroscopies, where the problem was infertility, were performed together with Laparoscopy (8\%IVF preparation). During the Operation first step was operative hysteroscopy ( $2.9 \mathrm{~mm}$ Karl Storz). polipectomy of small polyp was performed by grasping forceps, big polyp, endometrial hyperplasia or submucous myoma was managed by hysteroresectoscopy.

Results: 20/132 (15.15\%) woman had endometrial polyps;6/132 (4.54\%) endocervical polyps; 5/132 (3.78 \%) had submucous myoma; 27/132 (20.45\%) endometrial hyperplasia;2/132 (1.51\%) endocervical hyperplasia;24/132 (18.18 \%) adenomyosis; 1/132(0.75\%) unicornuate uterus; 48/132(36.36\%) normal uterine cavity; 2/132 (1.51\%) placental polyp; $5 / 132(3.78 \%)$

Discussion: there is no need of hysteroresectoscopy for small polyps and polypectomy by grasping forceps may perform. If there is presented a big polyp, endometrial hyperplasia or submucous myoma hysteroresectosopy is necessary.

200

\section{OUTPATIENT HYSTEROSCOPY IN WOMEN WHO HAVE UNDERGONE SURGERY TO THE CERVIX}

Operative Hysteroscopy

G. Papageorgiou*, A. Gkoutzioulis, G. Michos, A. Pinto, G. Halmos, M.D. Dacco', A. Magos

${ }^{[1]}$ Royal Free Hospital, Hampstead, London, UK. $\sim$ London United Kingdom

Summary (4 lines): Cervical surgery does not affect the success of outpatient diagnostic hysteroscopy.

Introduction: Outpatient diagnostic hysteroscopy has become the gold standard for evaluating the uterine cavity. We wanted to find out the feasibility of the investigation in women who have undergone cervical surgery.

Material and Methods: We identified 52/2069 (2.5\%) women seen in our OP Hysteroscopy Clinic between October 2002 and May 2012 who had a history of cervical surgery. We compared the outcome of hysteroscopy in this group with the rest of the patients (control group). All hysteroscopies were done using a $2.9 \mathrm{~mm}$ fore-oblique hysteroscopy, N/Saline for uterine distension, and a "no touch" (vaginoscopic) technique.

Results: The 52 women in our study group were similar to the controls in terms of age, parity, indication for hysteroscopy and uterine size. Seven $(13.4 \%)$ of the hysteroscopies could not be completed in the study group compared with $156(7.7 \%)$ in the control group. The main reason for not carrying or failing with hysteroscopy was pain (3/7 cases in the study group and 35/156 in the control group) and cervical stenosis (1/7 in the study group and 41/156 in the control group). None of these differences were statistically significant.

Discussion: We are not aware of any study which has addressed the effect of cervical surgery on the success of outpatient hysteroscopy. Our study shows that these patients are good candidates for hysteroscopy

\section{6}

OUTPATIENT RESECTOSCOPY: ANALYSE OF 589 CONSECUTIVE PROCEDURES

Operative Hysteroscopy 
V. Silva, C. Ferreira, N. Nogueira-martins*, A. Pipa, F. Nogueiramartins

${ }^{[1]}$ Department of Obstetrics and Gynecology - São Teotónio Hospital Viseu $\sim$ Portugal

Summary (4 lines): Review of 589 consecutive outpatient resectoscopies, including procedures and complications

Introduction: Resectoscopy is an endoscopic surgical procedure that uses a resectoscope for the treatment of benign intrauterine pathology. It can be performed in an outpatient setting (stay in hospital less than $24 \mathrm{~h}$ ).

Material and Methods: Between January-2008 and April-2012, 589 patients were admitted at the authors'Department to undergo an outpatient resectoscopy. All procedures were done with a bipolar resectoscope, under general anesthesia. Postmenopausal patients had done cervical preparation with misoprostol. Hegar bougies until $9 \mathrm{~mm}$ were used to cervical dilatation.

Results: In 10 cases it was impossible to enter the uterine cavity cervical stenosis (5) or false paths (5). 425 polypectomies (73\%), 83 myomectomies (14\%), 24 myomectomies plus polypectomies (4\%) and 19 endometrial ablations (3\%) were performed. The rate of complications was $2,8 \%: 5$ uterine perforations, 5 intraoperative bleeding,2 significant postoperative bleeding, 1 cervical laceration with need of local treatment, 1 pelvic infection, and 2 technical faults. No cases of intravasation syndrome. 6 cases of endometrial carcinoma were diagnosed.

Discussion: Our low prevalence of complications was probably related to high levels of training and experience of surgeons. In our experience, bipolar resectoscopy, performed in an outpatient setting, is a feasible and safe surgical technique to treat benign endocavitary pathologies.

14

\section{REPEAT RESECTOSCOPIC ENDOMETRIAL RESECTION AFTER FAILED PRIMARY RESECTOSCOPIC ENDOMETRIAL ABLATION: IS IT WORTH THE RISK? \\ Operative Hysteroscopy}

G.W. Yeung*, G.A. Vilos, M. Garcia-erdeljan, J. Marks, A.G. Vilos, B. Abu-rafea

${ }^{[1]}$ St. Joseph's Health Care, Department of Obstetrics and Gynecology, The University of Western Ontario $\sim$ London $\sim$ Canada

Summary (4 lines): Repeat resectoscopic endometrial ablation (RREA) is a feasible, safe alternative to hysterectomy (HYST) for abnormal uterine bleeding (AUB) from benign causes when performed by experienced surgeons.

Introduction: Following primary (PREA), $<=30 \%$ women require RREA or HYST for persistent AUB, uterine/pelvic pain or both. Since HYST is a major surgical procedure with significant morbidity, mortality, costs and resources, we routinely offer RREA as an alternative. We present patient characteristics, indications, and outcomes associated with RREA.

Material and Methods: After IRB approval, 183 women (university hospital) who failed PREA underwent RREA from 19932007. Follow-up included office visits and telephone interview. RREA was performed under general anesthesia using $1.5 \%$ glycine, $26 \mathrm{~F}$ resectoscope, $8 \mathrm{~mm}$ monopolar loop electrode at $120 \mathrm{~W}$ cut waveform $(74.7 \%), 5 \mathrm{~mm}$ rollerball coag waveform $(22.0 \%)$ or both $(3.3 \%)$.

Results: Corresponding median age (range) for PREA and RREA: 40 (26-70) and 43 (29-76)yrs. RREA indications: AUB (52.7\%), AUB/ pain $(25.8 \%)$, pain $(18.8 \%)$, other $(2.7 \%)$. RREA complication rate $=$ $5.5 \%(n=10)$ : uterine perforation (1); perforation/fluid absorption (2); fluid absorption (1); bleeding (2); false passage (3); partial ablation (1). Following RREA, $73.2 \%$ women avoided HYST.

Discussion: Median time to RREA was approx. 3 yrs. RREA is feasible and safe by experienced surgeons and obviated HYST in $73.2 \%$ women.

131

\section{REPRODUCTIVE OUTCOME OF SEPTATE UTERUS FOLLOWING HYSTEROSCOPIC SEPTUM RESECTION \\ Operative Hysteroscopy}

C. Sofoudis*, N. Vlahos, V. Grigoriou, G. Creatsas

${ }^{[1]} 2 N D$ DEPARTMENT OF OBSTERISC AND GYNECOLOGY UNIVERSITY OF ATHENS ARETAIEION HOSPITAL ATHENS Greece

Summary (4 lines): Hysteroscopic septum resection improves the live birth rates and the future fertility.

Introduction: To evaluate the reproductive outcome following hysteroscopic septum resection in patients with infertility.

Material and Methods: The reproductive efficiency in thirty two patients with septate uterus who underwent hysteroscopic septum resection in our institution from January 2005 to December 2010 was analyzed. The septum resection was performed using a bipolar versapoint system. Reproductive performance of these patients after septum resection was estimated. The main outcome measures were clinical pregnancy and live birth rates. Exclusions criteria were associated genital or pelvic diseases (myomas, endometriosis, adhesions and pelvic inflammatory disease.)

Results: Twenty-eight patients were included in the study. Twelve out of twenty-eight ( $42 \%$ ) eventually became pregnant. Eight out of twelve cases $(66 \%)$ had natural conception and four out of twelve cases $(33 \%)$ IVF. In one out of four IVF cases $(25 \%)$ was a twin pregnancy. In sixteen out of twenty-eight patients $(50 \%)$ did not get pregnant in a follow up period of three years after the hysteroscopic septum resection. In four out of thirty-two cases $(12.5 \%)$ we lost contact.

Discussion: IN OUR STUDY POPULATION THE HYSTEROSCOPIC RESECTION WAS ACCOMPAINED BY A SIGNIFICANT IMPROVEMENT IN PREGNANCY OUTCOME.

86

\section{RESULTS OF HYSTEROSCOPIC METROPLASTY IN THE MANAGEMENT OF UTERINE SEPTUM \\ Operative Hysteroscopy}

A. Hernandez*
${ }^{[1]}$ Hospital Quiron Barcelona $\sim$ Barcelona $\sim$ Spain

Summary (4 lines): We studied the hysteroscopic metroplasty in the management of uterine septum. in the Valle de Hebron Hospital in Barcelona.

Introduction: TheUterine septum, could cause adverse obstetric outcome. Uterine abnormalities are classified in, congenital and acquired. The management of uterine septum is an hysteroscopic metroplasty technique. We evaluate this technique in the Valle de Hebron Hospital in the last two years.

Material and Methods: We evaluate patients with septate uterus in the Vall d'Hebron Hospital, since 2010-2012, performed the hysteroscopy with resectoscope or Bettocchi hysteroscope. We evaluate the technical difficulties, complications and obstetric outcome.

Results: We selected 18 cases with diagnosis of uterine septumsubseptate. The age of the patients evaluated has a mean $33.7 \mathrm{y}$. The diagnostic methods were: Nuclear Magnetic Resonance, HSG and 
ecography, we performed diagnostic and operative hysteroscopies for the mamagement. The only complication that document was bleeding in 2 cases forced to stay proceedings in any diagnostic hysteroscopy was necessary to suspend pain.

Discussion: This findings confirms that this malformation produce adverse obstetric outcome. The finding of 3 patients with thrombophilia disorder in a very limited series draws attention to the possible relationship between the two findings.

\section{5}

\section{T SHAPED ENDOMETRIAL CAVITY AND INFERTILITY} Operative Hysteroscopy

\author{
M. Mounir* \\ ${ }^{[1]}$ Agial gynecology hospital $\sim$ Alexandria, Egypt $\sim$ Egypt
}

Summary (4 lines): Metroplasty of the $\mathrm{T}$ shaped uterus in infertile women has magnificent pregnancy results $(58 \%)$.

Introduction: $\mathrm{T}$ shaped uterine cavity is considered major obstacle facing both spontaneous and assisted pregnancies. What is the role of hysteroscopy?

Material and Methods: The study conducted for 36 months, including 88 women suffering from 1ry infertility for 3-8 years with exclusion of the male factors, their ages were 27 to 35 years, 50 of them had ICSI failure. Vaginal U/S revealed narrow endometrial cavity. The diagnoses had been confirmed by either 3D U/S or HSG. postmenstrual operative 90 degree resectopscope was used in all of them with monopolar current $25-30 \mathrm{~V}$ and glycine as a distension media, under anaesthesia. Postoperative cyclic treatment used for 2 cycles.

Results: Spontaneous pregnancy:19 women (21.6 \%) had became pregnant within 6 months, 13 cases(14.8\%)within 12 months, 17 (19.3\%)cases within 24 months , 3 cases of them had missed abortion after abortion one had IUFD due to severe PET.2 cases had preterm deliveries. The rest had Full Term deliveries. Assisted pregnancies:16 women $(18.18 \%)$ had ICSI 9 of them had success, 5 of them had twin pregnancies 4 of them had delivered 1 preterm and three full term and the other still followed up. Lost cases:11 cases.

Discussion: In our study it was clear that, metroplasty of T shaped and narrow infantile uterine cavity has marked success in the treatment of infertile women in addition to its effect in term pregnancies that discussed in previous studies.

\section{0}

THE EFFECT OF THE INITIAL MORPHOLOGY OF UTERINE SEPTUM AND HYALOBARRIER GEL® APPLICATION ON THE FINAL ANATOMICAL RESULTS AFTER COMPLETE HYSTEROSCOPIC METROPLASTY

Operative Hysteroscopy

\author{
L. Artur*, L. Inga, P. Kazimierz, B. Tomasz, M. Tomasz \\ ${ }^{[1]}$ Chair of Gynaecology and Obstetrics, Departament of Gynaecology \\ and Oncology, Jagiellonian University $\sim$ Krakow $\sim$ Poland
}

Summary (4 lines): The patients who benefit most from anti-adhesion prevention are women with a wide-based uterine septum (interostial distance)

Introduction: The aim was the assessment of the effect of the initial morphology of uterine septum and Hyalobarrier gel ${ }^{\circledR}$ application on the formation of intrauterine adhesions and final anatomical results after complete hysteroscopic septum resection

Material and Methods: Prospective study involved 93 women with uterus septus and a RA or infertility treated histeroscopically; group A -anti-adhesion prevention was not used and group B-an intrauterine application of Hyalobarrier gel ${ }^{\circledR}$. In preoperative diagnostics using 3DSHG to characterise the septal morphology (length, area, interostial distance). Hysteroscopic metroplasty was monitored by an intraoperative 3D/4D-TRUS. During postoperative diagnostics, 3D-SHG and second-look hysteroscopy were used.

Results: The interostial distance and the application of the Hyalobarrier gel ${ }^{\circledR}$ were independent factors influencing the final anatomical result and the frequency of repeated hysteroscopic interventions $(\mathrm{p}<$ 0.001). The threshold value of the interostial distance above which the observed results were worse was $4.30 \mathrm{~cm}$.

Discussion: We assumed that the morphological diversification of the septum can influence the formation of adhesions and be an indication for the use of the targeted anti-adhesion prevention.

\section{1}

\section{THE EFFICACY OF TRANSCERVICAL RESECTION OF BIG MYOMA COMBINED WITH GNRH-A THERAPY - ANALYSIS OF 75 CASES}

Operative Hysteroscopy

E. Xia*

${ }^{[1]}$ Fuxing Hospital, Capital medical University $\sim$ Beijing $\sim$ China

Summary (4 lines): TCRM is the golden standard treatment in the treatment of submucosal myoma of uterus. Preoperation GnRH-a therapy can be useful and necessary to enlarge selection of patients, decrease diffculty during operation, and prevention of complications. Introduction: To investigate the efficacy of transcervical resection of big myoma (TCRM) combined with drug therapy in the treatment of submucosal myoma of uterus.

Material and Methods: There were 75 cases of uterine submucosal myoma being treated by TCRM from October 2003 to October 2009. Their diameters were greater than $50 \mathrm{~mm}$. Among them 13 cases were type 0,30 type I and 32 type?. All cases were accepted therpy of $\mathrm{GnRH}-\mathrm{a}$ for three agents. The mean diameter of myoma decreased to $33 \pm 12 \mathrm{~mm}$ and were resected by monopolar resectoscope. Follow up 2 years.

Results: Seventy five cases myoma were totally resected. There diameter of myoma were $20 \sim 50 \mathrm{~mm}(33 \pm 12 \mathrm{~mm})$. The operating time was $37 \pm 14 \mathrm{~min}$. The intraoperative blood loss was $48 \pm 15 \mathrm{ml}$. No residual myoma was found and post-operative menstruation was improved obviously after one year.

Discussion: GnRH-a is quite useful to reduce the volume of big myoma (diameter of myoma $>5 \mathrm{~cm}$ ) which can be resected totally hysterocopically.

96

\section{THE IMPACT OF INHALATED SEVOFLURANE IN GENERAL ANESTHESIA ON GLYCINE ABSORPTION IN HYSTEROSCOPIC MYOMECTOMY \\ Operative Hysteroscopy}

M. Nonell*, M. Munmany, C. M, M. Pons, F. Carmona

${ }^{[1]}$ Hospital Clínic $\sim$ Barcelona $\sim$ Spain

Summary (4 lines): The use of sevoflurane in General Anesthesia (GA) seems to be associated with and increased glycine absorption.

Introduction: In this study we aim to analyze if the use of sevoflurane in General Anesthesia (GA) is associated with and increased and significant glycine absorption in hysteroscopic myomectomy. 
Material and Methods: Retrospective study of 41 women who underwent hysteroscopic myomectomy between 2010-2011; of those, 25 underwent GA with inhalated sevoflurane (GAsevo+)while 16 GA without sevoflurane (GAsevo-). The absorption of glycine, demographic, clinical and surgical characteristics were compared among the two groups.

Results: The median absorption was $352 \mathrm{ml}$ (10th-90th centile: 197.2 $1567.2 \mathrm{ml})$ for the GAsevo + and $215 \mathrm{ml}$ (10th-90th centile: 23.8 $979.8 \mathrm{ml})$ for the GAsevo- $(\mathrm{p}=0.09)$. Clinically significant absorption of glycine $(>500 \mathrm{ml})$ was increased in the GAsevo + compared to GAsevo- group (48\% vs $25 \%$; $=0.141)$ and $>1000 \mathrm{ml}(28 \%$ vs $6.2 \% ; p=0.08)$ but not significantly.

Demographic, clinical and surgical characteristics were comparable between the two groups with the exception of operative time which was significantly increased in the GAsevo- group ( 33.3 vs $26.1 \mathrm{~min} ; \mathrm{p}=0.023$ ). Discussion: The use of sevoflurane seems to be associated with more glycine absorption and the need of premature discontinuation of the surgery. Further studies with a major number of cases are needed to confirm our findings.

95

\section{THE IMPACT OF SEVOFLURANE ON GLYCINE ABSORPTION IN OPERATIVE HYSTEROSCOPY}

Operative Hysteroscopy

M. Munmany*, R. Nonell, M. Cardona, M. Pons, F. Carmona

${ }^{[1]}$ Hospital Clínic $\sim$ Barcelona $\sim$ Spain

Summary (4 lines): We aim to analyze if the use of sevoflurane in the general anesthesia is associated with and increased and significant glycine absorption.

Introduction: We aim to analyze if the use of sevoflurane in the general anesthesia is associated with and increased and significant glycine absorption.

Material and Methods: Retrospective study includes 115 women who underwent operative hysteroscopy with General anesthesia (GA) between 2010-2011; patients were classified in 2 groups: with (GAsevo+; $\mathrm{n}=77$ ) and without (GAsevo-; $\mathrm{n}=38$ ) inhalated sevoflurane. The absorption of glycine was compared among the two groups.

Results: Demographic, clinical and surgical characteristics were comparable between the two groups.

Women in the group GAsevo + had a higher median absorption of the glycine solution (10th-90th centile) compared with women in the GAsevogroup $(259 \mathrm{ml}(156-989 \mathrm{ml})$ vs $200 \mathrm{ml}(60.8-539 \mathrm{ml}) ; \mathrm{p}=0.007)$.

Secondary outcomes included clinically significant absorption of glycine; $>500 \mathrm{ml}(26.2 \%$ vs $8.8 \% \mathrm{p}=0.096)$ and $>1000 \mathrm{ml}(10 \%$ vs $2.7 \% \mathrm{p}=$ 0.147 ), and differences were not significant among the two groups.

Operative time was significantly longer in the GAsevo- group compared to GAsevo + (19.1 vs $12 \mathrm{~min} ; \mathrm{p}=0.03)$

Discussion: The use of sevoflurane is associated with more glycine absorption and the need of premature discontinuation of surgery, more studies should analyze if it should be avoided for general anesthesia for operative hysteroscopy.

213

\section{THE ROLE OF ANTIBIOTIC PROPHYLAXIS IN OPERATIVE HYSTEROSCOPY}

Operative Hysteroscopy

O. Gregoriou, N. Vlahos, P. Bakas, C. Grigoriadis*, V. Gregoriou, A. Liapis, G. Creatsas

${ }^{[1]}$ University of Athens, 2nd Department of Obstetrics and Gynecology, Aretaieion Hospital $\sim$ Athens $\sim$ Greece
Summary (4 lines): The aim of this study was to examine if antibiotic prophylaxis is necessary or not in operative hysteroscopy.

Introduction: Infections associated with hysteroscopy are uncommon and occur in $0.18-1.5 \%$ of cases. It is important to reduce the prevalence of iatrogenic surgical infections. On the other hand, indiscriminate antibiotic use results to development of antibiotic-resistant bacteria.

Material and Methods: This was an eight-year randomized controlled clinical study. The study group included 411 patients who underwent operative hysteroscopy between January 2003 and December 2010 in our Department. All cases diagnosed with intrauterine abnormality (submucosal myomas, endometrial polyps, adhesions, septa) during diagnostic hysteroscopy that led to operative hysteroscopy were analyzed. 206 (Group A) received intraoperatively or immediately preoperatively antibiotic prophylaxis, while 205 (Group B) underwent the operation without antibiotic prophylaxis. There were no significant differences between the two groups for age, parity, body mass index or previous surgical history.

Results: There were no significant differences in the prevalence of postoperative infections between women who received antibiotic prophylaxis $(0.48 \%)$ and those who did not $(0.49 \%)$.

Discussion: Routine antibiotic prophylaxis is not recommended in cases of operative hysteroscopy, because of a very low risk of infection associated with hysteroscopy and lack of evidence of prophylaxis efficacy.

51

\section{THE ROLE OF PLASMA BIPOLAR MUSHROOM ELECTRODE TRANSCERVICAL VAPORIZATION IN THE MANAGEMENT OF BENIGN INTRAUTERINE LESIONS}

Operative Hysteroscopy

E. Xia*

${ }^{[1]}$ Fuxing Hospital $\sim$ Beijing $\sim$ China

Summary (4 lines): Plasma bipolar mushroom electrode transcervical vaporization in the management of benign intrauterine lesions was save and efficiency especially to stop bleeding.

Introduction: To evaluate the effectiveness of plasma bipolar mushroom electrode transcervical vaporization in the management of benign intrauterine lesions.

Material and Methods: From Oct. 2011 to May 2012 there were 31 cases of benign intrauterine lesions treated by plasma bipolar mushroom electrode transcervical vaporization. They were small multiple submucous myoma 10 cases, Adenomyosis 4 cases, endometrial ablation 6 cases. polyps 9 cases and uterine adhesion 4 cases.

Results: All procedures performed smoothly. Operating time was shoter. Intraoperation bleeding was very rare. No venous air embolism was encountered.

Discussion: Although plasma bipolar mushroom electrode transcervical vaporization to treat benign intrauterine lesions can produce much more air bubbles but no venous air embolism was encountered.

\section{1}

\section{EMERGENCY LAPAROSCOPY IN GYNAECOLOGY: OUR EXPERIENCE \\ Operative Risk Management}

\author{
L. Mañalich, A. Gascón, L. Berlanga, E. Suárez*, J. Xercavins \\ ${ }^{[1]}$ Hospital Vall d'Hebron $\sim$ Barcelona $\sim$ Spain
}

Summary (4 lines): Surgical outcomes of the laparoscopic approach in the management of gynaecological emergencies. 
Introduction: The aim of our study was to evaluate the feasibility and the security of the laparoscopy for the diagnosis and the treatment of gynaecological emergencies.

Material and Methods: A retrospective descriptive study (Jan2010-Dec2011) was performed by a review of 91 cases of gynaecological emergencies managed by laparoscopy. Age, BMI, previous abdominal surgery, the indication, the presence of abdominal distension and hemodynamic instability, surgical-clinical correlation, the rate of operative complications and of conversion to laparotomy were evaluated.

Results: $17,6 \%$ of cases had some previous abdominal surgery. Ectopic pregnancy, ovarian torsion and pelvic inflammatory disease were the most common indications, with a correct surgicalclinical correlation in the $85,7 \%$. Abdominal distension and hemodynamic instability was noted in a $34,1 \%$ and a $33,3 \%$. The rate of intra and postoperative complications was $4,4 \%$ and $6,6 \%$. The conversion to laparotomy was needed in a $6,6 \%$ of cases.

Discussion: The experience in our centre shows that laparoscopy is a feasible and safe surgical approach for management of gynaecological emergencies, considering the low rate of operative complications and conversion to laparotomy; and, regarding the fact that abdominal distension, hemodynamic instability and previous abdominal surgery are not determining factors for the choice of the surgical approach.

\section{1}

\section{IMPACT OF OVER-NIGHT SHIFTS ON LAPAROSCOPIC SKILLS AND COGNITIVE FUNCTIONS AMONG NORWEGIAN GYNAECOLOGISTS}

Operative Risk Management

A. Veddeng*, T. Husby, I. Bøe Engelsen, H. Flaatten

${ }^{[1]}$ Haukeland University Hospital $\sim$ Bergen $\sim$ Norway

Summary (4 lines): Our study did not show impaired laparoscopic skills or cognitive functions among gynaecologists after 17.5 hours on site on call.

Introduction: It is common belief that the length of Gynaecologists' on calls is a threat to patient safety. The trial was based on both laparoscopic skills and cognitive function which were tested directly post call.

Material and Methods: A prospective cohort study of 28 gynaecologists trained to proficiency on a laparoscopic simulator. The participants performed three repetitive salpingectomies after 17.5 hours on call. Cognitive function was tested by a standardized cognitive testbattery (CANTAB). Baseline results in rest for both tests were gathered prior to the post call test.

Results: There were no significant differences in laparoscopic skills of the gynaecologists after 17.5 hours on site on call compared to their own baseline results. 1 of 4 tests on reaction time was significantly altered post call.

Discussion: Unlike several other studies we have not established in our study that gynaecologists have impaired operative skills or cognitive functions after 17.5 hours on site call. Although we did not register any differences in surgical performances, we believe that the study adds valuable information as we have tested gynaecologists in an emergency situation where they performed one of the most important surgical procedures. Additionally the test tool has been validated by the study group itself.

\section{9}

\section{LAPAROSCOPIC LYMPHOCELE FENESTRATION IN GYNAECOLOGICAL CANCER PATIENTS \\ Operative Risk Management}

R. Marc, D. Herbert*, M. Anke, A. Julia, R. Ingo Bernard

${ }^{[1]}$ Jena University Hospital Jena Germany

Summary (4 lines): We summarize our long-term evaluation of laparoscopic lymphocele fenestration with a total of 102 patients included. Introduction: The objective of this study was to evaluate laparoscopic lymphocele fenestration (LLF) as a first-line treatment in gynaecological cancer patients with a history of retroperitoneal lymph node dissection (LND)

Material and Methods: Surgical outcome of 102 patients who underwent LLF was analyzed. Main outcome measures included operating time, blood loss, conversion rate, intra- and post-operative complication rate, hospital stay and relapse rate

Results: A total of 132 lymphoceles were fenestrated in 102 patients per laparoscopy. Mean duration of surgery was 115.6 minutes and the average intra-operative blood loss per patient was $146 \mathrm{ml}$. Overall conversion rate to laparotomy was $7.8 \%$. Intra- and postoperative complication rates were estimated at $9.8 \%$ and $5.9 \%$ retrospectively. The rate of intraoperative and postoperative complications was significantly higher in patients after pelvic plus paraaortic LND $(23.8 \%)$ compared to those after pelvic LND only (3.6\%; $>>0.01)$. Mean follow-up time was 60.4 months and a total of seven symptomatic recurrences of lymphoceles were observed (recurrence rate: $6.9 \%$ ).

Discussion: LLF has been previously established as an efficient firstline treatment option in a post-transplant context. Our data suggest that these favourable results for LLF may be transferable to gynaecological cancer patients.

514

\section{LAPAROSCOPY IN GYNECOLOGICAL EMERGENCY A COMPARISON BETWEEN 2 DEPARTMENTS \\ Operative Risk Management}

M. Burtea*, R. Ganescu, L. Ples, N. Ples, I. Negoi, B. Gaspar, S. Paun ${ }^{[1]}$ Clinic of Obstetrics and Gynecology, "Bucur" $~$ Bucharest $\sim$ Romania

Summary (4 lines): Although gynecologic emergency is the privilege of gynecologists, there are cases when patients address first to general surgeons.

Introduction: The aim of the study is to compare a general surgery department and a gynecological department in terms of acute abdomen of gynecologic origin.

Material and Methods: A retrospectiv study is carried out for 20 patients with acute abdomen of gynecologic origin treated in General Surgery Department of Emergency Hospital Bucharest (Group I)and for 20 patients with the same pathology treated in Obs\&Gyn Department „Bucur”(Group II). The pathology includes: 15 complicated ovarian cysts(OC)and 5 ectopic pregnancies(EP)for each group.

Results: Mean age was 26,3(Group I)vs.24,7(Group II). In terms of localisation - 16 cases on right side (13OC and 3EP) in Group I vs. 12 cases on the right side(8OC and 4EP)in Group II. Time admission to OR - 6,45 hrs vs. 8,12 hrs. Mean operation time was $56,45 \mathrm{~min}$ in Group I vs. 74,5 min in Group II. In both Groups cystectomy and salpingectomy were performed.2 patients with EP needed blood transfusion in both Groups. There was no conversion, morbidity or mortality. Median hospital stay was4,74 days vs.6,2 days.

Discussion: Patients with right lower abdominal pain of gynecologic origin presented as acute abdomen address not allways to a gynecologist. In this study there are no significant differencies in terms of treatment and postoperative evolution between the 2 Groups. 
88

\section{SITE SPECIFIC ADHESION PREVENTION IN GYNAECOLOGIC ENDOSCOPIC SURGERY \\ Operative Risk Management}

L. Mettler*, T. Schollmeyer, I. Alkatout, G. Peters

${ }^{[1]}$ University Hospitals of Schleswig-Holstein $\sim$ Kiel, SchleswigHolstein Germany

Summary (4 lines): Adhesions either preoperatively existant or postoperatively coming into existance put every endoscopic surgical procedure at a certain risk level. Therefore risk protection by adhesion prevention is indicated.

Introduction: In every 5th laparoscopy we have to deal with adhesions.

Material and Methods: We performed a selective pubmed/medline search using ,adhesions“, „laparoscopy“ and „prevention of adhesions" as key words.

Results: The first generation of barriers consisted of meshes like „Interceed“. Later viscous solutions as „Intergel“ and „tissue col“ were propagated". In recent years sprayable liquids as polyethylene glycols=PEG's (SprayShield and Coseal) which polymerize to hydrogels with addition of colorants and without color, revealed $65-70 \%$ reduced adhesion formation.

Hydroflotation with liters of icodextrin solutions (4\%) for rinsing and instillation at the end of surgery gave on the surgical site a significant adhesion reduction. „HyaCorp endogel, BioScience, Germany“is a product on hyaluronate basis that is applied by simple squeezing through an applicator. It has recently gained attraction again and appeares to be effective.

Discussion: The level of evidence of the current available products on the market is limited. However, a combination of a site specific spray or gel together with hydroflotation and, possible assisted by an antiinflamatory medication seems promising.

374

TIMING OF FIRST ROBOTIC COMPLEX ONCOLOGICAL PROCEDURES. PRELIMINARY RESULTS.

Robotics

C. Goiri*, T. Bernal, L. Martinez, M. Arrue, L. Urbieta, A. Lekuona, M. Gorostidi

${ }^{[1]}$ Hospital Universitario Donostia $\sim$ San Sebastian $\sim$ Spain

Summary (4 lines): We describe the timing of our learning curve in robotic complex oncological procedures.

Introduction: Complex laparoscopic oncological procedures are exhausting and long. The setup and docking of the robot is time consuming, especially in first cases.

Material and Methods: We describe the first 9 complex robotic oncological procedures performed in our institution from 28th November 2011 to 18 th June 2012 .

We performed complete staging procedures for 1 ovarian cancer, 1 type II endometrial cancer, 1 advanced cervical cancer (with ovarian transposition), as well as 6 type I endometrial cancers.

Results: Our average time was $340 \mathrm{~min}$.

Factors influencing surgical time may include:

3 conversions from retroperitoneal to transperitoneal access

Dock changes in 2 patients

Average BMI of 34

2 full staging procedures with appendectomy and omentectomy

Previous surgeries, adhesiolysis required
1 ovarian transposition

Our learning curve

Table 1.

Discussion: Longer times in our first cases show our learning curve in complex oncological procedures.

197

\section{COMPARISON OF PERIOPERATIVE OUTCOME ASSOCIATED WITH ROBOTIC ASSISTED HYSTERECTOMY AND MYOMECTOMY}

Robotics

K. Huang*

${ }^{[1]}$ New York Hospital Queens $\sim$ New York $\sim$ United States

Summary (4 lines): We want to assess the relative safety of the two of the most commonly performed surgical procedures for the treatment of fibroid uterus. We found that RAH is associated with decreased blood loss and a shorter operative time when compared to RAM.

Introduction: To compare perioperative outcomes in patients with large uteri undergoing robotic-assisted hysterectomy (RAH) and robotic - assisted myomectomy (RAM) performed by a single surgeon.

Material and Methods: Using our hospital's surgical database, we identified patients who underwent either RAH or RAM from May 2010 to March 2012. Only patients with specimen weighing over $250 \mathrm{gm}$ were included in the analysis. We examined the relationship between the patients' perioperative outcomes and the type of surgery performed (RAH versus RAM).

Results: 93 patients undergoing RAH and 33 patients undergoing RAM were identified. Patients who underwent RAM were found to be significantly younger and more likely to be nulliparous. When compared to RAM, RAH was associated with a significant decrease in operative time $(127 \mathrm{~min}$ vs. $189 \mathrm{~min}, \mathrm{p}<0.0001)$ as well as blood loss $(50 \mathrm{~mL}$ vs. $100 \mathrm{~mL}, \mathrm{p}<0.01)$.

Discussion: RAH is associated with shorter operative time and decreased blood loss, especially in patients with large uteri. While both RAM and RAH are safe surgical modalities for treatment of symptomatic leiomyomas, we should continue to recommend RAH to patients who no longer desire fertility preservation.

39

\section{COST ANALYSIS OF ROBOTIC SURGERY FOR ENDOMETRIAL CANCER: HOW TO REDUCE COSTS. Robotics}

L. Mereu, G. Carri*, I. Arena, H. Khalifa, T. Zampetoglou, L. Mencaglia

${ }^{[1]}$ Centro Oncologico Fiorentino $\sim$ Sesto Fiorentino $\sim$ Italy

Summary (4 lines): The objective is to analyse the feasibility of a new robotic operative room set-up to reduce the costs for endometrial cancer treatment

Introduction: Robotic is a new technique which provides several advantages for surgeon \& patients, but it's high costs limits its use.

Material and Methods: 22 endometrial cancer patients undergoing robotic surgery at CFO were enrolled in the study from June'10 April'12. They were divided in 2 different approaches (A: standard, B: modified)in relations to numbers of operative robotic arms, OR staff composition (nurses, non-nurses, surgeons, anaesthetists), disposable $\&$ non-disposable instruments. Comparison of costs \& feasibility between the 2 groups was performed. 
Results: 8 patients were treated with standard robotics approach (A)with 4 robotic arms; 14 women with the modified approach(B)with 3 robotic arms, reduction of disposable laparoscopic instrumentation and OR staff. Pelvic lymphadenectomy was performed in 14 cases (5 in group A,9 in group B). Median operative time in group A 214 min (157-265), group B $185 \mathrm{~min}$ (126-219). No intra or postoperative major complications occurred in both groups. Median hospital costs per intervention (excluding robotic surgical system cost) were in group A $8.858 €$, in group B $7.244 €$, with a reduction of $18 \%$.

Discussion: The cost of robotics are mainly related to the purchase \& maintenance, we demonstrate they can be reduced by increasing the number of interventions and adopting a modified approach

\section{4}

\section{EVALUATION OF THE KYMERAX LAPAROSCOPY SYSTEM IN THE DRY LAB}

Robotics

F. Neis*

${ }^{[1]}$ Univerity of Tuebingen $\sim$ Tuebingen $\sim$ Germany

Summary (4 lines): The new Kymerax system for laparoskcopic surgery was tested in the dry lab.

Introduction: New instruments, such as the Kymerax try to push forward in the market of robotic instruments. The Kymerax consists of a handpiece with the possibility to rotate and curve the instrument head. Of interest is whether it has advantages compared to conventional laparoscopy, how hard and fast the learning process is.

Material and Methods: To evaluate the system 20 medical students and 10 surgeons were tested in dealing with the Kymerax in the Sarbo trainer box. The test sytem was, the LASTT model. The exercise was performed with conventional laparoscopic instruments and the Kymerax, 3 times by each participant.

Results: Everyone improved during the exercises. The chance to curve and rotate the tip of Kymerax was used more often by the experienced. The biggest drawback is the heavy handle of Kymerax which absorbed a part of the haptic feedback. Although the handling is not intuitive, the handling was more fluently with increased use and leads to faster times.

Discussion: As with any new instrument a period of addaption is necessary. With the introduction of 3D-look, the difficulty of using the curved tip will vanish. With the addition degree of freedom it's easier to assess difficult areas and to sew. The Kymerax is as a mobile, "roboterised" tool, not a replacement for the the conventional laparoscopy, but it can be a good extention.

\section{2}

\section{KYMERAX ${ }^{\circledR}$ : AN INNOVATION IN ROBOTIC SURGERY} Robotics

E. Berkes*, A. Hackethal, F. Oehmke, H. Tinneberg

${ }^{[1]}$ University of Giessen and Marburg $\sim$ Giessen $\sim$ Germany

Summary (4 lines): Here we are introducing a new system, which combines the advantages of conventional laparoscopy and robotics.

Introduction: The development of robotics in endoscopy began in the mid-1980s, simultaneously with the advent of minimal invasive surgery. Robot-assisted endoscopic surgery (DaVinci ${ }^{\circledR}$, Zeus ${ }^{\circledR}$ ) offers several advantages such as 3-D visualization, seven degrees of freedom, improved dexterity, elimination of the fulcrum effect, and intuitive instrument manipulation. However, some major disadvantages - high start-up and keep-up costs, size, lack of compatible instrumentsare limiting robotics' routine clinical use.

Material and Methods: In our presentation we are introducing an innovation of the Giessen School of Endoscopy, the KYMERAX ${ }^{\circledR}$ system, which combines the advantages of conventional laparoscopy and robotics. Results: KYMERAX ${ }^{\circledR}$ is a motor-driven hand-held device that comprises a console, handles, and instruments and provides articulation at the tip. In addition to the four degrees of freedom of conventional laparoscopy, KYMERAX ${ }^{\circledR}$ offers two additional degrees of freedom: a yaw movement to the left and right in 70 degrees and a rotation in 320 degrees. The system enables easier manipulation of target tissues in hard-to-reach areas, provides haptic sensation, and is compatible with conventional instruments.

Discussion: KYMERAX ${ }^{\circledR}$ with its advantages and lower costs fills the gap between conventional laparoscopy and high-end robotics and, thus, is ideally suited to daily clinical use.

17

\section{LEARNING CURVE FOR ROBOTIC-ASSISTED GYNECOLOGIC ONCOLOGY SURGERY \\ Robotics}

S. Yaribakht*

${ }^{[1]}$ Centre anticancéreux Alexis Vautrin $\sim$ Nancy $\sim$ France

Summary (4 lines): Robotic-assisted surgery allows a minimally invasive approach to gynecologic oncology surgery for uninitiated surgeons to conventional laparoscopy with a faster learning curve for the initial learning phase

Introduction: Evaluation of the learning curve of two robotic-assisted gynecologic oncology procedures performed by a single surgeon with no previous experience in laparoscopy

Material and Methods: Retrospective analysis of 73 procedures between 2002 and 2011.

Surgeon console time (SCT) and operative time (OT) learning curves analysis using CUSUM method for procedures including radical hysterectomy (RH) and RH with pelvic lymphadenectomy (RHPL)

Results: $30 \%$ endometrial carcinoma,36\% uterine cervix carcinoma,19\% high grade cervical neoplasia, $15 \%$ prophylactic surgeries treated by RH or RHPL. RH group: learning curve consisted in 2 phases (I:initial learning, II:increasing skills) with decrease in SCT from 10 procedures $(\mathrm{p}<.05)$. RHPL group:3 phases identified (III:more complex surgical cases) with decrease in OT from 13 procedures $(p<.05)$

Discussion: Initial learning phase (I) of robotic-assisted surgery seems faster compared with conventional laparoscopy. Robot-assisted laparoscopy is a relevant technical approach for uninitiated surgeons to conventional laparoscopy and could be an alternative pathway for young surgeons training without previous experience

\section{8}

\section{ROBOTIC COMPLEX ONCOLOGICAL PROCEDURES WITH PARAAORTIC LYMPHADENECTOMY. PRELIMINARY RESULTS.}

Robotics

C. Goiri*, T. Bernal, L. Martinez, M. Arrue, J.A. Navarrina, A. Lekuona, M. Gorostidi

${ }^{[1]}$ Hospital Universitario Donostia $\sim$ San Sebastián $\sim$ Spain

Summary (4 lines): We describe the results of our first serie of oncological patients treated robotically with several complex oncological procedures. 
Introduction: Complex oncological procedures are being increasingly performed robotically in hospitals where this technology and qualified surgeons are avaible.

Material and Methods: We describe the 9 first complex robotic oncological procedures performed in our institution from 28th November 2011 to 18th June 2012.

The same surgeon performed all the procedures. He had previous laparoscopic experience in the same procedures.

Results: One patient with a BMI of 34 required laparotomy due to very difficult intestinal mobilization.

The number of lymph nodes obtained was similar to other published series starting from the first case.

Many of our patients were obese. We do not base our selection of patients for robotic surgery on BMI.

All patients were discharged free of complications 2-3 days after the procedure, except the patient that required laparotomy, who remained hospitalized for 10 days due to intestinal ileus.

Our only late complication has been one patient who developed a symptomatic lymphocele 5 months after the procedure, and required laparoscopic peritoneal marsupialization to successfully resolved it.

Discussion: Trained laparoscopic oncological surgeons can successfully and safely perform complex robotic oncological surgery.

\section{3}

\section{ROBOTIC HYSTERECTOMY FOR BENIGN INDICATIONS}

Robotics

P. Gambadauro*, M.A. Martínez-maestre, C. González Cejudo, R. Torrejón

${ }^{[1]}$ Department of Obstetrics, Gynaecology and Breast Pathology "Virgen del Rocio" University Hospital, University of Seville Seville $\sim$ Spain

Summary (4 lines): Prospective controlled study comparing conventional total laparoscopic hysterectomy and robotic hysterectomy for benign indications.

Introduction: In spite of the advantages of minimally invasive approaches, most hysterectomies are still performed abdominally. Robotic assistance is thought to facilitate laparoscopic surgery. Our aim was to compare the surgical outcomes of robotic and conventional laparoscopic total hysterectomy.

Material and Methods: Patients candidate to hysterectomy for benign indications were allocated to either robotic or conventional laparoscopy and operated following a standardized protocol. Main outcome measures were surgical time, conversions to laparotomy, blood loss, hospital stay and complications.

Results: Fifty one patients underwent robotic hysterectomy and 54 conventional laparoscopy. The groups were homogeneous in age, BMI and uterine weight. Robotic assisted hysterectomies were significantly shorter(p0.0001). Patients in the robotic group also had a significantly smaller reduction in hemoglobin(p0.0012) and hematocrit(p0.008). No intraoperative conversions to laparotomy were required. Complication rate was low and similar in both groups. All patients were fully recovered at one month.

Discussion: Robotic hysterectomy for benign indications is safe. Significantly lower operative times and blood loss indicate that robotic assistance can facilitate surgery. Nevertheless, the cost-effectiveness of robotic hysterectomy for benign conditions is yet to be confirmed.

385

\section{ROBOTIC PARAAORTIC LYMPHADENECTOMY AND COMPLEX ONCOLOGICAL PROCEDURES. LEARNING CURVE. ADVANTAGE AND DISADVANTAGES \\ Robotics}

M. Gorostidi*, J.A. Navarrina, M. Arrue, A. Lekuona, L. Martinez, T. Bernal, C. Goiri

${ }^{[1]}$ Hospital Universitario Donostia $\sim$ San Sebastian $\sim$ Spain

Summary (4 lines): We describe our experience starting robotics in gynecology oncology.

Introduction: Complex oncological procedures are being increasingly performed robotically in hospitals where this technology and qualified surgeons are avaible.

Material and Methods: We describe our first complex robotic oncological procedures performed in our institution from November 2011.

The same surgeon performed all the procedures. He had previous laparoscopic experience in the same procedures.

Our procedures include complete staging ovarian cancer, type II and type I endometrial cancer, and 1 advanced cervical cancer (with ovarian transposition)

Results: The number of lymph nodes obtained was similar to other published series starting from the first case. The average number of nodes was 22 (10-31), with 13 pelvic (6-25) and 9 paraaortic (4-14) nodes.

Many of our patients were obese. The average BMI was 34 in endometrial cancer patients. We do not base our selection of patients for robotic surgery on BMI.

We gained better access to interaortic-cava nodes and left infrarenal nodes, and more difficoult intestinal mobilization in obese patients.

Discussion: Trained laparoscopic oncological surgeons can successfully and safely perform complex robotic oncological surgery.

\section{7}

\section{ROBOTICS: WHY NOT?}

Robotics

A. Setubal*
${ }^{[1]}$ HOSPITAL DA LUZ $\sim L I S B O N \sim$ Portugal

Summary (4 lines):

Advanced laparoscopic surgery marked the beginning of a new era in most of all gynecological procedures. Nevertheless, some procedures of third and fourth level of the ESGE Classification (special when suture is needed), makes Laparoscopy a high skill techniques.

The need for special training did hinder widespread acceptance.

In a certain way Robotics change this situation, bringing to smaller learning curves. On the other hand bulkiness of the device, costs, operative time and applications on all type of complex gynecological procedures, are issues that should be discuss on the pos and cons of Robotics.

\section{Introduction:}

Advanced laparoscopic surgery marked the beginning of a new era in most of all gynecological procedures. Nevertheless, some procedures of third and fourth level of the ESGE Classification (special when suture is needed), makes Laparoscopy a high skill techniques.

The need for special training did hinder widespread acceptance. In a certain way Robotics change this situation, bringing to smaller learning curves. On the other hand bulkiness of the device, costs, operative time and applications on all type of complex gynecological procedures, are issues that should be discuss on the pos and cons of Robotics.

Material and Methods: MAC

Results: -

Discussion: - 
183

\section{SYSTEMATIC SIDE-DOCKING IN GYNECOLOGIC ROBOTIC SURGERY: PRELIMINARY EXPERIENCE IN CHILE \\ Robotics}

\author{
D. Larraín, F. Marengo, F. González*, H. Buckel, J. Prado, I. Rojas \\ ${ }^{[1]}$ Clínica Santa María $\sim$ Santiago $\sim$ Chile
}

Summary (4 lines): Side-docking is easy, improves vaginal access and facilitate specimen retrieval.

Introduction: Most studies on robotic gynecologic surgery used traditional docking with the robot between the patient's legs. A significant limitation is limited vaginal access. Recently, side docking (SD) with the robot at $45^{\circ}$ angle to the lower torso has been described. To report our experience using the SD since the beginning of our robotic program.

Material and Methods: 22 consecutive patients who underwent robotic procedures for gynecological diseases were included. All surgeries were performed using SD. Data collected included demographics, surgical procedures, operative time, complications, conversion rate and hospital stay.

Results: All procedures were completed robotically with no conversion. Mean age was 46 years. Mean BMI was $26+/-5 \mathrm{~kg} / \mathrm{m}^{2}$. Mean docking time was $12+/-6$ minutes and the mean setup time was $12+/$ -4 minutes. Mean operative time was $111+/-60$ minutes. When our first 10 cases were compared with the others we found a statistically significant reduction in docking time $(\mathrm{p}=0.03)$. Surgical procedures were the following: Hysterectomy $(n=18)$, sacrocolpopexy $(n=1)$, annexectomy $(n=1)$, endometriosis resection $(n=1)$, pelvic lymphadenectomy $(\mathrm{n}=4)$, aortic lymphadenectomy $(\mathrm{n}=1)$. Two complications occurred: a bladder injury and a bowel perforation. Mean hospital stay was 36 hours.

Discussion: SD is easy, improves vaginal access and facilitate specimen retrieval.

181

\section{THE GREEK EXPERIANCE ON ROBOTICALLY ASSISTED LAPAROSCOPY IN ENDOMETRIAL ADENOCARCINOMA - SIDE DOCKING \\ Robotics}

V. Chatzirafail*, T. Aliki, A. Bakas

${ }^{[1]}$ EUROCLINIC hospital $\sim$ Athens $\sim$ Greece

Summary (4 lines): Robotic assisted hysterectomy and pelvic lymphadenectomy for endometrialCa (RA-TAH-PLNE) with the DaVinci$\mathrm{S}$ system during learning curve period, in 8 patients, is safe, oncologicaly approved, and satisfactory for patients

Introduction: Robotic technics have been successfuly used in the management of endometrialCa, even in obese patients

Material and Methods: July 2011-March 2012,8 patients with endometrial adenoca underwent RA-TAH-PLNE with DaVinci-S system. Patients age, BMI, histological type, surgical stage, operative time, hospitalization, blood transfusions, complications were recorded.

Five ports, plus DaVinci-S system placed on the side of the patient (side docking). Patients' age:35-65, mean BMI:31. Most patient had well differentiated adenoca(6/8), invaded $<1 \backslash 2$ myometrium. Mean size of tumor ranged: $0,5-5 \mathrm{~cm}$.

Results: No metastases in the cervix, ovaries, or pelvic lymph nodes (mean number 19 on the right side and 15 on the left). Mean operative time:285 min. Mean postoperative stay:1,6 days. None of the patients required blood transfusion. No conversion to open surgery. There were no intraoperative complications.1 patient, BMI 38, developed deep vein thrombosis and left pelvic lymphocyst.

Discussion: The RA-TAH-PLNE for endometrial adenocarcinoma is feasible. Postoperative hospital stay and morbidity were minimal even in this population of obese patients. Still the operative time and the cost of such procedure has to be discussed.

371

\section{TROCAR PLACEMENT IN ROBOTIC PARAAORTIC LYM- PHADENECTOMY. OUR PROPOSAL \\ Robotics}

T. Bernal*, C. Goiri, L. Martinez, M. Arrue, A. Lekuona, M. Gorostidi ${ }^{[1]}$ Hospital Universitario Donostia $\sim$ San Sebastian $\sim$ Spain

Summary (4 lines): We describe our proposal for trocar placement in robotic retroperitoneal paraaortic lymphadenectomy.

Introduction: Retroperitoneal Paraaortic lymphadenectomy provides excellent access to paraaortic lymphatics, particularly in obese patients. Few papers describe the technique in robotic surgery. Trocar placement is a concern due to the small surgical field and the risk of collision of the robotic arms.

Material and Methods: STUDIED POSSITIONS

We studied the trocar positions described by Dr. Antonio Gil in Barcelona and Dr. Vergote in Leuven and a third position tried by us. None of them were confortable for the assistant.

Results: OUR PROPOSAL

We place the $12 \mathrm{~mm}$ balloon trocar 2 finger widths above and medial to the Anterosuperior Iliac spine. This is the assistant's trocar. We put a robotic $8 \mathrm{~mm}$ trocar below the assistant balloon trocar as caudal as safe insertion allows. We put a second robotic arm as high as possible between the anterior axillar line and midclavicular line (with robotics we can approach the axillar line and safely avoid peritoneal recessions).

ACCESS TO PELVIS AFTER RETROPERITONEAL TIME

Pelvic procedures only require insertion of one additional trocar.

Discussion: Our trocar placement provides an alternative access route to the paraaortic region, from the common iliac artery to renal vein.

\section{8}

\section{TUBAL REANASTOMOSIS BY SURGICAL ROBOTIC SYSTEM} Robotics

F. Sendag*, A. Akdemir

${ }^{[1]}$ Ege University School of Medicine, Departmet of Obstetrics and Gynecology Izmir Turkey

Summary (4 lines): Surgical robotic systsems have been used for tubal reanastomosis for more than ten years.

Introduction: Robotic assistance in laparoscopy has been proposed to overcome the disadvantages of traditional laparoscopy in tubal anastomosis while still benefiting from the advantages of minimally invasive technique. Here were report our initial experience about robotic assisted tubal reanastomosis procedure.

Material and Methods: Laparoscopic tubal reanastomosis by using $\mathrm{Da}$ Vinci ${ }^{\circledR}$-SI system was performed to two patients with a history of tubal ligation. Three months after the procedure tubal patecy was assessed by hyterosalpingogram (HSG).

Results: Bilateral fallopian tubes were successfully reconstructed as confirmed by chromopertubation at the end of the procedure in both two patients. There were no major complications and the blood loss was minimal. The operation time (including also docking of the robotic 
arms to the patient) was 165 and 177 minutes, respectively. HSG after the procedure showed bilateral patency in two patient.

Discussion: Main advantage of robotic system is facilitating the intracorporeal knot tying with fine suture but preoperative preperation of robot and connection to patient leads to long operation time. Prospective randomized studies are needed to compare the robotic assisted tubal reanastomosis with invitro fertilization or conventional laparoscopic tubal reanastomosis.

388

\section{A CASE OF SINGLE PORT SURGERY FOR SYMPTOMATIC BILATERAL ADNEXAL MASSES IN PREGNANCY \\ Single Access Surgery}

D. Surico, L. Leo, I. Nupieri*, L. Izzo, A. Vigone, N. Surico

${ }^{[1]}$ Experimental Endoscopic Surgery Center, Department of Obstetrics and Gynaecology, University of Eastern Piedmont $\sim$ Novara $\sim$ Italy

Summary (4 lines): We show the possibility to perform LESS to treat symptomatic adnexal masses in pregnancy.

Introduction: LESS surgery is a minimally invasive alternative to traditional laparoscopy

Material and Methods: A 30-year-old pregnant at 9 weeks of amenorrhea presented for a severe pelvic pain and fever for two days. Laboratory tests found a leukocytosis and an increased of PCR; tumor markers were negative. Transvaginal ultrasound examination confirmed a regular intrauterine pregnancy but right and left ovary were occupied by a cyst of 11 and $4 \mathrm{~cm}$, respectively. We submitted the woman to an urgent Single Port LPS surgery. LESS Port (X-Cone; Storz) was placed in a $2.5 \mathrm{~cm}$ vertical umbilical incision. It was found a voluminous ovarian right cyst of $11 \mathrm{~cm}$, that completely occupied the pelvis and a left ovarian cyst of $4 \mathrm{~cm}$; uterus was corresponding to amenorrhea time. We performed a bilateral ovarian cysts enucleation. We placed Tacho Sil ${ }^{\circledR}$ (Nycomed) device on right ovary and Tissue$\mathrm{col}^{\circledR}$ (Baxter) on left ovary. Post-operative pain was managed using intravenous paracetamol and transvaginal progesterone $(200 \mathrm{mg})$ were administered daily.

Results: Operative time was 95 minutes. At discharge, after 48 hours, pregnancy was in the regular trend. Histopathological examination showed a mucinous cystadenoma of right ovary and a serous cystadenoma of the left ovary.

Discussion: LESS can be performed also to treat in urgency adnexal masses during pregnancy.

\section{6}

\section{EXTRAPERITONEAL SINGLE PORT LAPAROSCOPY FOR} PARA-AORTIC LYMPHADENECTOMY

Single Access Surgery

L. Mereu*, S. Tateo, G. Carri, H. Khalifa, I. Arena, T. Zampetoglou, L. Mencaglia

${ }^{[1]}$ Centro Oncologico Fiorentinio $\sim$ Sesto Fiorentino $\sim$ Italy

Summary (4 lines): single port extra peritoneal para-aortic lymphadenectomy is a feasible minimal invasive technique

Introduction: Extraperitoneal para-aortic lymphadenectomy performed by single port laparoscopy in case of bulky lymph nodes.

Material and Methods: A 56 years old woman with squamous carcinoma of the cervix, FIGO stage 1B2, with bulky common iliac and para-aortic lymph nodes in CT scan, underwent extraperitoneal paraaortic lympadenectomy. A $2 \mathrm{~cm}$ incision was made $2 \mathrm{~cm}$ above Querleu; surgeon's index finger used to bluntly develop the retroperitoneal space. $\mathrm{X}$ cone trocar was inserted and $12 \mathrm{mmHg}$ of $\mathrm{CO} 2$ gas was introduced, a $5 \mathrm{~mm}-30^{\circ}$ laparoscope was placed into the right channel, curved atraumatic S-portal forceps into the left access and Harmonic scalpel into the central channel. After retroperitoneal identification of psoas muscle, iliac vessels and ureter, blunt dissection of the adventitial tissue was performed and the identification of the left renal vein. Lymphadenectomy specifically targeted the left sided supra and infra-mesenteric para-aortic spaces to remove bulky lymph nodes. Results: Surgery was performed successfully with no intra- or postoperative complications. Operative time was $165 \mathrm{~min} .9$ lymph nodes were removed. Oral intake was started in day 0 . Patient was discharged on day 1. Discussion: In experienced hands, single port extra peritoneal paraaortic lymphadenectomy is feasible without particular differences \& technique from multi-access laparoscopy approach.

\section{1}

\section{IS SINGLE PORT LAPAROSCOPY FOR BENIGN ADNEXAL DISEASE LESS PAINFUL THAN CONVENTIONAL LAPAROSCOPY? : A SINGLE CENTRE RANDOMISED CONTROLLED TRIAL}

Single Access Surgery

C. Hoyer-sorensen*, I. Vistad, K. Ballard

${ }^{[1]}$ Sorlandet Hospital Kristiansand $\sim$ Kristiansand $\sim$ Norway

Summary (4 lines): Women having LESS surgery for adnexal disease experience similar levels of postoperative pain to women having conventional laparoscopy.

Introduction: To determine whether laparo-endoscopic single-site surgery results in less postoperative pain and a better cosmetic surgical scar when compared to conventional laparoscopy.

Material and Methods: A single center, prospective, randomised controlled trial of 40 patients with either a presumed benign adnexal disease or a hereditary cancer risk. The patients were scheduled to laparoscopic adnexal surgery and randomized to laparo-endoscopic single-site surgery or conventional laparoscopy.

Results: There was no difference in pain at 24 hours post-operatively, with a mean score of 3.0 (SD 2.1) in the laparo-endoscopic single-site surgery group and 2.5 (SD 1.5) in the conventional laparoscopy group $(p=0.35)$. Significantly more shoulder tip pain was reported by women undergoing laparo-endoscopic single-site surgery compare with those having conventional surgery at $6(p=0.01)$ and 24 hours $(p=0.03)$ postoperatively.

Discussion: Although similar levels of postoperative pain are experienced by women having LESS and conventional laparoscopic surgery, women having LESS report significantly more shoulder tip pain compared to those having conventional laparoscopic surgery.

\section{2}

\section{LAPARO-ENDOSCOPIC SINGLE SITE SURGERY: A CASE OF SUB-TOTAL HYSTERECTOMY USING LAP LOOP SYSTEM \\ Single Access Surgery}

D. Surico, L. Leo, L. Galli*, I. Nupieri, L. Izzo, A. Vigone, N. Surico ${ }^{[1]}$ Experimental Endoscopic Surgery Center, Department of Obstetrics and Gynaecology, University of Eastern Piedmont Novara Italy

Summary (4 lines): To the best our knowledge, we report the first case of sub-total hysterectomy in LESS surgery

Introduction: LSH is associated with fewer complications and a more favourable outcome to women. 
Material and Methods: A 44 -year-old woman was referred for pelvic pain and metrorrhagia On transvaginal US examination the uterus was deformed by multiple nodes myoma. Taking into account the young patient's age and her active sex life, we decided to submit the patient to a subtotal hysterectomy with conservation of ovaries in LESS Surgery. After $2.5 \mathrm{~cm}$ intraumbelical vertical skin incision we introduce the Gel Port System. Laparoscopy showed a massive adhesions complex between omentum and peritoneum; it confirmed an uterine fibromatosis and indirect signs of parametritis on the right side. Lap Loop System was used to separate the uterus corpus from the cervix at the level of isthmus and it was extracted from umbilical access. Endocervical canal and glands were coagulated to minimize the risk of developing a cancer of the cervical stump and to delete any portions of the endometrium in the cervical canal that can cause possible menstrual bleeding.

Results: Operative time was 150 minutes, estimated blod loss was $50 \mathrm{ml}$. Time of hospitalization was 48 hours.

Discussion: When indicated, LSH approach is safe and well tolerated. The use of Lap Loop system permits a fast resection of the uterin corpus from the cervix

\section{5}

\section{PROSPECTIVE COMPARISON OF SINGLE PORT LAVH TO CONVENTIONAL THREE PORTS LAVH}

Single Access Surgery

M.H. Jung, Y.I. Ji*

${ }^{[1]}$ Kyung Hee University Hospital $\sim$ SEOUL $\sim$ Korea, Republic of

Summary (4 lines): The single port LAVH could be a feasible alternative method for removal of the uterus with a less weight uterus and resulting in better postoperative pain score and cosmetic outcomes.

Introduction: To evaluate the efficacy and safety of single port LAVH comparing to conventional three ports LAVH in women with benign uterine diseases.

Material and Methods: Prospective analysis of 158 cases of single port $(\mathrm{N}=78)$ and three ports LAVH $(\mathrm{N}=78)$.

Setting: Kyung Hee University Hospital and Haeundae Paik Hospital, Tertiary teaching University Hospital

Patients or Participants: One hundred fifty eight patients with benign uterine diseases.

Interventions: Single port LAVH and three ports LAVH

Results: Data analysis was done for 78 patients in the single port LAVH group and 78 patients in the conventional LAVH group. No major complications, including ureteral or bladder injuries, occurred in any of the patients. No statistically significant differences were found in mean age, mean body mass index, mean operative time, mean anesthesia time, mean estimated blood loss, or mean postoperative days to passage of flatus, but the mean uterine weights on the pathologic reports and mean postoperative pain score were significantly reduced in the single port LAVH group.

Discussion: The single port LAVH could be a feasible alternative method for removal of the uterus with a less weight uterus and resulting in better postoperative pain score and cosmetic outcomes.

\section{0}

\section{SINGLE PORT LAPAROSCOPY FOR OVARIAN TISSUE CRYOPRESERVATION}

Single Access Surgery

L. Mereu, G. Carri, I. Arena*, H. Khalifa, T. Zampetoglou, L. Mencaglia

${ }^{[1]}$ Centro Oncologico Fiorentino $\sim$ Sesto Fiorentino $\sim$ Italy
Summary (4 lines): Single port laparoscopy could be an effective minimally invasive technique to get ovarian fragments for cryopreservation.

Introduction: $10 \%$ of cancers occur in fertile women. Oncological treatments improve patients survival but lead to high risk of ovarian failure. Ovarian tissue cryopreservation provides the chance to conserve fertility.

Material and Methods: From March'11 to March'12, 10 patients underwent single port laparoscopy for ovarian tissue cryopreservation. Clinical indications were: breast cancer(n.4); ovarian cancer(n.4); recurrent deep endometriosis(n.1); leiomiosarcoma(n.1). All patients were under general anaesthesia, in dorso-lithotomic position. Surgical equipment included: $\mathrm{X}$-cone trocar, $30^{\circ}$ laparoscope $(5 \mathrm{~mm}$ in diameter, $50 \mathrm{~cm}$ in length), coaxial curved forceps, straight scissor connected with $80 \mathrm{~W}$-cutting unipolar energy. By using a cold scissor, ovarian fragments of $1 \times 1 \mathrm{~cm}$ were removed, immersed in specific medium and rapidly transported to the lab where they were carefully dissected in slices of 1-2 mm thickness for cryopreservation. A specimen from both ovaries was sent also for histology. All procedures were carried out by an expert surgeon (LM).

Results: Mean operative time was $35 \pm 16 \mathrm{~min}$. No intra or postoperative complication occurred. Patients were discharged on day 1 in good condition and followed their oncologic program.

Discussion: We demonstrate single port laparoscopy is an effective minimally invasive technique to get ovarian fragments for cryopreservation.

\section{9}

\section{USING THE SINGLEPORT}

Single Access Surgery

C. Escayola*, Y. Vinyals Gutierrez, C. Gimenez Millan, V. Garcia Orihuela, L. GÜemes Domenech, M. Genis Selva, C. Delgado Torrens ${ }^{[1]}$ Pilar Sant Jordi Hospital $\sim$ Barcelona $\sim$ Spain

Summary (4 lines): The singleport is an instrument increasingly used in laparoscopic surgery, since it allows to perform the surgery with a single portal access. However, as it is still a novel instrument in many operating rooms, nurses need a clear and concise training on its use. Introduction: The single-port provide advantages in comparison to the usual access, with are: the decrease in the risk of infections, better and faster recovery and the positive assessment by the patient. Wich are factors that encourage the gynecologist to use it), it predisposes increasingly more gynecologists to use it.

Our hospital use the singleport much more than others hospitals in Spain. Therefore, our intention is to make known this method to the operating room staff, describing what it is, what preparation is required, what material is needed, what are the most appropriate indications, what advantages and disadvantages presents, and provide staff the training needed to perform the technique of safely and efficiently.

Material and Methods: Bibliography and medical practice.

Results: Development of a consultation tool for the continuing education of operating room staff in the management of the singleport.

Discussion: Provide a simple reference material on the singleport management for staff who works in the surgical area, allowing the practice of this surgery safely and efficiently

488

ASSESSMENT AND LEARNING CURVE EVALUATION OF TVUS SKILLS FOLLOWING SIMULATION AND CLINICAL TRAINING

Teaching and Training 
A. Alsalamah*, N. Pugh, N. Amso

${ }^{[1]}$ Cardiff University $\sim$ Cardiff $\sim$ United Kingdom

Summary (4 lines): ScanTrainer simulator is a virtual reality computerised system, which provides structured learning and a task-oriented assessment method that gives an immediate and comprehensive feedback to trainees.

Introduction: Aims: (1) Determine the changes found in a trainee's learning curves using (i) structured simulation-supported training and (ii) conventional training alone, by continuous evaluation of ultrasound skills and trainee's competency. (2) Monitor the trainees' engagement with and utilisation of the simulator during the trial.

Material and Methods: Study design: randomised controlled trial.

31 Subjects recruited primarily from specialist trainees in Obstetrics \& Gynaecology in the Welsh Deanery, UK.

At recruitment into the trial, the simulator was used to assess the baseline skills and then monthly assessment of skills' acquisition was undertaken during the trial period (within 6 months). Skill checklist score form and questionnaire were used.

Results: Preliminary results demonstrated a gradual change in participant's scores after simulation training. Higher number of hours spent on simulator indicated a higher improvement on trainees' scores.

Discussion: Using an ultrasound simulator leads to more rapid acquisition of skills in transvaginal ultrasound compared to conventional training, as assessed by ultrasound ScanTrainer simulator.

\section{1}

ASSESSMENT OR NOT ASSESSMENT. DO WE KNOW HOW WE ACQUIRE BASIC LAPAROSCOPIC SKILLS?

Teaching and Training

R. Rovira*, J. Estadella, R. Muñoz, O. Porta, J. Calaf

${ }^{[1]}$ Hospital de Sant Pau $\sim$ Barcelona $\sim$ Spain

Summary (4 lines): Laparoscopy needs to acquire specific psychomotor skills which can be got by a structured training. The authorities think that these habilities must be acquired out of the operation theater and it's necessary to validate the assessment of these skills.

Introduction: This study shows that attending a structured course allows to acquire basic laparospic skills, and theorical knowledge needed in this surgical approach

Material and Methods: Prospective study of 46 residents from the first year of Gynecology in Catalonia (Spain). We assessed before and after the course the practical (with LASTT developed by EAGS) and theorical knowledge (with a 20 question test exam).

Results: Results are expressed in mean. We observed a stadistically significative improvement $(\mathrm{p}<0,001)$ in all of the data. Residents improved form a 4,80 to 7,9 in the theorical exam. Exercise 1, the mean time to complete it was reduced from 117,9 seconds (s) to $89,9 \mathrm{~s}$, and the number of characters observed improved from 8,67 to 13,68. Exercise 2, the mean time to complete it was reduced from $125,52 \mathrm{~s}$ to $85,41 \mathrm{~s}$, and the number of objects placed improved from 5,51 to 5,94. Exercise 3, the mean time to complete it was reduced from $158,47 \mathrm{~s}$ to $117,27 \mathrm{~s}$ and the number of objects placed improved from 5,08 to 5,89 . Discussion: These results obtained allow us to say that theorical knowledge and basic laparoscopic skills can be acquired by participating in a well structured and designed course.

\section{9}

\section{AUTOMATIC CALCULATION OF COMPUTERISED PERFORMANCE METRICS USING STANDARD VIDEO SIMULATORS}

Teaching and Training
C. Harrity*, R. O'Sullivan, W. Prendiville

${ }^{[1]}$ RCSI $\sim$ Dublin $\sim$ Ireland

Summary (4 lines): LapApp adds the potential to automatically calculate performance metrics with any standard video simulator, in combination with a touchscreen device.

Introduction: A benefit of VR simulators over box systems is the ability to automatically score psychomotor skills and performance metrics. LapApp is the first program to combine instant calculation of performance metrics with video simulators, which are typically less expensive and more portable than VR alternatives.

Material and Methods: Trials were designed to correlate results with the validated ProMIS VR simulator, and to determine if the application can differentiate between participants of varying skill. The system has been trialled at conferences, teaching hospitals, and the National Clinical Skills Centre, and participants ranged from experienced surgeons to complete novices.

Results: Primary results $(n=17)$ from comparison with the ProMIS overall score indicate a strongly positive and significant correlation (Pearson $=0.85, \mathrm{p}<0.001)$. Mean overall scores between experienced and inexperienced surgeons differed significantly, $79.5 \%$ vs $55.3 \%$ (t-statistic $8.52, \mathrm{p}<0.001$ ), demonstrating the ability to detect difference in psychomotor skill levels of the user $(\mathrm{n}=30)$.

Discussion: This system could represent a cost-effective method of combining accurate quantitative assessment of psychomotor skill metrics and video simulators, without the need for more expensive VR or augmented reality equipment.

\section{4}

\section{CHANGING SURGICAL TRAINING OPPORTUNITIES \\ Teaching and Training}

E. Pavlidis*, D.H. Hunter

${ }^{[1]}$ Belfast Health Trust $\sim$ Belfast $\sim$ United Kingdom

Summary (4 lines): Surgical training opportunities are thought to have been diluted by changes in working pattern and changing surgical practice

Introduction: The introduction of Mirena and the 2nd generation endometrial ablation devices has had a profound impact on training opportunities for hysterectomy. A comparison of treatment numbers in 2000 and 2010 is presented.

Material and Methods: Theatre ledgers for the calendar years 2000 and 2010 were examined. The number of hysterectomies undertaken for DUB was recorded. Changing working patterns were examined to calculate number of days worked under an on call system and a shift system.

Results: In 2000, 69 hysterectomies were performed compared with 2010, when 48 hysterectomies were undertaken for DUB. Changing working patterns have meant that trainees can now be expected to be absent for 62 'potential working days' annually as opposed to 48 in 2000. In addition, the number of trainees in our unit has risen from 14 in 2000 to 26 in 2010 .

Discussion: The combination of reduction in number of straightforward hysterectomies performed, reduction in time spent in the hospital and increased number of trainees has significantly diluted a trainees exposure to straightforward hysterectomy.

IMPLEMENTATION OF MINIMALLY INVASIVE SURGERY IN A GYNECOLOGY DEPARTMENT.

Teaching and Training 
A. Ubeda*, E. Fernandez, M. Simon, A. Vazquez, P. Barri

${ }^{[1]}$ Institut Universitari Dexeus $\sim$ Barcelona $\sim$ Spain

Summary (4 lines): At present, Gynecologic departments should trend towards increasing ambulatory rates in surgucal procedures.

Introduction: To define gynaecological procedures to be safely performed under a minimally invasive approach in a private teaching hospital in Barcelona (Spain).

Material and Methods: In terms of gynaecologycal surgical procedures, the critical point is whether increase of ambulatory may lead to unsafe or risky attitudes towards patients. Along 2011, 1.988 gynaecological surgeries were carried out in our hospital. We analized hospital and office setting and the possibility of increasing ambulatory rates, related to other Spanish regions.

Results: 955 procedures (48\%) were performed in operating room under general anaesthesia, while the other 1.033 (52\%) were undertaken in the office or in an ambulatory setting. Among OR surgeries, $52.9 \%$ were laparoscopies. When comparing with other Spanish regions, our hospital in Cataluña stands as one of the greatest supporters of ambulatory gynaecological surgery $(63.9 \%$ of all procedures) (figure 1). In terms of laparoscopy, legal considerations forced us to be more cautious when taking decisions of ambulatory procedures.

Discussion: Even though present trends in national health services tend to force ambulatory indications for gynaecological procedures, final decisions should be taken by each team. Postoperative support and legal consequences are obliged conditions to be considered before increasing ambulatory rates.

401

INFLUENCE OF GENDER AND PROGRAM LOCATION IN MINIMALLY INVASIVE SURGERY TRAINING OF FRENCH RESIDENTS

Teaching and Training

A. Fazel*, M. Aout, E. Barranger, E. Vicaut

${ }^{[1]}$ HOPITAL LARIBOISERE $\sim$ PARIS $\sim$ France

Summary (4 lines): A french survey assessing academic and practical training of residents in laparoscopy (L)and hysterocsopy $(\mathrm{H})$ shows clear differences depending on gender and program location.

Introduction: Whether gender or program location are important in training is debated.

Material and Methods: National anonymous survey (900 residents). Academic training assessed by local or specific courses, congresses, diplomas, or homework. Practical training assessed by the number of $\mathrm{L}$ of level 1 to 4 or $\mathrm{H}$ of level 1 to 3 .

Results: $43.2 \%$ replied. $85.35 \%$ were female. $68.4 \%$ of men learned theories of L by themselves vs. $44.9 \%$ of women, $49.1 \%$ vs $31 \%$ for H.39.8 \% of women performed more than $10 \mathrm{~L}$ of Level $1,37.9 \%, 11 \%$, and $5.6 \%$ more than 10 cases of level 2 to 4 respectively. $60.3 \%$ of men performed more than 10 cases of level $1,60.5 \%, 26 \%$, and $11 \%$ more than 10 cases of level 2 to 4 . For $\mathrm{H}$ of level 1 to 3 , the rates were $65.6 \%, 39.8 \%$ and $7.1 \%$ respectively for women, and $62.6 \%, 56.3 \%$ and $19.6 \%$ for men. $65 \%$ of Parisian residents performed more than 10 level $1 \mathrm{~L}$ compared to $39 \%$ of non Parisians, $70 \%$ vs 33 for level 2, $50 \%$ vs 15.8 level 3 , and 14.6 vs $4 \%$ level 4 .

Discussion: Men performed more cases of $\mathrm{L}$ or $\mathrm{H}$ compared to women, and Parisians were more trained than non Parisians, especially in advanced cases. The reasons are debatable but we need to adapt the clinical and academic training in minimally invasive surgery since gender has largely switched towards females.

\section{0}

\section{LAPAROSCOPIC ELECTROSURGERY \\ Teaching and Training}

C. Escayola*, Y. Vinyals Gutierrez, C. Gimenez Millan, V. Garcia Orihuela, L. GÜemes Domenech, M. Genis Selva, C. Delgado Torrens

${ }^{[1]}$ Pilar Sant Jordi Hospital $\sim$ Barcelona $\sim$ Spain

Summary (4 lines): We want to present, in a comparative way, the laparoscopic surgical material of sealing, cutting and coagulating that we usually use in our hospital, in a way that serves for the training of operating room staff.

Introduction: The correct knowledge of the instruments used in endoscopic surgery leads to a better and more secure medical practice. The electrosurgery instrumental that uses energy for its operation need a knowledge more in-depth and updated. We want present this publication. So, through the comparison of different instruments (monopolar instruments, bipolar forceps, cutting forceps Gyrus, Ultracision, Ligasure and Thunderbeat), staff working in the operating room of endoscopic surgery can have a consultation tool to carry out its assistential work more safely and efficiently.

We want to introduce functions and main characteristics of such instruments, the type of energy that they work with, what indications have, what advantages and disadvantages present, what complications can occur and their cost/benefit, from a point of view of resource management

Material and Methods: Bibliography and medical practice.

Results: The development of a consultation tool for the continuing education of the staff in the operating room of endoscopic surgery.

Discussion: The development of a consultation tool for operating room staff, allows the practice of such surgery safely and efficiently. In addition, good knowledge of indications of each instrument, allows a more efficient use of the instrumental.

\section{4}

\section{LAPAROSCOPIC SALPINGO-OOPHORECTOMY: A VALID VIRTUAL REALITY TRAINING MODEL.}

Teaching and Training

R. Bharathan*, S. Vali, T. Setchell, T. Miskry, A. Darzi, R. Aggarwal ${ }^{[1]}$ Imperial College $\sim$ London $\sim$ United Kingdom

Summary (4 lines): Virtual reality (VR) training for laparoscopic salpingo-oophorectomy (LSO) demonstrates construct validity. This is an effective training tool for novice gynaecologists

Introduction: LSO is an important risk reducing or therapeutic procedure. VR training aids skill transference to the operating room. No studies have demonstrated a valid model for LSO. Validation of VR training for LSO will enable curriculum development.

Material and Methods: 34 gynaecologists with varying levels of experience were recruited. All participant performed 2 sessions of LSO using a tripolar device on a VR simulator. 9 novices performed 10 sessions permitting assessment of the learning curve (LC). The face validity (FV) of the task was reported by all but the novices.

Results: LSO demonstrated CV in terms of time taken $(p=0.05)$ total movements $(\mathrm{p}=0.02)$ and idle time of right $(\mathrm{p}=0.01)$ and left $(\mathrm{p}=0.03)$ 
instruments. LC analysis for LSO demonstrated a plateau in time taken at session (S) 7, and total movements at S6. Novices achieved the benchmark level as set by performance of experienced subjects at $\mathrm{S} 4$ for time taken and for total movements. The FV scores reported by intermediate and experienced subjects ranged from 7-9 on a ten point Likert scale.

Discussion: The LAP Mentor VR simulator is a valid, effective tool for training novice gynaecologists in LSO. The next phase in curriculum development will involve assessment of transference to preclinical or clinical stage.

\section{6}

\section{LEARNING CURVE OF HYSTEROSCOPIC TUBAL STERILIZATION ON A VIRTUAL REALITY SIMULATOR Teaching and Training}

J. Janse*, R. Goedegebuure, S. Veersema, F. Broekmans, H. Schreuder

${ }^{[1]}$ Antonius Hospital Nieuwegein $\sim$ Nieuwegein $\sim$ Netherlands

Summary (4 lines): The learning curve for hysteroscopic sterilization on a virtual reality simulator is demonstrated by large improvements of novices' performance in comparison to experts.

Introduction: The hysteroscopic placement of tubal sterilization micro-inserts is a relatively new and popular endoscopic technique and a valid training model is eligible. The aim of this study is to assess the learning curve of the Essure ${ }^{\circledR}$ sterilization method on a virtual reality simulator.

Material and Methods: Novices $(\mathrm{N}=30$, medical students) and experts $(\mathrm{N}=5$, performed $>150$ hysteroscopic sterilizations) voluntarily participated. Participants performed nine repetitions of a bilateral sterilization on the simulator. Novices returned for five repetitions. Structured observations by a Global Rating Scale (GRS) and parameters derived from the simulator provided measures for analysis. Effect sizes (ES) express the practical significance of the results (ES $>0.50$ indicates a large effect).

Results: Large improvements of novices' performance were found for time, path length, patient comfort, trauma, time the view obscured and GRS ( $<<0.001$, ES:0.51-0.96). Novices approached expert level within 9-15 repetitions.

Discussion: This study confirms construct validity and adds important evidence concerning the learning effects that can be achieved. Therefore, this virtual reality simulator can be recommended for training of hysteroscopic sterilization skills.

\section{5}

\section{ROLE OF SIMULATION IN DEVELOPING A UK EVIDENCE BASED TRAINING CURRICULUM IN LAPAROSCOPIC GYNAECOLOGY}

Teaching and Training

H. Haerizadeh*, J. Frappell

${ }^{[1]}$ Department of minimal access gynaecology, Derriford Hospital Plymouth United Kingdom

Summary (4 lines): We have developed an evidence based curriculum to optimise training in laparoscopic gynaecology. Literature has been thoroughly searched for the evidence to help develop a detailed structured programme tailored to trainees' from early years of training.

Introduction: Evidence suggests that in trainees without surgical experience, virtual reality simulation training improves technical accuracy while reducing operating time and errors. However, there is a definite need for an evidence based curriculum with detailed structure integrating simulation into laparoscopic training.

Material and Methods: The Cochrane Library, EMBASE, Medline, European Resource Information Centre (ERIC) and British Education Index (BEI) were searched for relevant papers using the relevant Medical Subject Heading (MESH) terms including "simulation, computer", "laparoscopy", and "gynecologic surgical procedures" combined with a keyword search using "laparoscopy", "virtual reality", and "box trainer".

Results: The initial search resulted in 420 articles and narrowed down to 89 articles after careful scrutiny. The results have been implemented in developing a training programme starting from first year of training with focus on practical tips for trusts, clinical supervisors and assessors.

Discussion: After implementation of this programme, further research is needed tomeasure the training outcome, quantify the effects and identify areas for further improvement.

498

\section{TEACHING TRENDS IN MANAGEMENT OF ECTOPIC PREGNANCY}

Teaching and Training

M. Mccomiskey*, D. Hunter

${ }^{[1]}$ Belfast Health Trust $\sim$ Belfast $\sim$ United Kingdom

Summary (4 lines): Indications for changing trends in the surgical management of ectopic pregnancy are discussed

Introduction: The RCOG recommended that haemodynamically stable patients undergoing surgical treatment forectopic pregnancy have this performed laparoscopically. This paper describes the change in practice in the treatment of ectopic pregnancy in Northern Ireland over a seven year period.

Material and Methods: Data of surgically managed ectopic pregnancies were collected over two time intervals from a tertiary referral teaching hospital and a third interval which included all units in Northern Ireland. Changes introduced during the study included provision of patient information and clinical algorithms, equipment changes, establishment of training courses, laparoscopically competent consultant supervision and emphasis on operating semi-electively.

Results: The proportion of patients undergoing laparoscopic surgery for the treatment of ectopic pregnacy has risen from $52 \%$ to $82 \%$ with a marked reduction in the number of intra-operative conversions to laparotomy. Detailed data will be presented

Discussion: Uptake of laparoscopic surgery has improved throughout study time period and factors contributing to this finding have been described.

478

TRAINING CENTER FOR GYNECOLOGIC ENDOSCOPY IN PERU - A 4-YEAR EXPERIENCE

Teaching and Training

H. Krentel*

${ }^{[1]}$ Hospital Eisenhüttenstadt $\sim$ Eisenhüttenstadt $\sim$ Germany

Summary (4 lines): The status quo of laparoscopy and hysteroscopy in Peru and Ecuador and the actual situation of education programs in endoscopic gynecology are discussed by the results of two studies. 
Introduction: More than 160 physicians from five countries participated successfully in the workshops for gynecologic laparoscopy and hysteroscopy since the foundation of CEGPA in 2008. A network in Peru and Ecuador has been established which is working on regional training concepts, national certification strategies and a wider distribution of laparoscopy and hysteroscopy in both countries. Anyway the minimally invasive surgery is not yet the approach of first choice.

Material and Methods: A questionnaire has been send to all participants of CEGPA workshops. In a parallel study the use of laparoscopy in gynecology in the public sector has been evaluated in the mayor hospitals in Quito, Ecuador.

Results: The results of both studies show the great motivation of ginecologists in both countries to use endoscopic gynecology, the effects of our training on the daily practice and the acceptance of the minimally invasive surgery, but also describe the main reasons why the endoscopic methods have not been yet implemented in more hospitals and why the access to teaching is limited for physicians in both countries.

Discussion: Based on our results the recent situation in both countries is discussed and the future goals in gynecologic endoscopy in the andean region are presented.

\section{9}

\section{ULTRASOUND SIMULATION TRAINING IN THE CLINICAL SETTING: IMPACT ON SKILLS DEVELOPMENT AND BARRIERS TO IMPLEMENTATION}

Teaching and Training

\author{
N. Amso*, S. Mullins, G. Langan, A. Alsalamah, N. Pugh \\ ${ }^{[1]}$ Cardiff University $\sim$ Cardiff $\sim$ United Kingdom
}

Summary (4 lines): Simulation is a technique whereby an environment is created that allows a virtual representation of a real process to be experienced.

Introduction: Objectives: (1) Evaluate the trainees' views on usefulness and the barriers to engagement with the ultrasound simulator (ScanTrainer) for training in core transvaginal ultrasound (TVUS) skills. (2) Determine the benefits and limitations of ultrasound simulation training.

Material and Methods: Design: Retrospective questionnaires in paper, electronic or phone interview format were analysed qualitatively and quantitatively. Participants in the randomised controlled trial (RCT) investigating the benefit of the simulator including those who have withdrawn from participation.

Results: Results: The main barriers to access to simulation training were conflict of commitments, lack of protected training time and location of the simulator. There were no statistically significant difference between the access and perceived ease of access between the centres or different levels of training.

Discussion: It is likely that the most effective method for improving utilisation of the simulator would be to implement protected training time, however there are staffing and time constraints to this

\section{5}

A USEFUL SURFACE ANATOMICAL LANDMARK TO AVOID INFERIOR EPIGASTRIC VESSEL INJURY AT LAPAROSCOPY

Technical Tricks and New Instrumentations

G. Michos*, I. Korkotzelos, I. Tsimpanakos, N. Gioulekas, C.

Stamatopoulos, M. Granata, A. Magos

${ }^{[1]}$ Royal Free Hospital $\sim$ London $\sim$ United Kingdom
Summary (4 lines): The anterior superior iliac spine (ASIS) is a useful landmark for placing ancillary laparoscopic ports.

Introduction: Injury to the inferior epigastric vessels is the commonest vascular injury at laparoscopy. We investigated if the ASIS is a useful guide for placement of the lateral accessory port during gynaecological laparoscopy.

Material and Methods: 56 patients undergoing laparoscopy were enrolled in a prospective study. Following creation of a pneumoperitoneum and insertion of the primary laparoscopic port and laparoscope, the distance between the inferior epigastric vessels and ipsilateral ASIS was measured in the coronal plane on both sides. Surgery then proceeded with insertion of ancillary laparoscopic ports lateral to the inferior epigastric vessels.

Results: Both inferior epigastric vessels were visualised in 48 cases and one at least one in two. The mean distance between the anterior superior iliac spine and the inferior epigastric vessels was $8.6 \mathrm{~cm}$ on both sides ( $95 \%$ CI $8.285-8.835 \mathrm{~cm}$ on the left, $8.35-8.88 \mathrm{~cm}$ on the right), with a range of $7-10.5 \mathrm{~cm}$ on the left and $7-10 \mathrm{~cm}$ on the right. There was no correlation between this distance and age, height, weight or body mass index.

Discussion: The ASIS is a useful landmark for the placement of the lateral ancillary laparoscopic ports. By placing the ports slightly lateral and superior to the ASIS $(<6 \mathrm{~cm})$, injury to the inferior epigastric vessels can be avoided.

\section{4}

ANTERIOR SUPERIOR ILIAC SPINE: A USEFUL ANATOMICAL LANDMARK FOR SYMMETRICAL ANCILLARY PORT PLACEMENT AT GYNAECOLOGICAL LAPAROSCOPIC SURGERY

Technical Tricks and New Instrumentations

M.D. Dacco'*, I. Korkontzelos, I. Tsimpanakos, N. Gioulekas, C. Stamatopoulos, M. Granata, A. Magos

${ }^{[1]}$ Royal Free Hospital $\sim$ London $\sim$ United Kingdom

Summary (4 lines): We assessed the usefulness of the anterior superior iliac spines as fixed reference points for achieving port symmetry between the left and right ancillary ports at laparoscopy.

Introduction: We are not aware of any study which has assessed the anterior superior iliac spine (ASIS) as a guide for symmetrical placing of lateral ancillary ports at gynaecological laparoscopy.

Material and Methods: We studied 30 women undergoing laparoscopic surgery. In the study group, we used the ipsilateral ASIS as a reference point to position the ancillary port on each side with the aim of achieving port symmetry. In the control group, symmetrical positioning of the two parts was judged visually. The position of the two ports sites was measured digitally in the longitudinal and transverse planes at the end of surgery. The surgeon always stood on the left of the patient.

Results: The right lateral port tended to be placed more caudally than the left in both groups. Port symmetry was better in both the longitudinal and transverse axes in the study group, although the difference only reached statistical significance in the longitudinal direction $(p=0.0344)$. We found differences $(>2 \mathrm{~cm})$ in the position of the ports in four patients in the control group $(27 \%)$ but none of the study group.

Discussion: The ASIS is an easily palpable landmark and a useful aid for placing the lateral ancillary laparoscopic ports symmetrically. 
211

\section{CESAREAN SCAR DEFECT. MANAGEMENT AND UPDATE.} A 10 YEAR EXPERIENCE

Technical Tricks and New Instrumentations

P.N. Barri-soldevila*, M.A. Pascual, A. Vazquez, I. Rodriguez

${ }^{[1]}$ Instituto Universitario Dexeus $\sim$ Barcelona $\sim$ Spain

Summary (4 lines): To evaluate our experience in the diagnostic and management of cesarean scard efect

Introduction: Retrospective evaluation of 38 consecutive patients diagnosed of cesarean scar defect by ultrasound in a single institution from January 2002 till December 2011.

Material and Methods: Most cases were diagnosed in the last five years. No cases of scar defect during pregnancy, uterine rupture or abnormal placentation were found. 5 patients were diagnosed of cesarean scar ectopic pregnancy, out of 7281 cesarean sections.

Results: They were treated by 2 hysterectomies, 2 local injections of metothrexate and one removal of ectopic tissue and defect suture. Out of the 33 non pregnant women diagnosed of cesarean scar defect, 7 patients underwent a histerectomy for clinical discomfort. (spotting, pelvic pain) 8 patients seeking pregnancy underwent defect repair. 3 of them had had preterm cesarean sections, but only one corioamnionitis was demonstrated. 4 of them had clinical discomfort. Two months after the operation, an ultrasound evaluation was performed and in one case the defect was still present. The patient had pelvic pain and requested a hysterectomy. Out of the remaining operations, all symptoms were solved. One patient underwent a cesarean section, and two of them 2 . All surgical procedures were performed laparoscopically without complications.

Discussion: Vaginal ultrasound and laparoscopy seem to be powerful tools to manage cesarean scar defects

\section{3}

EVALUATION OF THE NOVEL SPRAYABLE ADHESION BARRIER IN PORCINE LAPAROSCOPIC MODEL

Technical Tricks and New Instrumentations

M. Kai*, K. Maeda, S. Kira, S. Nakamura, M. Tasaki, N. Chino, H. Nishida, T. Kawanishi

${ }^{[1]}$ TERUMO CORPORATION R\&D Headquarters $\sim$ Kanagawa Japan

Summary (4 lines): In this study, the adhesion prevention effects of the novel adhesion barrier system were evaluated in a porcine laparoscopic model. It was found that our system significantly prevents adhesions of injured sites.

Introduction: Post-operative adhesions are considered a major cause of pelvic pain and infertility.

Therefore, we have developed the sprayable adhesion barrier system in the form of a dextrin based gel. The gel attaches to the surface of the tissue to work as a physical barrier to reduce postoperative adhesions. In this study, the adhesion prevention effects of our system were evaluated in a porcine adhesion model that was constructed with laparoscopic myomectomy.

Material and Methods: Eight female pigs were used, and the suture site of the uterine horn and of the peritoneal excision in both sides of each animal (total of two sites per animal) $(\mathrm{N}=16)$ were assessed. The surgery was performed laparoscopically. Spray was applied to the excision area and surrounding the suture site of the uterine horn.

Results: The rate of adhesion was 13/16 (81\%) in the control group and $2 / 16(13 \%)$ in the treated group.
Discussion: It was found in a model intended for myomectomy and simulating use under conditions of laparoscopic surgery that the gel formed by spraying significantly prevents adhesions of injured sites. With its ease of application and these results of adhesion prevention efficacy, our system may have significant potential for clinical use.

274

\section{LAPAROSCOPIC ANTERIOR UTERINE LIGAMENTOPEXY FOR PELVIC PAIN IN A SELECTED POPULATION OF PATIENTS}

Technical Tricks and New Instrumentations

L. Lise*, F. Emilie, A. Jaime, P. Marco, N. Joseph, W. Arnaud

${ }^{[1]}$ universitary Hospital of Strasbourg $\sim i R C A D /$ EITS Strasbourg France

Summary (4 lines): In women with retroverted uterus who have dysmenorrhea, dyspareunia or chronic pelvic pain, laparoscopic anterior uterine ligamentopexy will be an effective method of achieving symptom relief including treatment of endometriosis.

Introduction: The objectives of this retrospective study were to evaluate clinical outcome after laparoscopic anterior uterine ligamentopexy for retroverted uterus associated with a congestive pelvic syndrome and to compare patients with and without endometriosis as well as their satisfaction scores.

Material and Methods: This is a descriptive single center retrospective study which aims to analyze all patients who have benefited from a laparoscopic uterine anterior ligamentopexy for pelvic pain from February 2008 to April 2012 at the Gynecology Department of the University Hospital of Strasbourg.

Results: Thirty two patients treated with this technique were selected. Nineteen patients $(59.37 \%)$ had endometriosis associated with CPS (Conventional Pap Smear) (Endometriosis group) and thirteen $(40.63 \%)$ patients only had CPS (No Endometriosis group). In all cases, satisfaction was high $(75.26 \%$ in the Endometriosis group, and $91.53 \%$ in the No Endometriosis group).

Discussion: Laparoscopic anterior uterine ligamentopexy is a simple procedure that can be useful to reduce pain, even in case of endometriosis. Randomized controlled trials are mandatory to corroborate our results.

346

\section{AN ASSESSMENT OF EFFICACY IN SURGICAL TREATMENT OF FEMALE STRESS URINARY INCONTINENCE WITH RETROPUBIC AND TRANSOBTURATOR APPROACH Urogynaecology}

J. Szymanski*, P. Kukulski, G. Stokluska, M. Wieczorek, L. Lewczuk ${ }^{[1]}$ Snt. Sophia Hospital $\sim$ Warsaw $\sim$ Poland

Summary (4 lines): TVT and TOT procedures are characterised by a high cure rate and improved postoperative QoL. However, it seems that transobturator approach should be the preferred method of SUI treatment.

Introduction: The aim of the study was to assess intra-operative, early postoperative complications as well as efficacy of two methods: TVT anTOT used for treatment of female SUI.

Material and Methods: The analysis involved 91 TVT and 60 TOT procedures. The patient self-assessed efficacy evaluation was based upon interviews during follow-up visits, as well as using UDI-6, IIQ-7 and VAS. 
Results: The time of the TOT procedure was significantly shorter than TVT. The average intra-operative blood loss was lower for TOT procedure. Bladder perforation was only seen during TVT procedures-5\%. Postoperative urination difficulties occurred in $13 \%$ of TVT pts and $5 \%$ of TOT pts. A significantly lower risk of intra-operative and early postoperative complications was noted in TOT pts, as compared to those undergoing TVT.

A similar cure rate has been seen in both groups ( $88 \%$-TVT,95 \%TOT), as well as improvement ( $2 \%$-TVT, $2 \%$-TOT) and failure rates (10\%-TVT,3 \%-TOT). The change in postoperative QoL has been noted in both groups.

Discussion: Results are similar to those obtained in published studies.

216

\section{CHRONIC PELVIC PAIN AFTER VAGINAL SURGERY GYNECOLOGIC \\ Urogynaecology}

J. González*, J.A. Solano, G. Zapico, J.M. Menéndez, E. Martínez

${ }^{[1]}$ Hospital Universitario Principe de Asturias $\sim$ Alcala de Henares $\sim$ Spain

Summary (4 lines): Clinical case of a patient who developed a potential pudendal neuralgia after gynecologic surgery.

Introduction: The pudendal nerve neuralgia in pelvic floor surgery is a difficult to diagnose.

Material and Methods: Review case in our Center and the available literature on this topic.

Results: 67 years old patient, in her second, a history of hypertension and multiple lumbar disc herniation, diagnosed with genital prolapse. Exploration: cystocele, histerocele third degree. Normal urodynamic study. Treatment by simple total hysterectomy with anterior colporrhaphy vaginally without complications.

After discharge pelvic pain iterative, requiring admission for control of it, with good clinical response later. Pelvic pain symptoms recurred and appeared as proctalgia, coitalgia, difficulty in urination and defecation. By imaging (MRI pelvic, lumbar spine MRI, anorectal manometry, ultrasound of both inguinal canals) as well as digestive and urological studies, concluded the possible diagnosis of postoperative pudendal neuralgia. Currently being treated and monitored by the Pain Unit and the Department of Surgery.

Discussion: The pudendal nerve neuralgia in pelvic floor surgery is a rare complication and difficult to diagnose. Nerve damage can occur during surgery or after it (trapping due to postoperative fibrosis). Its diagnosis is clinical. Treatment is initially conservative, with perineural injections of local anesthetic, or surgical treatment if no response to release nerve entrapment.

\section{8}

\section{DIFFERENT SLING PROCEDURES FOR TREATMENT OF FEMALE SUI. LONG-TERM RESULTS \\ Urogynaecology}

A. Slobodyanyuk, I. Mazokhin, M. Suchalko, B. Slobodyanyuk*

${ }^{[1]}$ Zhukowsky Municipal Clinical Hospital $\sim$ Zhukowsky $\sim$ Russian Federation

Summary (4 lines): We represent the results of using 3 different sling procedures

Introduction: We've made comparsion of standard techniques with budget surgery in aspect of long-term outcome.
Material and Methods: Form January 2004 to December 2011, 92 sui patients was operated by single surgeon, $43 \%$ of cases with POP surgery. Retropubic approach was used in 25 cases in recurrent SUI. TOT in 38 cases. In 29 patients we performed polypropylene needle suprapubic minisling similar to Raz operation. Median follow up was 49 month.

Results: Subjective assessment of cure rate did not show any differences between groups. For TVT group cure rate was $92 \%$, improvement $8 \%$, failure $0 \%$. For TOT group cure rate was $89 \%$, improvement $8,5 \%$, failure $2,5 \%$. For minisling group cure rate was $76 \%$, improvement $20 \%$, failure $3,4 \%$. After the TVT procedure we've found protrusion of 1 side of the sling in the bladder. Minilaparotomy and transvesical resection of part of the sling was performed with no consequences. We also have 3 cases of obstructive urination. During postoperative assessment of patients we diagnosed 2 cases of OAB in TOT and minisling groups. All of these patients treated with cholinolytics with some improvement.

Discussion: Analysis of results shows high efficacy after 5 years (98\%) and little worsening (89\%) after 7 years regardless of type of sling, including "hand-made" budget minisling for poor people. Right patient selection and good surgical technique are essential.

306

INITIAL RESULTS OF LAPAROCOPIC SACROHYSTEROPEXY Urogynaecology

T. Smith Walker*, D.L. Byrne, W. Petro

${ }^{[1]}$ Royal Cornwall Hospital $\sim$ Truro $\sim$ United Kingdom

Summary (4 lines): Laparoscopic Sacrohysteropexy (LSP) resuspends the prolapsed uterus restoring level one support, whilst the traditional approach of vaginal hysterectomy only removes the uterus. We believe re-suspension is a better approach and present one surgeons results of the first 38 cases of LSP.

Introduction: The literature shows a $40 \%$ incidence of vault prolapse after vaginal hysterectomy. Growing evidence indicates preservation of the uterus and restoration of the anatomy is a superior approach to vaginal hysterectomy. A video of the surgical procedure will be shown.

Material and Methods: One surgeons cases of LSP between January 2010 and January 2012 were reviewed. Pre, and 3 month post, operative POP-Q assessment was carried out.

Results: The results show that the uterine position as measured by point $\mathrm{C}$ consistently improves post LSP. The average delta $\mathrm{C}$ of -6 . In addition the anterior vaginal support also improves in selected cases. Whilst the posterior wall appears unaffected.

Discussion: LSP is successful at restoring the uterus to its correct position. It is very well tolerated with no major complications reported in this series. Further evaluation is required, including quality of life assessment, but our initial results with LSP are very promising and we continue to offer it as our primary treatment for primary uterine prolapse.

\section{1}

\section{LAPAROSCOPIC PELVIC FLOOR RECONSTRUCTION (PFR): OBJECTIVE AND SUBJECTIVE OUTCOME} Urogynaecology

S.Z. Syed Abdul Jalil*, K. Johnston

${ }^{[1]}$ Antrim Area Hospital $\sim$ Antrim $\sim$ United Kingdom 
Summary (4 lines): Retrospective review of laparoscopic pelvic floor reconstruction (LPFR) in 23 patients (single operator). Improved objective and subjective outcome utilising POPQ, patient quality of life and satisfaction rate scores are reported.

Introduction: Superb visualisation and laparoscopic suturing make this an excellent approach for pelvic organ prolapse (POP) repair. Subjective and objective outcome measures were reviewed.

Material and Methods: Retrospective review of 23 patients who had LPFR (laparoscopic paravaginal repair) to address anterior compartment prolapse and total laparoscopic hysterectomy with uterosacral plication to address middle compartment prolapse. Pre and post op POPQ was analaysed, as was validated Quality of Life Questionnaires, P-QOL and PGI. Median time for objective follow up was 2.5 months. Subjective follow up ranged from 7 months to 4 years.

Results: Mean operating time was 135 mins. Eighteen patients (78.26\%) had no recurrence of same compartment prolapse. Six patients $(26.09 \%)$ had new compartment prolapse. POPQ scores in the anterior compartments improved from Stage II-III to Stage 0-II. PQOL score ranged from 35-135 (median: 56). Global patient satisfaction from PGI score ranged from 1-6 (median: 2)

Discussion: Laparoscopic PFR with the right expertise has a good outcome; overall success rate approaches $80 \%$. The subjective and objective outcome measures correlate well.

506

\section{LAPAROSCOPIC PROMONTOFIXATION FOR ADVANCED APICAL PELVIC ORGAN PROLAPSE: TECHNIQUE AND OUTCOME}

Urogynaecology

A.R. Mothes*, M.P. Radosa, I.B. Runnebaum

${ }^{[1]}$ Jena University Hospital $\sim$ Jena $\sim$ Germany

Summary (4 lines): To our knowledge this is the only study evaluating selected high degree apical defect pattern in POP after laparoscopic promontofixation.

Introduction: Life expectancy in industrial countries has reached its highest level and life time risk for POP at an age of 80 years is $11 \%$. The future demand for pelvic floor surgery is increasing.

Material and Methods: App.150 operations for POP are performed annually at Jena University Hospital. We report on selected 28 cases presenting advanced apical prolapse that underwent laparoscopic promontofixation. Patients were followed up 3 months and 1 year after surgery. Evaluation was done by POP-Q values and interviews for symptoms and subjective cure.

Results: In $64 \%$ laparoscopic colpopromontofixation was performed. Mean operating time was $156 \mathrm{~min}$.

In $29 \%$ LASH was combined with cervicopromontofixation. Mean operating time was $160 \mathrm{~min}$. In $7 \%$ hysteropromontofixation was performed.

In 11 patients extensive laparoscopic adhaesiolysis had to be done. No complications occurred. Mean hospital stay was 3 days.

All patients were re-examined after 3 months and 13 patients after 1 year. For POP-Q Aa, Ba, D, Ap and Bp significant differences were found. Objective and subjective cure was found $96 \%$. Functional cure ranged from $80-96 \%$.

Discussion: Laparoscopic surgery for advanced POP is valid. It goes ahead with very low morbidity and short hospital stay. The anatomic, functional and subjective results are excellent.
492

LAPAROSCOPIC SACROCOLPOPEXY FOR POSTHYSTERECTOMY PROLAPSE: OUR INITIAL EXPERIENCE Urogynaecology

H. Ferreira*, R. Cubal, S. Guimarães, A. Tomé

${ }^{[1]}$ Centro hospitalar do Porto $\sim$ Porto $\sim$ Portugal

Summary (4 lines): This retrospective descriptive analysis of our initial experience demonstrate that laparoscopic sacral colpopexy may be the procedure of choice for difficult and recurrent prolapses in patients who wish to maintain a functioning vagina Introduction: We wish to describe our initial experience of laparoscopic sacral colpopexy in an academic university hospital

Material and Methods: Five patients with recurrent prolapse of the apex (stage III-IV) underwent laparoscopic sacral colpopexy with Gynemesh (Ethicon) used as the graft material

Results: Five patients, from September 2010 through May 2012, underwent a colpo sacropexy. The patients had recurrent apical prolapse. Intraoperative and postoperative complications didn't occur. All patients were evaluated at 2 weeks and 6 weeks after surgery, and pelvic examination was performed. Median age was 67 years (range 52-78 years), and median body mass index was 27 (range 24-28). Laparoscopic sacral colpopexy was performed successfully in 5 patients. The median hospital stay was 2 day (range 1-3 days). The five patients are extremely happy and satisfied with the surgery. Postoperative recovery has been uneventful. Objective cure is $100 \%$. There have been no cases of colpopexy graft exposure or recurrence till now. Discussion: Our experience is recent and short, but our results are comparable with the majority of the literature publications. Laparoscopic sacral colpopexy is a safe and effective procedure.

12

MESH DESIGN FOR MINIMAL INVASIVE CORRECTION

OF PELVIC ORGAN PROLAPSE (POP): ANTERIOR

TRANSOBTURATOR (ATOM), POSTERIOR ISCHIORECTAL (PIRM), POSTERIOR ISCHIORECTAL TRANSOBTURATOR (PIRTOM) AND ANTERIOR TRANSOBTURATOR POSTERIOR ISCHIORECTAL MESH (ATOPIRM)

Urogynaecology

M. Lužnik*

${ }^{[1]}$ General Hospital Slovenj Gradec $\sim$ Slovenj Gradec $\sim$ Slovenia

Summary (4 lines): To correct pelvic organ static and pelvic floor $(\mathrm{PF})$ function with minimally invasive needle transvaginal intervention. Introduction: Use of appropriate designed mesh implants (MI) allow marked improvement in pelvic organ static and PF function.

Material and Methods: In 6 years 328 corrections of female POP and PF dysfunction with MI have been performed. The preoperative vaginal status was assessed as stage II-IV by the ICS system. Individually trimmed Gynemesh $10 \mathrm{~cm} \times 15 \mathrm{~cm}$ was used in 5 different forms; for ATOM with 6 arms and Intravaginal sling (IVS) 04 Tunneller (Tyco) was used for trans-obturator (TO) application; for PIRM with 4 arms for application of 2 long arms (LA) through ishiorectal (IR) fossa via an infraligamental way with IVS 02 and two short arms (SA) for perineal body (PB); for PIRTOM with 6 arms, IVS 04 was used for TO application of 2 apical arms, 2 IR LA to application by IVS 02 through IR fossa and 2 SA for PB; for ATOPIRM with 8 arms, IVS 04 was used for TO application of 4 arms and IVS 02 was used for application of 2 LA via IR fossa and 2 SA through PB. 
Results: Postoperatively all women had ICS stage 0.21 MI extrusions cured spontaneously after excision of denudated mesh part.

Discussion: Quality of life markedly improved after operation.

359

OPENING OF THE VAGINA DURING THE LEARNING CURVE AS MAIN PREDICTOR FOR TECHNIQUE FAILURE AFTER LAPAROSCOPIC SACRAL COLPOPEXY.

Urogynaecology

J. Gilabert-estelles*, J.J. Rodenas, B. Salas, R. Aliaga, J. Gilabertaguilar

${ }^{[1]}$ Hospital General Universitario $\sim$ Valencia $\sim$ Spain

Summary (4 lines): The objective of this study was to compare the risk of mesh erosion and recurrence of pelvic organ prolapse according to the type of approach for pelvic floor prolapse

Introduction: Laparoscopic sacral colpopexy is an effective technique in complex pelvic organ prolapse. Even if the risk of mesh erosion is low in comparison to vaginal approach to the prolapse it is mandatory to identify the possible factors that might increase of this complication.

Material and Methods: A retrospective cohort of 64 patients treated with two different techniques of sacral colpopexy was evaluated. Ten patients were treated with a combined vaginal and laparoscopic approach and 54 patients were treated with total laparoscopic colpopexy using polypropilene mesh.

Results: Opening of the vagina was the main predictor of mesh erosion. Results of total laparoscopic approach were superior to the combined technique in terms of recurrence, complications and patient satisfaction.

Discussion: Insertion of graft material should be avoided if the vagina is opened during the laparoscopic correction of pelvic floor defects.

13

\section{SURGICAL PROLIFT MESH REPAIR: AN AUDIT OF PATIENT OUTCOMES \\ Urogynaecology}

S.S. Syed Badrol*

${ }^{[1]}$ Newcastle University $\sim$ Newcastle Upon Tyne $\sim$ United Kingdom

Summary (4 lines): This audit aims to assess the implementation of the NICE IPG 267, patient's intra and post-procedural outcomes as well as long term efficacy.

Introduction: Long- term studies since the introduction of the transvaginal tape procedure have shown that the use of mesh reduces the risk of failure and is associated with a lower complication rate. However, there are insufficient studies on the efficacy and complications of the use of mesh causing growing concerns.

Material and Methods: All 77 procedures done on 75 patients who underwent Prolift Mesh surgery for repair of vaginal prolapse in University Hospital of North Durham from September 2009 to December 2011 were retrospectively identified. Relevant data were then collected from patient case notes and theatre documentation using the designed data collection proforma.

Results: Only $3.9 \%$ intra-procedural complication rate. Complications within 30 days of procedure were observed in $18.2 \%$ of patients. $78.6 \%$ of patients reported symptomatic benefit from baseline. $15.7 \%$ of patients suffered from mesh exposure within 12 months of procedure. $27.1 \%$ of patients had de novo or recurrent prolapse within 12 months of procedure.
Discussion: Found that both the rate of post-procedural follow up and percentage of patient receiving special consent to be lower than that of defined standards. Additionally, some of the complications seen within 12 months of the procedure were higher than that of defined standards.

474

\section{URODYNAMIC PARAMETERS ALTERATIONS AFTER LAPAROSCOPIC MANAGEMENT OF INCONTINENCE AND PELVIC ORGAN PROLAPSE \\ Urogynaecology}

A. Hosseini, A. Moeini*

${ }^{[1]}$ Department of Obstetrics and Gynecology, ShohadaTajrish Hospital, Shahid Beheshti University of Medical Science $\sim$ Tehran $\sim$ Iran, Islamic Republic of

Summary (4 lines): The aim of this study was to evaluate urodynamic parameter changes before and after laparoscopic Burch colposuspension.

Introduction: Urodynamic assessment is strongly recommended before management of incontinence and pelvic organ prolapse. The aim of this study was to evaluate urodynamic parameter changes before and after laparoscopic Burch colposuspension.

Material and Methods: A total of 35 patients who underwent extraperitoneal laparoscopic Burch colposuspensions were included. All cases underwent urodynamics with pressure flow and abdominal leak point pressure (ALPP) measurements before and 12 months postoperatively. Maximum cystometric capacity (MCC), ALPP, maximum flow rate (Qmax.), detrusor voiding pressure at Qmax., and postvoid residual urine volume (PVR) were assessed.

Results: Mean age was $51.8 \pm 7.4$ years and mean body mass index was $26.8 \pm 7.2 \mathrm{~kg} / \mathrm{m} 2$. No major complications were observed. Outcomes in regards to increase in ALLP and Qmax, show a significantly improvement compared with preoperative values $(57 \pm 16.4$ vs $75 \pm 22.4 \mathrm{~cm}$ $\mathrm{H} 2 \mathrm{O}$ and $13.2 \pm 2.3$ vs $20.1 \pm 4.1 \mathrm{mdL} / \mathrm{s}$ respectively). No PVR> $25 \mathrm{~mL}$, de novo reduced bladder compliance and hypo- or overactivity were observed.

Discussion: Laparoscopic Burch colposuspension is an effective and safe procedure for management of incontinence and improves urodynamic signs of compression or obstruction of the urethra without any adverse influence on voiding parameters.

\section{3}

\section{A VIDEO OF LAPAROSCOPIC HYSTERECTOMY USING THE NEW THUNDERBEAT® \\ Case Reports}

Video

H. Jan*, G. Araklitis, N. Narvekar

${ }^{[1]}$ king's College Hospital $\sim$ London $\sim$ United Kingdom

Summary (4 lines): A video of laparoscopic hysterectomy using the new Thunderbeat ${ }^{\mathbb{R}}$

Introduction: We present a video of laparoscopic hysterectomy using the new Thunderbeat ${ }^{\circledR}$, an integrated hand instrument that delivers the benefits of both advanced bipolar and ultrasonic energy in a single device.

\section{Material and Methods:}

Results: A 40 year old para 1 presented to clinic with a large 20 week size fibroid uterus and history of heavy menstrual bleeding requiring previous hospital admission for blood transfusions. Ultrasound scan 
confirmed multiple uterine fibroids with the largest being $42 \times 42 \times$ $38 \mathrm{~mm}$. After discussion of treatment options, she opted for hysterectomy. Total laparoscopic hysterectomy was performed using a supraumbilical $5 \mathrm{~mm}$ optical port, 2 iliac fossa ports and a $10 \mathrm{~mm}$ umbilical grasper for uterine traction and manipulation. A subtotal hysterectomy was performed initially using the $5 \mathrm{~mm}$ Thunderbeat device, followed by removal of the cervix. The specimen was morcelated using Gynecare morcellator. The patient had an uneventful recovery and was discharged the next day.

Discussion: This video demonstrates the thunderbeat device in a complex laparoscopic hysterectomy of a large uterus. This device has been shown to have better sealing abilities in comparison to a sole ultrasonic device and faster dissection time than an advanced bipolar device (Seehofer et al, 2012).

\section{6}

\section{CASE OF BIG OVARIAN CYSTECTOMY, FIBROID UTERUS AND A VERY RARE PARARECTAL DERMOID CYST Case Reports}

\section{Video}

P. Trompoukis*, A. Pachydakis, I. Frouradakis

${ }^{[1]}$ IASO Group of hospitals $\sim$ Athens $\sim$ Greece

Summary (4 lines): This is a video of a laparoscopic removal of a $10 \mathrm{~cm}$ dermoid cyst and two fibroids, and which during surgical dissection a pararectal dermoid cyst was discovered and removed.

Introduction: Video of a young patient who presented with a big $10 \mathrm{~cm}$ dermoid cyst and two fibroids for laparoscopic surgical removal. During surgery we noticed that the anatomy especially of the left adnexae was not normal. So after the first dermoid cystectomy and subsequent removal of fibroids we proceeded with extensive surgical dissection to restore normal anatomy. During dissection we mistakenly thought that accidentally we made a hole to the bowel, however it appeared to be a second dermoid cyst "buried" deep in the pelvis laterally to the rectum.

Material and Methods: 24 years old patient submitted for laparoscopic removal of big dermoid ovarian mass and fibroids.

Results: All dermoid cysts and fibroids were removed and sent for histological examination. The postoperative course was uneventful. We believe that is a good rare case that all surgeons ccould possibly face a similar situation. We analyse the technique and results.

Discussion: Retroperitoneal masses are rare, often malignant, and difficult to diagnose. Surgical removal is associated with significant morbidity and mortality since these masses are often very deep in the pelvis. The cyst has to be removed otherwise can be relapsed.

\section{8}

\section{FEASIBILITY OF LAPAROSCOPIC APPROACH IN PARAMETRIAL RECURRENCE OF SEROUS OVARIAN CARCINOMA}

Case Reports

Video

J. Gilabert-estelles*, D. Izquierdo, A. Bolos, G. Lazaro, A. Cano

${ }^{[1]}$ Hospital General Universitario $\sim$ Valencia $\sim$ Spain

Summary (4 lines): We present the laparoscopic technique in a case of pelvic recurrence of ovarian cancer.

Introduction: This approach is controversial and infiltration of bladder and ureter might be consider a limitation of the technique.
Material and Methods: A patient diagnosed of a FIGO IIIC ovarian serous carcinoma was treated with optimal debulking and adjuvant chemotherapy in 2001. She attended our unit after 132 months of disease free interval and an increase of CA125 was detected. The imaging showed a $33 \times 46 \mathrm{~mm}$ lesion located at the left parametrium without apparent infiltration of the bladder wall and left ureter. PETScan showed hypermetabolic pattern. A laparoscopic approach was indicated in this patient. Retroperitoneal dissection was performed and the ureter was dissected away from the surrounding tissue until the entrance in the bladder. A complete laparoscopic excision of the tumour was performed without opening of the bladder or ureter. Final examination showed no disease in the pelvis and adjuvant chemotherapy was indicated.

Results: Patient recovered uneventfully and presented normal oncological controls after 4 months follow up.

Discussion: Laparoscopic approach to parametrial recurrence of ovarian cancer is feasible and permits conservative approach in selected cases

367

\author{
HETEROTOPIC PREGNANCY \\ Case Reports \\ Video \\ J.C.R. Dr* \\ ${ }^{[1]}$ Clinica del Country $\sim$ Bogota $\sim$ Colombia
}

Summary (4 lines): 32 year old patient with a history of infertility of 2 years of evolution, it was decided to initiate ovulation induction, the patient presented limited genital bleeding and mild cramping pain in abdomen a week later, transvaginal ultrasound was performed, which revealed two gestational sacs, one with showed an intrauterine embriocardia and a second ectopic sac with positive embriocardia located in the left tube. It took the patient to laparoscopy, blood in the peritoneal cavity in about $200 \mathrm{cc}$ and ectopic pregnancy in left tube, with corpus luteum cyst approximately $4 \mathrm{~cm}$, left salpingectomy was performed with cavity lavage, extracted tube and ectopic ultrasound monitoring two days, and weekly thereafter for 4 more weeks, showing a favorable progression of pregnancy.

Introduction: 32 year old patient with a history of infertility of 2 years of evolution, transvaginal ultrasound was performed, which revealed two gestational sacs, one with showed an intrauterine embriocardia and a second ectopic sac with positive embriocardia located in the left tube.

Material and Methods: Case report Heterotopic Pregnancy

Results: We present this video showing a very rare disease which happens in 1 in 30000 pregnancies.

Discussion: This increased frequency may be associated with income of assisted reproductive techniques as in this case presented. Indeed, the combined incidence of pregnancy can be as high as $1 \%$ when using this technology. The ideal management is the minimum laparoscopic uterine manipulation and fast recovery.

\section{7}

\section{LAPAROSCOPIC APPROACH TO RECURRENT COMPLEX PELVIC ORGAN PROLAPSE WITH MESH EXCISION AND MODIFIED HIGH UTEROSACRAL COLPOPEXY}

Case Reports

\section{Video}

J. Gilabert-estelles*, C. Bauset, M. Gonzalez, A. Cano

${ }^{[1]}$ Hospital General Universitario $\sim$ Valencia $\sim$ Spain 
Summary (4 lines): We present the laparoscopic approach in a patient that attended our pelvic floor unit due to recurrent pelvic organ prolapse Introduction: Laparoscopic sacral colpopexy for pelvic floor defects is being used since two decades for pelvic floor defects in young patients but this technique is not free of complications.

Material and Methods: The patient has been operated in 2002 with a vaginal hysterectomy and anterior colporraphy. Anterior and apical prolapse recurred and a laparoscopic sacral colpopexy with polypropilene mesh was performed in may 2009. In the postoperative period the patient presented abdominal pain due to a retraction of the mesh and a new laparoscopy was performed in order to release the mesh tension. An anterior, apical and posterior grade IV defect occurred and at the time of evaluation at our unit a mesh erosion at the vaginal cuff was detected in the gynaecological examination. The patient underwent a laparoscopic complete removal of the remaining mesh with laparoscopic excision of the vaginal ulcer. Pelvic organ prolapse was corrected after wide dissection of both ureters and a modified high uterosacral colpopexy was performed using braided polypropylene sutures.

Results: Patient is free of symptoms at 6 months follow up visit.

Discussion: Laparoscopic high uterosacral colpopexy with removal of graft material is an effective approach to recurrent complicated pelvic organ prolapse

130

\section{LAPAROSCOPIC CERCLAGE PLACEMENT FOLLOWING A RADICAL TRACHELECTOMY \\ Case Reports}

Video

J.I. Einarsson*

${ }^{[1]}$ Harvard Medical School $\sim$ Boston $\sim$ United States

Summary (4 lines): The video shows a removal of an eroded cerclage in a patient who had a radical trachelectomy and the subsequent placement of a laparoscopic cerclage.

Introduction: 35 year old $\mathrm{G} 0$ had a radical trachelectomy for early cervical cancer. A cerclage was placed as part of the procedure. She had persistent vaginal spotting after surgery and was found to have a cerclage eroding into the vagina. She was extensively counseled and a decision was made to proceed with vaginal removal of the cerclage and placement of a new laparoscopic cerclage.

Material and Methods: The eroded cerclage was initially removed vaginally. Laparoscopy was then performed and extensive lysis of adhesions and bilateral ureterolysis was required to gain access to the pelvic anatomy. A new cerclage was then placed laparoscopically, paying close attention to the placement in the lower uterine segment.

Results: The surgical procedure took 2 hours and 20 minutes. There were no complications and she did well post-operatively. She has not become pregnant as of yet.

Discussion: We have seen two cases of cerclage erosion after a radical trachelectomy. In both cases, we were able to remove the cerclage vaginally and place another cerclage laparoscopically. While technically feasible, pregnancy outcomes in this scenario are uncertain. The video will highlight the steps of the procedure.

LAPAROSCOPIC EXCISION OF PELVIC RECURRENCE OF CERVICAL CARCINOMA AFFECTING RIGHT EXTERNAL ILIAC VESSELS

Case Reports

Video

J. Gilabert-estelles*, J. Gilabert-aguilar

${ }^{[1]}$ Hospital General Universitario $\sim$ Valencia $\sim$ Spain
Summary (4 lines): We present the laparoscopic technique in a pelvic recurrence in cervical cancer located at the caudal portion of external iliac vessels.

Introduction: A 36 year-old patient diagnosed of an invasive FIGO IB2 cervical carcinoma was treated in july 2010 with a radical hysterectomy without adjuvant radiotherapy.

Material and Methods: Dissection of the external iliac vessels was performed from the common iliac bifurcation and the ureter was freed from the surrounding tissue. Previous radiation made very difficult to identify the clivage planes. Both external iliac artery and vein were secured with a double vessel-loop in order to obtain hemostasis in the case the vessels were damaged during the dissection and vascular surgeons were available. Using bipolar scissors and forceps the mass was liberated from the vessel wall without opening it. Ultrasound sealing device was used to extract the mass from the fibrotic surrounding tissue. Results: The patient postoperative course is uneventful and the oncologic controls are normal after a 12 months follow up.

Discussion: Laparoscopic approach to pelvic recurrence in cervical cancer is feasiable and safe.

\section{4}

\section{LAPAROSCOPIC GENITAL REASSIGNMENT SURGERY FOR FEMALE-TO-MALE TRANSSEXUALISM Case Reports \\ Video \\ S. Ribeiro*, A. Valentim Lourenco, A. Henriques, A.L. Ribeirinho \\ ${ }^{[1]}$ Centro Hospitalar Lisboa Norte $\sim$ Lisboa $\sim$ Portugal}

Summary (4 lines): Female-to-male transsexualism has a demanding treatment that traditionally includes combined vaginal and abdominal genital reassignment surgery. In this case-series we report on an alternative to this procedure, in which the vaginectomy is also performed laparoscopically.

Introduction: We report on an alternative procedure to the traditional female-to-male transsexualism genital reassignment surgery in which the vaginectomy is also performed laparoscopically.

Material and Methods: We reviewed the charts of all (15 cases) patients submitted to genital reassignment surgery in our department between November 2007 and March 2011. In all cases the vaginectomy was also performed laparoscopically in continuum with the hysterectomy and bilateral salpingo-oophorectomy.

Results: The average operating time was 222 minutes. No serious complications were observed. Patients were released no later than 3 days after the procedure, except in 2 cases with prolonged postoperative urinary retention who were released on the seventh postoperative day with no need for bladder catheterization. One patient had a perineal hematoma 1 month after surgery, which resolved spontaneously.

Discussion: Total laparoscopic genital reassignment surgery appears to be an appropriate alternative to the traditional combined abdominal/ vaginal approach. This procedure provides better exposure of the anatomical structures and has the advantage of being executed in a continuous fashion.

\section{5}

\section{LAPAROSCOPIC MANAGEMENT OF INTRAUTERINE CONTRACEPTIVE MIGRATION TO UNUSUAL LOCATION Case Reports}

Video

E. Martínez Lamela*, J. Molero Vílchez, Y. Mena Buendía, A.B. Martin Escobedo, A.B. Ibañez Santamaría, J.J. Hernández Aguado

${ }^{[1]}$ Hospital Universitario Infanta Leonor $\sim$ Madrid $\sim$ Spain 
Summary (4 lines): Intrauterine contraception is generally welltolerated, but sometimes side-effects occur. The most important complication of IUD is uterine perforation (1 in 1000 procedures) and have been attributed to slow spontaneous IUD migration and it can go unnoticed. It normally appear in omentum rectosigmoid, peritoneum, bladder and, less frequently, in appendix, adnexal or iliac vein one.

Introduction: Removal of copper containing devices is recommended, due to the potential for inflammatory reactions which can cause bowel obstruction and perforation.

Material and Methods: A 23 -year-old asymptomatic woman who had IUD (Copper T) inserted at another center and theoretically extracted. She requested family planning with ESSURE sterilisation. Control Abdominal X-Ray showed two intratubal devices in minor pelvis and IUD at left pelvic wall.

Results: Laparoscopy was performed: IUD transversal branches are visible at intraparenchymal level of left ovary, broad ligament hilum and central Branch (Copper) in a $1.5 \mathrm{~cm}$ path in sigmo-rectal wall without mucus affectation. Hemisection of ovarian parenchyma is performed for its liberation and the IUD is removed by controlled traction inside the sigmo-rectal while checking the mucus integrity. No complication was observed.

Discussion: If the IUD is in the abdomen, operative laparoscopy is the preferred method of removal and must be performed electively in asymptomatic patients.

\section{0}

\section{LAPAROSCOPIC MANAGEMENT OF OVARIAN ECTOPIC PREGNANCY}

Case Reports

\section{Video}

D. Morgan*, A. Thompson

${ }^{[1]}$ Antrim Hospital $\sim$ Antrim $\sim$ United Kingdom

Summary (4 lines): This video shows an early ovarian ectopic and contralateral corpus luteum. The ectopic appears to have implanted on the ovarian cortex. This video illustrates the appearance of such early ectopics which may be mistaken for ovarian malignancy or functional cysts.

Introduction: This Para $0+0$ presented with LIF pain and vaginal bleeding at 7 weeks amenorrhoea. Mild tenderness only was elicited in the LIF. Scan demonstrated no intra uterine sac and no adnexal masses. B-HCG was 3616.

Material and Methods: At laparoscopy there was an unusual appearing right ovary with vascular lesion. The left ovary was slightly enlarged with a corpus luteum. Symptoms were left sided however, the right ovary appearance raised suspicion of ectopic gestation or other significant pathology.

Results: Using monoplar scissors and sharp dissection the lesion was excised with preservation of the right ovary. Histology showed chorionic villi and trophoblast embedded within the surface of the ovarian tissue.

Discussion: Early laparoscopy in the absence of definite adnexal mass facilitated timely treatment and preservation of ovary in this nulliparous woman

Early ovarian ectopics may not be clinically obvious but presence of corpus luteum on contralateral ovary raises index of suspicion and helps differentiate an early ectopic from a physiological corpus luteum.

Pain may not correlate with site of pathology and despite left sided ovulation, this pregnancy implanted on the right ovary
303

\section{LAPAROSCOPIC REPAIR OF COLOVAGINAL FISTULA USING STAPLING DEVICE}

Case Reports

Video

A. Siozos, T. Majmudar*, E. Manzo

${ }^{[1]}$ Hinchingbrooke Hospital $\sim$ Huntingdon $\sim$ United Kingdom

Summary (4 lines): We present a case of a colovaginal fistula which was repaired laparoscopically with resolution of patient's symptoms Introduction: A colovaginal fistula can present many years after an operation

Material and Methods: A 51 year old woman presented with symptoms of malodorous vaginal discharge and an occasional sensation of passing wind PV. The patient had undergone a laparoscopic hysterectomy and $\mathrm{BSO}$ for endometrial $\mathrm{Ca} 7$ years earlier.

During examination, a small opening on the left side of the posterior fossa of the vagina was identified. A CT scan of the pelvis demonstrated no obvious fistula or recurrence of cancer. A decision was made to perform a diagnostic laparoscopy.

Results: During laparoscopy, part of the sigmoid was seen attached firmly to the vault over a small area on the left side suggesting the presence of a fistula tract. The attached colon was separated from the vaginal vault with staples only as the fistula tract was small.

The diagnosis was confirmed histologically and the patient made a good recovery and was discharged home on the following day. Her symptoms completely resolved.

Discussion: A colovaginal fistula can develop postoperatively following pelvic surgery. The fistula repair can -in some cases- be undertaken laparoscopically with a stapling device only and with no need of laparoscopic suturing or laparotomy

\section{4}

\section{RETROPERITONEAL 12 CM FALLOPIAN TUBE TUMOR- LAPAROSCOPIC TREATMENT}

Case Reports

Video

O. Istre*, H. Springborg

${ }^{[1]}$ Aleris-Hamlet Hospital, Copenhagen $\sim$ Copenhagen $\sim$ Denmark

Summary (4 lines): We present a case which simulates a large ovarian cyst a hydrosalpings, and was embedded behind the bowel and attached to the pelvic sidewall

Introduction: Normally hydrosalpings presents as dilated fallopian tubes pre peritoneum, this case is post peritoneum

Material and Methods: A 37 year nullipara women presented with pain and a $12 \mathrm{~cm}$ large cyst opting for laparoscopic treatment

Results: During Laparoscopic surgery we found the fallopian tube under the peritoneum attached to the sigmoid and to the retro peritoneal structures. The ureter and the pelvic vessels were mobilized and visualized in order to remove the tumor. Histology showed large benign hydro salpinges. The patient was successfully treated laparoscopically and were discharged after 14 hours and returned to work after two weeks.

Discussion: Laparoscopic treatment is advantageous, compared to open surgery because of better visualization of the organs during surgery and shorter recovery. We recommend using a $5 \mathrm{~mm}$, 30 degree HD optic which makes it possible to perform port jumping. 
329

\section{TREATMENT OF INTRACTABLE VULVODYNIA: LAPAROSCOPIC RESECTION OF SACRAL NERVE SHWANNOM Case Reports}

Video

M. Possover*, P. Kostov

${ }^{[1]}$ Hirslanden Klinik $\sim$ Zürich $\sim$ Switzerland

Summary (4 lines): The etiologic treatment of vulvodynia is a challenging problem for many gynecologists. We present the laparoscopic resection of a shwannom of the sacral nerves roots in patient with intractable vulvodynia after "neuropelveologic" evaluation

Introduction: The chronic pelvic pain is a commonly encountered problem in many medical offices. When it produces genital pain (vulvodynia) the diagnosis becomes confusing when clinical examinations are inconspicuous

Material and Methods: Case: a 42-years old woman with intractable vulvodynia and normal colposcopic findings. By the gynecological examination a solid tumor has been palpated on the left pelvic wall. The US scan gave the suspect of an adnexal mass and the MRI described a solid tumor of $7 \mathrm{~cm}$ diameter. We performed a laparoscopic resection of the tumor which histological examination confirmed a shannom.

Results: There were no perioperative complications. The patient left the hospital after three days and after 7 days with a suprapubic catheter the patient could void normally the bladder. After 3 months of follow up the patient is pain free and no bladder or bowel dysfunction occurred

Discussion: Pathologies of the sacral nerves roots should always be considered and neuropelveological evaluation is recommended. We believe that the laparoscopy, offering the possibility of magnification and micro dissection is the best surgical procedure for the treatment of the organic pathology on the sacral nerve roots.

28

\section{UTERINE MANIFESTATIONS OF BOURNEVILLE'S DISEASE: CASE REPORT AND VIDEO PRESENTATION.}

Case Reports

Video

G. Pados, A. Makedos*, D. Tsolakidis, K. Almaloglou, B. Tarlatzis

${ }^{[1]} 1$ st Dept. OB-GYN, Aristotle University, "Papageorgiou" General

Hospital $\sim$ Thessaloniki $\sim$ Greece

Summary (4 lines): Video presentation of laparoscopic excision of uterine cysts in a patient with Bourneville's disease.

Introduction: Bourneville's disease, also known as tuberous sclerosis or tuberous sclerosis (TSC) complex is a rare multisystem genetic disease, affecting multiple organs such as the central nervous system, kidneys, eyes, skin and heart.

Material and Methods: A 33 year old Caucasian woman presented with primary infertility since 1.5 years. In her medical history the patient was diagnosed with Bourneville's disease in the brain and both kidneys and no gynecological history was reported other than dysmenorrhea. During the transvaginal ultrasound scanning a $4 \mathrm{~cm}$ in diameter left ovarian hypoechogenic cyst was detected, similar to endometrioma. The patient was decided to have a laparoscopic excision of the cyst. During the laparoscopy uterus was checked with multiple cysts of haemorhagic view, a left ovarian endometrioma and multiple endometriotic foci. The endometrioma was further removed, the scattered endometriosis was vaporized with CO-2 LASER and so the uterine cysts, after biopsies were taken.
Results: Histopathological report regarding the uterine cysts came as lymphomyoangiomatomas, a common finding of Bourneville's disease in the lungs.

Discussion: According to the existing literature is the first time that uterine manifestations of Bourneville's disease is reported based on histopathological report.

7

\section{APPLICATION OF THE KNOWLEDGE OF PELVIC ANATOMY FOR DIFFICULT SITUATIONS IN GYNAEC ENDOSCOPY- TIPS AND TRICKS \\ Complications}

Video

S. Puntambekar*, S. Puntambekar, R. Desai, A. Galagali

${ }^{[1]}$ Galaxy CARE Laparoscopic Institute $\sim$ Pune $\sim$ India

Summary (4 lines): The video shows how to apply the knowledge of pelvic anatomy to overcome any difficult situation in gynaec endoscopic surgery.

Introduction: Pelvic anatomy forms the basis of advanced laparoscopic gynaecological surgeries. Difficult situations in Gynaec laparoscopy can be tackled successfully if one learns the tips and tricks of handling vascular structures and the ureters.

Material and Methods: This video depicts various situations such as stage IV endometriosis, multiple laparotomies, previous caesarean section, large uterus etc where this approach helps to overcome the difficulties encountered.

Results: The video teaches one how to apply the knowledge of pelvic anatomy to tackle the vascular structures and ureters easily

Discussion: A good knowledge of pelvic anatomy is necessary for any gynaec endoscopic surgeon. Even with a theoretical knowledge, one needs to know how to apply it to a difficult case. This video demonstrates easy to do tips and simple tricks to tackle the ureters and the vascular structures during a difficult case.

10

\section{LAPAROSCOPIC TRANSVESICAL APPROACH FOR VESICO-VAGINAL FISTULA REPAIR \\ Complications}

Video

S. Puntambekar*, S. Puntambekar, A. Galagali, R. Desai

${ }^{[1]}$ Galaxy CARE Laparoscopic Institute $\sim$ Pune $\sim$ India

Summary (4 lines): Laparoscopic vesico vaginal fistula repair has comparable results to the vaginal approach. The benefit of good magnification and ease of suturing in deep pelvis are the main advantages of the laparoscopic approach.

Introduction: Laparoscopic repair of vesico vaginal fistulas is rarely undertaken due to its complexity and skill dependence. We present a laproscopic repair to establish the role of minimal access surgery in such cases.

Material and Methods: Patient with history of urinary leakage through vagina following vaginal hysterectomy. Five pelvic ports used. Surgical procedure carried out with the same principles as that of open surgery namely, separation of the vaginal wall from the bladder wall, repair of the fistula and interposition of omentum

Results: Early recovery of the patient in terms of continence and less chances of recurrence. Comparable results as that of vaginal approach.

Discussion: The use of minimally invasive surgery for the vesico vaginal fistula repair, helps ease the suturing deep in the pelvis and 
the magnification facilitates good identification of tissues planes and thus better mobilization of the vaginal and bladder walls and decreases the postoperative morbidity.

64

\section{LAPAROSCOPIC URETERAL REIMPLANTATION WITH PSOAS HITCH FOR IATROGENIC LOWER URETERAL INJURIES IN LAPAROSCOPIC HYSTERECTOMY \\ Complications}

Video

E. Martínez Lamela*, J. Molero Vílchez, E. Sáez Cerezal, J. Cambronero

Santos, S. Monasterio Vallejo, J.J. Hernández Aguado

${ }^{[1]}$ Hospital Universitario Infanta Leonor $\sim$ Madrid $\sim$ Spain

Summary (4 lines): To describe the surgical technique of laparoscopic ureteral reimplantation with psoas hitch.

Introduction: A 67 year-old female with an G1 endometrial cancer IA stage. During the adenocarcinoma staging surgery, ureteral affectation is noticed, why it is dissected in all the path, checking complete section of the ureter at 3-4 cm of its insertion in the bladder.

Material and Methods: Extravesical ureteroneocystostomy is performed as follows: Liberation of Visceral peritoneum; Liberation of ureter; Liberation of the bladder by sectioning the peritoneum up to the line of umbilical arteries and entering in the retzius space; Brepleinding to psoas muscle at right primitive iliac artery; level Check of tension-free ureter up to the bladder and sectioning up to the front part; Spatulation of the ureter and ureterovesical reimplantation with simple intracorporeal stitches; Bladder closure and integrity check through vesical repletion with methylene blue; double $\mathrm{J}$ catheter was inserted.

Results: The urinary catheter was removed after 2 weeks and the double $\mathrm{J}$ stent after 4 weeks. Follow-up urography showed good clearance of the kidney, ureter with normal radiographic features and bladder with good repletion and the patient remains free of symptoms

Discussion: Laparoscopic ureteral reimplantation with psoas hitch is a feasible procedure for the management of ureterovaginal lesion and minimizing the morbility.

\section{2}

\section{SUCCESSFUL LAPAROSCOPIC REANASTOMISIS OF UTERINE AVULSION FOLLOWING A COLD-KNIFE CONIZATION \\ Complications}

\section{Video}

S. Ribeiro*, A. Coelho, T. Diniz Da Costa, C. Alho, F. Osorio, C. Calhaz-jorge

${ }^{[1]}$ Centro Hospitalar Lisboa Norte $\sim$ Lisboa $\sim$ Portugal

Summary (4 lines): We report on a successful case of laparoscopic reconstruction following uterine avulsion, a previously unreported complication of an excisional reconization.

Introduction: Uterine avulsion following conization is an unreported surgical complication with no recommended management.

Material and Methods: We report on a successful case of laparoscopic reconstruction following an iatrogenic uterine avulsion.

Results: A 30-year-old nullipara was referred to our department with secondary amenorrhea and cyclic pelvic pain following two excisional conizations performed 10 and 9 months before in another institution. We were unable to identify the presence of a uterine cervix during both gynecologic and imagiologic examinations. The patient underwent a diagnostic laparoscopy which confirmed that the cervix had been completely ablated and that the uterine and vaginal cavities were no longer in contact. We reconnected the uterine and vaginal cavities with a foley catheter and vaginal-uterine end-to-end anastomosis with interrupted sutures. This procedure was complemented with a prophylactic cérclage. Six months after the laparoscopy the patient was asymptomatic. Discussion: To our knowledge, this is the first time uterine avulsion is reported as a complication of cervical conization. Although our surgical correction apparently repaired the anatomy and function, further follow-up is necessary to confirm to what extent reproductive function can be restored.

\section{3}

\author{
A STRUCTURED APPROACH TO LAPAROSCOPIC \\ DISSECTION OF THE FROZEN PELVIS \\ Endometriosis: Surgery \\ Video \\ T. Smith Walker*, P. Wipplinger, D.L. Byrne \\ ${ }^{[1]}$ Royal Cornwall Hospital $\sim$ Truro $\sim$ United Kingdom
}

Summary (4 lines): We present a structured approach to laparoscopic dissection of the frozen pelvis in a case of severe rectovaginal endometriosis.

Introduction: The frozen pelvis presents the greatest surgical challenge. Rectovaginal endometriosis or resolved pelvic sepsis are common causes.

Material and Methods: The approach begins on the patients left side, reflecting the sigmoid adherence to release the sigmoid completely from the abdomino-pelvic wall. This enables access to the left ureter and ureterolysis. Once the left ovary is released, it is suspended to the abdominal wall. Then a right ureterolysis and suture suspension of the right ovary is undertaken.

Peritoneum containing endometriosis is excised in sequence from each pelvic sidewall. The bowel is instrumented to allow manipulation, so bilateral pararectal incisions can be made and the rectum dissected free to the level of the rectovaginal septum. Careful dissection encroaching medially is performed until the central rectal adherence is reached. In conjunction with our colorectal surgeon, the bowel is dissected from the posterior uterus/vagina using sharp dissection.

Results: This results in a 'free pelvis' and the endometriosis within the peritoneum and rectovaginal septum is completely excised en bloc.

Discussion: Bowel preparation is required when the bowel is affected and down regulation with $\mathrm{GnRH}$ analogues where endometriosis is involved.

\section{2}

\section{CONSERVATIVE LAPAROSCOPIC APPROACH FOR BOWEL ENDOMETRIOSIS.}

Endometriosis: Surgery

Video

F. Emilie*, A. Jaime, P. Marco, W. Arnaud

${ }^{[1]}$ Universitary Hospital of Strasbourg IRCAD/EITS Strasbourg France

Summary (4 lines): This video presents a strategy to avoid in almost all cases a segmental bowel resection following a step by step procedure.

Introduction: We present the strategy used for the conservative treatment of bowel endometriosis and to avoid bowel resection in almost all cases. In all cases of endometriosis, we first have to follow a general strategy. Pelvic exploration

Exposure

Suspension of the ovaries

Ureters control 
Opening of the pararectal fossas

Material and Methods: Specific strategy

The rectovaginal space is opened

Shaving

Mucosal Skinning

Discoid resection

Results: Safety test

In all cases, safety tests are mandatory. The rectum is clamped and a gas test as well as a blue methylene test are performed.

Discussion: This strategy can be carried out step-by-step, and in most cases following this protocol allows to excise the disease and obtain good functional benefits, and to prevent functional complications linked to bowel resection.

382

\section{CONSERVATIVE MANAGEMENT OF BOWEL PERFORATION POST ENDOMETRIOSIS SURGERY}

Endometriosis: Surgery

Video

S. Andrikopoulos*, E. Dimitriou, G. Pistofidis

${ }^{[1]}$ Leukos Stauros Athinwn $\sim$ Athens $\sim$ Greece

Summary (4 lines): This is a video presentation of an interesting case of a bowel perforation which happened 25 days after extensive bowel surgery for deep infiltrating endometriosis and was managed conservatively with very good results

Introduction: The perforation was noted 25 days after the initial surgery which involved extensive resection and shaving of a big nodule off the anterior rectal wall. The patient presented with painless bright red rectal bleeding on two different bowel motions. TVS and pelvic MRI scans showed a collection between the rectum and the cervix containing small amount of free air. flexible sigmoidoscopy confirmed the communication of the collection with the rectum.

Material and Methods: Since the patient had no symptoms and signs of peritonitis or infection, a decision to proceed with conservative management with i.v antibiotics and low residue diet was made. The patient was discharged home 8 days later, on oral antibiotics, GnRH analogs and oral contraceptives.

Results: 2 months later MRI scan and flexible sigmoidoscopy showed complete healing of the dehiscence of the rectum and complete resolution of the cavibetas the collection. The patient remains symptom free with no PR or PV discharge.

Discussion: This is a very interesting case of a quite serious complication resulting from laparoscopic resection of deep infiltrating endometriosis which was managed conservatively with excellent outcome.

\section{3}

FEASIBILITY AND SURGICAL OUTCOME OF ROBOTICASSISTED LAPAROSCOPIC COLORECTAL RESECTION FOR DEEP INFILTRATING ENDOMETRIOSIS

Endometriosis: Surgery

Video

L. Freschi*, L. Morelli, N. Pluchino, G. Simi, M. Ruggiero, F. Papini, R. Tana, V. Cela

${ }^{[1]}$ Santa Chiara Hospital $\sim$ Pisa $\sim$ Italy

Summary (4 lines): An infertile patient underwent robotic rectosigmoidectomy for an infiltrating rectosigmoid endometriotic nodule.

Introduction: Deep infiltrating endometriosis is a gynecologic disorder that cause decreased quality of life, impaired sexual relation and infertility. Robotic surgery is a revolutionary approach with advantages such as 3-dimensional vision, decrease of surgeon's fatigue and tremors, improved dexterity and greater surgical precision.

Material and Methods: A 29-years-old infertile patient referring cyclic rectorragia, dyschezia and abdominal distention underwent an ultrasound examination revealing a rectal nodule of $2 \mathrm{~cm}$ of diameter. Pelvis MRI and radiologic colonrectal examination showed an infiltrating recto-sigmoid lesion. A rectosigmoidectomy was performed with the Da Vinci Surgical System by a multidisciplinary team. The procedure consisted of lateral to medial colon dissection releasing adherences and rectum section. The trocar sovrapubic incision was enlarged for the piece resection and intraperitoneal colorectal anastomosis was constructed and the pneumotic continence tested.

Results: Operative time lasted 4 hours and postoperative evolution was satisfactory. The patient left the hospital at the 5th postoperative day just for precaution and after 3 months is asymptomatic and attempting assisted reproduction technique.

Discussion: This approach is feasible and safe without conversion to laparotomy.

459

\section{LAPAROSCOPIC RESECTION OF RETROPERITONEAL ENDOMETRIOSIS - CASE REPORT \\ Endometriosis: Surgery}

Video

A. Almeida*, J. C Pereira, P. Ramoa

${ }^{[1]}$ Hospitais Privados de Portugal, Boavista $\sim$ Porto $\sim$ Portugal

Summary (4 lines): We reported a rare case of retroperitoneal endometriosis with two different localizations and the surgical treatment

Introduction: Extrapelvic endometriosis occurs in every organ system. Although a relatively common condition, endometriosis remains difficult to recognize, diagnose, and treat because of its extremely variable presentation, which is particularly true for extragenital endometriosis.

Material and Methods: Case report

Results: A 38 y women with a history of right acute low back pain. She was referred to our surgical department for treatment of subhepatic cystic lesion probably originated from liver.

Magnetic Resonance (RM) revealed a $4 \mathrm{~cm}$ cystic mass with subhepatic localization; it was directed percutaneous needle biopsy showed endometriosis.

At laparoscopy was found 2 independent lesions of endometriosis, one in the abdominal wall and other on the adrenal gland, both with retroperitoneal localization.

The patient was managed with surgical resection of endometriosis lesion in abdominal wall and resection of endometrioid lesion with upper region of adrenal gland.

Histopathology revealed two endometrioid lesions.

Discussion: Retroperitoneal endometriosis may coexist in multiple sites. A percutaneous biopsy guided by Computer Tomography allowed a multidisciplinary approach, followed by appropriate surgical eradication of visible disease, can successfully treat endometriosis even in such extreme cases.

98

LAPAROSCOPIC SIGMOIDECTOMY FOR ENDOMETRIOSIS WITH TRANSANAL SPECIMEN EXTRACTION

Endometriosis: Surgery

Video

P. Messori*, E. Faller, J. Albornoz, J. Leroy, A. Wattiez

${ }^{[1]}$ IRCAD/EITS $\sim$ Strasbourg $\sim$ France 
Summary (4 lines): In this video is presented the case of an elective sigmoidectomy for endometriosis

Introduction: The aim is to describe a less invasive and more conservative approach in case of colorectal resection for benign lesions like endometriosis

Material and Methods: A less invasive approach consists in the natural orifice specimen extraction (transanal via). A conservative surgical strategy in colorectal resection for benign lesions like endometriosis is possible dividing the mesentery close to the digestive tract in order to preserve the vascular-lymphatic vessels and the surrounding sympathetic and parasympathetic nerves

Results: This surgical technique, especially preservation of the superior rectal vessels, permits to maintain up to $80 \%$ of the vascularization of the rectum

Discussion: Preserving the superior rectal vessel is very important in order to maintain the best vascularization of the rectum, essential for intestinal anastomosis health. The transanal specimen extraction maximizes the benefits of laparoscopy by sparing the abdominal wall from an incision, with its associated complications

\section{3}

\section{MUCOSAL SKINNING FOR BLADDER ENDOMETRIOSIS Endometriosis: Surgery}

Video

F. Emilie*, A. Jaime, P. Marco, W. Arnaud

${ }^{[1]}$ Universitary Hospital of Strasbourg $\sim$ IRCAD/EITS Strasbourg France

Summary (4 lines): We present the case of a 33-year-old nulliparous woman who presents with a nodule of adenomyosis at the anterior uterine wall with an invasion of the vesicouterine pouch and of the bladder wall

Introduction: The procedure starts with the opening of the vesicouterine peritoneum lateral to the nodule. Dissection is performed with the help of monopolar scissors until the nodule is reached.

The bladder is then dissected carefully above the nodule trying to stay proximal to the uterus. The $\mathrm{RoBi}^{\circledR}$ forceps in the left hand is very useful to dissect and coagulate in turn while scissors are manipulated with the right hand.

Material and Methods: Excision of the adenomyotic nodule of the isthmus is performed by means of a monopolar hook The nodule of the bladder is grasped using the Manhes forceps and mucosal skinning is progressively performed using a monopolar hook.

Traction of the nodule and light monopolar coagulation allow to find the correct plane.

The nodule is progressively dissected without opening of the mucosa. The bladder is filled in with methylene blue to facilitate the procedure. Double J stents have not been placed in this case.

Results: The serosa of the bladder is sutured with one layer of separate stitches (monocryl 3/0). The angles are sutured first. The serosa is then closed taking care to invaginate the mucosa.

Discussion: Finally, the suture is controlled and the Foley catheter is left in place during 5 days.

\section{9}

RECTOVAGINAL ENDOMETRIOTIC NODULE: SURGICAL RESECTION AND STRATEGY

Endometriosis: Surgery

Video

P. Trompoukis*, A. Wattiez

${ }^{[1]}$ IASO Group of hospitals $\sim$ Athens $\sim$ Greece
Summary (4 lines): We present a video of a 32 years old patient with endometriotic rectovaginal nodule. We analyze in this video the surgical dissection and our strategy in dealing with these difficult cases.

Introduction: A young patient presenting with chronic pelvic pain, dyspareunia, severe dysmenorrhea and an MRI scan depicting rectovaginal endometriosis underwent surgical resection of disease. The nodule was deep extending from the rectal wall to vaginal mucosa. Material and Methods: A 32 years old patient presenting with chronic pelvic pain, severe dysmenorrhea and an MRI scan depicting rectovaginal endometriosis underwent laparoscopic treatment of disease.

Results: We resected the nodule dissecting deep into the retroperitoneal pelvic tissue and rectovaginal septum. The nodule was deep, penetrating the vaginal wall; we demonstrate resection from vaginal wall and shaving of endometriotic tissue from rectal wall. The surgical result and post op course was completely satisfactory.

Discussion: Laparoscopic treatment of rectovaginal endometriosis is always difficult and demanding and needs surgical strategy and expertise. We always follow specific steps to dissect and remove the endometriotic tissue. We believe this is a good and didactic video especially for colleagues facing similar cases. There is also an international debate about bowel shaving vs resection that we analyze.

RESECTION OF A RUDIMENTARY UTERINE HORN IN A PATIENT WITH SEVERE ENDOMETRIOSIS IN THE PELVIS AND THE DIAPHRAGM.

Endometriosis: Surgery

Video

S. Khazali*, S. Banerjee

${ }^{[1]}$ Centre for Endometriosis and Pelvic pain (St. Peter's Hospital) Chertsey, Surrey United Kingdom

Summary (4 lines): Our video demonstrates an interesting case of a young woman with a non-communicating uterine horn, covered with a large endometrioma and deep infiltrative endometriosis scattered within the pelvis, as well as endometriotic deposits over the diaphragm.

Introduction: A patient in her early $20 \mathrm{~s}$ with a cyclical pain (VAS score 10/10) and primary infertility. Imaging revealed, rudimentary right uterine horn, covered by large endometrioma and adhered to pelvic sidewall. Right kidney was absent.

Material and Methods: N/A

Results: Careful dissection and detatchment of the surrounding structures is demonstrated in the video, followed by resection of the horn and also resection of both uterosacral ligaments following ureterolysis of the patient's only ureter on the left.

Discussion: Knowledge of anatomical relationships in patients with uterine anomalies is paramount to perform a safe corrective surgery.

352

\section{ROBOT-ASSISTED LAPAROSCOPIC TREATMENT OF DIAPHRAGMATIC ENDOMETRIOSIS}

Endometriosis: Surgery

Video

G. Simi*, L. Freschi, N. Pluchino, L. Morelli, F. Papini, O. Di Berardino, R. Tana, V. Cela

${ }^{[1]}$ Santa Chiara Hospital $\sim$ Pisa $\sim$ Italy

Summary (4 lines): A 35 year old woman with ciclic abdominal pain irradiating up to the right shoulder underwent robotic excision of an endometriotic nodule on the right hemidiaphragm.

Introduction: Thoracic endometriosis is a rare disorder affecting women during their reproductive years. The etiopathogenesis is not 
well understood. Symptoms always appear connected to the periods of the woman affected.

Material and Methods: A 35 year old woman came to our clinic with an history of ciclic abdominal pain irradiating up to the right shoulder. Pelvic ultrasound examination and thorax X-ray were normal. Thoracic and abdominal pelvis MRI revealed a nodule on the right hemidiaphragm. Robotic surgery was used to perform surgical removal of the nodule due to its advantages such as 3-dimensional vision, decreased surgeon's fatigue and tremors, improved dexterity and greater surgical precision. The patient was put on the left lateral bedsore. Three robotic trocars of $7 \mathrm{~mm}$ diameter were introduced at the diaphragmatic level. The right hemidiaphragm presented adherence with the liver due to endometriosis disease. We removed the adhesion and performed the excision of the diaphragmatic endometriosic nodule that was extracted through endobag.

Results: Operative time lasted 2 hours. The postoperative evolution was satisfactory and the patient left the hospital at the 3 th postoperative. No complications occurred.

Discussion: We demonstrate that this approach is feasible and safe, without conversion to laparotomy.

177

\section{SEVERE ENDOMETRIOSIS IN AN ADOLESCENT PATIENT}

Endometriosis: Surgery

Video

P. Trompoukis*, I. Frouradakis

${ }^{[1]}$ IASO Group of hospitals $\sim$ Athens $\sim$ Greece

Summary (4 lines): This is a video of a 19 years old patient suffering from severe endometriosis that was operated for removal of big ( 8 and $10 \mathrm{~cm}$ ) "kissing ovaries" and extensive adhesiolysis.

Introduction: A case of a young patient that was suffering from dysmenorrhea without dyspareunia or chronic pelvic pain and so she was treated mistakenly for long periods of time symptomatically only with analgesics. Finally an ultrasound scan in our department revealed the extent of the disease and the need for subsequent surgical and pharmacological treatment.

Material and Methods: 19 years old patient, laparoscopic removal of bilateral endometriotic cysts together with extensive adhesiolysis.

Results: A strategic procedure for removal of all visible endometriotic tissue took place and of all possible adhesions. The post op course was uneventfull and she is now under pharmacologic treatment to avoid relapses.

Discussion: This is an important video demonstrating not only the surgical technique but also stimulating the discussion in order to keep a high index of suspicion in cases of young patients suffering from endometriosis. The pelvic pain in this age group is important not to be underestimated since these patients need surgical and special pharmacological treatment to avoid permanent sterility and severe chronic pelvic pain.

305

STRATEGIES TO THE RESECTION OF DEEP INFILTRATING ENDOMETRIOSIS INVOLVING THE URETERS, URINARY BLADDER AND RECTOVAGINAL SEPTUM

Endometriosis: Surgery

Video

M. Dassel*, R. Pasic

${ }^{[1]}$ University of Louisville $\sim$ Louisville, KY USA $\sim$ United States

Summary (4 lines): This video demonstrates strategies for the ressection of deep infiltrating endometriosis including review of relevant anatomy and ressective/reparative surgical techniques.
Introduction: Deep Infiltrating Endometriosis (DIE) is an invasive entity and can be very difficult to manage surgically despite the fact that studies have shown surgical intervention for DIE is more effective than conservative management.

Material and Methods: This video explores the surgical management of DIE with specific attention to the rectovaginal septum, ureters and urinary bladder. Relevant anatomy is discussed and tenets to resection of DIE are presented with the goal of restoring normal anatomy to the diseased pelvis.

Results: A review of the relevant anatomy of the urinary bladder, ureters and rectovaginal septum are discussed. Surgical techniques including entry into the space of retzius, ureterolysis, and dissection of the rectovaginal septum are demonstrated.

Discussion: Deep infiltrating endometriosis can invade important functional structures of the pelvis such as the urinary bladder, ureters and rectum. Since evidence points to resection of DIE as the optimal treatment strategy, it is important that the advanced laparoscopic surgeon is able to use the tools provided by laparoscopy to optimally resect this difficult pathology.

\section{9}

\author{
SURGICAL MANAGEMENT OF SMALL BOWEL \\ ENDOMETRIOSIS. \\ Endometriosis: Surgery \\ Video \\ E. Suárez-salvador*, O. Puig, L. Mañalich, J. Xercavins \\ ${ }^{[1]}$ Hospital Universitari Vall d'Hebron $\sim$ Barcelona $\sim$ Spain
}

Summary (4 lines): We report 2 surgical videos of patients with small bowel endometriosis. We described surgical findings and technique. Introduction: The incidence of small intestine endometriosis is $1 \%$. Symptoms are nonspecific; pain, bloating, sub-oclusive symptoms. Imaging tests have low sensitivity. In patients with severe symptoms unresponsive to medical treatment the surgical removal is the best alternative.

Material and Methods: 1

Results: The vídeo shows ileum terminal involvement for endometriosis. First step is mobilization of the small bowel mesentery as well as of the peritoneal attachments of the right colon. Tredenlenburg positioning, tilt to the left and cephalad medial traction of the cecum may facilitate mobilization of the small bowel away from the pelvis and the operative field. The ileal mesentery is mobilized from the retroperitoneum in a medial to lateral fashion using both blunt and sharp dissection. The endometriotic lesion is isolated with healthy margin cranial and caudal. The bowel is transected using linear endo stapler, with subsequent construction of a side-to-side functional end-to-end stapled anastomosis. The bowel anastomosis is constructed intracorporally. In these cases the vagina was opened, as a recto vaginal nodule was excised in the same act, so the specimen was extracted by vaginal route

Discussion: The laparoscopic approach is feasible and safe in cases of small bowel endometriosis, mainly when the surgery is made out of emergent presentations

\section{5}

SURGICAL STRATEGY FOR URETERAL ENDOMETRIOSIS Endometriosis: Surgery

Video

J. Albornoz*, E. Faller, M. Puga, A. Wattiez, T. Piechaud

${ }^{[1]}$ IRCAD $\sim$ Strasbourg $\sim$ France

Summary (4 lines): Report the surgical strategy to face difficult cases of deep infiltration endometriosis of the ureter. 
Introduction: Ureteral endometriosis is a rare condition and specific symptoms are not frequent, but as it can lead to hydronephrosis it can cause sever damage to the kidney function.

Material and Methods: Three patients complaining from dysmenorrhea, dyspareunia, chronic pelvic pain (CPP) and occasional urinary symptoms like hematuria and lumbar pain who underwent laparoscopic endometriosis excision.

Results: Pre-operative assessment includes abdominal ultrasound and magnetic resonance. Once the hydronephrosis has been diagnosed, a JJ Stent should be inserted into the ureter by cystoscopy before the endometriosis surgery. General surgical strategy includes proper exposure and careful ureterolysis. Specific surgical strategy will depend on the type of ureteral infiltration and includes JJ Stent insertion, partial ureteral excision and end-to-end anastomosis and in some cases ureteral reimplantation and vesicopsoas hitch.

Discussion: The laparoscopic approach for ureteral endometriosis is feasible, safe and provides long-term relief of symptoms.

212

\section{SURGICAL STRATEGY IN DEEP ENDOMETRIOSIS OF THE PELVIC SIDE WALL. TIPS AND TRICKS OF THE "SOCCER STRATEGY"}

Endometriosis: Surgery

Video

P.N. Barri-soldevila*, A. Vazquez, A. Ubeda, M.A. Lequerica

${ }^{[1]}$ Instituto Universitario Dexeus $\sim$ Barcelona $\sim$ Spain

Summary (4 lines): To share the experience of our deep endometriosis unit in the management of deep lateral involvement.

Introduction: Assuming that the Redwine technique and nerve sparing strategies are applied, we present an intuitive explanation of the approach to this difficult localisation.

Material and Methods: Once both ureters are dissected untill crossing uterine vessels and the ovaries suspended to the abdominal wall, it is the time to face the endometriotic nodule. In the case of a posterior central nodule, identification of hipogastric nerves and bilateral pararectal dissection are needed to reach rectovaginal septum. Such as a forward player reaching the goal through the sides.

Results: When the nodule is lateral, a 90 degree "mental"rotation is needed to get back to the same strategy. Presacral space dissection is helpful, not only in cases of bowel resection.

Discussion: In our experience, this trick is recomended in the standarisation of the technique. It may allow the surgeon to be at ease, save time, better indentify pelvic avascular planes, anatomical landmarks and preserve as many neural branches as possible.

\section{1}

\section{A NEW TECHNIQUE FOR RADICAL LAPAROSCOPIC HYSTERECTOMY FOR ENDOMETRIOSIS \\ Hysterectomy}

Video

J. English*

${ }^{[1]}$ Brighton and Sussex University Hospital Brighton United Kingdom

Summary (4 lines): Radical hysterectomy for endometriosis is a complex operation which frequently involves is very complex. This video demonstrates a reproducible technique for radical en bloc resection of the uterus and endometriosis including rectal segment as required.

Introduction: En bloc laparoscopic hysterectomy and segmental anterior rectal resection in the presence of cul-de-sac obliteration secondary to endometriosis presents a major surgical challenge: the ureters are frequently involved in the disease process; entry to the rectovaginal septum to mobilize the inferior rectum is difficult as the normal planes are destroyed.

Material and Methods: This technique involves dissection of the lateral pelvic side wall with division of the uterine vessels lateral to the ureters and opening of the bladder tunnel with ureterolysis; the anterior vagina is opened to determine the inferior extent of the rectal nodule and the rectovaginal septum opened and dissected leaving the uterus attached to the rectum for en bloc resection

Results: The technique is readily reproducible and may be used routinely to resect the uterus in the presence of severe endometriosis Discussion: The technique facilitates ureterolysis, entry into the rectovaginal septum and, if required, uterine excision en-bloc with the rectal segment.

\section{9}

\section{HYSTERECTOMY IN GIANT UTERUS}

Hysterectomy

Video

J.C.R.M. Dr*

${ }^{[1]}$ Clinica del Country. $\sim$ Bogota $\sim$ Colombia

Summary (4 lines): We report a 48 year old woman, who consults for heavy menstrual bleeding, cramping pain in hypogastrium, and feeling progressively growing abdominal mass, history of 2 vaginal deliveries, without other relevant data.

We performed ultrasonography and pelvic abdominal CT scan and found a severe fibroids that filled the mesogastrium and hypogastrium, was taken to laparoscopic hysterectomy, with modifications to the surgical technique, such as supraumbilical incision 7 inches of 10 MMTS, 2 incisions of 10 MMTS in left upper quadrant and right flank, in the laparoscopy we found a giant uterus, conventional dissection began, but given the lack of access to the uterine arteries, vasopressin boluses were applied diluted with morcellation in situ to identify the vascular pedicles, which are energetically electrocoagularon. Surgical procedure was ended after 140 minutes, with approximately $1000 \mathrm{cc}$ bleeding and uncomplicated, the patient had recovered adequate outlet for their home 48 hours postoperatively. The pathology report showed a weight of 1600 grams.

Introduction: Surgical procedure and technics for histerectomy in megauterus

Material and Methods: Case report Hysterectomy in giant uterus Results: Tips and Tricks for development hysterectomy in uterus bigger than 1000 grs

Discussion: We present this case to consider laparoscopic hysterectomy with giant uterus, is feasible, safe and making modifications to the conventional technique, its realization is possible, avoiding large surgical incisions in the abdomen.

\section{6}

\section{LAPAROSCOPIC ASSISTED MINILAPAROTOMY HYSTERECTOMY FOR A LARGE POSTERIOR LOWER UTERINE FIBROID CAUSING BILATERAL URETERIC OBSTRUCTION WITH ABNORMAL RENAL FUNCTION Hysterectomy}

\section{Video}

P. De Rosnay*, D. Chou, G. Cario, D. Rosen, L. Reyftmann, O. Baghlaf, S. Choi, G. Condous

${ }^{[1]}$ Sydney Women's Endosurgery Centre $\sim$ Sydney $\sim$ Australia

Summary (4 lines): This video demonstrates surgical management as well as technical challenges.

Introduction: A 30 year-old woman presented with repeated episodes of acute urinary retention. Ultrasound scan showed bilateral 
hydronephrosis and a posterior cervical fibroid $-19 \times 15 \times 11 \mathrm{~cm}$. Creatinine level was $136 \mathrm{umol} / \mathrm{L}$. Nephrostogram confirmed bilateral lower ureteric obstruction. Double-J ureteric stents and nephrostomy drains were inserted bilaterally.

Material and Methods: The cervix was displaced anteriorly and cephalad limiting uterine manipulation. The cervical fibroid occupied the majority of the pelvic cavity with minimal mobility. It extended retroperitoneally over the pelvic sidewalls and into the rectovaginal space. Superiorly it extended above the pelvic brim and over the sacral promontory. Bilateral uterine artery ligation was performed using LigaClip ${ }^{\circledR}$. The fibroid was freed from the retroperitoneal attachment, followed by debulking with amputation across the isthmus using a laparoscopic knife. The specimen was removed through a $6 \mathrm{~cm}$ transverse suprapubic incision and the remaining part of the cervical fibroid and cervix was excised.

Results: Uneventful postoperative recovery with normalisation of creatinine level.

Discussion: Bilateral obstructive uropathy secondary to large uterine leiomyomas is uncommon. In the presence of deranged renal function, hysterectomy may be required.

123

\section{LAPAROSCOPIC HYSTERECTOMY IN CERVICAL FIBROIDS Hysterectomy}

Video

O. Istre*, H. Springborg

${ }^{[1]}$ Aleris-Hamlet Hospital, Copenhagen and $\sim$ Copenhagen $\sim$ Denmark

Summary (4 lines): Laparoscopic treatment compared to open surgery because of better visualization of the organs during surgery and shorter recovery.

Introduction: Large cervical fibroids are rare and account for less than $2 \%$ of all fibroids. Symptoms are pain and bleeding. Treatment is difficult vaginally, damage to the bladder and ureter can occur and traditionally, abdominal hysterectomy has been the treatment of choice.

Material and Methods: The video shows the diagnostic procedure and the operation. The patient: 55 years old postmenopausal woman with a small uterus displaced by an $8 \mathrm{~cm}$ round myoma on the right side of the cervix. Symptoms were dyspareunia and abdominal pain. Uterine weight was $425 \mathrm{~g}$ (fibroid approximately $325 \mathrm{~g}$ ), peroperative bleeding $200 \mathrm{ml}$ and operating time 120 minutes. In this case we use the new thunderbeat system, a combination of ultrasound and bipolar. Results: The patient was successfully treated laparoscopically and were discharged after 14 hours and returned to work after two weeks. Discussion: Laparoscopic treatment is advantageous, compared to open surgery because of better visualization of the organs during surgery and shorter recovery. We recommend using a $5 \mathrm{~mm}, 30$ degree HD optic which makes it possible to perform port jumping.

\section{0}

LAPAROSCOPIC HYSTERECTOMY INTRAFASCIAL (LHI). IS IT TIME TO MODIFY THE CLASSIFICATION?

Hysterectomy

Video

A. Kent*

${ }^{[1]}$ MATTU $\sim$ Guildford $\sim$ United Kingdom

Summary (4 lines): LHi is now an established technique. The video will demonstrate the distinction between current techniques and highlight the benefits of adopting such an approach.

Introduction: We have previously described a simplified technique for intrafascial laparoscopic hysterectomy (LHi) using Harmonic ACE.
There have been descriptions of similar techniques using other energies but there is some doubt as to whether they are truly utilizing an intrafascial approach. I propose that the generally accepted simple LH classification of Garry and Reich be modified to include LHi.

Material and Methods: Harmonic ACE. Valtchev Uterine Manipulator (VUM)

Results: Illustrated in video.

Advantages of an intrafascial technique.

1) Increased distance from ureters.

2) Rapid access to vaginal vault.

3) Avoids need for forniceal delineators.

4) Increased vaginal length.

5) Leaves uterosacral arch intact - no effect on prolapse, afferent nerves from bladder left intact.

6) Allows colpopexy, sacrocolpopexy.

7) Possible reduction in vault dehiscence.

Discussion: I propose a simple modification of the benign classification.

Type I diagnostic laparoscopy.

Type II lap adhesiolysis, minor procedures.

Type III classic LAVH.

Type IV LH uterine pedicles.

Type IVi LH intra-fascial (LHi)

Type V TLH suturing vault laparoscopically.

Type VI Subtotal LH

178

LAPAROSCOPIC SUBTOTAL HYSTERECTOMY AND SACROCOLPOPEXY TOGETHER WITH A SUSPICIOUS ADNEXAL MASS

Hysterectomy

Video

P. Trompoukis*, I. Frouradakis, A. Pachydakis

${ }^{[1]}$ IASO Group of hospitals $\sim$ Athens $\sim$ Greece

Summary (4 lines): This is a case of a patient presenting with cystocele and Grade 1 uterine prolapse together with an adnexal mass. We performed a subtotal hysterectomy and a frozen biopsy to reassure that the mass is benign and thereafter we proceed to sacrocolpopexy with mesh. Introduction: we present a case of a 63 years old lady who presented with a cystocele without incontinence but recurrent cystitis together with Grade 1 uterine prolapse and an adnexal mass.

Material and Methods: 63 years old patient, laparoscopic removal of adnexal mass with subtotal hysterectomy and sacrocolpopexy.

Results: This is a good technique of subtotal hysterectomy and sacrocolpopexy that we analyze together with the possible complications. The postoperative period was uneventful and the overall patient satisfaction excellent.

Discussion: We performed a subtotal hysterectomy and a frozen biopsy and after we proceed to mesh fixation to the cervical vault and to the sacral promontory with subsequent burial of the mess to lateral pelvic peritoneum. This is a good example of optimal preoperative management together with an analytic operative strategy for this demanding procedure.

203

LAPAROSCOPIC SUBTOTAL HYSTERECTOMY WITH MONOPOLAR LOOP: OUR STANDARD TECHNIQUE Hysterectomy

Video

C. Barbed*, A. Vazquez, D. Amselem, P. Barri, M. Cusido, A. Ubeda ${ }^{[1]}$ INSTITUTO UNIVERSITARIO DEXEUS $\sim$ BARCELONA $\sim$ Spain 
Summary (4 lines): Standardization of subtotal laparoscopic hysterectomy can simplify this surgery

Introduction: Despite the unquestionable passion at the beginning, laparoscopic surgery did not developed as hoped. The major arguments against laparoscopic surgery are its difficulty and its length, or in other words, its technicality and low productivity. Probably due to these reasons only around $12 \%$ of hysterectomies are performed by laparoscopy (Wu JM et al., 2007). Subtotal hysterectomy could be a way to increase this productivity since a recent Cochrane review (Lethaby A et al., 2012) showed that length of operation was significantly reduced during subtotal hysterectomy when compared with total hysterectomy.

Material and Methods: Total laparoscopic subtotal hysterectomy with monopolar loop: Our standard technique

Results: Laparoscopic subtotal hysterectomy does not offer any clinical benefit when compared with total laparoscopic hysterectomy (Lethaby A et al., 2012), but considering that this technique is widely performed and has proven indications we believe that the standardization of subtotal laparoscopic hysterectomy can simplify this surgery, make it faster and also reproducible.

Discussion: In this video we present our technique for laparoscopic subtotal hysterectomy that can help any gynecological surgeon to safely reproduce the procedure.

187

TLH AND THE LARGE FIBROID UTERUS: SEQUENTIAL APPROACH TO ACHIEVE LAPAROSCOPIC SUCCESS

Hysterectomy

Video

R. Keedwell*, D. Byrne, T. Smith-walker, A. Borland, P. Wipplinger

${ }^{[1]}$ Royal Cornwall Hospital $\sim$ Truro $\sim$ United Kingdom

Summary (4 lines): Video presentation demonstrating myomectomy in order to facilitate subsequent hysterectomy.

Introduction: Uteri significantly enlarged by fibroids have long since presented a challenge to laparoscopic surgeons at hysterectomy. Various techniques are described in order to deal with this challenge, such as Palmer's point entry, isolation of uterine arteries at source to limit bleeding, and direct Morcellation.

Material and Methods: Herein we present the case of a 45 year old lady presenting with menorrhagia and symptoms of stress incontinence. Examination revealed an enlarged uterus, and a large $9 \mathrm{~cm}$ anterior fundal fibroid was noted on transvaginal scan. A laparoscopic hysterectomy was recommended, and so a surgical challenge was anticipated.

Results: A sequential approach was employed whereby the hysterectomy was performed in the following stepwise fashion;

1. Bilateral salpingo-oophorectomy initially in order to maximise the training opportunities available to junior surgeons.

2. Subsequent laparoscopic myomectomy to remove the large central fibroid en mass.

3. Laparoscopic hysterectomy of the 'debulked' uterus was then possible due to the reduced size.

Discussion: This video presentation demonstrates the efficiency of using combined surgical techniques to achieve a common operative goal. The efficient conduction of $\mathrm{BSO} /$ myomectomy/TLH is performed in order to avoid open hysterectomy and to preserve the benefits of the laparoscopic approach.

LAPAROSCOPIC BILATERAL NEOSALPINGOSTOMIES FOR PRIMARY SUBFERTILITY

Infertility and Reproductive Medicine
Video

R. Keedwell*, S. Bates, D. Byrne

${ }^{[1]}$ Royal Cornwall Hospital $\sim$ Truro $\sim$ United Kingdom

Summary (4 lines): Video presentation showing successful laparoscopic neosalpingostomies to achieve tubal patency.

Introduction: Neosalpingostomy is one of the less common laparoscopic procedures seen today. The advent of IVF and the urgency of some patient's need to conceive often obviate their need. However, in the carefully selected patient, this procedure may be the quickest, most efficacious and cost effective mode of achieving conception. Reported success rates are comparable to first cycle of IVF, and the laparoscopic procedure has shown to have significant economic benefits over laparotomy/microsurgery and IVF.

Material and Methods: Herein, we report the case of a 23 year old lady with primary infertility, and bilateral tubal occlusion demonstrated by diagnostic laparoscopy and Fallopian tube dye insertion.

Results: The video presentation demonstrates the adhesiolysis and tubal incision, performed by a trainee surgeon, needed to achieve tubal patency. Ongoing patency is then achieved by the suturing of the fimbrial end. Finally, successful tubal patency is demonstrated by tubal dye injection.

Discussion: This case demonstrates an easily achievable laparoscopic treatment of primary subfertility, which may be the treatment of choice in certain patient groups.

196

\section{LAPAROSCOPIC MANAGEMENT OF PELVIC ABSCESS}

Infertility and Reproductive Medicine

Video

P. Bhardwaj*

${ }^{[1]}$ Max Hospital $\sim$ Delhi $\sim$ India

Summary (4 lines): Untreated tuboovarian abscess (TOA) may rupture resulting in sequlae. Laparoscopy offers the possibility to diagnose \& manage PID early, safely \& cost effectively, improves primary recovery, \& prevents complications of delayed treatment in terms of fertility \& catastrophy.

Introduction: A TOA is the end stage of progression of genital tract infection. The treatment for it would be an approach that is safe, efficacious, cost effective, minimally invasive.

Material and Methods: TOA treated with broad-spectrum antibiotics require surgical intervention in $25 \%$ cases. Laparoscopy should be considered in such patients.

Laparoscopic drainage of pelvic abscess with lyses of adhesions, excision of necrotic inflammatory exudates allow host defenses to better control the infection with antibiotics.

Results: The combination of laparoscopic treatment \& antibiotics works best.

Surgery for pelvic abscess is technically difficult. Such surgeries, should be carried out in centers with good equipment \& surgical expertise

Discussion: Drainage, appropriate use of antibiotics is the treatment of TOA. Patients requiring surgery can be managed laparoscopically. The advantage of laparoscopic management of pelvic abscess allow for an accurate diagnosis, effective treatment with minimal complications, faster response rates \& shorter hospitalization. Early detection \& management improves fertility outcomes in desired cases

\section{6}

OVARIAN TISSUE CRYOPRESERVATION AND TRANSPLANTATION

Infertility and Reproductive Medicine 
Video

F. Salvagno*, E. Bianquin, F. Evangelista, A. Razzano, A. Revelli, G.L. Marchino, C. Benedetto

${ }^{[1]}$ University of Torino, S. Anna Hospital $\sim$ Torino $\sim$ Italy

Summary (4 lines): Different strategies to preserve fertility are the cryopreservation of embryos, oocytes, or ovarian tissue. The last requires laparoscopic retrieval of ovarian cortex and orthotopic transplantation of thawed ovarian tissue.

Introduction: Fertility preservation is very important for young women at risk of infertility. Ovarian cortex cryopreservation is the only option for pre-pubertal girls and women who cannot undergo ovarian stimulation.

Material and Methods: Ovarian tissue collection is usually performed in laparoscopy, with atraumatic scissors, avoiding the use of electrocoagulation to preserve as much as possible the remnant ovary. Then the tissue is stored in liquid nitrogen after the freezing procedure. When the patient is willing to get pregnant the transplantation is performed. Transplantation involves two surgical sessions: a first laparoscopy is performed to scar the grafting sites stimulating angiogenesis and neo-vascularisation; then, a few days later, a second laparoscopy is performed to suture the thawed ovarian tissue to the grafting sites.

During the first laparoscopy tubal patency is tested and biopsies of the remnant ovaries are sampled.

Results: Although still experimental, ovarian tissue transplantation has been demonstrated to restore endocrine ovarian function and twenty live births have been reported worldwide.

Discussion: Ovarian cortex cryopreservation requires a strict collaboration between reproductive medicine specialists and laparoscopic surgeons.

126

\section{SEPTUM REMOVAL WITH THE HYSTEROSCOPIC} MORCELLATOR: A FIRST SERIES.

Infertility and Reproductive Medicine

Video

T. Hamerlynck*, S. Weyers

${ }^{[1]}$ Women's Clinic, University Hospital $\sim$ Gent $\sim$ Belgium

Summary (4 lines): We report our first experience with the hysteroscopic morcellator as an alternative technique for uterine septum removal. Introduction: The hysteroscopic morcellator (HM) has been suggested as an alternative to resectoscopy for septum removal. This mechanical technique comprises no risk of thermal damage to the healthy endometrium, which is of major importance in patients with a desire to conceive. Since the recent introduction of the HM in our university centre we have applied it for this indication.

Material and Methods: The HM consists of a 4-mm, rotary or reciprocating blade, and a continuous flow 9-mm rigid hysteroscope with 0 -degree optic (TRUCLEAR, Smith \& Nephew, Andover, USA). Procedures were performed in the operating room under general anaesthesia.

Results: We used the HM for septum removal in a first series (ongoing) of 5 patients. Women were diagnosed with a uterine septum, covering more than $50 \%$ of the cavity, in the work-up for subfertility and/or recurrent miscarriage. We performed nearly complete septum removal in all patients, combined with hysteroscopic myomectomy in 1 patient. All procedures were uneventful. Repeat hysteroscopy performed in 3 patients revealed no adhesions. We will continue the evaluation of fertility outcome in these patients.

Discussion: We report a good experience with the HM for septum removal. Comparative trials with resectoscopy are needed to elucidate possible advantages of the HM in the treatment of uterine septa.
403

\section{CASE REPORT OF SINGLE PORT LAVH WITH GELPOINT ${ }^{\circledR}$ Innovation in Surgery}

Video

N. Martins, C. Ferreira, C. Andrade*, F. Nogueira-martins

${ }^{[1]}$ Hospital Sao Teotonio $\sim$ Viseu $\sim$ Portugal

Summary (4 lines): First Laparoscopically-Assisted Vaginal Hysterectomy (LAVH) performed with the Applied Medical's Gelpoint ${ }^{\circledR}$ single port (SP) at authors' Department.

Introduction: First documented SP procedures of significance occurred in late 1990s, but first hysterectomies were performed later. Advantages when compared to traditional multi-port laparoscopic techniques are claimed to include less pain, faster recovery and better cosmetic results. Material and Methods: The Gelpoint ${ }^{\circledR}$ platform seems to facilitate triangulation of standard instruments, offering greater freedom of movement and maximizing internal working diameter.

Results: 45-year-old, presenting with abnormal vaginal bleeding and anaemia. Hysteroscopy revealed a large submucous fibroid and patient opted for total hysterectomy without adnexectomy. Incision was $4 \mathrm{~cm}$ wide and standard instruments were used exclusively. Total operating time was 96 minutes and there were no intraoperative complications, except for uterine perforation with the manipulator. The patient was discharged after 48 hours. Reassessment at the outpatient setting revealed a good cosmetic effect.

Discussion: SP-LAVH is a feasible alternative for hysterectomy in selected patients, possibly resulting in a better cosmetic outcome related to the single wound. The learning curve for experienced laparoscopic surgeons seems to be short. It's likely that improvements in the materials used for SP surgeries will increase the general acceptance of this surgical option in upcoming years.

\section{1}

\section{FIRST EXPERIENCES WITH A NEW 5MM DEVICE THAT COMBINES ADVANCED BIPOLAR AND ULTRASOUND ENERGY FOR DISSECTION, SEALING AND CUTTING IN LAPAROSCOPIC SURGERY \\ Innovation in Surgery}

Video

M. Francx*, B. De Vree

${ }^{[1]}$ ZNA Campus Middelheim - Lindendreef 1, B2020 Antwerpen Belgium

Summary (4 lines): First we describe the technical aspects of the new $5 \mathrm{~mm}$ laparoscopic Thunderbeat device (Olympus).

The ultrasound energy, well known for its dissection and cutting properties, is combined with advanced bipolar energy producing a sealing of vessels up to $7 \mathrm{~mm}$ diameter.

We comment on our experiences using this device during 5 consecutive total laparoscopic hysterectomy procedures ( 3 minute video).

91

\section{LAPAROSCOPIC CONSERVATIVE MANAGEMENT OF HUGE TWISTED PARA-TUBAL CYST \\ Innovation in Surgery}

Video

A. Alobaid*, L. Aldakhil, S. Alobaid

${ }^{[1]}$ King Fahad Medical City $\sim$ Riyadh $\sim$ Saudi Arabia

Summary (4 lines): We present a video for laparoscopic salpingectomy for a patient that had adnexal torsion of huge adnexal cyst 
Introduction: It is unlikely to have torsion of huge adnexal cysts. Every effort has to be made to preserve healthy ovarian tissue

Laparoscopic management for such patients is feasible and should be carried out

Material and Methods: A 27 years old patient, presented with severe pain, nausea and vomiting

Exam revealed a huge pelvi-abdominal mass that was reaching till the xiphi-sternum. The mass was mobile but very tender

The ultrasound revealed a $28 \mathrm{~cm}$, unilocular simple looking ovarian cyst Results: Open technique was used to insert the camera Intraoperative evaluation revealed a huge unilocular cyst with smooth surface. 7.5 liters of serous fluid was drained at the beginning of the procedure under laparoscopic visualization. The cyst was found to be a para-tubal cyst with adnexal torsion and bluish discoloration of the ovary. The adnexa was untwisted and salpingectomy was done. At the end of the procedure the ovary had normal color. The procedure was done using three ports only. The blood loss was minimal and the patient was discharged the next day

The pathology report confirmed a benign para-tubal cyst

Discussion: Torsion should be considered in cases of acute pain and huge adnexal cyst

Before aspiration of huge cysts the malignancy index should be very low Conservative laparoscopic management should be attempted for huge cysts even in cases of torsion

\section{0}

\section{LAPAROSCOPIC LARGE OVARIAN CYSTECTOMY AND REMOVAL THROUGH A NATURAL ORIFICE IN A 16 YEAR OLD FEMALE}

Innovation in Surgery

Video

C. Nezhat*, A. Katz, A. Gojayev

${ }^{[1]}$ Atlanta Center for Special Minimally Invasive Surgery \& Reproductive Medicine $\sim$ Atlanta $\sim$ United States

Summary (4 lines): We present a video of laparoscopic excision of an $18 \mathrm{~cm}$ cyst via $5 \mathrm{~mm}$ abdominal ports and a $12 \mathrm{~mm}$ posterior colpotomy in a 16 year old female.

Introduction: We present a video of laparoscopic excision of an $18 \mathrm{~cm}$ cyst in a 16 year old female.

Material and Methods: The cystectomy was performed through $5 \mathrm{~mm}$ abdominal ports and a $12 \mathrm{~mm}$ posterior colpotomy.

Results: Controlled entry into a large ovarian cyst and no-spill evacuation of the cyst fluid are demonstrated.

Discussion: Laparoscopic management of benign ovarian cyst with preservation of the ovary is an acceptable and preferable method in reproductive age females.

375

\section{LAPAROSCOPIC MESH SACROCOLPOPEXY IN COMBINATION WITH ANTERIOR ELEVATE VAGINAL MESH REPAIR FOR A LARGE COMPLETE PROCIDENTIA IN A PATIENT WITH BLADDER EXSTROPHY \\ Innovation in Surgery \\ Video \\ D. Chou, P. De Rosnay*, G. Cario, D. Rosen, L. Reyftmann, S. Choi, O. Baghlaf \\ ${ }^{[1]}$ Sydney Women's Endosurgery Centre $\sim$ Sydney $\sim$ Australia}

Summary (4 lines): We present the surgical management of a 59 year-old woman with bladder exstrophy presenting with a large procidentia for 20 years.
Introduction: Bladder exstrophy is a developmental anomaly of the anterior abdominal wall characterised by a defect in the musculature as well as absence of the pubic symphysis and anterior bladder wall. These patients are particularly prone to genital prolapse.

Material and Methods: We performed a laparoscopic subtotal hysterectomy, sacrocolpopexy in combination with Anterior Elevate vaginal mesh repair and an extensive posterior colpoperineorrhaphy. Surgery was commenced with Anterior Elevate with mesh modification by creating a dorsal extension with GynaeMesh. This was rolled up and closed behind the vaginal mucosa at the cervico-vaginal junction. The mesh was not secured to the sacrospinous ligament as per standard procedure. The extension was later retrieved under the bladder laparoscopically and used as the anterior arm of the sacrocolpopexy. This modified mesh provided support long the entire length of the anterior vaginal wall to the level of the bladder neck and was dorsally joined to the posterior mesh arm of sacrocolpopexy.

Results: Correction of prolapse.

Discussion: Severe prolapse in the presence of distorted anatomy restricted the use of conventional surgical repair techniques. We share our experience of an innovative combination.

8

LAPROSCOPIC RADICAL HYSTERECTOMY DUPLICATED BY ORIFICE- ASSISTED SMALL INCISION SURGERY (OASIS) Innovation in Surgery

Video

S. Puntambekar*, S. Puntambekar, R. Desai, A. Galagali

${ }^{[1]}$ Galaxy CARE Laparoscopic Institute $\sim$ Pune $\sim$ India

Summary (4 lines): OASIS is a safe and feasible technique, which incorporates the benefits of single incision and natural orifice surgery. It overcome the challenges of single incision surgery. The oncological clearance is comparable to laparoscopic radical hysterectomy.

Introduction: Minimal access surgeries aim at scarless surgeries. Orifice-assisted small-incision surgery (OASIS) is a new milestone in MIS. It incorporates benefits of single-incision and natural-orifice surgery and overcomes the challenges of both, like instrument and optical access clashing and instrument crowding thus allowing the performance of advanced laparoscopic procedures with ease.

Material and Methods: In OASIS, the optical access is gained via the posterior cul-de-sac by placing a flexible $13 \mathrm{~mm}$ sigmoidoscope through a $15 \mathrm{~mm}$ trocar at this location. Two $5 \mathrm{~mm}$ working ports are placed on the either side of umbilicus. The steps of laparoscopic radical hysterectomy by the 'Pune technique' were duplicated by this approach.

Results: The oncological clearance achieved in the patients we operated by OASIS was $3 \mathrm{~cm}$ of paracervical margins and $2 \mathrm{~cm}$ of distal vaginal margins, with a average nodal yield of 16 lymph nodes.

Discussion: Based on preliminary case series experience, OASIS appears to be a safe and feasible. The important drawbacks of single incision surgery are overcome by this technique. The oncological clearance is comparable to laparoscopic radical hysterectomy.

\section{6}

NOTES TECHNIQUE FOR LAPAROSCOPIC RECTAL RESECTION AND PELVIC CLEARANCE DURING SURGERY FOR SEVERE ENDOMETRIOSIS

Innovation in Surgery

Video

N. Waters*, F. Shakir, A. Kent, T. Rockall

${ }^{[1]}$ Royal Surrey Hospital $\sim$ Guildford $\sim$ United Kingdom 
Summary (4 lines): NOTES technique is used for organ retrieval and mass extraction and therefore no need to perform a mini-laparotomy. The vaginal incision made as part of the hysterectomy is sufficient to extract the bowel

Introduction: 17 NOTES (natural orifice transluminal endoscopic surgery) cases were identified during 66 anterior rectal resection and pelvic clearance procedures for severe rectovaginal endometriosis

Material and Methods: Stapling of the rectum below the disease prior to total hysterectomy and bilateral salpingo-oophorectomy is performed. The specimen is removed through the vagina. This technique requires dissection of the sigmoid and descending colon up to the splenic flexure allowing delivery of the proximal part without tension. The portion of rectum is removed (average length: $101 \mathrm{~mm}$ ), an anvil is placed in the proximal part and replaced back to the abdomen. The vaginal vault is closed and the procedure is completed laparoscopically

Results: 17 cases of NOTES were compared to 49 cases of minilaparotomy. The average length of stay was 2 days and there was no difference in terms of complications, sexual function or infection. There were no cases of bowel obstruction or vault dehiscence

Discussion: In our institution this technique has become routine during pelvic clearance for severe endometriosis. There is extra cosmetic benefit with no increase in complications

405

\section{SIMULTANEOUS LAPAROSCOPIC TREATMENT OF APICAL PROLAPSE AND RECTOCELE TECHNIQUE (LAPSTAR) \\ Innovation in Surgery}

Video

P. Ramôa*, A. Almeida, C. Costa Pereira, C. Martinez, R. Themudo,

J. Costa Pereira

${ }^{[1]}$ Hospital da Boavista HPP $\sim$ Porto $\sim$ Portugal

Summary (4 lines): We propose a technique to reconstruct the normal pelvic anatomy in case of middle and posterior pelvic defects

Introduction: Symptomatic rectocele associated with obstructed defecation is often accompanied by descent of the perineum, enterocele, apical prolapse and intussusception of the rectum. The correction of the rectocele alone does not solve the defecation obstruction syndromes if anatomic changes associated are present. This video demonstrate the main steps of a technique for correction of middle and posterior compartment defects.

Material and Methods: Rectovaginal space dissection. Intracelomic introduction of polypropylene mesh Y-shaped and central fixation to the uterine isthmus with shaft abandon in the rectovaginal space. Abdominal incision. Introduction a dissecting forceps and subperitoneal progression to the rectouterine pouch previously exposed. Traction of the upper ends of the prosthesis, conditioning the repositioning of the uterus or the vaginal apex

Results: Easily reproducible technique, low morbidity, appropriate correction of anatomic changes with a resolution of the obstructed defecation

Discussion: Apropriate technique to treat middle and posterior compartment defects

320

\section{TOTAL LAPAROSCOPIC HYSTERECTOMY WITH 3MM} Innovation in Surgery

Video

M. Puga*, E. Faller, J. Albornoz, A. Wattiez

${ }^{[1]}$ IRCAD Strasbourg $\sim$ France
Summary (4 lines): We present a video of a Total Laparoscopic Hysterectomy (TLH) with $3 \mathrm{~mm}$ instrumentation. Feasibility and safety of this technique is presented as well as its advantages and disadvantages Introduction: To improve the benefits of MIS different technologies have been developed such as NOTES and single port. However, important limitations originate from ergonomics of the instrumentation and the new learning curve. On the other hand, smaller instruments allow us to perform surgeries in a precise and meticulous fashion, obtaining cosmetic benefit. Nevertheless, concerns are raised when we consider using them in more complex procedures. We tried to address these issues by performing a TLH with $3 \mathrm{~mm}$ instrumentation Material and Methods: Video shows different steps of surgery and proper use of instrumentation.

Results: OR time: $67 \mathrm{~min}$, Blood loss: $<20 \mathrm{cc}$

Discussion: TLH with $3 \mathrm{~mm}$ instruments is feasible and safe. The instruments are similar to the $5 \mathrm{~mm}$ ones, their performance identical and do not require a training phase. Cosmetic results are excellent and sutures are not required. They can be considered for both simple and complex procedures. Limitations are diminished resistance when used on rigid/fibrotic tissues (myomectomy, deep endometriosis) and possibly the longevity of scissors. Further analysis of advantages and drawbacks must be carried out to properly define the place of this new technology in the laparoscopic armamentarium.

446

UPDATE OF THE BEST VIDEO SELECTION OF THE IBS $®$ (INTEGRATED BIGATTI SHAVER) IN ACTION

Innovation in Surgery

Video

G. Bigatti*, M. Rosales, A. Baglioni, S. Franchetti, L. De Giambattista, S. Bianchi

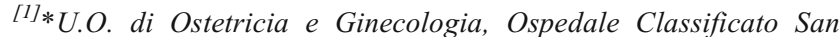
Giuseppe Via San Vittore, 12 - 20123 Milano - Italia g.big@tiscalinet.it Cuggiono $\sim$ Italy

Summary (4 lines): At present conventional bipolar resectoscopy is still recognized as first choice procedure for major hysteroscopic operations. Since 2009 we have proposed an alternative approach to operative hysteroscopy called IBS ${ }^{\circledR}$ Integrated Bigatti Shaver that improving the visualization during the procedure reduces several problems of conventional resectoscopy such as, fluid overload, water intoxication uterine perforation and long learning curve.

Introduction: In cooperation with Karl Storz $\mathrm{GmbH} \& \mathrm{Co}$. we have created a new shaving system that, introduced through a straight operative channel of a panoramic $90^{\circ}$ optic, allows performing all kinds of major hysteroscopic operations.

Material and Methods: At present we have performed 220 cases including all kinds of operative hysteroscopic procedures such as $n^{\circ} 135$ polypectomies, $\mathrm{n}^{\circ} 72$ submucosal myomas resection, $\mathrm{n}^{\circ} 3$ septum resection and $n^{\circ} 11$ endometrial ablation according to ESGE classification

Results: We present this update of the best videos performed with the IBS ${ }^{\circledR}$. Discussion: We confirm the several advantages offered by the IBS ${ }^{\circledR}$ that removing the tissue chips at the same time of resection, makes operative hysteroscopy safer, easier and faster.

\section{4}

\section{RETROPERITONEAL FIBROID LAPAROSCOPIC TREATMENT} Myomectomy

Video

O. Istre*, H. Springborg

${ }^{[1]}$ Aleris-Hamlet Hospital, Copenhagen and University of Southern Denmark $\sim$ Copenhagen $\sim$ Denmark 
Summary (4 lines): A 44 year old women para 1, Symptoms Pain, pressure Bladder and voiding problems Laparoscopic extirpation of retroperitonal and LSH

Introduction: The incidence of leiomyomata among primary retroperitoneal tumors is estimated at $1.2 \%$ The median size of was $12 \mathrm{~cm}(2.0$ 37.0 )and $77.8 \%$ of cases solitary tumors $73.1 \%$ of tumors were found pelvic retroperitoneum, $50 \%$ show some degree of degeneration.

$25 \%$ were asymptomatic $31.3 \%$ experienced abdominal fullness, $18.8 \%$ had urinary symptoms, $18.8 \%$ had weight loss and $18.8 \%$ had pelvic pain

Material and Methods: Laparoscopic extirpation of retroperitoneal fibroid are presented in this video

Results: Laparoscoic surgery with op time $90 \mathrm{~min}$, specimen weight 690 gram, discharge 14 hours The patient was successfully treated laparoscopically and were discharged after 14 hours and returned to work after two weeks.

Discussion: Laparoscopic treatment is advantageous, compared to open surgery because of better visualization of the organs during surgery and shorter recovery. We recommend using a $5 \mathrm{~mm}, 30$ degree HD optic which makes it possible to perform port jumping.

102

\section{APPROACH TO CERVICAL MYOMECTOMY AND TRACHELECTOMY \\ Myomectomy}

Video

J. Moulder, S. Cohen, K. Wang*

${ }^{[1]}$ Brigham and Women's Hospital, Department of Obstetrics and Gynecology, Division of Minimally Invasive Gynecologic Surgery Boston, MA United States

Summary (4 lines): This video illustrates a laparoscopic approach to cervical myomectomy and trachelectomy in a patient with a prior supracervical hysterectomy for fibroids.

Introduction: Patient is a 40-year-old woman with a history of uterine leiomyomata. She underwent a supracervical hysterectomy and right salpingo-oophorectomy 5 years ago. She now presents with pelvic pain and pressure, and heavy, irregular bleeding.

Imaging showed recurrence of a cervical fibroid.

Material and Methods: Patient underwent laparoscopic adhesiolysis, cervical myomectomy, and trachelectomy. The cervical stump was isolated, and the fibroid enucleated. The remnant uterine vascular pedicles were cauterized and lateralized.

Colpotomy was performed to resect the fibroid and perform the trachelectomy. The colpotomy was closed using a quill barbed suture.

Results: Adhesiolysis and dissection allowed for complete visualization of posterior cervical fibroid. Colpotomy allowed for delivery of the enucleated fibroid and cervical stump. No residual fibroid was present at end of procedure.

Discussion: Although infrequent, recurrence of leiomyomata following supracervical hysterectomy provides a particular challenge for gynecologists.

Restoring normal anatomy is essential for surgical treatment of symptoms.

Ultimately, a trachelectomy was necessary, however, cervical myomectomy was performed to better define the anatomy in order to successfully achieve the primary goal.

\section{8}

\section{LAPAROSCOPIC MYOMECTOMY WITH TRANSITORY CLIPPING OF UTERINE ARTERIES \\ Myomectomy}

Video

J. Prado, F. Gonzalez*, D. Larraín, F. Marengo, I. Rojas

${ }^{[1]}$ Clínica Santa María Santiago $\sim$ Chile

Summary (4 lines): We present a video showing our tecnique and instrumentation for transient uterine artery occlusion before laparoscopic myomectomy

Introduction: The goal of myomectomy is to treat symmtomatic fibroids while preserving fertility. Laparoscopy offers several advantages, such less postoperative pain and faster recovery.

Material and Methods: We describe our technique and instrumentation of transient uterine artery occlusion before performing laparoscopic myomectomy.

Results: Ten patients who underwent laparoscopic myomectomies with transitory clipping of uterine arteries. We describe the technical approach used, clipping both uterine arteries transiently with an endoscopic titanium clip, which is removed at the end of surgery. We also present an illustrative video of the procedure. Mean time to identify an clip uterine arteries was 14,5 minutes (range 9 to 21 minutes).

Discussion: Transient uterine artery occlusion is a safe and useful technique to decrease bleeding during laparoscopic myomectomy when performed with adecuate technique eand instrumentation.

\section{6}

\author{
LAPAROSCOPIC REMOVAL OF PRE-PERITONEAL \\ LIPOLEIOMYOMA \\ Myomectomy \\ Video \\ S. Cohen*, C. Hill-lydecker, K. Wang \\ ${ }^{[1]}$ Brigham and Women's Hospital $\sim$ Boston $\sim$ United States
}

Summary (4 lines): This is a video demonstration of laparoscopic management of a pre-peritoneal lipoleiomyoma found at the time of surgery.

Introduction: This was a case of a 40 year old G2P0020 with a history of Stage 4 endometriosis and fibroid who had undergone a previous abdominal myomectomy of a $10 \mathrm{~cm}$ fibroid and left salpingooophorectomy for an endometrioma by a vertical laparotomy incision in 2010 at an outside hospital. She presented to us with complaints of right lower quadrant dysmenorrhea, urinary symptoms, dyspareunia, and the desire for future conception.

Material and Methods: Pre-operative imaging was done and ultrasound showed a $9 \mathrm{~cm}$ solid mass adjacent to the right ovary which contained two endometriomas. Subsequent MRI showed an anterior $8.5 \mathrm{~cm}$ pedunculated fibroid and right ovary with an endometrioma.

Results: Findings at the time of surgery revealed a pre-peritoneal mass adjacent to the bladder, broad ligament fibroid, absent left ovary and tube, rectosigmoid adhesions to the posterior aspect of the uterus, and right ovary with an endometrioma. The endometrioma and fibroid were removed without complication.

Discussion: Despite having adequate pre-operative imaging in preparation for surgery, findings at the time of surgery were unexpected. Final pathology revealed an endometrioma and both fibroid specimens consistent with lipoleiomyoma.

\section{3}

\section{LAPAROSOPIC MYOMECTOMY USING ENDO LOOP DEVICE} Myomectomy

\section{Video}

H. Haerizadeh*, J. Frappell

${ }^{[1]}$ Department of minimal access gynaecology, Derriford Hospital Plymouth United Kingdom 
Summary (4 lines): This is a video presentation of laparoscopic myomectomy using Endo Loop device to achieve better haemostasis. Introduction: Pedunculated subserosal fibroids can cause pain by complete or incomplete torsion. Twisting of the fibroid along its blood supply can cause ischemia and subsequent pain.

Material and Methods: A 47 year old woman presented with episodic pain on her lower abdomen and right iliac fossa. Pelvic ultrasound revealed a 5 centimetre right dermoid cyst and a 5 centimetre subseroal fundal fibroid. Patient was counselled and booked for laparoscopic right oophorectomy and myomectomy.

Results: Laparoscopic right oophorectomy was completed using bipolar diathermy and scissors. Then, two Endo Loop devices were applied on the base of the fibroid and myomectomy was completed by using monopolar scissors. Both samples were retrieved through suprapubic incision. The procedure was completed in 45 minutes with an estimated blood loss of less than 50 millilitres.

Discussion: Endo Loop device is a safe instrument to perform myomectomy for pedunculated subserosal fibroids. The technique is easy to use with optimal haemostasis and is quite safe and practical.

\section{8}

\section{TECHNIQUE OF LAPAROSCOPIC MYOMECTOMY FOR THE} XXL INTRAMURAL FIBROID

Myomectomy

Video

E. Dimitriou*, P. Mpalinakos

${ }^{[1]}$ Leukos Stauros Athinwn $\sim$ Athens $\sim$ Greece

Summary (4 lines): We present the technique of enucleation of a $15 \mathrm{~cm}$ big intramural fibroid from the posterior wall of the uterus Introduction: This is a 39 year old nuliparus woman who presented to her general practitioner with intermenstrual spotting and abdominal swelling. A transabdominal ultrasound revieled a big fibroid uterus that measured $15 \mathrm{~cm}$. it was arising from the fundus and it was $7 \mathrm{~mm}$ deep in the myometrium

Material and Methods: Laparoscopic myomectomy was performed succesfully

Results: EBL was $350 \mathrm{mls}$. Operative time was $2 \mathrm{hrs} 15 \mathrm{~min}$. No disposable material was used apart from the sutures. The patient went home the next day. We also present ultrasound pictures of the uterus 2 months post operatively

Discussion: We aim to show step by step how one can achieve laparoscopic removal of bigger intramural fibroids

\section{0}

\section{UTERINE RUPTURE POST MYOMECTOMY: REVIEW OF 6 CASES}

Myomectomy

Video

E. Dimitriou*, P. Mpalinakos, N. Mpardis, G. Pistofidis

${ }^{[1]}$ Leukos Stauros Athinwn $\sim$ athens $\sim$ Greece

Summary (4 lines): Uterine rupture post myomectomy maybe a catastrophic event. We have collected and analysed the videos of the initial procedures of 6 cases and identified risk factors

Introduction: Uterine rupture during pregnancy is a rare but potentially catastrophic event. Laparoscopic myomectomy is a independent risk factor with incidence of 6-18\%.

Material and Methods: The whole videos of 6 cases that had had a uterine rupture during pregnancy following laparoscopic myomectomy were reviewed and analysed for risk factors by two experts.
Results: All cases of ruptures happened between 27 and 34 weeks and had favourable outcomes for the foetuses. None needed to have hysterectomy and primary repairs were performed. In all six cases extensive use of bipolar on the serosa was noted. 4 cases were sutured with bad technique and 2 had no sutures. Operative time and blood loss was also higher than average in 4 of the cases.

Discussion: Less or no use of bipolar cauterisation and good suturing technique may lower the incidence of uterine rupture after laparoscopic myomectomy

\section{8}

\section{WHO NEEDS A MYOMECTOMY SCREW? Myomectomy}

Video

A. Kent*

${ }^{[1]}$ MATTU $\sim$ Guildford $\sim$ United Kingdom

Summary (4 lines): Tips and tricks in laparoscopic myomectomy. A novel approach to fibroid enucleation.

Introduction: Enucleating a tenacious fibroid at laparoscopic myomectomy can be bothersome and time consuming. By their very design laparoscopic myomectomy screws have a habit of bending and are therefore really not fit for purpose, $5 \mathrm{~mm}$ instruments have their limitations.

Material and Methods: Harmonic ACE, a novel approach to fibroid enucleation, laparoscopic suturing techniques, morcellation

Results: See video

Discussion: See video

30

\section{ENDOMETRIAL POLYPS: EVALUATION AND TRATEMENT IN OFFICE HYSTEROSCOPY}

Office \& Diagnostic Hysteroscopy

Video

G. Germano*, C. Dörner, I. Posada

${ }^{[1]}$ Hospital Central de las Fuerzas Armadas $\sim$ Montevideo $\sim$ Uruguay

Summary (4 lines): OBJECTIVE: To evaluate the feasibility of treating endometrial polyps in Office in an efficient and safe manner.

Introduction: We make residents training in Office Hysteroscopy, and this procedures are made by residents under experienced endscopist supervision.

Material and Methods: MATERIAL AND METHODS: We analyze 198 hysteroscopy made during 2011 and 2012 in the Hysteroscopy Office of the Hospital Militar Montevideo Uruguay. We used a 2,9 mm Storz Optic; Bettocchi Sheath, and bipolar electrobisturi Versapoint.

Results: RESULTS: 109 endometrial polyps were found, in patients between 33 and 79 years old. The sizes oscillated between 0,5 and $5 \mathrm{~cm}$, being the $51 \%$ minor than $3,5 \mathrm{~cm}$. In 98 cases we made interventions with the Bettocchi set, with total polypectomies using bipolar Twizzle in 28 cases; total polypectomies using scissors and grasping in 29 cases, and we made directed biopsies in 34 cases. The limiting size for achieving the polypectomy was $3,5 \mathrm{~cm}$. The tolerance was very good, only 5 patients complained of pain, so the procedure was post posed. We had no complication in none of the procedures. In 2 cases the polyps had malignant aspect, what was confirmed by the biopsy. In all the rest cases the biopsies showed benignant polyps.

Discussion: CONCLUSSIONS: We conclude that the management of endometrial polyps with benign aspect and with less than $3,5 \mathrm{~cm}$ size can be achieved in a safe manner in office, and without the need of anesthesia in most patients. 
455

\section{HYSTEROSCOPIC ENLARGMENT OF CERVICAL CANAL BY TWIZZLE GYNECARE ${ }^{\circledR}$ TO IMPROVE THE FEASIBILITY OF THE POLIPECTOMY IN OFFICE HYSTEROSCOPY}

Office \& Diagnostic Hysteroscopy

Video

E. Castellacci*, S. Calzolari

${ }^{[1]}$ Palagi Hospital- Florence- Italy $\sim$ Florence $\sim$ Italy

Summary (4 lines): We report a new technical procedure in office hysteroscopy to improve the removal of endometrial polyps $<20 \mathrm{~mm}$ en bloc or sectioned in few parts throw a minimal enlargement of cervical canal by Twizzle instrument.

Introduction: We know very well that a limit of the office hysteroscopy can be the measure of the endocavital neoformation, expecially for the operative time and discomfort of the patient.

Material and Methods: an example of endometrial polipectomy by enlargment of cervical canal using twizzle Gynecare ${ }^{\mathbb{R}}$

Results: polipectomy en bloc

Discussion: With this thin $(3 \mathrm{~mm})$ modification of capacity of cervical canal is possible to remove endocavitary neoformazions without anesthesia and with low discomfort of the patient and operative time.

\section{8}

NEW TECHNICAL SETTING OF TWIZZLE GYNECARE TO OPTIMIZE THE HYSTEROSCOPIC METROPLASTY OF UTERUS SEPTUS

Office \& Diagnostic Hysteroscopy

Video

E. Castellacci, S. Calzolari*, V. Dubini

${ }^{[1]}$ Palagi Hospital- Florence- Italy $\sim$ Florence $\sim$ Italy

Summary (4 lines): we know very well that often is difficult to see both the tubal recesses in one image of the hysteroscopic camera, so may be difficult to be sure to difine a correct line from right to left of the uterine fondus

Introduction: to set points on the endometrial tissue by twizzle gyanecare, from right to left, improves the correct line of setting metroplasty

Material and Methods: to set points on the endometrial tissue by twizzle gyanecare, from right to left, improves the correct line of setting metroplasty

Results: a quickly and sure method to improve the hysteroscopical metroplasty

Discussion: it's a valid method to break the connectival structures of the endometrial septum

61

\section{OFFICE HYSTEROSCOPY IN WOMEN WITH FAILED IVF CYCLES.}

Office \& Diagnostic Hysteroscopy

Video

G. Keklikián*, M. Hermida, P. Marendazzo, A. Rubal, G. Antunez, A. Quartucci

${ }^{[1]}$ Tornu Hospital Buenos Aires $\sim$ Argentina

Summary (4 lines): Office hysteroscopy was performed in 78 patients with at least 2 failed IVF attempts. The most frequent findings were: intrauterine, endometrial and cervical benign pathologies. When necessary, operative hysteroscopy was performed in the same procedure.
Introduction: Nowadays, office hysteroscopy is recommended in patients with at least 2 failed IVF attempts. The objective of this report is to show our findings and to evaluate the feasibility, technique and patient compliance when correcting the pathologies during the same procedure. Material and Methods: 78 patients with recurrent implantation failure and previous normal hysterosalpingography and ultrasound underwent diagnostic and, if necessary, operative hysteroscopy in an outpatient setting with a $2.9 \mathrm{~mm}$ hysteroscope and a double sheath Bettocchi set.

Results: In 49 patients $(62.8 \%)$ no intrauterine abnormalities were found. The most frequent abnormal findings were: a)intrauterine pathology: polyps, myomas, adhesions $(19,2 \%)$, b) endometrial: endometritis, hypotrophia, hyperplasia $(17,9 \%)$, c)cervical abnormalities: polyps, adhesions $(15,4 \%)$. Surgical hysteroscopy was performed in 25 patients with either microscissors or bipolar electrode, with satisfactory patient compliance and no complications.

Discussion: A diagnostic hysteroscopy should be performed prior to the first IVF cycle. The "see and treat" procedure is likely to be carried out in an outpatient setting depending on the surgeon expertise and careful patient selection.

\section{5}

SMALL DIAMETER HYSTEROSCOPIC MORCELLATOR FOR OFFICE HYSTEROSCOPY: FIRST EXPERIENCE IN BELGIUM. Office \& Diagnostic Hysteroscopy

Video

T. Hamerlynck*, S. Weyers

${ }^{[1]}$ Women's Clinic, University Hospital $\sim$ Gent $\sim$ Belgium

Summary (4 lines): We report an excellent first experience with the small diameter hysteroscopic morcellator for the ambulatory treatment of intrauterine pathology.

Introduction: The hysteroscopic morcellator (HM) is a novel technique that was recently introduced in our hospital. After gaining experience with the classic HM in patients under anaesthesia, we started using the small diameter HM for the ambulatory treatment of smaller intrauterine pathologies. We were the first centre in Belgium that started using this instrument.

Material and Methods: The small diameter HM consists of a $2.9 \mathrm{~mm}$ rotary blade, and a 5 - $\mathrm{mm}$ hysteroscope, with a $5.6 \mathrm{~mm}$ sheath and 0 degree optic (TRUCLEAR, Smith \& Nephew, Andover, USA). This purely mechanic technique has the possibility to treat a wider range of abnormalities as compared to hysteroscopic scissors.

Results: We performed a first series (ongoing) of 6 ambulatory procedures in both pre- and postmenopausal women, aged 34 to 75 years, with evidence of one or more intrauterine polyps. Procedures were performed without or under local anaesthesia, and all were uneventful. Pathology analysis was performed in 5 cases, showing a benign intrauterine polyp. Discussion: The small diameter HM was successfully introduced in our centre, with high satisfaction amongst patients, hysteroscopists and assisting personnel. We believe that this technique will allow for a wider range of intrauterine pathologies to be treated in the office setting.

121

EXTRAPERITONEAL LAPAROSCOPIC APPROACH IN AN ISOLATED AORTIC NODE RECURRENCE OF CERVICAL CANCER

Oncology

Video

A. Correa-paris*, M. Carbonell Socias, A. Perez-benavente, B. Díaz-feijoo, M. Bradbury, A. Gil-moreno

${ }^{[1]}$ Hospital Materno-Infantil Vall D'hebron. Autonomous University of Barcelona $\sim$ Barcelona $\sim$ Spain 
Summary (4 lines): We report a case of a paraaortic lymph node recurrence of cervical cancer managed by extraperitoneal operative laparoscopy. This approach is a safe and feasible method for the diagnosis and treatment of retroperitoneal lymph node recurrence in cervical cancer

Introduction: Laparoscopic surgical staging is a useful technique for the assessment of nodal involvement, reducing laparotomy-associated complications as well as favoring quick recovery and prompt initiation of treatment. The extraperitoneal approach offers advantages over the conventional transperitoneal approach

Material and Methods: In may 2007 a patient underwent extraperitoneal laparoscopic paraaortic lymphadenectomy for suspected isolated lymph node recurrence in cervical cancer. The suspected diagnosis of recurrence was performed with computed tomography scanning and magnetic resonance imaging

Results: The patient's age was 70 years. The body mass index was $25 \mathrm{~kg} / \mathrm{m} 2$. The operative time was 150 minutes. The blood loss was $<100 \mathrm{~mL}$. The hospital stay was 2 days and no complications were observed. Recurrence was confirmed in the pathologic study

Discussion: Complete debulking of suspicious lymphadenopathy offers an exact diagnosis of malignancy, and it may have a therapeutic benefit in the case of being positive. The extraperitoneal laparoscopic approach for lymph node recurrence in cervical cancer is a safe and feasible procedure

63

\section{LAPAROSCOPIC FENESTRATION OF LYMPHOCELE Oncology}

\section{Video}

E. Martínez Lamela*, J. Molero Vílchez, V. Sobrino Mota, Y. Expósito Lucena, R. Lorente Ramos, J.J. Hernández Aguado

${ }^{[1]}$ Hospital Universitario Infanta Leonor $\sim$ Madrid $\sim$ Spain

Summary (4 lines): We report laparoscopic fenestration of a symptomatic lymphocele after staging laparotomy in ovarian cancer.

Introduction: Lymphocysts are uncommon after paraortic lymphadenectomy ( 1 to $3 \%$ ) and most of them are asymptomatic. Once a lymphocele has become symptomatic it has to be treated.

Material and Methods: A 45 year-old woman who undertakes optymal cytoreductive surgery after being diagnosed ovarian adenocarcinoma IC final stage. 6 weeks after surgery, she developes intense pain in left lumbar area radiated to crural area and which prevents deambulation. In Pelvic MRI is observed a $20 \times 14 \mathrm{~cm}$ lymphocele with is located in left retroperitoneal area, extended to the renal vein and left kidney.

Results: As first-line treatment percutaneous drainage is performed with persistent lymphocele, so it is decided debridement laparoscopy as follows: Adhesiolysis and lateral release of the descending colon to the psoas muscle : pelvic retroperitoneal dissection for location of ureter and left common iliac artery; anterior dissection of the psoas muscle to lymphocele; anterior approach to the lymphocele with two holds opening :one $8 \mathrm{~cm}$ wide in midline and another of $5 \mathrm{~cm}$ in the lateral; drainage of abundant lymphatic content. Patient has completed adjuvant chemotherapy treatment and no new complications have occurred.

Discussion: Laparoscopic drainage of a symptomatic lymphocele after a lymphadenectomy for gynaecologic malignancies constitutes a valid therapeutic option.

\section{2}

\section{LAPAROSCOPIC RETROPERITONEAL LYMPHADENECTOMY IN GYNAECOLOGICAL CANCER. \\ Oncology}

Video

P. Barri, D. Amselem*, M. Cusido, R. Fabregas, F. Fargas

${ }^{[1]}$ Institut Universitari Dexeus $\sim$ barcelona $\sim$ Spain
Summary (4 lines): The video presents the technique used at our institution since 2007 in 35 successive patients.

Introduction: We describe the technique of endoscopic retroperitoneal lymphadenectomy in gynecologic cancer.

Material and Methods: The following data were reviewed: pathological data and postoperative follow-up (length of hospital stay, blood loss).??

Results: The mean age was 57,2 years. Most cases 66,66 \% were endometrial cancers. Cervical cancer represent $(16,66 \%)$ of the cases, carcinosarcoma $(5,55 \%)$ and ovarian cancer $(2,22 \%)$. Operating time was 161.25. minutes in a single retroperitoneal aortic lymphadenectomy and 243 minutes when hysterectomy and pelvic/aortic lymphadenectomy were performed. The mean drop in haemoglobin in single aortic lymphadenectomy was 1.22 compared to 1.69 in the hysterectomy group. The mean length of hospital stay was 4 days. Blood transfusion was not required. There were no intra-operative complications but 3 cases presented minor postoperative complications: deep vein thrombosis in lower extremity, lymphocele and wound infection. The number of aortic lymph nodes analyzed varied from 4 to 29 (mean 13.11)

Discussion: Lower rate of intestinal adhesions and complications.? Reduced hospital stay and shorter recovery time.?-Do not delay the onset of adjuvant radiotherapy or chemotherapy.?-It requires a learning curve for the surgeon and the standardization of the technique?

\section{7}

\section{LAPAROSCOPIC TECHNIQUE FOR THE FRONT BOARDING OF THE URETER DURING RADICAL HYSTERECTOMY Oncology}

Video

J. Molero Vílchez*, E. Martínez Lamela, Y. Expósito Lucena, V. Sobrino Mota, J.L. Prieto Alonso, M. Rejas Gutierrez, J.J. Hernández Aguado

${ }^{[1]}$ Hospital Universitario Infanta Leonor $\sim$ Madrid $\sim$ Spain

Summary (4 lines): To describe our surgical technique for the dissection of the ureter tunnel during the parametrial resection in radical histerectomy Introduction: The laparoscopic route facilitates the front dissection of ureter. 44 patients with cervix cancer from April of 2008 to February of 2012 were revised. In $92.3 \%$ of the cases laparoscopic route was chosen (technique radical hysterectomy type III of Piver) with nerve sparing (Querleu-Morrow C1) in 13 cases.

Material and Methods: The average age was of 49.22 years. In $70.45 \%$ of cases, squamous cell carcinoma type was found.18 patients were surgically treated (Stages Ia1, 6 cases; IB1, 10 cases; IB2, 2 cases). In 12 cases pelvic linfadenectomy was made (average of 23.3 extirpated ganglia).

Results: Laparoscopic technique of front boarding of ureter is described: dissection of the vesicouterina plica up to $4-5 \mathrm{~cm}$ underneath the cervicovaginal union, dissected of the parauretral tissue to both sides of vagina and released both ureters from the vagina until its entrance in back of the bladder. Dissection of medial and lateral pararrectal spaces allows to identify and respect the hypogastric nerve. The upwards traction of parametrio allows to identify the entrance and exit of the ureter

Discussion: This technique allows a faster and safe resección of the uréter tunnel, without risk of ureteral injury and facilitates the nerve sparing of the uterovesical branches.

\section{2}

\section{LAPAROSCOPIC TECNIQUE FOR UPPER VAGINECTOMY IN RECIDIVED CERVICAL CANCER Oncology}

Video

J. Molero Vílchez*, E. Martínez Lamela, V. Sobrino Mota, E. Sáez Cerezal, S. Sancho Garcia, F.J. Salazar Arquero, J.J. Hernández Aguado

${ }^{[1]}$ Hospital Universitario Infanta Leonor $\sim$ Madrid $\sim$ Spain 
Summary (4 lines): The indication for this procedure is treatment of vaginal neoplasia and vaginal recurrence before radical hysterectomy for early cervix cancer or endometrial cancer.

Introduction: To describe this surgical technique by laparoscopy. Material and Methods: Case report a patient 67 years old with recidived epidermoide carcinoma in vaginal cupola. In the extension study, there was an abdomino-pelvic CT scan nonpathological reveals adenopathy.

Results: The laparoscopic route was elected to remove upper vagina and the adjacent tissues of the paracolpium. The patient is in the dorsal lithotomy position with to her buttocks off the table end $5-10 \mathrm{~cm}$. To bladder is emptied. The procedure began with the development of the straight-vaginal septum. Monopolar scalpe was used to incise the peritoneum in the region between the uterosacral ligaments. The rectal-vaginal space was developed facilitated by placing to sponge on to stick in the later fornix. Anteriorly the incision was carried down to bladder vesico-vaginal flap, thus facilitating the creation of the space and separate the bladder pillars. The completely separation was development for rolling both ureters laterally till we could see ones entering in to bladder. Upper vagina was free from all support tissues. Laparoscopic removal of this specimen ensuring adequate vaginal margins.

Discussion: Laparoscopic abording for vaginectomy allows one better anatomical dissection respect to the traditional vaginal approach.

135

\section{PRIMARY FALLOPIAN TUBE CARCINOMA DIAGNOSED LAPAROSCOPICALLY \\ Oncology}

Video

R. Mesquita Pinto*, C. Andrade, A. Monteiro, N. Nogueira Martins, F. Nogueira Martins

${ }^{[1]}$ Centro Hospitalar de Tondela-Viseu, Hospital S. Teotónio, Viseu Viseu $\sim$ Portugal

Summary (4 lines): Primary fallopian tube carcinoma (PFTC) is a very rare disease, with a difficult preoperative diagnosis.

Introduction: PFTC is one of the less common gynaecological cancers. Fewer than $5 \%$ of the patients are accurately diagnosed preoperatively due to the lack of specific symptoms and even the intraoperative diagnosis is missed in up to $50 \%$ of the cases. Tumor staging represents the most prognostic factor

Material and Methods: Sixty-three-year-old nulliparous admitted to Internal Medicine Department due to abdominal pain and ascites - undergoing investigation. Following request for Gynaecology Department's Cooperation, the patient underwent diagnostic laparoscopy.

Results: At surgery, cystic fallopian tube lesion was found bilaterally, reaching the fimbrial end, as well as peritoneal carcinomatosis and implants all over the abdominal cavity. The biopsies performed, including the left tube, revealed serous primary fallopian tube carcinoma. The patient was included in the stage IV of FIGO staging system and neoadjuvant chemotherapy was decided, using platinum/taxane combination.

Discussion: Gold standard initial management of PFTC is the same as for ovarian cancer that is complete surgery. Bilateral tubal involvement has been reported in 10-27 \% of cases and most are diagnosed in stage III/IV. Prognosis of tubal cancers seems to be better than ovarian cancers mainly because the most common histological type is serous carcinoma, very sensitive to chemotherapy.

140

ROBOTIC-ASSISTED EXTRAPERITONEAL LAPAROSCOPY FOR SURGICAL STAGING OF CERVICAL CANCER Oncology
Video

A. Correa-paris*, M. Carbonell Socias, A. Perez-benavente, B. Díaz-feijoo, J.L. Sanchez, A. Gil-moreno

${ }^{[1]}$ Hospital Materno-Infantil Vall D'hebron. Autonomous University of Barcelona $\sim$ Barcelona $\sim$ Spain

Summary (4 lines): We report the case of a robotic-assisted extraperitoneal laparoscopy for paraaortic lymphadenectomy. This approach is a safe and feasible method for surgical staging of locally advanced cervical cancer

Introduction: Laparoscopic surgical staging is a useful technique for the assessment of nodal involvement, reducing laparotomy-associated complications as well as favoring promt recovery and initiation of treatment with chemo-radiotherapy. The extraperitoneal approach offers advantages over the conventional transperitoneal approach and robotic assistance reduces the technical difficulty

Material and Methods: In april 2012 a patient underwent roboticassisted extraperitoneal laparoscopic paraaortic lymphadenectomy for surgical staging, after suspition of FIGO stage II-B cervical cancer given the findings in magnetic resonance imaging and positron emission tomography studies

Results: The patient was 61 years old, and previously treated with chemotherapy for Chronic Lymphoid Leukemia. Her body mass index was $29.6 \mathrm{~kg} / \mathrm{m} 2$. The operative time was 150 minutes. Blood loss was $10 \mathrm{~mL}$. The hospital stay was 2 days and no complications were observed. Histologic analysis revealed 18 negative lymph nodes

Discussion: The extraperitoneal laparoscopic lymphadenectomy for surgical staging in cervical cancer is a safe and feasible procedure. Robotic assistance and optimal visualization of retroperitoneal anatomy allows for adecuate management of most intraoperative findings

\section{3}

\section{ROLE OF LAPAROSCOPY IN ADVANCED OVARIAN CANCER Oncology}

\section{Video}

M. Cusido, D. Amselem*, A. Vazquez, R. Fabregas, F. Fargas

${ }^{[1]}$ Institut Universitari Dexeus $\sim$ Barcelona $\sim$ Spain

Summary (4 lines): Surgery is the standar treatment in ovarian cancer. Residual disease is the most importan prognostic factor.

Introduction: Suboptimal surgery involves a worse survival. In this cases neoadjuvant chemotherapy is a good approach with less morbidity than surgery.

Material and Methods:

Results: Laparoscopy for evaluation of resectability is emerging as the standard technique over the image in these cases, with minimal morbidity and the possibility of making a correct histological study that will guide subsequent chemotherapy.

Discussion: We present several examples of laparoscopy for evaluation of resectability showing different situations of differential diagnosis and possible complications in these cases.

\section{5}

TOTAL LAPAROSCOPIC RADICAL HYSTERECTOMY: THE TECHNIQUE AND THE RESULTS

Oncology

Video

M. Gol*, Z. Guner

${ }^{[1]}$ Izmir University Faculty of Medicine; Medical Park Hospital Izmir $\sim$ Turkey 
Summary (4 lines): Here we present our technique in three patients who underwent total laparoscopic radical hysterectomy at our instution (TLRH).

Introduction: TLRH was originally described in the 1990s by Spiritos et al. Accordingly; Puntambekar et al. presented their TLRH technique (Pune technique) in a large series of patients. With this method they showed reasonably short operative time (92 minutes) without any conversion to laparatomy. In contrast to their technique, we unroof the ureters at the later stage of the operation which we find it more easier. Similarly this technique is clearly demostrated by Prof. Dr. A. Wattiez at Web surg.

Material and Methods: We report three patients with cervical cancer who had underwent TLRH.

Results: A total of three patients with a cervical cancer stage IB1 underwent TLRH. The mean operative time was 200 minutes. The mean intraoperative loss was $350 \mathrm{ml}$. whereas the mean drop in Hb concentration was $2 \mathrm{mg} / \mathrm{dl}$. Extracted pelvic lymph node counts were 9, 12, and 15 respectively. There were no intraoperative nor postoperative complications. All patients discharged on the third postoperative day.

Discussion: TLRH is increasingly used in expert centers. In the hands of skillfull and experienced surgeons this operation seems to be a feasible and demanding operation. However, we need more data regarding outcomes of the effeciency and survival rates in these patients.

\section{5}

CONVENTIONAL TECHNIQUES OF RESECTOSCOPY COMPARED TO A NEW APPROACH USING THE NOVEL HYSTEROSCOPIC MORCELATOR TRUCLEAR ® ACCORDING TO THE "SCOOP \& SHAKE" TECHNIQUE Operative Hysteroscopy

Video

B.C. Schoot*

${ }^{[1]}$ Catharina Hospital $\sim$ Eindhoven $\sim$ Netherlands

Summary (4 lines): Aim of this video is to demonstrate three different techniques of hysteroscopically removing myoma using a resectoscope, and compare these approaches to a new technique used with a novel operative hysteroscopic device Truclear ${ }^{\circledR}$ using the scoop and shake method.

Introduction: The different techniques of classic electroresection of myoma are demonstrated using animations. a Different technique of hysteroscopic morcelation is demonstrated using the Truclear ${ }^{\circledR}$ device. Material and Methods: Compared to the demonstrated electroresection approaches, the Truclear ${ }^{\circledR}$ morcelating device (Smith and Nephew, Andover MA, USA) offers the possibility to cut small pieces of myoma. Chips are directed out of the uterus through the inner canal of the blade using continuous suction and collected in a filter outside the patient. Beside use of conventional techniques, the device can be used to enucleate myoma and free them out of their capsula. In contrast to conventional resectoscopy,

Results: morcelation of myoma using the Truclear ${ }^{\circledR}$ device can be done using the "scoop and shake method". This technique is different compared to classic resection. enucleation of the myoma can be advantages, and can improve the speed of resection.

Discussion: In our opinion the morcelation technique is a wellcontrolled safe way to remove large deep myoma.

\section{5}

HYSTEROSCOPIC RESECTION OF LARGE SUBMUCOSAL FIBROID USING BIPOLAR ELECTROSURGERY SYSTEM Operative Hysteroscopy

Video

H. Haerizadeh*, J. Frappell

${ }^{[1]}$ Derriford Hospital $\sim$ Plymouth $\sim$ United Kingdom
Summary (4 lines): This is a video presentation of hysteroscopic resection of a 7 centimetre submucosal fibroid using bipolar electrosurgery system. This was performed in a two step procedure taking into account the size of the fibroid and patient's clinical situation.

Introduction: Hysteroscopic bipolar energy delivery system has a better safety profile compared to monopolar system with less risk of hyponatremia. This is a serious complication that may need monitoring and treatment.

Material and Methods: A 36 year old nulliparous woman presented with 12 months history of menorrhagia. Ultrasound revealed a $7 \mathrm{~cm}$ submucosal fibroid. Patient wishes to preserve fertility.

She was booked for hysteroscopic resection of fibroid. Bipolar Versapoint resection loop was used and Versablend mode was chosen to achieve optimal cutting and adequate haemostatic effects.

Results: In 45 minutes, $50 \%$ of the fibroid was removed; about $2500 \mathrm{ml}$ of normal saline was absorbed. Minimal bubble formation provided good views intra operatively.

A second procedure was scheduled after 6 weeks when the rest of fibroid was removed. Patient made a good recovery with significant improvement in her symptoms and was discharged following the second procedure.

Discussion: Hysteroscopic resection of submucosal fibroids using bipolar electro surgery system is an effective treatment modality in patients who want to preserve fertility and has a better safety profile compared to traditional monopolar system.

\section{6}

\section{SEPTOPLASTY OF A COMPLETE UTERINE SEPTUM, CERVICAL AND VAGINAL SEPTUM. \\ Operative Hysteroscopy \\ Video \\ M. Simón*, C. Barbed, A. Úbeda, O. Salas, D. Amsalem \\ ${ }^{[1]}$ Dexeus $\sim$ Barcelona $\sim$ Spain}

Summary (4 lines): Complete uterine septum, including cervix and vagina, is a rare pathology with major problems of infertility, with easy handling hysteroscopic.

Introduction: Many of the complete septums including vagina go unnoticed, therefore it is very important to make a good clinical examination of the patient.

Material and Methods: A video of a hysteroscopic septum resection Results: The septoplasty is a safe minimally invasive outpatient surgery with excellent obstetrics results. We no consider necessary to respect the cervical septum. After two month of the intervention, it is important to do a diagnostic histerocopy.

Discussion: In conclution the hysteroscopy is a useful tool in the whole management of resortive uterine pathologies. In this video we want to present our technique for a complete septoplaty via histerocopy.

\section{5}

TUBAL PERFORATION WITH ESSURE® AND REMOVAL OF THE MICRO-IMPLANT BY LAPAROSCOPY - CASE REPORT Operative Hysteroscopy

Video

C. Andrade*, R.M. Pinto, C. Ferreira, J. Damasceno, N. Nogueira-martins ${ }^{[1]}$ São Teotónio Hospital Viseu $\sim$ Portugal

Summary (4 lines): Video case report of 34-year-old woman with tubal perforation after hysteroscopic Essure ${ }^{\circledR}$ sterilization.

Introduction: One of the major complications of hysteroscopic sterilization is tubal perforation, which may occur in up to $2 \%$ of the procedures. 
Material and Methods: Case report of 34-year old woman, gravida 3, para 3, who underwent Essure ${ }^{\circledR}$ sterilization. The follow up was performed three months later by pelvic X-ray, a trans-vaginal scan and an hysterosalpingogram (HSG).

Results: The Essure ${ }^{\mathbb{R}}$ placement was made only on the left tube (as there was an obstruction on the right side). On the follow-up appointment, the transvaginal scan and pelvic x-ray performed raised some doubts about the position of the device. An HSG confirmed the suspected diagnosis of misplacement. Diagnostic laparoscopy was done four months later and reinstated the pelvic position of the Essure ${ }^{\circledR}$ coils. Successful removal of the Essure ${ }^{\circledR}$ from an epiploic appendix of the sigmoid colon was performed in the same surgery, as well as the laparoscopic tubal ligation.

Discussion: Essure ${ }^{\circledR}$ sterilization is a safe and minimally invasive method of non-reversible contraception. Perforation is the most frequent complication and it should be suspected whenever the procedure is difficult. Laparoscopic removal seems to be the best approach to solve this complication, as it carries the advantage to perform the tubal ligation on the same procedure.

\section{8}

\section{LAPAROSCOPIC COLPOSACROPEXY, CONVENCIONAL} VERSUS ROBOT-ASSISTED.

Robotics

\section{Video}

I. Garcia Ruiz*, E. Suarez Salvador, O. Puig Puig, J.L. Poza Barrasus, L. Mañalich Barrachina

${ }^{[1]}$ Hospital Universitari Vall D'Hebron $\sim$ Barcelona $\sim$ Spain

Summary (4 lines): In this video we show our experience in laparoscopic colposacropexy, comparing convencional laparoscopic versus robot-assisted.

Introduction: Laparoscopic colposacropexy has become a substitute for open surgery in the treatment of pelvic organ prolapse. Robotic assisted surgery is a new step in the evolution of the procedure.

Material and Methods: All patients undergo surgery under general anesthesia. Four trocars are replaced. Initially, small intestine and colon in the pelvis can be brought into the abdominal cavity. The sacral promontory is identified by following the base of the sigmoid mesentery medially and the pulsatile iliac artery laterally. The right ureter is a helpful landmark. The peritoneum over the bony promontory is dissected until the anterior longitudinal ligament. All our patients have previous hysterectomy, so vaginal cuff is dissected. Following, a polypropylene mesh is secured to the anterior and posterior vaginal walls and the cephalad limb of the mesh is sutured at the promontory. Vaginal apex pushed in as far as possible to avoid any tension on the mesh. The peritoneum is then closed over the mesh

Results: Robot assisted is specially useful to fix the mesh to the promontory.

Discussion: From our experience, the major advantages of robotassisted over conventional laparoscopy are three-dimensional imaging of the operative field; and seven degrees of freedom in the instruments.

45

\section{ROBOTIC SINGLE PORT TRANSUMBILICAL TOTAL HYSTERECTOMY}

Robotics

Video

L. Mereu, G. Carri, H. Khalifa, I. Arena, T. Zampetoglou*, L. Mencaglia

${ }^{[1]}$ Centro Oncologico Fiorentino $\sim$ Sesto Fiorentino $\sim$ Italy
Summary (4 lines): Robotic single-port total hysterectomy technique in patients with endometrial cancer

Introduction: A technique to perform single access robotic laparoscopy for endometrial cancer treatment.

Material and Methods: 4 patients with diagnosis of endometrial adenocarcinoma G1 and instrumental FIGO stage IA or IB underwent single access robotic laparoscopic extrafascial hysterectomy and bilateral salpingo-oophorectomy. Procedure was performed through a single trans-umbilical incision of $35 \mathrm{~mm}$ using Da Vinci Robotic S System and Gelport device with one $12 \mathrm{~mm}$ trocar for robotic 3D optic, two $8 \mathrm{~mm}$ and one $5 \mathrm{~mm}$ trocars. Surgeries followed the classic laparoscopic technique using 0 degree robotic camera, Endo Wrist bipolar forceps, monopolar scissor, needle holder and laparoscopic suction irrigation and grasper. Vaginal cuff was repaired with an intra-corporeal suture using a barbed wound closure system.

Results: All surgical procedures were successfully. Two G1 endometrial cancer FIGO stage IA and IB were treated. Median BMI was 25,6 (22 - 29,3). Median total operative time was $218 \min (176-250)$. Median docking time was $14 \mathrm{~min}$ (11-17). Median blood loss was $50 \mathrm{~mL}(10-90)$. No cases required multiple abdominal access or conversion to laparoscopy or laparotomy. No intra or post-operative complications occurred. All patients were discharged on day two.

Discussion: Robotic single-port transumbilical total hysterectomy is technically feasible and reproducible in patients with low-stage endometrial cancer.

128

\section{LYMPHADENECTOMY PLUS HYSTERECTOMY FOR ENDOMETRIAL CANCER BY SINGLE PORT APPROACH WITH CONVENTIONAL EQUIPMENT}

Single Access Surgery

Video

A. Zapico*, A. Couso, P. Valenzuela, P. Fuentes, D. Rodrigulez Garnica

${ }^{[1]}$ Principe de Asturias $\sim$ Alcalá de Henares $\sim$ Spain

Summary (4 lines): Single port management of endmetrial cancer staging may be perform with conventional laparoscopic equipment Introduction: Single port has been widely reported for endometrial cancer staging. Specific devices are usually used

Material and Methods: One case of endometrial cancer managed by single port approach is showed using conventional laparoscopic equipment

Results: Video surgical technique is showed

Discussion: Single port procedure may be performed with conventional laparoscopic equipment even in oncological procedures

\section{7}

\section{SINGLE PORT AND SENTYNEL NODES BIOPSY IN ENDOMETRAL CANCER WITH CONVENTIONA EQUIPMENT \\ Single Access Surgery}

Video

A. Couso, A. Zapico*, P. Valenzuela, P. Fuentes, C. Del Valle

${ }^{[1]}$ Principe de Asturias $\sim$ Alcalá de Henares $\sim$ Spain

Summary (4 lines): Single port and sentynel nodes biopsy in endometrial cancer may be performed without specific devices

Introduction: Single port procedures are wide expanding. However some cases may be done without specific devices

Material and Methods: One case of sentynel nodes biopsy by single port approach is shown. Conventional trocar and forceps are used for te procedure 
Results: Video surgical procedure is shown

Discussion: Sentynel nodes may be performed without specific devices

263

\section{ECTOPIC PREGNANCIES - COMMON AND UNCOMMON LOCALIZATIONS}

Teaching and Training

Video

R. Oehler*, M. Mueller, M. Eberhard

${ }^{[1]}$ Frauenklinik Inselspital $\sim$ Bern $\sim$ Switzerland

Summary (4 lines): A short history of the diagnosis and treatment of ectopic pregnancies, typical localizations, standard treatment and rare cases are shown.

Introduction: Ectopic pregnancies occur in 1-2 \% of all pregnancies. The rate is increasing due to reproductive medicine and reoccurences. Treatment options are medical and surgical. In emergencies involving ectopic pregnancies, laparoscopy should be the surgical treatment of choice.

Material and Methods: In the narrated video a short historic overview is given, data on localization of ectopic is presented, laparoscopic videos of standard treatments are demonstrated and ultrasound and laparoscopic videos of rare case are shown.

Results: Modern medicine and surgery dramatically reduced maternal mortality caused be ectopic pregnancies. Emergency situations in the treatment of ectopic pregnancies can be managed laparoscopically, provided there is quick access to blood products, anaestesiology and trained laparoscopic surgeons.

Discussion: Medical or surgical treatment of ectopic pregnancies depends on the time of diagnosis. Laparoscopy is feasible in even difficult cases. If salpingotomy or salpingectomy has better future fertility rates is being studied by the ESEP study group

\section{7}

\section{FEASIBILITY STUDY 5MM MOTORIZED ARTICULATED NEEDLE DRIVER}

Technical Tricks and New Instrumentations

Video

A. Agostini, M. Marcelli*

${ }^{[1]}$ Hôpital de la Conception $\sim$ Marseille $\sim$ France

Summary (4 lines): This study has proven feasibility of use of a novel concept of robotic instrument in human in multiport and single port laparoscopic surgery.

Introduction: Minimally invasive surgery creates new challenges for the surgeon. The use of long rigid instruments through rigid cannulas limits intra-abdominal degrees of freedom. The objective is to test the clinical feasibility of a novel concept of robotic instrument to overcome these difficulties in multiport and single port laparoscopic surgery.

Material and Methods: The tested instrument offers two motorized distal degrees of freedom: bending of the shaft to adjust needle positioning into the appropriate plane and unlimited axial rotation of the end effector to drive the needle through the tissue, while keeping an outer diameter of $5 \mathrm{~mm}$.

In this study, the instrument has been used in human in a multiport myomectomy and for the first time in a single port sacralcolpopexy.

Results: Use of this robotic instrument has proven feasible for suturing the uterine wall and the anterior and posterior prosthetic band, with a better compliance with suture lines.

Discussion: The combination of distal shaft bending and rotation of the end effector makes these sutures easier and improves the surgeon's posture by reducing the movement of shoulder abduction.
JAiMY has allowed increased triangulation and precise control of the needle during suturing in any plane.

381

LAPAROSCOPIC PURSE - STRING SUTURE TECHNIQUE FOR MANAGMENT OF INTERSTITIAL GESTATION

Technical Tricks and New Instrumentations

Video

I. Miranda-mendoza*, M. Puga, A. Martic, N. Guiloff, C. Sandoval

${ }^{[1]}$ Hospital Clínico Universidad de Chile $\sim$ Hospital Clínico Universidad de Chile $\sim$ Chile

Summary (4 lines): We present a short video describing the laparoscopic purse - string hemostatic suture technique, for managment of interstitial pregnancy, during a cornual resection.

Introduction: Interstitial pregnancy is a rare condition occurring once in every 2500 to 5000 live births. Rich vascular anastomosis of the uterine and the ovarian arteries in this region makes surgery difficult. We present a short video describing the laparoscopic purse - string hemostatic suture technique, for managment of interstitial pregnancy, during a cornual resection.

Material and Methods: This is the case of a 35-year-old woman with a right interstitial pregnancy. She presented at admission with a BHCG 14593. US showed a gestational sac in the right uterine corn. In order to reduce blood loss, the myometrium was infiltrated with Adenosine by means of a 19-gauge spinal trocar. A second preventative hemostatic strategy is carried out. A Vicryl 0 suture is positioned around the ectopic pregnancy (purse-string). Monopolar hook electrode was used to make the cornual resection. Closure of the defect is performed with four figure-of-eight stitches of Vicryl 0 . The specimen is introduced in a finger glove and extracted.

Results: Post op two days, serum B-hCG levels declined to 4904 and normalized over the next four weeks.

Discussion: Purse - string, hemostatic suture technique, for managment of interstitial pregnancy is a feasible laparoscopic procedure.

\section{4}

\section{LAPAROSCOPIC TREATMENT OF INTERSTITIAL ECTOPIC PREGNANCY (IEP)}

Technical Tricks and New Instrumentations

Video

A. Stavroulis*, E. Saridogan

${ }^{[1]}$ UCLH NHS Foundation Trust $\sim$ London $\sim$ United Kingdom

Summary (4 lines): This technique for surgical treatment of IEP avoids cornual resection, minimises the risk of bleeding and allowe repair of the tube.

Introduction: Tubal interstitial pregnancy represents a challenging early pregnancy complication in $2-6 \%$ of all the ectopic pregnancies. The management options are expectant management, medical (Methotrexate) treatment or surgery depending the case.

Material and Methods: This video shows an IEP treated with salpingotomy. Dilute vasopressin was injected around the pregnancy to minimise bleeding and to maintain clear view during the removal of pregnancy so that meticulous suturing of the salpingotomy incision can be achieved. After an incision over the cornual part of the tube into the ectopic pregnancy, the products of conception were removed to reduce the risk of persistent ectopic pregnancy. After haemostasis, the incision was repaired using 2/0 monofilament polyglactin suture.

Results: The patient was discharged home the following morning and had a bhCG on day 6 postoperatively which confirmed complete excision. 
Discussion: Surgical treatment of IEP can be complicated by uncontrollable bleeding or incomplete excision of the pregnancy needing further treatment. Different surgical techniques have been prescribed including cornual resection and salpingotomy. The former carries a risk of uterine rupture in a subsequent pregnancy and the latter a risk of retained pregnancy tissue.

\section{1}

\section{TECHNICAL DIFFICULTIES IN REMOVING OVARIAN CYST AFTER A HYSTERECTOMY: VIDEO}

Technical Tricks and New Instrumentations

\section{Video}

N. Purandare*, A. Mchugh, S. Patel, Y. Jhala

${ }^{[1]}$ Rotunda Hospital $\sim$ Dublin $\sim$ Ireland

Summary (4 lines): We intend to illustrate through a video, the technical difficulties involved in the removal of tubo-ovarian masses (after a hysterectomy in the past) and to highlight the importance of avoiding ureteric injury during such procedures.

Introduction: Case Report

We present the case of a 40-year-old woman with a Tubo-Ovarian mass following a Total Abdominal Hysterectomy.

Material and Methods: Case Report

Results: Successful excision by surgical precision is possible. But identifying and careful dissection of the ureter prevents injury.

Discussion: We intend to illustrate through a video, the technical difficulties involved in the removal of such cysts and to highlight the importance of avoiding ureteric injury during such procedures. The video demonstrates the technique of ureteric dissection and cannulation.

377

\section{LAPAROSCOPIC BURCH PROCEDURE: TIPS AND TECHNIQUES}

Urogynaecology

Video

A. Azadi*, L. Lipetskaia, N. Guirguis, R. Pasic

${ }^{[1]}$ University of Louisville- $O B / G Y N \sim$ Louisville $\sim$ United States

Summary (4 lines): Retropubic colposuspension procedures have recently fallen out of favor with the advent of the tension free vaginal tape, despite demonstrating comparable efficacy for the treatment of stress urinary incontinence. This video aims to identify the steps in performing a Laparoscopic Burch procedure thus making a technically complex procedure simple and feasible

Introduction: With the rising popularity of midurethral slings for stress urinary incontinence, retropubic coloposuspension procedures are mostly being performed as part of concomitant laparoscopic procedures. The technical difficulty of laparoscopic suturing and inability to perform fine adjustments to the tension suture is a deterrent to many. In this case, our patient underwent a Laparoscopic sacro-colpopexy for stage $3 \mathrm{C}$ prolapse, and as a result a retropubic colposuspension was felt to be warranted

Material and Methods: Surgical film footage was edited to produce a video demonstrating the steps required to perform a laparoscopic Burch procedure. Visual aids are used to highlight important anatomical landmarks

Results: Two month follow up reveals no evidence of de novo detrusor over activity or persistent stress urinary incontinence

Discussion: By standardizing surgical techniques the laparoscopic Burch procedure can become a feasible option for the surgical management of stress urinary incontinence.
35

LAPAROSCOPIC RICHTER PROCEDURE AND PARAVAGINAL REPAIR WITH SUTURES VERSUS SACROCOLPOPEXY USING MESHES : A REALITY ?

Urogynaecology

Video

J. Dubuisson*, A. Samim, J. Dubuisson, J. Bouquet De Jolinière, A. Feki ${ }^{[1]}$ Department of Obstetrics and gynecology, Cantonal hospital, Fribourg, Switzerland $\sim$ Fribourg $\sim$ Switzerland

Summary (4 lines): A laparoscopic alternative to sacrocolpopexy is the laparoscopic Richter procedure, often associated with a paravaginal repair. Introduction: The laparoscopic sacrocolpopexy is the gold standard. It offers good long term results. This excellent technique has a low risk of complications but including mesh erosion, vascular injury during the promontory dissection. For laparoscopic Richter, two routes give access to the sacrospinous ligament. The anterior route is performed by opening the prevesical space, the posterior by a lateral rectal dissection.

Material and Methods: We prefer the anterior route if the vaginal vault (and rectal) prolapse is associated with a cystocele. Then, we associate a spinofixation and a paravaginal repair. We describe the technique in this video. After entrance into the prevesical space, the Cooper's ligament, the obturator nerve, the arcus tendineus fascia pelvi are exposed bilaterally. On one side, blunt dissection is continued until the ischial spine and the sacrospinous ligament. Spinofixation and paravaginal repair are then performed using 0 non-absorbable Ethibond Polyester sutures.

Results: The operation is an efficacious and safe procedure in selected cases of uterine or vaginal vault prolapse. The associated cystocele is treated with a paravaginal repair.

Discussion: The operation presented in this video is a possible alternative to the laparoscopic sacrocolpopexy, without using a mesh.

9

MANAGMENT OF URETRIC COMPLICATIONS DURING AND FOLLOWING LAPAROSCOPIC SURGERIES

Urogynaecology

Video

S. Puntambekar*, S. Puntambekar, A. Galagali, R. Desai

${ }^{[1]}$ Galaxy CARE Laparoscopic Institute $\sim$ Pune $\sim$ India

Summary (4 lines): Intra operative recognition of uretric injuries can help to reduce post operative morbidity. Once the injury is recognized it can be safely and effectively managed intra operatively laparoscopically. Delayed complications of uretric injuries can also be safely managed laparoscopically.

Introduction: As the limits of laparoscopy have widened to include advanced pelvic surgeries, injuries to the genitourinary tract are increasing. Intra operative recognization can solve half the problem. Bladder pathology, adhesions, previous surgery, inflammation, or endometriosis increase the risk of uretric injuries. We present to you tips and tricks of managing such complications.

Material and Methods: Cases, where there are small, focal injuries to the ureter, can be treated using a double-J-shaped catheter passed into the ureter or are sutured with polygalactin. More extensive injuries may require an end-to-end anastomosis or ureteral reimplantation.

Results: Intraoperative recognization of uretric injuries during laparoscopy can be safely and effectively managed. Delayed complications of uretric injuries can also be safely managed laparoscopically.

Discussion: Anticipation of injuries in difficult cases and efforts to recognize them help in decreasing post operative morbidity. These injuries can be managed laparoscopically without conversion to laparotomy. 